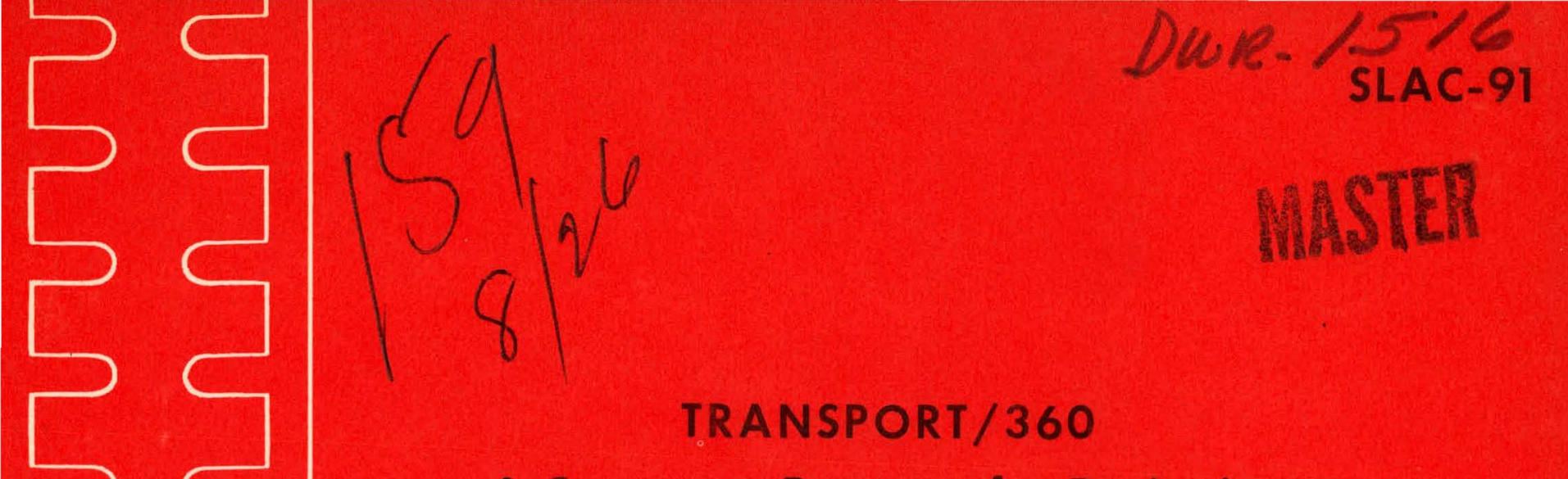

A Computer Program for Designing

Charged Particle Beam Transport Systems

\author{
Karl L. Brown \\ Sam K. Howry
}

SLAC Report No. 91

July 1970

AEC Contract AT(04-3)-515

STANFORD LINEAR ACCELERATOR CENTER Stanford University - Stanford, California 


\section{DISCLAIMER}

This report was prepared as an account of work sponsored by an agency of the United States Government. Neither the United States Government nor any agency Thereof, nor any of their employees, makes any warranty, express or implied, or assumes any legal liability or responsibility for the accuracy, completeness, or usefulness of any information, apparatus, product, or process disclosed, or represents that its use would not infringe privately owned rights. Reference herein to any specific commercial product, process, or service by trade name, trademark, manufacturer, or otherwise does not necessarily constitute or imply its endorsement, recommendation, or favoring by the United States Government or any agency thereof. The views and opinions of authors expressed herein do not necessarily state or reflect those of the United States Government or any agency thereof. 


\section{DISCLAIMER}

Portions of this document may be illegible in electronic image products. Images are produced from the best available original document. 
This report was prepared as an account of work

sponsored by the United States Government. Neither

the United States nor the United States Atomic Energy

STAC-91

Commission, nor any of their employees, nor any of

their contractors, subcontractors, or their employees,

makes any warranty, express or implied, or assumes any

legal liability or responsibility for the accuracy, com-

pleteness or usefulness of any information, apparatus,

product or process disclosed, or represents that its use

would not infringe privately owned rights.

TRANSPORT $/ 360$

A COMPUTER PROGRAM FOR DESIGNING CHARGED PARTICLE

BEAM TRANSPORT SYSTEMS

\author{
KARL L. BROWN, SAM K. HOWRY \\ STANFORD LINEAR ACCELERATOR CENTER \\ STANFORD UNIVERSITY \\ Stanford, California 94305
}

PREPARED FOR THE U.S. ATOMIC ENERGY

COMMISSION UNDER CONTRACT NO. AT(04-3)-515

July 1970

Reproduced in the USA. Available from the Clearinghouse for Federal Scientific and Technical Information, Springfield, Virginia 22151. Price: Full size copy $\$ 3.00 ;$ microfiche copy $\$ .65$. 
FOREWORD

This report (SLAC-91) represents the completion of an extensive documentation of the first-and second-order theory of Beam Transport Optics and of the, associated computer program (TRANSPORT) that has evolved at SLAC over the past several years. This report contains the following:

1) A Users Manual describing how to prepare data sets for a TRANSPORT computation.

2) Specific Examples of simple and complex TRANSPORT computations to illustrate first-order fitting techniques.

3) A set of Flow Charts for the benefit of the beginning user who needs to know what the program does.

4) An Appendix containing the underlying optics theory behind the program that is not adequately covered by other existing references. This also serves to introduce the user to TRANSPORT notation.

In addition to this report, other pertinent documents that may be useful to TRANSPORT users are the following:

5) SLAC-75: A First- and Second-Order Matrix Theory for the Design of Beam Transport Systems and Charged Particle Spectrometers.

6) A Matrix Element Test Deck (obtained from the SLAC program librarian). This has been prepared for the new user and is designed to check all of the first-and second-order matrix elements contained in the TRANSPORT program.

7) A TRANSPORT listing (also obtained from the SLAC program librarian).

For NEW users of TRANSPORT who desire a copy of the program and the appropriate backup documentation, please send your requesto to:

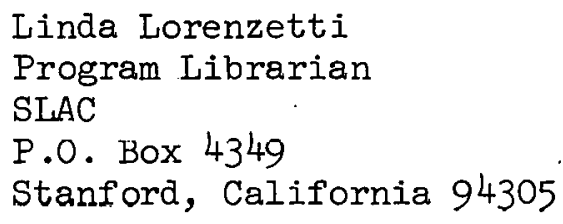

If all you require is the TRANSPORT manual (SLAC Report No. 9l) send your requests to:

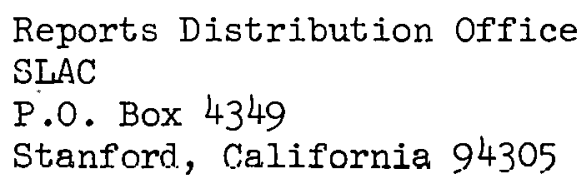


INTRODUCTION

Mathematical Formulation of TRANSPORT $\quad 0-3$

Input Format for TRANSPORT/360.

An Example of a TRANSPORT/360 Input Deck

$0-12$

Table of Data Input Parameters for TRANSPORT/360 $\quad 0-14$

Title Card

Indicator Card

Comment Cards

The Phase Ellipse Beam Matrix

Example of a Two Dimensional Phase Ellipse $\quad 1-6$

rms Addition to the Beam ' $1-7$

Type Code 2.0 Fringing-Fields and Pole Face Rotations 2-1

Type Code 3.0 A Drift Distance 3-1

Type Code 4.0 Wedge Bending Magnets . 4-1

Type Code 5.0 Quadrupoles 5-1

Type Code 6.0.1. Transform 1 Update . . 6-1

Type Code 6.0.2. Auxiliary Transformation Matrix . ' 6-2

Type Code $7.0 \quad$ Shift in the Beam Centroid . $7-1$

Type Code 8.0 Magnet Alignment Tolerances 8-1

Type Code 9.0 Repetition. 9-1

Type Code 10. First-Order Vary Codes and Fitting Constraints 10-1

Coupled Vary Codes 10-3

RI Matrix Fitting Constraints $10-4$

R2 Matrix Fitting Constraints $10-5$

BEAM Matrix Fitting Constraints $10-6$

System Length Constraint $\quad 10-8$

AGS Machine Constraint . 10-9

First Moment Constraint. 10-11

Internal Constraints 10-12

Type Code 11.0 Acceleration. 11-1

Type Code 12.0 Beam (Rotated Ellipse) 12-1 
Type Code $13.0 \quad$ Output Print Controls

Type Code 14.0 Arbitrary Transformation Matrix Input 14-1

Type Code 15.0 Input-Output Units 1 . . 15-1

Type Code 16.0 Special Input Parameters 16-1

Tilt-To-Focal Flane $\quad 16-6$

Type Code 17.0 Second-Order Calculation $\quad 17-1$

Type Code 18.0 Sextupole 18-1

Type Code 19.0' Solenoid 19-1

Type Code 20.0 Beam Rotation 20-1

Type Code 21.0 Stray Magnetic Field , 21-1

Sentinel Card 21-2

References $21-3$

Examples . E-1A:

Flow Charts

l'ransport $\quad$ F-1

Subroutine BKKIN $\quad$ F-2

Subroutine BKKPAK $\quad$ F-9

Subroutine BKKFX $\quad$ F-10

Subroutine BKKFL $\quad$ F-12

Subroutine SCANT $\quad$ F-15

Subroutine FITTIN $\quad$ F-16

Outfit Subprogram $\quad F-22$

Element Subroutine F-26

Solve Subprogram : : : F-38

Form Subroutine $\quad$ F-40

Alter Subprogram $\quad$ F-4I

Partials Subroutine F-44

Subroutine CFWK $\quad F-51$

Summary $\quad F-52$

Appendix

Introduction $\quad A=0$

1 - Beam Transport Optics (a set of lectures given to the SLAC technical staff')

Part I

$A-1$

Geometric Light Optics vs. Magnetic Optics A-I

Introduction of Momentum Dispersion into the

Matrix Formalism A-8

Second-Order Matrix Formalism A-12

Transformations Involving Many Trajectories A-13 
First-Order $3 \times 3$ Matrix Transformation

Example of a Beam Switchyard

TRANSPORT Notation

Second-Order Matrix

2 - The First-Order R Matrix and Sigma Matrix (Beam Ellipsoid) Formulation of TRANSPORT

First-Order Dispersion

First-Order Path Length

Achromaticity

Is ochronicity

$A-30$

First-Order Imaging Conditions

$A-30$

Focal Lengths

$A-31$

Zero Dispersion

$A-32$

Magnification

$A-32$

First-Order Momentum Resolution

$A-32$

First-Order Phase Ellipse Formalism of TRANSPORT

Description of the Sigma BEAM Matrix

$A-37$

The Phase Ellipse Matrix used by TRANSPORT

Physical Interpretation of Various Projections of the 2-dimensional BEAM Ellipse

$A-41$

The Upright Ellipse

A -44

Relationship between a Waist and Parallel-to-Point Image

Relationship between a Waist and Point-to-Point Image

A- 46

Relationship between a Waist and the Smallest Spot Size Achievable at a Fixed Target Position

$A-48$

Imaging from an Erect Ellipse to an Erect Ellipse

A -51

Relationship between a First-Order Point-to-Point Image and the Minimum Spot Size Achievable at a Fixed Target Position

$A-54$

Orientation of the Major Axes of a Phase Space Ellipse

A -55

3 - Second-Order Calculations and Corrections

$A-58$

Second-Order Phase Ellipsoid Formalism

A -59

A Systematic Procedure t'or Designing High Resolving Power Beam Transport Systems or Charged Particle Spectrometers

I Introduction

A -60

II Theory

$A-62$

A -63

Multipole Strengths for Pure Multipole Field

A -65

Multipole Strengths for a Non-Uniform Field Expancion

Multipole Strengths for a Contoured Entrance or Exit Boundary of a Magnet 
The Description of the Trajectories as a Taylor's Expansion

III Solution of the Equation of Motion

IV Interpretation and use of the Coupling Coefficient

V A Systematic Procedure for Designing High Resolution Systems

First-Order Considerations

First-Order Resolving Power

Dispersion

The Selection of the Optical Mode

Aberrations and their Correction.

References

$\Lambda-76$

4 - Theory of Magnet Alignment Tolerances

A -77

5 - First-Order Parameter Optimization and Covariance

$\Lambda-80$ 


\section{INTRODUCTION}

TRANSPORT/360 is a computer program for the design of static-magnetic beam transport systems.

This report ( $S L A C-91$ ) supercedes the original TRANSPORT instruction manual issued in October, 1963 by C. H. Moore, S. K. Howry, and H. S. Butler. Since the issuance of the original manual, several important modifications and additions have been made to the TRANSPORT program, among them are:

a) The second-order portion of the program has been extended to include the fringing-fields of bending magnets, second-order curvatures on the entrance and exit faces of bending magnets, and the second-order aberrations of solenoids. All of the second-order portion of the program has been thoroughly de-hugged using appropriate magnetic optical theorems, direct comparisons with hand calculations, and comparisons with results from ray-tracing programs.

b) Fringing-field corrections to the first-order transverse-optics of bending magnets have been added to the program via the Type Code 16 . elements.

c) The program has been translated from the original Balgol version to Fortran. (We hereby wish to acknowledge the important contribution made hy stan Kowalski of M.I.T. in effecting this translation).

d) A complete free-field format for data input has been added to the Fortran version therehy considerably simplifying the use of the program. 
e) A relativistically-correct first-order matrix for a traveling-wave linear accelerator section has been added to the program via Type Code 11.0 .

f) Provision has been made to introduce second-order corrections (curvatures) to the input and output faces of bending magnets via Type Code 16. elements to fäcilitate correcting for various second-order aberrations.

g) Provision has also been made, via the Type Code 16. elements, to enable the user to rotate to the focal plane of a system and print-out the second-order aberrations along this plane.

For the benefit of the new users of TRANSPORT, we shall now review briefly the mathematical basis of the program. From the outset, it is important to emphasize that TRANSPORT is not a ray tracing program in the usual sense, but rather is based upon a first-and second-order matrix theory of beam transport optics $(1,2)$. 


\section{Wathematical Formulation of TRANSPORT $(*)$}

The following of a charged particle through a system of magnetic lenses may be reduced to a process of matrix riultiplication. At any specified position in the system an arbitrary charged particle is represented by a vector (single column matrix), $x$, whose components are the positions, angles, and momentum of the particle with respect to a specified reference trajectory.

Definitions:

$$
\text { i.e. } x=\left[\begin{array}{l}
x \\
\theta \\
y \\
\varphi \\
\ell \\
\delta
\end{array}\right]
$$

$x=$ the radial displacement of the arbitrary ray with respect to the assuined central trajectory.

$\theta=$ the angle this ray makes in the radial plane with respect to the assumed central trajectory.

$y=$ the transverse displacement of the ray with rospect to the assumed central trajectory.

$\varphi=$ the transverse angle of the ray with respect to the assuried central trajectory.

(*) For a more complete descrintion of the mathematical hasis of TRANSPORT, refer to SLAC Report 75, the Appendix of this report and to other References 1 isted at the end of this report. 
$\ell=$ the path length difference between the arbitrary ray anit the central trajectory

$\delta=\Delta \mathrm{P} / \mathrm{P}$ is the fractional monentunil deviation of the ray from the assumed central trajectory.

The magnetic lens is represented by a square matrix, R, which describes the action of the magnet on the particle coordinates. Thus the passage of a charged particle through the systen may be represented by the equation :

$$
X[1]=R \times[0]
$$

where $x[0]$ is the initial coordinate vector and $x[1]$ is the final coordinate vector of the particle under consideration; $R$ is the transformation matrix for all such particles traversing the system (one particle differing. from another only by its initial coordinate vector $x:[0])$.

The traversing of several magnets and interspersing drift spaces is described by the same basic equation but with $R$ now being the product matrix $R=R(n) \ldots R(3) R(2) R(1)$ of the individual matrices of the system elements. The following of a charged particle via TRANSPORT through a system of magnets is thus analogous to tracing rays through a systern of optical lenses except that TRANSPORT is a matrix calculation which truncates the problem to either first or second-order in a Taylor's expansion about a central trajectory. For studying bean optics to greater precision than a second-order TRANSPORT calculation permits, 
-ay-tracing programs which directly integrate the basic differential equation of motion are recommended(6).

In accelerator and beam transport systems, the behavior of an individual particle is often of less concern than is the behavior of a hundle of particles (the BEAM) of which an individual particle is a member. An extension of the matrix algebra of equation(1) provides a convenient means for defining and manipulating this BEAM. TRANSPORT assumes that the bundle of rays constituting a BEAM may correctly be represented in coordinate phase-space by an ellipsoid whose coordinates are the position, angle, and momentum coordinates of the arbitrary rays in the heam about an assumed central trajectory. Particles in a BEAM are assumed to lie within the boundaries of the ellipsoid with each point within the ellipsoid representing a possible ray. The sum total of all phase points, the phase space volume, is commonly referred to as the "phase space" occupied by the BFAM. The validity and interpretation of this phase ellipse formalism must be ascertained for each system being designed. However, in general, for charged particle beams in, or emanating, from accelerators, the first-order phase ellipse fornalism of TRANSPORT is a reasonable representation of physical reality; but for other applications, such as charged particle spectrometers, caution is in order in its use and interpretation. 
The equation of an n-dimensional ellipsoid may be written in matrix form as follows:

$$
x[0]^{\top} \sigma[0]^{-1} \times[0]=1
$$

where $x[0]^{\top}$ is the transpose of the coordinate vector $x[0]$, and $\sigma[0]$ : is a real, positive definite, symmetric matrix.

$n$ The volume of the n-dimensional ellipsoid defined by sigma is $\frac{\pi \frac{n}{2}}{\Gamma\left(\frac{n}{2}+1\right.}(\operatorname{det} \sigma)^{1 / 2}$, the area of the projection in one plane is $A \stackrel{\left(\frac{n}{C}+1\right.}{=}=\pi(\operatorname{det} \sigma)^{1 / 2}$. This is the "phase space" occupied by the beam. As a particle passes through a system of magnets, it undergoes the matrix transformation of equation(1). Combining this transformation with the equation of the initial ellipsoit, and using the identity $R R^{-1}=I$ (the unity matrix), it follows that:

$$
x[0]^{\top}\left(R^{\top} R^{T^{-1}}\right) \sigma[0]^{-1}\left(R^{-1} R\right) \times[0]=1
$$

from which:

$$
(R \times|0|)^{T}\left(R \sigma|0| R^{T}\right)^{-1}(R \times|0|)=1
$$

The equation of the new ellipsoid after the transformation becomes:

$$
x[1]^{\top} \sigma[1]^{-1} \times[1]=1
$$


where:

$$
\sigma[1]=R \sigma[0] R^{\top}
$$

It can readily be shown that the square roots of the diagonal terms of the signa matrix are a measure of the "beam size" and the off-diagonal terms are a measure of the orientation of the ellipsoid in $n$-dimensional space (for TRANSPORT $n=6$ ).* Thus, we may specify the beam at any point in the system via equation(5), given the initial "phase space" represented by the matrix elements of $\sigma[0]$.

Several types of physical elements have heen incorporated in the program to facilitate the design of very general heam transport systeins, included are: an arbitrary drift distance, bending magnets, quadrupoles, sextupoles, solenoids, and an accelerator section. (to first-order only). Provision is made in the program to vary some of the physical parameters of the elements comprising the system and to impose first-order fitting conditions upon either the TRANSFORH ( $R$ ) matrix representing the transformation of an arbitrary ray through the system and/or to impose first-order fitting conditions upon the phase ellipse (signa) matrix representing the transfornation of a bundle of rays through the system. Thus, in principle, the program is capable of searching for and finding the first-order solution to any physically-reallzaljle problem. In practice, life is nnt quite so

*. See the appendix of this report for a derivation of these statements. 
simple, the user will find that an adequate knowledge of geometric magnetic optics principles is a necessary prerequisite to the successful use of TRANSPORT. In other words, the program is superb at solving the mathematics of the problem but not the physics. The user must provide a reasonable physical input if he expects complete satisfaction from the program. For this reason a brief review of magnetic optics along with a list of pertinent reprints and references and some representative TRANSPORT calculations are given in the appendix of this manual to provide assistance to the inexperienced as well as the experienced user.

A thorough understanding of the first-order matrix algebra of beam transport optics and of the physical interpretation of the various matrix elements is an essential prerequisite to the successful use of this program. 


\section{INPUT FORMAT FOR TRANSPORT/360}

The input format for TRANSPORT/360 is quite similar to that of the previous versions of the program. The input DATA SET consists of three kinds of cards: the TITLE card, the INDICATOR card, and the DATA cards.

The TITLE card has single quotes punched in any two columns on the card. Any information punched between the quotes will be used as a heading in the output of the TRANSPORT run.

The second card of the input is the INDICATOR card. If the data which follows describes a new prohlem, a zero(0) is punched in any column on the card. If the data which follows describes changes to be made in the previous problem, a one(1) is punched in any column on the card.

The rest of the cards in a deck contain the DATA describing a beam and the physical elements of the magnetic system. Each data set must be terminated by the word SENTINEL; the word SENTINEL need not be punched on a separate card. The input format of these cards is "free-field" which is described below. Each element uses a type code number which identifies the element; a field which indicates which physical parameters of the element are to be varied if there is to be any fitting; the parameters necessary to describe the physical element, such as length, magnetic field strength, etc. and, optionally, a one-to-four character label (enclosed between single quotes). The type code numbers and their interpretations are summarized in TABLE. I. If the type code number is negative, the element will be ignored in the calculation which follows, but may be user in a succeeding calculation. 
The vary field is punched immediately to the right of the decimal point of the type code number. The meaning is exactly the same as it was in the 7090 version of the TRANSPORT program. See section under Type Code 10. for an explanation of the use of vary codes.

The data parameters describing the element are punched in the order given in TABLE 1; in the same order as has been required for previous versions of TRANSPORT. Each data entry must contain a decimal point unless it is equal to zero.

The label, if present, is enclosed by single quotes. It may contain up to four characters. It will be printed in the output during the calculation and can be useful in problems with many elements and/or when :sequential fitting is used. Labels are essential if the data associated with the element is to be changed in succeeding problems.

Provision has heen made in the program to allow the user to iintroduce comments before any type code entry in the data deck. This is accomplished by enclosing the comments made on each card with single parentheses.

The data associated with each type-code entry must be followed by a semi-colon. If the program encounters a semi-colon before the expected number of parameters has been readin, the rest of the parameters are set to zero if the indicator card was a zero( 0$)$. If the indicator card was a one(1) then the numbers on the card are substituted for the numbers from the previous solution, but the remaining numbers are unchanged.

The "free-field" input format of the data cards make it considerably easier to prepare input than the standard fixed-field formats of 
FORTRAN. Numbers may be punched anywhere on the card. They must be separated by one or more blanks. A single number must be all on one card; it may not continue from one card to the next. The progran is limited to 500 data number entries and 200 type code entries.

A floating-point number e.g. (2.47) may he represented in any of the following ways:

$$
\begin{aligned}
& 2.47 \\
& .00247+3 \\
& .0247 E+02 \\
& 247 . E-2 \\
& 247000 .-5
\end{aligned}
$$

The sample problem input which is included below causes TRANSPORT to: do a first order calculation with fitting and then to do a second order calculation with the data that is the result of the fitting. The type ten element which specifies the fitting condition is labeled flTl. It is active for the first order calculation but is turned off for the second order calculation. The vary codes for elements DP. 1 are set to zero for the second-order problem. The second order element, SEC.1, is ineffective during the fitting, but causes the progran to compute the second-order matrices in the second calculation. The word SENTINEL need not he punched on 3 separate card. 


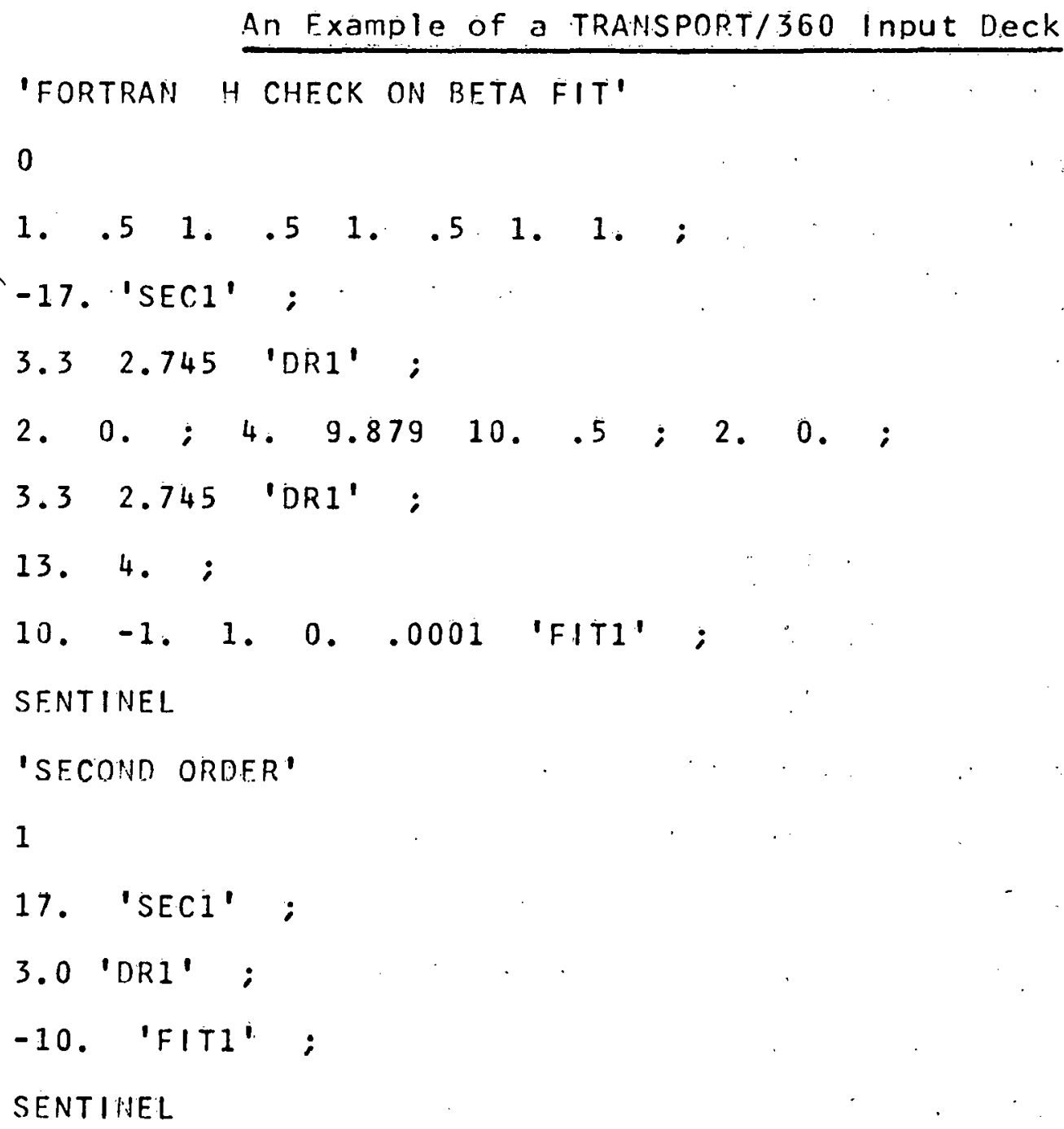


The figure below shows the 360 . TRANSPORT deck setup. If the data are punched on an 026 keypunch instea:t of an 029 keypunch, one should precede the title card with a card on which the characters KEYgo are punched in any column. No inbedded blanks are allowed in this control statement.

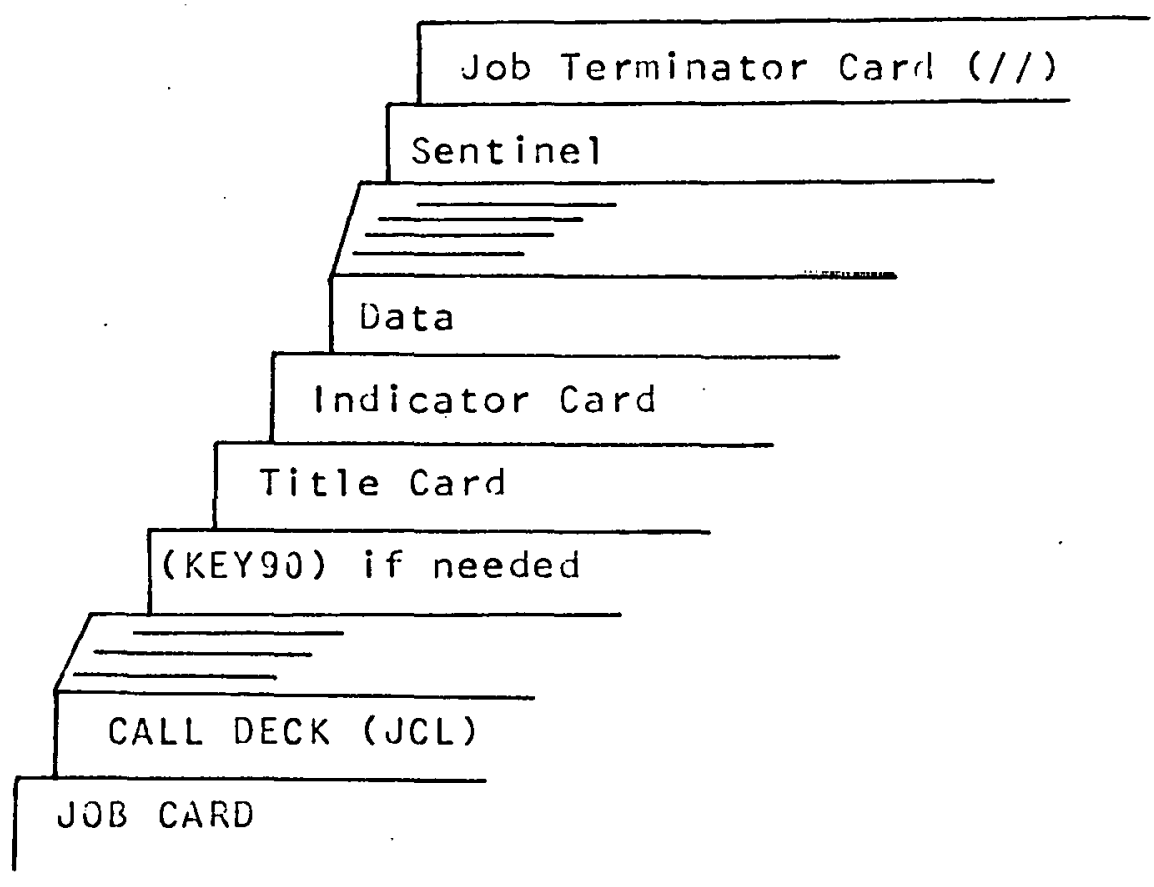

ilote that as many problems as one wishes may be stacker in one job. 
TABLE I

\begin{tabular}{|c|c|c|c|c|c|c|c|c|c|}
\hline PHYSICAL ELEMENT & $\begin{array}{r}\text { TYPE } \\
\text { CODE } \\
\end{array}$ & $\begin{array}{l}\text { 2nd } \\
\text { EMTRY }\end{array}$ & $\begin{array}{c}\text { 3rd } \\
\text { EnITRY }\end{array}$ & $\begin{array}{c}4 \text { th } \\
\text { ENTRY } \\
\end{array}$ & $\begin{array}{l}5 \text { th } \\
\text { ENTRY }\end{array}$ & $\begin{array}{c}\text { 6th } \\
\text { EVIRY }\end{array}$ & $\begin{array}{c}7 \text { th } \\
\text { ENIRY }\end{array}$ & $\begin{array}{c}\text { Bth } \\
\text { ENTRY }\end{array}$ & $\begin{array}{c}9 \mathrm{th} \\
\text { ENTRY } \\
\end{array}$ \\
\hline BEAM & 1.vrrurvo & $x(\mathrm{~cm})$ & $\theta(m r)$ & $y(\mathrm{~cm})$ & $\varphi(m r)$ & $\ell(\mathrm{cm})$ & $\delta$ (percent) & Po & \\
\hline $\begin{array}{l}\text { R.M.S. ADDITION TO } \\
\text { BEAM ENVELOPE }\end{array}$ & 1. & $\left.\Delta x^{\prime} \mathrm{cm}\right)$ & $\Delta \theta(\mathrm{mr})$ & $\Delta y(\mathrm{~cm})$ & $\Delta \varphi(\Delta r)$ & $\Delta I(\mathrm{~cm})$ & $\Delta \delta$ (percent) & $\begin{array}{c}\Delta \mathrm{P}_{\mathrm{o}} \\
(\mathrm{GeV} / \mathrm{s})\end{array}$ & 0 \\
\hline POLE FACE ROTATION & $2 . v$ & $\begin{array}{c}\text { ARTLE OF } \\
\text { ROTATI N Degrees }\end{array}$ & 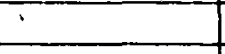 & & & & & & \\
\hline DRIFT & $3 . v$ & LDMGTH(meters). & & & & & & & \\
\hline BENDTNS MAGNET & $4.0 \mathrm{vv}$ & LENGTH(meters) & FIELD $(\mathrm{k} 8)$ & $\begin{array}{l}\text { FIDLD ONADTint } \\
\text { (n-value) }\end{array}$ & & & & & \\
\hline QUADRUPOLE & 5.v00 & LEWGTH(meters) & FIEID (48) & HALF -APERTURE $(\mathrm{cW})$ & & & & & \\
\hline TRANSFORM 1 UPDATE & 6. & 0. & 1. & & & & & & \\
\hline TRANSFOPM 2 UPDATE & 6. & 0. & 2. & . & $\dot{-}$ & & & & \\
\hline BEAM CENTROID SHIFT & 7. & $\operatorname{SHIFT}(\mathrm{x})(\mathrm{cm})$ & $\operatorname{SHIFT}(\theta)(\pi r)$ & $\operatorname{SHIFT}(y)(\mathrm{cm})$ & $-\operatorname{SHIFT}(\varphi)(\underline{\mathrm{ar}})$ & $\operatorname{SHIFT}(\ell)(\mathrm{cm})$ & SHIFT( $(\delta)$ (goroont) & & \\
\hline ALIGNMENT TOLERANCE & 8.vrvurvo & DISPLACEMENT $(x) \mathrm{cm}$ & ROTATION $(\theta) \mathrm{mr}$ & DISPLACEMENT $(y) \mathrm{cm}$ & ROTATION $(\varphi)_{\mathrm{mr}}$ & DISFLACEMETTI $(\ell) \mathrm{cm}$ & $\operatorname{ROTATION}(\alpha)_{\mathrm{mr}}$ & $\begin{array}{c}\text { CODE } \\
\text { NUMBER }\end{array}$ & \\
\hline REPEAT CONRROL & 9. & $\begin{array}{c}\text { WUNBER OF } \\
\text { REPEATS } \\
\end{array}$ & & & & & & & \\
\hline FITTING CONSTRAINTS & 10. & $\pm I$ & $\mathrm{~J}$ & $\begin{array}{l}\text { DES IRED VALUE OF } \\
(\mathrm{I}, \mathrm{J}) \text { MATTR IX } \\
\text { ELEMEITS }\end{array}$ & $\begin{array}{l}\text { ACCURACY OF } \\
\text { FIT }\end{array}$ & & & & \\
\hline \multicolumn{10}{|c|}{$\begin{aligned} \text { Note: } & + \text { I is used for fitting a bean (o) matrix element. - I Is used for fitting an RI matrix element. } \\
& -(I+20) \text { is used for fltting an R2 matrix element. }\end{aligned}$} \\
\hline ACCELERATOR & 11. & LENGTH( meters) & $\begin{array}{c}\text { E(energy gain) } \\
(\mathrm{GeV})\end{array}$ & $\varphi\left(\begin{array}{l}\text { phase 1ag }) \\
\text { (Degrees) }\end{array}\right.$ & (WAVELENGTH) $(\mathrm{cm})$ & $\dot{-}$ & & & \\
\hline $\begin{array}{l}\text { BEAM } \\
\text { (Rotated Ellipse) }\end{array}$ & 12. & THE FIFTEEN CORRF & ELLATIONS AMONG THE SI & $X$ ELEMENTS (This entr & $\chi$ must be preceded & Code $1 . e$ & ntry). & & \\
\hline $\begin{array}{l}\text { IMPUT/OUTPUT } \\
\text { OPTIONS }\end{array}$ & 13. & $\begin{array}{l}\text { CONTROL } \\
\text { CODE NUMBER }\end{array}$ & & & & & & & \\
\hline ARB FTRARY $R$ MATR $I X$ & $14 . \mathrm{rrrvrvo}$ & $R(J, 1)$ & $\mathrm{R}(\mathrm{J}, 2)$ & $\mathrm{R}(\mathrm{J}, 3)$ & $\mathrm{R}(\mathrm{J}, 4)$ & $\mathrm{R}(\mathrm{J}, 5)$ & $R(J, 6)$ & $J$ & \\
\hline $\begin{array}{l}\text { UNTTS CONRROL } \\
\text { (Trunsport } \\
\text { Dimensions) }\end{array}$ & 15. & $\operatorname{CODE}$ & LABEE & $\begin{array}{l}\text { SCALE FACTOR } \\
\text { (1f required) }\end{array}$ & & & & & \\
\hline $\begin{array}{l}\text { QUADRATIC TERM } \\
\text { OF EENDING FIELW }\end{array}$ & 16. & 1. & $\begin{array}{c}\epsilon(1)=\beta\left(\frac{x}{\rho}\right)^{2} \\
(\text { dimensionless) } \\
x=1\end{array}$ & & & & & & \\
\hline $\begin{array}{l}\text { MASS OF PARTTCLES } \\
\text { II BEAM }\end{array}$ & 16. & 3. & $\mathrm{M} / \mathrm{m}$ (d1mensionless) & & & $\cdot$ & & & \\
\hline $\begin{array}{l}\text { HALF -APERTURE OF } \\
\text { BEID DNO MAC. In } x \\
\text { rLALE }\end{array}$ & 16. & 4. & $w / 2(\mathrm{~cm})$ & & & & & & \\
\hline $\begin{array}{l}\text { HALF-APERTURE OF } \\
\text { BERD NNC MAG. in y } \\
\text { PLANE (gap) }\end{array}$ & 16. & 5. & $\mathrm{~g} / 2(\mathrm{~cm})$ & & & & & & \\
\hline LENGTH OF SYSTEM & 16. & 6. & L. (meters) & & & & & & \\
\hline $\begin{array}{l}\text { FRINGE FIELD CORR- } \\
\text { ECTION COEFFICIENTI }\end{array}$ & 16. & 7. & KL (aimensionless) & & & & & & \\
\hline $\begin{array}{l}\text { FR INGE FIELD CORR- } \\
\text { ECTION COEFFIC IENT }\end{array}$ & 16. & 8. & K2 (dimcnoionlcos) & & & & & & \\
\hline 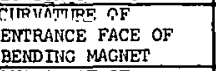 & 16. & 12. & (1/R1) (1/meters) & & & & & & \\
\hline $\begin{array}{l}\text { CURY } T \text { TURE OF } \\
\text { EXTT FACE OF } \\
\text { BEND ING MAGNET }\end{array}$ & 16. & 13. & $(1 / R 2)(1 /$ meters $)$ & & & & & & \\
\hline $\begin{array}{l}\text { FOCAL PLANE } \\
\text { ROTATION }\end{array}$ & 16. & 15. & $\begin{array}{l}\text { angle of focal plane } \\
\text { Scc acction } 16 \text { for a }\end{array}$ & $\begin{array}{l}\text { rotation (Degrees) } \\
\text { etuils. }\end{array}$ & & & & & \\
\hline CALCULATIONS & 17. & & & & & & & & \\
\hline SEXIUPOLE & 18. & LENGUTH(meters) & FIELD (kg) & HALF-APERTURE (cm) & & & & & \\
\hline 3OLENOID & $19 . v v$ & LENGTH(meters) & FIEZD $(\mathrm{kg})$ & & & & . & & \\
\hline JEAM ROTATION & $20 . v$ & $\begin{array}{c}\text { ANGLE OF } \\
\text { ROTATIOH( Degrees) }\end{array}$ & & & & & & & \\
\hline 3 TRAY FIEID & 21. & See later section & of report. & -1 & & & - & & \\
\hline $\begin{array}{l}\text { Note: The v's follo } \\
\text { The units are }\end{array}$ & $\begin{array}{l}\text { ing the } t \\
\text { tandara }\end{array}$ & $\begin{array}{l}\text { pe codes ind cate } \\
\text { LANSPORT units (as }\end{array}$ & $\begin{array}{l}\text { he parameters which } \\
\text { shown) unless chang }\end{array}$ & $\begin{array}{l}\text { ay be varied. See } \\
\text { via type code } 15.0\end{array}$ & $\begin{array}{l}\text { ction under type } \\
\text { ntries. }\end{array}$ & ode $10.0 \mathrm{f}$ & led explan & $y$ & es. \\
\hline
\end{tabular}


TRANSPORT is stored on disc in the program libraries of both the SLAC $360 / 91$ and the Stanford Campus 360/67 computers. The call decks for these cases are listed below:

For batch or CRBE runs on the 360/91, use the following JCL if you wish the output to be printed on the line printers:

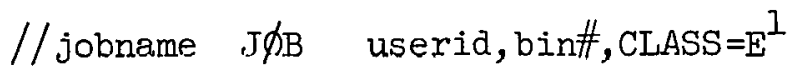

$/ / J \emptyset B L I B$ DD DSNAME $=S Y S I$.USERLIB, DISP $=S H R$

$/ /$ STEPI EXEC PGM=TRANS

$/ /$ FTO4FOO1 DD SYS $\phi \mathrm{UT}=\mathrm{B}$

$/ /$ FTO6F001 DD SYS $\varnothing \mathrm{UT}=\mathrm{A}$

$/ /$ FTOSFOOL DD *

If you wish the output to be returned to the terminal for a CRBE run change SYS $\phi U T=A$ to SYS $\phi U T=R$ on line 5 of JCL.

If you desire to use the Campus $360 / 67$ for either a Batch or Wylbur run, the JCL is as follows:

// jobname 'J $\phi \mathrm{B}$ (acct\#, bin\#,1.0,05,001, , $1, \mathrm{X}$ ), 'your name', MSGLEVEL=1

$/ / J \phi B L I B \quad D D \quad D S N A M E=S Y S 2 . P R \phi G L I B, D I S P=(\phi L D, P A S S)$

//STEPI EXEC PGM=TRNSP $\phi R T$

$/ /$ FTO6FOO1 DD SYS $\phi U \mathrm{~T}=\mathrm{A}$

$/ /$ FTO4FOO1 DD UNIT $=$ SYSCP, $D C B=($ RECFM $=F, B L K S I E=80)$

$/ /$ H'LOSFUOI DD *

The job terminator card for the $360 / 67$ has a /* in columns 1,2 .

1),$T M M E=(m, s s)$ should be included if your run will take over 30 seconds of CPU time. This will rarely be necessary, even if a large number of problems are included in the run. This JCL is valid for MVT, Release 18, HASP . 


\section{TITLE CARD}

The title card is the first card in every TRANSPORT data set. The Title Card is always required and must be followed by a 0 or 1 card (see next section) to indicate whether the data to follow is new (0 card) or a continuation of a previous data set (1 card).

The title must be enclosed within single quotation marls on a single card. For TRANSPORT/360, the quotes may be entered in any column (Free field format).

Example of a DATA SET:

Ist card

'SLAC $20 / \mathrm{GEV} / \mathrm{C}$ SPECTROHETER'

2nd card

0

data

Last card - SENTINEL $^{-}$

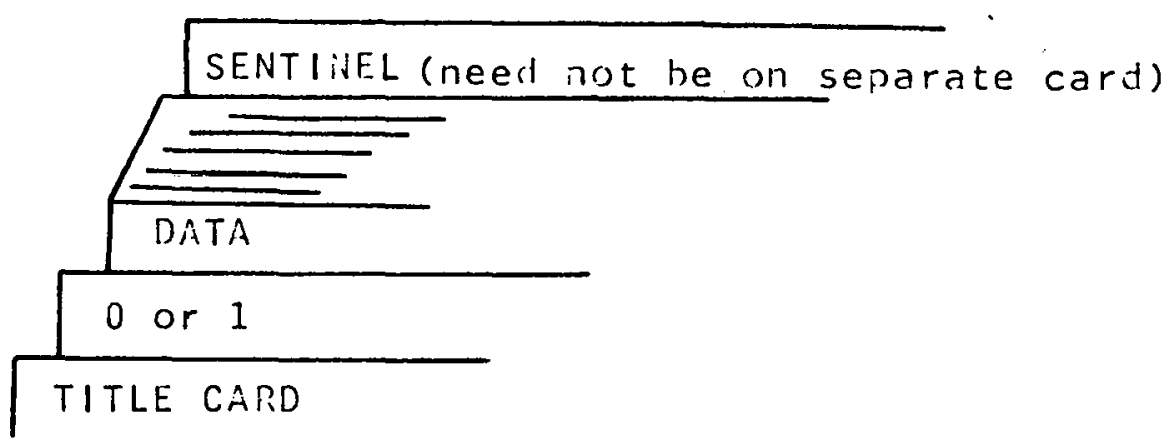




\section{INDICATOR CARD ( 0 or 1$)$}

The second card of the input is the indicator card. If the data which follow describe a new problem, a zero( 0$)$ is punched in any column on the card. If the data which follow describe changes to be made in the previous problem, a one(1) is punched in any columin on the card. No other entries are allowed on this card.

The sample problem input which is included below causes TRANSPORT to do a first order calculation with fitting ( 0 indicator card) and then to do a second order calculation (I indicator card) with the data that is the result of the fitting.

'FORTRAIN H CHECK OAN BETA FIT'

0) (Indicator Card)

1. .5. $1 . \quad .5$ 1. .5 1. 1. ;

-17. 'SEC1';

3.32 .745 'DRI';

2. $0 . ; 4.9 .879$ 10. $.5 ; 2.0$. ;

3.32 .745 'DRI' ;

13. 4. :

10. -1. 2. 0. .0001 'FIT1';

SEINT I NEL

'SECOHD ORDER'

1 (Indicator Card)

17. 'SEC1' ;

3.0 'DR1';

-10. 'FIT1';

SENTINEL 


\section{COMMENT CARDS}

Comment cards may be introduced in the data deck before any type code entry by inclosing the comments made on each card by single parentheses. No parentheses or quotation marks are allowed within the parentheses of any cornment card.

Example of the use of comment cards in a data set:

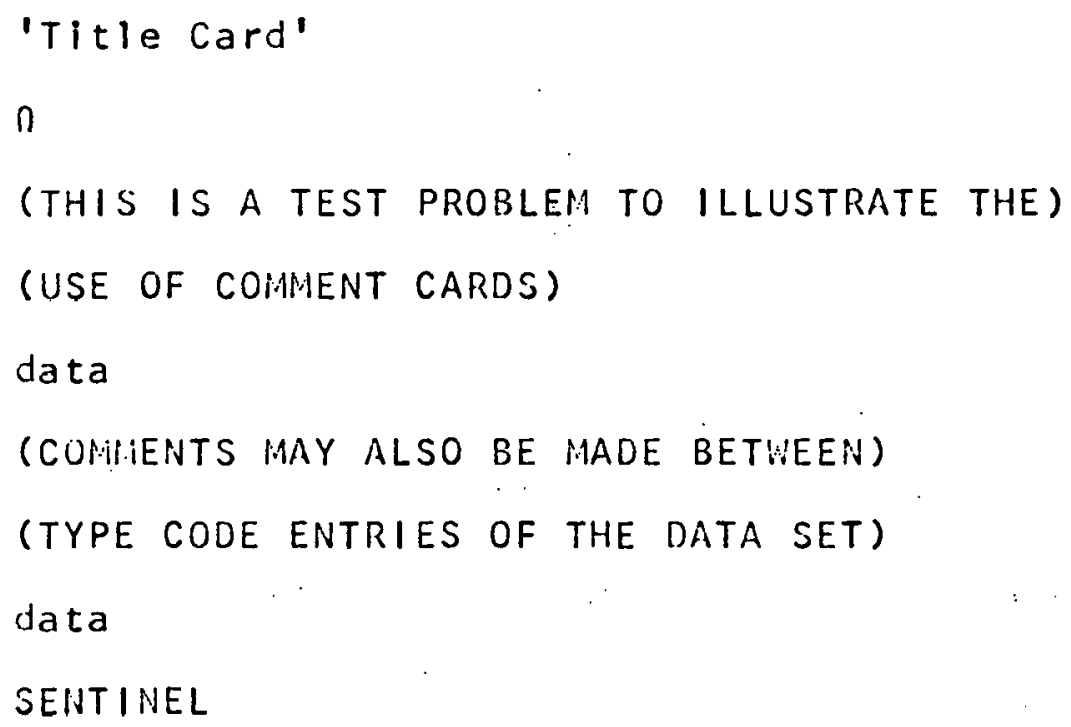




INPUT BEAM Type Code 1.0

This element specifies the phase space and the average momentum of the input beam for a TRANSPORT calculation. The input is specified by the semi-axes of a 6 -dimensional erect phase space variahles $x, \theta, y, \varphi, l$, and $\delta$. Each of these six parameters is entered as a positive quantity, but should be thought of as $\pm x, \pm \theta$, etc; hence, the total beam width is $2 x$, the total horizontal heam divergence is $2 \theta$ and so forth.

Normally, the BEAM card is the third card in the deck if standard TRANSPORT units are to be used; otherwise it should immediately follow any special units cards (Type Code 15.) chosen for the calculation to be nade. Standard TRANSPORT units for $x, \theta, y, \varphi, l$, and $\delta$ are $\mathrm{cms}, \mathrm{mr}, \mathrm{cms}$, $\mathrm{mr}, \mathrm{cms}$, and percent. The standard unit for the momentum $P(0)$ is $\mathrm{GeV} / \mathrm{c}$. There are eight entries (all positive) to be made on the BEAM card.

1- The Type Code 1.0 (Specifies a BEAM entry follows)

2- One-half the horizontal beam extent ( $x$ ) (cms in standard units)

3-One-half " " divergence $(\theta)(\mathrm{mr})$.

4- One-half the vertical beam extent (y) (cms).

5-One-half " " divergence $(\varphi)(\mathrm{mr})$.

6- One-half the longitudinal beam extent ( $l$ ) (cms).

7- One-half the momentum spread ( $\delta$ ) (in units of percent $\Delta P / P$ )

8- The momentum of the Central Trajectory $(P(0))(G e V / C)$.

*For a rotated (non-erect) phase ellipsoid input, see Type Code 12 . 
All eight entries must be made even if they are zero( 0$)$ and the last entry must be followed by a semicolon. Thus a typical BEAM entry might be.

$$
\begin{array}{lllllllll}
1.0 & 0.5 & 2.0 & 1.3 & 2.5 & 0 . & 1.5 & 10.0 & \text {; }
\end{array}
$$

meaning, $x= \pm 0.5 \mathrm{cms}, \theta= \pm 2.0 \mathrm{mr}, y= \pm 1.3 \mathrm{cms}, \varphi= \pm 2.5 \mathrm{mr}$, $\ell= \pm 0.0$ cins, $\delta= \pm 1.5$ percent $\Delta P / P$, and the central momentum $P(U)=10.0 \mathrm{GeV} / \mathrm{c}$.

The units of the tabulated matrix elements in either the 1 st order $R$ or sigma matrix or second order $T$ matrix of a TRANSPORT printout will correspond to the units chosen for the BEAM card. For the above, exarnple, the $R(12)=(x / \theta)$ matrix element will have the dimensions of $\mathrm{cms} / \mathrm{mr}$; and the $T(236)=(\theta / y \delta)$ matrix element will have the dimensions $\mathrm{mr}$ per $\mathrm{cm}$ per percent $\triangle P / P$ and so forth.

The longitudinal extent $\ell$ is useful for pulsed beams. It indicates the spread in length of particles in a pulse. It does not interact with any other component and may be set to zero if the pulse length is not important .

The phase ellipse (sigma matrix) beam parameters are normally printed as output after each data entry unless suppressed by a (13. 2. ;) type code entry. The projection of the semi-axes of the ellipsoid upon each of its six coordinate axes is printed in a vertical array and the correlations among these components indicating the phase ellipse orientations are printed in a triangular array (see the following pages). 
THE PHASE ELLIPSE BEAM MATRIX

The beam matrix carried in the computer has the following construction:

\begin{tabular}{c|cccccc} 
& $\mathrm{x}$ & $\theta$ & $\mathrm{y}$ & $\varphi$ & $\ell$ & $\delta$ \\
\hline $\mathrm{x}$ & $\sigma(11)$ & & & & \\
$\theta$ & $\sigma(21)$ & $\sigma(22)$ & & & \\
$\mathrm{y}$ & $\sigma(31)$ & $\sigma(32)$ & $\sigma(33)$ & & \\
$\varphi$ & $\sigma(41)$ & $\sigma(42)$ & $\sigma(43)$ & $\sigma(44)$ & \\
$\ell$ & $\sigma(51)$ & $\sigma(52)$ & $\sigma(53)$ & $\sigma(54)$ & $\sigma(55)$ & \\
$\delta$ & $\sigma(61)$ & $\sigma(62)$ & $\sigma(63)$ & $\sigma(64)$ & $\sigma(65)$ & $\sigma(66)$
\end{tabular}

The matrix is symmetric so that only a triangle of elements is needed.

In the printed output this matrix has a somewhat different format for ease of interpretation:

\begin{tabular}{|c|c|c|c|c|c|c|c|}
\hline & & & $x$ & $\theta$ & $y$ & $\varphi$ & $\ell$ \\
\hline$x$ & $\sqrt{\sigma}(11)$ & $\mathrm{CM}$ & & & & & \\
\hline$\theta$ & $\sqrt{\sigma}(22)$ & MR & $r(21)$ & & & & $\because$ \\
\hline$y$ & $\sqrt{\sigma}(33)$ & $\mathrm{CM}$ & $r(31)$ & $r(32)$ & & & \\
\hline$\varphi$ & $\sqrt{\sigma}(44)$ & MR & $r(41)$ & $r(42)$ & $r(43)$ & & \\
\hline$\ell$ & $\sqrt{\sigma}(55)$ & $\mathrm{CH}$ & $r(51)$ & $r(52)$ & $r(53)$ & $r(54)$ & \\
\hline$\delta$ & $\sqrt{\sigma}(66)$ & $P C$ & $r(61)$ & $r(62)$ & $r(63)$ & $r(64)$ & $r(65)$ \\
\hline
\end{tabular}


where: $\quad r(i j)=\frac{\sigma(i j)}{[\sigma(i i) \sigma(j j)]^{1 / 2}}$

As a result of the fact that the $\sigma$ matrix is positive definite, the $r(i j)$ 's satisfy the relation

$$
|r(i j)| \leqslant 1
$$

The full significance of the $\sigma(i j)$ 's and the $r(i j)$ 's are discussed in detail in Appendix I ("Description of Beam Matrix"). The units are always printed with the matrix.

In brief, the meaning of, the $\sqrt{\sigma}(i i)$ 's is as follows:

$\sqrt{\sigma}(11)=x_{\max }=$ The maximum (haif)-width of the beam envelope in the $x$ (bend) plane at the point of the printout.

$\sqrt{\sigma}(22)=\theta_{\max }=$ The maximum (half)-angular divergence of the beam envelope in the $x$ (hend) plane.

$\sqrt{\sigma}(33)=y_{\max }=$ The maximum (half)-height of the beam envelope.

$\sqrt{\sigma}(44)=\varphi_{\max }=$ The maximum (half)-angular divergence of the beam envelope in the $y($ non-bend)-plane.

$\sqrt{\sigma}(55)=l_{\max }=1 / 2$ the longitudinal extent of the bunch of particles.

$\sqrt{\sigma}(66)=\delta=$ The half-width $1 / 2(\Delta P / P)$ of the momentum interval being transmitted by the system.

The units appearing next to the $\sqrt{\sigma}(i i)$ 's in the TRANSPORT printout sheet are the units chosen for coordinates $x, \theta, y, \varphi, l$ and $\delta=\Delta P / P$ ' respectively. 
To the immediate left of the listing of the beam envelope size in a TRANSPORT printout, there appears a column of numbers whose values will normally be zero. These numbers are the coordinates of the centroid of the heam phase ellipse (with respect to the initially assumed central trajectory of the system). They may become non-zero under one of three circumstances:

1) When the misalignment (Type Code 8. ) is used.

2) When a Beam Centroid shift (Type Code 7.) is used.

or 3) When a 2nd-order calculation (Type Code 17.) is used. 
To aid in the interpretation of the phase ellipse parameters liste above, an example of an $(x, \theta)$ plane ellipse is lllustrated below. For further details the reader should refer to the appendix of this report.

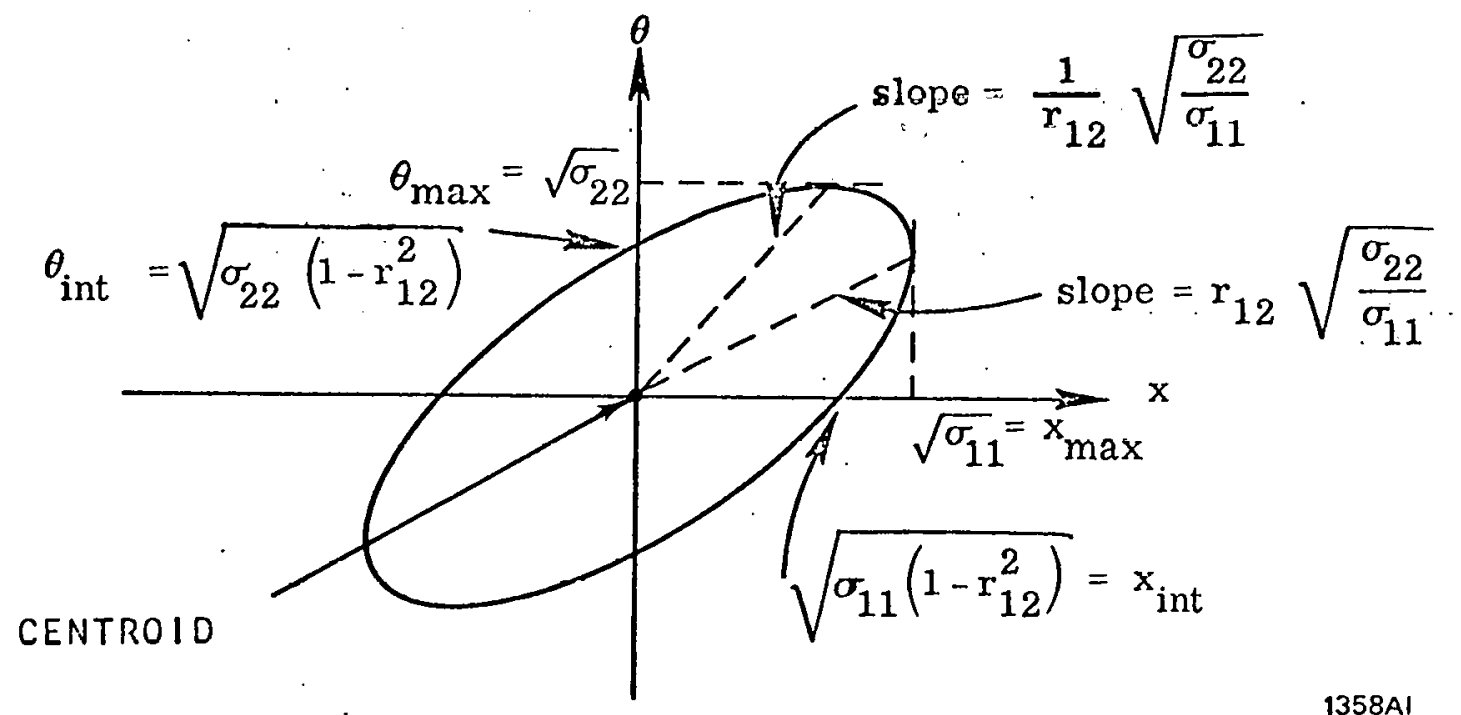

A Two Dimensional BEAM Phase Ellipse

The area of the ellipse is given by:

$$
A=\pi(\operatorname{det} \sigma)^{1 / 2}=\pi x_{\max } \theta_{\text {int }}=\pi x_{\text {int }} \theta_{\max }
$$




rms ADDITION TO THE BEAM Type Code 1.0

To allow for physical phenomena such as multiple-scattering, provision has been made in the program to permit an rms addition to the beam envelop to be made. There are nine entriés to be included:

1 - Type Code 1.0 (specifying a BEAM entry follows)

2 - one-half the rms addition to the horizontal beam extent $(\Delta x)(\mathrm{cms})$ :

3 - one-half the rms addition to the horizontal beam divergence $(\Delta \theta)(\mathrm{mr})$.

4 - one-half the rms addition to the vertical beam extent $(\Delta y)(\mathrm{cms})$.

5 - one-half the rms addition to the vertical beam divergence $(\Delta \varphi)(\mathrm{mr})$.

6 - one-half the rms longitudinal beam extent $(\Delta l)(\mathrm{cms})$.

7 - one-half the rms momentum spread $(\Delta \delta)$ (in percent $\Delta P / P)$.

8 - the momentum change in the central trajectory $(\Delta P(0))$ in $(\mathrm{GeV} / \mathrm{C})$.

9 - the code digit 0 . indicating an rms addition to to the beam is being made. 
The units for the r.m.s. addition are the same as those selected for a regular beam Type Code 1.0 entry. Thus a typical r.m.s. addition to the beam would appear as follows:

$$
\begin{array}{llllllllll}
1.0 & 0.1 & 0.2 & 0.15 & 0.3 & 0 . & 0.13 & -0.1 & 0.0
\end{array}
$$

where the last entry $(0.0)$ preceding the semicolon signifies an r.m.s. addition to the beam is being made and the next to the last entry indicates a central momentum change of $-0.1 \mathrm{GeV} / \mathrm{c}$. 
To provide for fringing fields and/or pole-face rotations on bending magnets; the Type Code 2.0 element is used.

There are two parameters:

$$
\begin{aligned}
& 1 \text { - Type Code } 2 . \\
& 2 \text { - angle of pole-face rotation (degrees). }
\end{aligned}
$$

The Type Code 2. element must either immediately precede a bending magnet (Type Code 4.) element (in which case it indicates an entrance fringing field and pole-face rotation) or immediately follow a Type Code 4. element (exit fringing field and pole-face rotation) with no other data entries between.* A positive sign of the angle on either entrance or exit pole-faces corresponds to a transverse focusing action and radial defocusing action.

For example, a symmetrically oriented rectangular bending magnet whose total bend is 10 degrees would be represented by the three entries: 2. 5.; 4.---; 2. 5.;

The angle of rotation may be varied. For example, the element 2.15. ; would allow the angle to vary from an initial guess of 5 degrees to a final value which would, say, satisfy a vertical focus constraint imposed upon the system. See the Type Code 10. section for a complete discussion of vary codes.

Even if the pole-face rotation angle is zero, 2. 0.; entries must he included in the data set before and after a Type Code 4.0 entry if fringing-field effects are to he calculated.

* It is extremely important that no data entries be made between a Type Code 2. and a Type Code 4. entry. If this occurs, it may result in an incorrect matrix multiplication in the program and hence an incorrect physiral answer. 
A single Type Code 2. entry that follows one bending magnet and precedes another will be associated with the latter.

Should it be desired to misalign such a magnet, an update must be forced prior to the first type 2. code entry and the convention appropriate to misalignment of a set of elements applied, since, indeed, three separate transformations are involved. See section under Type Code 8. for a discussion of misalignment calculations. The type code signifying a rotated pole-face is 2.0 . The input format is:

$$
2.0 \text { B. 'RO' }
$$

The units for $\beta$ are degrees. 


\section{POLE-FACE ROTATION MATRIX}

The first-order $R$ matrix for a pole-face rotation used in a TRANSPORT calculation is as follows:

$$
R=\left(\begin{array}{cccccc}
1 & 0 & 0 & 0 & 0 & 0 \\
\frac{\tan \beta}{\rho} & 1 & 0 & 0 & 0 & 0 \\
0 & 0 & 1 & 0 & 0 & 0 \\
0 & 0-\frac{\tan (\beta-\psi)}{\rho} & 1 & 0 & 0 \\
0 & 0 & 0 & 0 & 1 & 0 \\
0 & 0 & 0 & 0 & 0 & 1
\end{array}\right)
$$

Definitions: $\beta=$ angle of rotation of pole-face (see figure on following page for sign convention of $\beta$ ) $\rho$ = bending radius of central trajectory $g$ - total gap of magnet $\psi=$ correction term resulting from finite extent of fringing fields.**

where

$$
\psi=K_{1}\left(\frac{g}{\rho}\right)\left(\frac{I+\sin ^{2} \beta}{\cos \beta}\right)\left[I-K_{1} K_{2}\left(\frac{g}{\rho}\right) \tan \beta\right]^{*}
$$

*See Type Code 16 . for input formats for $g, K(1)$, and $K(2)$ TRANSPORT entries.

** See SLAC-Report No. 75 (page 74) for a discussion of $\psi$. 


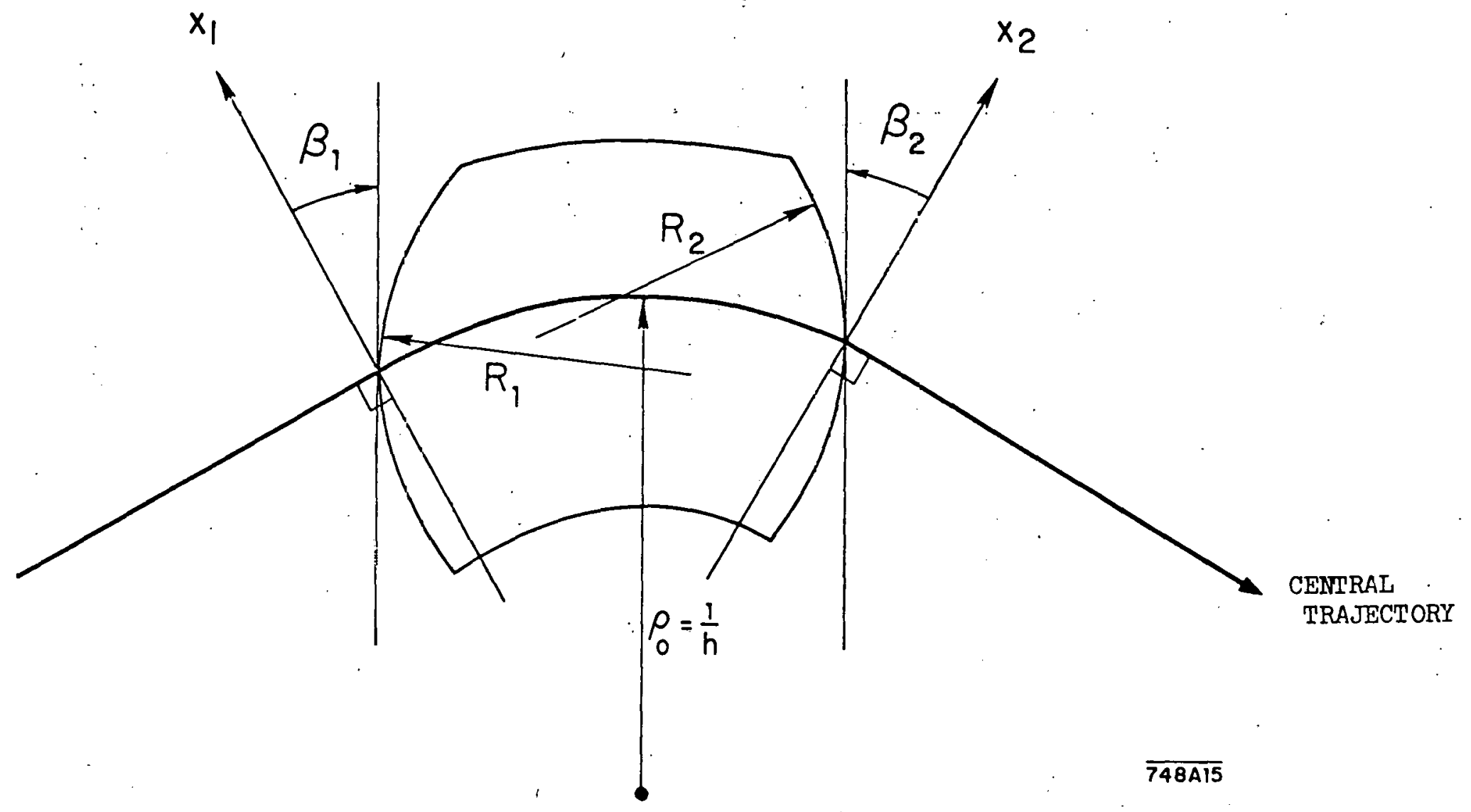

FIELD BOUNDARIES FOR BENDING MAGNETS

The TRANSPORT sign conventions for $x, \beta, R$ and $h$ are all positive as shown in the figure. The positive $y$ direction is out of the paper. Positive $\beta^{\prime}$ s imply transverse focusing. Positive R's (convex curvatures) represent negative sextipole comporients of strength $S=\left(-\frac{h}{2 R}\right) \sec ^{3} B . \quad$ (See SLAC-75, page 7I). 
DRIFT Type Code 3.0

A drift space is a field-free region through which the beam passes. There are two parameters:

1 - Type Code 3. (specifying a drift length).

2 - (effective) drift length (meters).

Typical Input Format for a DRIFT

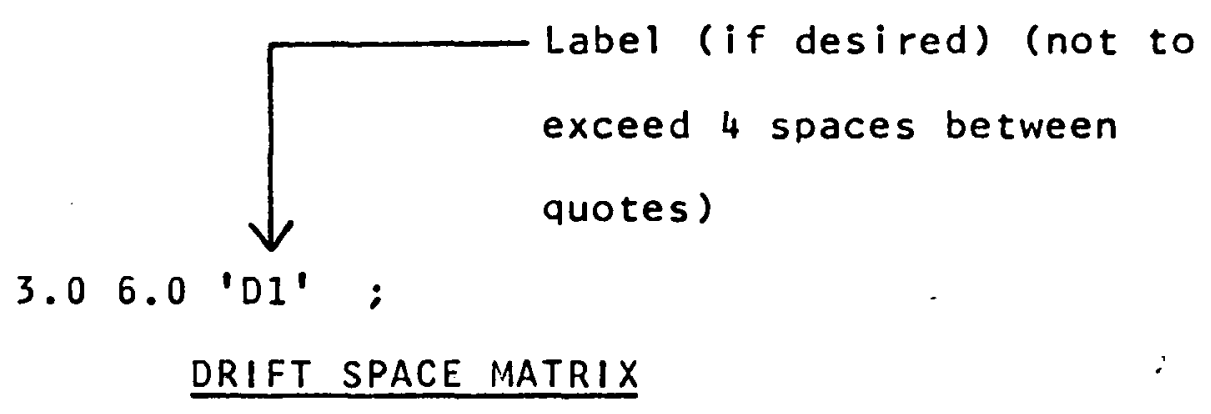

The first-order $R$ matrix for a DRIFT space is as follows:

$\left(\begin{array}{llllll}1 & L & 0 & 0 & 0 & 0 \\ 0 & 1 & 0 & 0 & 0 & 0 \\ 0 & 0 & 1 & L & 0 & 0 \\ 0 & 0 & 0 & 1 & 0 & 0 \\ 0 & 0 & 0 & 0 & 1 & 0 \\ 0 & 0 & 0 & 0 & 0 & 1\end{array}\right)$

where

$L$ - the length of the drift space. 
The dimensions of $L$. are those chosen for longitudinal length via a

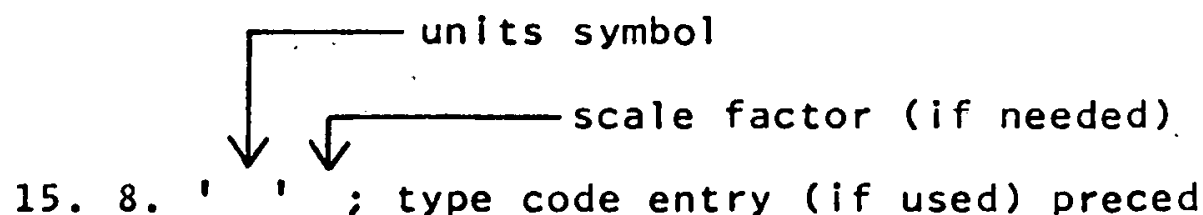

15. 8. ' ' ; type code entry (if used) preceding the BEAM

(Type Code 1.) card. If no 15. 8. entry is made, the units of L. will automatically be in METERS (Standard TRANSPORT/360 units). 


\section{WEDGE BENDING MAGNET TYPe Code 4.0}

A wedge bending magnet implies that the central trajectory of the beam enters and exits perpendicularly to the pole-face boundarles (to include fringing-field effects and non-perpendicular entrance or exit boundarles--see Type Codes 2.0 and 16.0 ).

There are four first-order parameters to be specified for the wedge magnet via Type Code $4.0 * *$

1 - Type Code 4.0 (specifying a wedge bending magnet)

2 - The (effective) length $L$ of the central trajectory. (meters)

3 - The central field strength $B(0)$ in $K G$, $B(0)=33.356(P / P)$

where $P$ is the momentum in GeV/c and $\rho$ is the bending radius of the central trajectory in meters.

4 - The field gradient ( $n$-value) dimensionless; where $n$ is defined by the equation $B_{y}(x, 0, t)=B_{y}(0,0, t)[1-n h x+\ldots]$,

where

$$
h=\frac{1}{\rho_{0}} \text {. See SLAC-75 (page } 31 \text { ). }
$$

$B(0)$, and $n$ may be varied for lst order fitting (see section 10.0 for a discussion of vary codes).

A typical first-order TRANSPORT/360 input for a wedge magnet is:

$$
\text { 4.0 L. B. n. } \sqrt{\text {, }} \text { Label (not to exceed } 4 \text { spaces) }
$$

* See Type Code 16. for special parameter entries affecting fringing field and/or second-order calculations for bending magnets. 
- If fringing field effects are to he included, a Type Code 2. entry must precede and follow the pertinent Type Code 4 . entry (even if there are no pole-face rotations.)** Thus a typical. TRANSPORT/360 input for a hending magnet including fringing fields might be:

$$
\begin{aligned}
& \text { 2. } 0 ., \text { Labels (not to exceed } 4 \text { spaces) if desired } \\
& \text { 4. L. B }(0) \text {. n. , , ; } \\
& \text { 2. 0. , , }
\end{aligned}
$$

For non-zero pole-face rotations a typical data input might be:

2. 10. : 4. L. B(0). n. ; 2. 20.;

note that the use of labels is ontional and that all data. entries may be made on one line if desired. 
The sign conventions for bending magnet entries are lllustrated in the following figure (748A15). For TRANSPORT/360, a positive bend is to the right looking in the direction of particle travel. To represent a bend in another sense, the coordinate rotation matrix (Type Code 20.) should be used as follows:

$A$ bend up is represented by rotating the $(x, y)$ coordinates by - 90.0 degrees about the $(z)$ beam axis as follows:

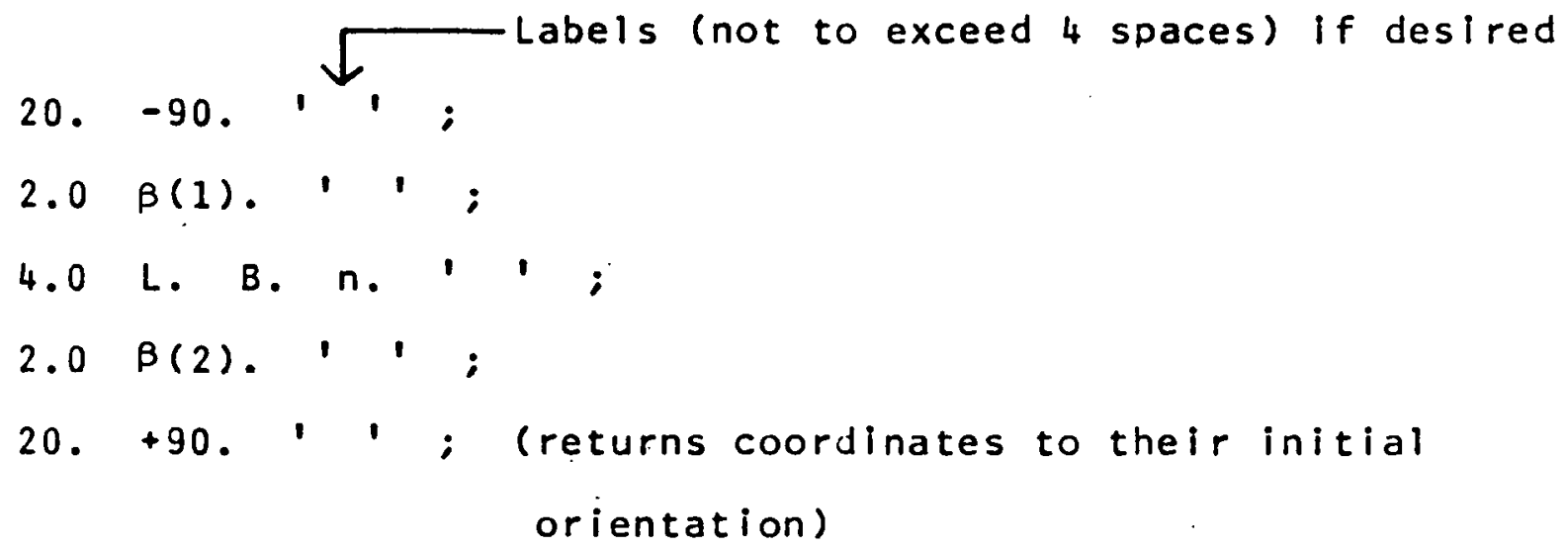

A bend down is accomplished via:

20. +90. ' ' :

2.

4.

2.

20. $-90.1,1$; 
A bend to the left (looking in the direction of beam travel) is accomplished by rotating the $x, y$ coordinates by 180 degrees, e.g. 20. 180.' ' ;

2 .

4 .

2 .

20. -180 ' '; 


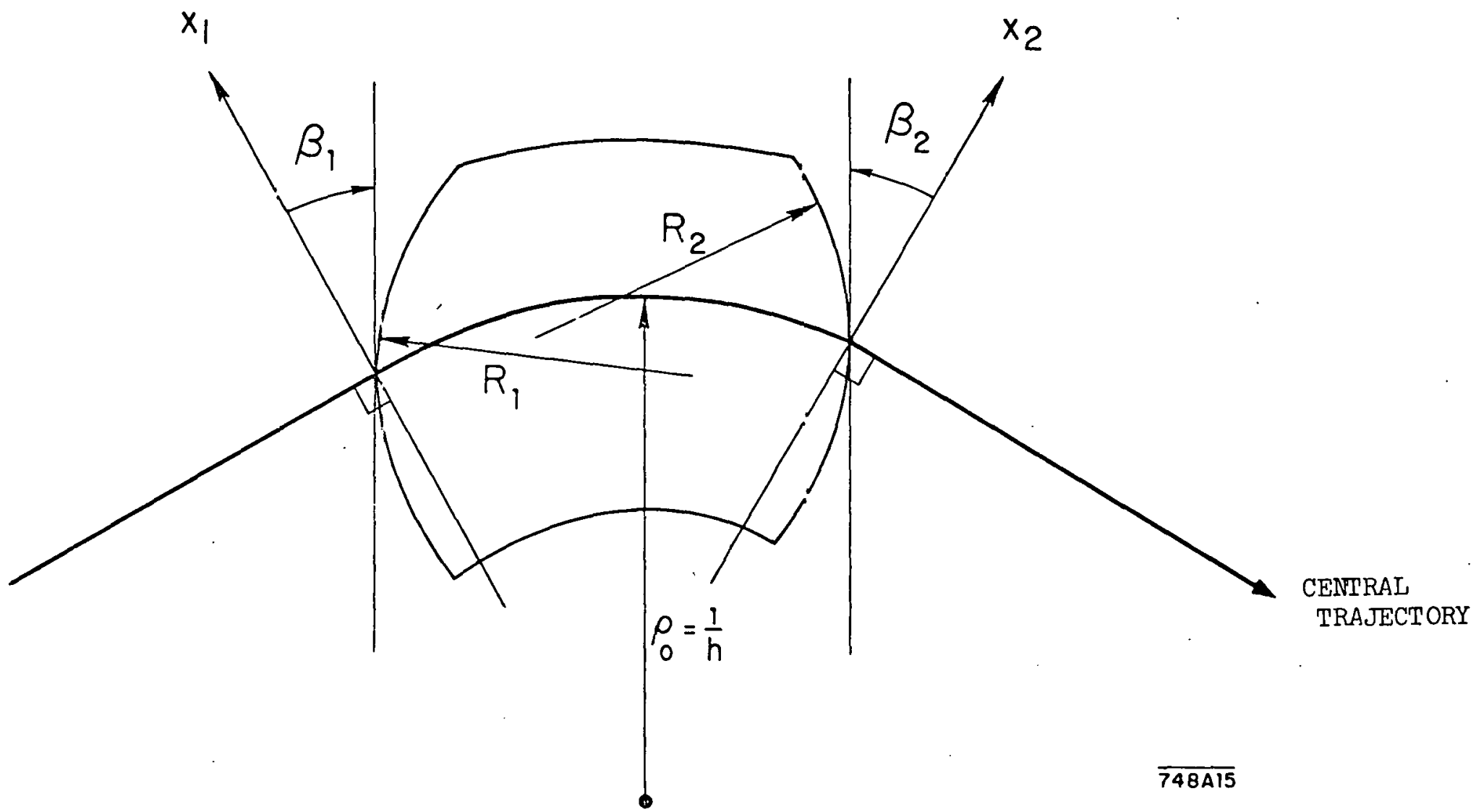

FIFID BOUNDARIES ICR BENDING MAGNETS

The TRANSPORT sign conventions for $x, \beta, R$ and $h$ are all positive as shown in the figure. The positive $y$ direction is olit of the paper. Positive $\beta^{\prime \prime}$ imply transverse focusing. Positive R's (convex. curvatures) represent negative sextupoie components of strength $S=\left(-\frac{h}{R}\right) \sec ^{3} \beta . \quad$ (See SLAC-75, page 71): 
WEDGE BENDING MAGNET MATRIX

\begin{tabular}{|c|c|c|c|c|c|}
\hline $\cos k_{x} L$ & $\frac{1}{k_{x}} \sin k_{x} L$ & 0 & 0 & 0 & $\frac{h}{k^{2}}\left[1-\cos k_{x} L\right]$ \\
\hline$-k_{x} \sin k_{x} L$ & $\cos k_{x} L$ & 0 & 0 & 10 & $\left(\frac{h}{k_{x}}\right) \sin k_{x} L$ \\
\hline 0 & 0 & $\cos k_{y} L$ & $\frac{1}{k_{y}} \sin k_{y} L$ & 0 & 0 \\
\hline 0 & 0 & $-k_{y} \sin k_{y} L$ & $\cos k_{y} L$ & 0 & 0 \\
\hline$-\frac{h}{k_{x}} \operatorname{in} k x^{L}$ & $-\frac{h}{k_{x}^{2}}\left[1-\cos k_{x} L\right]$ & 0 & 0 & 1 & $-\frac{h^{2}}{k_{x}^{3}}\left[k_{x} L-\sin k_{x} L\right]$ \\
\hline 0 & 0 & 0 & 0 & 0 & 1 \\
\hline
\end{tabular}

Definitions: $\quad h=\frac{I}{\rho_{0}}, k_{x}^{2}=(1-n) h^{2}, k_{y}^{2}=n h^{2}$

$$
\begin{aligned}
& \alpha=h L=\text { The angle of bend } \\
& L=\text { path length of the central trajectory }
\end{aligned}
$$

The field expansion for the midplane of a bending magnet is taken from eq(18) page 31 of SLAC-75 thereby defining the dimensionless quantities $n$ and $\beta$ as follows:

$$
B_{y}(x, 0, t)=B_{y}(0,0, t)\left[1-n h x+\beta h^{2} x^{2}+\gamma h^{3} x^{3}+\ldots\right]
$$

The type code signifying a BEND is 4.0. The input format for a TRANSPORT/360 calculation is: 


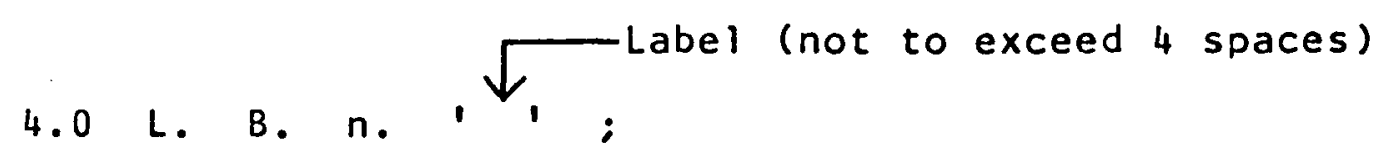

If $n$ is not included in the data entry, the program assumes it to be zero. A $\beta$ entry for a second order calculation is made via the 16. 1. element. (Do Not confuse this $\beta$ with a pole-face rotation)

The standard units for L. and B. are Meters and KG. If desired, these units may be changed by 15. 8. and 15. 9. type code entries preceding the BEAM Card. 


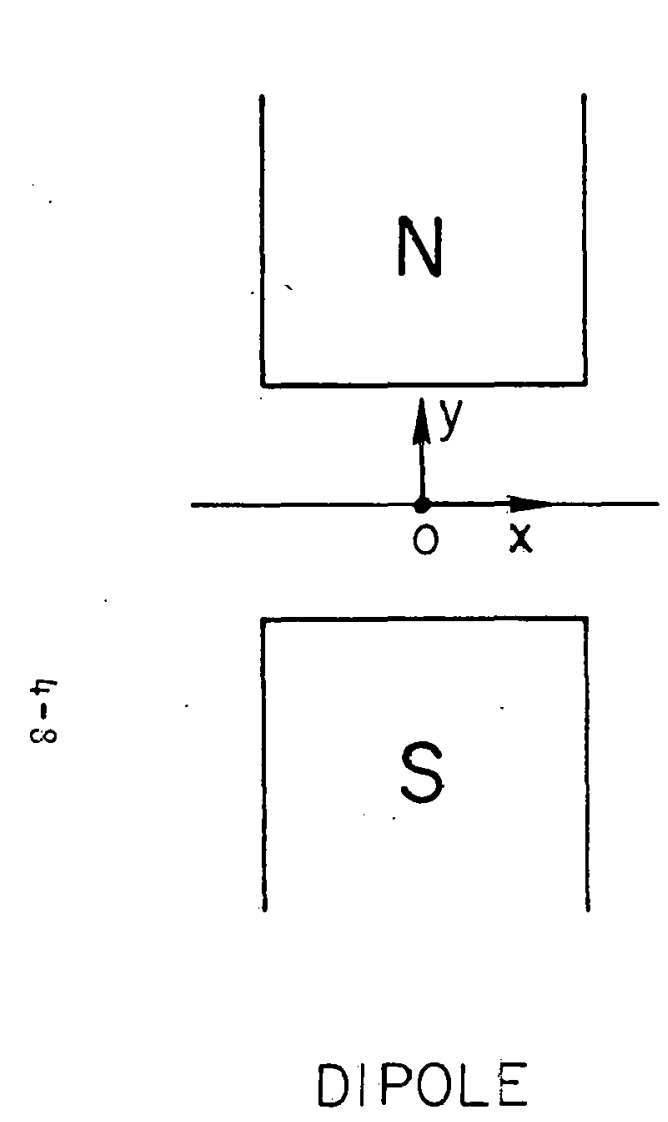

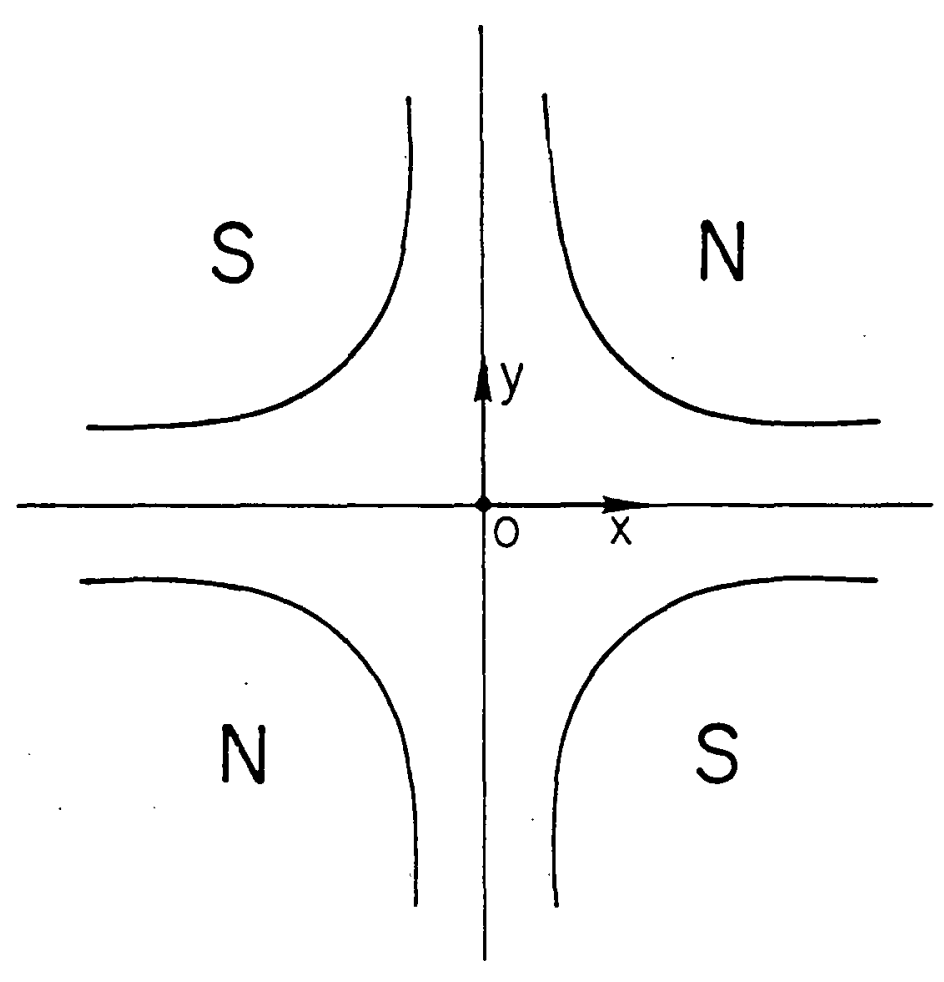

QUADRUPOLE

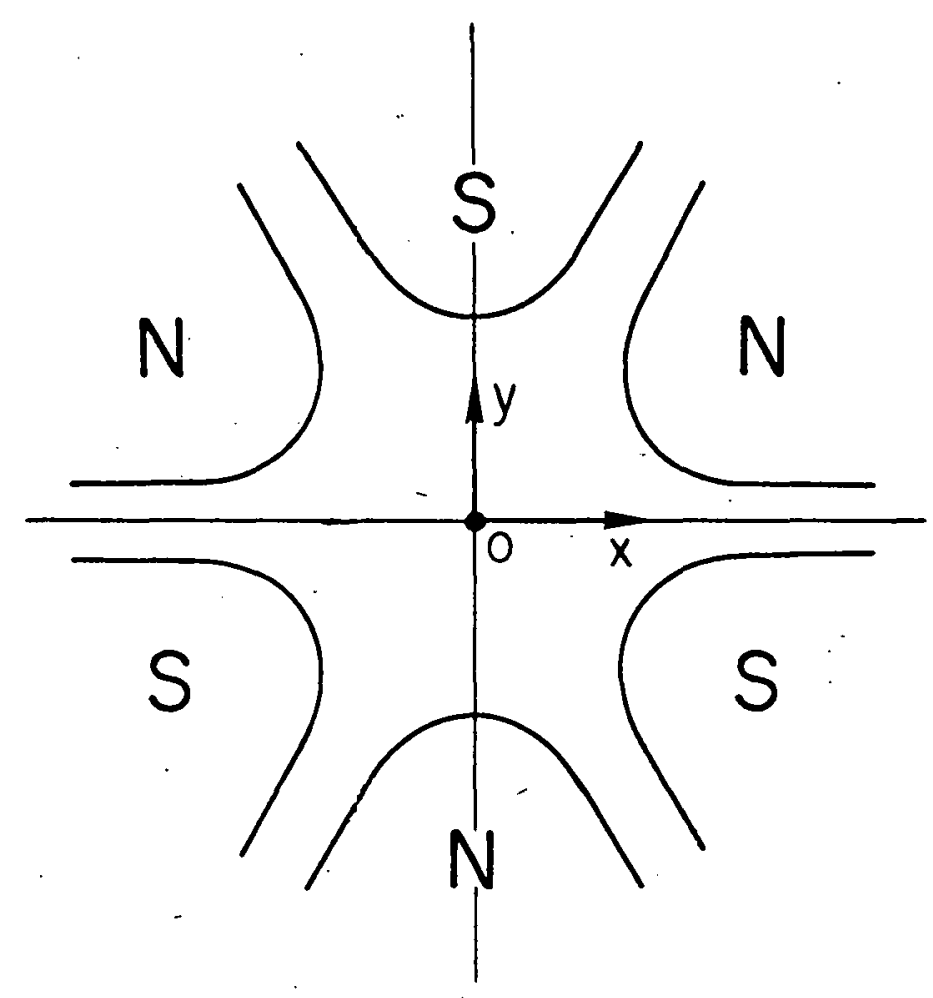

SEXTUPOLE

ILLUSTRATION OF THE MAGNETIC MTPEANE ( $x$ AXIS) FOR DIPOLE, QUADRUPOLE AND SEXTUPOLE ELEMENTS. THE MAGNET POLARITIES INDICATE MULTIPOLE ELEMENTS THAT ARE POSITIVE WITH RESPECT TO EACH OTHER. 


\section{QUADRUPOLE TyPe Code 5.0}

A quadrupole provides focusing in one transverse plane and defocusing in the other.

There are four parameters to be specified for a TRANSPORT calculation:

1 - Type Code 5. (specifying a quadrupole).

2 - (effective) magnet length L (in meters).

3 - Field at pole tip $B$ (in $K G)$. A positive field implles horizontal focusing; a negative field, vertical focusing.

4. Half-aperture a (in $\mathrm{cm})$. Radius of the circle tangent to the pole tips.

The strength of the quadrupole is computed from its field, aperture and length. The horizontal focal length is printed as output; a positive focal length indicates horizontal focusing and a negative focal length indicates horizontal defocusing.

The type core for a QUAD is 5.0. The input format for a typical data set is:

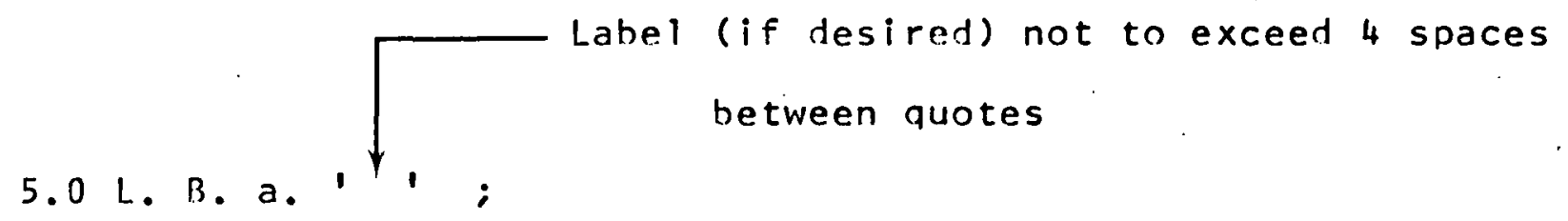

The data may be entered In FREE FIELD format. The standard TRANSPORT/360 units for L. B. and a. are Meters, KG, and cms respectively. If other units are desired they must be chosen via the appropriate 15. type code entries preceding the BEAM (Type Code 1.) card. 


\section{Ist ORDER \\ QUADRUPOLE MATRIX}

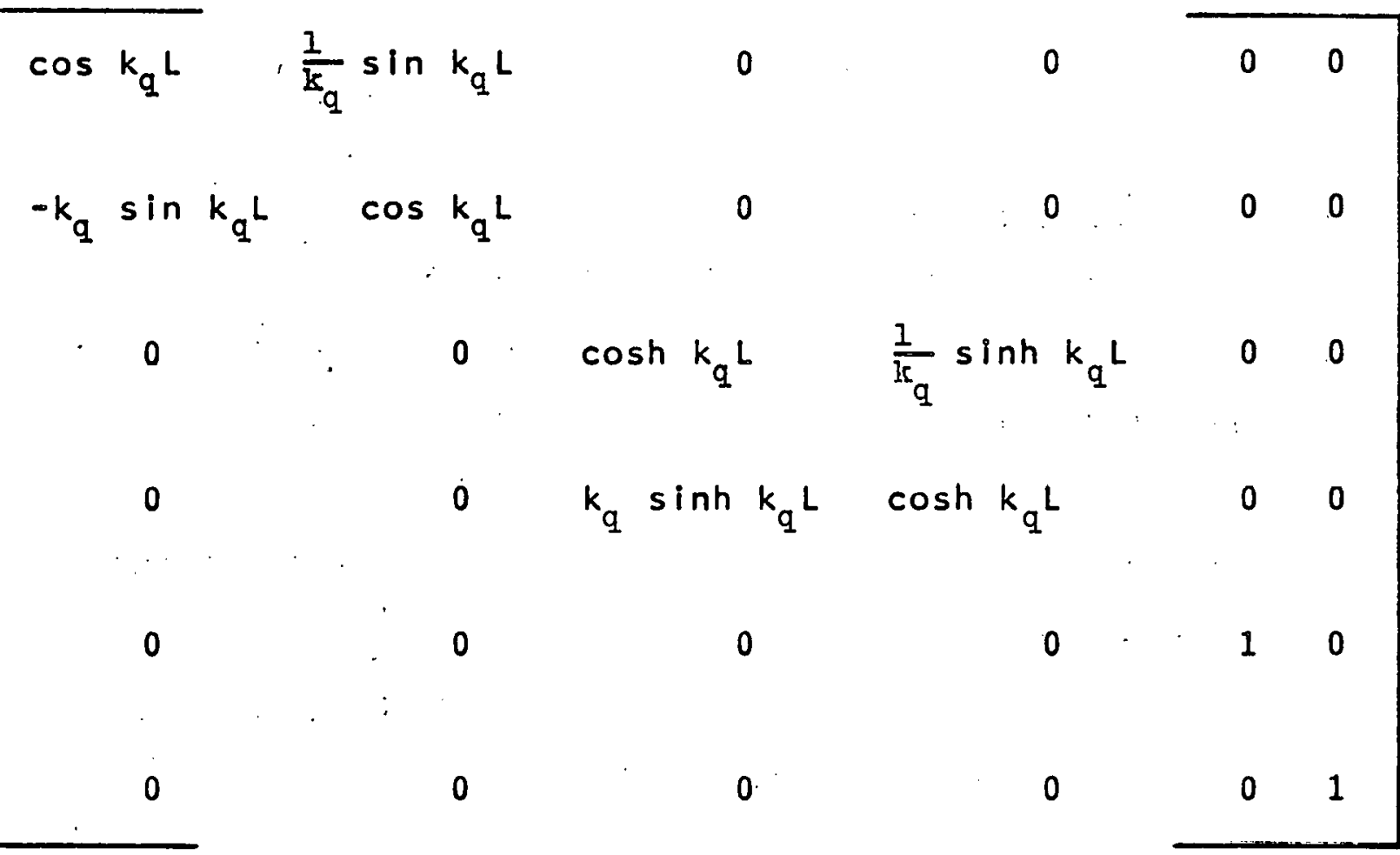

These elements are for a quadrupole which focuses in the horizontal (x) plane (B positive). A vertically (y plane) focusing quadrupole ( $B$ negative) has the two partial matrices interchanged.

Definitions: $L=$ the effective length of the quadrupole

$$
\begin{aligned}
& a=\text { the radius of the aperture } \\
& B_{0}=\text { the field at radius a } \\
& k_{q}^{2}=\left(\frac{B_{0}}{a}\right)\left(\frac{1}{B p}\right)
\end{aligned}
$$

where $(B \rho)=$ the magnetic rigidity (momentum) of the central trajectory. 

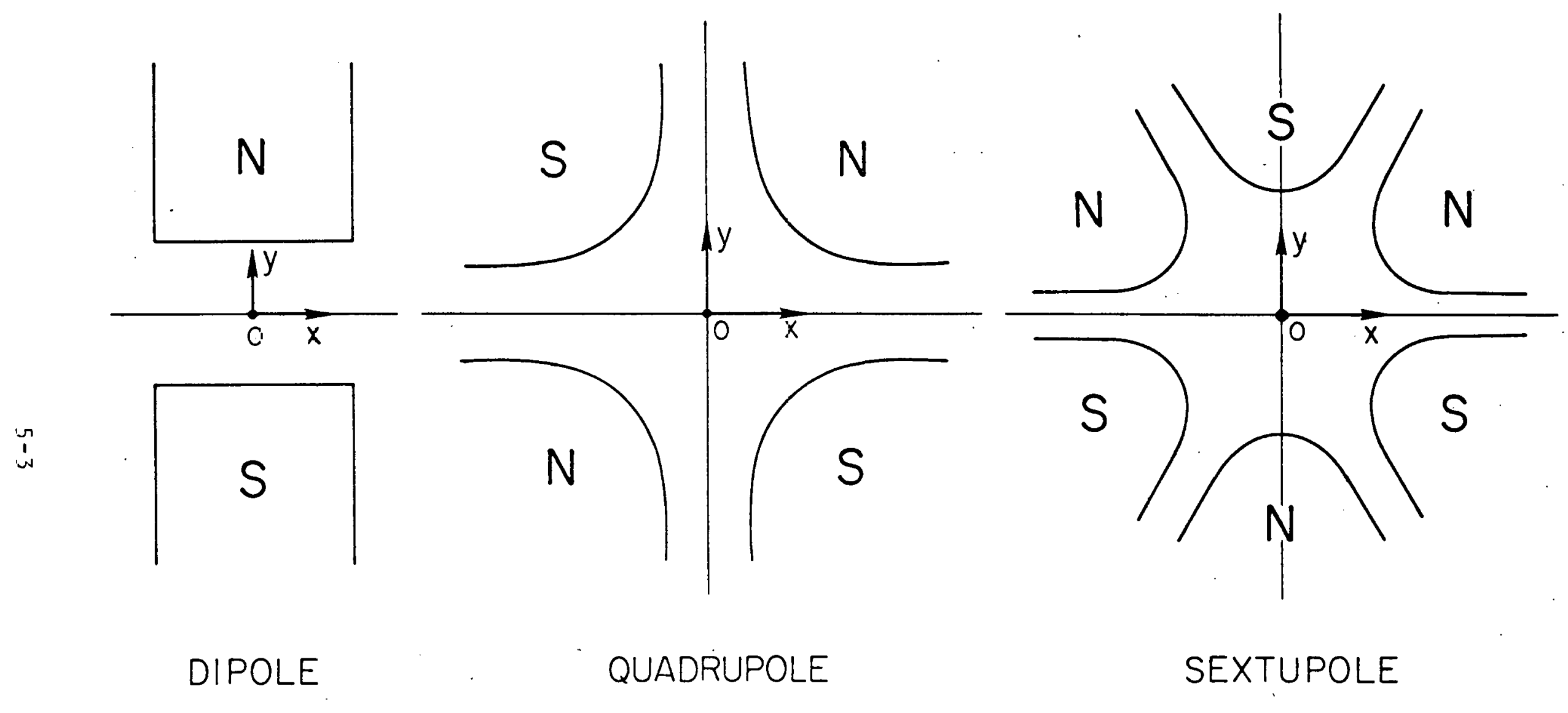

$748 A 10$

ILLUSTRATION OF THE MAGNETIC MDPLANE ( $x$ AXIS) FOR DIPOLE, QUADRUPOLE AND SEXTUPOLE ELEMENTS. THE MAGNE? POLARITIES INDICATE MULTIPOLE ELEMENTS THAT ARE POSITIVE WITH RESPECT TO EACH OTHER. 
TRANSFORM 1 UPDATE* Type Code 6.0.1.

To provide a facility for updating TRANSFORM 1 (the product of the $R$ matrices, R1) use Type Code 6.0. Thus a $(6,0.1 . ;)$ forces an update of the RI matrix and initiates the accumulation of a new product matrix initialized from the point of the update. This facility is useful in conjunction with misaligning a set of magnets or fitting only a portion of a system.

The complete list of elements which update TRANSFORM 1 is:

1) The (6.,0.1. ;) entry

2) Any fitting constraint imposed upon the beam (sigma) matrix (See Type Code 10. for a discussion of this)

3) A misalignment Type Code 8.0 entry.

4) A beam Type Code 1.0 entry

A TRANSFORM 1 matrix will be printed at any position in the data set where a (13.4. ;) entry is inserted.

See the following section for the introduction of an auxiliary transformation matrix R2 (TRANSFORM 2) to circumvent the problems arising from TRANSFORM 1 updates.

* By "updating" we mean initiating a new starting point for the accumulation (multiplication) of the $R$ matrix. l.e. at the point of update, the $R$ matrix is assumed to he reset to the unity matrix and all previous accumulations are forgotten. 


AUXILIARY TRANSFORMATION MATRIX (R2) TyPe Code 6.0.2.

To circumvent the difficulties caused by frequent heam updates and consequent redefinition of the cumulative transformation matrix, $R I$, provision has been made for introducing an independent transformation natrix, the R2 matrix.

This matrix is not normally accumulated, and must be introduced by defining its starting point with a $(6.0 .2$. ;) card. This variant of the Type 6.0 code also serves to redefine the starting point of the $R 2$ matrix (update $i t$ ) when desired.

R2 has no effect upon the BEAM (sigma) matrix as does R1 nor do any constraints imposed upon the BEAM (sigma) matrix react upon it. It is solely a convenience for the user. It may be printed by a $(13,24$. ; ) entry. Any components of R2 may be constrained by a Type Card 10. entry with code digits: $-(i+20$.$) and j$.

For example, 10. -1. 2. 0. .01; and 10. -21. 2. 0. .01: are identical constraints applied to the R1 and R2 matrices, respectively.

R2 is updated by a beam (1.) element, provided R2 has been introduced, but by no other elements. No provision has been made for suppressing $R 2$ once it is introduced. 


\section{SHIFT IN THE BEAM CENTROID Type Code 7.0}

Sometimes it is convenient to redefine the BEAM centroid** such that it does not coincide with the TRANSPORT reference trajectory. Provision has been made for this possibility via Type Code 7.0. Seven parameters are required:

\section{1 - Type Code 7.}

( 2 to 7 )- the coordinates $x, \theta, y, \varphi, l$, and $\delta$ defining the initial conditions of the new beam centroid with respect to the reference trajectory. The units for $x, \theta, y, \varphi, \ell, \delta$ are the same as those chosen for the beam (Type Code 1.0 entry), normally $\mathrm{cm}, \mathrm{mr}, \mathrm{cm}, \mathrm{mr}, \mathrm{cm}$, and percent.

Only the beam $(\sigma)$ matrix is affected by this code. The transformation matrices $R 1$ and $R 2$ are unaffected.

In order for this code to function properly, the initial BEAM entry (Type Code 1.0 ) must have a non-zero phase space volume.

e.g. a

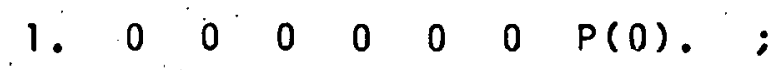

beam entry is not permissible when calculating a shlft in the beam centroid; whereas a

$$
1.0 \text { 1. 1. 1. 1. 1. 1. } P(0) \text {; }
$$

entry (finite phase volume) will work.

* By "Beam Centroid" we mean the center of the beam ellipsoid. 


MAGNET ALIGNMENT TOLERANCES TyPe Code 8.0

The misalignment of a magnet to first order affects only the centroid of the beam. Two situations are commonly encountered: the magnet is displaced and/or rotated by a known amount; or the actual position of the magnet is uncertain within a given tolerance. Both these effects may be simulated through use of the 'align' element.

There are eight parameters to be specified:

1 - Type Code 8. (specifying alignment).

2 - the magnet displacement in the horizontal direction $(\mathrm{cm})$.

3 - a rotation about the horizontal axis (mr).

4 - a displacement in the vertical direction $(\mathrm{cm})$.

5 - a rotation about the vertical axis (mr).

6 - a displacement in the beam direction $(\mathrm{cm})$.

7 - a rotation about the beam direction ( $m r$ ).

8 - a three digit code number (defined below) specifying the type of misalignment.

The coordinate system employed is that to which the beam is referred at the point it enters the magnet. For example, a rotation of a bending magnet about the heam direction (parameter 7 above) is referred to the direction of the beam where it enters the magnet. The units employed are the standard TRANSPORT units shown above unless redefined by Type Code 15. entries. If the units are changed, the units of the misalignment displacements are those determined by the 15 . 1. type code entry; the units for the misalignment rotations are those determined by the 15.2 . type code entry. 
Only the misalignments of bending magnets or quadrupoles may be simulated. The Type Code 8.0 align element(s) must directly follow the magnetic element(s) to be misaligned.

When the actual position of the magnet(s) is uncertain within a given tolerance, the BEAM card entry at the beginning of the system should correspond to a zero phase space input, i.e., the BEAM card should appear as follows:

$$
1.0 \text { 0. } 0.0 .0 .0 .0 . P(0) \text {; }
$$

The phase ellipse printouts following the misalignment will then possess a finite phase volume which represents the uncertainty in the position of the beam centrold resulting from the uncertainty in the knowledge of the positioning of the magnet(s).

When the misalignment is a known amount, the BEAM card entry at the beginning of the system should possess a non-zero phase volume. (e.g. a

$$
\text { 1.0 1. 1. 1. 1. 1. 1. P(0).; }
$$

is a permissible Type Code 1.0 entry.)

The beam centroid printouts following the misalignment will then show a definite shift in the position of the beam centroid resulting from the misalignment of the magnet( $s)$. 
An align element updates the BEAM (sigma) matrix and the RI matrix but not the R2 matrix.

The following three digit code numbers provide several alternative misalignment situations:

A. The units position distinguishes between a single type code entry and a set of entries:

\begin{tabular}{|c|c|}
\hline NUMBER & INTERPRETATION \\
\hline$\times \times 0$ & $\begin{array}{l}\text { In this case, the } 0 \text { means that the information contained } \\
\text { on the align card refers only to the single magnet (type code } \\
\text { entry) iminediately preceding it. } \\
\text { With thls option the } 1 \text { means that all of the type code : } \\
\text { elements since the last Rl matrix update (a BEAM CARD entry, } \\
\text { a constraint upon the BEAM (sigma) matrix or a } 6 \text {. } 0 \text {. } 1 . \text {; } \\
\text { type code entry) are treated as a unit and the misalignnent } \\
\text { information on the card is applied to the unit as a } \\
\text { whole. This option should be used for the misalignment of } \\
\text { a bending magnet when fringing-fields or pole face } \\
\text { rotations (Type Code } 2.0 \text { ) are included. See Examples } \\
1 \text { and } 2 \text { below for an illustration of this. Only one } \\
\text { bending magnet may be included in the array. Another } \\
\text { example of the use of this option is in the misalignment } \\
\text { of an array of quadrupoles. By successive application of } \\
\text { align elements, for example, the elements of a quadrupole } \\
\text { doublet could be misaligned relative to each other and } \\
\text { then the doublet as a whole could be misaligned. }\end{array}$ \\
\hline
\end{tabular}


XX2. With this option the 2 means that all of the type core elements since the last R2 matrix update (a 6. 0. 2. ; type code entry) are treated as a unit. This code digit, for example, is necessary for studying misalignment of quadrupole triplets and other combinations involving more than two quadrupole magnets. It makes use of the fact that the R2 matrix remains unaffected by the usual update codes.

B. The tens position defines the system axis along which the succeeding magnets are positioned:

\begin{tabular}{l|l}
$x 0 x$. & The 0 means they are positioned along the same axis as \\
& if the magnet were not displaced. \\
$x 1 x$ & The 1 means they are positioned along the axis defined
\end{tabular} by the magnetic axis of the displaced magnet.

For instance, if a quadrupole is rotated, the remainder of the system may be left alone $\left(X_{0} X_{\text {. }}\right)$ or rotated with the quadrupole $X I X$.

c. The hundreds position distinguishes between an uncertainty in position $\left(0 x x_{.}\right)$or a known displacement $\left(1 \times x_{.}\right)$.

Any combination of digits may be used to define the exact circumstances intended. Thus, code 111. $(=1 .+10 .+100$.$) indicates$ the deliberate displacement of a set of magnets and the remainder of the system (referred to the point the beam enters the set). Code 101. would leave the remainder of the system alone. 
Typical code numbers for magnet alignment tolerances might be:

\begin{tabular}{|c|c|}
\hline Code Number & Interpretation \\
\hline 000 & $\begin{array}{l}\text { The reference trajectory is unchanged. Only } \\
\text { the preceding magnet is misaligned } \\
\text { and by an uncertain amount (but } \\
\text { within the specified tolerances). }\end{array}$ \\
\hline 001. & $\begin{array}{l}\text { The reference trajectory is unchanged. All } \\
\text { magnets (type code entries) between the last } \\
\text { R1 matrix update and the Type code } 8 \text {. entry are } \\
\text { misaligned by an uncertain amount (but within } \\
\text { the specified tolerances). }\end{array}$ \\
\hline 002 . & $\begin{array}{l}\text { Same as above except replace } R 1 \text { with } R 2 \text { in the } \\
\text { statement }\end{array}$ \\
\hline 100 . & $\begin{array}{l}\text { The reference trajectory is unchanged. Only } \\
\text { the preceding magnet is misaligned by the } \\
\text { specified ámount. }\end{array}$ \\
\hline 101 & $\begin{array}{l}\text { The reference trajectory is unchanged. All } \\
\text { magnets between the last R1 matrix update and } \\
\text { the type code } 8 \text { entry are misaligned by the } \\
\text { specified amount. }\end{array}$ \\
\hline 102. & $\begin{array}{l}\text { Same as above except replace } R 1 \text { with } R 2 \text { in the } \\
\text { statement }\end{array}$ \\
\hline
\end{tabular}


111.

The axis of the misaligned magnet(s) is the new reference trajectory for all subsequent magnets. All magnets between the last R1 matrix update and the type code 8 alignment entry are misaligned by the specified amount

The tolerances may he varied. Thus, type-vary code 8.111111 permits any of the six parameters ( 2 through 7 above) to be adjusted to satisfy whatever BEAM constraints may follow. (See the section under Type Code 10. for a discussion of the use of vary-codes.)

\section{Example No. 1: A misaligned hending magnet}

A bending magnet (including fringing-fields) misaligned by a known amount might be represented as follows:

$$
\begin{aligned}
& 3.0 \mathrm{~L}(1) . ; \\
& \text { 6. 0. 1. ; } \\
& 2.00 .0 ; 4.0 \text { L. B. n. ; } 2.00 .0 \text {; }
\end{aligned}
$$

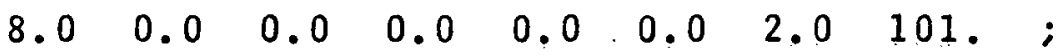

$$
\begin{aligned}
& 3.0 \mathrm{~L}(2) . ;
\end{aligned}
$$

This represents a known rotation of the bending magnet about the incoming beam direction (z-axis) by $2.0 \mathrm{mr}$. The result of this misalignment will be a definite shift in the beam centroid. The 6 . 0. 1. ; transform 1 update is necessary because of the use of the 2.0 0.0 ; entries hefore and after the bending magnet. The code number 
$x \times 1$. is required because the magnetic array (bending magnet + fringing-fields) consists of three type code elements instead of one.

Example No. 2: A misaligned bending magnet

A bending magnet having an uncertainty of $2 \mathrm{mr}$ in its angular positioning about the incoming beam (z-axis) would be represented as follows:

$$
\begin{aligned}
& 3.0 \mathrm{~L}(1) \text {; } \\
& \text { 6. 0. 1. ; } \\
& 2.00 .0 ; 4.0 \text { L. B. n.; } 2.00 .0 \text {; }
\end{aligned}
$$

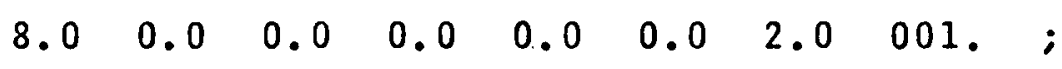

$$
\begin{aligned}
& 3.0 L(2) \text {; }
\end{aligned}
$$

The result of this misalignment will be an uncertainty in the knowledge of the outcoming beam centroid - hence to observe this, the input Beam Card should have zero phase space volume as follows:

$$
\text { 1.0 0. 0. 0. 0. 0. 0. } P(0) \text {; }
$$


Example No. 3: A misaligned quadrupole triplet

One typical use of the $R 2$ matrix is to permit the

misalignment of a triplet. For example, an uncertainty in the position of the following triplet

$$
\begin{array}{llll}
\text { 5. } & \text { 1. } & -8 . & 10 . ; \\
\text { 5. } & \text { 2. } & +7 . & \text { 10.; } \\
\text { 5. } & \text { 1. } & -8 . & \text { 10.; }
\end{array}
$$

may he induced by approprlate 8 . elements as noted.

$$
\begin{aligned}
& \text { 6. } 0.2 \text {. ; } \\
& \text { 5. 1. }-8.10 \text {, ; } \\
& \text { 6. 0. 1. : } \\
& \text { 5. 2. +7. 10.: } \\
& \text { 5. 1. -8. 10.; }
\end{aligned}
$$

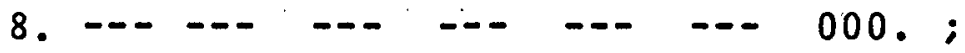

$$
\begin{aligned}
& \text { 8. -- - - - - - - - - } 001 \text {. ; } \\
& \text { 8. - - - - - - - - - }
\end{aligned}
$$

The first 8 . card in the list refers to misalignment of the third magnet only. The second 8 . refers to misalignment of the second and third magnets as a unit via the R1 matrix update (the 6. 0.1 ; entry). The last 8 . refers to misalignment of the whole triplet as a unit via the R2 matrix update (the 6. 0.2.; entry).

The BEAM card (Type Code 1.0 entry) should have zero phase space volume as shown in Example 2 above. 


\section{REPETITION Type Code 9.0}

Many systems include a set of elements that are repeated several times. To minimize the chore of input preparation, a 'repeat' facility has heen added.

There are two parameters:

$$
1 \text { - Type Code } 9 .
$$

2 - code ligit. If non-zero, it states the number of repetitions desired from the point it appears. If zero it marks the end of a repeating unit.

For example, a total bend of 12 degrees composed of four 3-degree bending magnets each separated by 0.5 meters could be represented by 9.0 4.; 4.-.; 3. 5 ; 9. 0.; Those elements ( $i n$ this case a hend and drift) between the 9. 4.; and 9.0.; would be employed four times.

There is no indication of the 9. cards in the printed TRAUSPORT output, save the repeated listing of the elements they control. Vary codes nay be used within a repeating unit in the usual fashion.

Repeat cards may be nested four deep. By "nesting" we mean a repeat within a repeat. An example is given below. 
Example of Nesting:

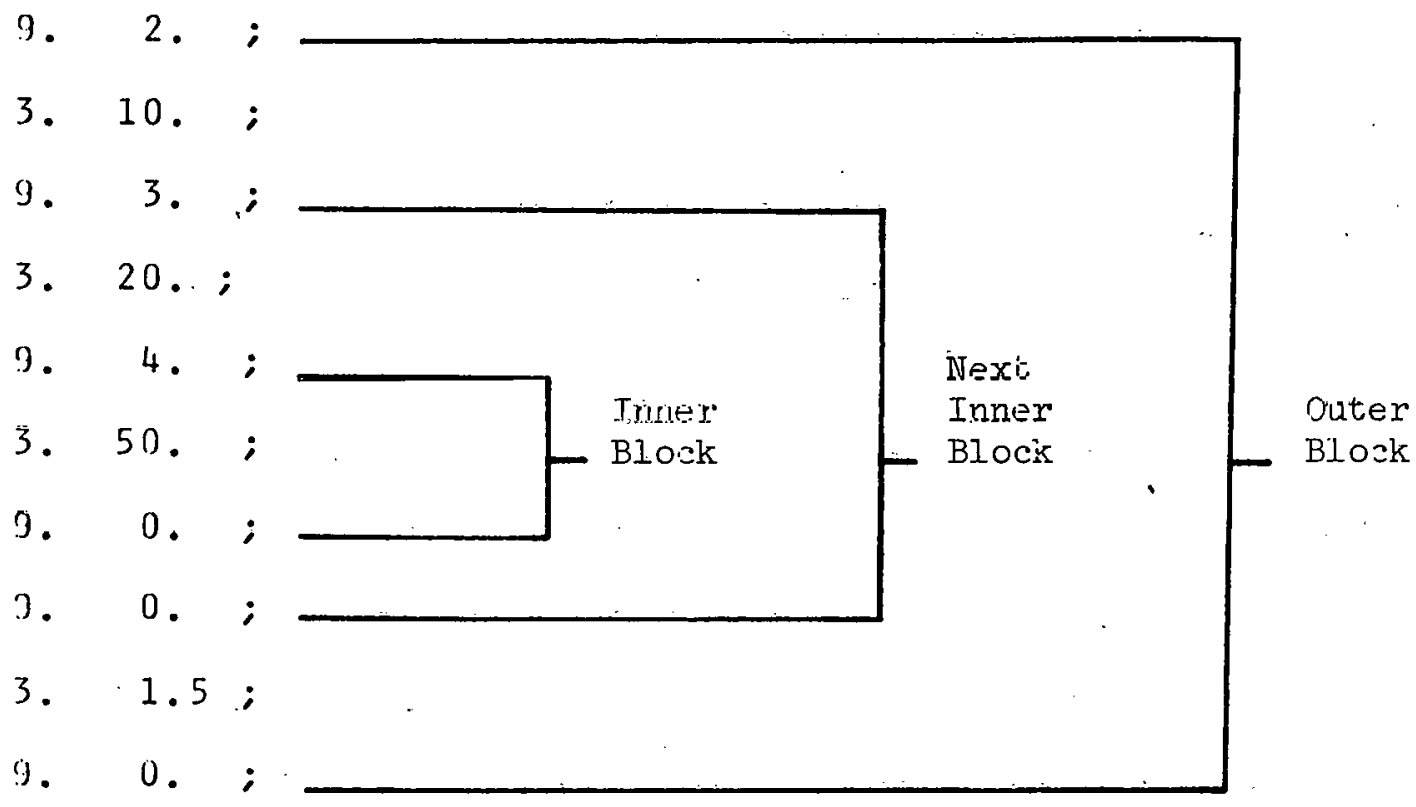

The total length of this sequence is:

$$
2 *(10+3 *(20+4 * 50)+1.5)=1343 .
$$




\section{FIRST-ORDER VARY CODES AND FITTING CONSTRAINTS}

Type Code 10.

TRANSPORT/360 has the capability to vary some (not all) of the physical parameters of the elements comprising a beam transport system in order to fit selected matrix elements of either the R1 or R2 transformation matrices or the BEAM (sigma) matrix to prescribed values. Special constraints are also available. The physical parameters to be varied are selected via 'Vary Codes' attached to the Type Codes of the elements comprising the system; and the fitting constraints imposed upon the $R$ and/or $\sigma$ matrix elements are selected via Type code 10. entries made at the location in the system where the constraint is to be imposed.

\section{Vary Codes}

Associated with the physical elements comprising a system is a vary code which specifies which physical parameters of the element may be varied. This code occupies the fraction portion of the type code specifying the element. It has one digit for each parameter, the digits having the same order in the code as the physical parameters have on the card. A ' 0 ' indicates the parameter may not be varied; a ' 1 ' that it may he. For instance, 3.0 is the combined type( 3 ) and vary code( 0 ) for a drifte length which is to remain fixed; 3.1 indicates a drift length that may be varied (by the virtue of the .1). The type code 4.010 indicates a bending magnet with a variable magnetic field. In punching the code 3.0, the zero need not be punched. In punching the 4.010 code, the first zero must be punched but the second zero need not be. 
The following paraneters may be varied ( 0 or 1 may be placed in positions marked $v$; only 0 may be placed in positions marked 0 ):

BEAM.... 1.vvVrvv - all components of the input heam may be varied.

ROTAT... 2.V - the pole-face angle of a bending magnet may be varied.

DRIFT... 3.v - the drift length may be varied.

BEND.... 4.0vV - the length may not be varied; the field (first $v$ ) and/or the n-value. (second $v$ ) may be varied.

QUAD.... 5.vVO - the length may be varied; the field may be, the aperture may not be.

ALIGN... 8. 8vvrvv0 - any of the alignment parameters may be varied.

MATRIX....14. VVVVVVO0 - any of the matrix elements may be varied.

SOLENOID.... 19.vv - the length and/or field may be varied.

BEAM ROTATION.... 20.v - the angle of rotation may be varied.

The use of the permissive 'may' rather than the imperative 'will' in discussing variables is meaningful. The program will. choose the parameters it will vary from among those that it may vary. In general it chooses to vary those parameters that have the greatest influence upon the conditions to be fit. 


\section{Coupled Vary Codes}

It is possible to apply the same correction to each of several variables. This may be done by replacing the digit 1 in the vary code with one of the digits 2 through 9. All variables whose vary digit has one of these values (and appears in the same position within the vary code) will receive the same correction. For example, the three type-vary codes $(5.02,5.01,5.02)$ might represent a symmetric triplet. The same correction will be made to the first and third quadrupoles, guaranteeing that the triplet will remain symmetric. Variables whose vary digits appear in different positions will not be tied together (the vary digit positions are completely independent). Thus, the codes $(8.666666,3.6)$ apply the same correction to the drift length as to the horizontal misalignment, but do not restrict the six misalignment parameters with respect to each other. (Such a combination would never arise in practice.)

If the vary digit 9 is used, the correction associated with the vary, digit 4 will be subtracted rather than added to this variable. Thus, the type-vary codes $(3.4,5.01,3.9)$ will slide the quadrupole back and forth within a prescribed drift length, but will not change the total drift length. The vary digits $(8$ and 3$)$, and $(y$ and 2$)$ are coupled in a similar manner as are ( 9 and 4$)$. This option may not be used with Type Code elements 1 . or 8 .

The total number of independent variables in a run is 1 imited to 10 by reasons of the mathematical method of fitting. So far as this limit is concerned, variables that are tied together count as one variable. Also note that variables within repeat elements (Type Code 9.) count only once. 


\section{RI MATRIX FITTING CONSTRAINTS}

There are five parameters to be specified when imposing a constraint upon the $(i, j)$ element of an Rl matrix.

$$
\begin{aligned}
& 1 \text { - Type Code } 10 . \text { (specifying that a fitting constraint } \\
& 2 \text { - collows) } \\
& 3 \text { - code digit }(j) . \\
& 4 \text { - desired value of the }(i, j) \text { matrix elenent. } \\
& 5 \text { - desired accuracy of fit (standard deviation). }
\end{aligned}
$$

\begin{tabular}{|c|c|}
\hline Desired Optical Condition & Typical Fitting Constraint \\
\hline point to point focus: & \\
\hline Radial plane $R(12)=0$ & 10. - - 2.0 \\
\hline $\begin{array}{l}\text { Transverse plane } R(34)=0 \\
\text { 'parallel' to point focus: }\end{array}$ & $.0001^{\prime} \mathrm{F} 2^{\prime} ;$ \\
\hline Radial plane $R(11)=0$ & 10. -1.1.0. .0001 'F3'; \\
\hline $\begin{array}{l}\text { Transverse plane } R(33)=0 \\
\text { achromatic beam: }\end{array}$ & $10 .-3.3 .0$ \\
\hline Radial plane $R(16)=R(26)=0$ & 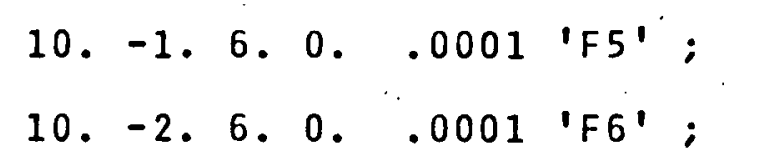 \\
\hline
\end{tabular}

Some typical R1 matrix constraints are as follows:

Note that any fitting constraint on an R1 matrix element is from the preceding update of the $R 1$ matrix. An $R I$ matrix is updated by a (6. 0 . 1. ;) entry or by any fitting constraint imposed upon the o (beam) matrix. 


\section{R2 MATRIX FITTING CONSTRAINTS}

There are five parameters to be specified when Imposing a constraint upon the $(i, j)$ element of an $R 2$ matrix.

1 - Type Code 10.

2 - Code digit $-(20+i)$.

3 - Code digit $(j)$.

4 - desired value of the $(i, j)$ matrix element.

5 - desired accuracy of fit (standard deviation).

Some typical R2 matrix constraints are as follows:

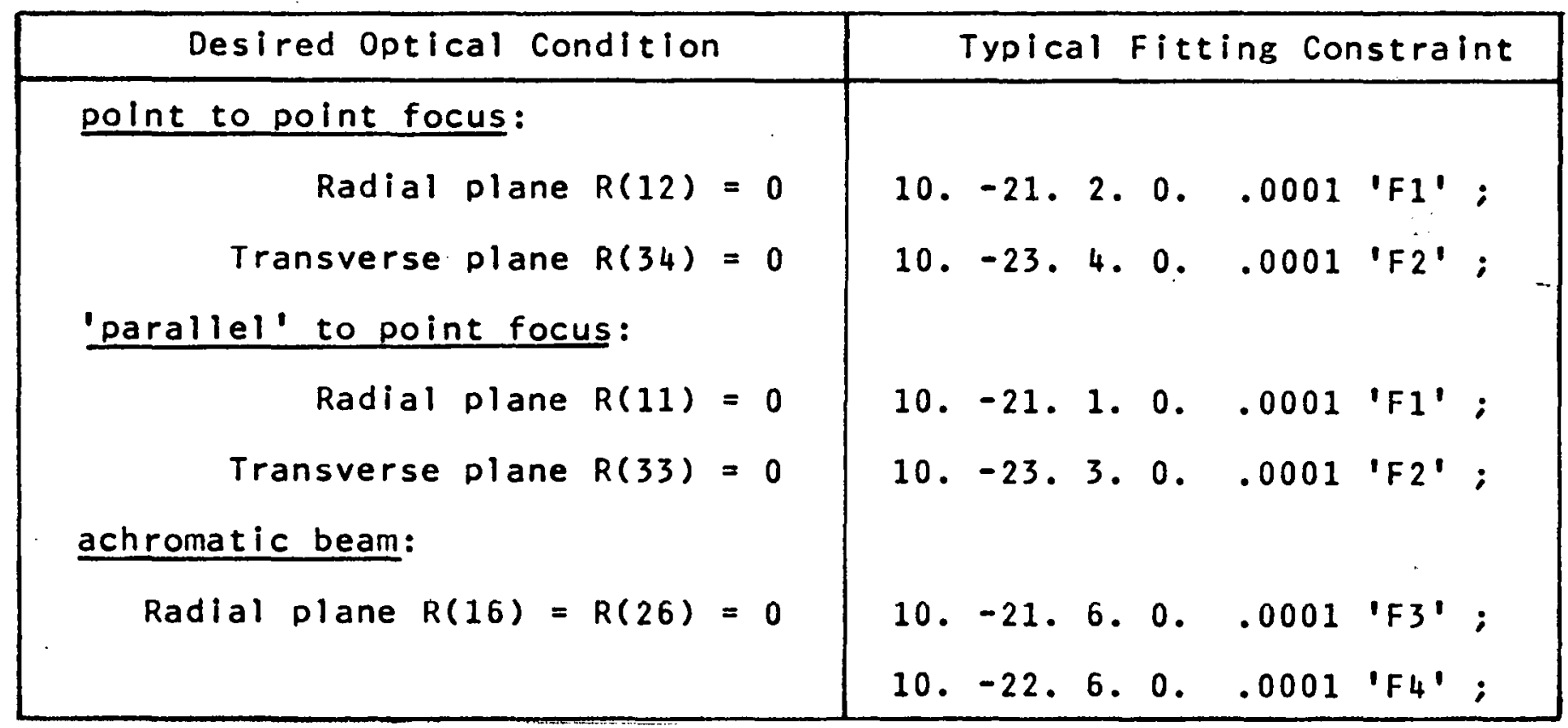

Note that the $R 2$ matrix is updated by a $(6,0.2$; ) type code entry only. 


\section{$\sigma(B E A M)$ MATRIX FITTING CONSTRAINTS}

There are five parameters to be specifled when imposing a constraint upon the $(i, j)$ element of a $\sigma$ (BEAM) matrix.

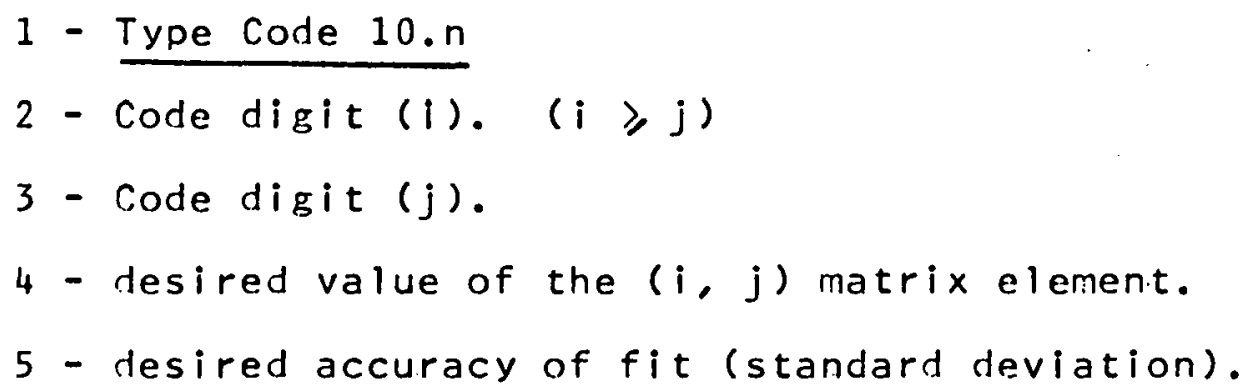

$(n)$ is normally zero or blank. If $n=1$, then entry 4 is taken to be a lower limit on the matrix element. If $n=2$, entry 4 is taken to be an upper limit. If $\mathbf{i}=j$, then the value inserted in entry 4 is the desired beam size $(\sigma(i i))^{1 / 2}$ e,g. $x(\max )=(\sigma(11))^{1 / 2}$ etc.

Hote:

Any constraint imposed upon the $\sigma$ (BEAM) matrix has an important side effect, it always updates the R1 matrix (but not the R2 matrix) at the location where the constraint is imposed.

Some typical $\sigma$ matrix constraints are as follows:

\begin{tabular}{|c|c|}
\hline Desired Optical Condition & Typical Fitting Constraint \\
\hline \multicolumn{2}{|l|}{ Beain Waist } \\
\hline Radial Waist $\sigma(21)=0$ & 10. 2. 1.0. .001 'Fl'; \\
\hline Transverse Waist $\sigma(43)=0$ & 10.4.3.0. .001 'F2'; \\
\hline Fit Beam size to $x(\max )=1 \mathrm{~cm}$ & 10.1.1.1. .001 ' $\mathrm{F}^{\prime}$; \\
\hline Fit Beam size to $Y(\max )=2 \mathrm{~cm}$ & 10.3.3.2. .001 ' $\mathrm{F}^{\prime}$ ': \\
\hline Limit Max beam size to $X=2 \mathrm{~cm}$ & $10.21 .1 .2 . .01$ \\
\hline Limit Min beam size to $Y=1 \mathrm{~cm}$ & $10.13 .3 .1 . .01$ ' $^{\prime} \mathrm{F}^{\prime}$; \\
\hline
\end{tabular}


In general, It will be found that achleving a satisfactory 'beam' fit with TRANSPORT is more difficult than achieving an $R$ matrix fit. When difficulties are encountered, it is suggested that the user 'help' the program by employing sequential (step by step) fitting procedures when setting up his data set. More often than not a "failure to fit" is caused by the user requesting the program to find a physically unrealizable solution. 
System Lengthi Constraint

A running total of the lengths of the various elements encountered is kept by the program and may be fit. The code digits are $i=0$. , $j=0$.

Thus the element $(10.0 .0 .150,5, ;)$ would make the length of the system prior to this element equal to' $150 \pm 5$ meters. Presumably there would be a variable drift length somewhere in the system. By redefining the cumulative length via the (16.6. L. ;) element, partial system lengths nay be accurnulated and fit. 


\section{AGS Machine Constraint*}

Provision has been made in the program for fitting the betatron phase shift angle, $\mu$, associated with usual AGS treatment of magnet systems.

In the horizontal plane: use code digits $i=-11 \ldots j=2$. and specify:

$$
\begin{aligned}
\Delta=\frac{1}{2 \pi} \cos ^{-1}\left[0.5\left(R_{11}+R_{22}\right)\right] & =\frac{\mu}{2 \pi} \text { (horiz) } \\
& =\text { freq/(no. of periode) } .
\end{aligned}
$$

In the vertical plane: $i=-13 \ldots, j=4 .$, and

$$
\Delta=\frac{1}{2 \pi} \cos ^{-1}\left[0.5\left(R_{33}+R_{44}\right)\right]=\frac{\mu}{2 \pi} \text { (vert) }
$$

For example, if there are 16 identical sectors to a proposed AGS machine and the betatron frequencies per revolution are to be 3.04 and 2.14 for the horizontal and vertical planes respectively, then the last element of the sector should be followed by the constraints:

$$
\begin{aligned}
& \text { 10. -11. 2. .190.0001; } \\
& \text { 10. }-13.4 . .134 .0001 \text {; } \\
& \text { i.e. } \frac{3.04}{16}=0.190 \text { and } \frac{2.14}{16}=0.134
\end{aligned}
$$

* See Courant and Snyder "Theory of the Alternating-gradient synchrotron". Annals of Physics 3; ppl-48 (1958). 
For example: A typical data listing might be:

$$
\begin{array}{lll}
9.0 & 16.0 ; \\
5.01 & \ldots ; \\
3.0 & -\ldots ; \\
5.01 & -\cdots ;
\end{array}
$$

10. -11. 2. 0.190 .0001 ;

10. -13. 4. 0.134 .0001 ;

$9.0 \quad 0.0 ;$ 


\section{First Moment Constraint}

Misalignments and second order effects cause the center (centroid). of the phase ellipsoid to be shifted from the reference trajectory, i.e., they cause the beam to have a non-zero first moment. The first moments appear in a vertical array to the left of the vertical array which give the $\sqrt{\sigma}(i i)$ 's The units of the corresponding quantities are the same.

It is perhaps helpful to emphasize that the origin always lies on the reference trajectory. First moments refer to this origin. However, the ellipsoid is defined with respect to its center, so the covariance matrix, as printed, defines the second moment about the mean.

First moments may be fitted. The code digits are $i=7$. and $j$, where $j$ is the index of the quantity being fit. Thus 10.7.1..1.01; constrains the horizontal (1.) displacement of the ellipsoid to be $0.1+0.01 \mathrm{~cm}$.

This constraint is useful in deriving the alignment tolerances of a system or in warning the system designer to offset the element in order to accommodate a centroid shift. 


\section{Internal Constraints}

A set of upper and lower bounds on the value of each type of paraneter is in the memory of the program. If a correction is computed for a parameter which would take its value outside this range, it is reset to the 1 init of the range, and a constraint constructed to keep it within range. The current limits are:

\begin{tabular}{c|c} 
Type Code & Limits \\
\hline 3.0 & $0.1<$ drift $<1000(\mathrm{~m})$ \\
5.0 & $.01<$ quad length $10(\mathrm{~m})$ \\
5.0 & $-14<$ quad field $<14(\mathrm{~kg})$ \\
4.0 & $-20<$ bend field $20(\mathrm{~kg})$ \\
4.0 & $-500<$ bend gradient $<500$ \\
2.0 & $-60<$ pole-face rotation $60(\mathrm{deg})$ \\
8.0 & $-1<$ alignment displacement $25(\mathrm{~cm})$ \\
8.0 & $-50<$ alisnment rotation $<50(\mathrm{mr})$ \\
1.0 & $0.01<$ input beam $<1000($ for all parameters) \\
20.0 & $-360<$ beam rotation $360($ deg $)$
\end{tabular}

These limits apply only when a parameter is being varied. Fixed values that exceed this range may he used as desired.

These constraints were included to avoid physically meaningless solutions. However, they are ratiner ineffective since systems that require values outslide these limits usually have some basic desirn difficulty.

The limits are not adjusted for different input units. So if drift lengths are expressed in inches, the effective 1 imits are $0.1<\mathrm{drift}$ $<1000$ (in.) etc. 


\section{ACCELERATION Type Code 11.0}

An energy gain is reflected in both the divergence and the width of the beam. This element provides a simulation of a traveling wave linear accelerator energy gain over a field free drift length. (i.e. no externally applied magnetic field)

There are five parameters:

$$
\begin{aligned}
& 1 \text { - Type Code } 11 . \\
& 2 \text { - accelerator length (meters). } \\
& 3 \text { - energy gain (GeV). } \\
& 4 \text { - } \varphi \text { (phase lag in degrees). } \\
& 5 \text { - } \lambda \text { (wavelength in cms). }
\end{aligned}
$$

The new beam energy is printed as output.

The energy of the reference trajectory is assumed to increase linearly over the entire accelerator length. If this is not the case, an appropriate model may be constructed by combining separate 11 . elements. An 11. element with a zero energy gain is identical to a drift length.

None of the parameters may he varied.

Second order matrix elements have not been incorporated in the program for the accelerator section. 
ACCELERATOR SECTION MATRIX

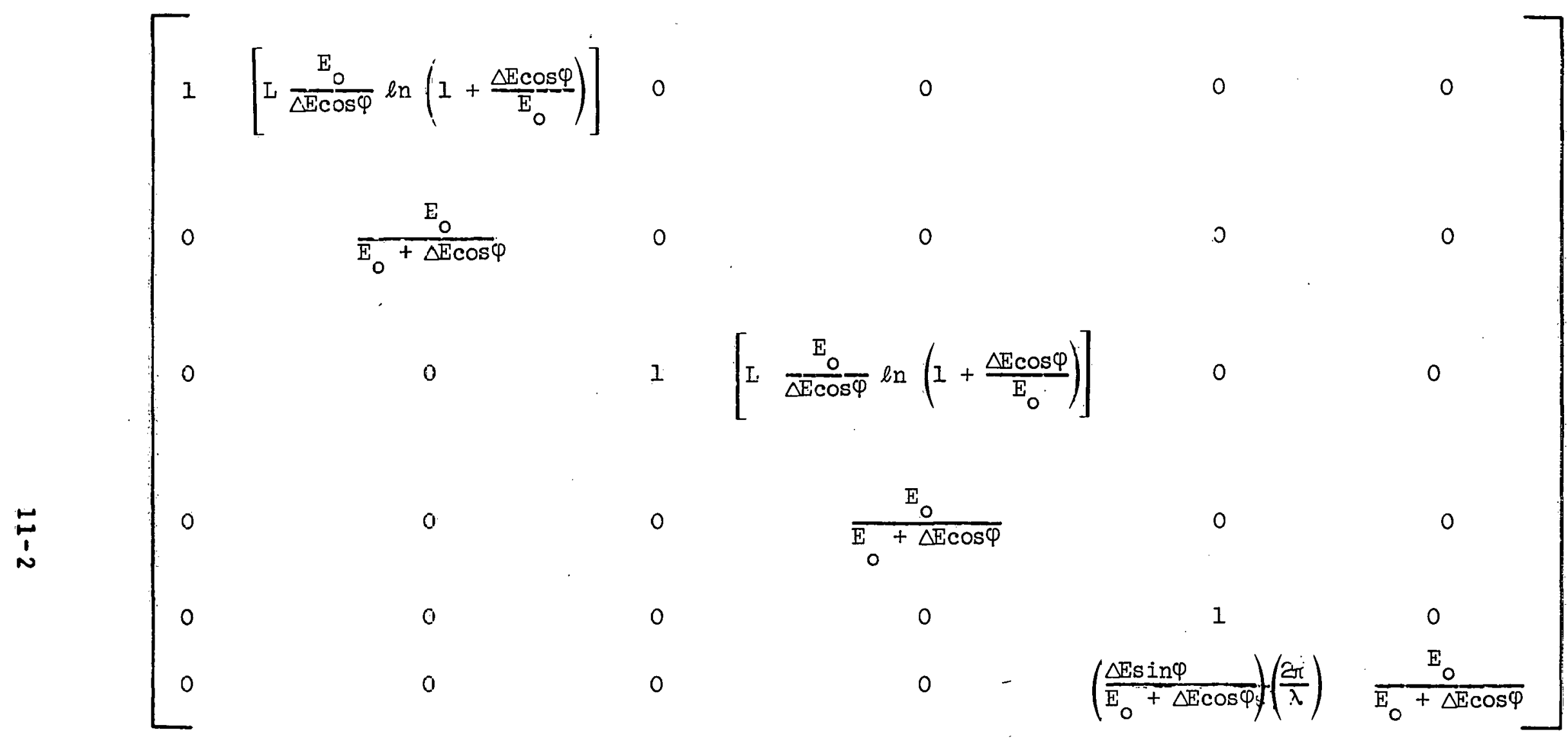

Definitions:

$L=$ effective length of accelerator sector

$\mathrm{E}_{\mathrm{O}}=$ pariicle energy at start of sector

$\Delta E=$ energy gain over sector length

$\varphi=$ phase lag of the reference particle behind the crest of the accelerating wave. i.e., if $\varphi$ is positive then for some $z>0$ the particles having this ralue are riding the crest of the wave. The units of $\varphi$ are degrees.

$\lambda=$ wave length of accelerating wave. The units of $\lambda$ are those of $z$ (normally $\mathrm{cm}$ ). This matrix element assumes that $E_{c} \gg m_{0} c^{2}$ (fully relativistic). 


BEAM (ROTATED ELLIPSE) Type Code 12.0

To allow the output beam from some point in a system to become the input heam of some succeeding system, provision has been made for. re-entering the correlation matrix which appears as a triangular matrix in the beam output. (See section under Type Code 1.0 and/or the appendix for definitions.)

There are 16 parameters:

$$
1 \text { - Type Code } 12 .
$$

2 to 16 - the 15 correlations ( $r(i j)$ 's) among the 6 beam components - in the order printed (by rows).

Several cards may be used to insert the 15 correlations, if necessary.

Since this element is solely an extension of the beam input, a 12 . element must be preceded by a 1 . (heam) element entry. 


\section{OUTPUT PRINT CONTROL INSTRUCTIONS TyPe Code 13.0}

A number of control codes which transmit output print instructions to the orogram have been consolidated into a single type code:

There are two parameters:

$$
1 \text { - Type Code } 13 .
$$

The effects of the various code numbers will be described below (not in numerical order).

PRINTED OUTPUT CONTROLS $1, \ldots 2,2,34,24$.

Several codes are available to control various aspects of the printed output. Most type codes produce a line of output that advertises their existence. Those that do not, usually have an obvious effect upon the remainder of the output and thus make their presence clear.

(13. 2. ;): This code instructs the program to suppress the printing of the beam $(\sigma)$ matrix.

(13. 1. ;): This code temporarily overrides the (13. 2. ;) suppress code. It causes a single beam matrix to be printed at that point in the sys tem.

(13. 3. ;): This code permanently overrides the $(13,2$, i) suppress code and restores the normal code in which every heam matrix is printed.

(13. 4. ;): This code instructs the program to print the current transformation matrix R1 (TRANSFORM 1). If the program is computing a second order matrix, this second order transformation matrix will he included in the print out. This matrix is cumulative from the last RI (TRANSFORM 1) update. The units of the elements of the printed matrix 
are consistent with the input units associated with the Type Code 1. (BEAM) entry.

(13. $24 . ;):$ This code causes the TRANSFORM 2 matrix, R2, to be printed. The format and units of $R 2$ are identical with those of $R 1$ which is printed by the (13.4. ;) code. The calculation of the R.2 matrix is started by a 6. 0. 2. ; entry (see Type Code 6.).

The units of the tabulated matrix elements in either the lst order $R$ or sigma matrix or second order T matrix of a TRAMSPORT printout will correspond to the units chosen for the BEAM card. For example, the $R(12)=(x / \theta)$ matrix element will have the dimensions of crns/mr; and the $T(236)=(\theta / y \delta)$ matrix element will have the dimensions mr per cm per percent $\triangle P / P$ and so forth.

PUNCHED OUTPUT CONTROLS 29, 30, 31, 32, 33, 34, 35, 36

If the control is equal to 29 , all of the terms in the first order matrix and the $x$ and $y$ terms of the second order matrix are punched.

If the control is equal to 30 , all of the terms of the first order matrix and all second order matrix elements are punched out.

If the control, $n$, is greater than 30, all of the first order terms are punched and the second order matrix elements which correspond to $(n-30$.$) i.e.., if n=32$, the second order theta matrix elements are punched out. If $n=31$, the second order $x$ matrix elements are punched and so forth. 


ARB ITRARY TRANSFORMATION INPUT TyPe Code 14.0

To allow for the use of empirically determined fringing fields and other specific (perhaps non-phase-space-conserving), transformations, provision has been made for reading in an arbitrary transformation matrix. The first-order $6 \times 6$ matrix is read in row by row.

There are eight parameters for each row of a first-order matrix entry:

$$
1 \text { - Type Code } 14 .
$$

2 to 7 - the six numbers comprising the row. The units must be those used to print the transfer matrix; in other words, consistent with the BEAM input/output.

8 - row number (1. to 6.)

A complete matrix must be read and applied one row at a time. Rows that do not differ from the unit transformation need not be read.

For example, (14.-..1.9 0.0.0.0.2.;) introduces a transformation matrix whose second row is given but which is otherwise.a unit matrix. Note that this transformation does not conserve phase space because $R(22)=0.9$ i.e. the determinant of $R \neq 1$.

Any of the components of a row may be varied; however, there are several restrictions.

Type Code 14. elements that immediately follow one another will all be used to form a single transformation matrix. If distinct matrices are desired, another element must be inserted to separate the Type Code 14. cards. Several do-nothing elements are available; for example, a zero length drift (3.0. ;) is a convenient one. 
Note that

$$
\left(\begin{array}{ll}
1 & 0 \\
a_{21} & a_{22}
\end{array}\right)\left(\begin{array}{ll}
a_{11} & a_{12} \\
0 & 1
\end{array}\right) \neq\left(\begin{array}{ll}
a_{11} & a_{12} \\
a_{21} & a_{22}
\end{array}\right)
$$

Hence, a matrix formed by successive $14 .(3,0 . ;), 14$. - elements is not always equal to the one formed by leaving out the $(3.0 . ;)$ element.

If components of a 14. card are to be varied it must be the last 14. card in its matrix. This will force a matrix to be split into factors if more than one row has variable components.

If it is desired to read in the second-order matrix coefficients for the $i$ th row, then the following 22 additional numbers may be read in: ** 9 - continuation Code 0 .

10 to 30 - the twenty-one coefficients:

$T(i 11) T(i 12) T(i 13) T(i 14) T(i 15) T(i 16)$

$T(i 22) T(i 23) T(i 24) T(i 25) T(i 26) T(i 33)$

$T(i 34) T(i 35) T(i 36) T(i 44) T(i 45) T(i 46)$

$T(i 55) T(i 56) T(i 66)$

in that order, where $i$ is the row number. It is necessary to read in the first order matrix row which corresponds to the second order natrix row being read in.

**This feature frees the user from making repetitive, expensive, second-order runs through a fixed portion of his systern while experimenting with other magnets. This is done by reading the full matrix of this portion (obtained from a previous run) back into the machine as a single "arbitrary matrix." 
As in the first-order case, full rows not different from the identity matrix (i.e., $R(i i)=1$, all other $R(i j)=0$, and all $T(i j k)=0$ ) need not be read in. 


$$
\text { INPUT - OUTPUT UNITS TyPe Code } 15.0
$$

TRANSPORT is designed with a standard set of units that have been used throughout this manual. However, to accommodate other units conveniently and to relieve certain parameter-fitting problems, provision has been made for redefining the units to be employed. This is accomplished with the following type code elements.

There are 4 parameters to be specified:

1 - Type Code 15.

2 - code digit

3 - the ahbreviation of the unit. (see examples below) This will be printed on the output listing. It must he enclosed in single quotes and is a maximum of 3 characters long ( 4 for energy).

4 - the scale factor (if needed) that multiplies a parameter expressed in the new units to convert it to the approprlate reference unit. The examples and tables helow should clarify this point. 
The various units that may be changed are:

\begin{tabular}{|c|c|c|c|}
\hline $\begin{array}{l}\text { Code } \\
\text { Digit }\end{array}$ & Quantity & $\begin{array}{l}\text { Standard } \\
\text { Reference Unit }\end{array}$ & $\begin{array}{l}\text { Symbols used } \\
\text { in SLAC }-75 \text { ". }\end{array}$ \\
\hline 1. & $\begin{array}{l}\text { horizontal and vertical } \\
\text { transverse dimensions }\end{array}$ & $\mathrm{cm}$ & $x, y$ \\
\hline 2. & $\begin{array}{l}\text { horizontal and vertical } \\
\text { angles }\end{array}$ & $\mathrm{mr}$ & $\theta, \varphi \cdot \cdots$ \\
\hline 3. & vertical beam extent (only) & $\mathrm{cm}$ & y \\
\hline 4. & $\begin{array}{l}\text { vertical beam divergence } \\
\text { (only) }\end{array}$ & $\mathrm{mr}$ & $\varphi$ \\
\hline 5. & pulsed beam length & $\mathrm{cm}$ & $\ell$ \\
\hline $\begin{array}{l}6: \\
7 .\end{array}$ & $\begin{array}{l}\text { momentum spread } \\
\text { undefined }\end{array}$ & $\%(P C)$ & $\delta$ \\
\hline 8. & length (longitudinal.) & meters $(M)$ & $\mathrm{t}$ \\
\hline 9. & magnetic fields & $\mathrm{kg}$ & B \\
\hline 10 & mass & electron mass & m \\
\hline 11 . & momentum & $\mathrm{GeV} / \mathrm{c}$ & $P(0)$ \\
\hline
\end{tabular}

Units are not normally restored at the end of a run. Once changed, they remain the same for all succeeding runs in an input deck, unless specifically changed. The units may be reset to standard units at any time by inserting a $(15 . ;)$ Type Code entry. The 15. elements are normally the first cards in a deck, (immediately following the title card and the 0 or 1 indicator card) or perhaps the last cards--in the event units are being restored to avoid disturbing succeeding runs. 
Example: To change length to feet, width to inches, and momentum to MeV/c, add to the front of the deck the elements

$$
\begin{aligned}
& \text { 15. 8. ' } F T^{\prime} 0.3048 ; \\
& \text { 15. 1. 'IN' 2.54; } \\
& \text { 15. 11. 'MEV' 0.001; }
\end{aligned}
$$

The scale factor, 0.3048 , multiplies a length expressed in the new unit, feet, to convert it to the reference unit, meters etc.

For the conventional units listed below, it is sufficient to stop with the label: (the conversion factor is automatically calculated by the program). If units other than those listed below are desired, then your label and the appropriate conversion factor must be included. If the automatic feature is used, there must be no blank spaces between the quotes and the labels. 
liSPUT-OUTPUT UNITS (Type Code 15.0)

CONVERSION FACTORS FCR DIMENSION CHANGES VS. CODE DIGIT AND LABEL

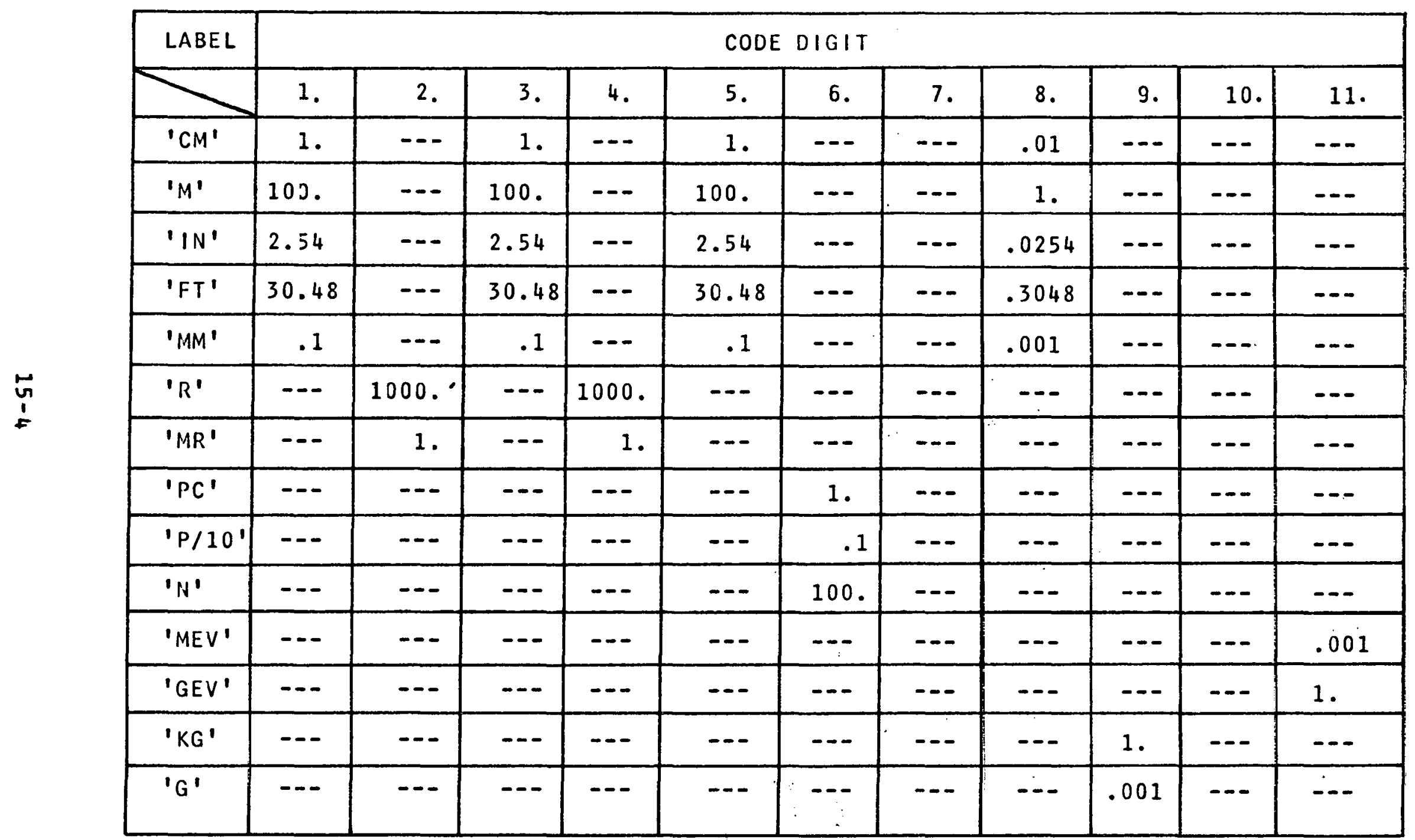


SPECIAL INPUT PARAMETERS

Type Code 16.0

A number of constants are used by the program which do not appear as parameters on any of the other type code element cards. A special element has been provided to allow the designer to set their values. These special parameter entries must always precede the physical element (s) to which they apply. Once introduced, they apply to all succeeding elements in a data set unless reset to zero or to new values.. There are three parameters:

1 - Type Code 16.

2 - code digit

3 - value of constant

$16-1$ 
A number of such constants have been defined in this manner. All have a normal value that is initialized at the beginning of each run.

\section{Code Digit}

1 .

$\epsilon(1)$ - a second-order measure of magnetic field inhomogeneity in bending magnets. If

$$
B(x)=B(0)\left[1-n\left(\frac{x}{\rho}\right)+\beta\left(\frac{x}{\rho}\right)^{2}-\cdot \cdot \cdot\right]
$$

is the field.expansion in the median $(y=0)$ plane). Then $\epsilon(1)$ (dimensionless) is defined as

$$
\epsilon(1)=\beta\left(\frac{x}{\rho_{0}}\right)^{2}
$$

evaluated at $x=1$ (in units of horizontal beam width - normally $\mathrm{cm}$.$) . This parameter$ affects secondourder calculations only. Normally the value is 0 .

3. $(M / m)$ - Mass of the particles comprising the beam, in units of the electron mass; normally 0. A non-zero mass introduces the dependence of pulse length on velocity, an important effect in low energy pulsed beams. 
4. W/2 - Horizontal half-aperture of bending magnet, in the same units as horizontal beam width. normally 0. (i.e.. effect of finite horizontal half aperture is ignored).

5. $g / 2$ - Vertical half-aperture of bending magnet, in the same units as vertical beam height; this parameter must be inserted if the finite extent of the fringing fields upon transverse focusing is to be taken into account. (see Type Codes 2.0 and 4.0 as a cross reference) normally 0 .

6.

$L$ - Cumulative length of system, in the same units as system length. It is set to'zero initially, then increased by the length of each element, and finally printed at end of system. This element allows the cumulative length to be reset as desired.

12. $1 / R 1$ - Where $R 1$ is the radius of curvature (in units of longitudinal length, normally meters) of the entrance face of bending magnets. (See Fig. 748 A15 page 2-4)

13. $1 / R 2$ - Where $R 2$ is the radius of curvature (in units of system length such as meters) of the exit face of bending magnets. (See Fig. 748A15, page 2-4) 


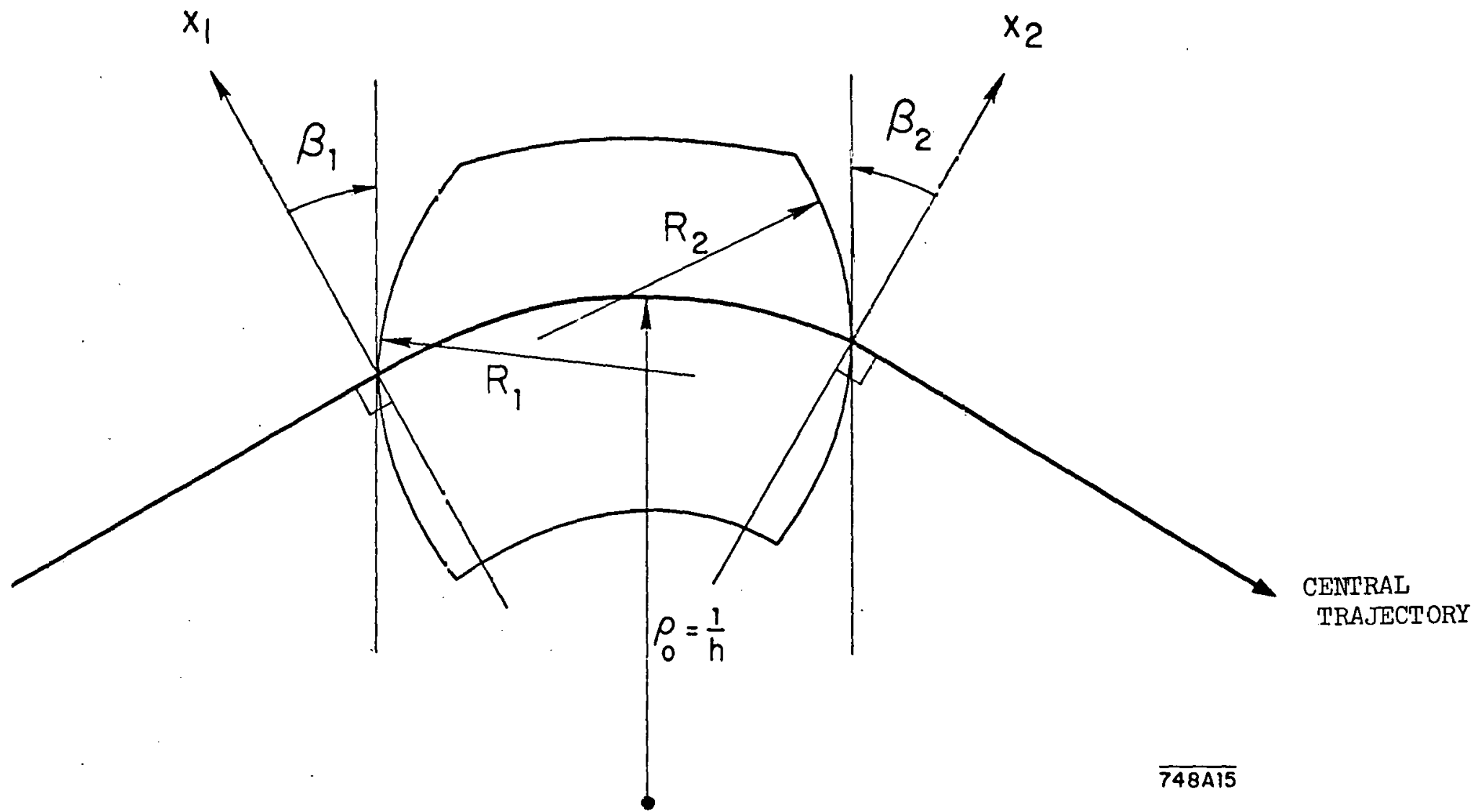

FIELD 3OUNDARITS FOR BENDING MAGNETS

The TRANSPORT sign conventions for $x, \beta, R$ and $h$ are all positive as shown in the figure. The positive $y$ direction is out of the paper. Positive $\beta^{\prime}$ 's imply transverse focusing. Positive R's (convex curvatures) represent negative sextupoie components of strength $S=\left(-\frac{h}{2 R}\right) \sec ^{3} \beta . \quad$ (See SLAC-75, page 71 ). 
R1 and R2 affect the system only in second-order, creating an effective sextupole component in the neighborhood of the magnet. If the parameters are not specified; they are assumed to be zero, i.e., no curvature and hence no sextupole component.

Programming for Fringe-Field Corrections to the Transverse Focal Length

Insert: 16.5 . $\mathrm{g} / 2$ elemient, then

16. 7. $k(1) \cdot[k(1)=1 / 2$ if 16.7 . element is not inserted but 16.5 . element is inserted.]

16. 8. $k(2) \cdot \mid k(2)=0$ if 16.8 . element is not inscrted.]

where

$$
\frac{1}{f}=-\frac{1}{\rho} \tan (\beta-\psi)
$$

and

$$
\psi=\frac{1+\sin ^{2} \beta}{\cos \beta} \cdot\left[k_{1}\left(\frac{g}{\rho}\right)-k_{2}\left(\frac{k_{1} g}{\rho}\right)^{2} \tan \beta\right]
$$

Fringing-field corrections will only be made where the pole-face rotation element ( $2 . \quad \beta$. ;) has been inserted in conjunction with a bending magnet ( $4 . .$. element.)

* See SLAC-75, page 74 for a discussion of and a definition. of $k(1)$. 
Typical values of $k 1$ and $k 2$ are given below for three types of fringing field boundaries:

a) a linear drop-off of the field.

b) a clamped "Rogowski" fringing-field.

and c) a "square-edged" non-saturating magnet.

\begin{tabular}{|c|c|c|c|}
\hline Model & Linear & Clamped Rogowski & Square Edged Magnet \\
\hline$k 1$ & $1 / 6$ & 0.3 to 0.4 & 0.45 \\
$k 2$ & 3.8 & 4.4 & 2.8 \\
\hline
\end{tabular}




$$
\text { Tilt-to-Focal Plane (16. 15. } \alpha . ;) \text { element }
$$

Very often it is desired to have a listing of the second order aberrations along the focal plane of a system rather than perpendicular to the optic axis i.e. along the $x$ coordinate. If the focal plane makes an angle alpha with respect to the $x$ axis (measured clockwise) then provision has been made to rotate to this focal plane and print out the second-order aberrations. This is achieved by the following procedures:

Alpha is the focal-plane tilt angle (in degrees) measured from the perpendicular to the optic axis ( $\alpha$ is normally zero).

The programming procedure for a tilt in the $x$ (bend)-plane (rotation about $y$ axis) is:

$$
\begin{aligned}
& \text { 16. 15. } \alpha . \text {; } \\
& \text { 3. } 0 . ; \text { (a necessary do-nothing element) } \\
& \text { 13. } 4 . ; \\
& \text { 16. } 15 .-\alpha . \text {; (Rotate back to zero) } \\
& \text { 3. } 0 . \text {; (a necessary do-nothing element) } \\
& \text { 16. } 15.0 . \text {; (to turn off rotation element) }
\end{aligned}
$$


The programming procedure for a tilt in the y-plane (rotation abou $x$ axis) is:

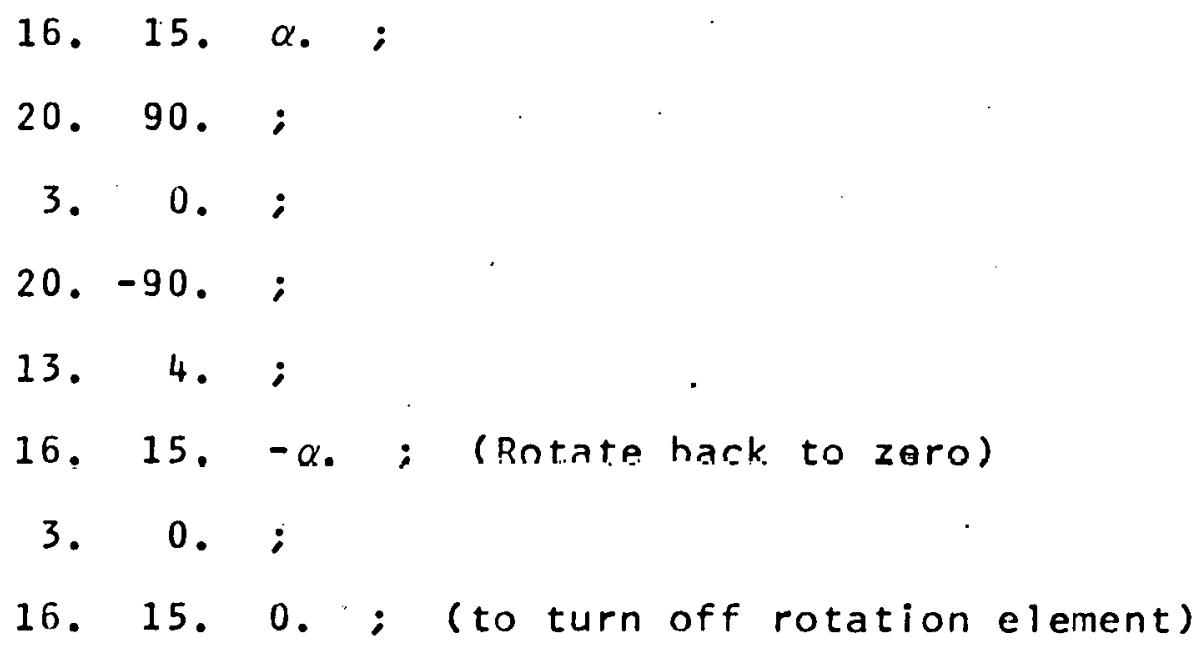




SECOND ORDER CALCULATION Type Code 17.0

A second order calculation may be obtained provided neither parameter fitting nor alignment elements are employed. A special element instructs the program to calculate the second order matrix elements. It must be inserted immediately following the beam ( 1 . element).

Only one parameter need be specified:

1 - Type Code 17. ; (signifying a 2nd order calculation is to be made)

The values of the BEAM (sigma) matrix components may be perturbed from their first order value by the second order aberrations. To print out the second order T matrix terms at a given location in the system, the (13. 4. ;) print control card is used. The update rules are the same as those for the RI first-order matrix.

See SLAC-75 for definitions of subscripts in the second order T(ijk) matrix elements.

Second-order matrices are included in the program for quadrupole, bending magnets (including fringing-fields), sextupoles, and solenoids. They have not been calculated for the acceleration (Type Code 11.0) element. 


\section{SEXTUPOLE TyPe Code 18.0}

Sextupole (Hexapole) magnets are used to modify second-order aberrations in beam transport systems. The action of a sextupole on beam particles is a second and higher order effect, so in first order runs (absence of the 17. card) this element will act as a drift space.

There are 4 parameters:

1 - Type Code 18.

2 - effective length (ineters).

3 - field at pole tips $(\mathrm{kg})$. Both positive and negative fields are possible (see figures below).

4 - Half-aperture $(\mathrm{cm})$. Radius of circle tangent to pole tips.

other orientations of the sextupole may be obtained using the beam rotation element (Type Code 20.). None of the parameters of the sextupole may he varied since it is a second order element.

See SLAC-75 for a tabulation of sextupole matrix elements.

The TRANSPORT input format for a typical data set is:

18. L. B. a. ' 


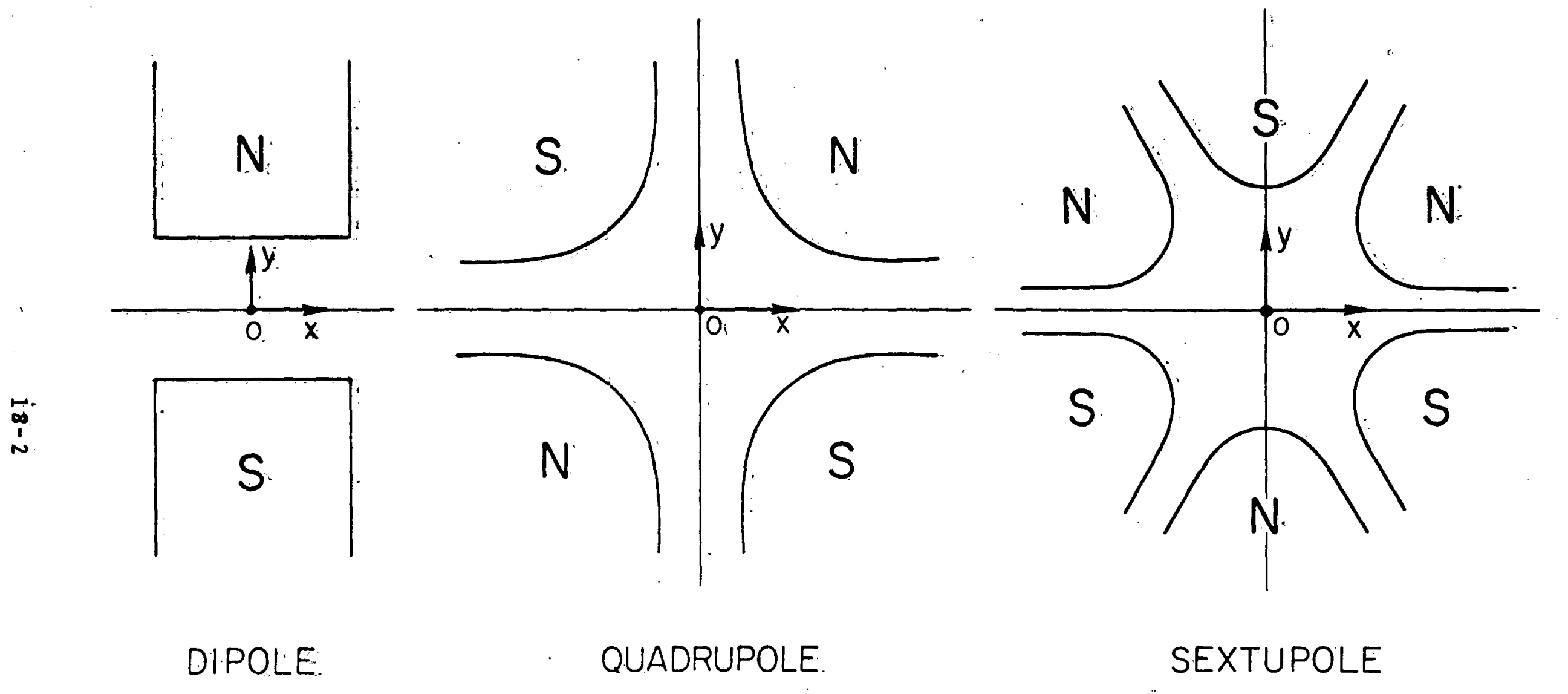

ILLUSTRATION OF THE MAGNETIC MIDPEANE ( $x$ AXIS) FOR DIPOLE, QUADRUPOLE AND SEXTUPOLE ELEMENTS. THE MAGNET POIARITIES INDICATE MUETIFOIE ELENENTS THAT ARE POSITIVE WITH RESPECT TO EACH OTHER. 


\section{SOLENOID. Type Code 19.0}

The solenoid is most often used as a focusing element in systems passing low energy particles. Particles in a solenoidal field travel helical trajectories. The solenoid fringing field effects necessary to produce the focusing are included.

There are three parameters:

1 - Type Code 19.0

2 - effective length of the solenoid (meters)

3 - the field $(\mathrm{kg})$. A positive field by convention points in the direction of positive $z$ for positively charged particles.

The length and the field may be varied by using 19.1 or 19.01 vary codes respectively. Both 1s.t and 2 nd order matrix calculations are available for the solenoid.

A typical input format is:

19. L. B. $\sqrt{\text { l }}$; 


\section{ST ORDER \\ SOLENOID MATRIX ELEMENTS}

Solenoid R Matrix

Definitions: $L=$ effective length of solenoid.

$$
\begin{aligned}
K= & B(0) /(2 B \rho) \text { where } B(0) \text { is the field inside the } \\
& \text { solenold and }(B \rho) \text { is the (momentum) of the } \\
& \text { Central Trajectory. } \\
C= & \cos K L \\
S= & \sin K L
\end{aligned}
$$

For a derivation of this transformation see SLAC-4 report by R. Helm.

Alternate Forms of Matrix Representation of the Solenoid.

$$
R \text { (Solenoid) }=\left(\begin{array}{l|l|l|l|l|l}
c^{2} & \frac{1}{\mathrm{~K}} S c & s c & \frac{1}{\mathrm{~K}} S^{2} & 0 & 0 \\
-K S C & c^{2} & -k s^{2} & s c & 0 & 0 \\
-S c & -\frac{1}{K} S^{2} & c^{2} & \frac{1}{K} S c & 0 & 0 \\
K S^{2} & -s c & -k S C & c^{2} & 0 & 0 \\
0 & 0 & 0 & 0 & 1 & 0 \\
0 & 0 & 0 & 0 & 0 & 1
\end{array}\right)
$$


Rotating the transverse coordinates about the $z$ axis by an angle $=-K L$ decouples the $x$ and $y$ lst order Terms, i.e.

$$
R(-K L) \cdot R(\text { Solenoid })=\left(\begin{array}{l|l|l|l|l|l|l}
C & \frac{1}{K} S & 0 & 0 & 0 & 0 \\
-K S & C & 0 & 0 & 0 & 0 \\
0 & 0 & C & \frac{1}{K} S & 0 & 0 \\
0 & 0 & -K S & C & 0 & 0 \\
0 & 0 & 0 & 0 & 1 & 0 \\
0 & 0 & 0 & 0 & 0 & 1
\end{array}\right)
$$




\section{BEAM ROTATION Type Code 20.0}

The transverse beam coordinates $x$ and $y$ may be rotated through an angle $\alpha$ ahout the $z$ axis (the axis tangent to the central trajectory at the point in question).** Thus a rotated bending magnet, quadrupole, or sextupole may be inserted into a beam transport system by preceding and following the element with the approprlate coordinate rotation. (See examples below). The positive sense of rotation is clockwise about the positive $z$ axis.

There are two parameters to he specified for a Beam Rotation:

1 - Type Code 20. (signifying a beam coordinate rotation)

2 - the angle of rotation $\alpha$. (degrees)

The angle of rotation may he varied in a ist order fitting (see vary Codes Section 10.)

\section{Note}

This transformation assumes that the units of ( $x$ and $y$ ) and ( $\theta$ and $\varphi$ ) are the same. This is always true unless a 15. 3. or a 15. 4. type code has been used.

** See SLAC-75, page 45 and Fig. 4, page 12 for definitions of $x, y$, and $z$ coordinates. 


\section{Examples:}

For a bending magnet, the Beam Rotation matrix may be used to specify a rotated magnet.

\section{Example No. 1}

A bend up is represented by rotating the $x$, $y$ coordinates by -90.0 degrees as follows:

Labels (not to exceed 4 spaces) if desired

$$
\begin{aligned}
& \text { 20. }-90 \text {. } 1 \text {; } \\
& 2.0 \beta(1) .1 \text {, ; } \\
& 4.0 \text { L. B. n. ' ' ; } \\
& 2.0 \beta(2) . \quad 1, \\
& \text { 20. +90. ' ' ; (returns coordinates to their initial } \\
& \text { orientation) }
\end{aligned}
$$

A bend down is accomplished via a +90 degree rotation.

$$
\begin{array}{lll}
20 .+90 . & 1 \text {; } \\
2 . \\
\text { 4. } \\
2 . \\
20 . \quad-90 . \quad 1,
\end{array}
$$

A bend to the left (looking in the direction of beam travel) is accomplished by rotating the $x$, y coordinates by 180 degrees, e.f.,

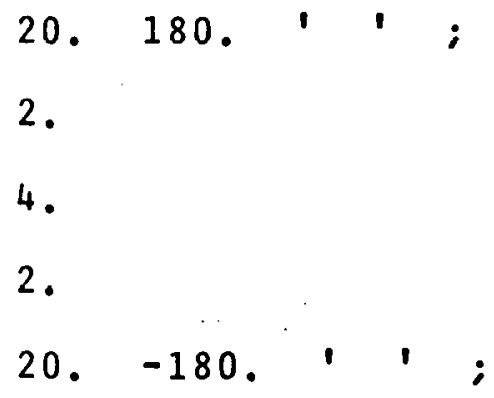




\section{Example No. 2}

A quadrupole rotated clockwise by 60 degrees about the positive $z$ axis would be specified as follows:

$$
\begin{aligned}
& \text { 20. } 60 . \text { ' ; } \\
& \text { 5. L. B. a. ' } \\
& 20.60 . \text { ' }
\end{aligned}
$$

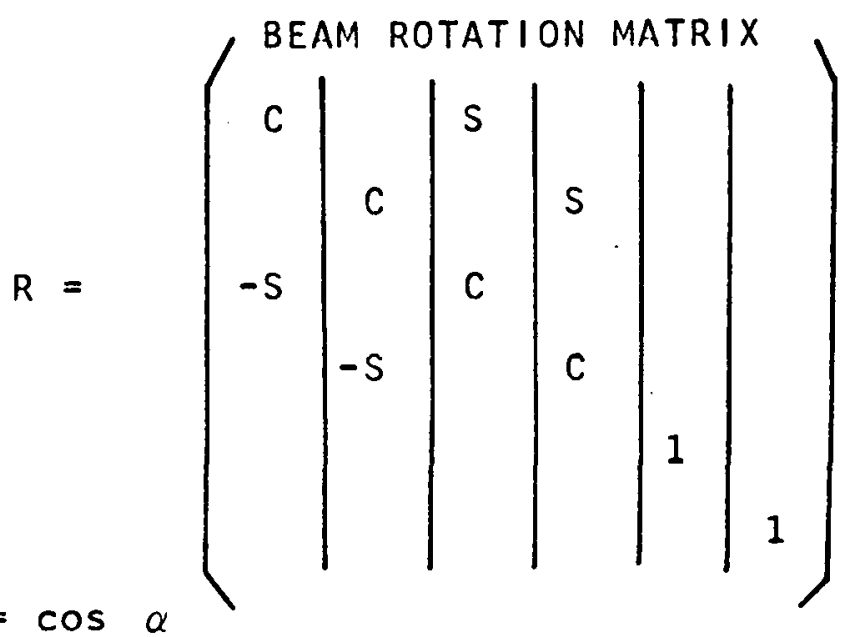

Where $\quad C=\cos \alpha$

$$
s=\sin \alpha
$$

\begin{tabular}{|c|c|c|c|c|c|c|c|c|c|c|c|c|}
\hline 0 & 0 & 1 & 0 & $R(11)$ & $R(12)$ & $R(13)$ & $R(14)$ & & $R(31)$ & $R(32)$ & $R(33)$ & $R(34)$ \\
\hline 0 & 0 & 0 & 1 & $R(21)$ & $R(22)$ & $R(23)$ & $R(24)$ & & $R(41)$ & $R(42)$ & $R(43)$ & $R(44)$ \\
\hline-1 & 0 & 0 & 0 & $R(31)$ & $R(32)$ & $R(33)$ & $R(34)$ & & $-R(11)$ & $-R(12)$ & $-R(13)$ & $-R(14)$ \\
\hline 0 & -1 & 0 & 0 & $R(41)$ & $R(42)$ & $R(43)$ & $R(44)$ & & $-R(21)$ & $-R(22)$ & $-R(23)$ & $-R(24)$ \\
\hline
\end{tabular}

$\alpha=$ angle of coordinate rotation about the beam axis.

Blank spaces are zeros.

e.g.; for $\alpha=+90$ degrees, this matrix interchanges rows 1 and 2 with 3 and 4 of the accumulated $R$ matrix as follows:

(The rest of the matrix is unchanged). 
1 - Element No. 21.

2 - Code No. n. $n=4$ : horizontal deflection

$n=2:$ vertical deflection

$3-\langle\overline{B L}\rangle$ mean value of $\int B d z$

$4- \pm\langle\delta$ BL $\rangle$

+: gaussian random number generator; effects beam first moment.

-: uncertainty in $\int B d z$ - effects beam second moment.

Uses the misalignment element ( 8. ) to calculate an angular deflection equal to $\frac{\int_{\mathrm{Bdz}}}{(\mathrm{B} \bar{\rho})}$

Random number generator

16. 14. $(2 n+1$.$) .$

The odd number $(2 n+1)$ resets the random number generator to a specified initial point in the $r$. $n$. sequence. 
SENTINEL

Each data set must he terminated with the word SENTINEL. The word SENTINEL need not be on a separate card. 


\section{REFERENCES}

1. K. L. Brown, "A First and Second-Order Matrix Theory for the Design of Beam Transport Systems and Charged Particle Spectrometers" SLAC Report No. 75 (revised), July 1967.

2. S. Penner, "Calculations of Properties of Magnetic Deflection Systems," Rev. Sci. Instr. 32, No. 2, 150-160 (February 1961).

3. A. P. Banford, The Transport of Charged Particle Beams, (E. and F. N. Spon, Ltd., London, 1966).

4. K. L. Brown (with R. Belbeoch and P. Bounin), "First- and Second- Order Magnetic Optics Matrix Equations for the Midplane of Uniform-Field Wedge Magnets, Rev. Sci. Instr. 35, 481 (1964).

5. K. G. Steffen, High Energy Beam Optics, Interscience Monographs and Tests in Physics and Astronomy, Vol. 17 (John Wiley and Sons, 1965).

6. Harold Enge and Stan Kowalski at M.I.T. have developed an effective Ray Tracing program using essentially the same terminology as TRANSPORT. Hence any experienced user of TRANSPORT should find it relatively easy to adapt to the M.I.T. program. 
Table of Contents

PAGE

A Quadrupole Triplet used to achieve Point-to-Point Imaging

$E-I A$

A Quadrupole Triplet used to achieve Waist-to-Waist Imaging

$E-7 A$

A Quadrupole Triplet used to achieve a Specified Spot Size at a Target

E-13A

A SLAC Beam Switchyard (an attempt to fit all variable parameters in one step)

E-19A

A SLAC Beam Switchyard (using sequential fitting of the variable parameters to achieve the desired result)

$\mathrm{E}-37 \mathrm{~A}$

Misalignment of a Quadrupole Triplet

$\mathrm{E}-114 \mathrm{~A}$ 
'USE TPIPLET TO FOCUS BENIA FROM SOURCE ONTO TARGET'

(THIS PRORLEH IIAS A UNITS CONVERSION FOR THE BEAH EXTENT FROH CM TO IN AUD)

(FOR THE LENGTH FROH METERS TO FFET. THE JUTER T:HO QUADRUROLES OF THE QUAD)

(TRIPLET HAVE VARV CODES SUCII TIIAT THEIR FIFLDS HILL VARY TOSETHFR THE FIELD)

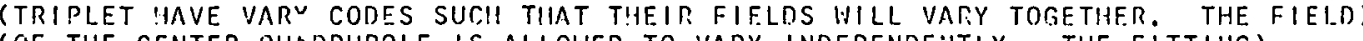

(OF T!HE CENTER QUADRUPOLE IS ALLOHED TO VARY THINEPENDE.NTLY. THE FITTIIIS)

(CONDITIOHS SPF.CIFY A FOCUS IN BOT! RIMENSIOHS ON THE TAPSET.)

15.001 .0 : $111^{3}$ :

$\begin{array}{llllllll}1.0 & 0.5 & 17.5 & 0.5 & 17.5 & 0.0 & 3.0 & 1.05\end{array}$

3.012 .0

$5.021 .5-1.04 .0$

3.00 .5 ;

$5.013 .01 .04 .0:$

3.00 .5

$5.02 i .50-1.04 .0$.

3.07 .0

$10.0-1.02 .00 .0 .001$ 'FITI';

$10.0-3.04 .00 .0 .001$ 'FIT2'?

13.011 .0

-15.0 'UAT';

15.0 
USE TRIPLET TO FOCUS BFAM FR[M SOURCE DINTI] TARGET

THIS PRUBLEM HAS A LNITS CONVERSIJN FUR THE BEAM EXTENT FRCM CM TO IN AND FUR THE LENGit FREM METERS TO FEET. THE OUTER TWO QUADRUPCLES CF THE QUAD

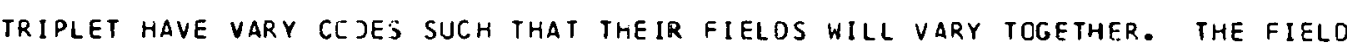
of the center guadrupjle is allowed to vary independently. THE fitting CONOITIONS SPECIFY : FOCLS IN BOTH DIMENSIONS CN THE TARGET.

\begin{tabular}{|c|c|c|c|c|c|c|c|}
\hline $15.000 \mathrm{co}$ & 1.00000 & IN & $2.5400 \mathrm{C}$ & & & & \\
\hline 15.00000 & 8.00000 & $\mathbf{F T}$ & 0.30480 & & & & \\
\hline 1.00000 & $0.5000 \mathrm{C}$ & 17.49598 & 0.50000 & 17.49998 & 0.0 & 3.00000 & 1.05000 \\
\hline 3.00000 & 12.00000 & & & & & & \\
\hline $\begin{array}{l}5.0 C 000 \\
\text { VARY COOE }\end{array}$ & $\begin{array}{r}1.50006 \\
02000 C\end{array}$ & -1.00000 & 4. 00000 & & & & \\
\hline 3.00000 & C. 50000 & & & & & & \\
\hline $\begin{array}{l}5.00000 \\
\text { VARY COCE }\end{array}$ & $\begin{array}{r}3.00000 \\
010000\end{array}$ & 1.00000 & 4.00000 & & & & \\
\hline 3.00000 & C. $5000 \mathrm{C}$ & & & & & & \\
\hline $\begin{array}{l}5.00000 \\
\text { VARY CODE }\end{array}$ & $\begin{array}{r}1.5000 C \\
02000 C\end{array}$ & -1.20000 & 4.00000 & & & & \\
\hline 3.00000 & $7.00 \mathrm{COC}$ & & & & & & \\
\hline $\begin{array}{c}10.00000 \\
\text { LABEL }=\end{array}$ & $\begin{array}{l}-1.00006 \\
\text { FIr }\end{array}$ & 2.00000 & 0.0 & $0 . \operatorname{col} 0 \mathrm{C}$ & & & \\
\hline $\begin{array}{c}10.00000 \\
\text { LABEL }=\end{array}$ & $\begin{array}{l}-3.000 \mathrm{CC} \\
\text { FIT } 2\end{array}$ & $4.0000 \mathrm{C}$ & 0.0 & 0.00100 & & & \\
\hline 13.00000 & $4 . \mathrm{CcCOC}$ & & & & & & \\
\hline $\begin{array}{l}-15.00000 \\
\angle A B E L=\end{array}$ & UNT & & 0.0 & & & & \\
\hline \multicolumn{8}{|l|}{ SENTINEL } \\
\hline 的 & $\therefore$ & : & . & $\cdots$ & : & & \\
\hline & . & & & & & $E-1$ & \\
\hline
\end{tabular}


* BEAM* $1.000000 \quad 1.05 \mathrm{GEV}$

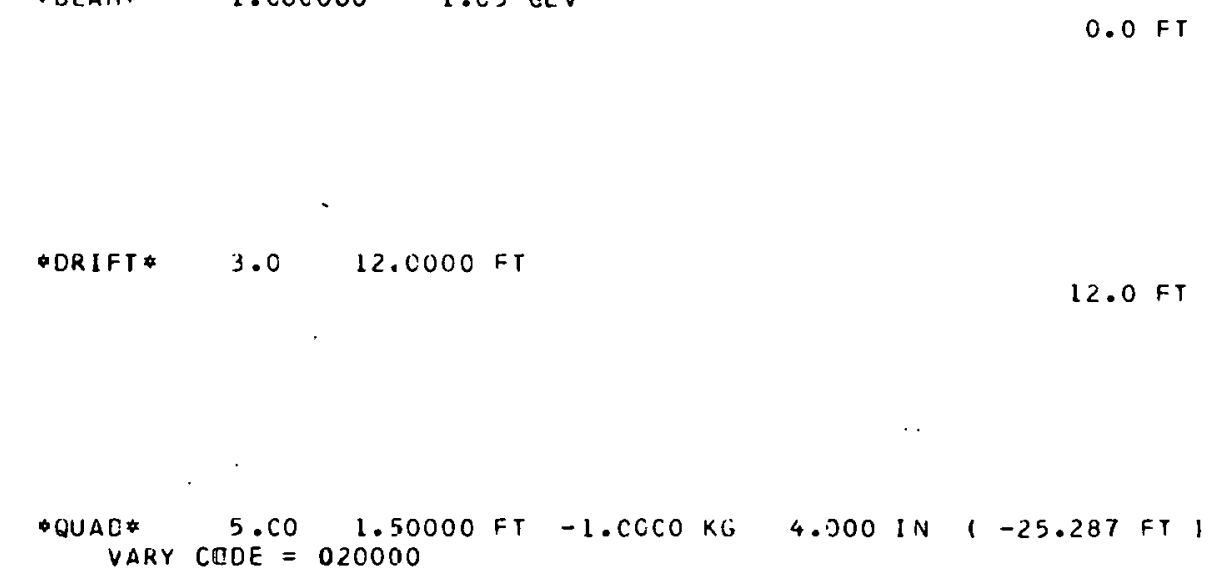

VARY CEOE $=020000$

$13.5 \mathrm{Fr}$

QURIFT $3.0 \quad$ C. $5000 \mathrm{FT}$

$5.00 \quad 3.00000 \mathrm{FT} 1.0000 \times \mathrm{KG} 4.000 \mathrm{IN} \mid 13.282 \mathrm{FT}$ VARY CODE $=010000$

DDRIFT* 3.C C. $5000 \mathrm{FT}$

$\begin{array}{cccccccc}0.0 & 0.500 & \text { IN } & & & & & \\ 0.0 & 17.500 & M R . & 0.0 & & & & \\ 0.0 & 0.500 \text { IN } & 0.0 & 0.0 & & & \\ 0.0 & 17.500 \text { MR } & 0.0 & 0.0 & 0.0 & & \\ 0.0 & 0.0 & \text { CM } & 0.0 & 0.0 & 0.0 & 0.0 & \\ 0.0 & 3.000 \text { PC } & 0.0 & 0.0 & 0.0 & 0.0 & 0.0 \\ & & & & & & & \\ & & & & & & & \\ 0.0 & 2.569 \text { IN } & & & & & \\ 0.0 & 17.500 \text { MR } & 0.981 & & & & \\ 0.0 & 2.569 \text { IN } & 0.0 & 0.0 & & & \\ 0.0 & 17.500 \text { MR } & 0.0 & 0.0 & 0.981 & & \\ 0.0 & 0.0 & \text { CM } & 0.0 & 0.0 & 0.0 & 0.0 & \\ 0.0 & 3.000 \text { PC } & 0.0 & 0.0 & 0.0 & 0.0 & 0.0\end{array}$

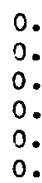

0.0
0.0
0.0
0.0

0.0

0.0

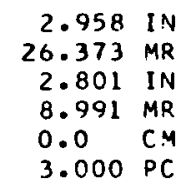

3.000 PC

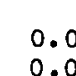

0.0
0.0
0.0

0.0

0.0
0.0
0.0

\title{
10. 994
}

$\begin{array}{ll}0.0 & 0.0\end{array}$

\section{$\begin{array}{lll}0.0 & 0.0 & \\ 0.0 & 0.0 & 0.938 \\ 0.0 & 0.0 & 0.0\end{array}$}

$\begin{array}{llll}0.0 & 0.0 & 0.0 & 0.0 \\ 0.0 & 0.0 & 0.0 & 0.0\end{array}$

0.0

\subsection{0}

$\begin{array}{lll}0.994 & & \\ 0.0 & 0.0 & \\ 0.0 & 0.0 & 0.940 \\ 0.0 & 0.0 & 0.0\end{array}$

$\begin{array}{llll}0.0 & 0.0 & 0.0 & 0.0\end{array}$

0.0

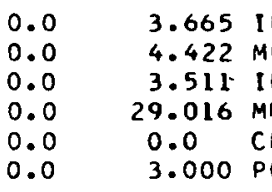

0.842

$$
0.0
$$

$$
\begin{aligned}
& 0.0 \\
& 0.0 \\
& 0.0
\end{aligned}
$$$$
0.0
$$

0.0
0.0
0.0

$0.0 \quad 0.0$

$3.000 \mathrm{PC}$

0.0

$0.0 \quad 0.0$

\footnotetext{
DRT
}

\begin{abstract}
$17.5 \mathrm{Fr}$
\end{abstract}
0.0

3.687 IN

$\begin{array}{rr}0.0 & 4.422 \mathrm{MR} \\ 0.0 & 3.685 \mathrm{IN}\end{array}$

0.844

0.844
0.0

0.0

0.997 


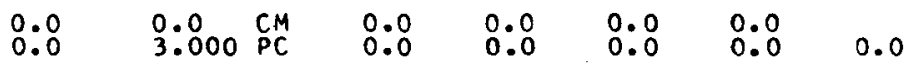

*QUAD* $5.00 \quad 1.50000 \mathrm{FT}-1.00 \mathrm{CO}$ KG $4.00 \mathrm{C}$ IN $(-25.287 \mathrm{FT})$ VARY CODE $=02000^{\circ}$

$19.0 \mathrm{FT}$

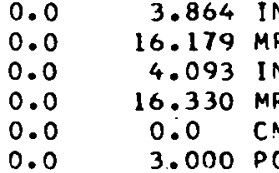

\subsection{0}

\section{0}

0.0

0.991

$\begin{array}{llll}0.0 & 0.0 & 0.0 & 0.0\end{array}$

*DR I F T *

3.C $\quad 7.0 .000 \mathrm{FT}$

$26.0 \mathrm{FT}$

0.0

$5.213 \mathrm{IN}$ $16.179 \mathrm{NPR}$ 5.455 IN
$16.330 \mathrm{MR}$

$C .995$
0.0

$\begin{array}{lll}0.0 & 0.0 & \mathrm{CM} \\ 0.0 & 3.000 \mathrm{FC}\end{array}$

$0.0 \quad 0.0 \quad 0.995$

0.0

$0.00 \quad 0.0$

* FIT*

$\begin{array}{lll}10.0 & -1.2 . & 0.0,0.001 \\ \text { LABEL }=\text { FITI } & 0.297\end{array}$

*F IT *

$$
\begin{array}{lll}
10.0 & -3.4 .0 & 0.0 \\
\text { LABEL }=\text { FIT2 } & 0.311
\end{array}
$$

*TRANSFORM 1

$\begin{array}{cll}0.949 .20 & 0.29666 & 0.0 \\ -0.0413 .06 & 0.92442 & 0.0 \\ 0.0 & 0.0 & 0.55425 \\ 0.0 & 0.0 & -0.35299 \\ 0.0 & 0.0 & 0.0 \\ 0.0 & 0.0 & 0.0\end{array}$

$\begin{array}{lll}0.0 & 0.0 & 0.0 \\ 0 . c & 0.0 & 0.0 \\ 0.311554 & 0.0 & 0.0 \\ 0.53307 & 0.0 & 0.0 \\ 0 . c & 1.00000 & 0.0\end{array}$

*LENGTH* $26.0000 \mathrm{FT}$

\section{*CORRECTIGNS*}
(3.036BE C $\bar{c}$
$-18 . \$ 39$
17.940
(2. 1600 E C31
2. 929
$-12.178$
$17.1664 E_{021}$
2.578
1.313
(1.5780E 021
0.379
c. 313 


\begin{tabular}{|c|c|c|c|c|}
\hline O. $\angle S U B E \quad 001$ & \multicolumn{3}{|c|}{0.024} & c.011 \\
\hline $1.6005 E-021$ & & & & 0.000 \\
\hline $\begin{array}{l}\text { *COVARI ANCE } \\
0.014 \\
0.869 \\
0.0004\end{array}$ & & FIT & 0.0 & $i$ \\
\hline
\end{tabular}

$$
E-4
$$


* bear* 1.000000 1.05 GEV

\#DRIFT * $\quad 3.0 \quad 12.0 \mathrm{OCC}$ FT

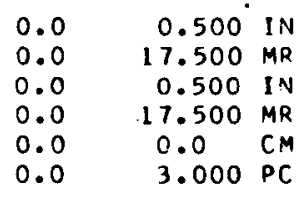

$12.0 \mathrm{FT}$

*QUAD* $5.00 \quad 1.50000 \mathrm{FT}-14.5080 \mathrm{~kg} \quad 4.00 \mathrm{C}$ IN $1-1.533 \mathrm{FT}$ । VARY COOE $=020000$

$13.5 \mathrm{FT}$

$14.0 \mathrm{FT}$

\section{\#DRIFT * $\quad 3.0 \quad 0.5000 \mathrm{FT}$}

* UUAD* 5.00 $3.00000 \mathrm{FT} \quad 8.3975 \mathrm{Kg} 4.000 \mathrm{IN}(2.166 \mathrm{FT}$ ) VARY CODE $=015000$

$17.0 \mathrm{FT}$

$\begin{array}{ccc}0.0 & 5.005 & \text { IN } \\ 0.0 & 168.545 & \text { MP } \\ 0.0 & 1.966 \text { IN } \\ 0.0 & 111.721 & \text { MR } \\ 0.0 & 0.0 & \text { CM } \\ 0.0 & 3.000 & \text { PC }\end{array}$

2.569 IN
$17.500 \mathrm{MR}$
$2.569 \mathrm{IN}$
$17.500 \mathrm{MR}$
$0.0 \mathrm{CM}$
$3.000 \mathrm{PC}$

\subsection{1}

$\begin{array}{ll}0.0 & 0.0 \\ 0.0 & 0.0\end{array}$

$\begin{array}{ll}0.0 & 0.0 \\ 0.0 & 0.0\end{array}$

0.0

\subsection{1}

$0.0 \quad 0.0$

$0.0 \quad 0.0$

$0.0 \quad 4.099$ IN $0.0164 .756 \mathrm{MR}$ $0.0 \quad 1.818$ IN $0.0 \quad 94.749 \mathrm{MR}$

$\begin{array}{lll}0.0 & 0.0 & C M \\ 0.0 & 3.000 P C\end{array}$

\subsection{0} $0.0 \quad 0.0$ $0.0 \quad 0.0$

$\begin{array}{llll}0.0 & 0.0 & 0.0 & 0.0\end{array}$

$\begin{array}{lllll}0.0 & 0.0 & 0.0 & 0.0 & 0.0\end{array}$

$\begin{array}{cr}0.0 & 5.088 \text { IN } \\ 0.0 & 164.756 \text { MR } \\ 0.0 & 1.250 \text { IN } \\ 0.0 & 94.749 \text { MR } \\ 0.0 & 0.0 \quad \text { CM } \\ 0.0 & 3.000 \text { PC }\end{array}$

1.000 $\begin{array}{ll}0.0 & 0.0\end{array}$ $\begin{array}{lll}0.0 & 0.0 & -0.997\end{array}$

$\begin{array}{cccc}0.0 & 0.0 & 0.0 & 0.0\end{array}$

$\begin{array}{llll}0.0 & 0.0 & 0.0 & 0.0\end{array}$

\section{$-1.000$}

$0.0 \quad 0.0$

$\begin{array}{lll}0.0 & 0.0 & 0.999\end{array}$

$\begin{array}{llll}0.0 & 0.0 & 0.0 & 0.0\end{array}$

$\begin{array}{lllll}0.0 & 0.0 & 0.0 & 0.0 & 0.0\end{array}$

\#ORIFT * $3.0 \quad$ C.5000 F $\pi$

$17.5 \mathrm{FT}$

$\begin{array}{rr}0.0 & 3.994 \text { IN } \\ 0.0 & 168.545 \text { MR } \\ 0.0 & 2.636 \text { IN } \\ 0.0 & 111.721 \text { MR }\end{array}$

$-1.000$

$\begin{array}{cc}-1.000 \\ 0.0 & 0.0\end{array}$

$\begin{array}{lll}0.0 & 0.0 & \\ 0.0 & 0.0 & 1.000\end{array}$




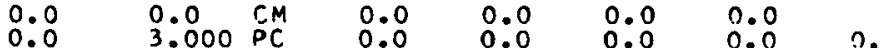

\#GUAD* $5.00 \quad 1.50000 \mathrm{FT}-14.5080 \mathrm{KG}$ VARY CCOE $=020000$

\#DRIFT* $\quad 3.0 \quad 7.000 \mathrm{CFT}$

\begin{tabular}{|c|c|c|c|c|}
\hline$* F I T *$ & $\begin{array}{l}10.0 \\
\angle A B E L=\end{array}$ & $\begin{array}{l}-1.20 \\
\text { FII I }\end{array}$ & $\begin{array}{c}0.0 \\
-0.000\end{array}$ & 10.001 \\
\hline IT* & 10.0 & -3.4. & $\begin{array}{l}0 . c \\
0.000\end{array}$ & 10.001 \\
\hline
\end{tabular}

*trarisform *

$\begin{array}{ccclll}\text { - } & & & & & \\ -15548 & -0.00000 & 0.0 & 0.0 & 0.0 & 0.0 \\ -15.63603 & -1.57364 & 0.0 & 0.0 & 0.0 & 0.0 \\ 0.0 & 0.0 & 0.046156 & 0.00000 & 0.0 & 0.0 \\ 0.0 & 0.0 & 28.41595 & 2.16653 & 0.0 & 0.0 \\ 0.0 & 0.0 & 0.0 & 0.0 & 1.00000 & 0.0 \\ 0.0 & 0.0 & 0.0 & 0.0 & 0.0 & 1.00000\end{array}$

*LENGTH* $26.0000 \mathrm{FT}$
$4.000 \mathrm{IN},-1.533 \mathrm{FT}$,

$19.0 \mathrm{FT}$

$\begin{array}{lcl}0.0 & 2.338 \text { IN } \\ 0.0 & 28.627 \text { MR } \\ 0.0 & 3.327 \text { IN } \\ 0.0 & 40.489 \text { MR } \\ 0.0 & 0.0 & \text { CM } \\ 0.0 & 3.000 \text { PC }\end{array}$

$26.0 \mathrm{FT}$

$\begin{array}{lcc}0.0 & 0.318 & \text { IN } \\ 0.0 & 28.627 & M R \\ 0.0 & 0.231 & \text { IN } \\ 0.0 & 40.489 & M R \\ 0.0 & 0.0 & \text { CM } \\ 0.0 & 3.000 & \text { PC }\end{array}$

0.273

$0.0 \quad 0.0$

$\begin{array}{lll}0.0 & 0.0 & 0.351\end{array}$

$0.0 \quad 0.0 \quad 0.0$

0.0 
'USE TRIPLET TO PUT WAIST ON TARGET'

(TIIIS PROBLFM DIFFERS FROM THE PRE:FOING ONE IN THAT A WAIST ON THE TARGET)

(IS REQUESTED. NOTE THE USE DF THE I NS THE IMDICATOR CARD SIGNIFYING)

(THAT THIS RUN IS A CONTINUATION O = THE PRFVIOUS ONE.)

10.02 .01 .00 .0 .001 ' $F I T 1$ '?

10.04 .03 .00 .0 .001 'FIT2'.

SENTINEL 
USE IKIPLET TO PUT HAIST ON TARGET

THIS PROBLEM CIFFERS FROM THE PRECEDINS ONE IN THAT A WAIST ON THE TARGET

IS REQUESTEC. NGTE THE USE OF THE I AS THE INOICATOR CARD SIGNIFYING

THAT THIS RUN IS A CONTINUATION OF the PREVIOUS ONE.

\begin{tabular}{|c|c|c|c|c|c|c|c|}
\hline 15.00000 & 1.00000 & IN & 2.54000 & & & & \\
\hline 15.00000 & 8.001000 & $F T$ & 0.30480 & & & & \\
\hline 1.00000 & C. 50000 & 17.49998 & 0.50000 & 17.47998 & 0.0 & $3: c 0000$ & $1.0500 \mathrm{C}$ \\
\hline $3 . c c 000$ & 12.00000 & & & & & & \\
\hline $\begin{array}{l}5 . C D O O 0 \\
\text { VARY CODE }\end{array}$ & $=\begin{array}{r}1.50000 \\
020000\end{array}$ & -14.50800 & 4. $c 0000$ & & & & \\
\hline 3.00000 & c. 50000 & & & & & & \\
\hline $\begin{array}{l}5.00000 \\
\text { VARY CODE }\end{array}$ & $\begin{array}{r}3.00000 \\
010000\end{array}$ & 8.39750 & 4. 100000 & & & & \\
\hline 3.00000 & c. 50000 & & & & & & \\
\hline $\begin{array}{l}5.0 C O O O \\
\text { VARY CODE }\end{array}$ & $\begin{array}{r}1.50000 \\
020000\end{array}$ & $-14.5080 C$ & 4.00000 & & & & \\
\hline 3.00000 & 7.00000 & & & & & & \\
\hline $\begin{array}{c}10.00000 \\
\text { LABEL }=\end{array}$ & $\begin{array}{l}2.00000 \\
\text { FIT } 1\end{array}$ & 1.00000 & 0.0 & 0.00100 & & & \\
\hline $\begin{array}{l}10.00000 \\
\text { LABEL }=\end{array}$ & $\begin{array}{l}4.00000 \\
\text { FIT }\end{array}$ & 3.00000 & 0.0 & 0.00100 & & & \\
\hline 13.00000 & 4.00000 & & & & & . & \\
\hline $\begin{array}{c}-15.00000 \\
\text { LABEL }=\end{array}$ & $\begin{array}{l}0.0 \\
\text { UNT }\end{array}$ & ' & 0.0 & & & & \\
\hline SENT INEL & & & & & & & \\
\hline
\end{tabular}


* BEAM $1.000000 \quad 1.05$ GEV

*ORIFT* $\quad 3.0 \quad 12.000 \mathrm{CFT}$

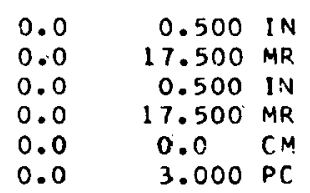

12. O FT

$0.0 \mathrm{FT}$
12.0 FT

$\begin{array}{ccc}0.0 & 2.569 & \text { IN } \\ 0.0 & 17.500 & M R \\ 0.0 & 2.569 & \text { IN } \\ 0.0 & 17.500 & M R \\ 0.0 & 0.0 & C M \\ 0.0 & 3.000 \mathrm{PC}\end{array}$

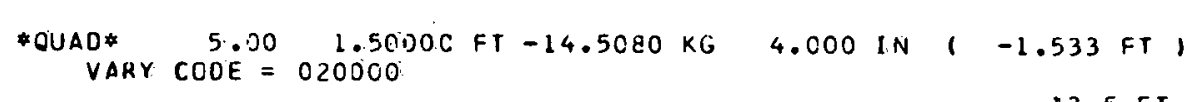

$13.5 \mathrm{FT}$

\#URIFT $\quad 3.0 \quad$ C.5000 FT

$14.0 \mathrm{~F} \mathrm{~T}$ *QUAD VARY CODE $=010000$.00 3.00000 FT $8.3575 \mathrm{KG} 4.000 \mathrm{IN} 1,2.166 \mathrm{FT}$,

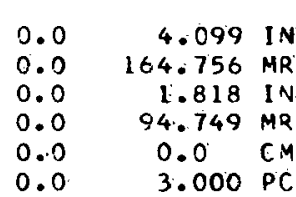

$0.0 \quad 3.000 \mathrm{PC}$

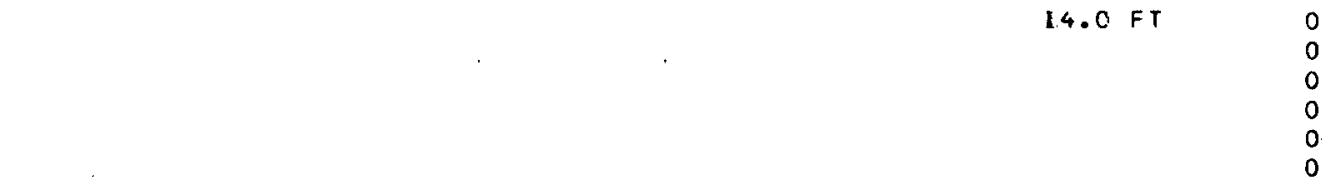

$\begin{array}{lcc}0.0 & 5.088 & \text { IN } \\ 0.0 & 164.756 & \text { MR } \\ 0.0 & 1.250 & \text { IN } \\ 0.0 & 94.749 & \text { MR } \\ 0.0 & 0.0 & \text { CM } \\ 0.0 & 3.000 & \text { PC }\end{array}$

\section{0}

0.0

0.0
0.0
0.0

0.0

0.0

0.0

0.0
0.0

0.0

0.0

0.981
0.0

$$
\begin{aligned}
& 0.0 \\
& 0.0 \\
& 0.0
\end{aligned}
$$

0.0
0.0
0.0

0.0
0.0 0.0
0.0 $0.0 \quad 0.0$

$\begin{array}{lll}0.0 & 0.0 & 0.0 \\ 0.0 & 0.0 & 0.0\end{array}$

1.000 $\begin{array}{ll}1.000 \\ 0.0 & 0.0\end{array}$ $\begin{array}{lll}0.0 & 0.0 & -0.999\end{array}$

$\begin{array}{cccc}0.0 & 0.0 & -0.999 & \\ 0.0 & 0.0 & 0.0 & 0.0\end{array}$ $\begin{array}{lllll}0.0 & 0.0 & 0.0 & 0.0 & 0.0\end{array}$

\subsection{0}

$\begin{array}{lll}1.000 & & \\ 0.0 & 0.0 & \\ 0.0 & 0.0 & -0.997\end{array}$

$\begin{array}{cccc}0.0 & 0.0 & -0.997 & \\ 0.0 & 0.0 & 0.0 & 0.0\end{array}$

$\begin{array}{llll}0.0 & 0.0 & 0.0 & 0.0\end{array}$

0.0

$1.7 .0 \mathrm{~F} \mathrm{~T}$

$\begin{array}{lrl}0.0 & 5.005 & \text { IN } \\ 0.0 & 168.545 & M R \\ 0.0 & 1.966 & \text { IN } \\ 0.0 & 111.721 & M R \\ 0.0 & 0.0 & C M \\ 0.0 & 3.000 & P C\end{array}$

\section{$-1.000$}

$\begin{array}{lll}0.0 & 0.0 & \\ 0.0 & 0.0 & 0.999\end{array}$

$\begin{array}{llll}0.0 & 0.0 & 0.0 & 0.0\end{array}$

$\begin{array}{llll}0.0 & 0.0 & 0.0 & 0.0\end{array}$

0.0

*DRIFT * $3.0 \quad$ C.5:00 F T

$17.5 \mathrm{FT}$ 
*UUAD* $5.00 \quad 1.50000 \mathrm{FT}-14.5080 \mathrm{KG}$ VARY CGOE $=020000$

\#DRIFT $\quad 3.0 \quad 7.0000 \mathrm{FT}$

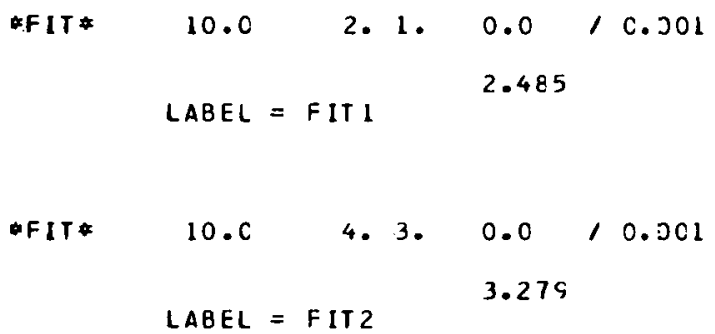

*IRANSFDRM 1

$\begin{array}{ccc}-0.63548 & -0.06000 & 0.0 \\ -15.63603 & -1.57364 & 0.0 \\ 0 . C & 0.0 & 0.46156 \\ 0 . C & 0.0 & 28.41595 \\ 0 . C & 0.0 & 0.0 \\ 0 . C & 0.0 & 0.0\end{array}$

\section{0}

0.0

0.00000

$2.16653 \quad 0.0$

$0.0 \quad 1.00000$
$19.0 \mathrm{FT}$

$26.0 \mathrm{FT}$

0.0
0.0
0.0

0.0

0.0

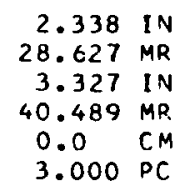

$-0.991$

$$
0.0
$$

$0.0 \quad 0.0$

$3.000 \mathrm{PC}$

0.0

0.0
0.0

0.0
0.0

0.0

0.0

0.318 IN
28.627 MR
0.231 IN
40.489 MP
$0.0 \quad$ CM
3.000 PC

0.273

0.0

0.0

0.0
0.0

*LENGTH* $\quad 26.0000 \mathrm{FT}$

\section{*CORRECT IONS*}

$\begin{array}{rrrr}(2.9092 E(3) & 0.063 & -0.026 \\ (1.1744 E(02) & 0.003 & -0.001 \\ (8.0844 E-01) & 0.000 & -C .000 \\ (3.0664 E-01) & -0.000 & C .000\end{array}$


* Covariance I fit 0.3)

0.000 R

$\begin{array}{lll}-0.786 & 0.000\end{array}$

$$
E-10
$$


* eeam 1.c00000 1.C5 GE V

$0.0 \mathrm{FT} \quad 0.0 \quad 0.500 \mathrm{IN}$

\begin{tabular}{|c|c|c|c|c|c|c|}
\hline $\begin{array}{l}0.0 \\
0.0 \\
0.0 \\
0.0 \\
0.0\end{array}$ & $\begin{array}{r}17.500 \\
0.500 \\
17.500 \\
0.0 \\
3.000\end{array}$ & $\begin{array}{l}\text { MR } \\
\text { IN } \\
\text { MR } \\
\text { CM } \\
\text { PC }\end{array}$ & $\begin{array}{l}0.0 \\
0.0 \\
0.0 \\
0.0 \\
0.0\end{array}$ & $\begin{array}{l}0.0 \\
0.0 \\
0.0 \\
0.0\end{array}$ & $\begin{array}{l}0.0 \\
0.0 \\
0.0\end{array}$ & $\begin{array}{l}0.0 \\
0.0\end{array}$ \\
\hline
\end{tabular}

*CRIfT* $\quad 3.0 \quad 12.000 \mathrm{CFT}$

$12.0 \mathrm{FT}$

$\begin{array}{lll}0.0 & 2.569 \text { IN } \\ 0.0 & 17.500 & \text { MR } \\ 0.0 & 2.569 \text { IN } \\ 0.0 & 17.500 \text { MR } \\ 0.0 & 0.0 & \text { CM } \\ 0.0 & 3.000 \mathrm{PC}\end{array}$

0.981

$\begin{array}{ll}0.0 & 0.0\end{array}$

0.0

$\begin{array}{ll}0.0 & 0.0 \\ 0.0 & 0.0\end{array}$

0.981

$\begin{array}{lll}0.0 & 0.0 & \\ 0.0 & 0.0 & 0.0\end{array}$

*UUAD* $5.00 \quad 1.5000 \mathrm{FT}-14.4423 \mathrm{KG} \quad 4.000 \mathrm{IN}(-1.541 \mathrm{FT}$ ) VARY CODE $=020000$

$13.5 \mathrm{FI}$

$\begin{array}{ccc}0.0 & 4.093 & \text { IN } \\ 0.0 & 164.000 & M R \\ 0.0 & 1.822 \text { IN } \\ 0.0 & 94.316 & M R \\ 0.0 & 0.0 & C M \\ 0.0 & 3.000 & P C\end{array}$

\subsection{0}

$0.0 \quad 0.0$

$0.0 \quad 0.0 \quad-0.999$

$\begin{array}{llll}0.0 & 0.0 & 0.0 & 0.0\end{array}$

\begin{tabular}{|c|c|}
\hline 0.0 & 0.0 \\
\hline
\end{tabular}

*URIFT* $3.0 \quad$ C.5000 FT

$14.0 \mathrm{FT}$

$\begin{array}{ccc}0.0 & 5.077 & \text { IN } \\ 0.0 & 164.000 & M R \\ 0.0 & 1.257 & \text { IN } \\ 0.0 & 94.316 \mathrm{MR} \\ 0.0 & 0.0 & \mathrm{CM} \\ 0.0 & 3.000 \mathrm{PC}\end{array}$

1.000 $\begin{array}{ll}0.0 & 0.0\end{array}$ $\begin{array}{lll}0.0 & 0.0 & -0.997\end{array}$

$0.0 \quad 0.0$

\section{0} 0.0 0.0

*QUAD* $\quad 5.00 \quad 3.00000 \mathrm{FT}$
VARY COOE $=010000$ $8.37 \mathrm{CG} \mathrm{KG}$ 4.000 IN

\section{$2.170 \mathrm{FT} 1$}

$17.0 \mathrm{FT}$

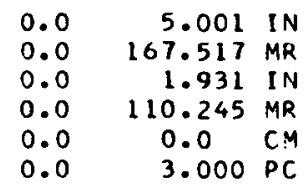

-1.000
0.0
0.0
0.0
0.0

\section{0}

0.0

0.0

0.0

0.999

$\begin{array}{ll}0.0 & 0.0\end{array}$

0.0

*DRIFT* $\quad 3.0 \quad 0.5000 \mathrm{FT}$

$\begin{array}{rrr}17.5 \mathrm{FT} & 0.0 & 3.996 \mathrm{IN} \\ & 0.0 & 167.517 \mathrm{MR} \\ 0.0 & 2.592 \mathrm{IN} \\ & 0.0 & 110.245 \mathrm{MR}\end{array}$


\#QUAD* VARY CCCE $=020000$

FDRIFT* 3.C 7.CDOO FT

\footnotetext{
* FIT* $10 . \mathrm{C} \quad$ 2. $2.0 .0,0.001$

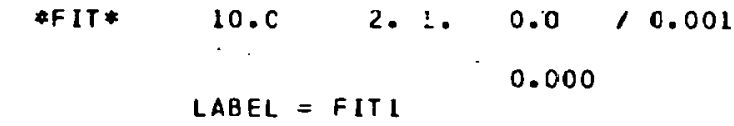

* FIT $10.0 \quad 4.3 .0 .0,0.001$

LABEL $=F I T .2$
}

4.000 IN ( $-1.541 \mathrm{FT})$

$19.0 \mathrm{FT}$

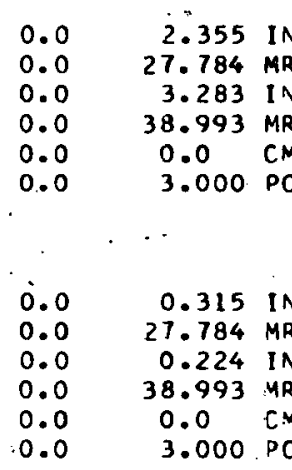

\section{$-0.991$

0.0

$0.0 \quad 0.0$

$\begin{array}{lll}0.0 & .0 .0 & -0.998\end{array}$

$\begin{array}{llll}0.0 & 0.0 & 0.0 & 0.0 \\ 0.0 & 0.0 & 0.0 & 0.0\end{array}$

$0.0 \quad 0.0$

0.0

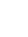

$\begin{array}{llll}0.000 & & & \\ 0.0 & 0.0 & & \\ 0.0 & 0.0 & -0.000 & \\ 0.0 & 0.0 & 0.0 & 0.0 \\ 0.0 & 0.0 & 0.0 & 0.0\end{array}$

0.0

\section{\#TRANSFORN}

$\begin{array}{ccccll}-C .60539 & 0.06497 & 0.0 & 0.0 & 0.0 & 0.0 \\ -15.34218 & -1.52592 & 0.0 & 0.0 & 0.0 & 0.0 \\ 0.0 & 0.0 & 0.41949 & -0.00456 & 0.0 & 0.0 \\ 0.0 & 0.0 & 27.71928 & 2.08264 & 0.0 & 0.0 \\ 0.0 & 0.0 & 0.0 & 0.0 & 1.00000 & 0.0 \\ 0.0 & 0.0 & 0.0 & 0.0 & 0.0 & 1.00000\end{array}$

*LENGTH* $20.0000 \mathrm{FT}$ 
'USE TRIPLET TO FIT CEFTAI!I SIZE BEAH OINTO TARGET'

1

(THIS PROBLEM IS THE SAME AS THE THO PRECEDING ONES EXCEPT A PARTICULAR)

(SIZF. OF THE BEAM IS ASKED FOR. THE BLANK TYPE 15 CARD WHICH RESFTS THE UNIT.S)

(IS TURAED ON.)

10.01 .01 .00 .6 .001 'FIT1',

10.03 .03 .00 .6 .001 'FIT2';

15.0 UNT'

SENTIHEL 
USE TRIPLET TC FIT CEFTAIN SILE BEAM ONTO TARGET

THIS PROBLEM IS THE SAME AS THE THO PRECEDING JNES EXCEPT a PARTICULAR

SILE OF THE BEAM IS ASKED FOR. THE BLANK TYPE 15 CARD WHICH RESETS THE UNITS

IS TURAED CN.

\begin{tabular}{|c|c|c|c|c|c|c|c|}
\hline 15.00000 & 1.00000 & IN & 2.54000 & & & & \\
\hline 15.00000 & 8.00000 & FT & 0.30480 & & & & \\
\hline 1.00000 & C. 50000 & 17.49598 & 0.50000 & 17.49998 & 0.0 & 3.00000 & 1.05000 \\
\hline 3.00000 & $12.000 \mathrm{CO}$ & & & & & & \\
\hline $\begin{array}{l}5.0 C 000 \\
\text { VARY CODE }\end{array}$ & $\begin{array}{r}1.50000 \\
020000\end{array}$ & $-14.4423 \mathrm{C}$ & 4. 10000 & & & & \\
\hline 3.00000 & C. 50000 & & & & & & \\
\hline $\begin{array}{l}5.00000 \\
\text { VARY CODE }\end{array}$ & $=\begin{array}{r}3.00000 \\
010000\end{array}$ & 8.37058 & 4. 00000 & & & & \\
\hline 3.00000 & C.50000 & & & & & & \\
\hline $\begin{array}{l}5.00000 \\
\text { VARY CDDE }\end{array}$ & $\begin{array}{r}1.50000 \\
020000\end{array}$ & $-14.4423 c$ & 4. 10000 & & & & \\
\hline 3.00000 & .7 .00000 & & & & & & \\
\hline $\begin{array}{c}10.00000 \\
\text { LABEL }=\end{array}$ & $\begin{array}{l}1.00000 \\
\text { FIT I }\end{array}$ & 1.00000 & 0.60000 & 0.00100 & & & \\
\hline $\begin{array}{c}10.00000 \\
\text { LABEL }=\end{array}$ & $\begin{array}{l}3.00000 \\
\text { FIT2 }\end{array}$ & $3.0000 \mathrm{C}$ & 0.60000 & 0.00100 & & & \\
\hline 13.00000 & 4.00000 & & & & & & \\
\hline $\begin{array}{l}15.00000 \\
\text { LABEL }=\end{array}$ & $\begin{array}{l}0.0 \\
\text { UNT }\end{array}$ & & 0.0 & & & & \\
\hline SENTINEL & & - & & & & & \\
\hline
\end{tabular}


* EEAM * 1.COOCJ0 I.C5 GEV

*LRIFT $* 3.0 \quad 12.0000 \mathrm{FT}$
$0.0 \mathrm{FT}$

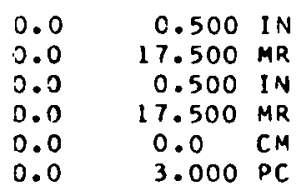

$12.0 \mathrm{FT}$

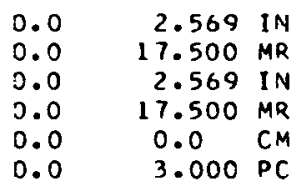

*QUAD* $\quad 5.00 \quad 1.50300 \mathrm{FT}-14.4423 \mathrm{H}: \mathrm{G} \quad 4.0 \mathrm{CO}$ IN $1-1.541 \mathrm{FT}$, VARY CODE $=020000$

$13.5 \mathrm{FT}$

$\begin{array}{ccc}0.0 & 4.093 & \text { IN } \\ 0.0 & 164.000 & \text { MR } \\ 0.0 & 1.822 \text { IN } \\ 0.0 & 94.316 \text { MR } \\ 0.0 & 0.0 & \text { CM } \\ 0.0 & 3.000 & \text { PC } \\ & & \\ & & \\ & & \\ 0.0 & 5.077 & \text { IN } \\ 0.0 & 164.000 & \text { MR } \\ 0.0 & 1.257 & \text { IN } \\ 0.0 & 94.316 & \text { MR } \\ 0.0 & 0.0 & \text { CM } \\ 0.0 & 3.000 & \text { PC } \\ 0.0 & & \end{array}$

\subsection{1}

0.0

0.0

$0.0 \quad 0.0$

$0.0 \quad 0.0$

0.98

0.0

$0.0 \quad 0.0$

0.0

\#LRIFT $3.0 \quad$ C. $500 \mathrm{CFT}$

$14.0 \mathrm{FT}$

0.0

$17.0 \mathrm{FT}$

VARY COOE $=010000$

$8.37 \mathrm{C6}$ : 4.000 IN ( $2.170 \mathrm{FT})$

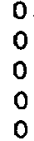

0.0
0.0
0.0
0.0

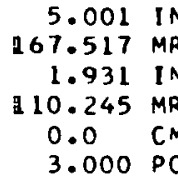

$-1.000$

0.0

$$
\begin{aligned}
& 0.0 \\
& 0.0
\end{aligned}
$$

0.0

0.0

0.0

0.999

$3.000 \mathrm{PC}$

0.0

0.0

0.0

0.0
0.0

$\begin{array}{rrr}17.5 \mathrm{FT} & 0.0 & 3.996 \mathrm{IN} \\ & 0.0 & 167.517 \mathrm{MR} \\ & 0.0 & 2.592 \mathrm{IN} \\ & 0.0 & 110.245 \mathrm{MR}\end{array}$

$\begin{array}{rr}0.0 & 2.592 \mathrm{IN} \\ 0.0 & 110.245 \mathrm{MR}\end{array}$

-1.000
0.0

$0.0 \quad 0.0$

0.0

1.000 


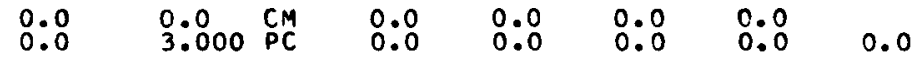

*QUAD* $5.00 \quad 1.50000 \mathrm{FT}-14.4423 \mathrm{KG} 4.000$ IN $(-1.541 \mathrm{FT}$ VARY $C C D E=0.20000$.

*DR IFT * $3.0 \quad 7.0000 \mathrm{FT}$

* FIT 10.0 1. 1. $0.600 / 0.001$

$L A B E L=F I T$

0.215

\# IT $10.0 \quad 3.3 .0 .600 / 0.001$

$\angle A B E L=F I T 2$

$\begin{array}{ccccll}1 & & & & \\ -C .6 C 539 & 0.00497 & 0 . c & 0.0 & 0.0 & 0.0 \\ -15.34218 & -1.52592 & 0.0 & 0.0 & 0.0 & 0.0 \\ 0.0 & 0.0 & 0.41949 & -0.00456 & 0.0 & 0.0 \\ c .0 & 0.0 & 27.71928 & 2.08254 & 0.0 & 0.0 \\ c .0 & 0.0 & 0 . c & 0.0 & 1.00000 & 1.0 \\ 0.0 & 0.0 & 0.0 & 0 . c & 0.0 & 1.0000 C\end{array}$

LAEEL = UNT

*LENGTH*

7.9248

*CCRRECTICNS*

$\begin{array}{lll}(2.3856 E(2) & 5.286 & -1.786 \\ (1.7593 E 03) & 1.335 & -0.401 \\ (1.4466 E 02) & 0.115 & -0.018 \\ (1.4665 E 01) & 0.012 & -0.0 C 1\end{array}$

$(1.4665 \mathrm{E} 01) \quad 0.012 \quad-0 . \mathrm{CCl}$
$19.0 \mathrm{FT}$

$\begin{array}{lcccccc}0.0 & 2.355 & \text { IN } & & & & \\ 0.0 & 27.784 & \text { MR } & -0.991 & & & \\ 0.0 & 3.283 \text { IN } & 0.0 & 0.0 & & \\ 0.0 & 38.993 \text { MR } & 0.0 & 0.0 & -0.998 & \\ 0.0 & 0.0 & \text { CM } & 0.0 & 0.0 & 0.0 & 0.0 \\ 0.0 & 3.000 & \text { PC } & 0.0 & 0.0 & 0.0 & 0.0\end{array}$

$26.0 \mathrm{FT}$

$\begin{array}{lll}0.0 & 0.315 & \text { IN } \\ 0.0 & 27.784 & \text { MR } \\ 0.0 & 0.224 & \text { IN } \\ 0.0 & 38.993 & \text { MR } \\ 0.0 & 0.0 & \text { CM } \\ 0.0 & 3.000 & \text { PC }\end{array}$

$\begin{array}{llll}0.000 & & & \\ 0.0 & 0.0 & & \\ 0.0 & 0.0 & -0.000 & \\ 0.0 & 0.0 & 0.0 & 0.0 \\ 0.0 & 0.0 & 0.0 & 0.0\end{array}$


(2.5497E-C1) $0.000 \quad$ C.COO

acovariance ( FIT 0.3 )

$C .001$
$-C .9180 .001$

$E-16$ 
* BEAM* $\quad 1.000000 \quad 1.05 \mathrm{GEV}$

*DRIFT* $\quad 3.0 \quad 12.000 \mathrm{CFT}$
$0.0 \mathrm{FT}$

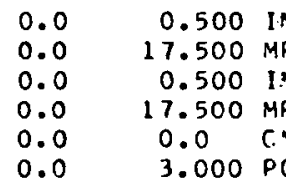

$12.0 \mathrm{FT}$

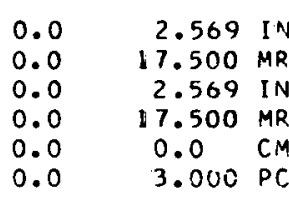

* QUAO* $5.00 \quad 1.50 \mathrm{COC}$ FT $-7.7144 \mathrm{KG} 4.000 \mathrm{IN}(-3.073 \mathrm{FT}$ ) VARY CODE $=0 ? 0000$

$13.5 \mathrm{FT}$

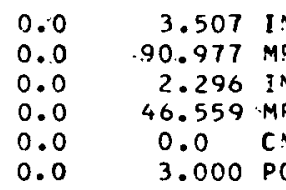

*DRIFT* $\quad 3.0 \quad$ C.5000 FT

vart CODE $=010000$ $.0 .1646 \mathrm{KG}$ I $2.671 \mathrm{FT})$ 17. $0 \mathrm{FT}$ $\begin{array}{ccc}0.0 & 3.995 \mathrm{IN} \\ 0.0 & 93.798 .11 \\ 0.0 & 1.574 \mathrm{IN} \\ 0.0 & 19.690 \mathrm{MR} \\ 0.0 & 0.0 \mathrm{CN} \\ 0.0 & 3.000 \mathrm{PC}\end{array}$ $\begin{array}{ccc}0.0 & 4.053 & \text { IN } \\ 0.0 & 90.977 & M R \\ 0.0 & 2.017 & \text { IN } \\ 0.0 & 46.559 & M R \\ 0.0 & 0.0 & \text { CH } \\ 0.0 & 3.000 & \text { PC }\end{array}$ $14.0 \mathrm{FT}$

1.000

0.0

0.0

0.0

$14.0 \mathrm{FT}$

$17.0 \mathrm{FT}$
0.0

0.0

0.0
0.0

0.0
0.0

0.0

$0.0 \quad 0.0$

$\begin{array}{lll}0.0 & 0.0 & 0.0\end{array}$ 0.0

\subsection{8}

$\begin{array}{ll}0.981 & \\ 0.0 & 0.0\end{array}$
0.0

$\begin{array}{llll}0.0 & 0.0 & 0.0 & 0.0 \\ 0.0 & 0.0 & 0.0 & 0.0\end{array}$

$0.0-0.997$

$0.0 \quad 0.0$

$0.0 \quad 0.0$

\#DRIFT $\quad 3.0 \quad$ C. $5000 \mathrm{FT}$

$17.5 \mathrm{FT}$
1.000

$$
\begin{aligned}
& 0.0 \\
& 0.0
\end{aligned}
$$

0.0
0.0

0.0
0.0

0.0
0.0 $0.0-0.996$

$\begin{array}{lll}0.0 & 0.0 & 0.0 \\ 0.0 & 0.0 & 0.0\end{array}$

\section{$-1.000$}

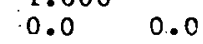

$\begin{array}{lll}0.0 & 0.0 & 0.959\end{array}$

$\begin{array}{llll}0.0 & 0.0 & 0.0 & 0.0 \\ 0.0 & 0.0 & 0.0 & 0.0\end{array}$

$\begin{array}{lrrrrrr}17.5 \text { FT } & 0.0 & 3.432 \text { IN } & & & \\ & 0.0 & 93.798 & \text { M. } & -1.000 & & \\ & 0.0 & 1.687 \text { IN } & 0.0 & 0.0 & \\ & 0.0 & 19.690 .4 F & 0.0 & 0.0 & 0.965 \\ & & & & & & \\ \text { E }-17 & & & & & & \end{array}$




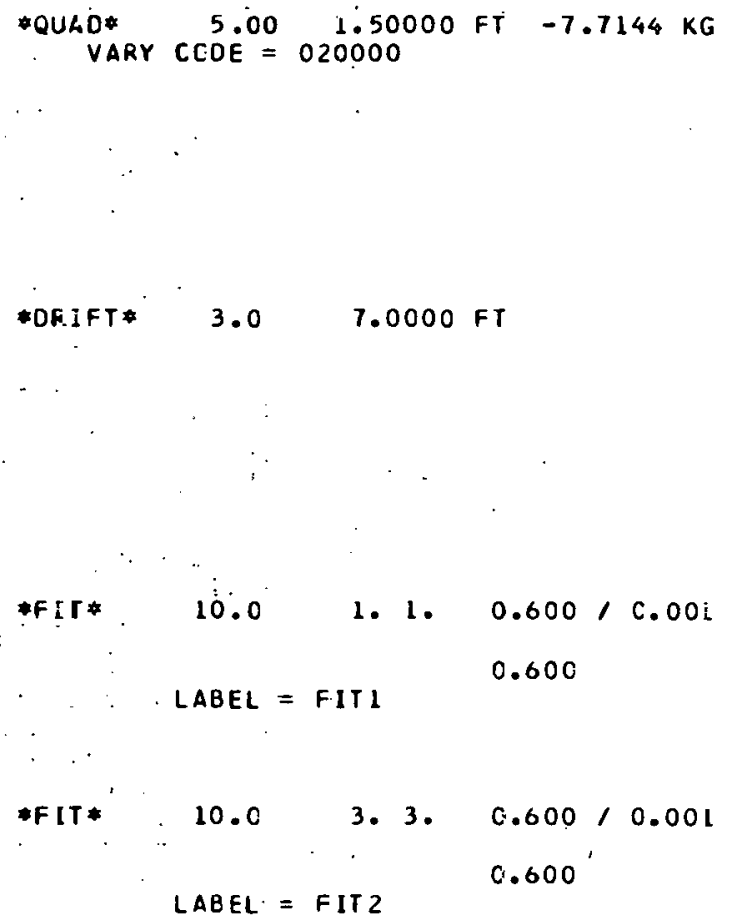

$\begin{array}{llll}0.0 & 0.0 & 0.0 & 0.0\end{array}$


'EFAM SHITCHYARD END STATIOH A ALL FITTING AT ONCE'

(THIS SYSTEM WAS ONE OF TUE DESIGNS FOR TUE SHITCHYARD ELEMENTS WHICH)

(TRAIISPORT THE SLAC BEAIA TO END STATION $\Lambda$. THE NUTCHATIC BEAM PRINTOUT IS)

(TURIIFD DFF BY THF 13.2. FLEMENT. THE MAGNETIC SYSTEM BFGIHS WITH A BENDING)

(MAGMFT WHICH DIVERTS THE BFAM BY 5 DECREFS. THIS MARNET IS FOLLOWFO BY AH)

(EIGITY MFTER ORIFT TO ALLO! THF BEAM TO CLEAR SUFFICIFWTLY THE ACCELERATCR)

(CENTER I.INF. TO ALLOH THE INSTALLATION OF A OUNDRUPCLE. DOUBLET. THE DOUBLET)

(IS FOLLOWED BY FOUR BENDINF MIATNETS WHICH ARE ENTFFED WITH THE USE OF T!IF. TYPF.)

(IINE RFPEAT CODE. THE INITIAL OUAO DOUBLET, Q1, IS ALLONF. TO VARY SO TIIAT A)

(FOCUS IS ORTAIINED AT TIIE FXIT OF THE SYMHETRY QUADEUPOLE, Q2. BOT!I T!HE)

(POSITION AHD TIHE FIEI.D $0=22$ ARF. ALLOHED TO VARY SC AS TO GET AN ACHROHATIC.)

(CONDITION AT THE EXIT OF THF SECOND FOUR BENDI:IG MARNFTS. A SFCOND DOUBIET 23)

(IS NLLOWFO TO VAFY TO PP.ONUCE $\Lambda$ EFRTAIH BEAM SIZF NT THE FNTPANCE TO THE FIMIL) (DOUBIET, 24. THIS DOIBBLET IS NLLOHED TO VARY TO GET A IMIST AND A CEPTAIN SPOT) (SIZF AT THE TARRET.)

(THIS IS AN FXAMPLE OF A OROFRAM HIHICH WILL NOT FIT ALL OF THF CONSTRAIHTS)

(AT ONCE. THE SUBSFOUFIT EXAMPLES SHOA THE USF OF SFRUENTINL. FITTING AND THE)

(IJSE OF THE TYPF ONE I:IDICATOR CAPD,)

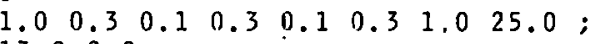

13.02 .0 ;

2.00 .0

$4.05 .0 \quad 1.45500 \quad 0.0$

2.00 .5 ;

$13.01 .0 ;$

13.01 .0

$5.012 .0-2.25 .0$ ' $211^{\prime}$;

3.02 .0 ;

$5.312 .0^{\prime} 2.45 .0$ '.21';

13.0 1.0; $;$

9.1 4.0 :

$3.01 .0 ;$

2.01 .5

4.10 3.014 .55 .0 .0

2.1) 1.5 ;

9.1) 0.0 ;

13.01 .0

3.930 .0 IDRl

5.012 .05 .015 .0 ' $22^{\prime}$.

13.01 .0

$10.0-1.02 .00 .00 .001$ 'FITI'.

$10.0-3.04 .00 .00 .001$ 'FIT1'?

13.04 .0

3.420 .0 'nR1'

13.01 .0

$3.04 .0 ;$

2.01 .5

$\begin{array}{llll}4.0 & 3.0 & 14.55 & 0.0\end{array}$

$2.01 .5 ;$

$3.001 .0 ;$

13.01 .0

$10.0-1.0 \quad 6.0 \quad 0.0 \quad 0.001$ 'FIT2'

$10.0-2.0 \quad 6.0 \quad 0.00 .001$ 'FIT3'

13.04 .0 ;

$5.012 .0-1.95 .0$ ' 23 ';

3.02 .0 .

$5.012 .0^{\prime} 1.855 .0$ ' 23 '; 
3.) 200.0 ;

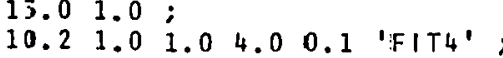

$10.23 .03 .0 \quad 1.50 .1$ 'FIT4'?

$13.04 .0 ;$

5.j1 $2.04 .5 \quad 5.0$ ' $0.44^{\prime}$;

3. 002.0

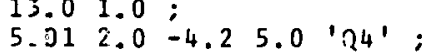

13.01 .0

3.015 .0

13.01 .0 :

$10.02 .0 \quad 1.0 \quad 0.0 \quad 0.001$ 'FIT5',

$10.04 .0 \quad 3.0 \quad 0.0 \quad 0.001$ 'FIT6'

$10.2 \quad 1.01 .000 .02 \quad 0.01$ 'FIT7',

10.23 .03 .00 .020 .01 'FIT7'

I3. 0 HT I IIEL 
BEAM SWITCHYARO END STATICN A ALL FITTING AT ONCE

THIS SYSTEM hAS ONE DF THE OESIGNS FOR THE SWITCHYARD ELEMENTS WHICH

TRANSPORT THE SLAC 8EAM TO END STATION A. THE AUTCMATIC BEAM PRINTOUT IS

TURNED OFF BY THE 13. 2. ELEMENT. THE MAGNETIC SYSTEM BEGINS WITH A BENDING

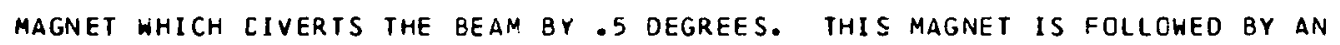
EIGHTY METER DRIFT TO ALLOW THE BEAM TO CLEAR SUFFICIENTLY THE aCCELERATOR CENTER LINE tC allow the installaticn of a quadrupole doublet. the duUbLet IS FOLLUWED OY FOUR BENDING MAGNETS WHICH ARE ENTERED WITH THE USE OF THE TYPE NINE REPEAT CODE. THE INITIAL QUAD DOUBLET,OL. IS allowed TO VARY SO THAT A FuCUS IS OBTAINED AT THE EXIT OF THE SYMMETRY QUAGRUPULE,Q2. BOTH THE POSITICN AND THE FIELD GF Q2 ARE aLlOWED TO VARY SO AS TO GET aN ACHRCMATIC CONOITION AT THE EXIT OF THE SECOND FOUR BENOING MAGNETS. A SECOND DOURLET,Q3 IS allgweo tu Vary to produce a certain beam SIZE at the entrance to the final DOUBLET, G4. THIS DOUBLET IS ALLUWED TO VARY TO GET A WAIST ANO A CERTALN SPOT SIZE AT THE TARGET.

IHIS IS AN EXAMPLE DF A PRGGRAM WHICH WILL NOT FIT ALL JF THE CCNSTRAINTS at unCE. THE SUBSEQUENT EXAMPLES SHOW THE USE DF SEQUENTIAL FITTING aNd the USE OF THE TYPE ONE INOTCATUR CARD.

$$
0
$$

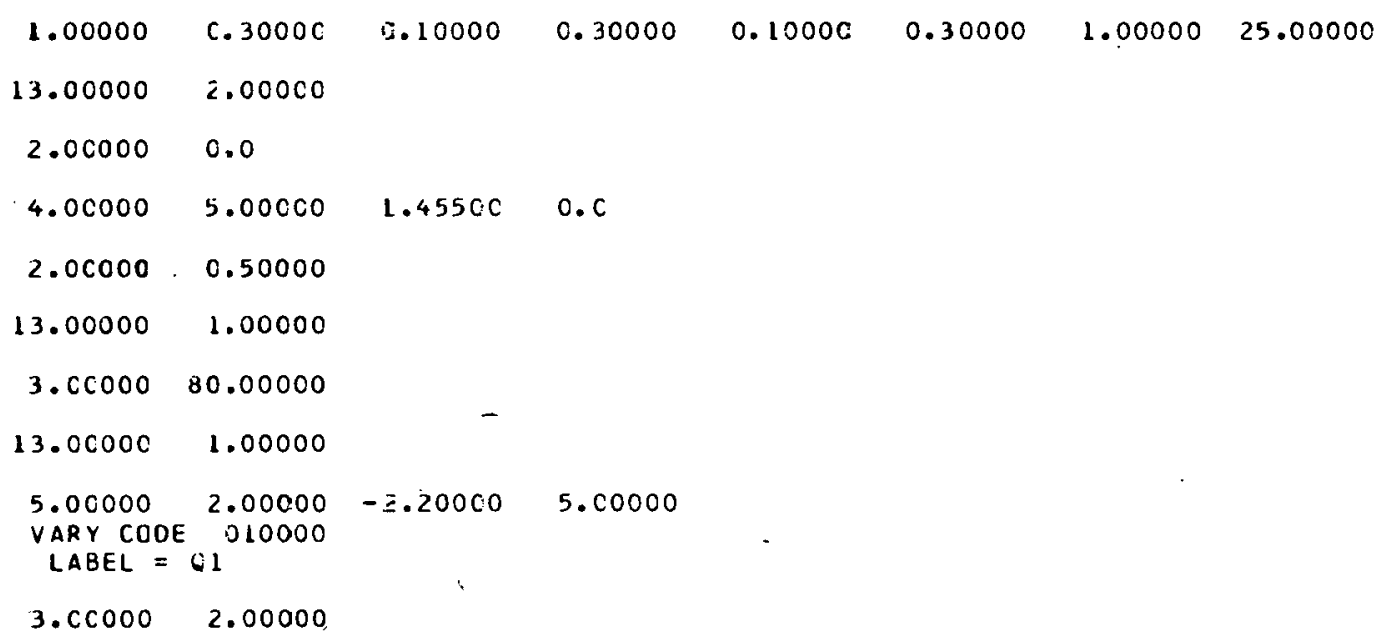




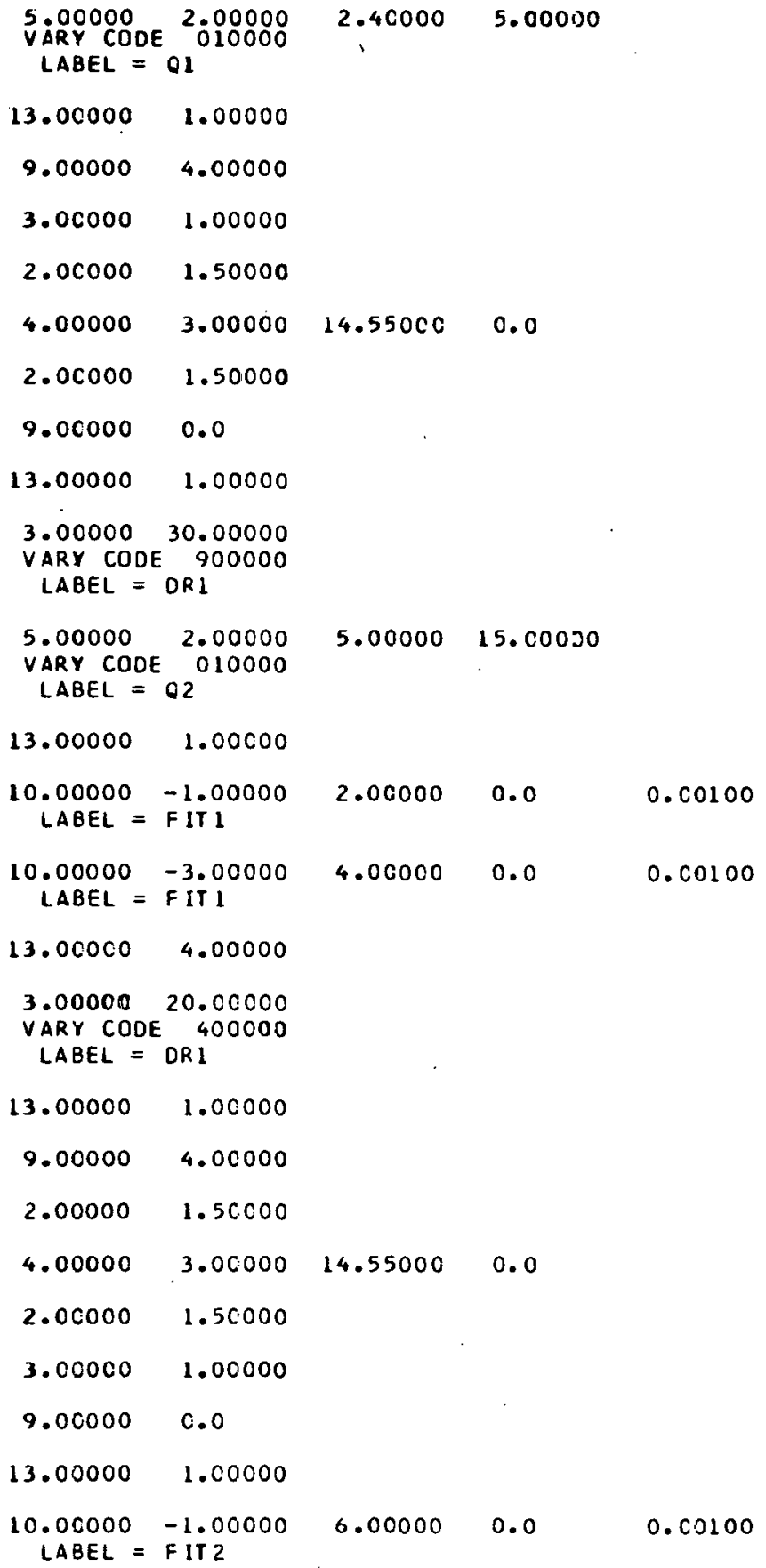




\begin{tabular}{|c|c|c|c|}
\hline 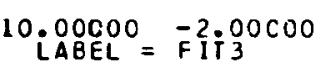 & 5.00000 & 0.0 & $0 . \cos 0 \mathrm{C}$ \\
\hline $13.00000 \quad 4.00000$ & & & \\
\hline $\begin{array}{l}5.00000 \quad 2.00000 \\
\text { VARY CODE } 010000 \\
\text { LABEL = } 63\end{array}$ & -1.90000 & 5.00000 & \\
\hline $3.00000 \quad 2.00000$ & & & , \\
\hline $\begin{array}{l}5.00000 \quad 2.00000 \\
\text { VARY CODE } \$ 10000 \\
\text { LABEL }=Q 3\end{array}$ & 1.85000 & 5.00000 & \\
\hline 3.00000200 .00000 & $\cdots$ & & \\
\hline $13.00000 \quad 1.00000$ & & & \\
\hline $\begin{array}{c}10.00000 \quad 1.00000 \\
\text { VARY CODE } 200000 \\
\text { LABEL }=\text { FIT4 }\end{array}$ & I. $c 0000$ & 4.00000 & $0.1000 C$ \\
\hline $\begin{array}{l}10.00000 \quad 3.00 C 00 \\
\text { VARY COOE } 200000 \\
\text { LABEL = FIT4 }\end{array}$ & 3.00000 & 1.50000 & $0.1000 \mathrm{C}$ \\
\hline $13.00000 \quad 4.00000$ & & & \\
\hline $13.00000 \quad 1.00000$ & & & . \\
\hline $\begin{array}{l}\begin{array}{l}5.00000 \\
\text { VARY CODE }\end{array} 2.000000 \\
\text { LABEL }=64\end{array}$ & $\therefore .5 \operatorname{coc} c$ & 5.00000 & \\
\hline $3.00000 \quad 2.00000$ & & & \\
\hline $13.00000 \quad 1.00000$ & & & \\
\hline $\begin{array}{l}5.00000 \\
\text { VARY CODE } 2.00000 \\
\text { LABEL }=64\end{array}$ & -4.20000 & 5.00000 & \\
\hline 13.00000 & & & \\
\hline $3.00000 \quad 15.00000$ & & & \\
\hline $13.00000 \quad 1.00000$ & & & \\
\hline $\begin{array}{l}10.00000 \\
\text { LABEL }=\text { FIT'S }\end{array}$ & 1. $\cos 00$ & $0 . \mathrm{C}$ & $0.0010 \mathrm{C}$ \\
\hline $\begin{array}{c}10.00000=4.00000 \\
\text { LABEL }=\text { FIT } 6 .\end{array}$ & $3 . c 0000$ & $c .0$ & $0.0010 \mathrm{C}$ \\
\hline $\begin{array}{l}\text { 10. CCOOO } 1.00000 \\
\text { VARY CODE } 200000 \\
\text { LABEL = FII }\end{array}$ & $1 . \cos c 0$ & 0.02000 & $0 . C 100 \mathrm{C}$ \\
\hline 10.00000 & 3.00000 & c. $C 2000$ & $0 . c 100 C$ \\
\hline
\end{tabular}


$z \tau-3$

7 $\exists$ I IN 1 S

00000•h $00000^{\circ E 1}$

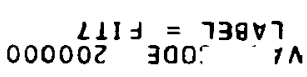


BEAM SHITCHYARD END STATION A ALL FITIING AT ONCE

* BEAM $\quad 1.000000 \quad 25.00 \mathrm{GEV}$

$0.0 \mathrm{M}$

0.0
0.0
0.0
0.0
0.0
0.0

$0.300 \mathrm{CM}$

$0.100 \mathrm{MR}$

$0.300 \mathrm{CM}$

$0.100 \mathrm{MR}$

$0.300 \mathrm{CM}$
$1.000 \mathrm{PC}$

0.0

0.0
0.0
0.0

0.0

$\begin{array}{ll}0.0 & 0.0 \\ 0.0 & 0.0\end{array}$

0.0
0.0
0.0

0.0
0.0

0.0

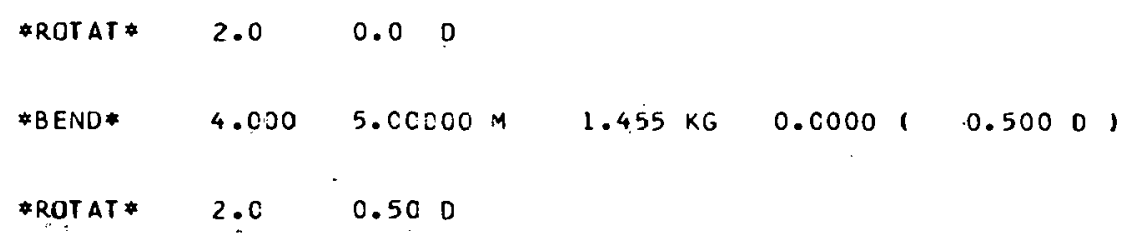

\subsection{1}

$\begin{array}{lll}0.0 & 0.0 & 0.164\end{array}$

$\begin{array}{rrrrrr}1.000 \mathrm{PC} & -0.009 & -0.001 & 0.0 & 0.0 & \\ 0.071 & 0.657 & 0.0 & 0.0 & -9.000\end{array}$

*ORIFT* $\quad 3.0 \quad 8 C .00 J C \mathrm{M}$

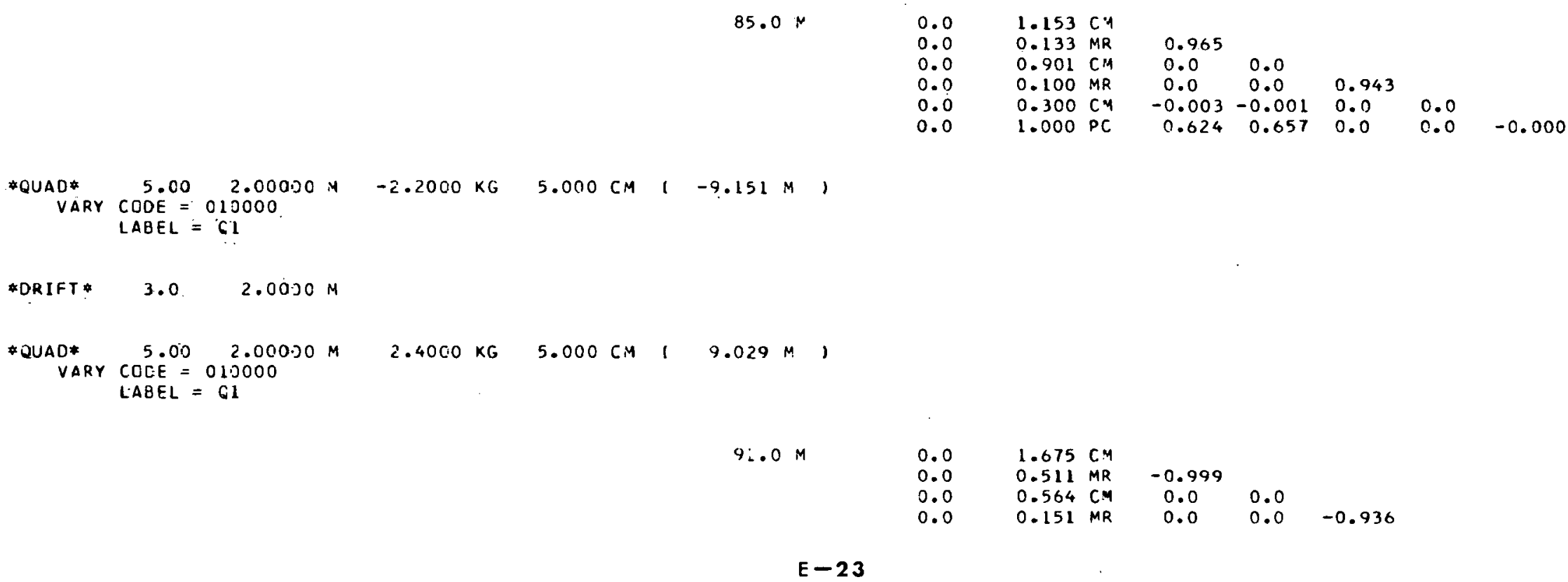

$85.0 \mathrm{~N}$

$\begin{array}{ll}0.0 & 1.153 \mathrm{C} \\ 0.0 & 0.133 \mathrm{MR} \\ 0.0 & 0.901 \mathrm{C} \\ 0.0 & 0.100 \mathrm{MR} \\ 0.0 & 0.300 \mathrm{C} \\ 0.0 & 1.000 \mathrm{PC}\end{array}$




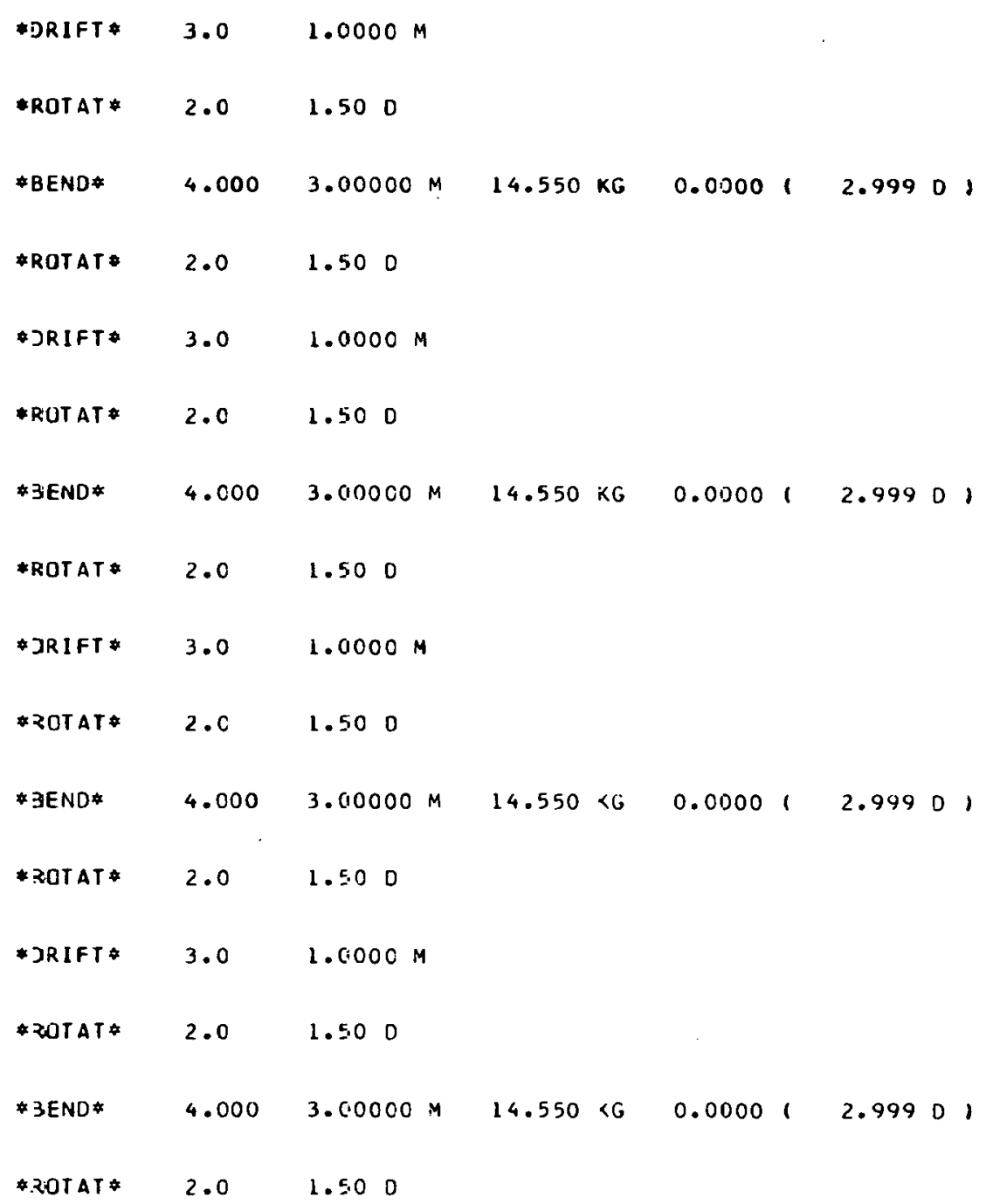




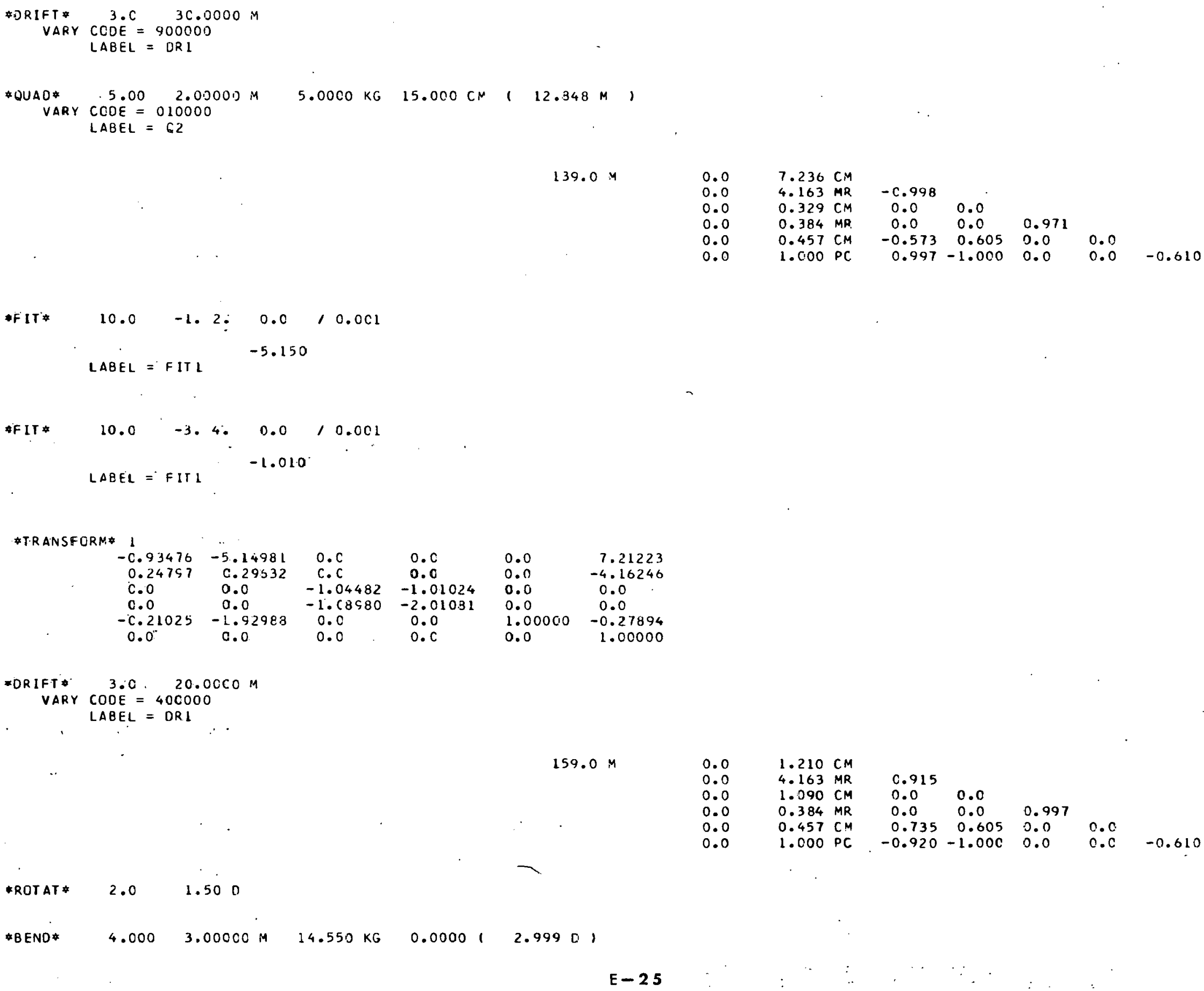

$139.0 \times 4$

* I IT*
10.0

$-1$

LABEL $=F I T L$

$$
-5.150
$$

IF IT*

$\begin{array}{cc}10.0 & -3.4 \% \\ L A B E L=F I I L & -1.010\end{array}$

\#TRANSFORM

$\begin{array}{cccclc}-C .93476 & -5.14981 & 0 . C & 0.0 & 0.0 & 7.21223 \\ 0.24757 & C .29532 & C . C & 0.0 & 0.0 & -4.16246 \\ c .0 & 0.0 & -1.04482 & -1.01024 & 0.0 & 0.0 \\ 0.0 & 0.0 & -1.08980 & -2.01031 & 0.0 & 0.0 \\ -c .21025 & -1.92983 & 0.0 & 0.0 & 1.00000 & -0.27894 \\ 0.0 & 0.0 & 0.0 & 0.0 & 0.0 & 1.00000\end{array}$

ODRIFT $\quad 3: 0, \quad 20.0060 \mathrm{M}$

VARY $\operatorname{CODE}=400000$

$\angle A B E L=D R L$

$159.0 \mathrm{M}$

0.0
0.0
0.0
0.0
0.0
0.0

$7.236 \mathrm{CM}$

$4.163 \mathrm{MR}$

$0.329 \mathrm{CM}$
$0.384 \mathrm{MP}$

$0.457 \mathrm{CM}$

$1.000 \mathrm{PC}$

0.0

$\begin{array}{lll}-0.573 & 0.605 & 0.0\end{array}$

$\begin{array}{llll}0.997-1.000 & 0.0 & 0.0\end{array}$

*Rotat* $2.0 \quad 1.500$

*BEND* 


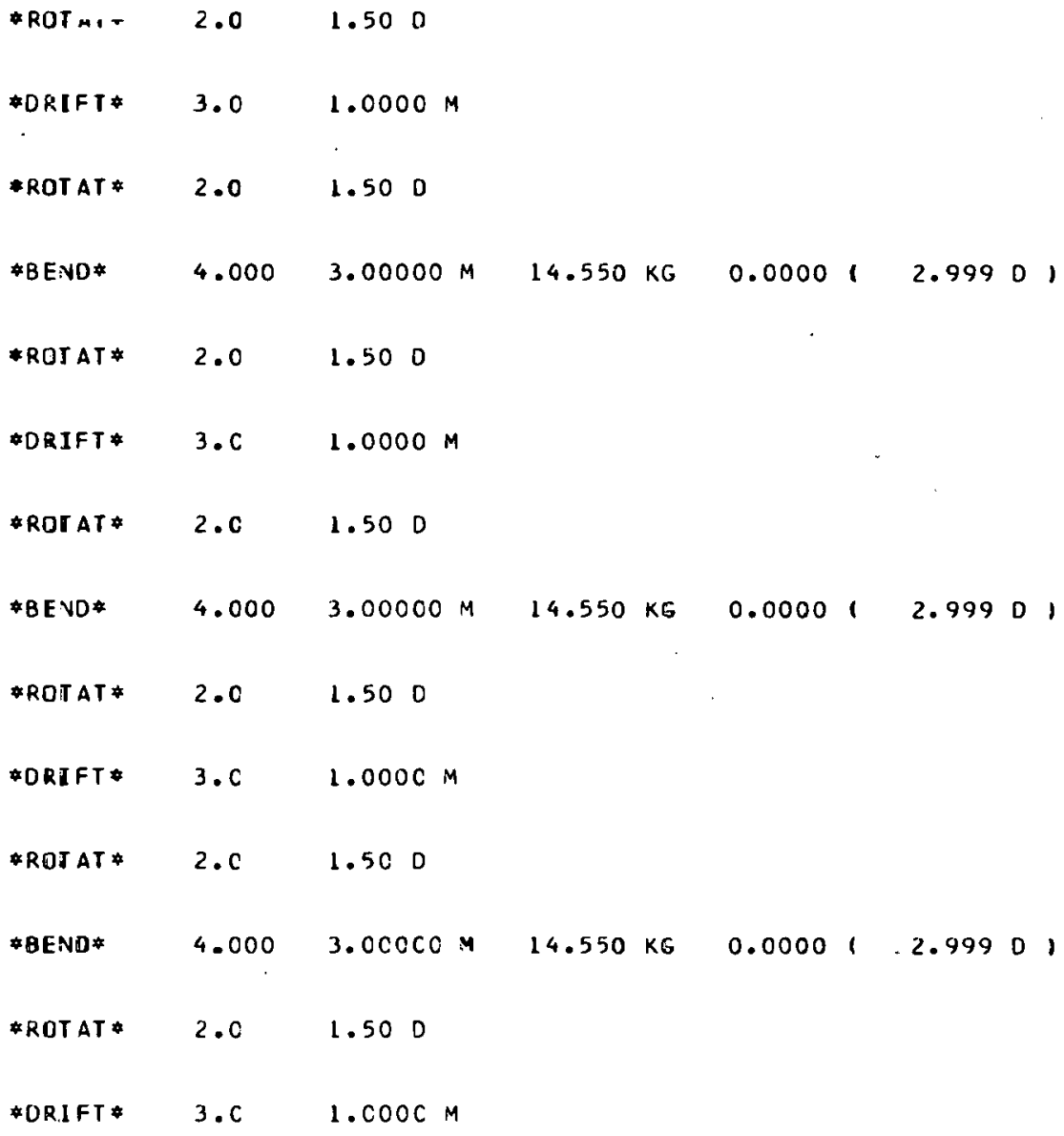




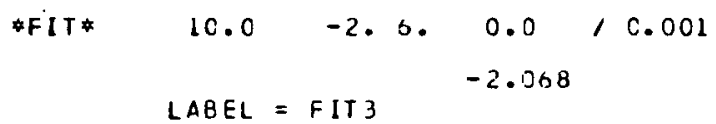

$\begin{array}{llllll}\text { \#TRANSFORN } 1 . & & & \\ -0.04222 & -4.08323 & 0 . c & 0 . c & 0.0 & -5.99086 \\ 0.24796 & 0.29627 & c . c & 0.0 & 0.0 & -2.06827 \\ C .0 & 0.0 & -4.85194 & -8 . C 5319 & 0.0 & 0.0 \\ 0.0 & 0.0 & -0.94335 & -1.77381 & 0.0 & 0.0 \\ -0.15728 & -1.02203 & 0.0 & 0.0 & 1.000 c 0 & 0.49253 \\ 0.0 & 0.0 & 0.0 & 0.0 & 0.0 & 1.00000\end{array}$

* QuAd* $5.00 \quad 2.0000 \mathrm{M}-1.9000 \mathrm{ke} 5.000 \mathrm{CM}(-10.646 \mathrm{M})$

VARY CCDE $=0100 C 0$

$\angle A E E L=63$

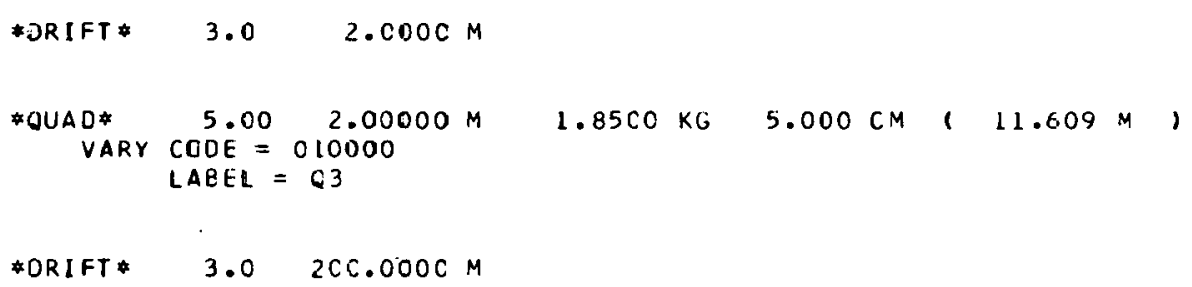

*DRIFT* $\quad 3.0 \quad 20 \mathrm{C} .000 \mathrm{C} \mathrm{M}$

$381.0 M$

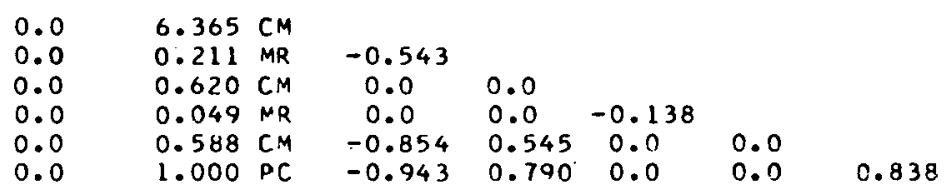

\#FIT* 10.0 1. 1. 4.600/0.100

VARY COCE $=200000$

6.365

$\angle A B E L=F I T 4$

\footnotetext{
*FIT* $\quad 10.0 \quad 3.3 .1 .500 / 0.100$

VARY CODE $=200000$

0.620

$\mathrm{LABEL}=\mathrm{FIT} 4$
}

*TRANSFORM *

$\begin{array}{rrrrrr}3.42558 & 28.46803 & 0.0 & 0.0 & 0.0 & -6.00397 \\ C .16690 & 1.15030 & 0.0 & 0.0 & 0.0 & 1.16643 \\ & & & & & \\ & & & & & E-27\end{array}$




$\begin{array}{llllll}0.0 & 0.0 & -0.30513 & -6.13656 & 0.0 & 0.0 \\ 0.0 & 0.0 & 0.16271 & -0.00492 & 0.0 & 0.0 \\ -0.15728 & -1.02203 & 0.0 & 0.0 & 1.00000 & 0.49253 \\ 0.0 & 0.0 & 0.0 & 0.0 & 0.0 & 1.00000\end{array}$

$381.0 \mathrm{M}$

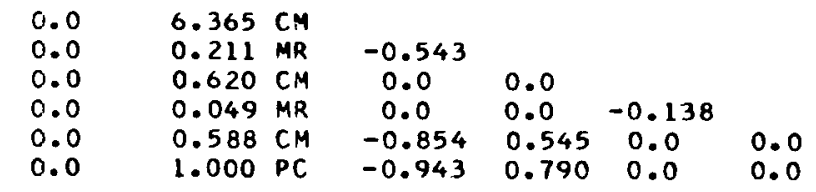

0.838

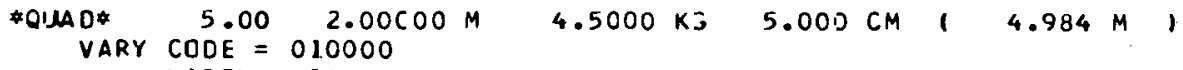

\#DIFT $\quad 3.0 \quad 2.0000 \mathrm{M}$

$385.0 M$

$387.0 \mathrm{M}$

$402.0 \mathrm{M}$

*DFi I FT *

3.C $15.000 \mathrm{CM}$

$$
\begin{aligned}
& 0.0 \\
& 0.0 \\
& 0.0 \\
& 0.0 \\
& 0.0 \\
& 0.0
\end{aligned}
$$

0.0
0.0
0.0
0.0
0.0
0.0
$2.447 \mathrm{CM}$
$12.863 \mathrm{MR}$ $1.044 \mathrm{CM}$ $1.431 \mathrm{MR}$.

1. $000 \mathrm{PC}$ $-0.999$ $\begin{array}{ll}0.0 & 0.0 \\ 0.0 & 0.0\end{array}$ $\begin{array}{lll}0.0 & 0.0 & 1.050\end{array}$ $\begin{array}{llll}-0.935 & 0.947 & 0.0 & 0.0\end{array}$

0.838

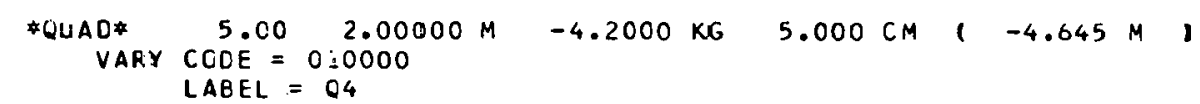
CCDE $=0: 0000$

$\begin{array}{lr}0.0 & 0.232 \mathrm{CM} \\ 0.0 & 10.281 \mathrm{MR} \\ 0.0 & 1.108 \mathrm{CM} \\ 0.0 & 0.813 \mathrm{MR} \\ 0.0 & 0.588 \mathrm{CM} \\ 0.0 & 1.000 \mathrm{PC}\end{array}$

$-0.881$

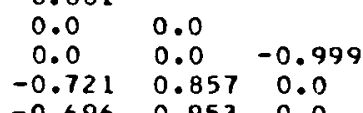

$\begin{array}{llll}-0.721 & 0.857 & 0.0 & 0.0 \\ -0.696 & 0.953 & 0.0 & 0.0\end{array}$

0.838

*FIT* $10.0 \quad$ 2. $1.0 .0,0.0 C 1$

$\begin{array}{lr}0.0 & 15.217 \mathrm{CM} \\ 0.0 & 10.281 \mathrm{MR} \\ 0.0 & 0.119 \mathrm{CM} \\ 0.0 & 0.813 \mathrm{MR} \\ 0.0 & 0.588 \mathrm{CM} \\ 0.0 & 1.000 \mathrm{PC}\end{array}$

1.000

$0.0 \quad 0.0$

$0.0 \quad 0.0 \quad 0.950$

$\begin{array}{llll}0.857 & 0.857 & 0.0 & 0.0\end{array}$

$\begin{array}{llll}0.955 & 0.953 & 0.0 & 0.0\end{array}$

0.838 


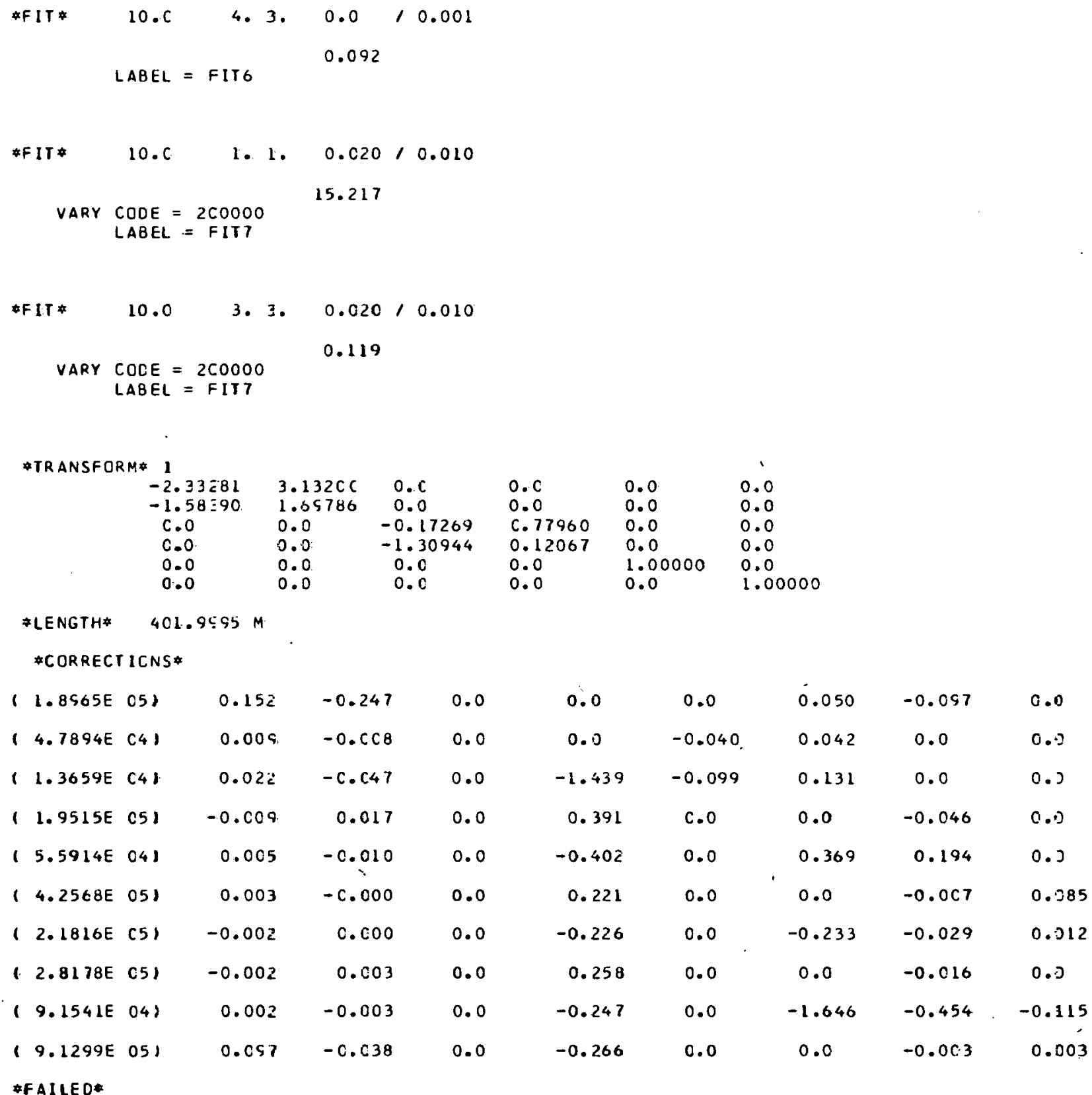


\# BEAM* $1.000000 \quad 25.00$ GEV

* Ror AT* $2.0 \quad 0.0 \quad 0$

\#BEND* 4.000 5.c0000 M $\quad 1.455<G \quad 0.000010 .50001$

* ROTAT* $2.0 \quad 0.500$

0.0
0.0
0.0
0.0
0.0
0.0

$0.300 \mathrm{CM}$ $0.100 \mathrm{MR}$ $0.300 \mathrm{CM}$ $0.100 \mathrm{MR}$ $0.300 \mathrm{CM}$ 0.0 $0.0 \quad 0.0$ 0.0 $\begin{array}{llll}0.0 & 0.0 & 0.0 & 0.0\end{array}$ 0.0

0.0
0.0
0.0
0.0
0.0
0.0
$0.305 \mathrm{CM}$ $0.133 \mathrm{MR}$ $0.304 \mathrm{CM}$

$0.100 \mathrm{MR}$

$0.300 \mathrm{CM}$ $1.000 \mathrm{PC}$

0.171

$\begin{array}{ll}0.0 & 0.0\end{array}$

$0.0 \quad 0.0 \quad 0.164$

$\begin{array}{llll}-0.009 & -0.001 & 0.0 & 0.0\end{array}$ $\begin{array}{rrrr}0.071 & 0.657 & 0.0 & 0.0\end{array}$

$-0.000$
$85.0 \mathrm{M}$

$$
\begin{aligned}
& 0.0 \\
& 0.0 \\
& 0.0 \\
& 0.0 \\
& 0.0 \\
& 0.0
\end{aligned}
$$

$1.153 \mathrm{CM}$ $0.133^{\prime} \mathrm{MR}$ $0.901 \mathrm{CM}$ $0.100 \mathrm{MR}$

$0.300 \mathrm{CM}$

\subsection{5}

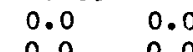

0.943

$\begin{array}{rrr}0.003 & -0.001 & 0.0 \\ 0.624 & 0.657 & 0.0\end{array}$

0.0

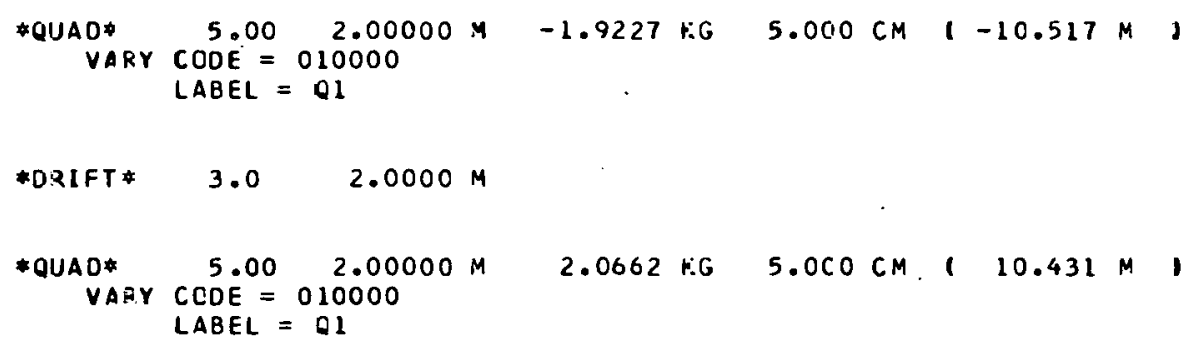

$91.0 \mathrm{M}$

0.0
0.0
0.0
0.0

$1.625 \mathrm{CM}$

$0.356 \mathrm{MR}$

$0.615 \mathrm{CM} \quad-0.998$

$0.094 \mathrm{MR}$

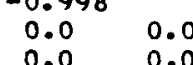

0.0
0.0 


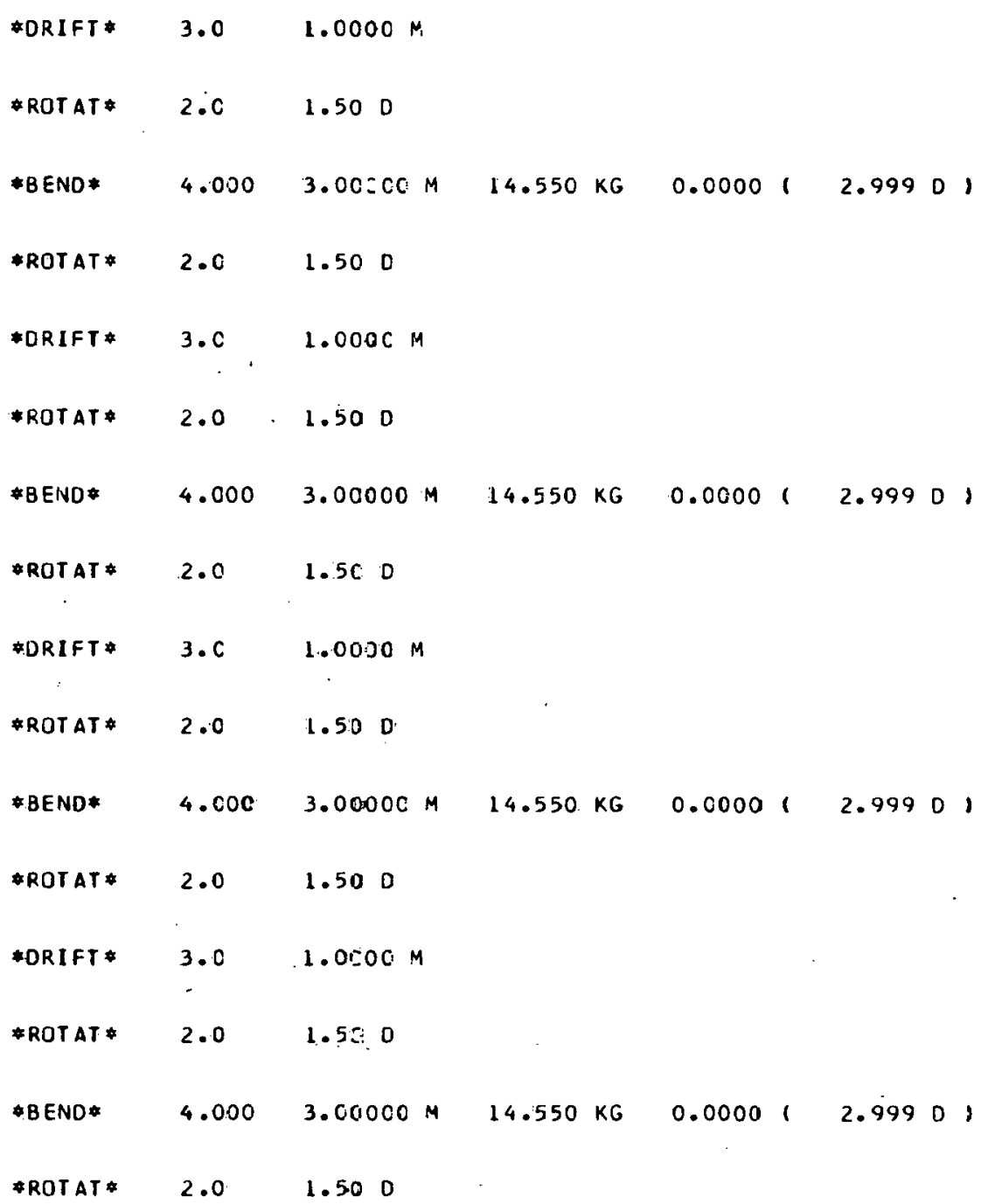


\#ORIFT* $3.0 \quad 30.0500 \mathrm{M}$

VARY CCDE $=900000$

$\angle A B E L=\cdot J R L$

* QUAD*

$5.00 \quad 2.00500 \mathrm{M}$

$3.29 C 9$ K.G

$15.060 \mathrm{~cm}$

$19.342 M \quad$

VARY $C O D E=0.10000$

$\angle A B E L=22$

$139.0 \mathrm{M} \quad 0.0$

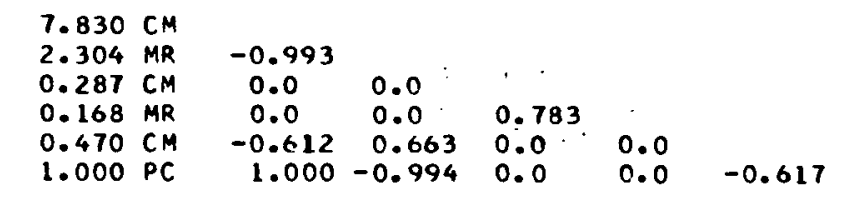

*FIT*

$10.0-1.2$. 0.c, 0.001

LABEL $=F I T I$

$-0.405$

*F IT *

$$
\begin{array}{lll}
10.0 & -3.4 . & 0.0 \\
\text { LABEL }=\text { FIT } 1 & 2.223
\end{array}
$$

* TRANSFCRM*

$\begin{array}{ccllll}1 & & & & \\ -0.42470 & -0.4 C 498 & 0.0 & 0.0 & 0.0 & 7.82867 \\ -0.16137 & -2.5 C 845 & 0.0 & 0.0 & 0.0 & -2.29023 \\ 0.0 & 0.0 & -0.60594 & 2.22344 & 0.0 & 0.0 \\ 0.0 & 0.0 & -0.54673 & 0.35586 & 0.0 & 0.0 \\ -0.22360 & -2.05654 & 0.0 & 0.0 & 1.00000 & -0.28970 \\ 0.0 & 0.0 & 0.0 & 0.0 & 0.0 & 1.00000\end{array}$

*DRIFT* $3.0 \quad 20.0000 \mathrm{M}$

VARY $C O D E=400000$

LAEEL = DRI

$159.0 \mathrm{M}$

$$
\begin{aligned}
& 0.0 \\
& 0.0 \\
& 0.0 \\
& 0.0 \\
& 0.0 \\
& 0.0
\end{aligned}
$$

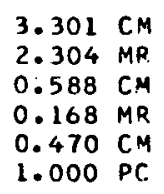

$-0.959$

0.0

$0.0 \quad 0.0$

$\begin{array}{llll}0.984 & -0.963 & 0.0 & 0.0\end{array}$

*ROTAT $2.0 \quad 1.500$

\#EEND* 


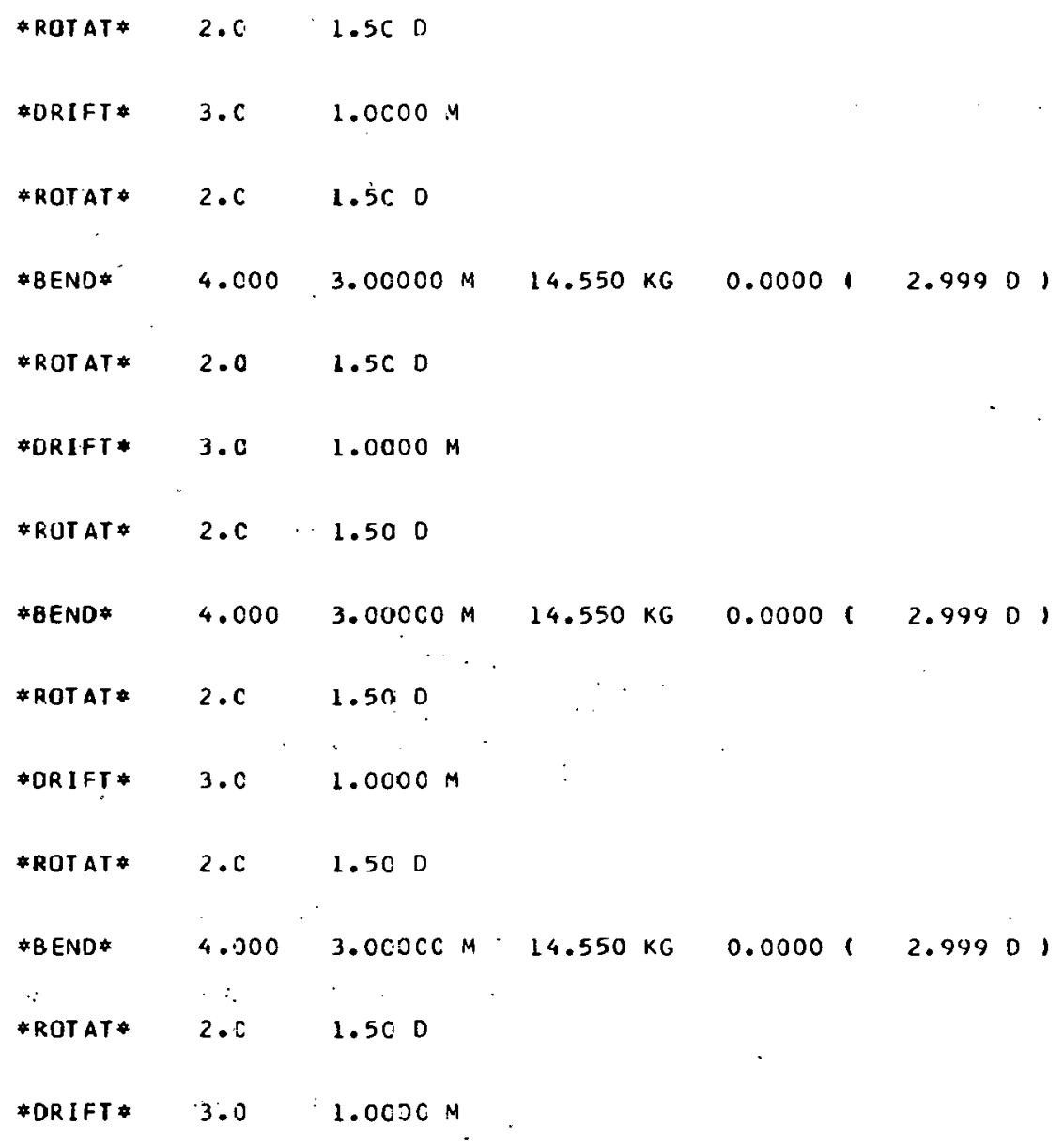




$\begin{array}{cccccc}C .0 & 0 . c & 27.77695 & -46.09175 & 0.0 & 0.0 \\ 0.0 & 0.0 & 1.47038 & -2.40389 & 0.0 & 0.0 \\ -0.04 .73 & -0.52722 & 0.0 & 0.0 & 1.00000 & -0.72547 \\ c .0 & 0.0 & 0.0 & 0.0 & 0.0 & 1.00000\end{array}$

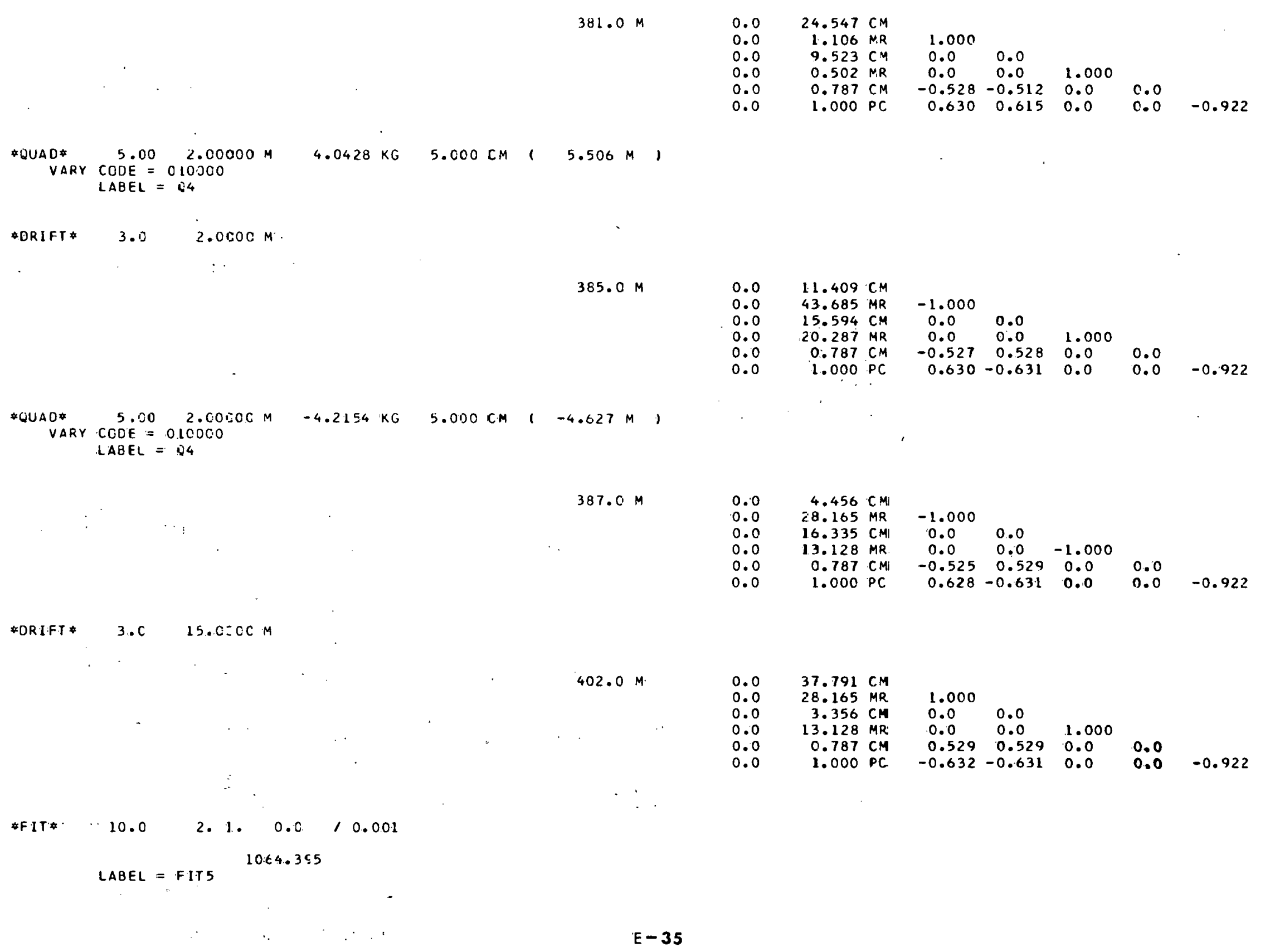




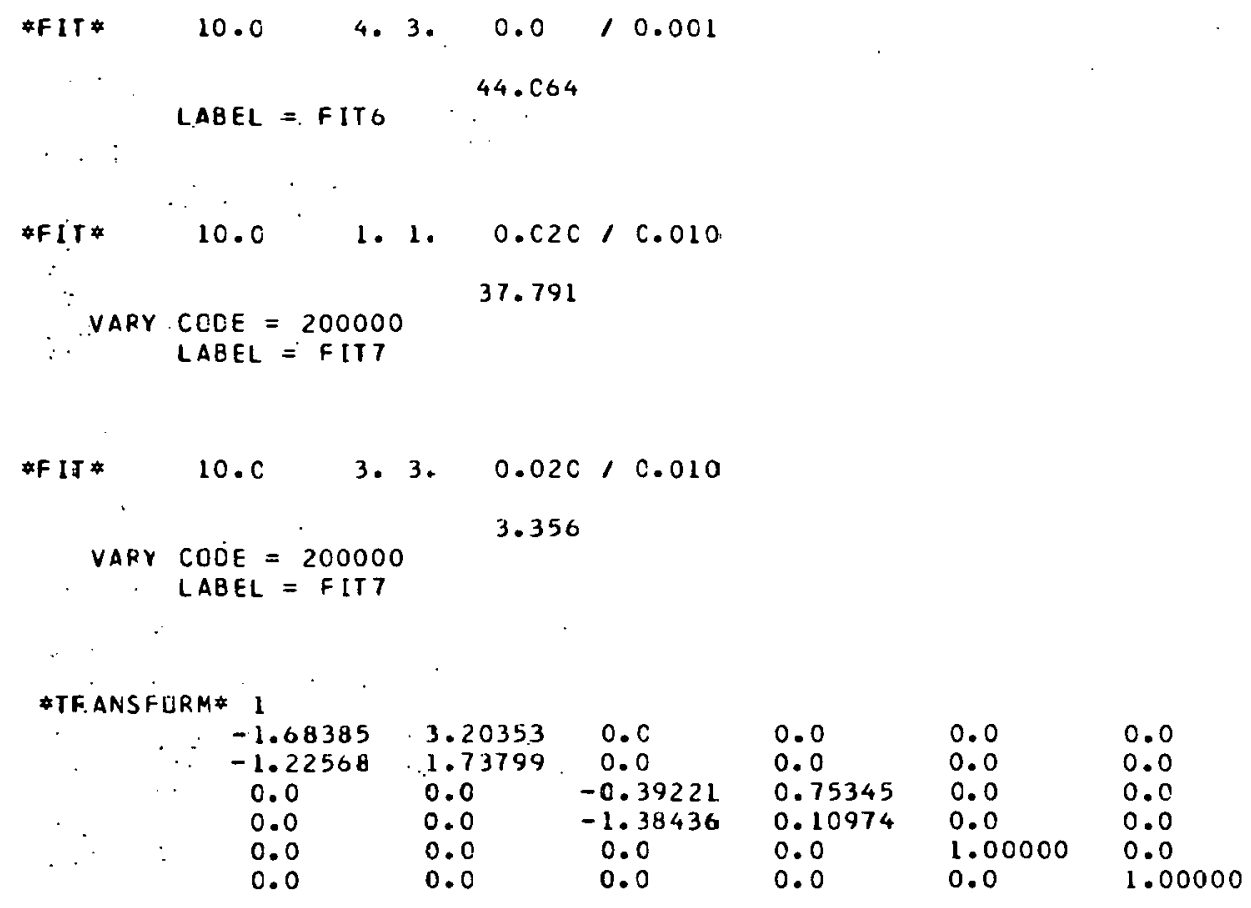

\#LENGTH* $401 . S S S 5 M$ 
'BEAI TRANSPORT SYSTEM FOR SLAC BEAM SINITCHYARD EI: STATIOI! A I'

(THE FIRST STEP TO FITTING IS TO ALLOW ONLY THE PQSITION AND FIELD OF THE)

(SYMMETRY QUAD, O2, TO VGRY IN ORDER TO GET AN ACHFCMATIC CONDITION BETWEEN THE)

(FIRST SET OF BENDING MGGNETS AND THE SECOND QUND C.OUBLET, Q3.)

$\begin{array}{llllllllllll}1.0 & 0.3 & 0.1 & 0.3 & 0.1 & 0.3 & 1.0 & 25.0\end{array}$

13.02 .0 ;

$4.05 .0 \quad 1.455000 .0$;

$2.00 .5 ;$

$13.01 .0 ;$

3.080 .0

13.01 .0

$5.02 .0-2.25 .0$ '.21',

3.02 .0

5.02 .02 .45 .0 'O1':

$13.01 .0 ;$

3.01 .0 ;

2.01 .5

4.03 .0 í 4.550000 .0 ;

2.01 .5

9.0000

3.930 .0 inR1'

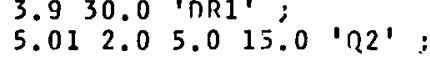

13.01 .0 ;

$\begin{array}{lllllll}-10.0 & -1.0 & 2.0 & 0.0 & 0.001 & \text { 'FITI' }\end{array}$

$-10.0-3.04 .00 .00 .001$ 'FITI';

13.04 .0 ;

3.420 .0 'DRI':

13.01 .0 :

2.04 .05

$4.03 .014 .55000 \quad 0.0$;

2.03 .5

3. 01.0

9.00 .0 ;

13.01 .0 ;

$10.0-1.06 .00 .00 .001$ 'FIT2';

$10.0-2.06 .00 .00 .001$ 'FIT3':

13.04 .0 :

$5.02 .0-1.95 .0$ '0.3';

3.02 .0

5.0 2.01 .850005 .0 ' $233^{\prime}$.

3.0200 .0 ;

13.01 .0 :

-10.21 .0 ' 1.04 .00 .1 'FIT4',

$-10.23 .0 \quad 3.0$ 1.5 0.1 'FIT4';

13.04 .0 ;

13.01 .0 ;

$5.02 .04 .55 .0{ }^{\prime} 24^{\prime}$;

$3.102 .0 ;$

5.0 2.04 .25 .0124 '

13.01 .0 :

3.015 .0

13.01 .0 :

-10.02 .01 .00 .00 .001 'FIT5';

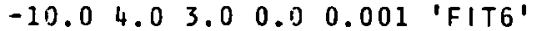

$-10.21 .01 .000 .02000 \quad 0.01000$ 'FIT7" :

-10.23 .03 .00 .020000 .01000 'FIT7:

13.04 .0

SENTIHEL 
THE FIRST.STEP TO FITTING IS TO ALLOW ONLY THE POSITION ANO fiELD OF the. SYMMETRY QUAC,Q2, TO VARY IN ORDER TO GET AN ACHRGMATIC CONDITION BETHEEN THE FIRST SET OF BENOING MagNetS AND THE SECONO QUAO DOUBLET,Q3.

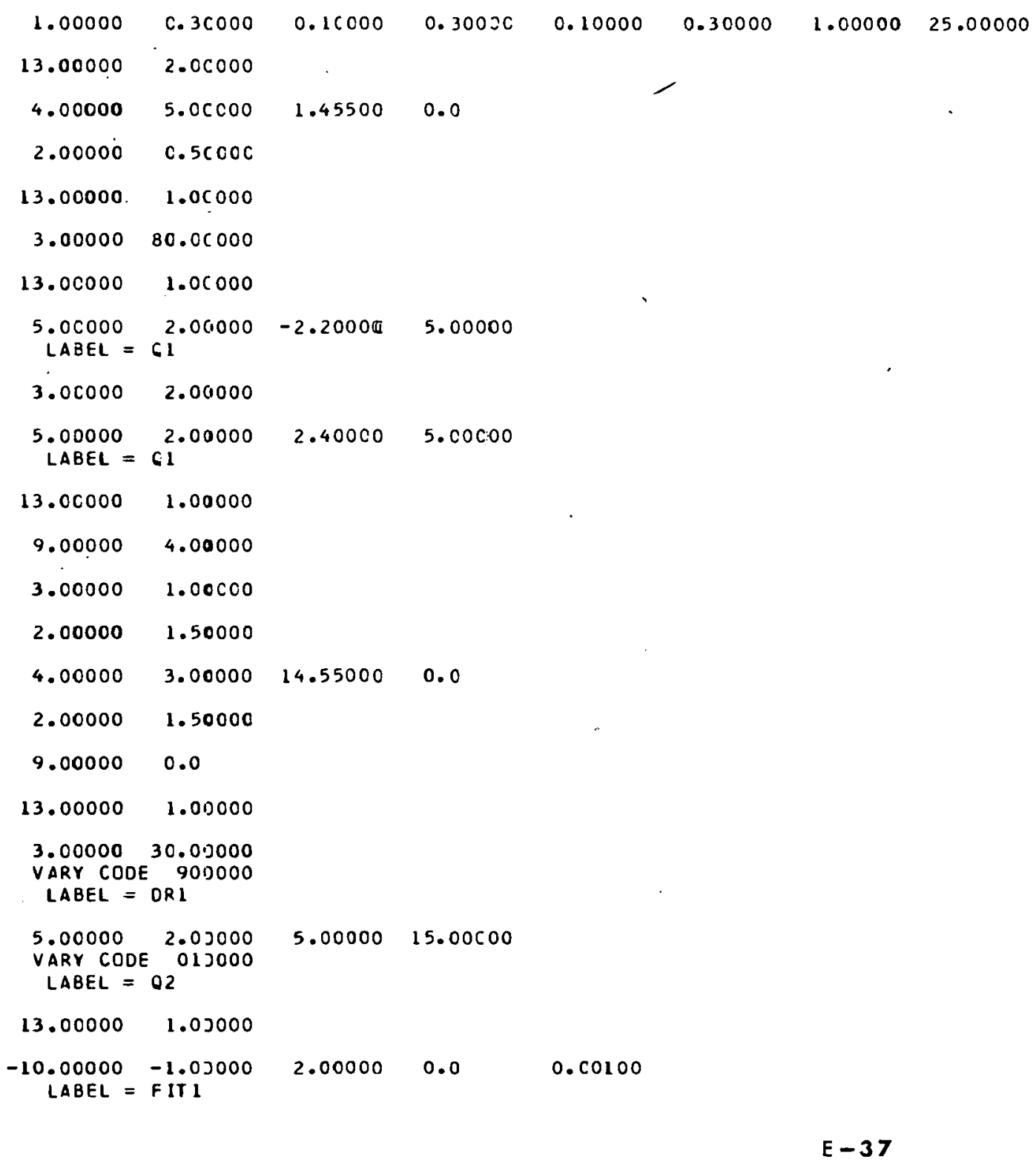




\begin{tabular}{|c|c|c|c|c|}
\hline $\begin{array}{c}-10,00000 \\
\text { LABEL }=\end{array}$ & FiTi & 4.00000 & 0.0 & 0.00100 \\
\hline 13.00000 & 4.00000 & . & - & \\
\hline $\begin{array}{l}3.0 C O 00 \\
\text { VARY CODE } \\
\text { LABEL = }\end{array}$ & $\begin{array}{l}20.00000 \\
400000 \\
\text { DRI }\end{array}$ & & & \\
\hline 13.00000 & 1.00000 &. & & \\
\hline 9.00000 & 4.00000 & & & \\
\hline $2: 00000$ & $1: 50000$ & & & \\
\hline 4.00000 & 3.00000 & 14.55000 & 0.0 & \\
\hline 2.00000 & 1.50000 & & & \\
\hline $3.0 \mathrm{COOO}$ & 1.00000 & & & \\
\hline 9. $c 0000$ & 0.0 & & & \\
\hline 13.00000 & 1.00000 & & & \\
\hline $\begin{array}{l}10.00000 \\
\text { LABEL = }\end{array}$ & $\begin{array}{l}-1.00000 \\
\text { F1T2 }\end{array}$ & 0.00000 & 0.0 & $0 . \operatorname{CCl} 100$ \\
\hline $\begin{array}{c}10.00000 \\
\text { LABEL = }\end{array}$ & $\begin{array}{l}-2.00000 \\
\text { FiT3 }\end{array}$ & 0.00000 & 0.0 & 0.00100 \\
\hline 13.00000 & 4.00000 & & & \\
\hline $\begin{array}{l}5.00000 \\
\text { LABEL = }\end{array}$ & 2.00000 & -1.90000 & 5.00000 & \\
\hline 3.00000 & $\overline{c .00000}$ & & & \\
\hline $\begin{array}{l}5.00000 \\
\text { LABEL }=\end{array}$ & $\begin{array}{l}2.00000 \\
63 \ldots\end{array}$ & 1.35000 & 5.00000 & \\
\hline 3.000002 & 200.00000 & & & \\
\hline 13.00000 & 1.00000 & & & \\
\hline $\begin{array}{c}-10.00000 \\
\text { VARY CODE } \\
\text { LABEL = }\end{array}$ & $\begin{array}{r}1.00000 \\
=200000 . \\
\text { FIT } 4 .\end{array}$ & $1.0000 \mathrm{C}$ & 4.00000 & 0.10000 \\
\hline $\begin{array}{c}-10.00000 \\
\text { VARY CODE } \\
\text { LABEL = }\end{array}$ & $\begin{array}{r}3.00000 \\
200000 \\
\text { FIT } 4\end{array}$ & 3.00000 & 1.50000 & 0.10000 \\
\hline 13.00000 & 4.00000 & - & & \\
\hline 13.00000 & 1.00000 & & & \\
\hline $\begin{array}{l}5.00000 \\
\text { LABEL }=\end{array}$ & 64.00000 & $4.5 \mathrm{COOC}$ & 5.00000 & \\
\hline
\end{tabular}




\begin{tabular}{|c|c|c|c|c|}
\hline 3.00000 & 2.00000 & & & \\
\hline 13.00000 & 1.00000 & & & \\
\hline $\begin{array}{l}5.00000 \\
\text { LABEL }=\end{array}$ & 64.00000 & -4.20000 & 5.00000 & \\
\hline 13.00000 & 1.00000 & & & \\
\hline 3.00000 & 15.00000 & & & \\
\hline 13.00000 & 1.00000 & & & \\
\hline $\begin{array}{c}-10.00000 \\
\angle A B E L=\end{array}$ & $\begin{array}{l}2.00000 \\
\text { Firs }\end{array}$ & 1.00000 & $0 . c$ & 0.00100 \\
\hline $\begin{array}{c}-10.000000 \\
\text { LABEL }\end{array}=$ & $\begin{array}{l}4.00000 \\
\text { FIT6 }\end{array}$ & $3 . c 0000$ & 0.0 & 0.00100 \\
\hline $\begin{array}{c}-12.00000 \\
\text { VARY CODE } \\
\text { LABEL }=\end{array}$ & $\begin{array}{l}1.00000 \\
200000 \\
\text { FIT7 }\end{array}$ & 1.00000 & 0.02000 & 0.01000 \\
\hline $\begin{array}{c}-10.00000 \\
\text { JARY CODE } \\
\text { LABEL = }\end{array}$ & $\begin{array}{l}3.00000 \\
200000 \\
\text { FIT }\end{array}$ & $3 . c \operatorname{coc} c$ & 0.02000 & $0 . c 1000$ \\
\hline 13.00000 & 4.00000 & & & \\
\hline
\end{tabular}


BEAM TRANSPORT SYSTEM For SLAC hEAM SWITCHYARD END STATICN A I

* BEAM* $\quad 1.000300 \quad 25.00$ GEV

$0.0 \quad 0.300 \mathrm{CM}$

$0.0 \quad 0.100 \mathrm{MR}$

$0.0 \quad 0.300 \mathrm{CM}$

$0.0 \quad 0.100 \mathrm{MR}$

$0.0 \quad 0.300 \mathrm{CM}$

$0.0 \quad 1.000 \mathrm{PC}$

0.0

0.0

$\begin{array}{lll}0.0 & 0.0 & 0.0\end{array}$

$0.0 \quad 0.0 \quad 0.0$

$\begin{array}{llll}0.0 & 0.0 & 0.0 & 0.0\end{array}$ 0.0

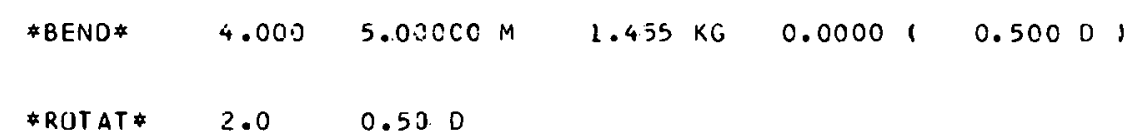

$5.0 \mathrm{M}$

$\begin{array}{ll}0.0 & 1.153 \mathrm{CM} \\ 0.0 & 0.133 \mathrm{MR} \\ 0.0 & 0.901 \mathrm{CM} \\ 0.0 & 0.100 \mathrm{MR} \\ 0.0 & 0.300 \mathrm{CM} \\ 0.0 & 1.000 \mathrm{PC}\end{array}$

$0.305 \mathrm{CM}$ $0.133 \mathrm{MR}$ $0.304 \mathrm{CM}$ $\begin{array}{llccc}0.0 & 0.100 \mathrm{MR} & 0.0 & 0.0 & 0.164 \\ 0.0 & 0.300 \mathrm{CM} & -0.009 & -0.001 & 0.0\end{array}$ $\begin{array}{ccccc}0.0 & 0.100 \mathrm{MR} & 0.0 & 0.0 & 0.164 \\ 0.0 & 0.300 \mathrm{CM} & -0.009 & -0.001 & 0.0\end{array}$ 0.171 $\begin{array}{ll}0.0 & 0.0\end{array}$ $\begin{array}{llccl}0.0 & 0.100 \mathrm{MR} & 0.0 & 0.0 & 0.164 \\ 0.0 & 0.300 \mathrm{CM} & -0.009 & -0.001 & 0.0\end{array}$ $\begin{array}{lllll}0.0 & 1.000 \mathrm{PC} & 0.071 & 0.657 & 0.0\end{array}$ 0.0 $0.0-0.000$

\#ORIFT * $3.0 \quad 80.0000 \mathrm{M}$

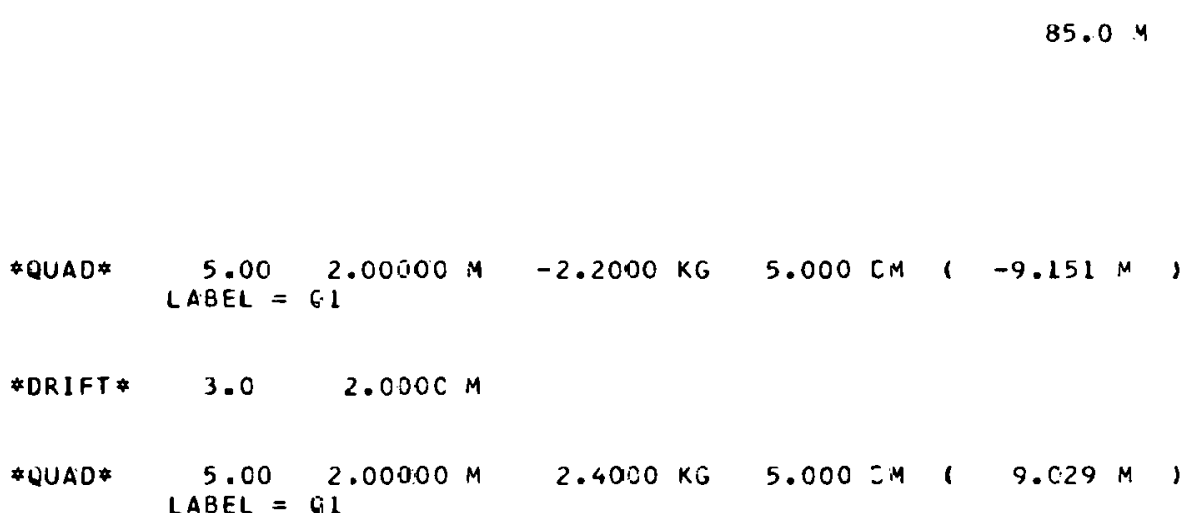

$91.0 \mathrm{M}$

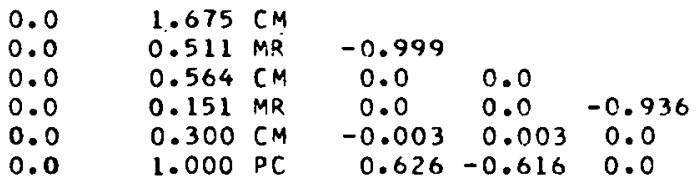
$\begin{array}{lll}c .965 & & \\ 0.0 & 0.0 & \\ 0.0 & 0.0 & 0.943 \\ -0.003 & -0.001 & 0.0\end{array}$ $\begin{array}{lll}-0.003 & -0.001 & 0.0\end{array}$ 0.0 $-0.000$ LABEL $=9$ 


\begin{tabular}{|c|c|c|c|c|c|c|c|c|}
\hline * ROT AT * & 2.0 & 1.500 & & & & & & \\
\hline \#BEND* & 4.000 & $3.00000 \mathrm{M}$ & 14.550 & $K G$ & 0.0000 & 1 & 2.9990 & 01 \\
\hline *ROTAT* & 2.0 & 1.500 & & & & & & \\
\hline *DRIFT* & 3.0 & $1.000 \mathrm{CM}$ & & & & & & \\
\hline * ROTAT* & 2.0 & $1.50 \quad 0$ & & & & & & \\
\hline *BEND* & 4.000 & $3.00000 \mathrm{M}$ & 14.550 & KG & 0.0000 & 1 & 2.9990 & 01 \\
\hline *ROTAT * & $2 \cdot C$ & 1.500 & & & & & & \\
\hline ADRIFT* & 3.0 & $1.0000 \mathrm{M}$ & & & & & & \\
\hline "ROTAT* & 2.0 & 1.500 & & & & & & \\
\hline \#BEND* & 4.000 & $3.0 \mathrm{COCCM}$ & 14.550 & KG & 0.0000 & 1 & $2.999 \mathrm{D}$ & 01 \\
\hline ORUTAT* & 2.0 & 1.500 & & & & & & \\
\hline WORIFT* & 3.0 & $1.000 \mathrm{CM}$ & & & & • & & \\
\hline "ROTAT* & 2.0 & $1.50 \mathrm{D}$ & & & & & & \\
\hline ه BEND\# & 4.000 & $3.000 \mathrm{COM}$ & 14.550 & $K G$ & 0.0000 & 1 & 2.9990 & 01 \\
\hline ROTAT* & 2.0 & 1.500 & & & . & & & \\
\hline
\end{tabular}

\begin{tabular}{|c|c|c|c|c|c|c|c|}
\hline $107.0 \mathrm{~N}$ & $\begin{array}{l}0.0 \\
0.0 \\
0.0 \\
0.0 \\
0.0 \\
0.0\end{array}$ & $\begin{array}{l}2.218 \\
1.824 \\
0.336 \\
0.165 \\
0.457 \\
1.000\end{array}$ & $\begin{array}{l}C M \\
M R \\
C M \\
M R \\
C M \\
P C\end{array}$ & $\begin{array}{l}0.865 \\
0.0 \\
0.0 \\
-0.715 \\
0.954\end{array}$ & $\begin{array}{c}0.0 \\
0.0 \\
-0.497 \\
0.975\end{array}$ & $\begin{array}{c}-0.841 \\
0.0 \\
0.0\end{array}$ & $\begin{array}{l}0.0 \\
0.0\end{array}$ \\
\hline
\end{tabular}

-DRIFI * $\quad 3.0 \quad 30.0000 \mathrm{M}$

VARY CODE $=900000$

LABEL $=$ DR I 


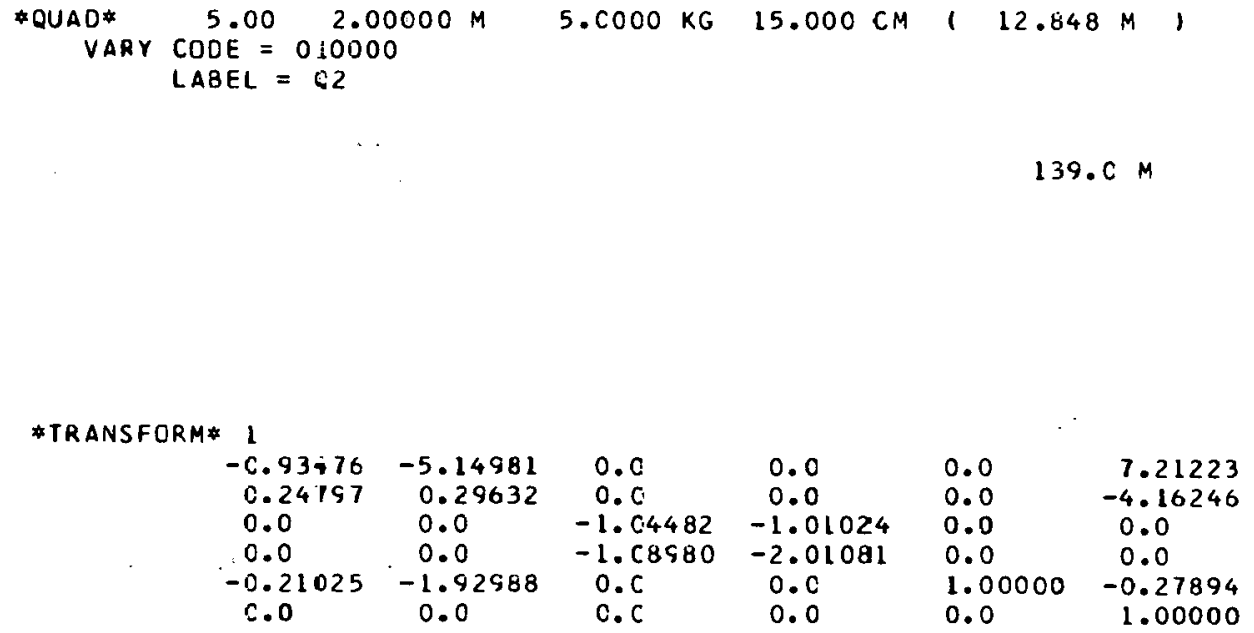

*ORIFT* $\quad 3.0 \quad 20.0000 \mathrm{C}$

VARY $C$ COE $=4110000$

$\angle A B E L=D R I$

$\begin{array}{llllllll}0.0 & 7.236 \mathrm{CM} & & & & & \\ 0.0 & 4.163 \mathrm{MR} & -0.998 & & & & \\ 0.0 & 0.329 \mathrm{CM} & 0.0 & 0.0 & & & \\ 0.0 & 0.384 \mathrm{MR} & 0.0 & 0.0 & 0.971 & & \\ 0.0 & 0.457 \mathrm{CM} & -0.573 & 0.605 & 0.0 & 0.0 & \\ 0.0 & 1.000 \mathrm{PC} & 0.997 & -1.000 & 0.0 & 0.0 & -0.610\end{array}$

$159.0 . M$

0.0
0.0
0.0
0.0
0.0
0.0

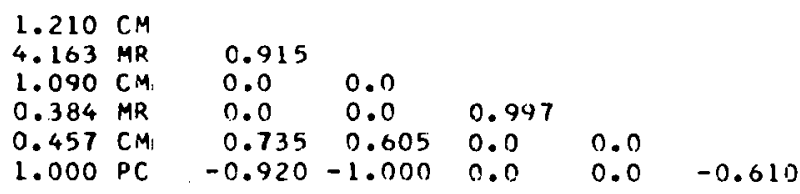

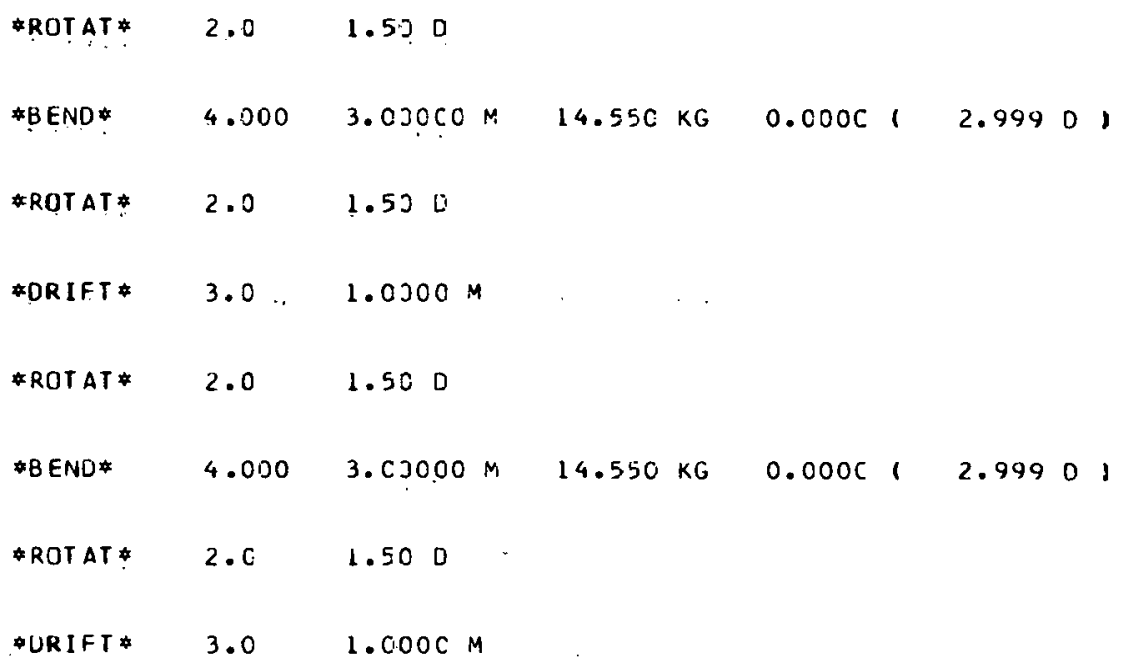




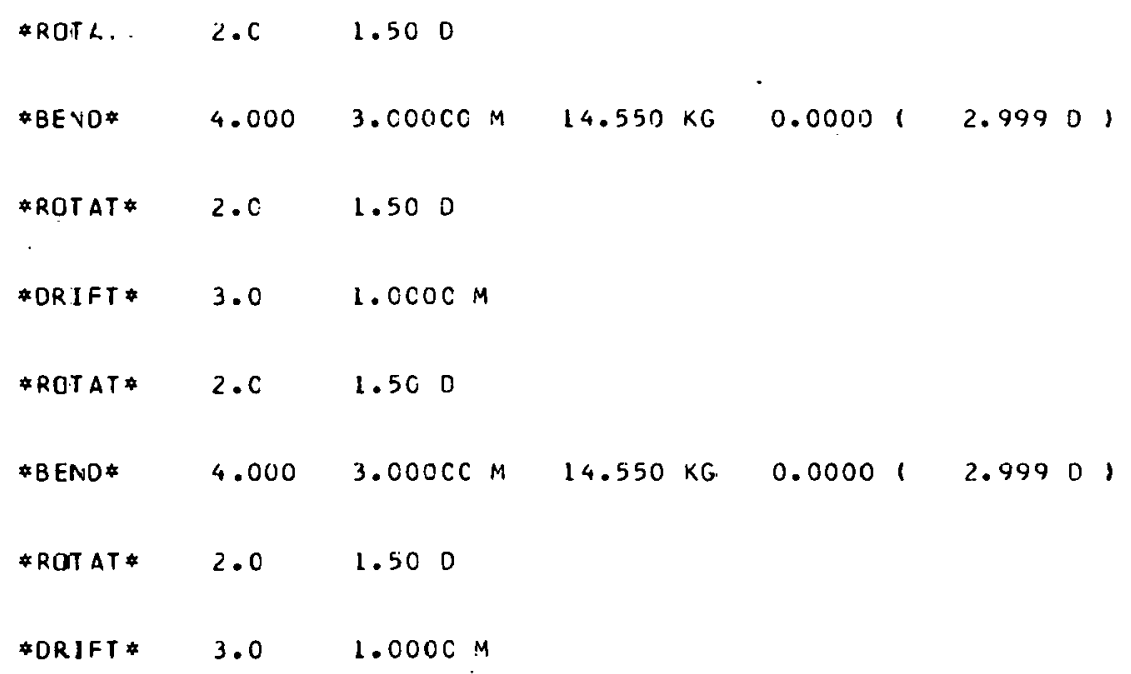

$175.0 \mathrm{M}$

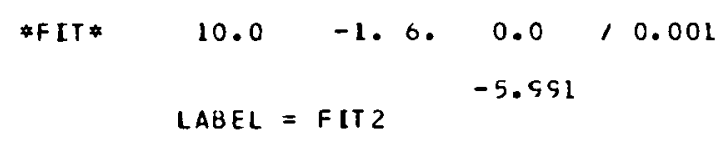

\#F ET *

$$
10.0-2.6 .0 .0,0.001
$$$$
\text { LABEL }=\text { FIT } 3
$$

\#TRANSFORM* 1

$$
\begin{array}{llllll}
-0.04222 & -4.68323 & 0.0 & 0.0 & 0.0 & -5.99086 \\
0.24796 & 0.29627 & 0.0 & 0.0 & 0.0 & -2.06827 \\
0.0 & 0.0 & -4.85154 & -8.06319 & 0.0 & 0.0 \\
0.0 & 0.0 & -0.94335 & -1.77381 & 0.0 & 0.0 \\
-0.15728 & -1.02203 & 0.0 & 0.0 & 1.00000 & 0.49253 \\
0.0 & 0.0 & 0.0 & 0.0 & 0.0 & 1.00000
\end{array}
$$

*QUAD* $5.00 \quad 2.00000 \mathrm{M} \quad-1.9000 \mathrm{KG} \quad 5.000 \mathrm{CM}(-10.646 \mathrm{M})$ 
$\angle A B E L=03$

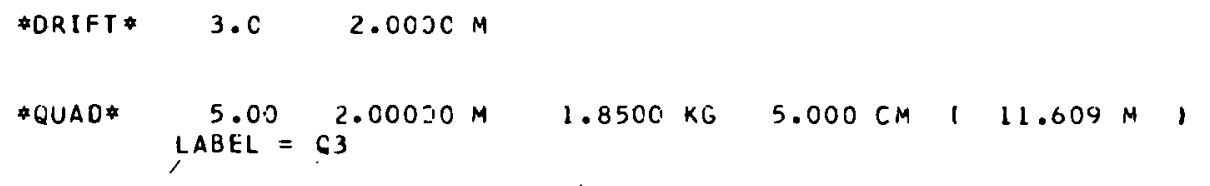

*DRIFT * $\quad 3.0 \quad 200.00: 0 \mathrm{M}$

$381.0 \mathrm{M}$

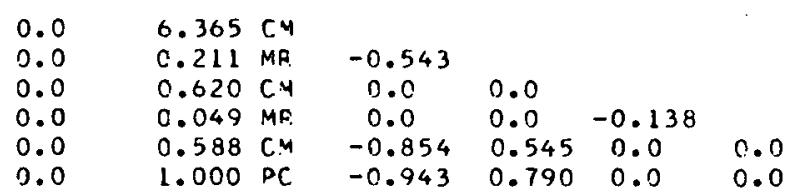

0.838
*TRANSFCRM*

$\begin{array}{llllll}1.42958 & 18.46803 & c .5 & 0.0 & 0.0 & -6.00397 \\ 0.15690 & 1.19030 & 0.5 & 0.0 & 0.0 & 0.16643 \\ c .0 & 0.0 & -0.30513 & -6.13656 & 0.0 & 0.0 \\ 0.0 & 0.0 & 0.16271 & -0.00492 & 0.0 & 0.0 \\ -0.15728 & -1.02203 & 0.0 & 0.0 & 1.00000 & 0.49253 \\ 0.0 & 0.0 & 0.0 & 0.0 & 0.0 & 1.00000\end{array}$

$381.0 \mathrm{M}$

$\begin{array}{ll}0.0 & 6.365 \mathrm{CM} \\ 0.0 & 0.211 \mathrm{MP} \\ 0.0 & 0.020 \mathrm{CM} \\ 0.0 & 0.049 \mathrm{MR} \\ 0.0 & 0.588 \mathrm{CH} \\ 0.0 & 1.000 \mathrm{PC}\end{array}$

$-0.543$

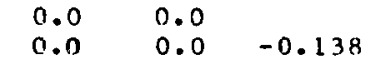

$\begin{array}{lll}0.0 & 0.0 & -0.13\end{array}$

$\begin{array}{llll}-0.854 & 0.545 & 0.0 & 0.0\end{array}$

$\begin{array}{llll}-0.943 & 0.790 & 0.0 & 0.0\end{array}$

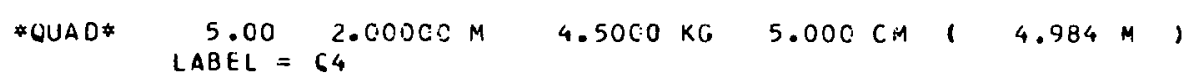

EORIFT* $\quad 3.0 \quad 2.000 C \mathrm{M}$

$385.0 \mathrm{M}$

$\begin{array}{lrllllll}0.0 & 2.447 & C M & & & & & \\ 0.0 & 12.863 & M R & -0.999 & & & & \\ 0.0 & 1.044 \mathrm{CM} & 0.0 & 0.0 & & & \\ 0.0 & 1.431 & M R & 0.0 & 0.0 & 1.000 & & \\ 0.0 & 0.588 \mathrm{CP} & -0.851 & 0.855 & 0.0 & 0.0 & \\ 0.0 & 1.000 \mathrm{PC} & -0.935 & 0.947 & 0.0 & 0.0 & 0.838\end{array}$

\#QUAD* $\quad \begin{aligned} & 5 . C O \\ & \text { LABEL }=64\end{aligned} 2.00000 \mathrm{H}-4.20 C 0 \mathrm{KG} 5.000 \mathrm{CM},-4.645 \mathrm{M}$,

$\begin{array}{rrrr}3.87 .0 \mathrm{M} & 0.0 & 0.232 \mathrm{CM} & \\ & 0.0 & 10.281 \mathrm{MP} & -0.881\end{array}$


- TR ANSFCRM* 1

$\begin{array}{ccllll}-7.47783 & -39.35443 & c .0 & 0 . C & 0.0 & 14.52741 \\ -5.14875 & -27.23064 & 0.0 & 0.0 & 0.0 & 9.79630 \\ 0.0 & 0.0 & c .17954 & 1.05592 & 0.0 & 0.0 \\ 0.0 & 0.0 & 0.41918 & 8.03487 & 0.0 & 0.0 \\ -0.15728 & -1.02203 & 0.0 & 0.0 & 1.00000 & 0.49253 \\ 0.0 & 0.0 & 0.0 & 0.0 & 0.0 & 1.00000\end{array}$

*LENGTH* $401.9945 \mathrm{M}$

$\begin{array}{llcc}(4.4815 E 031 & 2.801 & -1.453 \\ (1.5177 E 021 & 1.457 & 0.043 \\ (1.5083 E 011 & 0.037 & 0.005 \\ (1.2714 E-02) & -0.000 & 0.000 \\ \text { \#COVAFIANCE } & \text { ( FIT } 0.01 \\ 0.011 & & \\ 0.480 \quad 0.000 & & \end{array}$

$402.0 M$

$\begin{array}{lr}0.0 & 15.217 \mathrm{CM} \\ 0.0 & 10.281 \mathrm{MR} \\ 0.0 & 0.119 \mathrm{CM} \\ 0.0 & 0.813 \mathrm{MR} \\ 0.0 & 0.588 \mathrm{CM} \\ 0.0 & 1.000 \mathrm{PC}\end{array}$

1.000

c. 0

$\begin{array}{ll}0.0 & 0.0\end{array}$

$\begin{array}{llll}0.857 & 0.857 & 0.0\end{array}$

$\begin{array}{llll}0.955 & 0.953 & 0.0\end{array}$

0.0

\section{*C.ORRECTION'S*}


BEAM TRANSPORT SYSTEM FOF: SLAC BEAM SWITCHYARO END STATICN A I

\# BEAM* $1.000000 \quad 25.00 \mathrm{GEV}$

$0.0 \mathrm{M}$

$\begin{array}{ll}0.0 & 0.300 \mathrm{CM} \\ 0.0 & 0.100 \mathrm{MR} \\ 0.0 & 0.300 \mathrm{CM} \\ 0.0 & 0.100 \mathrm{MR} \\ 0.0 & 0.300 \mathrm{CM} \\ 0.0 & 1.000 \mathrm{PC}\end{array}$

$$
\begin{aligned}
& 0.0 \\
& 0.0 \\
& 0.0 \\
& 0.0 \\
& 0.0
\end{aligned}
$$

$0.0 \quad 0.0$

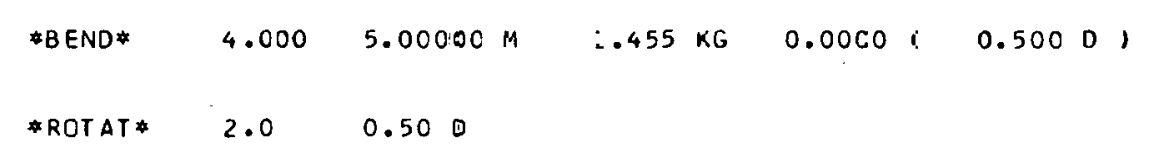

$5.0 M$

85.0 PA

$\begin{array}{ll}0.0 & 1.153 \mathrm{CM} \\ 0.0 & 0.133 \mathrm{MR} \\ 0.0 & 0.901 \mathrm{CM} \\ 0.0 & 0.100 \mathrm{MR} \\ 0.0 & 0.300 \mathrm{CM} \\ 0.0 & 1.000 \mathrm{PC}\end{array}$

$0.305 \mathrm{CH}$ $0.133 \mathrm{MR}$ $0.304 \mathrm{CM}$

$0.100 \mathrm{MR}$.

$0.300 \mathrm{CM}$
$1.000 \mathrm{PC}$

\subsection{1}

$0.0 \quad 0.0$

$\begin{array}{lll}0.0 & 0.0 & 0.164\end{array}$ $\begin{array}{rrrr}-0.009 & -0.001 & 0.0 & 0.0 \\ 0.071 & 0.657 & 0.0 & 0.0\end{array}$

$-0.000$

\#DRIFT 3.C $\quad 8 C .0000 \mathrm{M}$

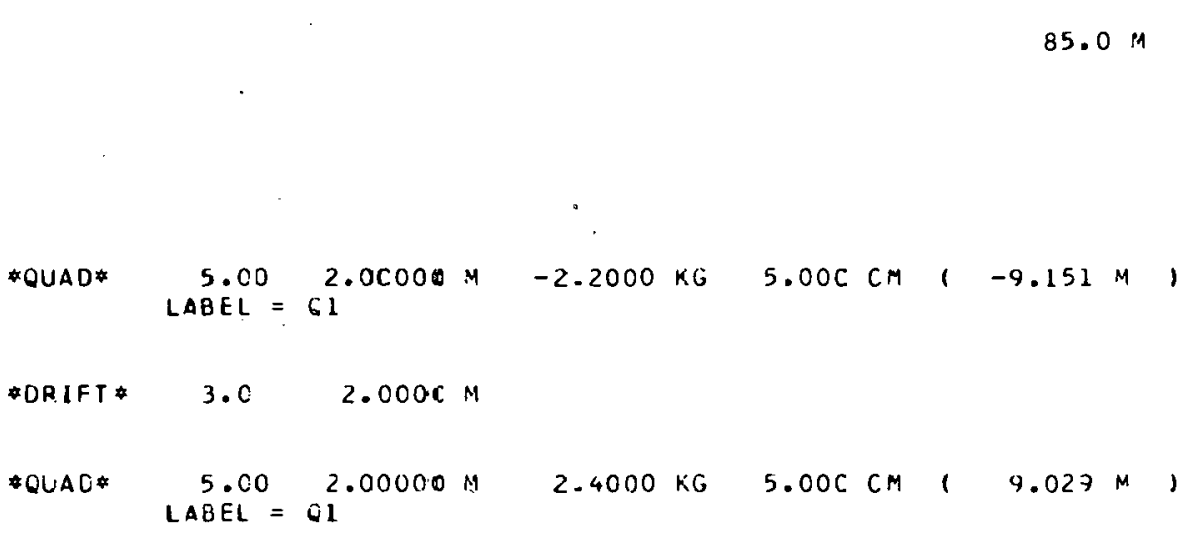

$91.0 M$

$1.675 \mathrm{CM}$ $0.511 \mathrm{MR}$ $0.564 \mathrm{CM}$
$0.151 \mathrm{MR}$

$0.151 \mathrm{MR}$
$0.300 \mathrm{CM}$

$1.000 \mathrm{PC}$ $-0.999$ $\begin{array}{lll}0.0 & 0.0 & \\ 0.0 & 0.0 & -0.936\end{array}$ $\begin{array}{clc}0.0 & 0.0 & -0.936 \\ -0.003 & 0.003 & 0.0 \\ 0.0226 & -0.616 & 0.0\end{array}$ $\begin{array}{llll}0.026 & -0.616 & 0.0 & 0.0 \\ 0.0 & 0.0\end{array}$

$-0.000$ 


\begin{tabular}{|c|c|c|c|c|c|c|c|c|}
\hline - RGIAI* & 2.0 & 1.500 & & & & & & \\
\hline *BEND* & $4.00 \mathrm{C}$ & $3.00000 \mathrm{M}$ & 14.550 & KG & 0.0000 & 1 & 2.999 & 01 \\
\hline *ROTAT* & 2.0 & 1.500 & & & & & & \\
\hline *ORIFT* & 3.0 & $1.0000 \mathrm{M}$ & & & & 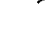 & & \\
\hline - & & & & & & & & \\
\hline *ROT AT* & 2.0 & 1.500 & & & & & & \\
\hline \#BEND* & 4.000 & $3.00000 \mathrm{M}$ & 14.550 & KG & 0.0000 & 1 & 2.999 & D 1 \\
\hline *ROTAT: & 2.0 & 1.500 & & & & & & \\
\hline *DRIFT* & 3.0 & $1.0000 \mathrm{M}$ & & & & & & \\
\hline \#ROTAT* & $2 \cdot C$ & 1.500 & & & & & & \\
\hline *3END* & 4.000 & $3.000 \mathrm{COM}$ & 14.550 & KG & 0.0000 & 1 & 2.999 & 01 \\
\hline * ROT AT* & 2.0 & 1.500 & & & & & & \\
\hline *DR IFT & 3.0 & $1.0000 \mathrm{M}$ & & & & & & \\
\hline * RUTAT* & $2 . c$ & 1.500 & & & & & & \\
\hline :BEND* & 4.000 & $3.0000 \mathrm{CM}$ & 14.550 & KG & 0.0000 & 1 & 2.999 & 01 \\
\hline *ROTAT* & 2.0 & .500 & & & & & & \\
\hline
\end{tabular}

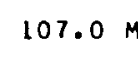

$E-47$ 


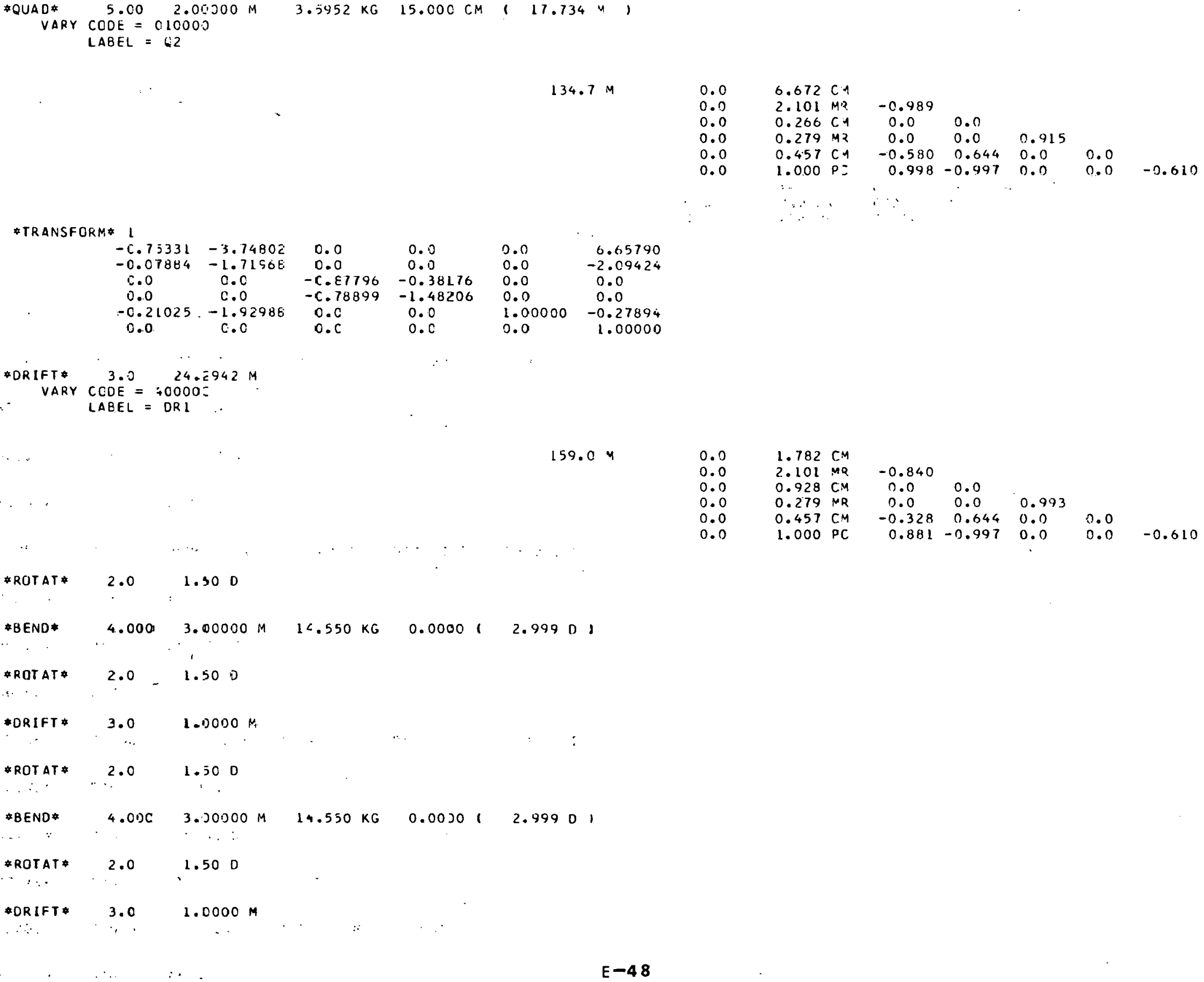

159.04

$6.672 \mathrm{CA}$

$2.101 M^{2}$

$0.266 \mathrm{CA}$

$0.279 \mathrm{Mz}$

$\begin{array}{rrrr}-0.580 & 0.644 & 0.0 & 0.0 \\ 0.998 & -0.997 & 0.0 & 0.0\end{array}$

$-0.989$

$-0.610$

$1.782 \mathrm{CM}$ $0.928 \mathrm{CM}$ $0.279 \mathrm{MR}$ $1.000 \mathrm{PC}$ 


\begin{tabular}{|c|c|c|c|c|c|c|c|c|c|}
\hline \#ROTAT* & 2.0 & 1.500 & & & & . & & & \\
\hline$=B E N D *$ & 4.000 & 3.00000 & i. & 14.550 & KG & 0.0000 & 1 & 2.9990 & 01 \\
\hline$=$ ROTAT* & 2.0 & 1.500 & - & & & & & & \\
\hline * DQR I F T * & 3.0 & 1.0000 & M & & & & & & \\
\hline FROT AT * & 2.0 & 1.500 & & & & & & & \\
\hline \#BEND* & 4.000 & $3.0000 \mathrm{C}$ & $M$ & 14.550 & $K G$ & 0.0000 & 1 & 2.9990 & 01 \\
\hline =ROT AT * & 2.0 & $1.50 \mathrm{D}$ & & & & & & & \\
\hline \#DR IFT & 3.0 & $1.0000 \mathrm{~N}$ & M & & & & & & \\
\hline
\end{tabular}

$175.0 M$

$\begin{array}{ll}0.0 & 1.115 \mathrm{CM} \\ 0.0 & 0.174 \mathrm{MR} \\ 0.0 & 1.340 \mathrm{CM} \\ 0.0 & 0.238 \mathrm{MR} \\ 0.0 & 0.495 \mathrm{CM} \\ 0.0 & 1.000 \mathrm{PC}\end{array}$

C. 988

$\begin{array}{ll}0.0 & 0.0 \\ 0.0 & 0.0\end{array}$

$\begin{array}{lll}0.0 & 0.0 & 0.996\end{array}$

$\begin{array}{rrr}-0.000 & -0.000 & 0.0 \\ 0.000 & 0.000 & 0.0\end{array}$

*TRANSFORM\# 1

\begin{tabular}{|c|c|c|c|c|c|}
\hline $\begin{array}{l}-1 . c 7094 \\
-c . c .7885 \\
0.0 \\
0 . c \\
-0.010000 \\
0.0\end{array}$ & $\begin{array}{l}-10.67648 \\
-1.71978 \\
0.0 \\
0.0 \\
-0.00001 \\
0.0\end{array}$ & $\begin{array}{l}0.0 \\
0.0 \\
-3.55881 \\
-0.66530 \\
0.0 \\
0.0\end{array}$ & $\begin{array}{l}0.0 \\
0.0 \\
-6.2 C 807 \\
-1.29747 \\
0.0 \\
0.0\end{array}$ & $\begin{array}{l}0.0 \\
0.0 \\
0.0 \\
C .0 \\
1.00000 \\
0.0\end{array}$ & $\begin{array}{l}0.00001 \\
0.00000 \\
0.0 \\
0.0 \\
-0.39405 \\
1.00000\end{array}$ \\
\hline
\end{tabular}

-QUAO*

5.0

2.00000

$-1.9000 K G$

$5.000 \mathrm{CM} /-10.646 \mathrm{M}$

$E-49$ 
$\angle A B E L=03$

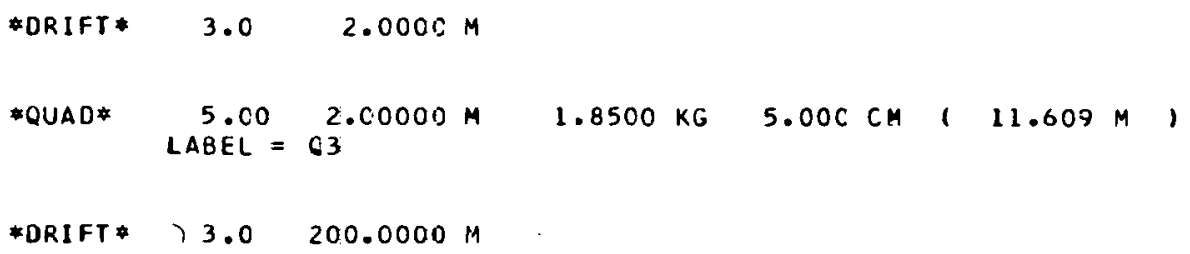

$381.0 \mathrm{M}$

0.0
0.0
0.0
0.0
0.0
0.0

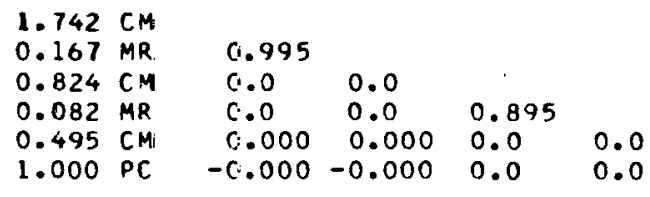
0.0 0.0
* TRANSFORM*

$\begin{array}{ll}1.77343 & 15.3011 \mathrm{C} \\ 0.21337 & 1.5377 \mathrm{C} \\ 0.0 & 0.0 \\ 0.0 & 0.0 \\ -0.00000 & -\mathrm{C} .00001 \\ 0.0 & 0.0\end{array}$

0.0
0.0
2.57533
$0.27 C 88$
0.0
C. 0

\section{0}

0.0

-2.86791
0.08736

$0 . \mathrm{C}$

0.0 a.

$\begin{array}{ll}0.0 & -0.00001\end{array}$

$-0.00000$

$0.0 \quad 0.0$

$1.00000-0.39405$

$0.0 \quad 1.00000$

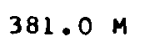

385.0 M

0.0
0.0
0.0
0.0
0.0
0.0

$0.737 \mathrm{CM}$

$3.364 \mathrm{MR}$

$1.424 \mathrm{CH}$
$1.999 \mathrm{MR}$

$0.495 \mathrm{CM}$

$1.000 \mathrm{PC}$

$-1.000$

$$
\begin{array}{ll}
0.0 & 0.0 \\
0.0 & 0.0
\end{array}
$$

$\begin{array}{rrr}-0.000 & -0.000 & 0.0 \\ -0.000 & 0.0\end{array}$

\#QUAD* $\quad 5 . C O=2.0000 D \mathrm{M} \quad-4.2000 \mathrm{KG} \quad 5.000 \mathrm{CM},-4.645 \mathrm{M}$,

\begin{tabular}{|c|c|c|c|c|}
\hline $387.0 \mathrm{M}$ & $\begin{array}{l}0.0 \\
0.0\end{array}$ & $\begin{array}{l}0.172 \\
2.478\end{array}$ & $\begin{array}{l}C M \\
M R\end{array}$ & -0.998 \\
\hline & & & & \\
\hline
\end{tabular}




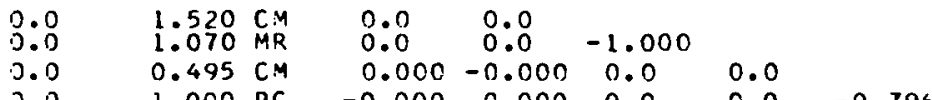

$402.0 \mathrm{M}$

$\begin{array}{ll}0.0 & 3.545 \mathrm{CM} \\ 0.0 & 2.478 \mathrm{MR} \\ 0.0 & 0.090 \mathrm{CM} \\ 0.0 & 1.070 \mathrm{MR} \\ 0.0 & 0.495 \mathrm{CM} \\ 0.0 & 1.000 \mathrm{PC}\end{array}$

\subsection{0}$$
0.0
$$$$
0.0 \quad 0.0
$$

$0.000 \quad 0.000$
*TF.ANSFORM* 1

$\begin{array}{ccclll}-5.80162 & -30.87849 & 0.0 & 0.0 & 0.0 & 0.00003 \\ -4.03053 & -21.62466 & 0.0 & 0.0 & 0.0 & 0.00002 \\ 0.0 & 0.0 & -0.23357 & 0.56217 & 0.0 & 0.0 \\ 0.0 & 0.0 & -3.33957 & 3.75673 & 0.0 & 0.0 \\ -6.00002 & -0.00001 & 0.0 & 0.0 & 1.00000 & -0.39405 \\ 0.0 & 0.0 & 0.0 & 0.0 & 0.0 & 1.00000\end{array}$

*LENGTH 4CL.S4S3 M 
'BEAM TRAHSPORT SYSTEM FOR SEAC BEAM SHITCHYARD EHD STATION A 11 '

(THE SECOND STFP TO FITTING IS TO ALLOW THE FIELDS OF THE FIRST QUAD)

(DOUBLET, ? 1, TO VARY AS WFLL AS THE SYMMETRY QUAD IN DRDER TO GET AN IMAGE AT)

(THE FXIT OF THE SYMHETPY ?UAD, D2, AS HELL AS THE PREVIOUSLY MENTIONED)

(ACHROMATIC, CONDITION.)

5.01 ' $Q 1$ ' 10.0 ' $\mathrm{FITI}$;

SENTIUEL 
BEA. ...ANSPORT SYSTEM FOR SLAC BEAM SWITCHYARD FNO STATION A II

1

THE SECONO STEP TO FITTING IS TO ALLOW THE FIELDS OF THE FIRST QULD

UCUBLET,OL, TC VARY AS WELl AS THE SYMMETRY QLAO IN OROER TO GET fiN IMAGE at

THE EXIT OF THE SYMMETRY GUAO,Q2, AS WELL AS THE PREVIOUSLY MENTICNFD

ACHRCMATIC CCNOITION.

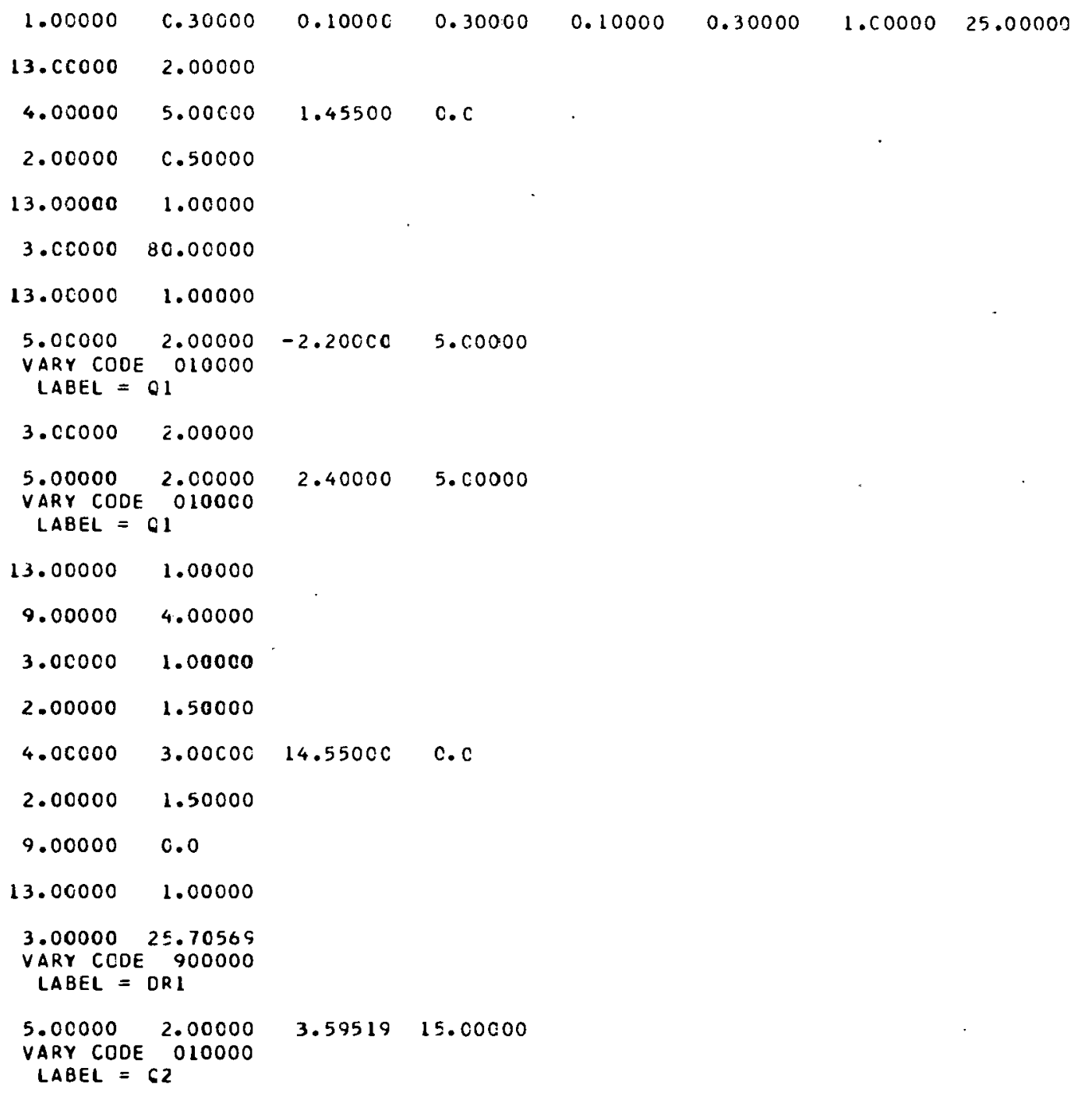




\begin{tabular}{|c|c|c|c|c|}
\hline 13.00000 & 1.00000 & & & \\
\hline $\begin{array}{c}10.00000 \\
\text { LABEL }=\end{array}$ & $\begin{array}{l}-1.00000 \\
F I T I\end{array}$ & 2.00000 & 0.0 & 0.00100 \\
\hline $\begin{array}{c}10.00000 \\
\text { LABEL }=\end{array}$ & $\begin{array}{l}-3.00000 \\
\text { FIT I }\end{array}$ & 4.00000 & 0.0 & 0.00100 \\
\hline 13.00000 & $4.0000 \mathrm{C}$ & & & \\
\hline $\begin{array}{l}3.00000 \\
\text { VARY CODE } \\
\text { LABEL = }\end{array}$ & $\begin{array}{l}24.29425 \\
\quad 400000 \\
C R 1\end{array}$ & & & \\
\hline 13.00000 & 1.00000 & & & \\
\hline 9.00000 & 4.00000 & & & \\
\hline 2.00000 & 1.50000 & & & \\
\hline 4.00000 & 3.00000 & $14.5500 \mathrm{C}$ & 0.0 & \\
\hline 2.00000 & 1.50000 & & & \\
\hline 3.00000 & 1.00000 & & & \\
\hline 9.00000 & $c .0$ & & & \\
\hline 13.00000 & 1.00000 & & & \\
\hline $\begin{array}{c}10.00000 \\
\text { LABEL }=\end{array}$ & $\begin{array}{l}-1.00000 \\
\text { FIT } 2\end{array}$ & 0.00000 & 0.0 & 0.00101 \\
\hline $\begin{array}{c}10.00000 \\
\text { LABEL }=\end{array}$ & $\begin{array}{l}-\bar{c} .00000 \\
\text { FIT3 }\end{array}$ & 0.00000 & 0.0 & 0.00100 \\
\hline 13.00000 & 4.00000 & & & \\
\hline $\begin{array}{l}5.00000 \\
\text { LABEL }=\end{array}$ & 63 & -1.90000 & 5.00000 & \\
\hline 3.00000 & 2.00000 & & & \\
\hline $\begin{array}{l}5.06000 \\
\text { LABEL }=\end{array}$ & $63^{2.00000}$ & 1.85000 & 5.00000 & \\
\hline 3.000002 & 200.00000 & & & \\
\hline 23.00000 & 1.00000 & & & \\
\hline $\begin{array}{c}-10.00000 \\
\text { VARY CODE } \\
\text { LABEL = }\end{array}$ & $\begin{array}{rl} & 1.00000 \\
2 & 200000 \\
F 1 T 4\end{array}$ & 1.00000 & 4.50000 & 0.10000 \\
\hline $\begin{array}{c}-10.00000 \\
\text { VARY CODE } \\
\text { LABEL = }\end{array}$ & $\begin{array}{l}3.00000 \\
200000 \\
\text { FIT4 }\end{array}$ & 3. $c 0000$ & 1.50000 & 0.10000 \\
\hline 13.00000 & 4.00000 & . & & \\
\hline
\end{tabular}




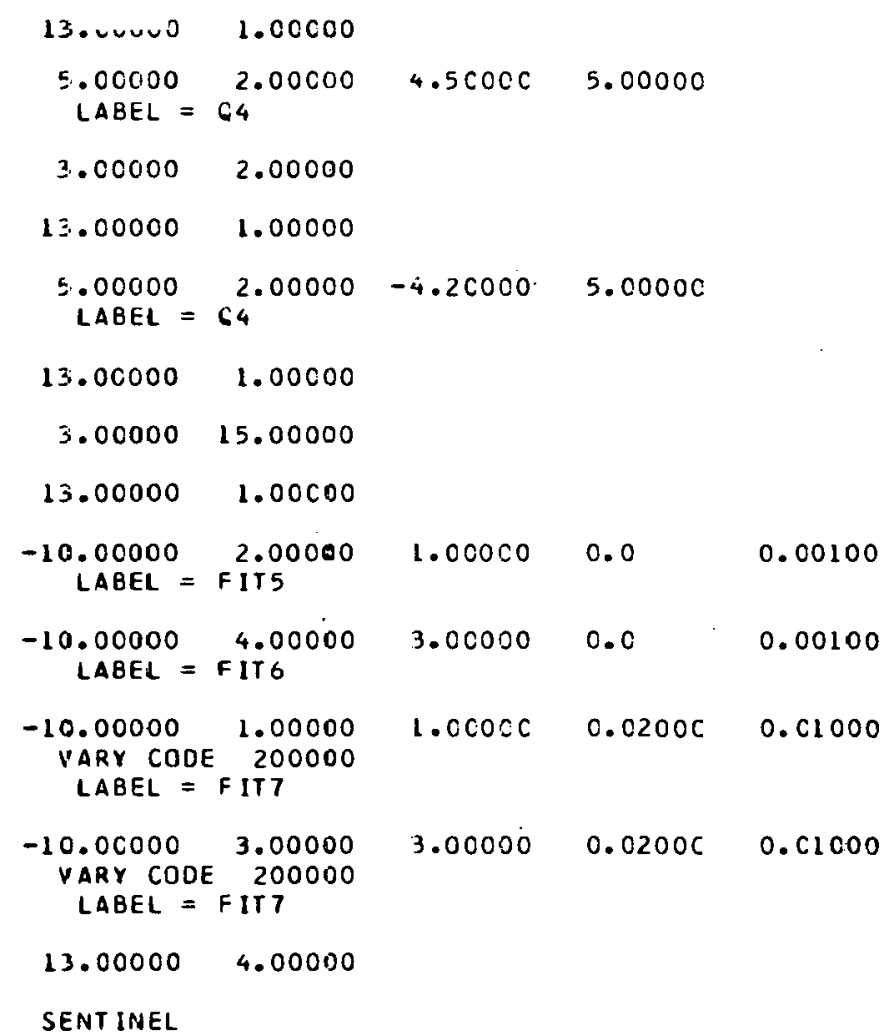


BEAM TRANSPORT SYSTEM FIR SLAC BEAM SWITCHYARO ENO STATION A II

* BEAM* $\quad 1.000000 \quad 25.00 \mathrm{GEV}$

$0.0 \mathrm{M}$

$\begin{array}{ll}0.0 & 0.300 \mathrm{CM} \\ 0.0 & 0.100 \mathrm{MR} \\ 0.0 & 0.300 \mathrm{CM} \\ 0.0 & 0.100 \mathrm{MR} \\ 0.0 & 0.300 \mathrm{CM} \\ 0.0 & 1.000 \mathrm{PC}\end{array}$

0.0

0.00 .0

$0.0-0.0$

1.000 PC

0.0

$0.0 \quad 0.0$

0.0

0.0

*BEND* $4.000 \quad 5.00=0 C$ M 1.455 KG $0.00001 \quad 0.500 \mathrm{D} 1$

* ROTAT * $2.0 \quad 0.500$

$5.0 \mathrm{M}$

$85.0 \mathrm{M}$

*URIFT* $\quad 3.0 \quad 8 \mathrm{C}, 0000 \mathrm{M}$

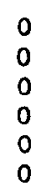

0.0
0.0
0.0
0.0

$0.305 \mathrm{CM}$ $0.133 \mathrm{MR}$ $0.304 \mathrm{CM}$ $0.100 \mathrm{MR}$ $0.300 \mathrm{CM}$
$1.000 \mathrm{PC}$ 0.171 $0.0 \quad 0.0$ $0.0 \quad 0.0 \quad 0.164$ $\begin{array}{cccc}-0.009 & -0.001 & 0.0 & 0.0\end{array}$ $\begin{array}{lll}0.071 & 0.657 & 0.0\end{array}$ $0.0-0.000$

$\begin{array}{ll}0.0 & 1.153 \mathrm{CM} \\ 0.0 & 0.133 \mathrm{MR} \\ 0.0 & 0.901 \mathrm{CM} \\ 0.0 & 0.100 \mathrm{MR} \\ 0.0 & 0.300 \mathrm{CM} \\ 0.0 & 1.000 \mathrm{PC}\end{array}$

$\begin{array}{ccc}0.965 & & \\ 0.0 & 0.0 & \\ 0.0 & 0.0 & 0.943 \\ -0.003 & -0.001 & 0.0 \\ 0.624 & 0.657 & 0.0\end{array}$
0.0 $-0.000$

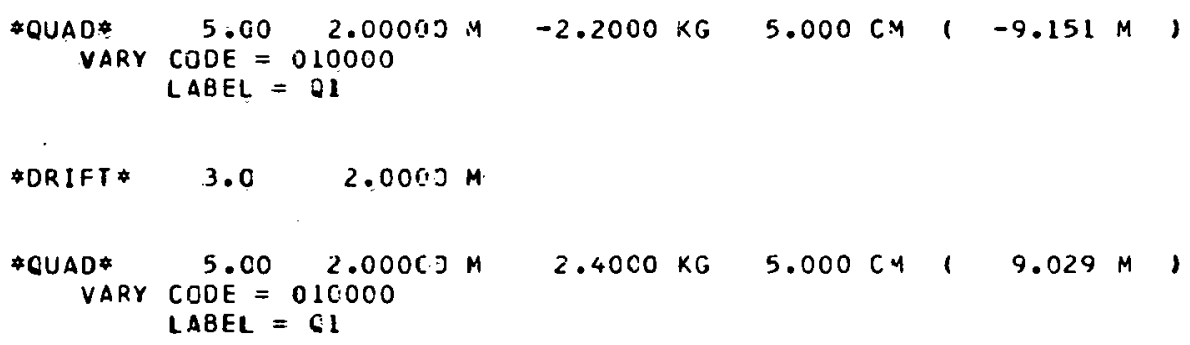




\begin{tabular}{|c|c|c|c|c|c|c|c|c|c|}
\hline$* D R I F I *$ & 3.0 & $1.000 \mathrm{C}$ & M & & & & & & \\
\hline \#ROTAT* & 2.0 & $1.50 \mathrm{D}$ & & & & & & & \\
\hline *BEND & 4.000 & $3.000 \mathrm{co}$ & $M$ & 14.550 & $K G$ & 0.0000 & 1 & 2.9990 & 01 \\
\hline \#ROTAT* & 2.0 & $1.50 \mathrm{D}$ & & & & & & & \\
\hline \#DRIFT & 3.0 & 1.0000 & $M$ & & & & & & \\
\hline \#ROTAT & 2.0 & 1.500 & & & & & & & \\
\hline *BEND* & 4.000 & 3.00000 & $M$ & 14.550 & $K G$ & 0.0000 & 1 & 2.999 & D 1 \\
\hline * ROTAT* & 2.0 & $1.50 \mathrm{D}$ & & & & & & & \\
\hline *DRIFT* & 3.0 & 1.0000 & M & & & & & & \\
\hline *ROTAT* & 2.0 & 1.500 & & & & & & & \\
\hline \#B END * & 4.000 & $3.0000 \mathrm{C}$ & $M$ & 14.550 & KG & 0.0000 & 1 & 2.999 & D 1 \\
\hline \#ROTAT * & 2.0 & 1.500 & & & & & & & \\
\hline *DRIFT* & 3.0 & 1.0000 & M & & & & & & \\
\hline \#ROT AT * & 2.0 & 1.500 & & & & & & & \\
\hline \#BEND* & 4.000 & 3.00000 & $M$ & 14.550 & KG & 0.0000 & 1 & 2.999 & D) \\
\hline *ROT AT * & 2.0 & 1.500 & & & & & & & \\
\hline
\end{tabular}

\begin{tabular}{|c|c|c|c|c|c|c|c|}
\hline $107.0 \mathrm{M}$ & $\begin{array}{l}0.0 \\
0.0 \\
0.0 \\
0.0 \\
0.0 \\
0.0\end{array}$ & $\begin{array}{l}2.218 \\
1.824 \\
0.336 \\
0.165 \\
0.457 \\
1.000\end{array}$ & $\begin{array}{l}C M \\
M R \\
C M \\
M R \\
C M \\
P C\end{array}$ & $\begin{array}{l}0.865 \\
0.0 \\
0.0 \\
-0.715 \\
0.954\end{array}$ & $\begin{array}{c}0.0 \\
0.0 \\
-0.497 \\
0.975\end{array}$ & $\begin{array}{c}-0.841 \\
0.0 \\
0.0\end{array}$ & $\begin{array}{l}0.0 \\
0.0\end{array}$ \\
\hline
\end{tabular}


VARY $\mathrm{COOE}=900000$

*Quad $\quad 5.00 \quad 2.00300 \mathrm{M} \quad 3.5952 \mathrm{~kg} \quad 15.000 \mathrm{CM} \quad 1 \quad 17.734$,

VARY $\mathrm{CODE}=010000$

$\angle A B E L=02$

$134.7 \mathrm{M}$

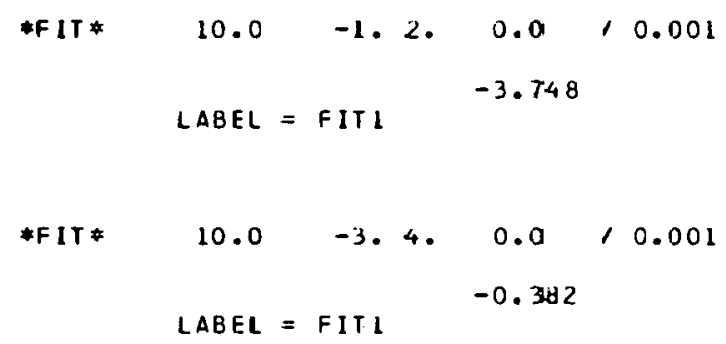

\#TRANSFORM 1

\begin{tabular}{|c|c|c|c|c|c|}
\hline $\begin{array}{l}-0.75331 \\
-0.07884 \\
0.0 \\
0.0 \\
-0.21025 \\
0.0\end{array}$ & $\begin{array}{l}-3.74802 \\
-1.71963 \\
0.0 \\
0.0 \\
-1.92983 \\
0.0\end{array}$ & $\begin{array}{l}3.0 \\
0.0 \\
-3 . E 7796 \\
-0.78399 \\
0.0 \\
0.0\end{array}$ & $\begin{array}{l}0.3 \\
0.3 \\
-0.38176 \\
-1.48206 \\
0.3 \\
0.3\end{array}$ & $\begin{array}{l}0.0 \\
0.0 \\
0.0 \\
0.0 \\
1.00000 \\
0.0\end{array}$ & $\begin{array}{l}6.65790 \\
-2.09424 \\
0.0 \\
0.0 \\
-0.27894 \\
1.00000\end{array}$ \\
\hline
\end{tabular}

\#DRIFT* $\quad 3.0 \quad 24.2942 \mathrm{M}$

VARY COOE $=40000$ :

$\angle A B E L=D R I$

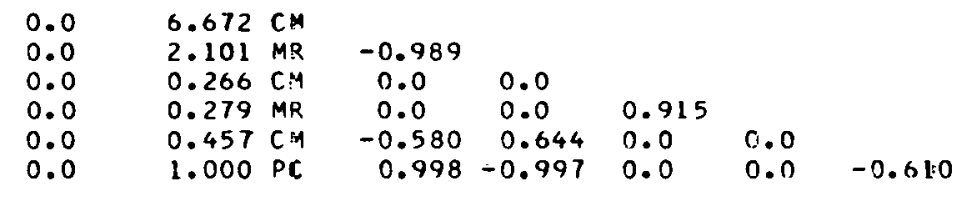

0.0
$-0.6150$

, 


\begin{tabular}{|c|c|c|c|c|c|c|c|c|c|}
\hline *ROT AI $=$ & 2.0 & 1.500 & & & & & & & \\
\hline *ORIFT & 3.0 & $1.0000 \mathrm{~N}$ & $M$ & & & & & & \\
\hline *ROT AT* & 2.0 & 1.500 & & & & & & & \\
\hline *BEND* & 4.000 & 3.00000 & $M$ & 14.550 & $K G$ & 0.0000 & 1 & $2.999 \mathrm{D}$ & D I \\
\hline \#ROTAT* & 2.0 & $1.50 \mathrm{D}$ & & & & & & & \\
\hline *DRIFT * & 3.0 & $1.0000 \mathrm{M}$ & M & & & & & & \\
\hline *ROT AT * & 2.0 & $1.50 \mathrm{D}$ & & & & & & & \\
\hline *BEND & 4.000 & 3.00300 & $M$ & 14.550 & KE & 0.0000 & 1 & 2.9990 & D 1 \\
\hline *ROTAT* & 2.0 & 1.500 & & & & & & & \\
\hline *DR IF T * & 3.0 & $1.0000 \mathrm{M}$ & M & & & & & & \\
\hline *ROT AT* & 2.0 & $1.50 \mathrm{D}$ & & & & & & & \\
\hline \#BEND * & 4.000 & $3.0000 \mathrm{C}$ & M & 14.550 & KG & 0.0000 & 1 & $2.999 \mathrm{D}$ & D 1 \\
\hline *ROT AT* & 2.0 & 1.500 & & & & & & & \\
\hline RIFT* & .0 & $0000 \mathrm{M}$ & M & & & & & & \\
\hline
\end{tabular}

$\begin{array}{llllllll}175.0 \mathrm{M} & 0.0 & 1.115 \mathrm{CM} & & & & & \\ & 0.174 \mathrm{MR} & 0.988 & & & & \\ & 0.0 & 0.174 & & & & \\ & 0.0 & 1.340 \mathrm{CM} & 0.0 & 0.0 & & & \\ 0.0 & 0.238 \mathrm{MR} & 0.0 & 0.0 & 0.996 & & \\ & 0.0 & 0.495 \mathrm{CM} & -0.000 & -0.000 & 0.0 & 0.0 & \\ & 0.0 & 1.000 \mathrm{PC} & 0.000 & 0.000 & 0.0 & 0.0 & -0.796\end{array}$

*FIT* $10.0 \quad-1 . t .0 .0,0.001$

LABEL $=$ FIT 2 
$\# \mathrm{FIT}$

$\begin{array}{lll}10 . C \quad-2.6 . & 0.0,0.001 \\ & 0.000\end{array}$

LABEL $=$ FIT3

$$
0.000
$$

\#TRANSFOFM\#

\begin{tabular}{|c|c|c|c|c|c|}
\hline $\begin{array}{l}1.07094 \\
0.07885 \\
0.0 \\
0.0 \\
0.00000 \\
0.0\end{array}$ & $\begin{array}{c}-16.67648 \\
-1.71978 \\
C .0 \\
C .0 \\
-6.00001 \\
C .0\end{array}$ & $\begin{array}{l}0.0 \\
0.0 \\
-3.95881 \\
-0.66630 \\
0.0 \\
C .0\end{array}$ & $\begin{array}{l}0.0 \\
0.0 \\
-6.2080 i \\
-1.2574 ? \\
0.0 \\
0.0\end{array}$ & $\begin{array}{l}3.0 \\
0.0 \\
0.0 \\
0.0 \\
1.00000 \\
0.0\end{array}$ & $\begin{array}{l}0.00001 \\
0.00000 \\
0.0 \\
0.0 \\
-0.39405 \\
1.00000\end{array}$ \\
\hline
\end{tabular}
\#QUAD* $\quad \begin{aligned} & 5 . C O \\ & \angle A B E L=63\end{aligned}$

\#URIFT* $\quad 3.0 \quad 2.0050 M$ 5.00
$\angle A B E L=63$

\#ORIFT $\quad 3.0 \quad 20 \mathrm{C.OCECM}$

$38: 0 \mathrm{M}$

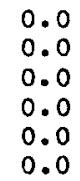

*TRANSFORM

$$
\begin{array}{ll}
1 & \\
2.77343 & 1 \equiv .30110 \\
0.21337 & 1.5377 C \\
0.0 & \Xi .0 \\
0.0 & \equiv .0 \\
-0.00000 & -\equiv .00001 \\
C .0 & =.0
\end{array}
$$

0.0

0.0

2.57533

C. $27 C 88$

0.0

0.0
0.0
-2.86091
0.08736
0.0
0.0

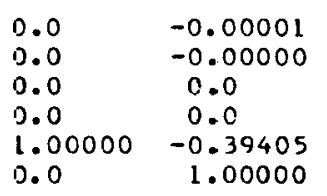

$381.0 \mathrm{M}$

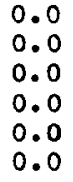

$1.742 \mathrm{~cm}$ $0.167 \mathrm{MR}$ $0.824 \mathrm{CH}$ $0.082 \mathrm{MR}$ $0.495 \mathrm{CM}$ $1.000 \mathrm{PC}$ 0.995 $0.0 \quad 0.0$ $\begin{array}{lll}0.0 & 0.0 & 0.895\end{array}$ $\begin{array}{lll}0.000 & 0.000 & 0.0\end{array}$ $\begin{array}{llll}-0.000-0.000 & 0.0 & 0.0 & -0.796\end{array}$ 
$385.0 \mathrm{M}$

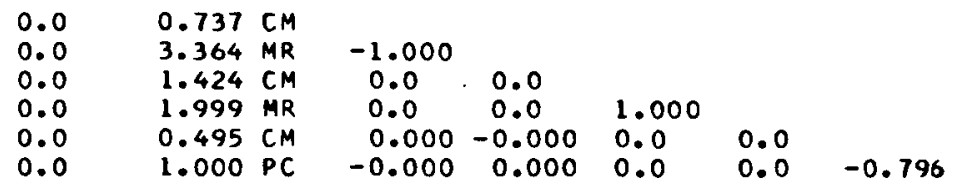

*QUAD* $\quad 5.00 \quad 2.00000 \mathrm{Ni}-4.2000 \mathrm{KG} 5.000 \mathrm{CM}(\mathbf{- 4 . 6 4 5 \mathrm { M } )}$

$387.0 \mathrm{M}$

\begin{tabular}{|c|c|c|c|c|c|c|}
\hline $\begin{array}{l}0.0 \\
0.0 \\
0.0 \\
0.0 \\
0.0 \\
0.0\end{array}$ & $\begin{array}{l}0.172 \mathrm{C} \\
2.478 \mathrm{M} \\
1.520 \mathrm{C} \\
1.070 \mathrm{M} \\
0.495 \mathrm{C} \\
1.000 \mathrm{P}\end{array}$ & $\begin{array}{l}C M \\
\text { YR } \\
C M \\
\text { CM } \\
C M \\
\text { PC }\end{array}$ & $\begin{array}{l}-0.998 \\
0.0 \\
0.0 \\
0.000 \\
-0.000\end{array}$ & $\begin{array}{c}0.0 \\
0.0 \\
-0.000 \\
0.000\end{array}$ & $\begin{array}{c}-1.000 \\
0.0 \\
0.0\end{array}$ & $\begin{array}{l}0.0 \\
0.0\end{array}$ \\
\hline
\end{tabular}

\#DRIFT $\quad 3 . \mathrm{C} \quad 15 . \cos \mathrm{M}$

$402.0 \mathrm{M}$

$\begin{array}{lllllllll}402.0 \mathrm{M} & 0.0 & 3.545 \mathrm{CM} & & & & & \\ & 0.0 & 2.478 & \mathrm{MR} & 1.000 & & & & \\ & 0.0 & 0.090 & \mathrm{CM} & 0.0 & 0.0 & & & \\ 0.0 & 1.070 & \mathrm{MR} & 0.0 & 0.0 & 0.950 & & \\ & 0.0 & 0.495 & \mathrm{CM} & -0.000 & -0.000 & 0.0 & 0.0 & \\ & 0.0 & 1.000 & \text { PC } & 0.000 & 0.000 & 0.0 & 0.0 & -0.796\end{array}$

*TRANSFCRM*

$\begin{array}{ccclll}-5.8 C 162 & -30.47849 & 0.0 & 0.0 & 0.0 & 0.00003 \\ -4.03058 & -21.62460 & 0.0 & 0.0 & 0.0 & 0.00002 \\ 0.0 & 0.0 & -0.23357 & 0.56217 & 0.0 & 0.0 \\ 0.0 & 0.0 & -3.33957 & 3.75673 & 0.0 & 0.0 \\ -0.00000 & -0.00001 & 0.0 & 0.0 & 1.00000 & -0.39405 \\ 0.0 & 0.0 & 0.0 & 0.0 & 0.0 & 1.00000\end{array}$

\#LENGTH* 401.9953 M

\section{\#CORGECTIONS*}

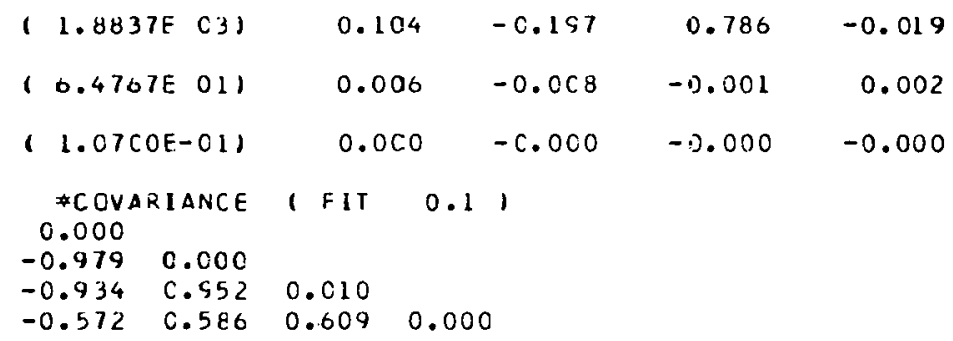

$\begin{array}{lllll}-0.572 & 0.586 & 0.609 & 0.000\end{array}$ 
*BEAM* $\quad 1.000000 \quad 2.5 .00$ GEV

*BEND*

$4.000 \quad 5.002 C C M$

$1.455 \mathrm{KG}$

0.00001

0.50001

* ROTAT*

$2 \cdot C$

0.500

* DR IFT \#

3.0

$80.0000 \mathrm{M}$

$85.0 \mathrm{M}$

0.0
0.0
0.0
0.0
0.0
0.0

0.0

0.0

0.0

0.0
0.0
0.0

$0.305 \mathrm{CM}$

$0.133 \mathrm{MR}$

$0.304 \mathrm{CM}$
$0.100 \mathrm{FR}$

$0.300 \mathrm{CM}$

$0.300 \mathrm{CM}$
$1.000 \mathrm{PC}$

0.171

$\begin{array}{ll}0.0 & 0.0 \\ 0.0 & 0.0\end{array}$

0.164

$\begin{array}{rrr}-0.009 & -0.001 & 0.0 \\ 0.071 & 0.657 & 0.0\end{array}$

0.0

$-0.000$

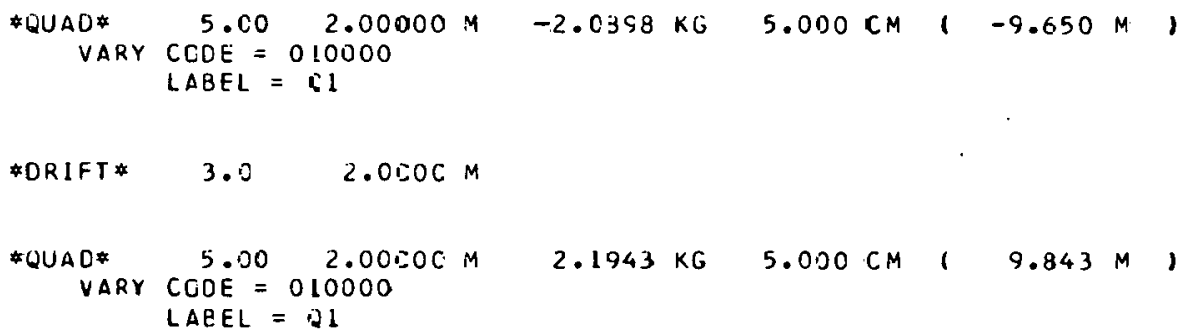

$91.0 M$ 


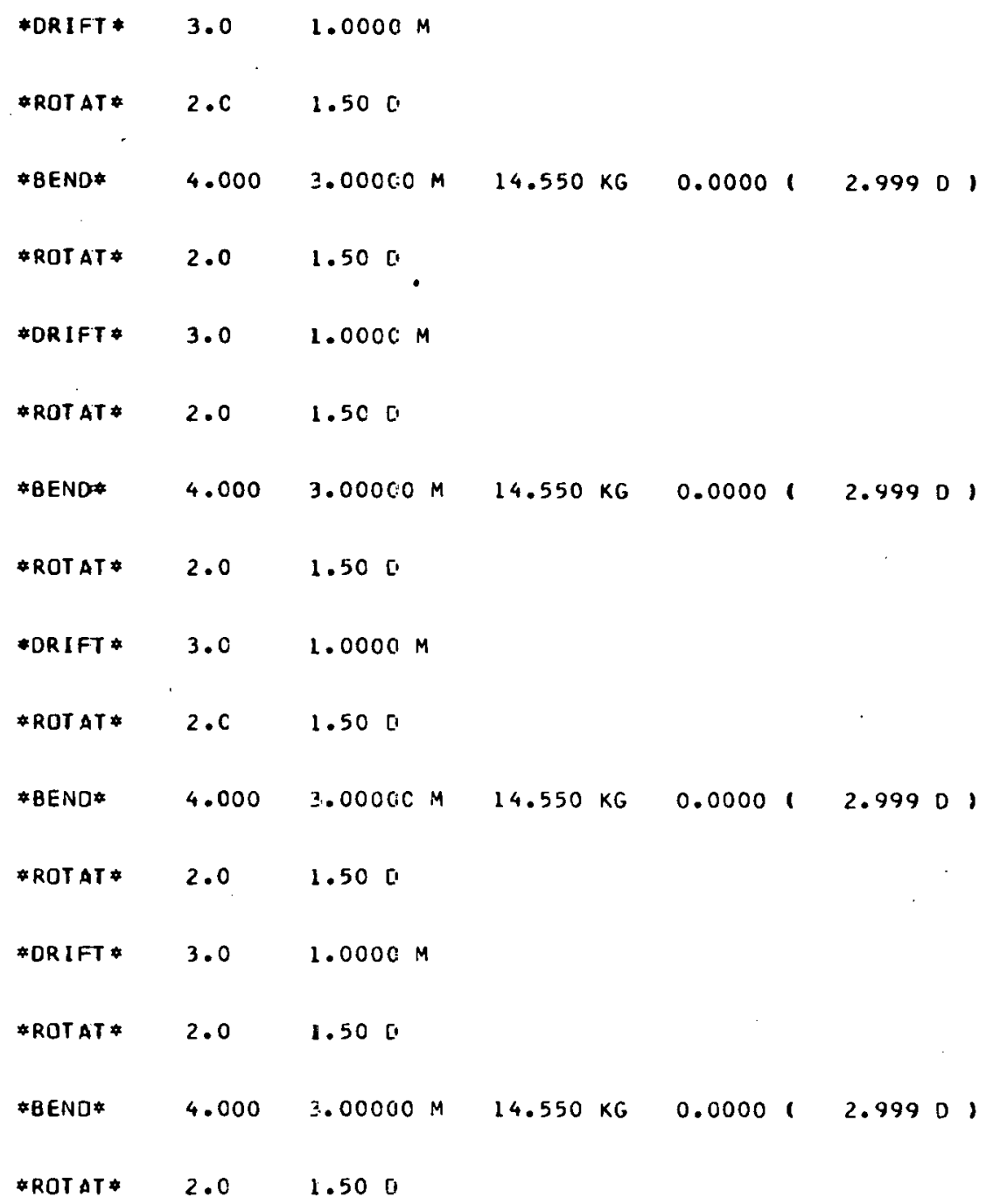


VARY CGCE $=900000$

*QUAD* b.CO 2.0000C M 3.5785 KG $15.000 \mathrm{CM}(17.815 \mathrm{M})$ VARY COUE $=010000$ $\angle A E E L=02$

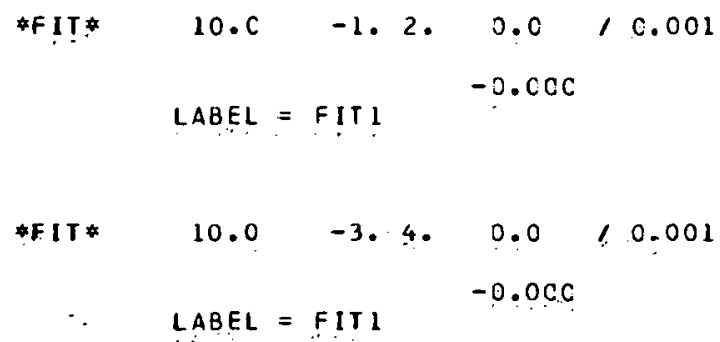

$\begin{array}{cccclc}-0.33045 & -0.30002 & 0.0 & 0.0 & 0.0 & 0.82236 \\ -0.22846 & -3.02617 & 0.0 & 0.0 & 0.0 & -2.09424 \\ 0.0 & 0.0 & -0.79757 & -0.00002 & 0.0 & 0.0 \\ 0.0 & 0.0 & -0.73609 & -1.25380 & 0.0 & 0.0 \\ -0.22507 & -2.06457 & 0.0 & 0.0 & 1.00000 & -0.29037 \\ 0.0 & 0.0 & 0.0 & 0.0 & 0.0 & 1.00000\end{array}$

\#ORIFT $3.0 \quad 25.0795 \mathrm{M}$

VARY COCE $=400000$ $\angle A B E L=O R 1$

\#ROTAT $2.0 \quad 1.5 \mathrm{CO}$

\#BEND* $\quad 4.000 \quad 3.00000 \mathrm{~N} \quad 14.553 \mathrm{KG} \quad 0.0000$ (. $2.999 \mathrm{D})$

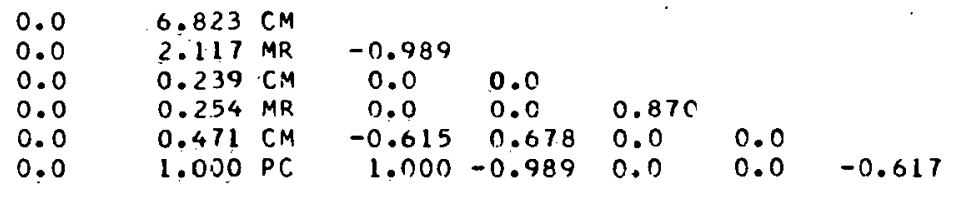

$159.0 \mathrm{M}$

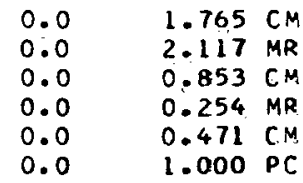

$-0.814$

$0.0 \quad 0.0$

$\begin{array}{lll}0.0 & 0.0 & 0.990\end{array}$

$\begin{array}{llll}-0.338 & 0.678 & 0.0 & 0.0\end{array}$

$0.0-0.617$ 


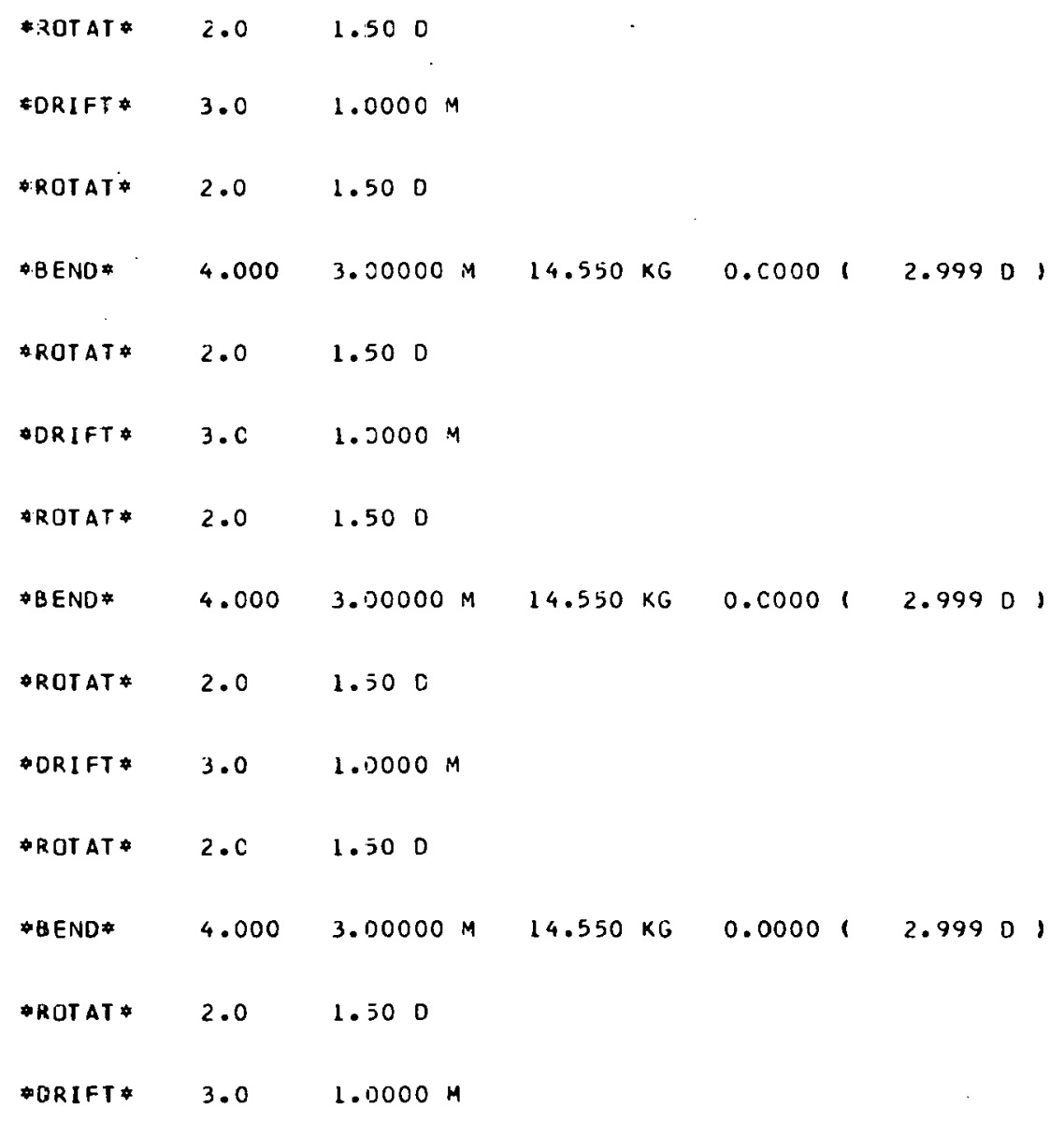

\begin{tabular}{|c|c|c|c|c|c|c|c|}
\hline $175.0 \mathrm{M}$ & $\begin{array}{l}0.0 \\
0.0 \\
0.0 \\
0.0 \\
0.0 \\
0.0\end{array}$ & $\begin{array}{l}1.300 \\
0.310 \\
1.227 \\
0.216 \\
0.504 \\
1.000\end{array}$ & $\begin{array}{l}C M \\
M R \\
C M \\
M R \\
C M \\
P C\end{array}$ & $\begin{array}{l}0.997 \\
0.0 \\
0.0 \\
-0.000 \\
0.000\end{array}$ & $\begin{array}{l}0.0 \\
0.0 \\
0.000 \\
0.000\end{array}$ & $\begin{array}{l}0.994 \\
0.0 \\
0.0\end{array}$ & $\begin{array}{l}0.0 \\
0.0\end{array}$ \\
\hline
\end{tabular}
* IT* $10.0 \quad-1.6 .0 .0 / 0.001$
LABEL = FIT


*FIT* $\quad 10.0 \quad-2.6 .0 .0 / 0.001$

$\angle A B E L=F I T 3$

*TRANSFORM 1

\begin{tabular}{|c|c|c|c|c|c|}
\hline $\begin{array}{l}-1.26882 \\
-0.22847 \\
0.0 \\
0.0 \\
-0.00000 \\
0.0\end{array}$ & $\begin{array}{c}-1 \bar{c} .42980 \\
-\bar{\vdots} .02623 \\
\overline{1} .0 \\
0.0 \\
-0.00001 \\
0.0\end{array}$ & $\begin{array}{l}0.0 \\
c .0 \\
-3.72852 \\
-0.62031 \\
0.0 \\
0.0\end{array}$ & $\begin{array}{l}0.0 \\
0.0 \\
-5.03435 \\
-1.10576 \\
0.0 \\
0.0\end{array}$ & $\begin{array}{l}0.0 \\
0.0 \\
0.0 \\
0.0 \\
1.00000 \\
0.0\end{array}$ & $\begin{array}{l}0.00001 \\
0.00000 \\
0.0 \\
0.0 \\
-0.40547 \\
1.00000\end{array}$ \\
\hline
\end{tabular}

*QUAD 5.CO 2.00000 M -1.9CCO KG $5.000 \mathrm{CM}(-10.646 \mathrm{M})$ $\angle A B E L=63$

*ORIFT* $\quad 3.0 \quad 2.0000 \%$

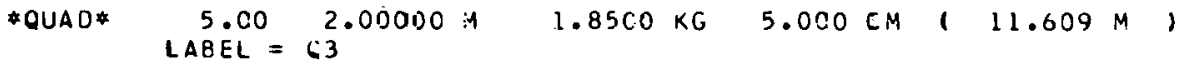

\#ORIFI $\quad 3.0 \quad 20 \mathrm{C} .0000$ i

$381.0 \mathrm{M}$
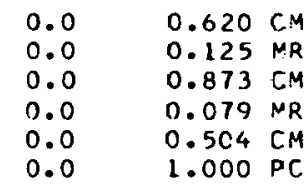

C. 922

$0.0 \quad 0.0$

$\begin{array}{lll}0.0 & 0.0 & 0.902\end{array}$

$\begin{array}{llll}0.000 & 0.000 & 0.0\end{array}$ $\begin{array}{rrrr}-0.000-0.000 & 0.0 & 0.0 & -0.804\end{array}$
¿TRANSFCRM*

\begin{tabular}{|c|c|c|c|c|c|}
\hline $\begin{array}{l}1.49989 \\
0.16754 \\
c .0 \\
0.0 \\
-0.00000 \\
0.0\end{array}$ & $\begin{array}{l}4.27131 \\
1.14380 \\
0.0 \\
0.0 \\
-0.00001 \\
0.0\end{array}$ & $\begin{array}{l}0.0 \\
0 . C \\
2.52306 \\
0.26479 \\
0.0 \\
0.0\end{array}$ & $\begin{array}{l}0.0 \\
0.0 \\
-3.78386 \\
-0.00074 \\
0.0 \\
0.0\end{array}$ & $\begin{array}{l}0.0 \\
0.0 \\
0.0 \\
0.0 \\
1.00000 \\
0.0\end{array}$ & $\begin{array}{l}-0.00001 \\
-0.00000 \\
0.0 \\
0.0 \\
-0.40547 \\
1.00000\end{array}$ \\
\hline
\end{tabular}

$381.0 \mathrm{M}$

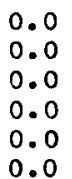

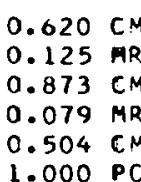

0.922

$0.0 \quad 0.0$

0.902

$\begin{array}{rrrr}0.0000 & 0.000 & 0.0 & 0.0 \\ -0.000 & -0.000 & 0.0 & 0.0\end{array}$

$0.0 \quad-0.804$

*QUAD* $5.00 \quad 2.00000 \mathrm{M} \quad 4.5000 \mathrm{KG} \quad 5.000 \mathrm{CM}(4.984 \mathrm{~N})$ $\angle A B E L=64$ 
\#DRIFT * $3.0 \quad 2.0005 \mathrm{M}$

$385.0 \mathrm{M}$

$\begin{array}{lllll}0.0 & 0.282 \text { CM. } & & & \\ 0.0 & 1.154 \text { MR } & -0.996 & & \\ 0.0 & 1.506 \text { CM } & 0.0 & 0.0 & \\ 0.0 & 2.111 \text { MR } & 0.0 & 0.0 & 1.000 \\ 0.0 & 0.504 \text { CM } & 0.000 & -0.000 & 0.0 \\ 0.0 & 1.000 \text { PC } & -0.000 & 0.000 & 0.0\end{array}$

* QUAD*

$5.00 \quad 2.00000 \mathrm{M} \quad-4.2000 \mathrm{KG}$ $\angle A B E L=64$

$5.000 \mathrm{CM}$

$-4.645 M$

$387.0 \mathrm{M}$

$\begin{array}{ll}0.0 & 0.098 \mathrm{CM} \\ 0.0 & 0.791 \\ 0.0 & 1.607 \mathrm{CM} \\ 0.0 & 1.135 \mathrm{MR} \\ 0.0 & 0.504 \mathrm{CM}\end{array}$

$-0.923$

$\begin{array}{lll}0.0 & 0.0 & \\ 0.0 & 0.0 & -1.000 \\ 0.000 & -0.000 & 0.0\end{array}$

$\begin{array}{lllll}-0.000 & 0.000 & 0.0 & 0.0 & -0.804\end{array}$
\#DRIFT $\quad 3.0 \quad 15.00 \mathrm{CC} \mathrm{M}$

*TRANSFORM *

$\begin{array}{llllll}-2.97423 & -6.38180 & 0.0 & 0.0 & 0.0 & 0.00002 \\ -2.09122 & -4.82334 & 0.0 & 0.0 & 0.0 & 0.00001 \\ 0.0 & 0.0 & -0.24656 & 0.05288 & 0.0 & 0.0 \\ 0.0 & 0.0 & -3.40280 & 4.55466 & 0.0 & 0.0 \\ -0.00000 & -0.00001 & 0.0 & 0.0 & 1.00000 & -0.40547 \\ 0.0 & 0.0 & 0.0 & 0.0 & 0.0 & 1.00000\end{array}$

*LENGTH\# $401.9993 \mathrm{M}$
$402.0 \mathrm{M}$

$\begin{array}{lllllll}0.0 & 1.097 \mathrm{CM} & & & & & \\ 0.0 & 0.791 & M R & 0.999 & & & \\ 0.0 & 0.099 \mathrm{CM} & 0.0 & 0.0 & & & , \\ 0.0 & 1.135 \mathrm{MR} & 0.0 & 0.0 & 0.963 & & \\ 0.0 & 0.504 \mathrm{CM} & -0.000 & -0.000 & 0.0 & 0.0 & \\ 0.0 & 1.000 \mathrm{PC} & 0.000 & 0.000 & 0.0 & 0.0 & -0.804\end{array}$


'BEAH TRANSPORT SYSTEM FJR SLAC BEAH SHITCHYARD EN) STATIOH A IIIA'

(IN THE THIRD STEP THE FIRST QLIAD DOUBLET, R1, AND THE SYMMETRY QUAD, C, 2, )

(ARE NOT ALLOHEC TC VAR'Y. THF FIFLLS DF THE SECONJ RUAD DOUBLET, R3, ARE)

(ALLOHED TO VAFY TC GET \& CERTAIN BEAM SIZF AT THF ENITRANCE TO THE T!:IRD NUAD)

(DOUBI.FT,, 4 .)

5.0 in :

3.0 , 22 '

$3 . .0 \mathrm{CRR}^{\prime}$;

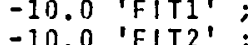

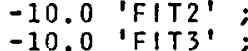

$5.01{ }^{\prime} Q 3{ }^{\prime} \cdot$

10.2 'FIT4'

SFNTINEL 
BEA.. I MANSPORT SYSTEM FOR SLAC BEAM SW:TCHYARO END STATICN A IIIA

IN THE THIRD STEP THE FIRST QUAD DOUBLET,Q1, AND THE SYMMETRY QUAD,QZ,

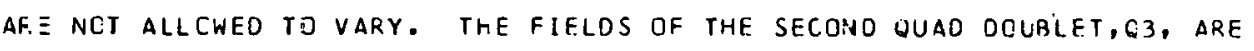

ALLUWEC TO VAKY TJ get a CERTAIN beam SIZE AT THE ENTRANCE TO THE THIRD QUAO

DOUBLET, 64 .

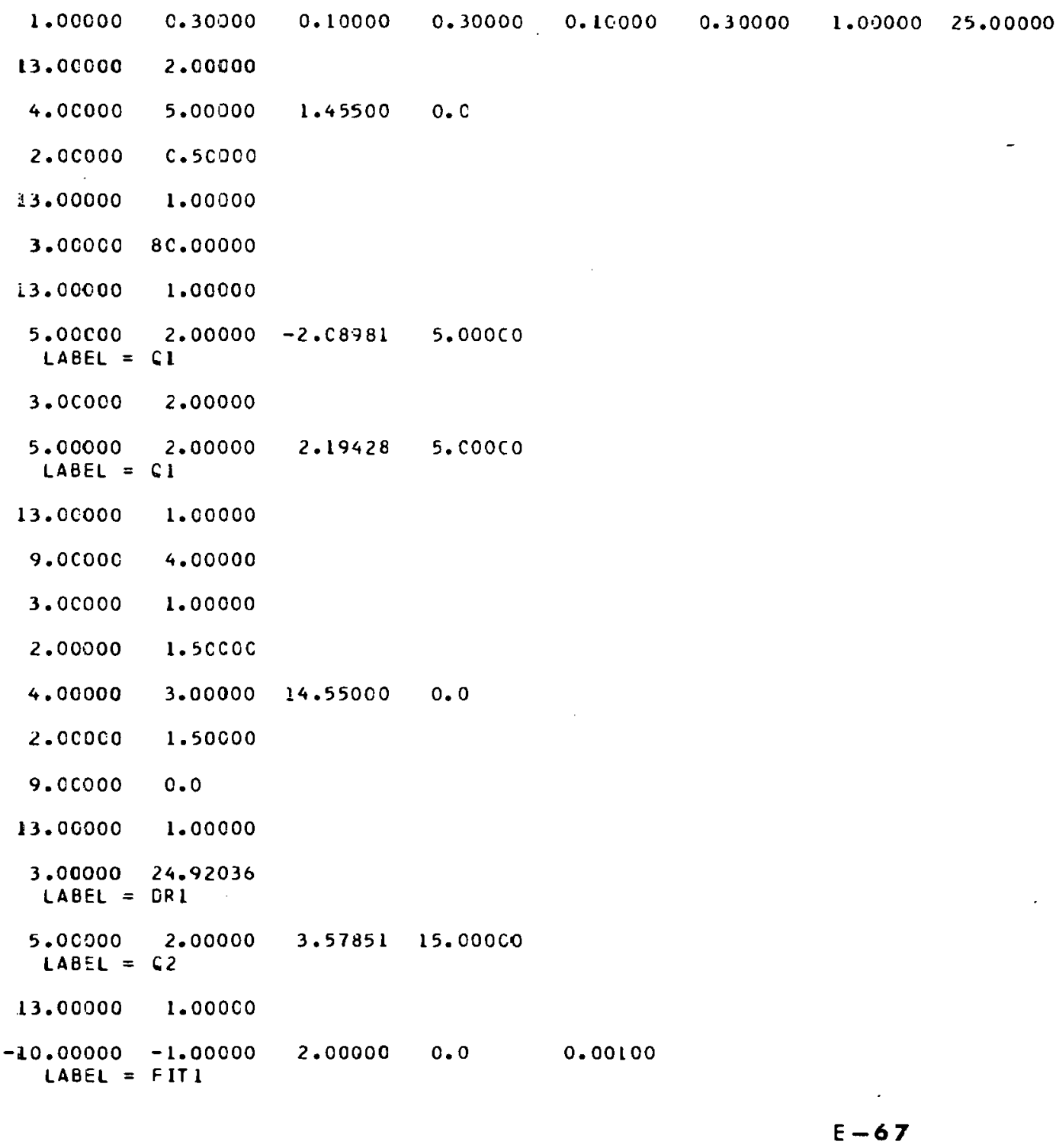




\begin{tabular}{|c|c|c|c|c|}
\hline-10.00000 & $\begin{array}{l}-3.00000 \\
\text { Fiti }\end{array}$ & 4.00000 & C. 0 & 0.00100 \\
\hline 13.00000 & 4.00000 & & & \\
\hline $\begin{array}{l}3.00000 \\
\angle A B E L=\end{array}$ & $\begin{array}{l}25.07953 \\
\text { ORI }\end{array}$ & & & \\
\hline 13.00000 & 1.00000 & & & \\
\hline 9.00000 & .4 .00000 & & & \\
\hline 2.00000 & 1.50000 & & & \\
\hline 4.00000 & 3.00000 & 1.4 .55000 & c. 0 & \\
\hline .2 .00000 & 1.50000 & & & \\
\hline 3.00000 & 1.00000 & & & \\
\hline 9.00000 & c.o & & & \\
\hline 13.00000 & 1.00000 & & & \\
\hline $\begin{array}{c}-10.00000 \\
\text { LABEL }=\end{array}$ & $\begin{array}{l}-1.00000 \\
\text { FIT } 2\end{array}$ & 0.00000 & 0.0 & 0.00100 \\
\hline $\begin{array}{c}-10.00000 \\
\text { LABEL }=\end{array}$ & $\begin{array}{l}-2.00000 \\
\text { FIT } 3\end{array}$ & 0.00000 & 0.0 & 0.00100 \\
\hline 13.00000 & 4.00000 & & & \\
\hline $\begin{array}{l}5.00000 \\
\text { VARY CODE } \\
\text { LABEL }=\end{array}$ & $E_{63}^{2.00000} \begin{array}{l}010000 \\
63\end{array}$ & -1.90000 & 5.00000 & \\
\hline 3.00000 & 2.00000 & & & \\
\hline $\begin{array}{l}5.00000 \\
\text { V.ARY CODE } \\
\text { LABEL = }\end{array}$ & $\begin{array}{r}2.00000 \\
010000\end{array}$ & $i .85000$ & 5.00000 & \\
\hline $3.0 c 0002$ & 200.00000 & & & \\
\hline 13.00000 & 1.00000 & & & \\
\hline $\begin{array}{l}10.00000 \\
\text { VARY CODE } \\
\text { LABEL }=\end{array}$ & $\begin{array}{l}1.00000 \\
200000 \\
\text { FIT }\end{array}$ & 1.00000 & 4. 00000 & 0.10000 \\
\hline $\begin{array}{l}10.00000 \\
\text { VARY CODE } \\
\text { LABEL }=\end{array}$ & $\begin{array}{l}3.00000 \\
200000 \\
\text { FIT } 4\end{array}$ & 3.00000 & 1.50000 & 0.10000 \\
\hline 13.00000 & 4.00000 & & & \\
\hline 13.00000 & 1.00000 & & & \\
\hline $\begin{array}{l}5.00000 \\
\text { LABEL = }\end{array}$ & $64^{2.00000}$ & 4.50000 & 5. 100000 & \\
\hline
\end{tabular}




\begin{tabular}{|c|c|c|c|c|}
\hline 3. Cuuvd & 2.00000 & & & \\
\hline 13.00000 & 1.00001 & & & \\
\hline $\begin{array}{l}5.00000 \\
\text { LABEL }=\end{array}$ & 2.00000 & -4.20000 & 5.00000 & \\
\hline 13.00000 & 1.00000 & & & \\
\hline 3.00000 & 15.00000 & & & \\
\hline 13.00000 & 1.00001 & & & \\
\hline $\begin{array}{c}-1 C .00000 \\
\text { LABEL }=\end{array}$ & $\begin{array}{l}2.00000 \\
\text { FITS }\end{array}$ & 1.00000 & 0.0 & 0.00100 \\
\hline $\begin{array}{c}-1 C .00000 \\
\text { LABEL }=\end{array}$ & $\begin{array}{l}4.000010 \\
F 116\end{array}$ & 3.00000 & 0.0 & 0.00100 \\
\hline $\begin{array}{l}\text { - IC. } 00000 \\
\text { VARY CODE } \\
\text { LABEL = }\end{array}$ & $\begin{array}{l}1.00001 \\
200001 \\
\text { FIT7 }\end{array}$ & $1.0000 \mathrm{C}$ & 0.02000 & 0.01000 \\
\hline $\begin{array}{c}-16.00000 \\
\text { VIRY CODE } \\
\text { LABEL }=\end{array}$ & $\begin{array}{l}3.0000 .0 \\
20000.0 \\
\text { FIT }\end{array}$ & 3.00000 & 0.02000 & 0.01000 \\
\hline 13.00000 & 4.c0003 & & & \\
\hline SE NT I NEL & & & & \\
\hline
\end{tabular}


BEAM TRANSPORT SYSTEM FOR SLAC GFAM SIITCHYARO END STATICN A IIIA

* BEAM $\quad$ 1. COOODO 25.0C GEV

$0.0 \mathrm{M} \quad 0.0 \quad 0.300 \mathrm{cM}$

$0.0 \quad 0.100 \mathrm{MR}$

$0.0 \quad 0.300 \mathrm{CM}$

$0.0, \quad 0.100 \mathrm{MR}$

$\begin{array}{ll}0.0 & 0.300 \mathrm{CM} \\ 0.0 & 1.000 \mathrm{PC}\end{array}$

$\begin{array}{ll}0.0 \\ 0.0 & 0.0\end{array}$

$0.0 \quad 0.0 \quad 0.0$

$0.0 \quad 0.0 \quad 0.0 \quad 0.0$

0.0

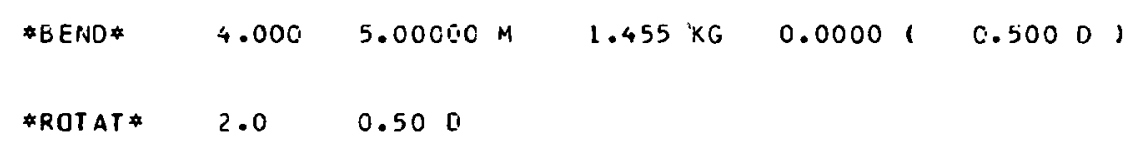

*ROTAT* $2.0 \quad 0.500$

$5.0 M$

$\begin{array}{lllllll}0.0 & 0.305 \mathrm{CM} & & & & & \\ 0.0 & 0.133 \mathrm{MR} & 0.171 & & & & \\ 0.0 & 0.304 \mathrm{CM} & 0.0 & 0.0 & & & \\ 0.0 & 0.100 \mathrm{MR} & 0.0 & 0.0 & 0.164 & & \\ 0.0 & 0.300 \mathrm{CM} & -0.009 & -0.001 & 0.0 & 0.0 & \\ 0.0 & 1.000 \mathrm{PC} & 0.071 & 0.657 & 0.0 & 0.0 & -0.000\end{array}$

\#ORIFT* 3.C $\quad 80 . \mathrm{COOOCM}$

$85.0 \mathrm{M}$

$\begin{array}{ll}0.0 & 1.153 \mathrm{CM} \\ 0.0 & 0.133 \mathrm{MR} \\ 0.0 & 0.901 \mathrm{CM} \\ 0.0 & 0.100 \mathrm{MR} \\ 0.0 & 0.300 \mathrm{CM} \\ 0.0 & 1.000 \mathrm{PC}\end{array}$
0.965
$0.0 \quad 0.0$
$\begin{array}{lll}0.0 & 0.0 & 0.943\end{array}$
$\begin{array}{llll}-0.003 & -0.001 & 0.0 & 0.0\end{array}$

$-0.000$

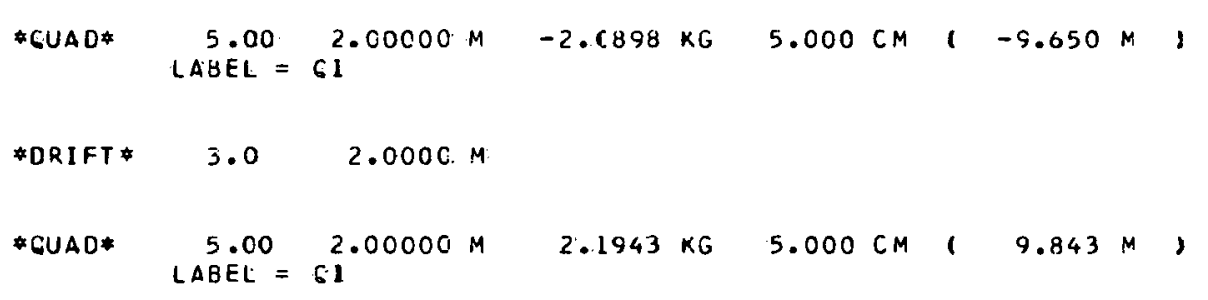

$91.0 M$

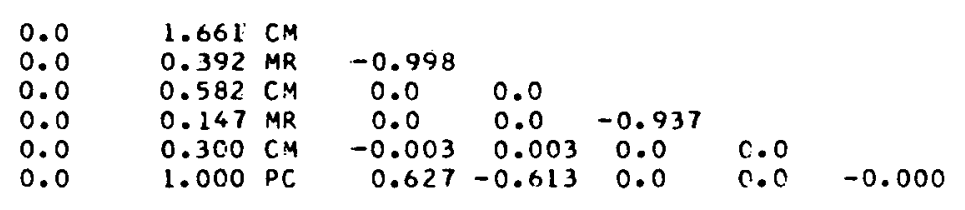

\#DRIFT* $\quad 3.0 \quad 1.0000 \mathrm{M}$ 


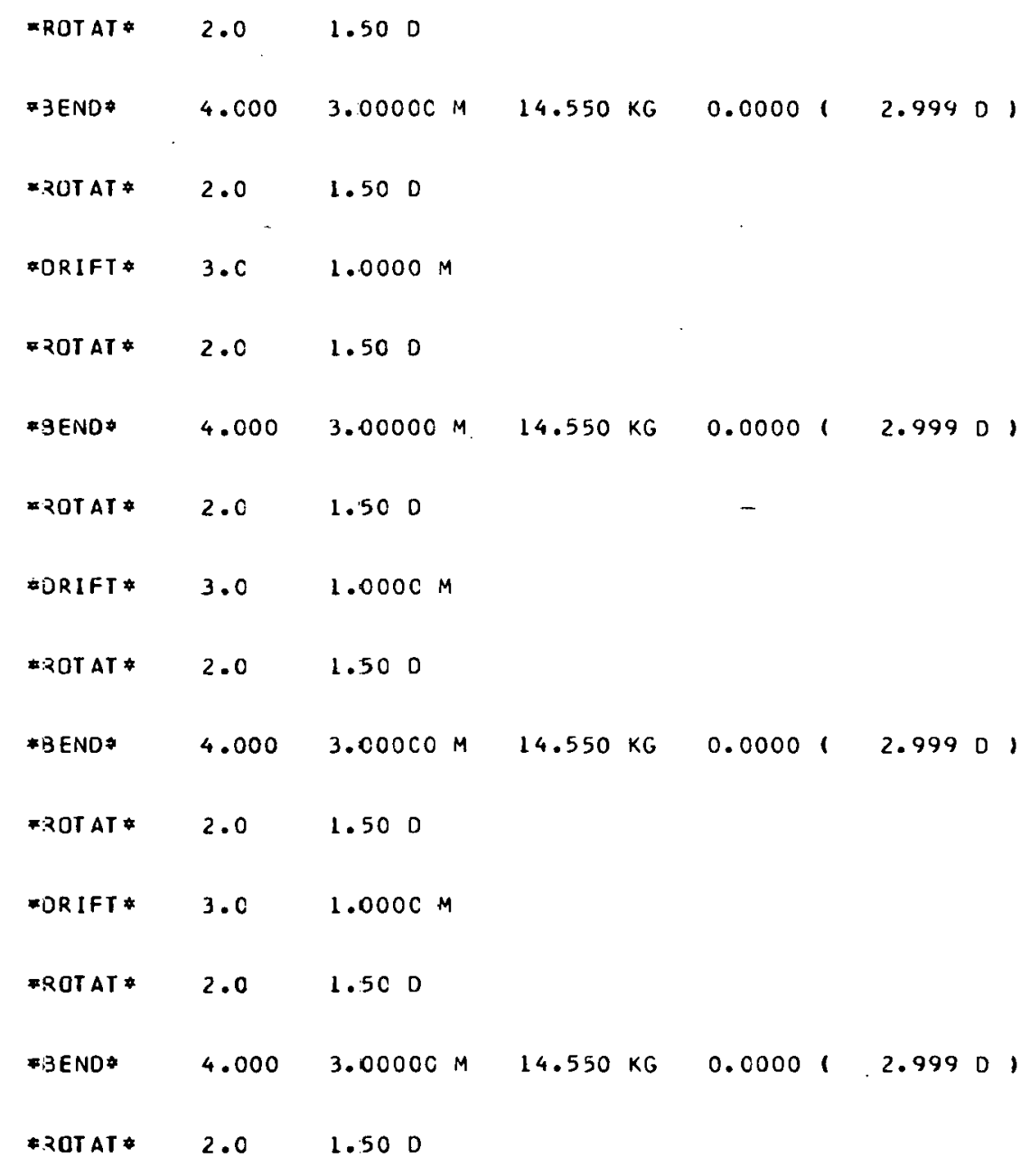

$107.0 \mathrm{M}$

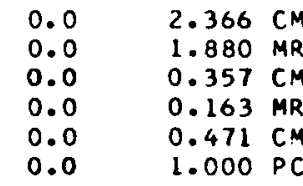

0.873

$0.0 \quad 0.0$

$\begin{array}{lll}0.0 & 0.0 & -0.856\end{array}$ $\begin{array}{llll}-0.737 & -0.533 & 0.0 & 0.0\end{array}$ $\begin{array}{lll}0.941 & 0.986 & 0.0\end{array}$

\#DRIFT $\quad \begin{aligned} & 3.0 \\ & \text { LABEL }=\end{aligned}$ 
*QUAD* $\quad \stackrel{5}{5} \cdot 00=2.00000 \mathrm{M} \quad 3.5785 \mathrm{KG} 15.000 \mathrm{CM} \quad 1 \quad 17.815 \mathrm{M})$

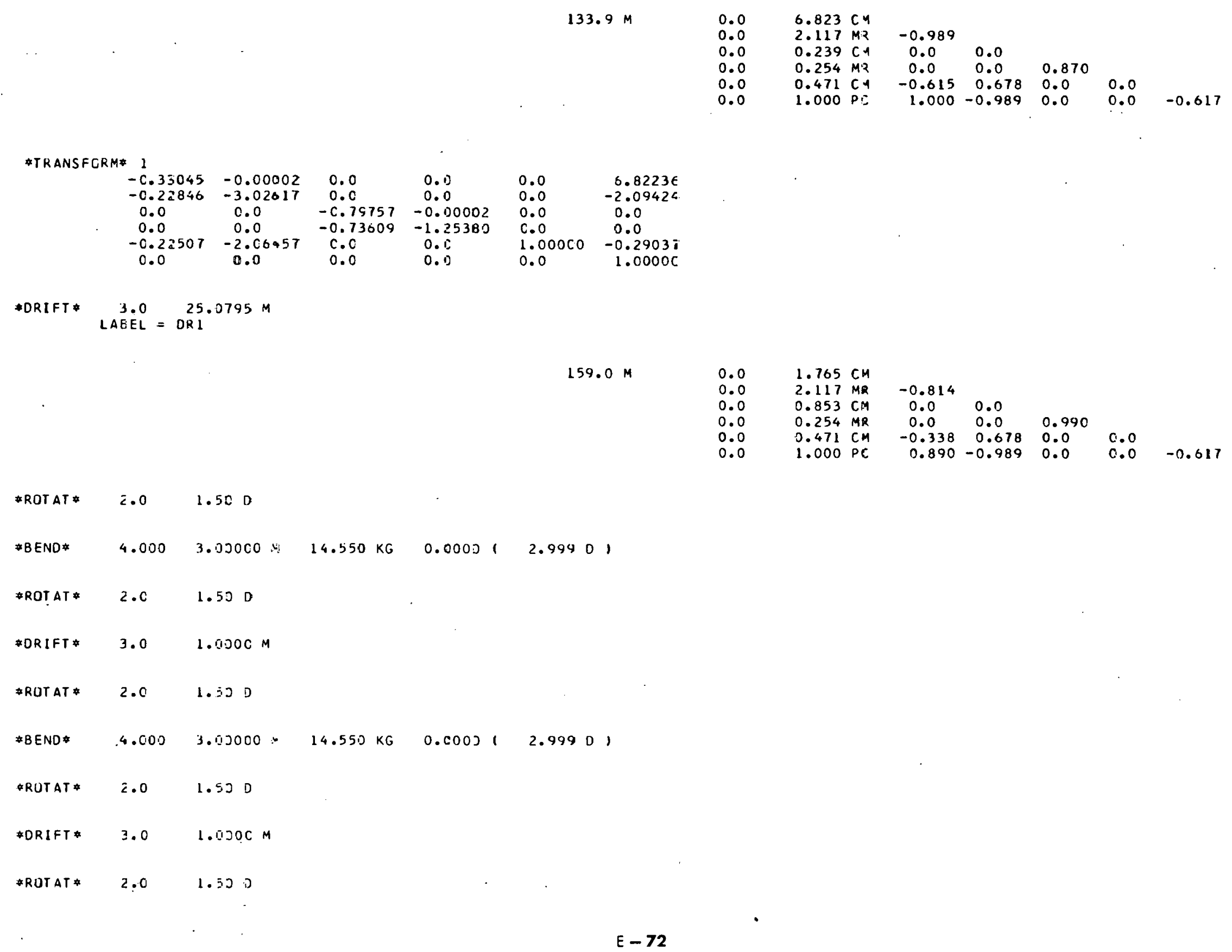




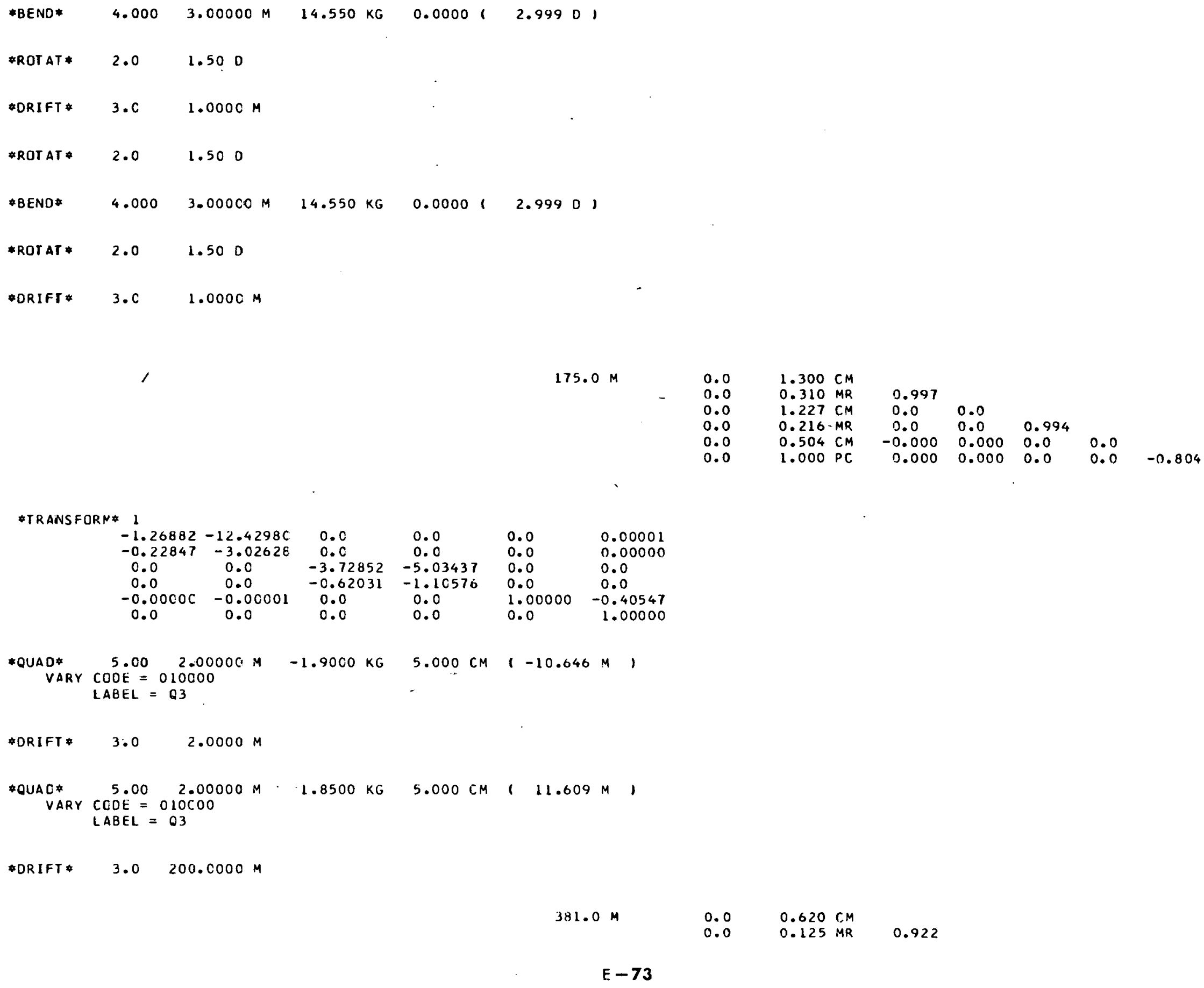

$\begin{array}{llll}381.0 \mathrm{M} & 0.0 & 0.620 \mathrm{CM} & \\ & 0.0 & 0.125 \mathrm{MR} & 0.922 \\ \mathrm{E}-73 & & & \end{array}$




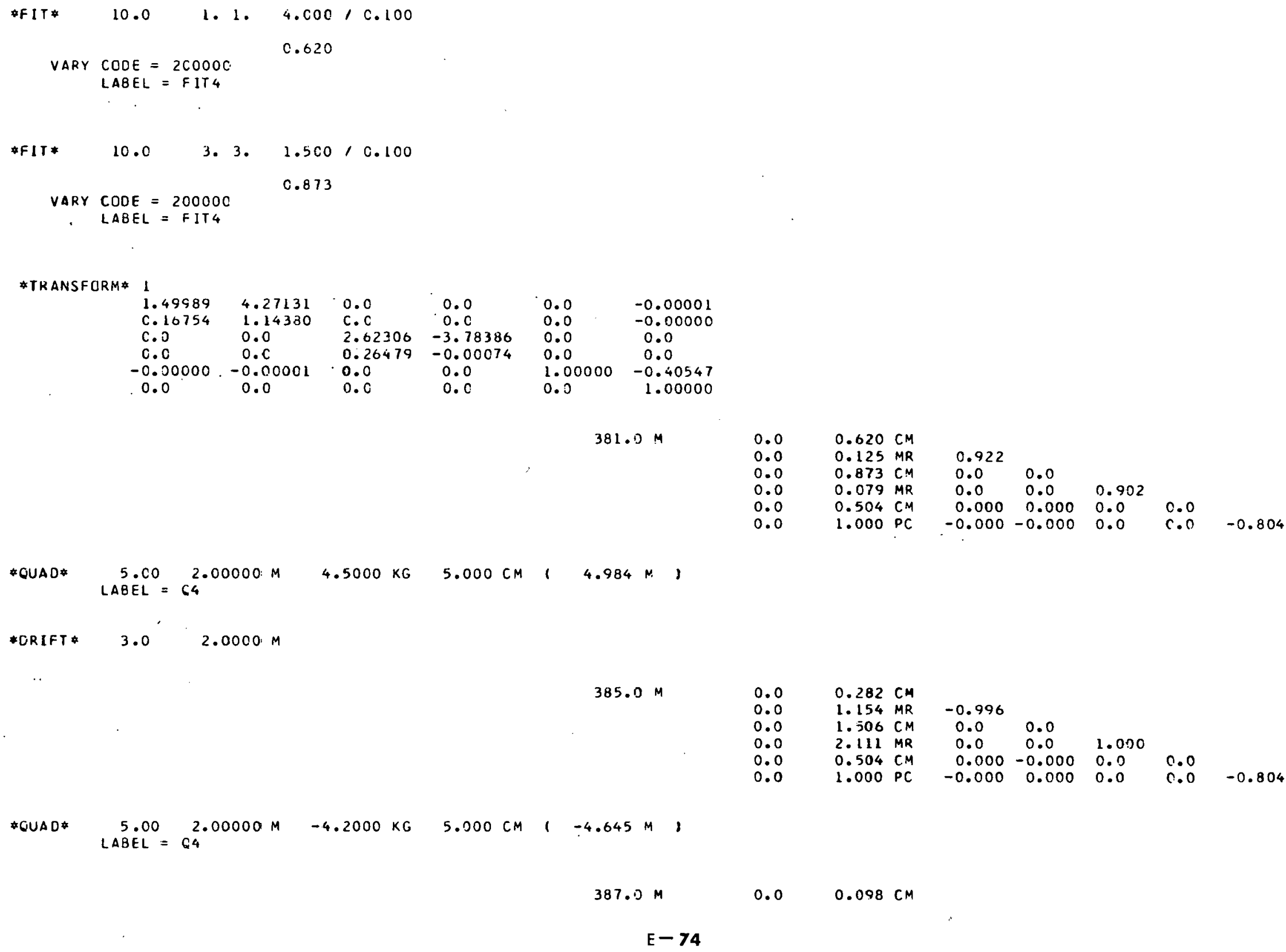


\#DIFT $\quad 3.0 \quad 15 . \operatorname{coc} \mathrm{M}$

$402.0 M$




0.0
0.0
$0 . C$
$0 . C$
$0 . C$
$1 . C 0000$

DLENTH* $401.9993 \mathrm{~N}$

\section{*CORRECTIONS*}

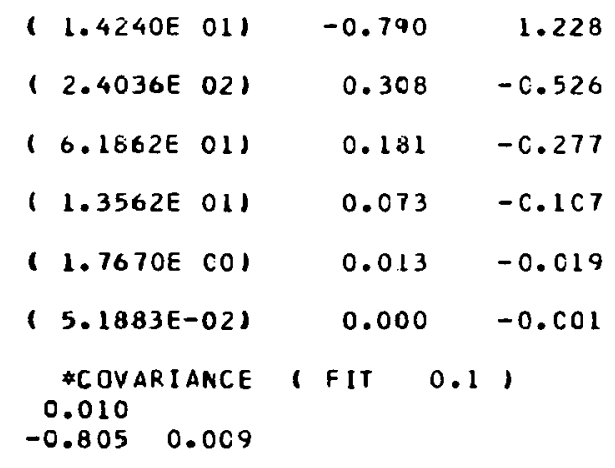

- TRANSFCRM 1

$\begin{array}{llllll}1-2.32281 & 3.1320 \mathrm{C} & 0.0 & 0.0 & 0.0 & 0.0 \\ -1.58390 & 1.65786 & 0.0 & 0.0 & 0.0 & 0.0 \\ 0.0 & 0.0 & -0.17269 & 0.77960 & 0.0 & 0.0 \\ 0.0 & 0.0 & -1.30944 & 0.12067 & 0.0 & 0 . \mathrm{C} \\ 0.0 & 0.0 & 0.0 & 0.0 & 1.00000 & 0 . \mathrm{C} \\ 0.0 & 0.0 & 0 . \mathrm{C} & 0 . \mathrm{C} & 0.0 & 1.0000\end{array}$

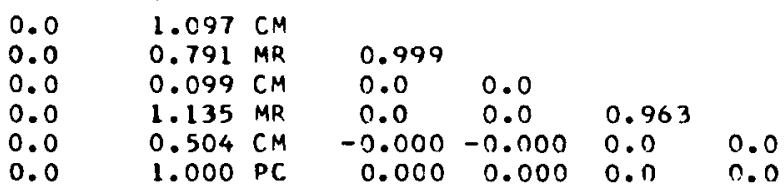


BEAM TRANSPORT SYSTEM FUR SLAC BEAM SHITCHYARD END STATION A IIIA

* Geam* 1. $000000 \quad 25.00 \mathrm{GEV}$

$0.0 \mathrm{M}$

$0.300 \mathrm{CM}$

$0.0 \quad 0.100 \mathrm{MR}$

$0.0 \quad 0.300 \mathrm{CM}$

$0.0 \quad 0.100 \mathrm{MR}$

$0.0 \quad 0.300 \mathrm{CM}$

$0.300 \mathrm{CM}$

$\begin{array}{llll}0.0 & 0.0 & & \\ 0.0 & 0.0 & 0.0 & \\ 0.0 & 0.0 & 0.0 & \\ 0.0 & 0.0 & 0.0 & 0.0 \\ 0.0 & 0.0 & 0.0 & 0.0\end{array}$

0.0

\begin{tabular}{|c|c|c|c|c|c|}
\hline *EEND* & 4.000 & $5.0000 \mathrm{CM}$ & $1.455 \mathrm{KG}$ & $0.0000 \mathrm{l}$ & 0.50001 \\
\hline *ROTAT* & 2.0 & $0.50 \mathrm{D}$ & & & \\
\hline
\end{tabular}

$5.0 \mathrm{M}$

$\begin{array}{ll}0.0 & 0.305 \mathrm{CM} \\ 0.0 & 0.133 \mathrm{MR} \\ 0.0 & 0.304 \mathrm{CM} \\ 0.0 & 0.100 \mathrm{MR} \\ 0.0 & 0.300 \mathrm{CM} \\ 0.0 & 1.000 \mathrm{PC}\end{array}$

\#DRIFT* $\quad 3.0 \quad 8 C .0 C 50 \mathrm{M}$
$85.0 \mathrm{M}$

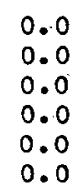

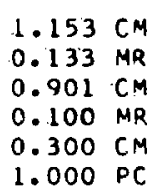

$0.133 \mathrm{MR}$

$0.100 \mathrm{MR}$

$0.300 \mathrm{CM}$

$\begin{array}{cccc}0.965 & & & \\ 0.0 & 0.0 & & \\ 0.0 & 0.0 & 0.943 & \\ -0.003 & -0.001 & 0.0 & 0.0 \\ 0.624 & 0.057 & 0.0 & 0.0\end{array}$

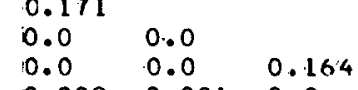

$\begin{array}{cccc}0.0 & 0.0 & 0.164 & \\ -0.009 & -0.001 & 0.0 & 0.0\end{array}$

$\begin{array}{llll}0.071 & 0.657 & 0.0 & 0.0\end{array}$

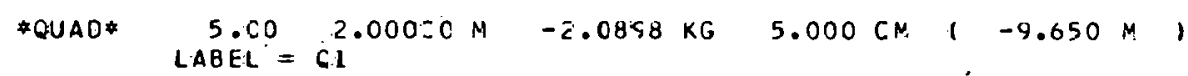

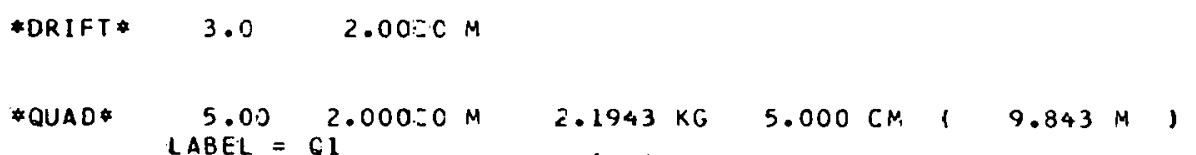

$91.0 \mathrm{M}$

\section{0}

0.0
0.0

0.0

0.0
0.0

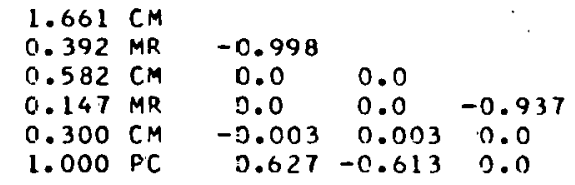




\begin{tabular}{|c|c|c|c|c|c|c|c|}
\hline \#RIIAI = & 2.0 & $1.50 \mathrm{D}$ & & & & & \\
\hline *BEND* & 4.000 & $3.00000 \mathrm{M}$ & 14.550 & KG & 0.0000 & 1 & $2.999 \mathrm{D} 1$ \\
\hline $\begin{array}{c}\text { *R JT AT } \\
:\end{array}$ & 2.0 & 1.500 & & & & & \\
\hline$\star D R I F T *$ & 3.0 & $1.0000 \mathrm{M}$ & & & & & \\
\hline *RDTAT * & 2.0 & 1.500 & & & . & & \\
\hline \$BEND* & 4.000 & $3.00000 \mathrm{M}$ & 14.550 & KG & 0.0000 & 1 & 2.99901 \\
\hline \#ROTAT & 2.0 & 1.500 & & & & & \\
\hline *DRIFT & 3.0 & $1.0000 \mathrm{M}$ & & & & & \\
\hline *ROT AT * & $2 . c$ & 1.500 & & & & & \\
\hline *BEND* & 4.000 & $3.00000 \mathrm{M}$ & 14.550 & KG & 0.0000 & 1 & 2.99901 \\
\hline *ROTAT* & 2.0 & 1.500 & & & & & \\
\hline$* D: R I F T *$ & 3.0 & $1.0000 \mathrm{M}$ & & & & & \\
\hline * RIOTAT A & 2.0 & 1.500 & & & & & \\
\hline$\# B E N D *$ & 4.000 & $3 . \mathrm{C0000 \textrm {M }}$ & 14.550 & $<G$ & 0.0000 & 1 & $2.999 \mathrm{D} 1$ \\
\hline * ROT AT* & 2.0 & 1.500 & & & & & \\
\hline
\end{tabular}

$107.0 \mathrm{M}$

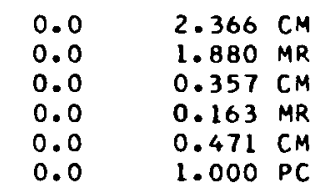

0.873

$\begin{array}{ll}0.0 & 0.0\end{array}$

$\begin{array}{lll}0.0 & 0.0 & -0.856\end{array}$

$\begin{array}{llll}-0.737 & -0.533 & 0.0 & 0.0\end{array}$

$\begin{array}{lllll}0.941 & 0.986 & 0.0 & 0.0 & -0.617\end{array}$

*ORIFT $\quad 3.0 \quad 24.9203 \mathrm{M}$ 
\#QUAD $\quad \angle A B E L=62.000030 \mathrm{M} \quad 3.5735 \mathrm{KG} 15.000 \mathrm{gM}, 17.815 \mathrm{M})$

\begin{tabular}{|c|c|c|c|c|c|c|c|c|}
\hline $133.9 \mathrm{M}$ & $\begin{array}{l}0.0 \\
0.0 \\
0.0 \\
0.0 \\
0.0 \\
0.0\end{array}$ & $\begin{array}{l}6.823 \\
2.117 \\
0.239 \\
0.254 \\
0.471 \\
1.000\end{array}$ & $\begin{array}{l}C M \\
M R \\
C M \\
M R \\
C M \\
F C\end{array}$ & $\begin{array}{l}-0.989 \\
0.0 \\
0.0 \\
-0.615 \\
1.000\end{array}$ & $\begin{array}{c}0.0 \\
0.0 \\
0.678 \\
-0.989\end{array}$ & $\begin{array}{l}0.870 \\
0.0 \\
0.0\end{array}$ & $\begin{array}{l}0.0 \\
0.0\end{array}$ & -0.617 \\
\hline
\end{tabular}

*TRANSFORN 1

$\begin{array}{cccclc}1 & & & & \\ -c .33045 & -0.00002 & 0.0 & 0.0 & 0.0 & 6.82236 \\ -C .22840 & -3.02617 & C . C & 0.0 & 0.0 & -2.09424 \\ 0.0 & 0.0 & -0.79757 & -0.00002 & 0.0 & 0.0 \\ 0.0 & 0.0 & -C .73639 & -1.25380 & 0.0 & 0.0 \\ -c .22507 & -2.06457 & 0.0 & 0.0 & 1.00000 & -0.29037 \\ 0.0 & 0.0 & 0.0 & 0.0 & 0.0 & 1.00000\end{array}$

3.0
\#DRIFT $25.0795 \mathrm{M}$

$159.0 \mathrm{M}$

0.0
0.0
0.0

$1.765 \mathrm{CM}$ $2.117 \mathrm{MR}$ $0.853 \mathrm{CM}$

$0.471 \mathrm{CM}$

1. 000 PC
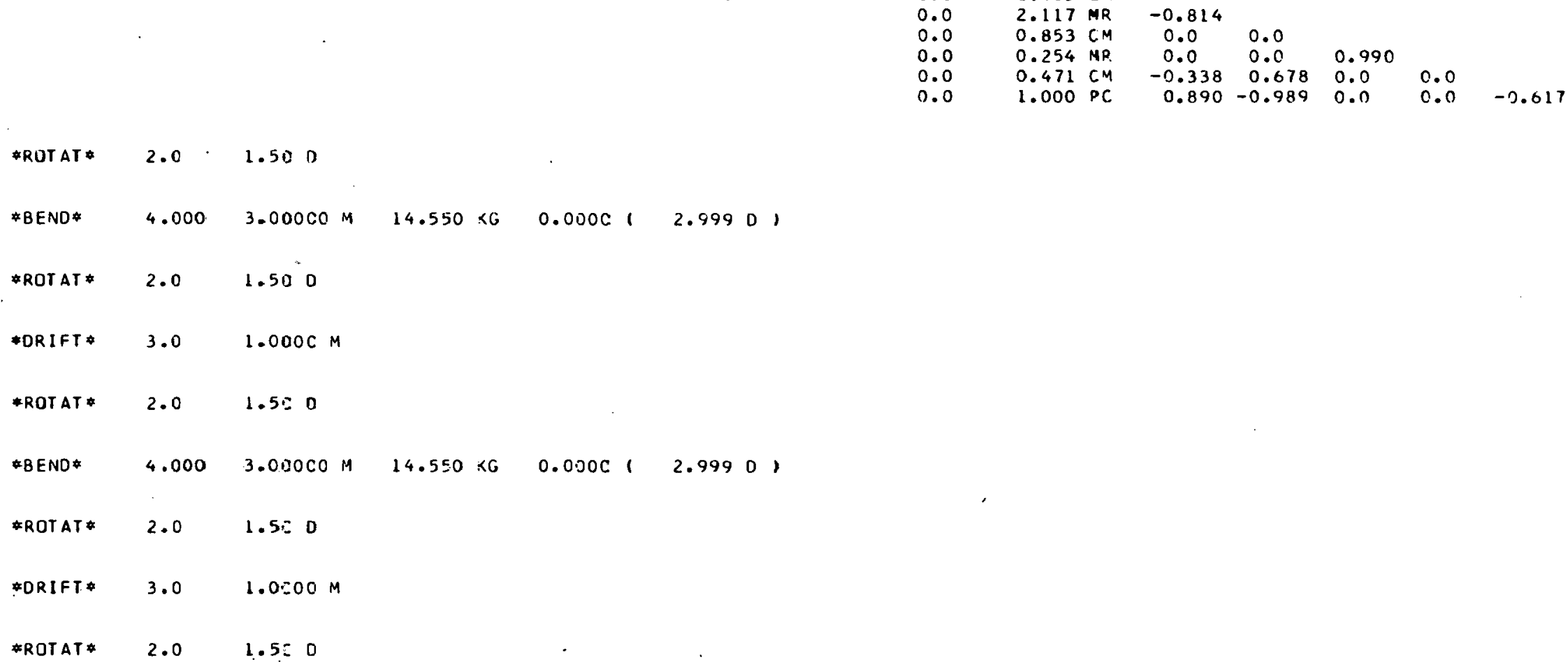


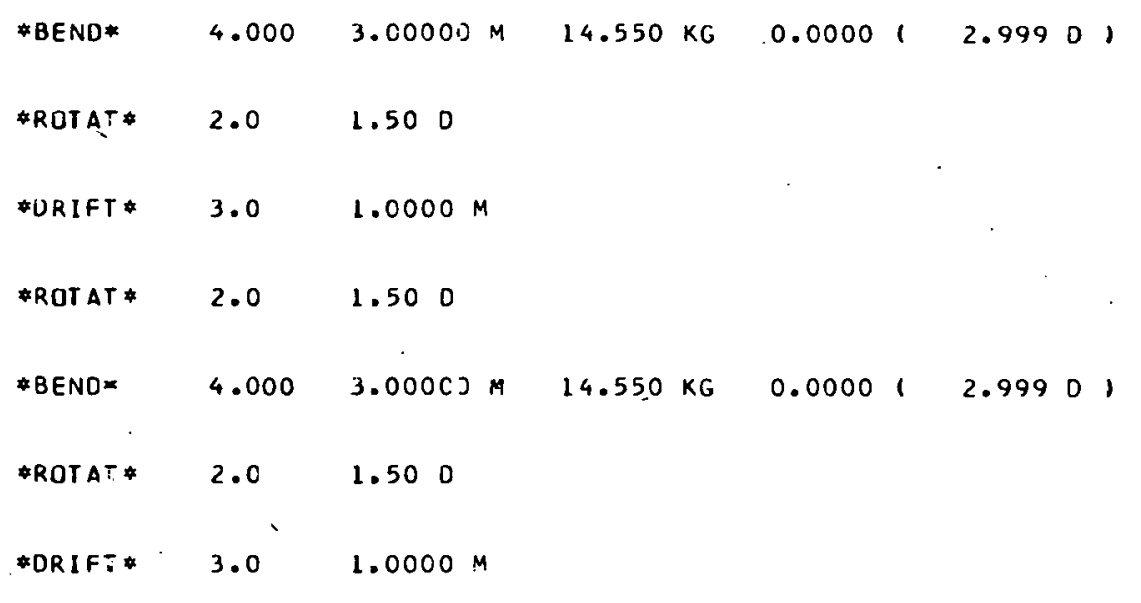

\begin{tabular}{|c|c|c|c|c|c|c|c|}
\hline $175.0 \mathrm{~m}$ & $\begin{array}{l}0.0 \\
0.0 \\
0.0 \\
0.0 \\
0.0 \\
0.0\end{array}$ & $\begin{array}{l}1.300 \\
0.310 \\
1.227 \\
0.216 \\
0.504 \\
1.000\end{array}$ & $\begin{array}{l}C M \\
M R \\
C M \\
M R \\
C M \\
P C\end{array}$ & $\begin{array}{l}0.997 \\
0.0 \\
0.0 \\
-0.000 \\
0.000\end{array}$ & $\begin{array}{l}0.0 \\
0.0 \\
0.000 \\
0.000\end{array}$ & $\begin{array}{l}0.994 \\
0.0 \\
0.0\end{array}$ & $\begin{array}{l}0.0 \\
0.0\end{array}$ \\
\hline
\end{tabular}

*transform* 1

$\begin{array}{cccclc}1 & & & & \\ -1.26882 & -12.42980 & 0.0 & 0.0 & 0.0 & 0.00001 \\ -0.22847 & -3.02628 & 0.0 & 0.0 & 0.0 & 0.00000 \\ 0.0 & 0.0 & -3.72852 & -5.03437 & 0.0 & 0.0 \\ 0.0 & 0.0 & -0.62031 & -1.10576 & 0.0 & 0.0 \\ -0.00000 & -0.50001 & 0.0 & 0.0 & 1.00000 & -0.40547 \\ 0.0 & 0.0 & 0.0 & 0.0 & 0.0 & 1.00000\end{array}$

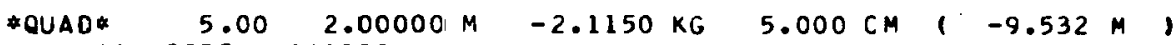

YARY CODE $=010000$

$\angle A B E L=03$

*DRIFT* $\quad 3.0 \quad 2.0000 \mathrm{M}$

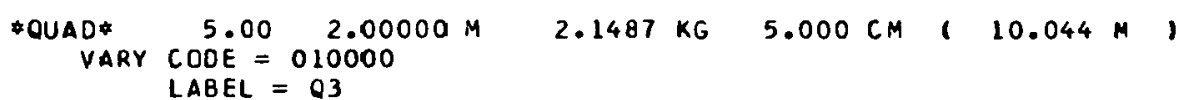

\#DRIFI * $\quad 3.0 \quad 200.0000 \mathrm{M}$

$\begin{array}{llll}381.0 M & 0.0 & 4.000 \mathrm{CM} & \\ & 0.0 & 0.299 \mathrm{MR} & 1.000 \\ & & & \end{array}$


VARY CODE $=200000$

4.000

$\angle A B E L=F I T 4$

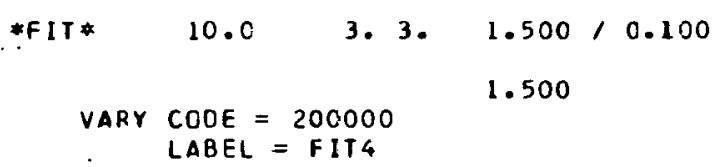

*TRANSFORM 1

$\begin{array}{lcllll}4.77122 & 37.35149 & 0.0 & 0.0 & 0.0 & -0.00003 \\ 0.33310 & 2.81727 & 0.0 & 0.0 & 0.0 & -0.00000 \\ C .0 & C .0 & 4.59169 & -0.89239 & 0.0 & 0.0 \\ 0.0 & c .0 & 0.37533 & 0.13324 & 0.0 & 0.0 \\ -c .00000 & -C .010001 & 0.0 & 0.0 & 1.00000 & -0.40547 \\ 0.0 & 0.0 & 0.0 & 0.0 & 0.0 & 1.00000\end{array}$

$381.0 M$

$4.000 \mathrm{CM}$ $0.299 \mathrm{MP} \quad 1.000$ $1.500 \mathrm{CH}$ $\begin{array}{llll}0.113 \text { MP } & 0.0 & 0.0 & \\ 0.0 & 0.0 & 0.984\end{array}$ $\begin{array}{lllllllllllllllll}0.504 \mathrm{CM} & 0.000 & 0.000 & 0.0 & 0.0\end{array}$ 1.000 PC $\cdot-0.000-0.000 \quad 0.0 \quad 0.0$

$-0.804$

*QUAD*

$5.00 \quad 2.00000$ in

$5.000 \mathrm{Cid}$

$4.984 \mathrm{M} \quad 1$

*DR I F T * 3. $\quad 2.0000 \mathrm{M}$

$385.0 M$

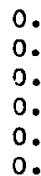

$1.665 \mathrm{CM}$ $7.789 \mathrm{MR}$ $2.582 \mathrm{CH}$

$0.504 \mathrm{CM}$

$1.000 \mathrm{PC}$

$-1.000$ $0.0 \quad 0.0$ $\begin{array}{lll}0.0 & 0.0 & 1.000\end{array}$ $\begin{array}{llll}0.000 & -0.000 & 0.0 & 0.0\end{array}$ $\begin{array}{lll}-0.000 & 0.000 \quad 0.0\end{array}$

$5.00 \quad 2.00000 \mathrm{H}-4.20 \mathrm{COKG} 5.000 \mathrm{CA}(-4.645 \mathrm{M})$ $\angle A B E L=\$ 4$ 
*CIRIFT* $\quad 3.0 \quad 15.0000 \mathrm{M}$

$402.0 \mathrm{M}$

$\begin{array}{ll}0.0 & 8.395 \mathrm{CM} \\ 0.0 & 5.828 \mathrm{MR} \\ 0.0 & 0.173 \mathrm{CM} \\ 0.0 & 1.951 \mathrm{MR} \\ 0.0 & 0.504 \mathrm{CM} \\ 0.0 & 1.000 \mathrm{PC}\end{array}$

$5.828 \quad M R$
$2.754 \mathrm{CM}$

$1.951 \mathrm{MR}$

$\begin{array}{lll}0.0 & 0.0 & -1.000\end{array}$

$0.504 \mathrm{CM}$
$1.000 \mathrm{PC}$

$0.000-0.000 \quad 0.0$

0.0

$-0.804$
1.000
0.0

$\begin{array}{ll}0.0 & 0.0 \\ 0.0 & 0.0\end{array}$

$0.0 \quad 0.996$

$0.000-0.000 \quad 0.0$

$0.000 \quad 0.000 \quad 0.0$

0.0

* TRANSFORM *

$\begin{array}{lll}-2.33281 & 3.1320 C & 0 . C \\ -1.58390 & 1.65786 & 0.0 \\ 0.0 & 0.0 & -0.17269 \\ 0.0 & 0.0 & -1.30944 \\ 0.0 & 0.0 & 0 . C \\ 0.0 & 0.0 & 0.0\end{array}$

0.0

C. 77960

$0.12067 \quad 0.0$

$\begin{array}{ll}0.0 & 0.0 \\ 0.0 & 0.0\end{array}$

0.

0.0

1.00000

0.0

0.0

0.0

$401.5793 \mathrm{M}$

-LENGTH*

$401.5793 \mathrm{M}$ 
'BFAM TRANSPOFT SYSTEM FOR SLAC BEAM SIYITCHYARO END STATION A IIIB'

(IN THIS CASE THE VARY CONES FJR THE QUAD DOUBLET, 23 , NRE TURMED OFF, AND)

(THE THIRD QUAD DOUBLET, 24 , IS ALLOWED TO VARY TS GET A WAIST WITH A CERTAIN)

(SPOT SIZE AT THE TARGET.)

5.0 ' $Q 33^{\prime}$;

10.0 I FIT

10.0 'FITG'

10.5 'FIT7',

10.2 STITT 
BEAM TRANSPORT SYSTEN: FOR SLAC BEAM SNITCHYARD ENO STATICN A IIIB

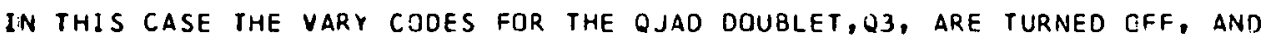

JHE THIRO QUAC DOUBLET,Q4, IS ALLOWEO TO VARY TO GET A WAIST WITH A CERTAIN

SPOT SILE AT THE TARGET.

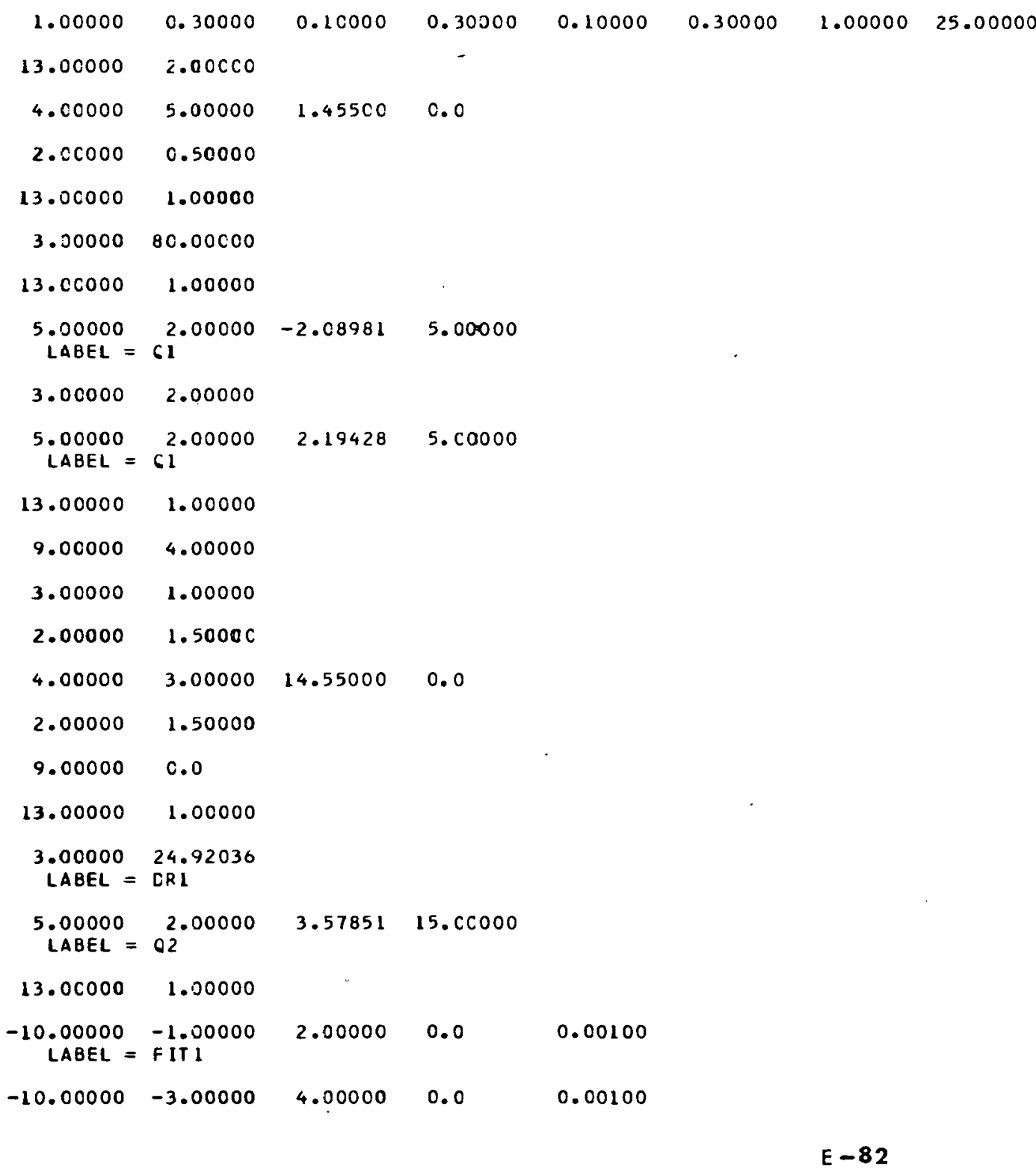




\begin{tabular}{|c|c|c|c|c|}
\hline 13.00000 & 4.00000 & & & \\
\hline $\begin{array}{l}3.00000 \\
\text { LAEEL = }\end{array}$ & $\begin{array}{l}25.07953 \\
\text { CF. } 1\end{array}$ & & & \\
\hline 13.00000 & 1.00000 & & & \\
\hline 9.00000 & 4.00000 & & & \\
\hline 2.00000 & 1.50000 & & & \\
\hline 4.00000 & 3.00000 & 17.55000 & 0.0 & \\
\hline 2.00000 & 1.50000 & & & . \\
\hline 3.00000 & 1.00000 & & & \\
\hline 9.00000 & 0.0 & & & \\
\hline 13.00000 & 1.00000 & & & \\
\hline $\begin{array}{c}-10.00000 \\
\text { LABEL }=\end{array}$ & $\begin{array}{l}-1.00000 \\
\text { FIT2 }\end{array}$ & $5.000 c c$ & 0.0 & 0.00100 \\
\hline $\begin{array}{r}-10.00000- \\
\text { LABEL }=\end{array}$ & $\begin{array}{l}-2.00000 \\
\text { FIT3 }\end{array}$ & $5 . c 00 c c$ & 0.0 & 0.00100 \\
\hline 13.00000 & 4.00000 & & & \\
\hline $\begin{array}{l}5.00000 \\
\angle A B E L=\end{array}$ & 63.00000 & -2.11497 & 5.00000 & \\
\hline 3.00000 & 2.00000 & & & \\
\hline $\begin{array}{l}5.00 C C O \\
\text { LABEL }=\end{array}$ & $c 3^{2.00000}$ & 2.14873 & 5.00000 & \\
\hline 3.000002 & 20.00000 & & & \\
\hline 13.00000 & 1.00000 & & & \\
\hline $\begin{array}{l}10.00000 \\
\text { VARY CODE } \\
\text { LABEL = }\end{array}$ & $\begin{array}{l}1.00000 \\
=200000 \\
\text { FIT4 }\end{array}$ & 1.00000 & 4. 00000 & 0.10000 \\
\hline $\begin{array}{l}10.00000 \\
\text { VARY CODE } \\
\text { LABEL = }\end{array}$ & $\begin{array}{l}3.00000 \\
=200000 \\
\text { F } 114\end{array}$ & 3.00000 & 1.50000 & 0.10000 \\
\hline 13.00000 & 4.00000 & & & \\
\hline 13.00000 & 1.00000 & & & \\
\hline $\begin{array}{l}5.00000 \\
\text { VARY CODE } \\
\text { LABEL = }\end{array}$ & $=\begin{array}{r}2.00000 \\
010000 \\
64\end{array}$ & $7.500 \mathrm{CO}$ & 5.00000 & \\
\hline 3.00000 & 2.00000 & & & \\
\hline
\end{tabular}




\begin{tabular}{|c|c|c|c|c|}
\hline 13.06 & 1.00000 & & & \\
\hline $\begin{array}{l}5.00000 \\
\text { VARY CODE } \\
\text { LABEL = }\end{array}$ & $\begin{array}{r}2.00000 \\
010000 \\
04\end{array}$ & -4.20000 & 5.00000 & \\
\hline 13.00000 & 1.00000 & & & \\
\hline 3.00000 & 15.00000 & & & \\
\hline 13.00000 & 1.00000 & & & \\
\hline $\begin{array}{c}10.00000 \\
\text { LABEL }=\end{array}$ & $\begin{array}{l}2.00000 \\
\text { FIr5 }\end{array}$ & 1.00000 & 0.0 & 0.00100 \\
\hline $\begin{array}{l}10.00000 \\
\text { LABEL }=\end{array}$ & $\begin{array}{l}4.00000 \\
\text { FIT6 }\end{array}$ & 3.20000 & 0.0 & 0.00100 \\
\hline $\begin{array}{l}10.00000 \\
\text { VARY CODE } \\
\text { LAEEL }=\end{array}$ & $\begin{array}{l}1.00000 \\
200000 \\
\text { FIT }\end{array}$ & $1.2000 \mathrm{C}$ & $0 . c 2000$ & $0 . \mathrm{Cl} 000$ \\
\hline $\begin{array}{l}10.0 C O O O \\
\text { VARY CODE } \\
\text { LABEL }=\end{array}$ & $\begin{array}{l}3.00000 \\
200000 \\
\text { FIT }\end{array}$ & 3.00000 & 0.02000 & 0.61000 \\
\hline
\end{tabular}
LABEL = FIT 7

$13.00000 \quad 4.00000$

SENT INEL 
BEAM TRANSPORT SYSTEI FOF: SLAC GEAM SWITCHYARD END STAIICN A IIIB

* BEAM* $\quad 1$. CCO000 $25.00 \mathrm{GEV}$

$0.0 \mathrm{M} \quad 0.0 \quad 0.300 \mathrm{CM}$

$0.0 \quad 0.100 \mathrm{MR}$

$0.0 \quad 0.300 \mathrm{CM}$

$0.0 \quad 0.100 \mathrm{MR}$

$0.0 \quad 0.300 \mathrm{CM}$

$1.000 \mathrm{PC}$

0.0

C. 0.0

$\begin{array}{lll}0.0 & 0.0 & 0.0\end{array}$

$0.0 \quad 0.0 \quad 0.0$

$\begin{array}{ll}0.0 & 0.0 \\ 0.0 & 0.0\end{array}$

0.0

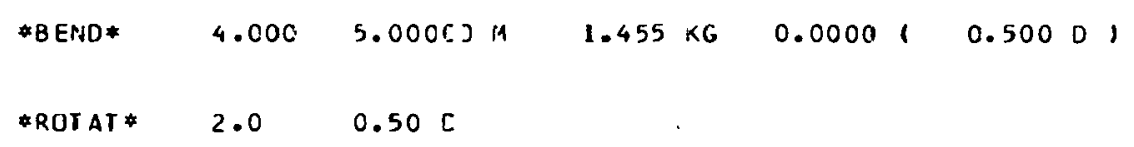

$91.0 \mathrm{M}$

0.0
0.0
0.0
0.0
0.0
0.0

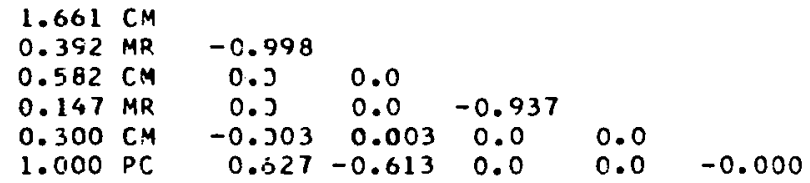

*DRIFT* $\quad 3.0 \quad 1.0000 \mathrm{M}$ 


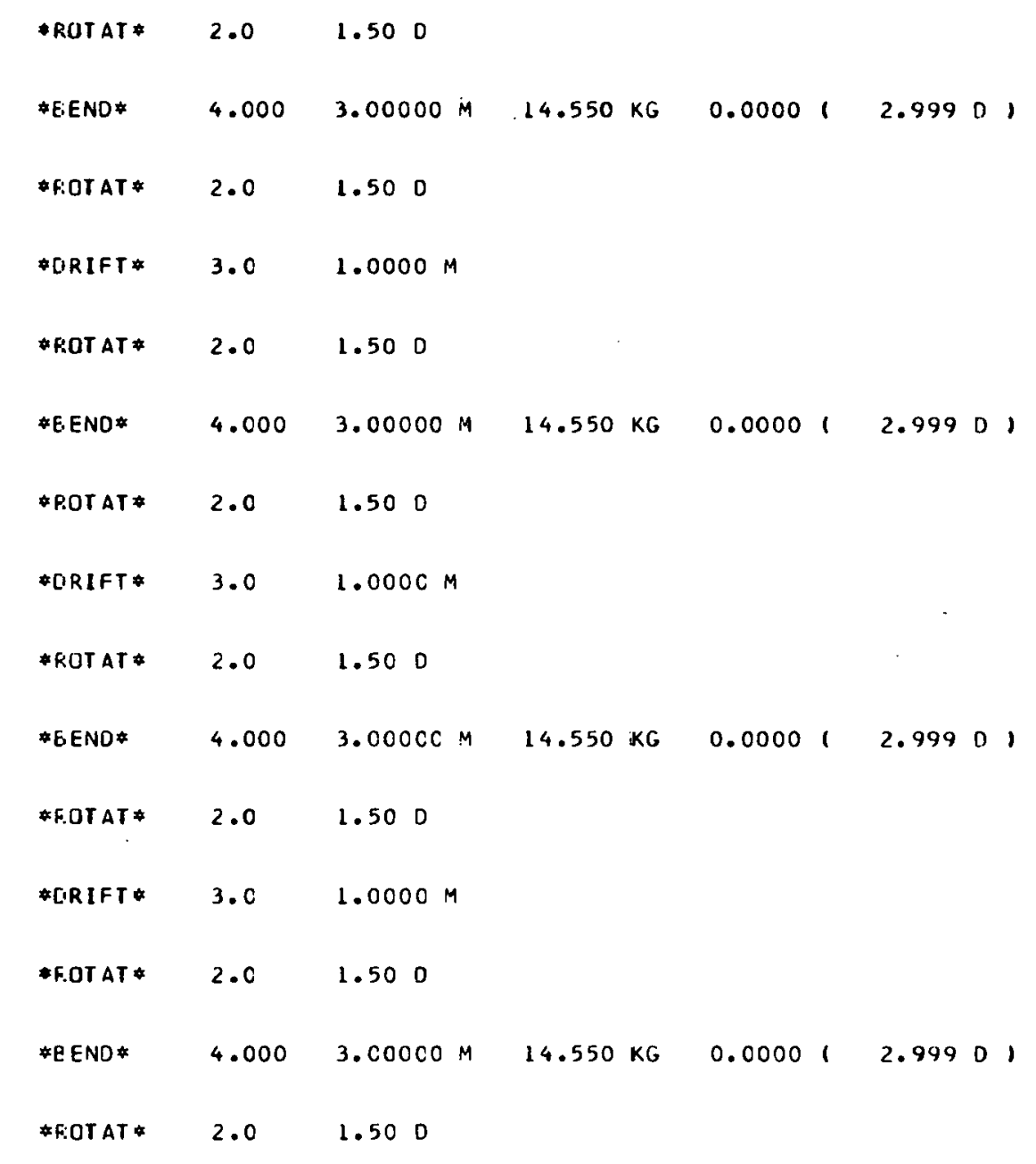

$\begin{array}{lllllllll}107.0 M & 0.0 & 2.366 & C M & & & & & \\ & 0.0 & 1.880 & M R & 0.873 & & & & \\ & 0.0 & 0.357 & C M & 0.0 & 0.0 & & & \\ 0.0 & 0.163 & M R & 0.0 & 0.0 & -0.356 & & \\ 0.0 & 0.471 & C M & -0.737 & -0.533 & 0.0 & 0.0 & \\ 0.0 & 0.0 & 1.000 & \text { PC } & 0.941 & 0.986 & 0.0 & 0.0 & -\Omega .617\end{array}$

*LRIFT* $\quad 3.0 \quad 24.9203 \mathrm{M}$ LABEL $=$ ORI 
*QUAD $\quad \stackrel{5}{5 . C O}=62.00000 \mathrm{M} \quad 3.5785 \mathrm{KG} 15.000 \mathrm{GM}(17.815 \mathrm{~N})$

\begin{tabular}{|c|c|c|c|c|c|c|c|}
\hline $133.9 \mathrm{H}$ & $\begin{array}{l}0.0 \\
0.0 \\
0.0 \\
0.0 \\
0.0 \\
0.0\end{array}$ & $\begin{array}{l}6.823 \\
2.117 \\
0.239 \\
0.254 \\
0.471 \\
1.000\end{array}$ & $\begin{array}{l}C M \\
M Q \\
C M \\
N R \\
C M \\
F C\end{array}$ & $\begin{array}{l}-0.989 \\
0.0 \\
0.0 \\
-0.615 \\
1.000\end{array}$ & $\begin{array}{c}0.0 \\
0.0 \\
0.678 \\
-0.989\end{array}$ & $\begin{array}{l}0.970 \\
0.0 \\
0.0\end{array}$ & $\begin{array}{l}0.0 \\
0.0\end{array}$ \\
\hline
\end{tabular}

- TRANSFORM* 1

\begin{tabular}{|c|c|c|c|c|c|}
\hline $\begin{aligned}-0.33045 \\
-0.22846 \\
0.0 \\
0.0 \\
-0.22507 \\
0.0\end{aligned}$ & $\begin{array}{l}-0.00002 \\
-3.02617 \\
0.0 \\
0.0 \\
-2.06457 \\
0.0\end{array}$ & $\begin{array}{l}0.0 \\
0.0 \\
-0.79757 \\
-C .73609 \\
0.0 \\
0.0\end{array}$ & $\begin{aligned} & 0.0 \\
& 0.0 \\
&-0.00002 \\
&-1.2538 .0 \\
& 0.0 \\
& 0.0\end{aligned}$ & $\begin{array}{l}0.0 \\
0.0 \\
0.0 \\
0.0 \\
1.00000 \\
0.0\end{array}$ & $\begin{array}{l}6.82236 \\
-2.09424 \\
0.0 \\
0.0 \\
-0.29037 \\
1.00000\end{array}$ \\
\hline
\end{tabular}

*DRIFT* $\begin{array}{r}3.0 \\ L A B E L=O R L\end{array}$

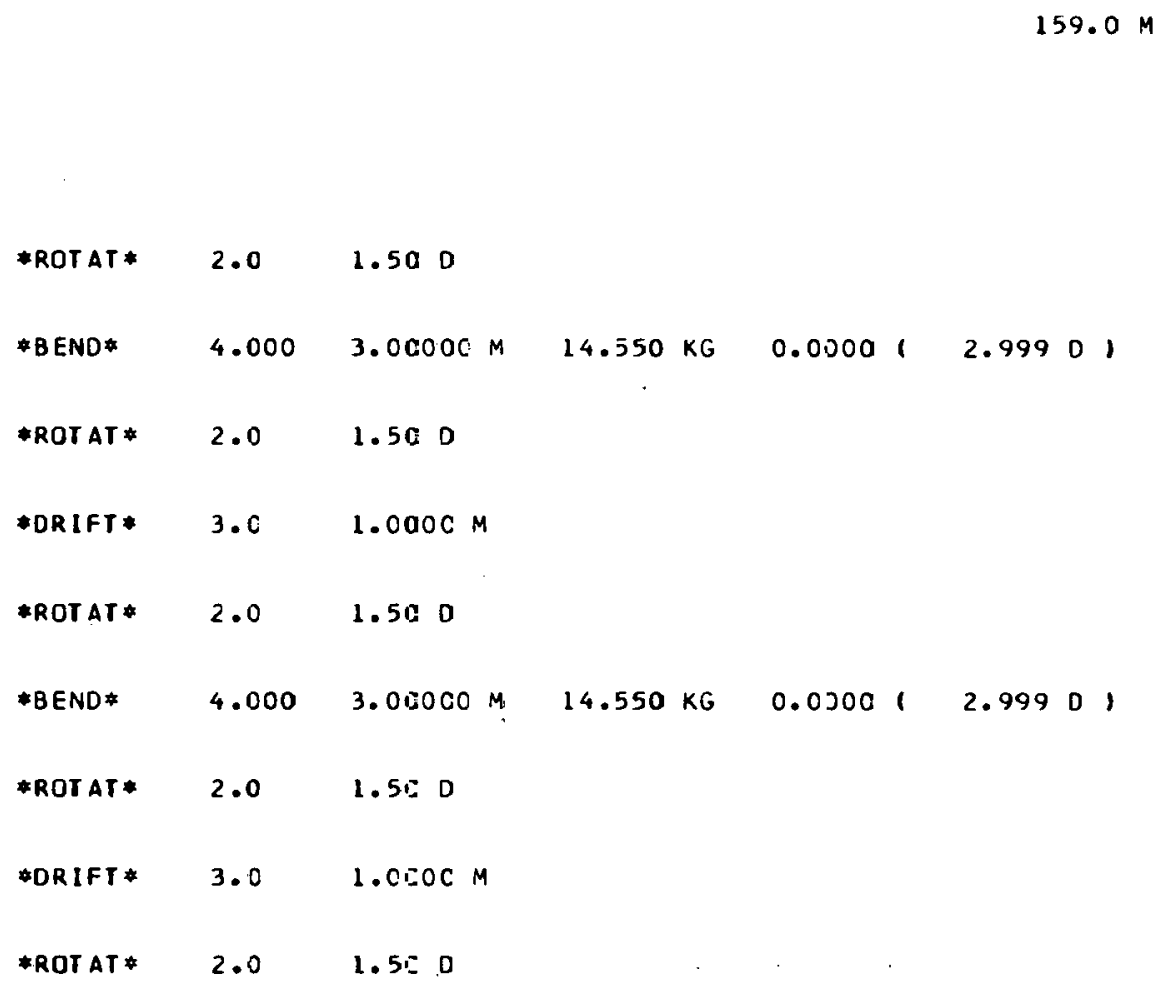

$159.0 M$

\begin{tabular}{|c|c|c|c|c|c|c|}
\hline *ROT AT * & 2.0 & 1.500 & & & & \\
\hline \#BEND* & 4.000 & $3.00000 \mathrm{M}$ & 14.550 & KG & 0.00001 & $2.999 \mathrm{D}$ \\
\hline *ROT AT * & 2.0 & 1.500 & & & & \\
\hline *DRIFT* & 3.0 & $1.000 \mathrm{CM}$ & & & & \\
\hline *ROTAT * & 2.0 & 1.500 & & & & \\
\hline *BEND* & 4.000 & $3.000 \mathrm{COM}$ & 14.550 & $K G$ & 0.03001 & $2.999 \mathrm{D})$ \\
\hline *ROTAT* & 2.0 & 1.500 & & & & \\
\hline *DRIFT* & 3.0 & $1.000 \mathrm{CM}$ & & & & \\
\hline *ROT AT* & 2.0 & 1.50 .0 & & & & \\
\hline
\end{tabular}

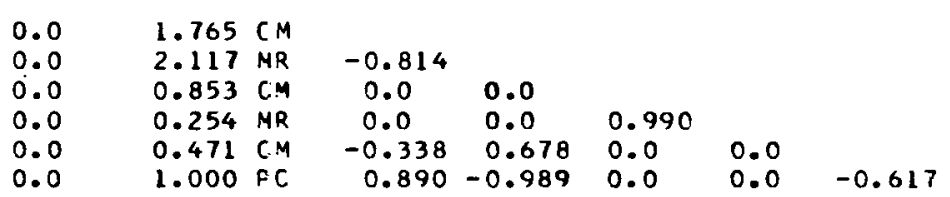




\begin{tabular}{|c|c|c|c|c|c|c|c|}
\hline *BEND* & 4.000 & $3.00000 \mathrm{M}$ & 14.550 & KG & 0.0000 & 1 & 2.9990 \\
\hline *FOT AT * & 2.0 & 1.500 & & & & & \\
\hline *ORIFT* & 3.0 & $1.0000 \mathrm{M}$ & & & & & \\
\hline *F.OT AT * & 2.0 & 1.500 & & & & & \\
\hline *EEND* & 4.000 & $3.00000 \mathrm{M}$ & 14.550 & KG & 0.0000 & 1 & 2.9990 \\
\hline *ROT AT* & $2 \cdot 0$ & 1.500 & & & & & \\
\hline *DRIFT* & 3.0 & $1.000 \mathrm{C} \mathrm{M}$ & & & & & \\
\hline
\end{tabular}

$\begin{array}{llllllll}175.0 M & 0.0 & 1.300 \mathrm{CM} & & & & & \\ & 0.0 & 0.310 \mathrm{MR} & 0.997 & & & \\ 0.0 & 1.227 \mathrm{CM} & 0.0 & 0.0 & & \\ 0.0 & 0.216 \mathrm{MR} & 0.0 & 0.0 & 0.994 & & \\ 0.0 & 0.504 \mathrm{CM} & -0.000 & 0.000 & 0.0 & 0.0 & \\ 0.0 & 0.00000 & 0.000 & 0.000 & 0.0 & 0.0 & -0.804\end{array}$

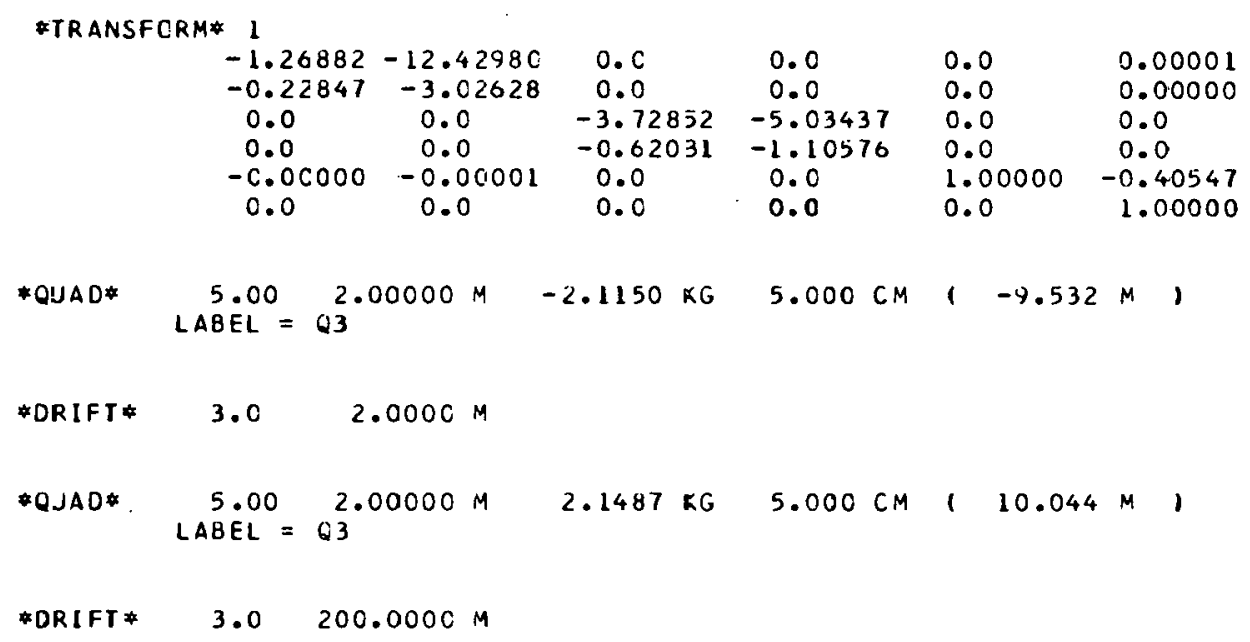

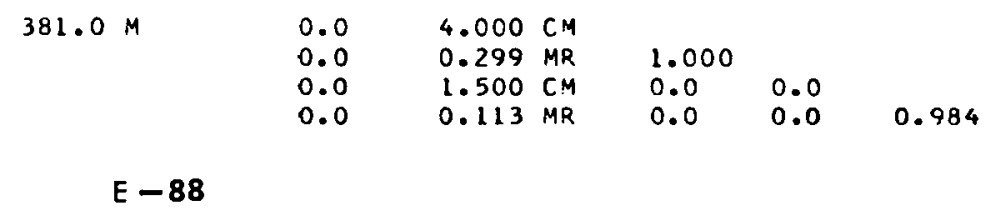


\#FIT $\quad 10.0 \quad$ 1. 1. $4.000 / 0.100$

VARY CODE $=200000 \quad 4.000$

$\mathrm{LABEL}=\mathrm{FIT} 4$

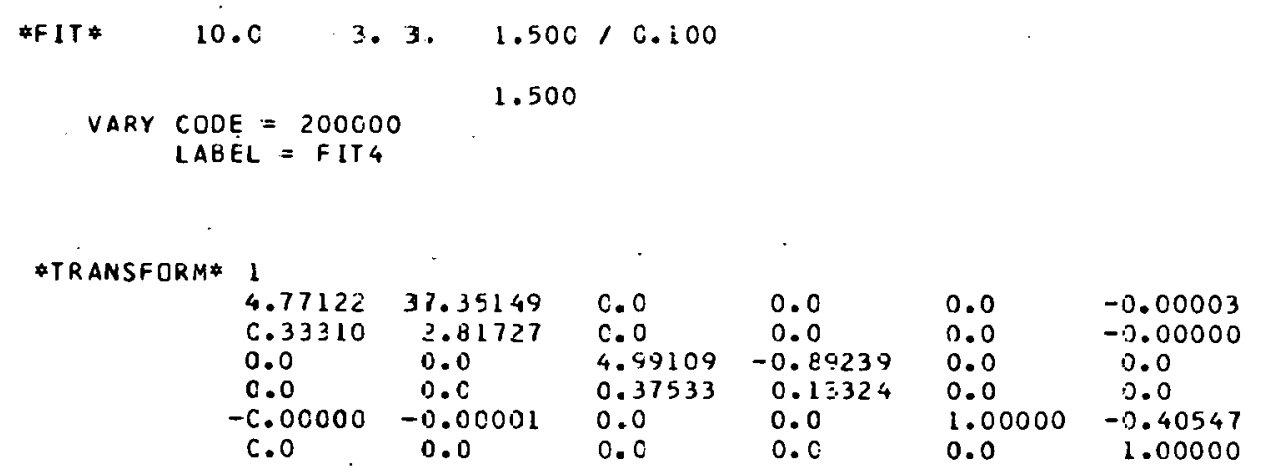

$381.0 \mathrm{M}$

$\begin{array}{ll}0.0 & 4.000 \mathrm{CM}\end{array}$

$0.0299 \mathrm{MR}$

$0.0 \quad 0.113 \mathrm{MR}$

$0.0 \quad 0.504 \mathrm{CM}$

$0.0 \quad 1.000$ PC
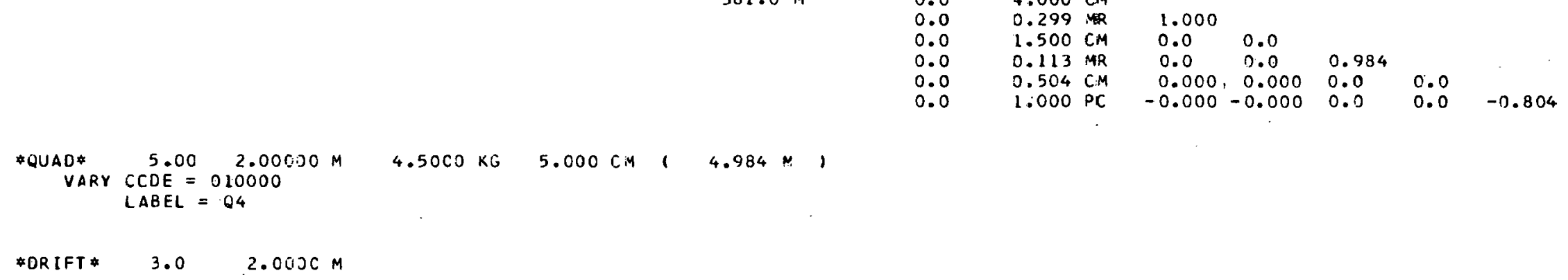

*QUAD* $5.00 \quad 2.00000 \mathrm{M}$

$-4.2000 K G$

$5.000 \mathrm{CM},-4.645 \mathrm{M}$,

$\begin{aligned} \text { V.ARY } & \text { CODE }=010000 \\ \text { LABEL } & =64\end{aligned}$ 
\#DRIFT $\quad 3.0 \quad 15.0100 \mathrm{CM}$

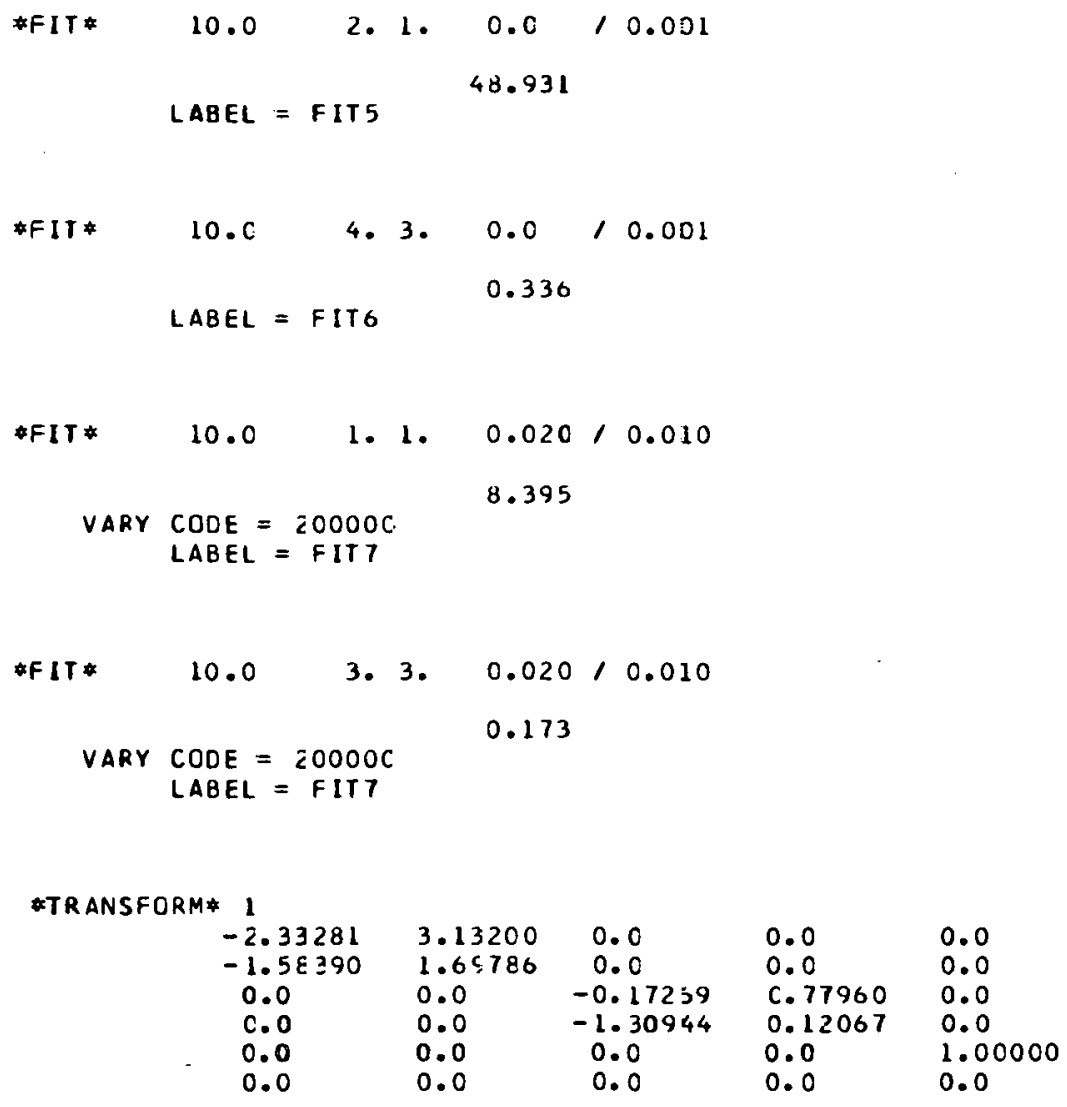

-2.33281
$-1.58 \geq 90$
0.0
0.0
0.0
0.0

$\begin{array}{ll}3.13200 & 0.0 \\ 1.65786 & 0.0 \\ 0.0 & -0.17259 \\ 0.0 & -1.30944 \\ 0.0 & 0.0\end{array}$

0.0

0.0

0.0
0.0
0.0

$0.12067 \quad 0.0$

$0.0 \quad 1.00000$

0.0

0.0

0.0

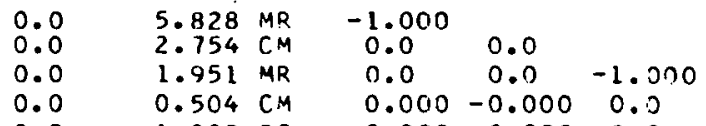

$\begin{array}{llll}0.504 \mathrm{CM} & 0.000-0.000 & 0.0\end{array}$

$\begin{array}{lllllllll}0.0 & 1.000 \text { PC } & -0.000 & 0.000 & 0.0 & 0.0\end{array}$
$402.0 M$

$\begin{array}{llllllll}0.0 & 8.395 & C M & & & & & \\ 0.0 & 5.828 & M R & 1.000 & & & & \\ 0.0 & 0.173 \text { CM } & 0.0 & 0.0 & & & \\ 0.0 & 1.951 & M R & 0.0 & 0.0 & 0.996 & & \\ 0.0 & 0.504 & C M & -0.000 & -0.000 & 0.0 & 0.0 & \\ 0.0 & 1.000 & P C & 0.000 & 0.000 & 0.0 & 0.0 & -0.804\end{array}$

*LENGTH $\quad 401.5993$

*CORRECTIONS* 


\begin{tabular}{|c|c|c|c|c|c|}
\hline & $7.4658 \mathrm{E}$ & $(4)$ & & -0.78 .0 & 0.213 \\
\hline & $1.9087 E$ & 041 & & -0.431 & 0.237 \\
\hline & $5.0201 E$ & (3) & & -0.240 & 0.163 \\
\hline & $1.3456 \mathrm{E}$ & (3) & & $-0.13 i$ & 0.095 \\
\hline & $3.7784 E$ & (2) & & -0.068 & 0.045 \\
\hline 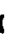 & $1.0482 \mathrm{E}$ & 021 & & $-c .032$ & 0.018 \\
\hline & $1.6754 E$ & 011 & & -0.0017 & 0.003 \\
\hline & $3.2649 E-$ & .011 & & $-c .000$ & 0.000 \\
\hline & $\begin{array}{l}\text { \#COVARIA } \\
0.000 \\
0.793^{\circ} \quad 0 .\end{array}$ & ANCE & 1 & FIT & 1 \\
\hline
\end{tabular}


BEAM IRANSPORT SYSTEM FOR SLAC BEAM SHITCHYARO ENO STATION A IIIB

* BeAm* 1.C00000 25.0C GEV

0.0

0.0

0.0

0.0

0.0

$0.300 \mathrm{CM}$

$0.100 \mathrm{MR}$

$0.300 \mathrm{CM}$

$0.100 \mathrm{MR}$

$0.300 \mathrm{CM}$

0.0

0.0
0.0
0.0

0.0

$1.000 \mathrm{PC}$

\section{*3END*}

$=$ ROT AT * $2.0 \quad 0.50 \mathrm{D}$

$5.0 M$

0.0
0.0
0.0

0.0

0.0

0.0

$0.305 \mathrm{CM}$

$0.133 \mathrm{MR}$

$0.304 \mathrm{CM}$
$0.100 \mathrm{MR}$

$0.300 \mathrm{CM}$

$1.000 \mathrm{PC}$

0.171

$\begin{array}{ll}0.0 & 0.0 \\ 0.0 & 0.0\end{array}$

$\begin{array}{rrrr}0.071 & 0.657 & 0.0 & 0.0\end{array}$

$-0.000$

=DRIFT $\quad 3.0 \quad 80.0000 \mathrm{M}$

$85.0 M$

$0.0 \quad 1.153 \mathrm{CM}$

$0.0 \quad 0.133 \mathrm{MR}$ $0.901 \mathrm{CM}$

$0.100 \mathrm{MR}$

$0.100 \mathrm{MR}$

$0.300 \mathrm{CM}$
$1.000 \mathrm{PC}$

0.965

$0.0 \quad 0.0$

$\begin{array}{ccc}0.0 & 0.0 & 0.943\end{array}$

$\begin{array}{cccc}-0.003 & -0.001 & 0.0 & 0.0\end{array}$

$\begin{array}{llll}0.624 & 0.657 & 0.0 & 0.0\end{array}$

$-0.000$

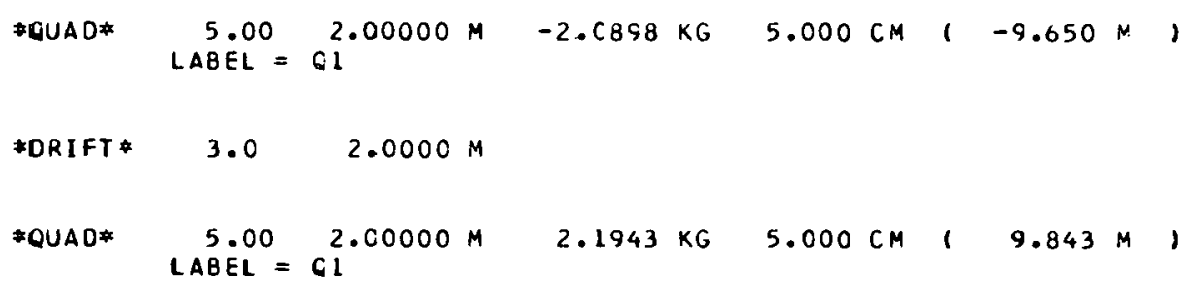

\section{$91.0 \mathrm{M}$}

. 


\begin{tabular}{|c|c|c|c|c|c|c|c|c|c|}
\hline * ROTAT* & 2.0 & 1.ES D & & & & & & & \\
\hline - BEND & 4.000 & $3 . c 5000$ & in & 14.550 & KG & 0.0000 & 1 & 2.999 .0 & D 1 \\
\hline *ROTAT * & $2 \cdot c$ & 1.500 & & & & & & & \\
\hline \#DRIFT* & 3.0 & $1.0000 \mathrm{M}$ & $M$ & & & & & & \\
\hline * ROT AT * & 2.0 & 1.500 & & & & & & & \\
\hline \# BEND * & 4.000 & 3.00000 & $M$ & 14.550 & $K G$ & 0.0000 & 1 & $2.999 \mathrm{D}$ & D 1 \\
\hline *ROTAT * & 2.0 & 1.50 & & & & & & & \\
\hline *DRIFT* & 3.0 & $1.0000 \mathrm{M}$ & $M$ & . & & . & & & . \\
\hline \&ROTAT & $2 \cdot C$ & $1.50 \mathrm{D}$ & & & & & & & \\
\hline \#BEND & 4.000 & 3.05000 & .4 & 14.550 & KG & 0.0000 & 1 & 2.9990 & 01 \\
\hline *ROTAT* & 2.0 & $1.50 \mathrm{D}$ & & & & & & & \\
\hline *ORIFT* & 3.0 & $1.0000 \mathrm{M}$ & $M$ & & & & & & \\
\hline *ROTAT* & 2.0 & 1.500 & & & & & & & \\
\hline \#BEND & 4.000 & $3.000 \mathrm{CC}$ & $M$ & 14.550 & KG & 0.0000 & 1 & $2.999 \mathrm{D}$ & D i \\
\hline \#ROTAT \# & 2.0 & 1.500 & & & & & & & \\
\hline
\end{tabular}

\begin{tabular}{|c|c|c|c|c|c|c|c|c|}
\hline $107.0 \mathrm{M}$ & $\begin{array}{l}0.0 \\
0.0 \\
0.0 \\
0.0 \\
0.0 \\
0.0\end{array}$ & $\begin{array}{l}2.366 \\
1.880 \\
0.357 \\
0.163 \\
0.471 \\
1.000\end{array}$ & $\begin{array}{l}C .4 \\
M R \\
C . M \\
M .2 \\
C .4 \\
P C\end{array}$ & $\begin{array}{l}0.873 \\
0.0 \\
0.0 \\
-0.737 \\
0.941\end{array}$ & $\begin{array}{c}0.0 \\
0.0 \\
-0.533 \\
0.986\end{array}$ & $\begin{array}{c}-0.856 \\
0.0 \\
0.0\end{array}$ & $\begin{array}{l}0.0 \\
0.0\end{array}$ & -0.617 \\
\hline
\end{tabular}

\#DRIFT $\begin{array}{rl}3.0 & 24.9203 \mathrm{M} \\ \text { LABEL } & =\text { ORI }\end{array}$ 
$133.9 M$

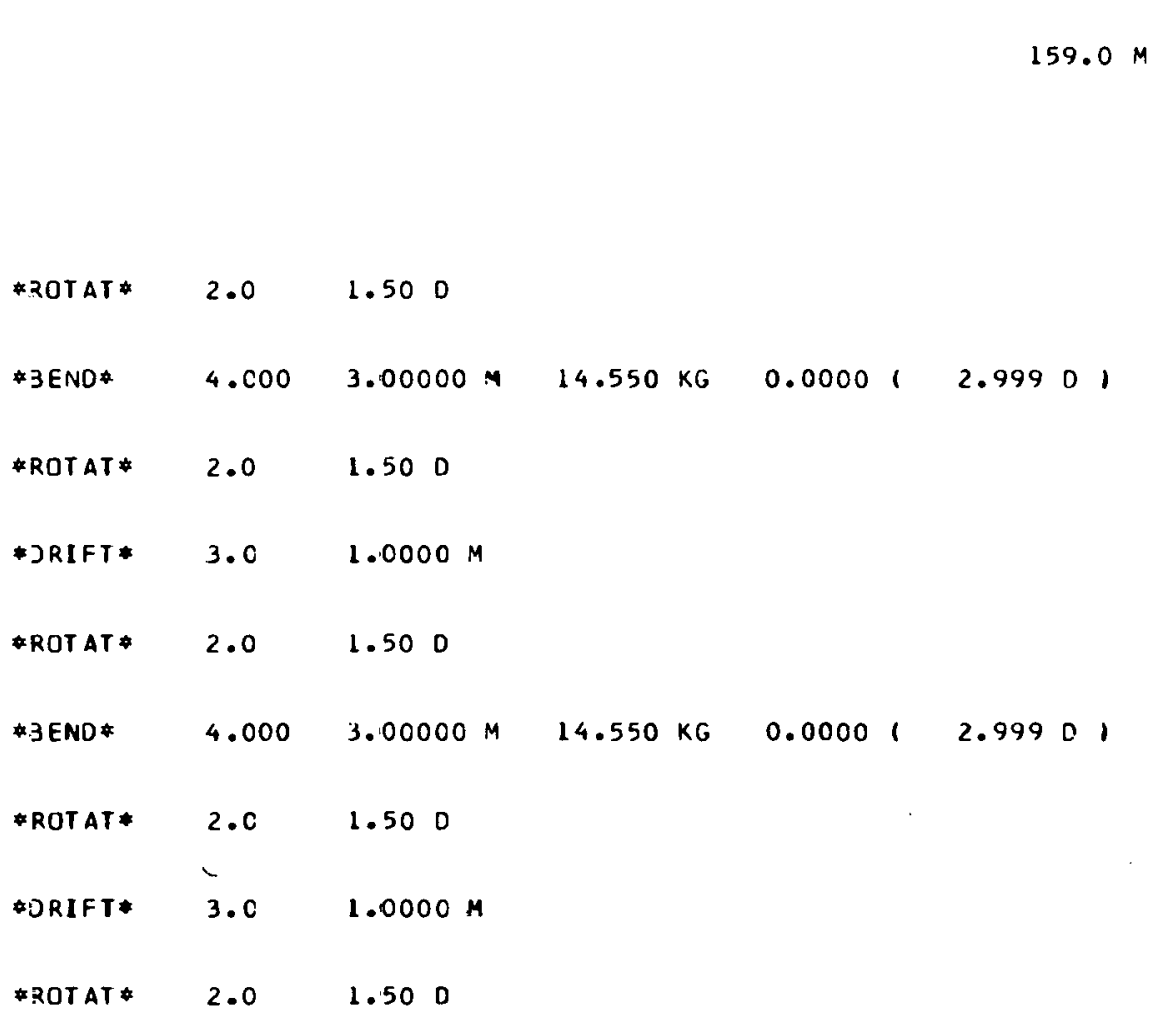

*DRIFT* $\begin{array}{rl}3.0 & 25.0795 \mathrm{M} \\ \text { LABEL } & =\text { ORI }\end{array}$

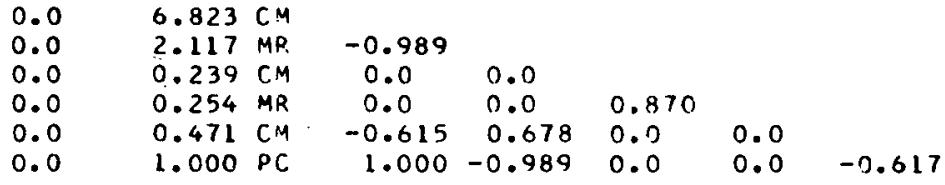




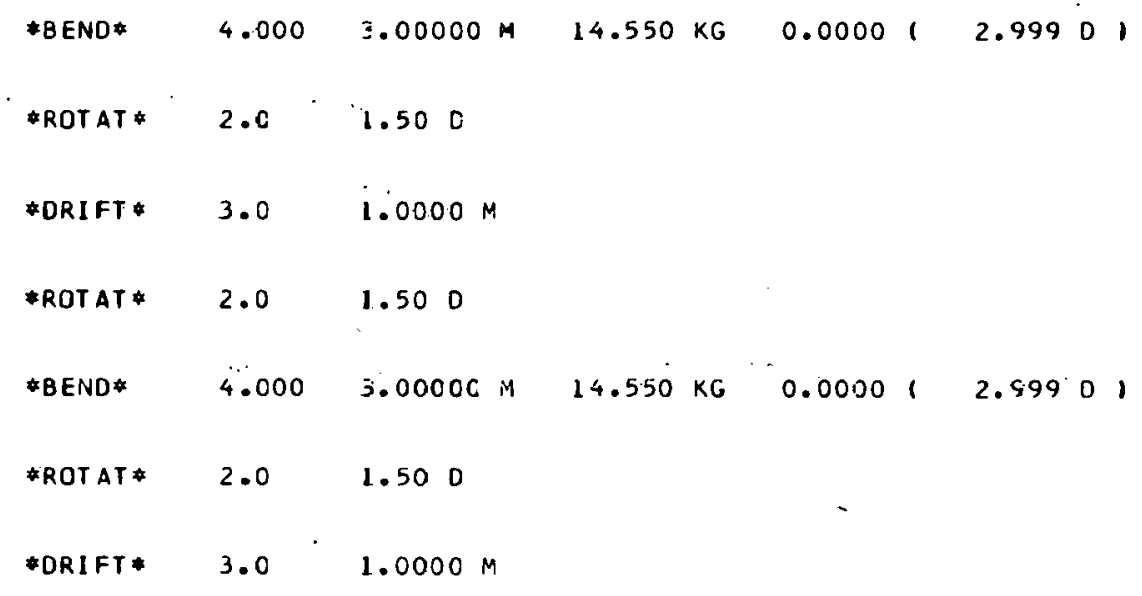

$\begin{array}{lllllll}175.0 \mathrm{M} & 0.0 & 1.300 \mathrm{CM} & & & & \\ & 0.0 & 0.310 \mathrm{MR} & 0.997 & & & \\ 0.0 & 1.227 \mathrm{CM} & 0.0 & 0.0 & & \\ 0.0 & 0.216 \mathrm{MR} & 0.0 & 0.0 & 0.994 & \\ & 0.0 & 0.504 \mathrm{CM} & -0.000 & 0.000 & 0.0 & 0.0 \\ & 0.0 & 1.000 \mathrm{PC} & 0.000 & 0.000 & 0.0 & 0.0\end{array}$

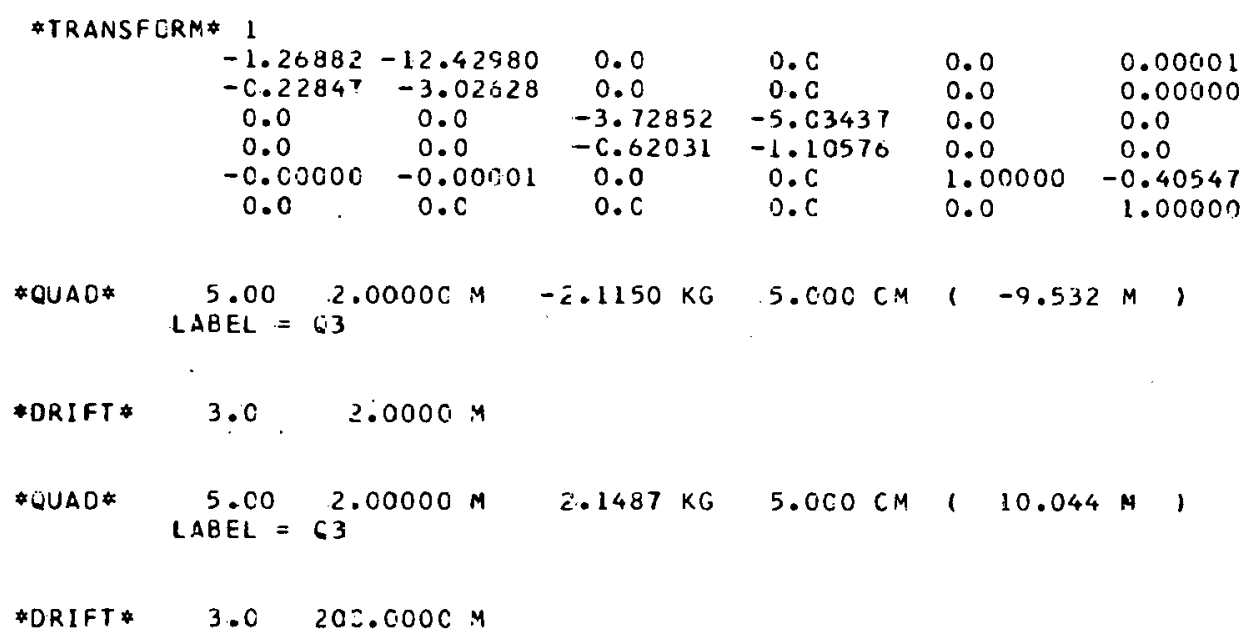

\begin{tabular}{|c|c|c|c|c|c|c|}
\hline $381.0 \mathrm{M}$ & $\begin{array}{l}0.0 \\
0.0 \\
0.0 \\
0.0\end{array}$ & $\begin{array}{l}4.000 \\
0.299 \\
1.500 \\
0.113\end{array}$ & $\begin{array}{l}C M \\
M R \\
C M \\
M R\end{array}$ & $\begin{array}{l}1.000 \\
0.0 \\
0.0\end{array}$ & $\begin{array}{l}0.0 \\
0.0\end{array}$ & 0.984 \\
\hline
\end{tabular}




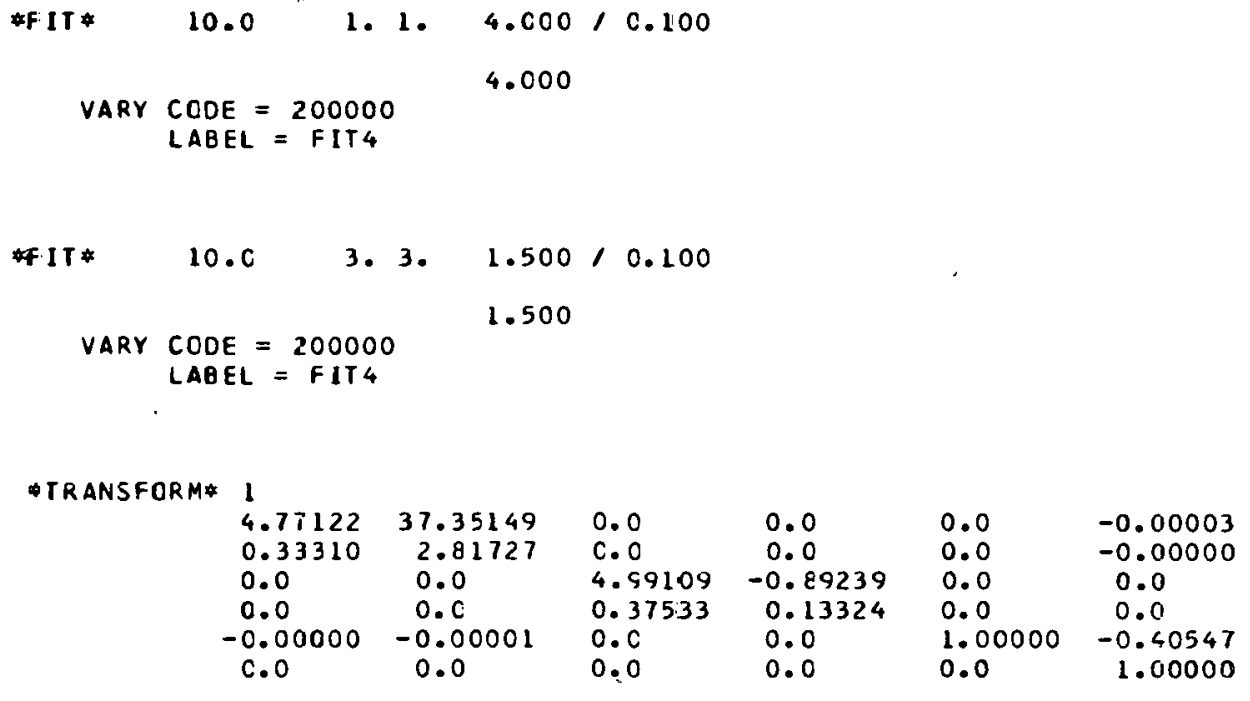

- TRANSFORM*

$\begin{array}{ccllll}4.77122 & 37.35149 & 0.0 & 0.0 & 0.0 & -0.00003 \\ 0.33310 & 2.81727 & 0.0 & 0.0 & 0.0 & -0.00000 \\ 0.0 & 0.0 & 4.59109 & -0.89239 & 0.0 & 0.0 \\ 0.0 & 0.0 & 0.37533 & 0.13324 & 0.0 & 0.0 \\ -0.00000 & -0.00001 & 0.0 & 0.0 & 1.00000 & -0.40547 \\ 0.0 & 0.0 & 0.0 & 0.0 & 0.0 & 1.00000\end{array}$

$381.0 \mathrm{M}$

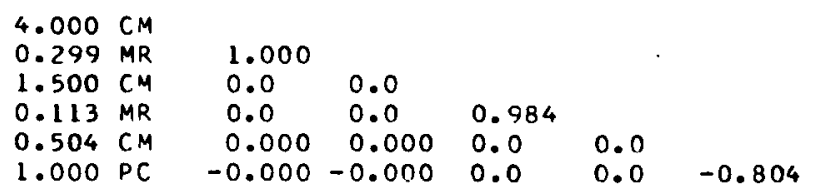

*QUAD* $5.00 \quad 2.00000 \mathrm{M} \quad 2.8115 \mathrm{KG} 5.000 \mathrm{CM} / 7.759 \mathrm{M}$, VARY COOE $=C 10000$ $\mathrm{LABEL}=04$

*ORIFT* 3.C $\quad 2.0000 M$

$385.0 M$

\begin{tabular}{|c|c|c|c|c|c|}
\hline $\begin{array}{l}0.0 \\
0.0 \\
0.0 \\
0.0 \\
0.0\end{array}$ & $\begin{array}{l}2.551 \mathrm{C} \\
4.896 \mathrm{MP} \\
2.179 \mathrm{CH} \\
2.242 \mathrm{MF} \\
0.504 \mathrm{C} \\
1.000 \mathrm{P}\end{array}$ & $\begin{array}{l}-1.000 \\
0.0 \\
0.0 \\
0.000 \\
-0.000\end{array}$ & $\begin{array}{l}0.0 \\
0.0 \\
-0.000 \\
0.000\end{array}$ & $\begin{array}{l}1.000 \\
0.0 \\
0.0\end{array}$ & $\begin{array}{l}0.0 \\
0.0\end{array}$ \\
\hline
\end{tabular}

"QUAD* $5.00 \quad 2.00000 \mathrm{M}-3.4253 \mathrm{Kg} 5.000 \mathrm{CM} /-5.765 \mathrm{M})$

VARY CCDE $=010000$ $\angle A B E L=6.4$ 
\#ORIFT * $\quad 3 . \mathrm{C} \quad \because 15.000 \mathrm{C}$ i1

$\begin{array}{lrcccccc}0.0 & -1.293 & M R & -1.000 & & & & \\ 0.0 & 2.255 & C .4 & 0.0 & 0.0 & & & \\ 0.0 & 1.503 & M R & 0.0 & 0.0 & -1.000 & & \\ 0.0 & 0.504 & C M & 0.000 & -0.000 & 0.0 & 0.0 & \\ 0.0 & 1.000 & \text { PC } & -0.000 & 0.000 & 0.0 & 0.0 & -0.804\end{array}$

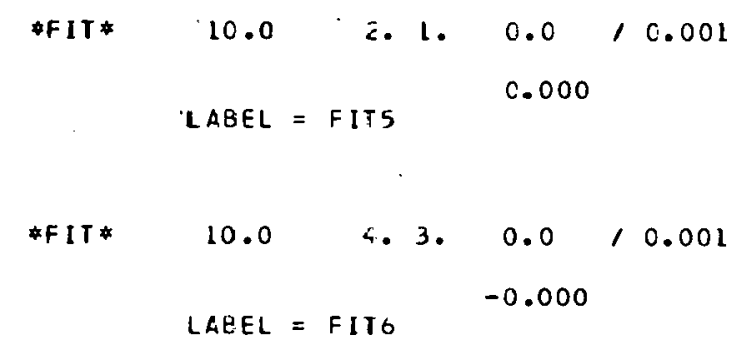

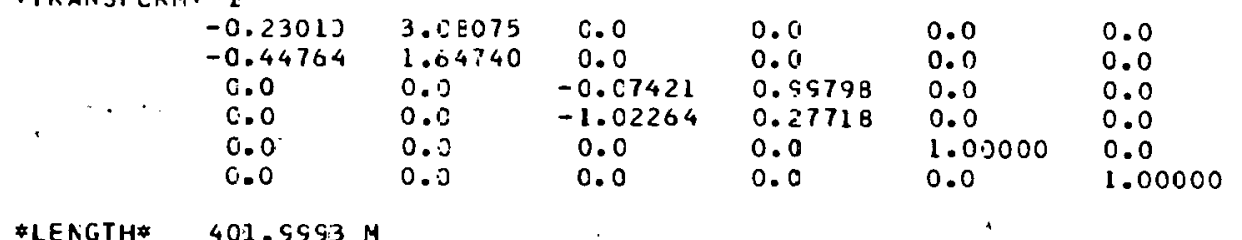

\#LENGTH* 401. S993 M

$\begin{array}{lllllll}402.0 \mathrm{M} & 0.0 & 0.023 \mathrm{CM} & & & \\ & 0.0 & 1.298 \mathrm{MR} & 0.000 & & \\ 0.0 & 0.020 \mathrm{CM} & 0.0 & 0.0 & \\ 0.0 & 1.503 \mathrm{MR} & 0.0 & 0.0 & -0.000 \\ & 0.0 & 0.504 \mathrm{CM} & -0.000 & -0.000 & 0.0 \\ & 0.0 & 1.000 \mathrm{PC} & 0.000 & 0.000 & 0.0\end{array}$

\section{0}

$-0.834$ 
'BFAM TRANSPORT SYSTEM FOR SLAC BEAM SWITCHYARD END STATION A IIIC'

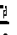

IN TIE FOURTH STEP THE LAST TWO RUAD DOUBLETS ARE BOTH ALLOWED TO VARY)

FOR A FINAL ADJUSTMENT TO GET THE WAIST AND THE SPOT SIZE AT THE TARIET.)

SENTINEL 
BEAM TRANSPORT SYSTEM FOR SLAC GEAM SWITCHYARD END STATICN A IIIC

IN THE FOURTH STEP THE LAST TWO QUAO DOUBLETS aRe BOTH allCWEO TO VARY

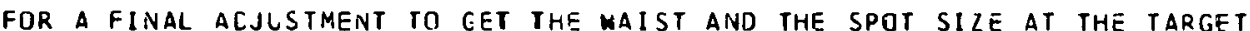

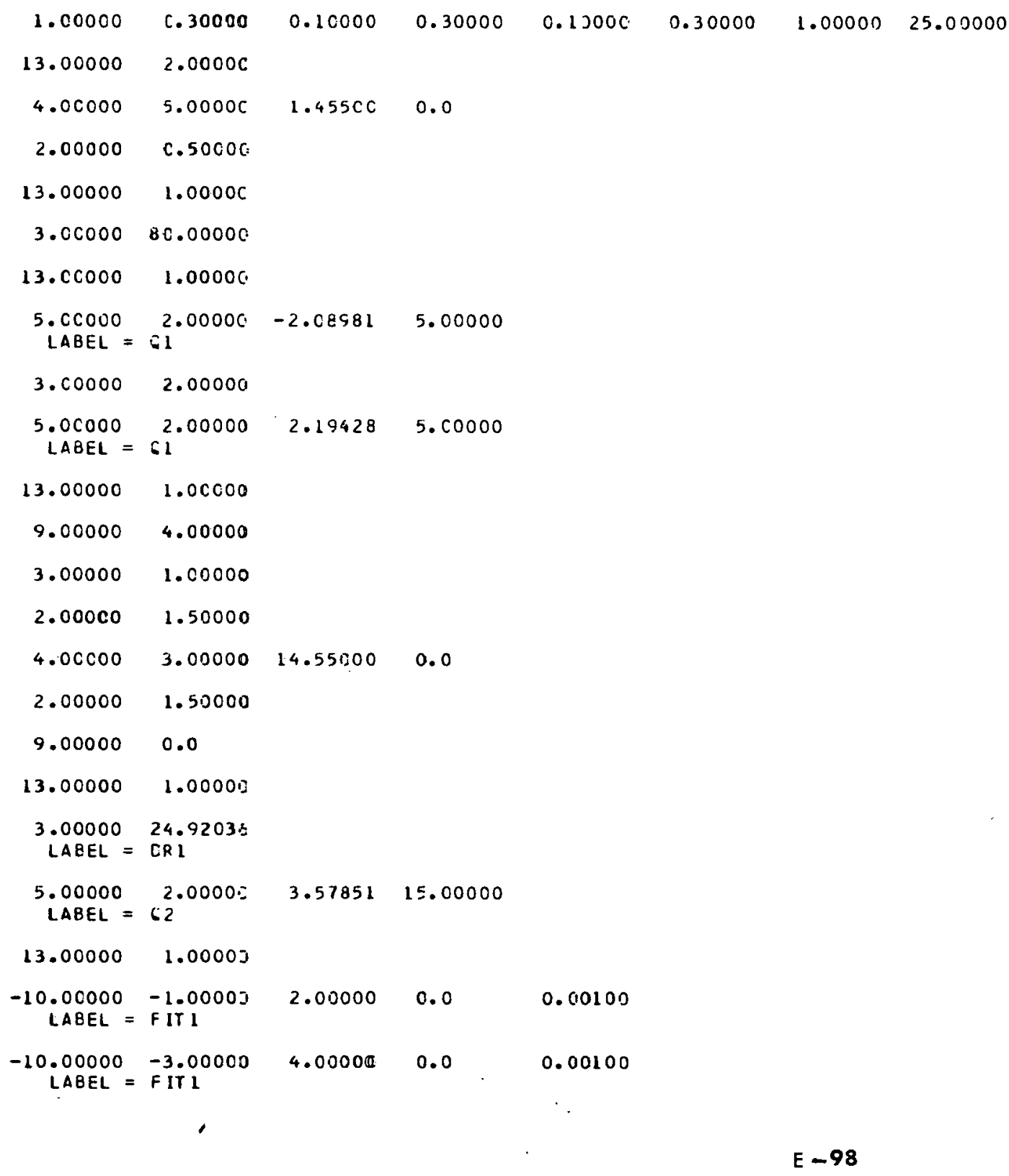




\begin{tabular}{|c|c|c|c|c|}
\hline 13.00000 & 4.00000 & & & - \\
\hline $\begin{array}{l}3.00000 \\
\text { LABEL }=\end{array}$ & $\begin{array}{l}25.07953 \\
\text { ORI }\end{array}$ & & & \\
\hline 23.00000 & 1.00000 & & & \\
\hline 9.00000 & 4.00000 & & & \\
\hline-2.00000 & 1.50000 & & & \\
\hline 4.00000 & 3.00000 & $14.5500 \mathrm{C}$ & 0.0 & \\
\hline 2.00000 & 1.50000 & & & \\
\hline 3.00000 & 1.00000 & & & \\
\hline 9.00000 & 0.0 & & & \\
\hline 13.00000 & 1.00000 & & & \\
\hline $\begin{array}{c}-10.00000 \\
\text { LABEL }=\end{array}$ & $\begin{array}{l}-1.00000 \\
\text { FIT2 }\end{array}$ & $6 . c c 000$ & 0.0 & 0.00100 \\
\hline $\begin{array}{c}-10.00000 \\
\angle A B E L=\end{array}$ & $\begin{array}{l}-2.00000 \\
\text { FIT } 3\end{array}$ & 6.00000 & 0.0 & 0.00100 \\
\hline 13.00000 & 4.00000 & & & \\
\hline $\begin{array}{l}5.00000 \\
\text { VARY CDOE } \\
\text { LAEEL }=\end{array}$ & $=\begin{array}{l}2.00000 \\
610000\end{array}$ & -2.11497 & $5 . \cos 00$ & \\
\hline 3.00000 & 2.00000 & & & \\
\hline $\begin{array}{l}5.00000 \\
\text { VARY CODE } \\
\text { LAEEL }=\end{array}$ & $\underbrace{2.00000}_{63} 010000$ & $2.14 \varepsilon 73$ & 5.00000 & \\
\hline 3.000002 & .00 .00000 & & & \\
\hline 13.00000 & 1.00000 & & & \\
\hline $\begin{array}{l}10.00000 \\
\text { VARY COOE } \\
\text { LABEL }=\end{array}$ & $\begin{array}{l}1.00000 \\
200000 \\
\text { FII } 4\end{array}$ & 1.00000 & 4.00000 & 0.10000 \\
\hline $\begin{array}{l}10.00000 \\
V A R Y C O D E \\
\text { LABEL = }\end{array}$ & $\begin{array}{l}3.00000 \\
200000 \\
\text { FIT }\end{array}$ & 3.00000 & 1.50000 & 0.10000 \\
\hline 13.00000 & $4.0 \mathrm{COCO}$ & & & \\
\hline 13.00000 & 1.00000 & & & \\
\hline $\begin{array}{l}5.0 C C O O \\
\text { VARY CODE } \\
\text { LABEL = }\end{array}$ & $\begin{array}{r}2.00000 \\
010000 \\
64\end{array}$ & 2.81145 & 5.00000 & \\
\hline $3.0 c 000$ & 2.00000 & & & \\
\hline
\end{tabular}




\begin{tabular}{|c|c|c|c|c|}
\hline 13.00000 & 1.00000 & & & \\
\hline $\begin{array}{l}5.00 C O 0 \\
\text { VARY CODE } \\
\text { LABEL = }\end{array}$ & $\begin{array}{r}2.00000 \\
010000 \\
64\end{array}$ & -3.42531 & 5.00000 & \\
\hline 13.00000 & 1.00000 & & & \\
\hline 3.00000 & 15.00000 & & & \\
\hline 13.00000 & 1.00000 & & & \\
\hline $\begin{array}{c}10.00000 \\
\text { LABEL }=\end{array}$ & $\begin{array}{l}2.00000 \\
\text { FIT5 }\end{array}$ & 1.00000 & 0.0 & 0.00100 \\
\hline $\begin{array}{c}10.00000 \\
\text { LABEL }=\end{array}$ & $\begin{array}{l}4.00000 \\
\text { Fito }\end{array}$ & 3.00300 & 0.0 & 0.00100 \\
\hline $\begin{array}{l}10.00000 \\
\text { VARY CODE } \\
\text { LABEL = }\end{array}$ & $\begin{array}{rl} & 1.00000 \\
2 & 200000 \\
\text { FII } 7\end{array}$ & 1.00000 & 0.02000 & 0.01000 \\
\hline $\begin{array}{l}10.00000 \\
\text { VARY CODE } \\
\text { LABEL }=\end{array}$ & $\begin{array}{l}3.00000 \\
200000 \\
\text { FIT7 }\end{array}$ & 3.00000 & 0.02000 & 0.01000 \\
\hline
\end{tabular}

$13.00000 \quad 4.00000$

SENTINEL 
* BEAM* $\quad 1.000000 \quad 25.00$ GEV

*BENO* 4.000

$5.0000 \mathrm{CH}$

\#ROTAT* 0.500

\section{\#DRIFT* $\quad 3.0 \quad 80.0000 \mathrm{M}$}

0.0
0.0
0.0
0.0
0.0
0.0

$0.300 \mathrm{CM}$ $0.100 \mathrm{MR}$ $0.300 \mathrm{CM}$ $0.100 \mathrm{MR}$ $0.300 \mathrm{CM}$ $1.000 \mathrm{PC}$ 0.0 $0.0 \quad 0.0$ $0.0 \quad 0.0$ 0.0
0.0 $\begin{array}{llll}0.0 & 0.0 & 0.0 & 0.0\end{array}$ 0.0
$5.0 \mathrm{M}$

$$
\begin{aligned}
& 0.0 \\
& 0.0 \\
& 0.0 \\
& 0.0 \\
& 0.0 \\
& 0.0
\end{aligned}
$$

$0.305 \mathrm{CM}$ $0.133 \mathrm{MR}$ $0.304 \mathrm{CM}$ $0.100 \mathrm{MR}$ $0.300 \mathrm{CM}$ $1.000 \mathrm{PC}$

\subsection{1} $\begin{array}{ll}0.0 & 0.0\end{array}$ $\begin{array}{lll}0.0 & 0.0 & 0.164\end{array}$ $\begin{array}{cccc}-0.009 & -0.001 & 0.0 & 0.0\end{array}$ $-0.000$
$85.0 \mathrm{M}$

$\begin{array}{ll}0.0 & 1.153 \mathrm{CM} \\ 0.0 & 0.133 \mathrm{MR} \\ 0.0 & 0.901 \mathrm{CM} \\ 0.0 & 0.100 \mathrm{MR} \\ 0.0 & 0.300 \mathrm{CM} \\ 0.0 & 1.000 \mathrm{PC}\end{array}$

*QUA D* $5.00 \quad 2.00000 \mathrm{M}-2 . \mathrm{CB98} \mathrm{KJ} \quad 5.000 \mathrm{CM} \quad(-9.650 \mathrm{M})$ $\angle A B E L=61$

\#DRIFT* $\quad 3.0 \quad 2.000 \mathrm{CM}$

*QUAC* $\stackrel{5.00}{L A B E L}=61.00000 M$ $2.1943 \mathrm{Kij}$ $5.000 \mathrm{CM}$ 9.843 M ।

$91.0 \mathrm{M}$

\#DRIFT* $\quad 3.0 \quad 1.0000 \mathrm{M}$

$$
91.0 M
$$

0.0
0.0
0.0
0.0
0.0
0.0

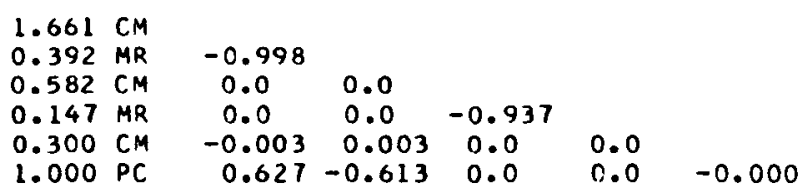




\begin{tabular}{|c|c|c|c|c|c|c|c|c|}
\hline *ROTAT * & 2.0 & 1.500 & & & & & & \\
\hline *BEND* & 4.000 & $3.00000 \mathrm{M}$ & 14.550 & KG & 0.0030 & 1 & 2.999 & 01 \\
\hline \#ROTAT* & 2.0 & 1.500 & & & & & & \\
\hline *DRIFI* & 3.0 & 1.0000 .4 & & & & & & \\
\hline *ROT AT * & 2.0 & 1.500 & & & & & & \\
\hline *BEND* & 4.000 & $3.00000 \mathrm{in}$ & 14.550 & KG & $0.000 J$ & 1 & 2.949 & 01 \\
\hline \#RUTAT* & 2.0 & 1.500 & & & & & & \\
\hline \#DRIFT * & 3.0 & $1.0000 \mathrm{M}$ & & & & & & \\
\hline *ROT AT * & $2 \cdot c$ & 1.500 & & & & & & \\
\hline *BENO* & 4.000 & $3.00000 \mathrm{M}$ & 14.550 & KG & 0.6900 & 1 & 2.999 & 01 \\
\hline *ROT AT * & 2.0 & 1.500 & & & & & & \\
\hline *DRIFT* & $3 \cdot c$ & $1.0000 \mathrm{M}$ & & & & & & \\
\hline \#ROT AT \# & $2 \cdot c$ & $1.5 \mathrm{C} \mathrm{D}$ & & & & & & \\
\hline \#BEND* & 4.000 & $3.00000 \mathrm{in}$ & 14.550 & KG & 0.0000 & 1 & 2.999 & 01 \\
\hline * ROT AT* & 2.0 & 1.500 & & & & & & \\
\hline
\end{tabular}

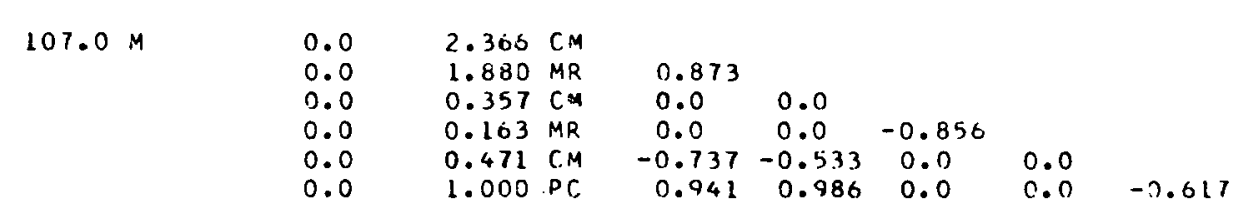

\#DRIFT* $\begin{array}{rl}3 . C & 24.9203 .4 \\ \angle A B E L & =2 R 1 .\end{array}$ 
*EUAO* $\quad$ LABEL $=02.00000 \mathrm{M} \quad 3.57 \varepsilon 5 \mathrm{KG} 15.000 \mathrm{CM} / 17.815 \mathrm{M}$,

$133.9 \mathrm{M}$

ATRANSFORM

$\begin{array}{cccclc}-C .33045 & -0.00002 & 0.0 & 0.0 & 0.0 & 6.82236 \\ -0.22846 & -3.02617 & 0.0 & 0.0 & 0.0 & -2.09424 \\ 0.0 & 0.0 & -c .79757 & -0.00002 & 0.0 & 0.0 \\ 0.0 & 0.0 & -0.73609 & -1.25380 & 0.0 & 0.0 \\ -0.22507 & -2.06457 & c .0 & 0.0 & 1.00000 & -0.29037 \\ c .0 & 0.0 & 0.0 & 0.0 & 0.0 & 1.00000\end{array}$

*LRIFT* $\begin{array}{rl}3.0 & 25.0795 M \\ \text { LABEL } & =\text { DRI }\end{array}$

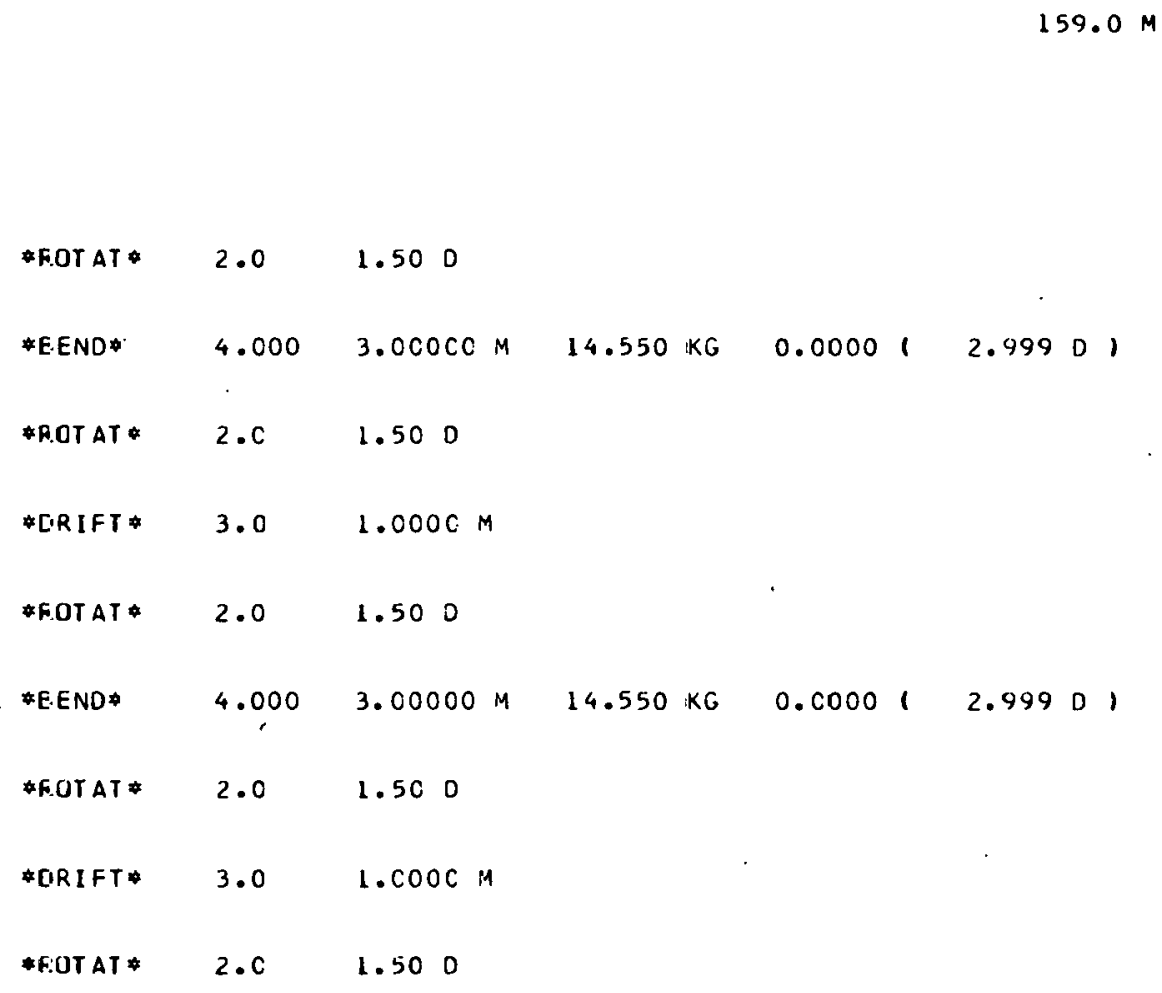

\begin{tabular}{|c|c|c|c|c|c|c|}
\hline * F.OT AT * & 2.0 & 1.500 & & & & \\
\hline *EEND & 4.000 & $3.0 \mathrm{COCOM}$ & $14.550 \mathrm{KG}$ & 0.0000 & 1 & $2.999 \mathrm{D}$ \\
\hline & . & & & & & \\
\hline *R.OT AT * & $2 \cdot c$ & 1.500 & & & & \\
\hline$\#[R I F T *$ & 3.0 & $1.000 \mathrm{CM}$ & & & & \\
\hline \#FOTAT * & 2.0 & 1.500 & & & & \\
\hline *E.END * & 4.000 & $3.00000 \mathrm{M}$ & $14.550 \mathrm{KG}$ & 0.0000 & 1 & $2.999 \mathrm{D}$ \\
\hline \#F.OTAT \# & 2.0 & 1.500 & & & & \\
\hline *QRIFT & 3.0 & $1 . \operatorname{coOCM} \mathrm{M}$ & & & & \\
\hline *EOUT AT* & $2 \cdot C$ & 1.500 & & & & \\
\hline
\end{tabular}

*EOTAT* $2 . \mathrm{C} \quad 1.500$

\begin{tabular}{|c|c|c|c|c|c|c|}
\hline $\begin{array}{l}0.0 \\
0.0 \\
0.0 \\
0.0 \\
0.0 \\
0.0\end{array}$ & $\begin{array}{l}6.823 \\
2.117 \\
0.239 \\
0.254 \\
0.471 \\
1.000\end{array}$ & $\begin{array}{l}C M \\
M R \\
C M \\
M R \\
C M \\
P C\end{array}$ & $\begin{array}{l}-0.989 \\
0.0 \\
0.0 \\
-0.615 \\
1.000\end{array}$ & $\begin{array}{c}0.0 \\
0.0 \\
0.678 \\
-0.989\end{array}$ & $\begin{array}{l}0.870 \\
0.0 \\
0.0\end{array}$ & $\begin{array}{l}0.0 \\
0.0\end{array}$ \\
\hline
\end{tabular}

$-0.617$ 


\begin{tabular}{|c|c|c|c|c|c|c|}
\hline *BEND* & 4.000 & $3.00000 \mathrm{M}$ & 14.550 & KG & 0.00001 & 2.9990 \\
\hline * ROTAT * & 2.0 & 1.500 & & & & \\
\hline \#DRIFT* & 3.0 & $1.000 C M$ & & & & \\
\hline \#ROT.AT * & 2.0 & 1.500 & & & & \\
\hline \#BEND* & 4.000 & 3. $\operatorname{coc} 00 \mathrm{H}$ & 14.550 & $K G$ & 0.00011 & 2.9 .9901 \\
\hline ROTAT * & $2 . c$ & 1.500 & & & & \\
\hline FDRIFT & $3 \cdot C$ & $1.0000 \mathrm{M}$ & & & & \\
\hline
\end{tabular}

$\begin{array}{llllllll}175.0 \mathrm{M} & 0.0 & 1.300 \mathrm{CM} & & & & & \\ 0.0 & 0.310 & \mathrm{MF} & 0.997 & & & & \\ 0.0 & 1.227 & \mathrm{CM} & 0.0 & 0.0 & & & \\ 0.0 & 0.216 & \mathrm{MF} & 0.0 & 0.0 & 0.994 & & \\ 0.0 & 0.504 & \mathrm{CM} & -0.000 & 0.000 & 0.0 & 0.0 & \\ 0.0 & 0.00000 & 0.000 & 0.0 & 0.0 & -0.84\end{array}$

\begin{tabular}{|c|c|c|c|c|c|c|}
\hline \#TRANSFORM & $\begin{array}{l}1 \\
-1.26882 \\
-0.22847 \\
\text { c. } 0 \\
\text { C. } 0 \\
- \text { C. } 0 \\
\text { a. } 0\end{array}$ & $\begin{array}{l}-12.42780 \\
-3.02628 \\
0.0 \\
0.0 \\
-0.00001 \\
0.0\end{array}$ & $\begin{array}{l}0.0 \\
0.0 \\
-3.72852 \\
-0.62031 \\
0.0 \\
0.0\end{array}$ & $\begin{array}{l}0 . C \\
0.0 \\
-5.03437 \\
-1.10576 \\
0.0 \\
0.0\end{array}$ & $\begin{array}{l}0.0 \\
0.0 \\
0.0 \\
0.0 \\
1.00000 \\
0.0\end{array}$ & $\begin{array}{l}0.00001 \\
0.00000 \\
0.0 \\
0.0 \\
-0.40547 \\
1.00000\end{array}$ \\
\hline
\end{tabular}

FOUAO* $5.002 .0 \mathrm{COOOJM}-2.1150 \mathrm{KG} 5.000 \mathrm{CH},-9.532 \mathrm{M}$, $\begin{aligned} \text { VARY CCLE } & =010006 \\ \text { LABEL } & =6.3\end{aligned}$

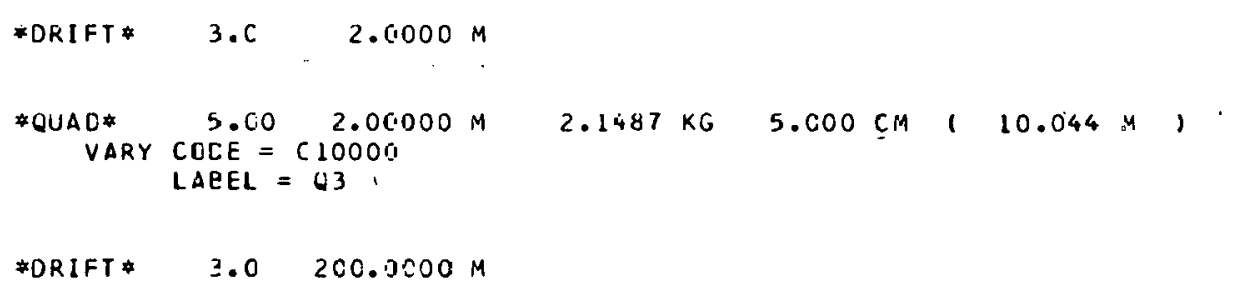

\#DRIFT \# $\quad 3.0 \quad 200.0000 M$

$\begin{array}{llll}381.0 M & 0.0 & 4.000 \mathrm{CA} & \\ & 0.0 & 0.299 \mathrm{MR} & 1.000 \\ & & & \end{array}$


*FIT* 10.0 1. 1. 4.000/0.100

VARY CODE $=200600$

$\angle A B E L=F I T 4$

*FIT * 10.C 3. 3. 1.500/C. 100

VARY CODE $=2000.00$

$\angle A B E L=$

*TRANSFORM * 1

$\begin{array}{ccllll}1.77122 & 37.35149 & 0.0 & 0.0 & 0.0 & -0.00003 \\ 0.3331 \mathrm{C} & 2.81727 & 0.0 & 0.0 & 0.0 & -0.00000 、 \\ 0.0 & 0.0 & 4.97109 & -0.89239 & 0.0 & 0.0 \\ 0.0 & 0.0 & 0.37533 & 0.13324 & 0.0 & 0.0 \\ -0.0000 \mathrm{C} & -0.00001 & 0.0 & 0.0 & 1.00000 & -0.40547 \\ 0.0 & 0.0 & 0.0 & 0.0 & 0.0 & 1.00000\end{array}$

$381.0 \mathrm{M}$

$0.0 \quad 4.000 \mathrm{CM}$

$0.0 \quad 0.299 M R$

$0.0 \quad 1.500 \mathrm{CM}$

$0.0 \quad 0.113 \mathrm{MR}$

$0.0 \quad 0.504 \mathrm{CM}$

1.000 PC

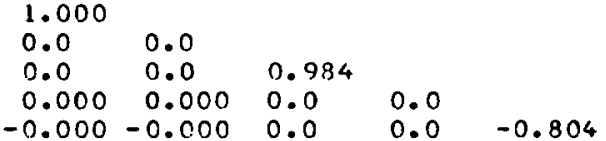

*QUAD*

$$
\begin{aligned}
& \text { VARY CCDE }=010000 \\
& \text { LABEL }=04
\end{aligned}
$$

\#DRIFT* $\quad 3.0 \quad 2.000 \mathrm{C} \mathrm{M}$
$385.0 \mathrm{M}$

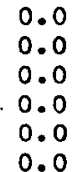

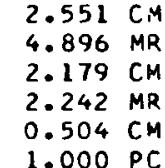

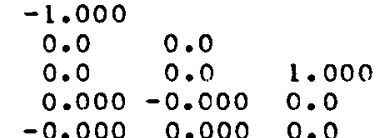

0.0

QQUAD* $\quad 5.00 \quad 2.0000 \mathrm{C}$ M $-3.4253 \mathrm{KG} 5.000 \mathrm{CM} /-5.765 \mathrm{M}$,

VARY COOE $=010000$

$\angle A B E L=04$ 


$\begin{array}{lllllll}0.0 & 1.948 C M_{1} & & & & & \\ 0.0 & 1.298 \mathrm{MR} & -1.000 & & & & \\ 0.0 & 2.255 \mathrm{CM} & 0.0 & 0.0 & & & \\ 0.0 & 1.503 \mathrm{MR} & 0.0 & 0.0 & -1.000 & & \\ 0.0 & 0.504 \mathrm{CM} & 0.000 & -0.000 & 0.0 & 0.0 & \\ 0.0 & 1.000 \mathrm{PC} & -0.000 & 0.000 & 0.0 & 0.0 & -0.804\end{array}$

\#DRIFT $\quad 3.0 \quad 15.0000 \mathrm{M}$

$402.0 \mathrm{M}$

$\begin{array}{lllll}0.0 & 0.023 \mathrm{CM} & & & \\ 0.0 & 1.298 \mathrm{MR} & 0.000 & & \\ 0.0 & 0.020 \mathrm{CM} & 0.0 & 0.0 & \\ 0.0 & 1.503 \mathrm{MF} & 0.0 & 0.0 & -0.000 \\ 0.0 & 0.504 \mathrm{CH} & -0.000 & -0.000 & 0.0 \\ 0.0 & 1.000 \mathrm{PC} & 0.000 & 0.000 & 0.0\end{array}$

0.0

$0.0-0.804$

\begin{abstract}
\#FIT $\quad 10 . \mathrm{C} \quad 2.1 .0 . \mathrm{C} / \mathrm{C.001}$
\end{abstract} $L A B E L=F I T S$
\#FIT $\quad 10.0 \quad 4.3 .0 .0,0.001$ LAEEL $=F 1 T 6$

\#F IT * IC. 0 1. 1

VARY CODE $=200000$ $\angle A G E L=F I T T$
*FIT*
10.0
3. 3.
$0.020 / c .010$
VARY COOE $=200000$
0.020 LABEL $=$ FITT

* TR ANSFORM . I

$\begin{array}{clllll}-0.23010 & 3.0507 b & 0.0 & 0.0 & 0.0 & 0.0 \\ -0.44764 & 1.04740 & 0 . C & 0.0 & 0.0 & 0.0 \\ 0.0 & 0.0 & -0.07421 & 0.59798 & 0.0 & 0.0 \\ 0.0 & C .0 & -1.02264 & 0.27718 & 0.0 & 0.0 \\ 0.0 & 0.0 & 0.0 & 0.0 & 1.00000 & 0.0 \\ 0.0 & 0.0 & 0.0 & 0.0 & 0.0 & 1.00000\end{array}$

*LENGTH* $401.5993 \%$
1.00000 
*Correct IGNS*

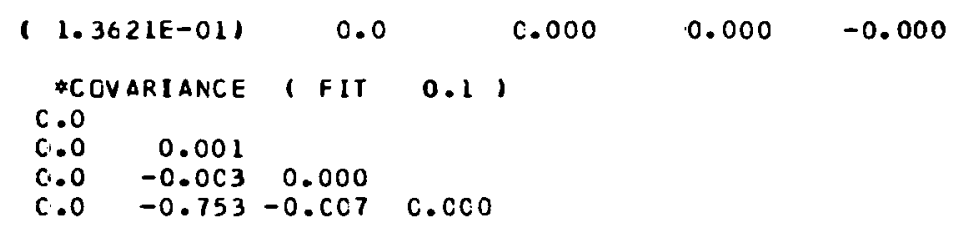


*BEAM* $1.000000 \quad 25.00 \mathrm{GEV}$

*BEND*

4.000

5.00000 it

$1.455 \mathrm{KG}$

0.6000

0.50001

* ROT AT

2.0

C. 50 D

\#DRIFT $\quad 3.0 \quad 8 \mathrm{C.JCCC} \mathrm{M}$

$85.0 \mathrm{M}$

0.0
0.0
0.0
0.0
0.0
0.0

$1.153 \mathrm{CM}$ $0.133 \mathrm{MR}$ $0.90 \mathrm{i} C .4$ $0.100 \mathrm{MP}$ $0.300 \mathrm{CM}$ $1.000 \mathrm{PC}$

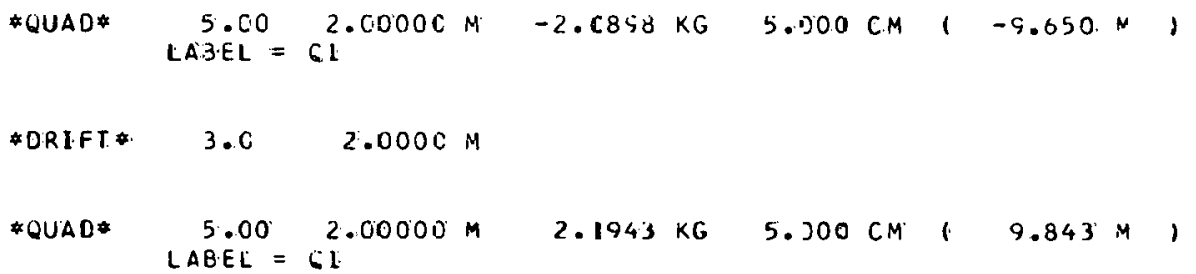

$\begin{array}{ll}0.0 & 1.661 \mathrm{CM} \\ 0.0 & 0.39 .2 \mathrm{MR} \\ 0.0 & 0.5 .82 \mathrm{CM} \\ 0.0 & 0.147 \mathrm{MR} \\ 0.0 & 0.300 \mathrm{CM} \\ 0.0 & 1.000 \mathrm{PC}\end{array}$

$-0.998$

$0.0 \quad 0.0$

$\begin{array}{lll}0.0 & 0.0 & -0.937\end{array}$ $\begin{array}{cccc}-0.003 & 0.003 & 0.0 & 0.0\end{array}$ 

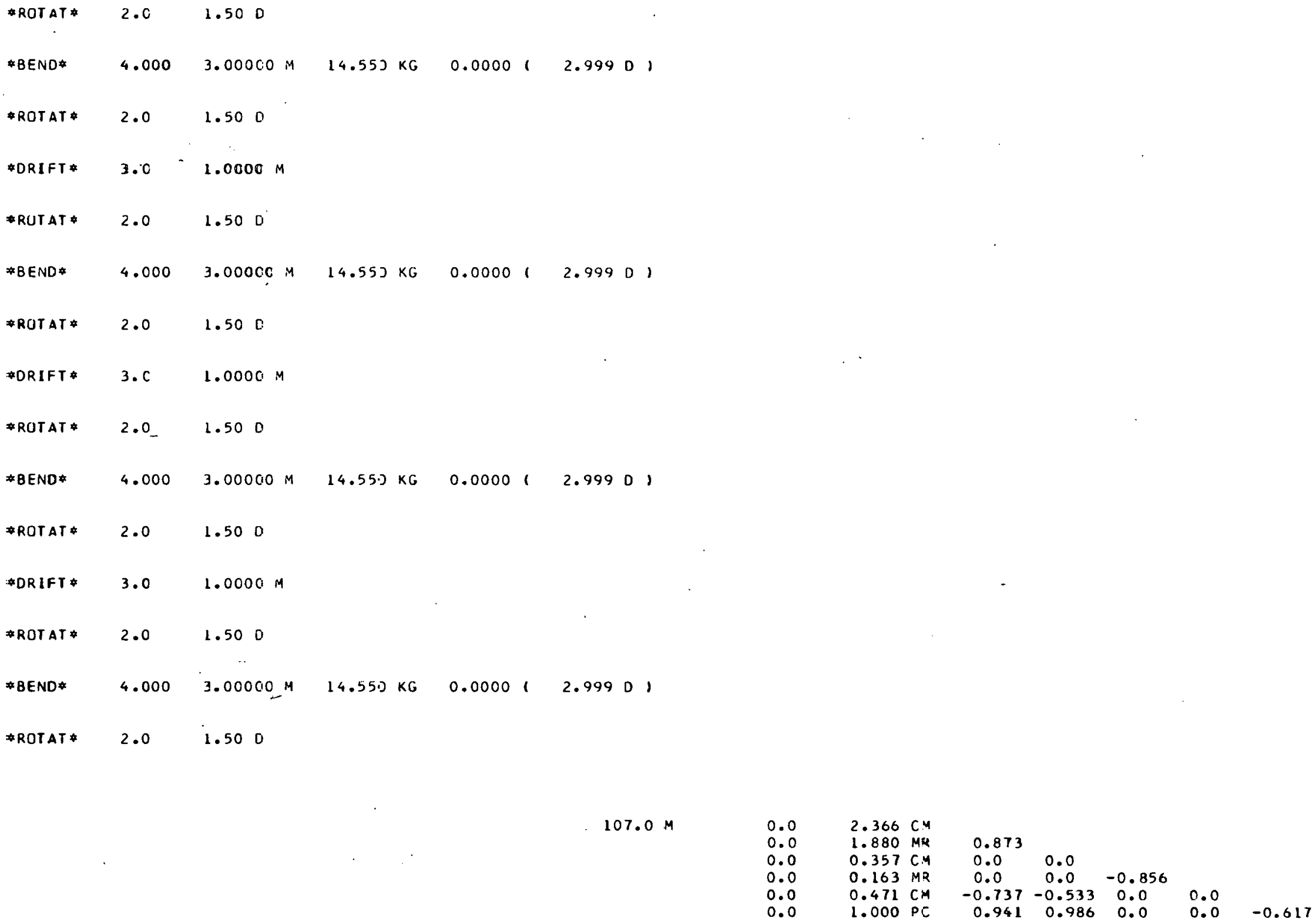
*OR IFT * $\begin{array}{rl}3.0 & 24.9203 \mathrm{M} \\ \text { LABEL } & =\text { DRI }\end{array}$ 
$133.9 M$

*IRANSFURM*

$$
\begin{array}{ll}
-C .33045 & -0.00002 \\
-C .22846 & -3.02617 \\
0.0 & 0.0 \\
0.0 & 0.0 \\
-0.22507 & -2.06457 \\
c . c & 0.0
\end{array}
$$

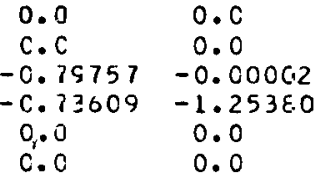

0.0
0.0
0.0
0.0
1.00000
0.0
6.82236
$-2.09424$
0.0
$-0.029037$
1.00000

$159.0 \mathrm{M}$

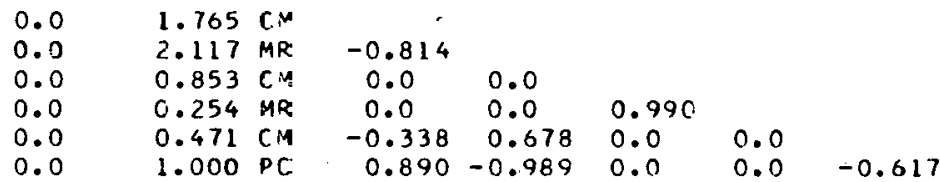

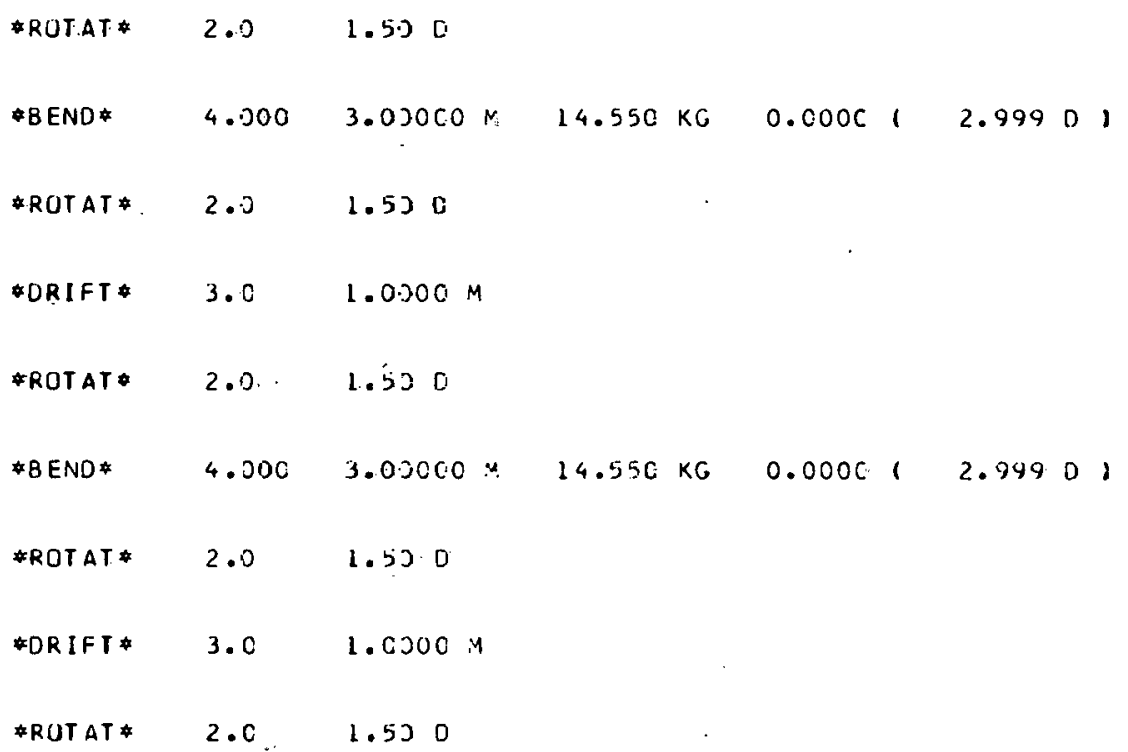




\begin{tabular}{|c|c|c|c|c|c|c|}
\hline \#BEND* & 4.000 & $3.00000 \mathrm{M}$ & 14.550 & KG & 0.00001 & 2.9990 \\
\hline *ROTAT* & 2.0 & 1.500 & & & & \\
\hline *ORIFT * & 3.0 & $1.0000 \mathrm{M}$ & & & & \\
\hline * ROTAT * & 2.0 & 1.500 & & & . & \\
\hline \#BEND* & 4.000 & $3.000 \mathrm{COM}$ & 14.550 & $K G$ & 0.00001 & 2.99901 \\
\hline * ROTAT* & 2.0 & $1.50 \mathrm{D}$ & & & & \\
\hline$* D R I F T *$ & $3 . c$ & $1.0000 \mathrm{M}$ & & & & \\
\hline
\end{tabular}

$\begin{array}{llllllll}175.0 M & 0.0 & 1.300 \mathrm{CM} & & & & & \\ & 0.0 & 0.310 \mathrm{MR} & 0.997 & & & & \\ & 0.0 & 1.227 \mathrm{CM} & 0.0 & 0.0 & & & \\ & 0.0 & 0.216 \mathrm{MR} & 0.0 & 0.0 & 0.994 & & \\ & 0.0 & 0.504 \mathrm{CM} & -0.000 & 0.000 & 0.0 & 0.0 & \\ & 0.0 & 1.000 \mathrm{PC} & 0.000 & 0.000 & 0.0 & 0.0 & -0.804\end{array}$

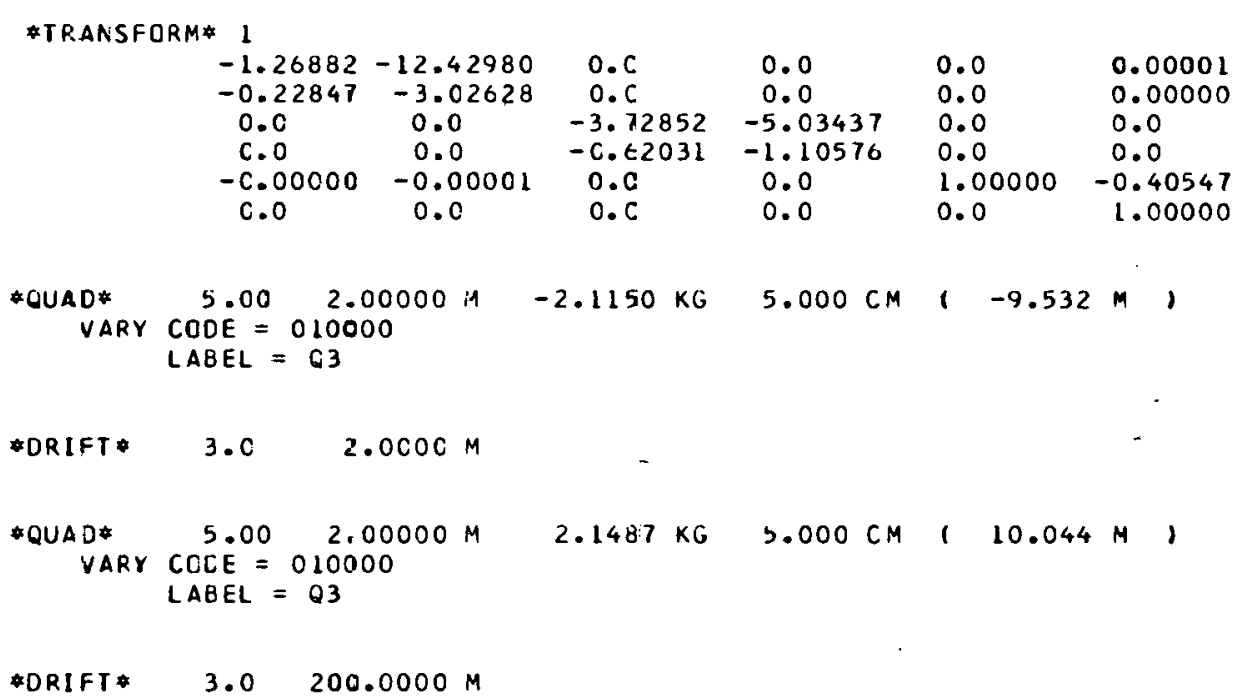

$\begin{array}{llll}381.0 M & 0.0 & 4.000 \mathrm{CM} & \\ & 0.0 & 0.299 \mathrm{MR} & 1.000 \\ & & & \end{array}$




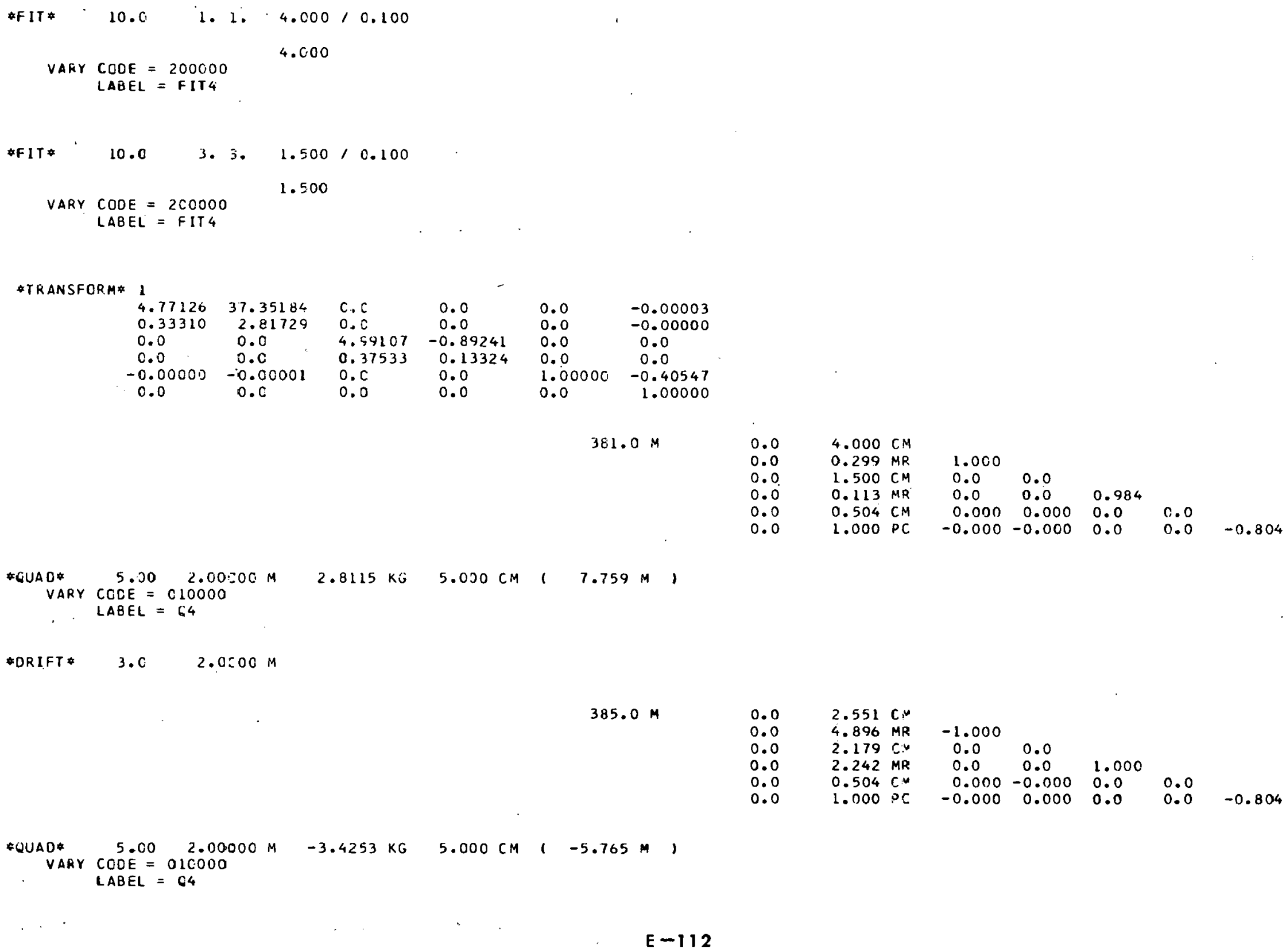


$387.0^{\circ} \mathrm{M}$
$10 . C$
z.
$0 . \mathrm{C}$

LABEL = FIT

*FIT*
10.
4. 3
0.0
0.001

LABEL $=F I T 6$

F I T*

10.0

1.1.

$0.020 / 0.010$

VARY CCDE $=200000$

$\mathrm{LABEL}=\mathrm{FITT}$

*FIT*

3. 3 .

$0.020 / 0.010$

VARY CODE $=200000$

0.020

LABEL = FIT T

- TRANSFORM* 1

$\begin{array}{llllll}-0.23010 & 3.08075 & 0.0 & 0.0 & 0.0 & 0.0 \\ -0.44764 & 1.64740 & 0.0 & 0.0 & 0.0 & 0.0 \\ 0.0 & 0.0 & -0.07421 & 0.99798 & 0.0 & 0.0 \\ 0.0 & 0.0 & -1.02264 & 0.27718 & 0.0 & 0.0 \\ 0.0 & 0.0 & 0.0 & 0.0 & 1.00000 & 0.0 \\ 0.0 & 0.0 & 0.0 & 0.0 & 0.0 & 1.00000\end{array}$

*LENGTH* $401.9993 \mathrm{~m}$ 
'UNCFRTAINTY IN MISALITMMENT OF MAGNETIS, TRIPLET'

(THIS IS AH EXAMPLE OF AN UNCERTAIHTY IN THE MISALIGNMENT OF A TRIPLET.)

(ZERO PHASE SPACE IS JSED. IT IS SIHILAR TO EXAYLLE THREE IN SECTION EIGHT OF) (SLAC, 91. THE. URCERTRINTIES APPEAR III THE PHASE SPACE ELLIPSF PRINTOLT.)

1.00 .00 .00 .00 .00 .00 .01 .0 :

$3.03 .66 ;$

$5.00 .45730-14.50300 \quad 10.15$

3.00 .15250

3.00 .15250

$5.0001 .0 \div 3975010.15$

3.00 .915250

$5.00 .45730-14.5080010 .15$

$5.0-0.45730-14.5030010 .15$

$\begin{array}{lllllllll}8.0 & 0.1 & 0.1 & 0.1 & 0.1 .0 .1 & 0.1 & 000.0 \text {; }\end{array}$

$8.0 \quad 0.1 \quad 0.1 \quad 0.1 \quad 0.1 \quad 0.1 \quad 0.1 \quad 001.0$

$\begin{array}{llllllllll}8.0 & 0.1 & 0.1 & 0.1 & 0.1 & 0.1 & 0.1 & 002.0 \text {; }\end{array}$

3.02 .13500

$13: 04.0 ;$

SFNTINEL 
UHCERTAINTY IN MISALIIGNMENT OF MAGNETIC TRIPLET

IHIS IS AN EXAMPLE OF AN UNCERTAINTY IN THE MISALIGNMENT OF a TRIPLET.

zERO PF́ÁSE SPACE IS USED. It IS SIMILAR TO EXAMPLE THREE IN SECTION EIGHT OF slac 91. the uncertainties appear in the phase space ellipse printout.

\begin{tabular}{|c|c|c|c|c|c|c|c|}
\hline 1.00000 & 0.0 & 0.0 & 0.0 & $0 . c$ & 0.0 & 0.0 & 1.00001 \\
\hline 3.00000 & 3.60000 & & & & & & \\
\hline 6.00000 & C.o & 2.00000 & & & & & \\
\hline 5.00000 & $c .45730$ & $-14.5 C 8 O C$ & 10.15000 & & & & \\
\hline 3.00000 & C. 15250 & & & & & & \\
\hline 6.00000 & 0.0 & 1.00000 & & & & & \\
\hline 5.00000 & C. 91400 & 8.35750 & 10.15600 & & & & \\
\hline 3.00000 & $c .15250$ & & & & & & \\
\hline 5.00000 & 0.45730 & -14.50800 & 10.15000 & & & & \\
\hline 8.00000 & C.10000 & 0.10000 & $0.10 c 00$ & 0.10000 & 0.10000 & 0.10000 & 0.0 \\
\hline 8.00000 & $0.1000 \mathrm{C}$ & 0.10000 & $0.100,00$ & 0.10000 & 0.10000 & 0.10000 & 1.00000 \\
\hline 8.00000 & 0.10000 & 0.10000 & 0.10000 & 0.10000 & 0.10000 & 0.10000 & 2.00000 \\
\hline 3.00000 & 2.13500 & & & & & & \\
\hline $13 . c 0000$ & 4.00000 & & & & & & \\
\hline ENT INEL & & & & & & & \\
\hline
\end{tabular}


UNEERTAINTY IN MISAL IGIJMENT UF MAGNETIC TRIPLET

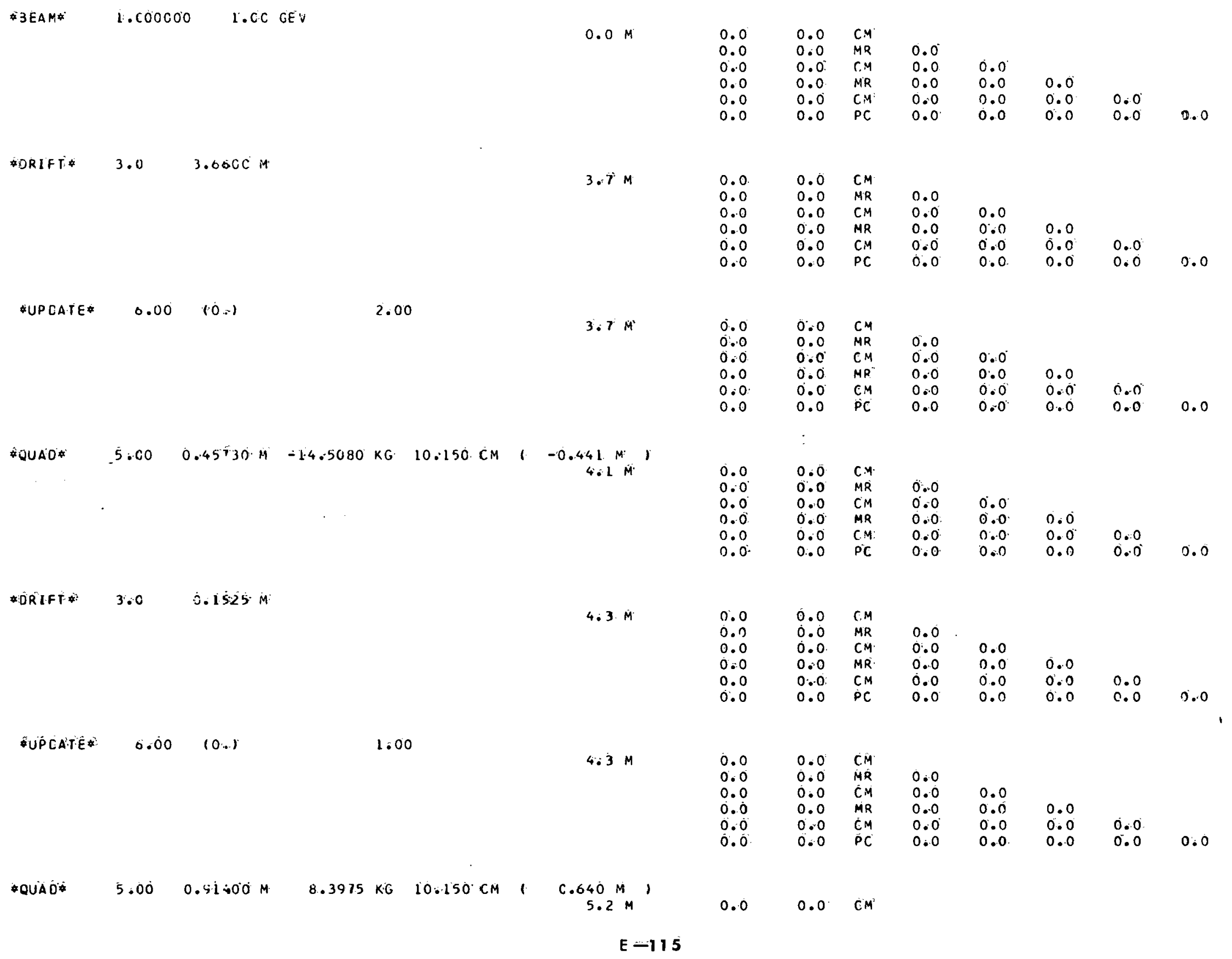




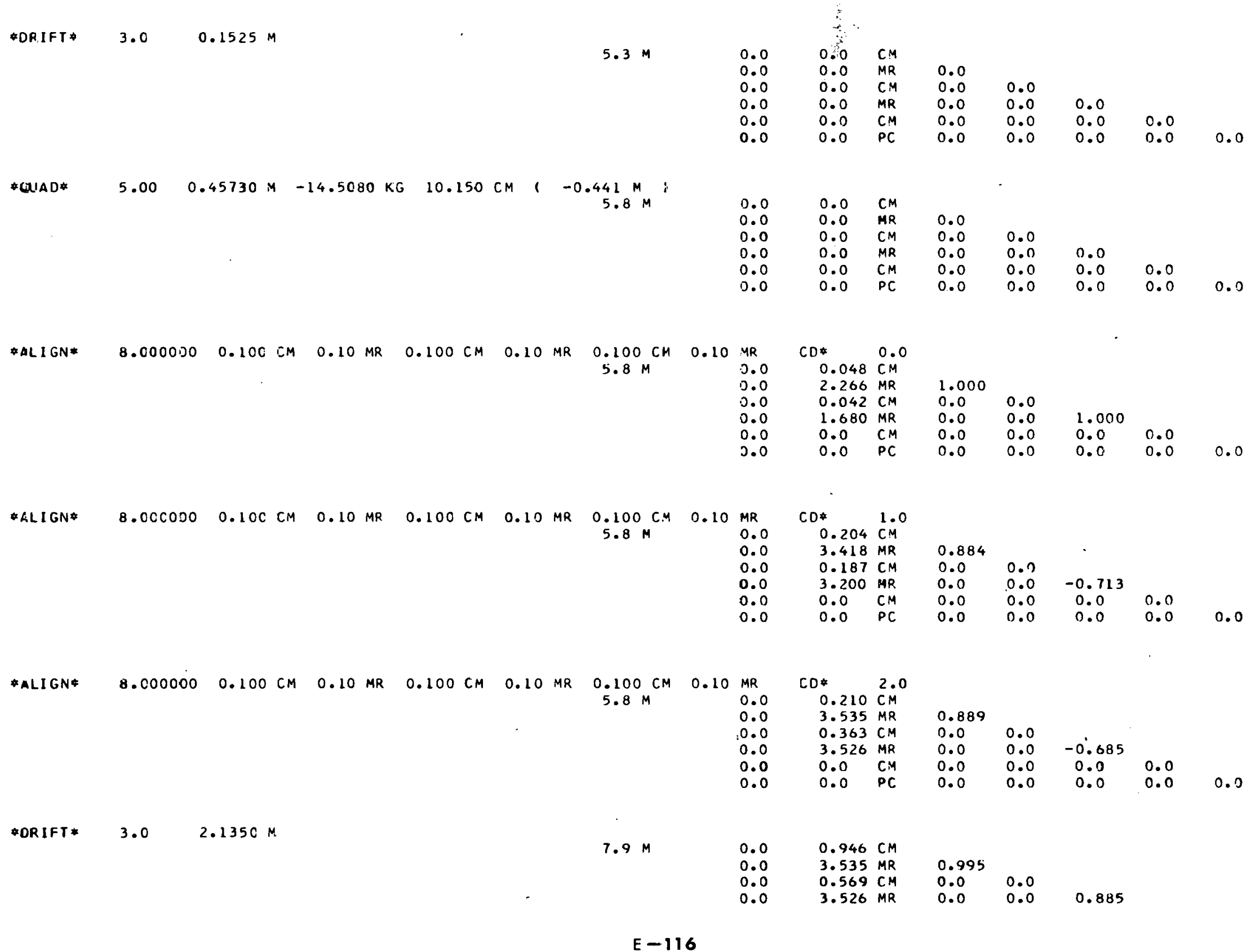


$\begin{array}{llllllll}0.0 & 0.0 & \text { CM } & 0.0 & 0.0 & 0.0 & 0.0 & \\ 0.0 & 0.0 & \text { PC } & 0.0 & 0.0 & 0.0 & 0.0 & 0.0\end{array}$

* TRANSFCRM*

$\begin{array}{llllll}1.00000 & 0.21350 & C . c & 0.0 & 0.0 & 0.0 \\ 0.0 & 1.00000 & 0.0 & 0.0 & 0.0 & 0.0 \\ 0.0 & 0.0 & 1.00000 & 0.21350 & 0.0 & 0.0 \\ 0.0 & 0.0 & 0.0 & 1.0000 C & 0.0 & 0.0 \\ 0.0 & 0.0 & 0.0 & 0.0 & 1.00000 & 0.0 \\ 0.0 & 0.0 & 0.0 & 0.0 & 0.0 & 1.00000\end{array}$

*LENGTH* $\quad 7.9286 \mathrm{M}$ 
'LELIBERATE DISPLACEMENT OF A TRIPLET'

(THIS IS AN EXAMPLE OF A KNOWN DISPLACEMENT IN A TRIPLET. A FINITE PHASE)

(SPACF IS ENTERED, AND THE DISPLACEMENT APPEARS IN THE BFAM CENTROID.)

$\begin{array}{llllllllll}1.0 & 0.1 & 0.2 & 0.3 & 0.4 & 0.50 & 0.6 & 7.0\end{array}$

$3.03 .66:$

$5.00 .45730-14.50800 \quad 10.15$

3.00 .15250

6.00 .15250

5.00 .974008 .3975010 .15

3.00 .915250

.

$8.0 .45730-14.5080010 .15$

100.0

0.1101 .0

$8.00 .10 .10 .10 .10 .10 .1 \quad 102.0$

3.02 .13500 :

15.04 .0

SF.NTIMEL 
DELIBERATE CISPLACEMENT OF A TRIPLE T

0

THIS IS AN EXAMPLE OF A KNCWN DisplaCEMENT IN A TRIPLET. a finite PHASE

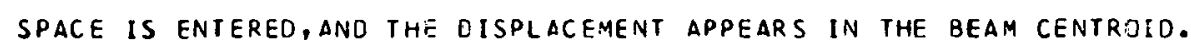

\begin{tabular}{|c|c|c|c|c|c|c|c|}
\hline 1.00000 & C.10000 & 0.20000 & 0.30000 & $0.4(001)$ & 0.50000 & 0.60000 & 7.00000 \\
\hline 3.00000 & 2.66000 & & & & & & \\
\hline 6.00000 & c.o & 2.00000 & & & & & \\
\hline 5.00000 & .0 .45730 & $-14.5080 \mathrm{C}$ & $1 C .15000$ & & & & \\
\hline 3.00000 & C. 15250 & & & & & & \\
\hline 6.00000 & 0.0 & $\therefore .02000$ & & & & & \\
\hline 5.c0000 & 0.91400 & $8.3575 c$ & $1 C .15000$ & & & & \\
\hline 3.00000 & 0.15250 & . & & & & & \\
\hline 5.00000 & 0.45730 & -14.50800 & 10.15000 & & & & \\
\hline 8.00000 & 0.10000 & 0.10000 & 0.10000 & 0.10000 & 0.10000 & 0.10000 & 100.00000 \\
\hline $8 . c 0000$ & c. 10000 & 0.10000 & 0.10000 & 0.10000 & 0.10000 & 0.10000 & 101.00000 \\
\hline 8.00000 & c. 10000 & 0.10000 & 0.10000 & 0.10000 & 0.10000 & 0.10000 & 102.00000 \\
\hline 3.00000 & 2.13500 & & & & & & \\
\hline 13.00000 & 4.00000 & & & & & & \\
\hline ENT INEL & & & & & & & \\
\hline
\end{tabular}


DELIBERATE CISPLACEMENT OF A TRIPLET

* BeAm* $1.000000 \quad 7.00 \mathrm{GEV}$

$0.0 \mathrm{M} \quad 0.0 \quad 0.100 \mathrm{CM}$

$0.0 \quad 0.200 \mathrm{MR}$

$\begin{array}{ll}0.0 & 0.200 \mathrm{CM} \\ 0.0 & 0.300 \mathrm{CM}\end{array}$

$0.0 \quad 0.400 \mathrm{MR}$

$0.0 \quad 0.50 \mathrm{C} \mathrm{CM}$

$0.60 \mathrm{CPC}$

0.0

$0.0 \quad 0.0$

$\begin{array}{llll}0.0 & 0.0 & 0.0 & \\ 0.0 & 0.0 & 0.0 & 0.0 \\ 0.0 & 0.0 & 0.0 & 0.0\end{array}$

$\begin{array}{llll}0.0 & 0.0 & 0.0 & 0.0\end{array}$

0.0

*URIFT* 3.C $\quad 3.660 \mathrm{CM}$

$3.7 \mathrm{M}$

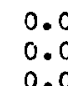

$0.124^{\circ} \mathrm{CH}$ $0.200 \mathrm{MR}$ $0.334 \mathrm{CH}$ $\begin{array}{ll}0.0 & 0.400 \mathrm{MR} \\ 0.0 & 0.500 \mathrm{CM}\end{array}$ $\begin{array}{ll}0.0 & 0.400 \mathrm{MR} \\ 0.0 & 0.500 \mathrm{CM}\end{array}$ 0.591

$\begin{array}{llll}0.591 & & & \\ 0.0 & 0.0 & & \\ 0.0 & 0.0 & 0.439 & \\ 0.0 & 0.0 & 0.0 & 0.0 \\ 0.0 & 0.0 & 0.0 & 0.0\end{array}$
0.0

*UPCATE* $0.00 \quad 10.1 \quad 2.00$

$$
3.7 M
$$

$$
\begin{aligned}
& 0.0 \\
& 0.0 \\
& 0.0 \\
& 0.0 \\
& 0.0 \\
& 0.0
\end{aligned}
$$

$0.124 \mathrm{CM}$ $0.200 \mathrm{MP}$ $0.334 \mathrm{CM}$
$0.400 \mathrm{MR}$ $0.400 \mathrm{MR}$
$0.500 \mathrm{CM}$ $0.500 \mathrm{CM}$
$0.600 \mathrm{PC}$ $\begin{array}{ll}0.591 & \\ 0.0 & 0.0\end{array}$ $\begin{array}{lll}0.0 & 0.0 & 0.439\end{array}$ $\begin{array}{llll}0.0 & 0.0 & 0.0 & 0.0\end{array}$

$\begin{array}{llll}0.0 & 0.0 & 0.0 & 0.0\end{array}$
0.0

*QUAD* $5.00 \quad 0.45730 \mathrm{M}-14.5580 \mathrm{KG} 10.150 \mathrm{CM} /-3.497 \mathrm{M}$,

$0.0 \quad 0.138 \mathrm{CM}$ $0.0 \quad 0.510 \mathrm{MR}$ $0.0 \quad 0.321 \mathrm{CM}$ $0.0 \quad 0.822 \mathrm{MR}$ $\begin{array}{ll}0.0 & 0.822 \mathrm{MR} \\ 0.0 & 0.500 \mathrm{CM}\end{array}$ 0.959 $\begin{array}{ll}0.959 & \\ 0.0 & 0.0\end{array}$ $\begin{array}{lll}0.0 & 0.0 & \\ 0.0 & 0.0 & -0.891\end{array}$

\#UPDATE* $6.00 \quad 10.1$

1.00

$\begin{array}{ll}0.0 & 0.145 \mathrm{CM} \\ 0.0 & 0.510 \mathrm{MR} \\ 0.0 & 0.310 \mathrm{CM} \\ 0.0 & 0.822 \mathrm{MR} \\ 0.0 & 0.500 \mathrm{CM} \\ 0.0 & 0.600 \mathrm{PC}\end{array}$

$4.3 M$

$$
\begin{gathered}
.246 \mathrm{M}^{\prime} \\
5.2 \mathrm{M}
\end{gathered}
$$

$\begin{array}{llll}0.963 & & \cdots & \\ 0.0 & 0.0 & & \\ 0.0 & 0.0 & -0.882 & \\ 0.0 & 0.0 & 0.0 & 0.0 \\ 0.0 & 0.0 & 0.0 & 0.0\end{array}$




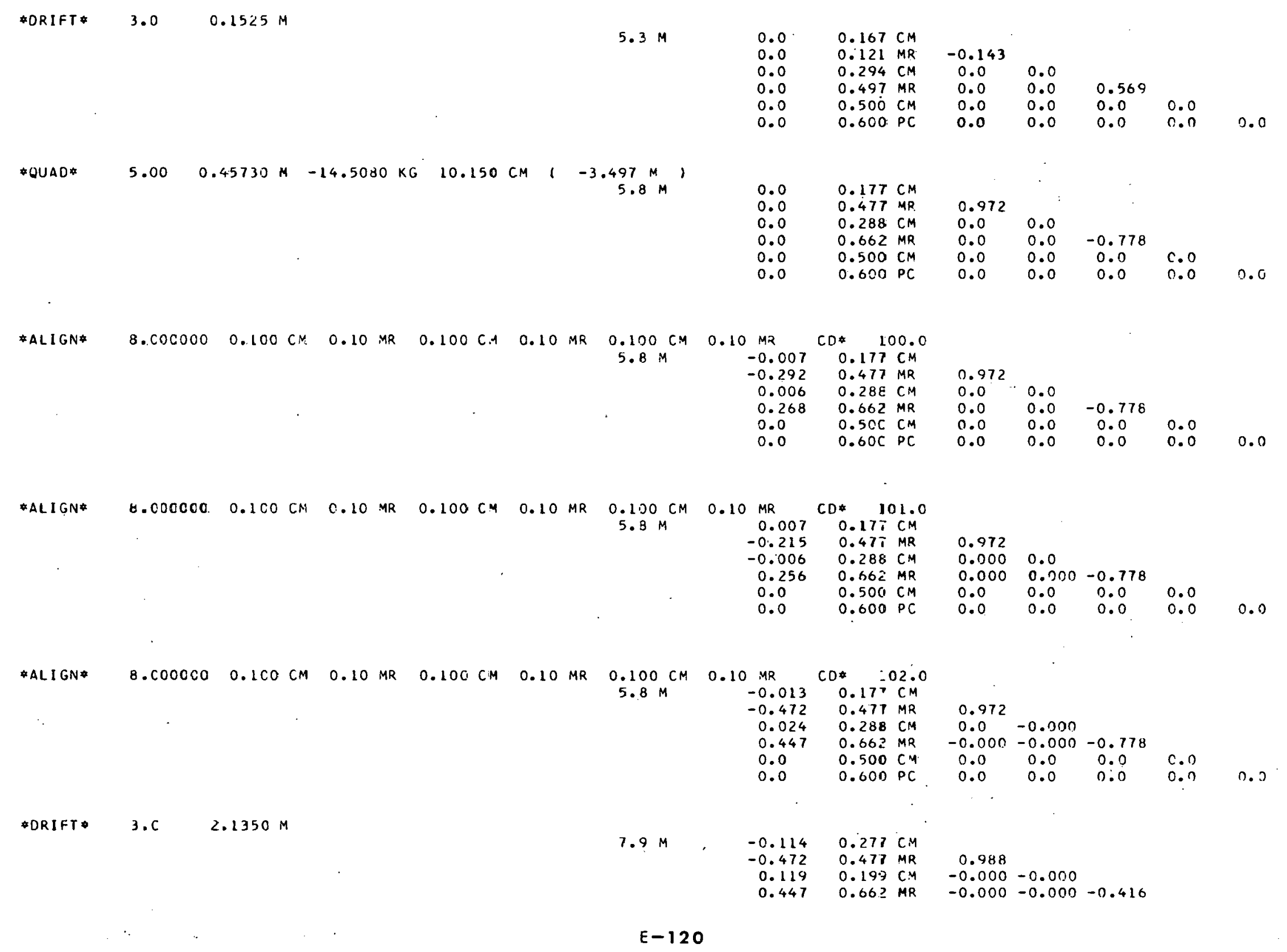


* TRANSFORM

$\begin{array}{llllll}1.00000 & 0.21350 & 0.0 & 0.0 & 0.0 & 0.0 \\ 0.0 & 1.0000 \mathrm{C} & \mathrm{C.C} & 0.0 & 0.0 & 0.0 \\ \mathrm{C} .0 & 0.0 & 1.00000 & 0.21350 & 0.0 & 0.0 \\ \mathrm{C} .0 & 0.0 & 0.0 & 1.00000 & 0.0 & 0.0 \\ 0.0 & 0.0 & 0.0 & 0.0 & 1.00000 & 0.0 \\ c .0 & 0.0 & C .0 & 0.0 & 0.0 & 1.00000\end{array}$

*LENGTH* $\quad 7.9286 \mathrm{M}$

IHC2L7I FIOCS - END OF DATA SET ON LNIT 5

\begin{tabular}{|c|c|c|c|c|c|c|}
\hline \multirow[t]{5}{*}{ TRACEBACK FOL } & $\begin{array}{l}\text { ROUT INE } \\
\text { IBCOM }\end{array}$ & I SN & $\begin{array}{l}\text { REG. } \quad 14 \\
000755 F C\end{array}$ & $\begin{array}{l}\text { REG. } \quad 15 \\
000700 E 8\end{array}$ & $\begin{array}{l}\text { REG. } 0 \\
00000001\end{array}$ & $\begin{array}{l}\text { R.EG. } \\
00065 F 04\end{array}$ \\
\hline & SKKIN & 0013 & 40066060 & 00075248 & 00000000 & 00065 FD4 \\
\hline & TITLE & 0029 & $4007806 A$ & $00065 F 50$ & 00000000 & $0007883 C$ \\
\hline & FITTIN & $004 t$ & $4007856 \mathrm{~A}$ & 00078628 & $00000 \mathrm{COF}$ & 00078414 \\
\hline & MAIN & & $00015 C 8 A$ & 01078338 & FDO000C08 & OOOADFF 8 \\
\hline
\end{tabular}

ENTRY POINT $=0.1078338$

SUMMARY OF ERRORS FOR THIS JUB ERROR NUMBER NUMBER OF ERRORS

$217 \quad 1$


TRANSPORT FLOW CHART 
TABLE OF CONTENTS

TRANSPORT

F-1

SUBROUTINE BKKIN

$\mathrm{F}-2$

SUBROUTINE BKKPAK

$F-9$

SUBROUTINE BKKFX

$F-10$

SUBROUTINE BKKFL

$F-12$

SUBROUTINE SCANT

$F-15$

SUBROUTINE FITTIN

F -16

OUTFIT SUBPROGRAM

$F-22$

ELEMENT SUBROUTINE

F-26

SOLVE SUBPROGRAM

$F-38$

FORM SUBROUTINE

$F-40$

ALTER SUBPROGRAM

F-4I

PARTIALS SUBROUTINE

$F-44$

SUBROUTINE CHEK

$F-51$

SUMMARY

F-52 
SET STE $\theta F$ R MATRICES TO SIX FOR FIRST ORDER (RESET TO 42 IF TYPE 17 ENCOUNTERED)

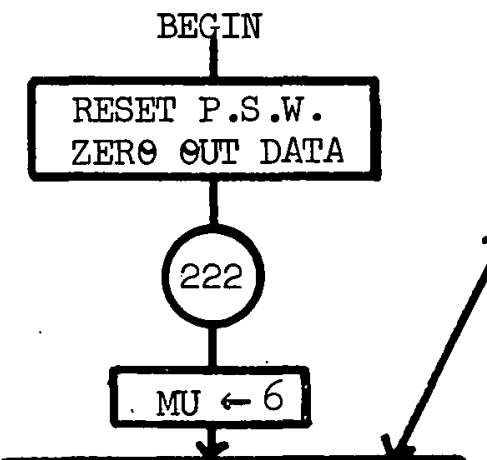

BKKIN

READ IN

AND INTERPRET

DATA

OUTFIT

CAILS ELEMEINT

AND PRINTS

iv. RESULTS

CALLS BKKIN, SETS

UP TIE ARRAY FOR

VARY CODES AND

CAIISS OUTFIT

ELMENT

CALCULATE $R$ MATRICES AND

PREDUCT $\theta F$ R's

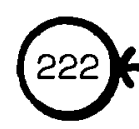

Ne

FITTING NEEDED?
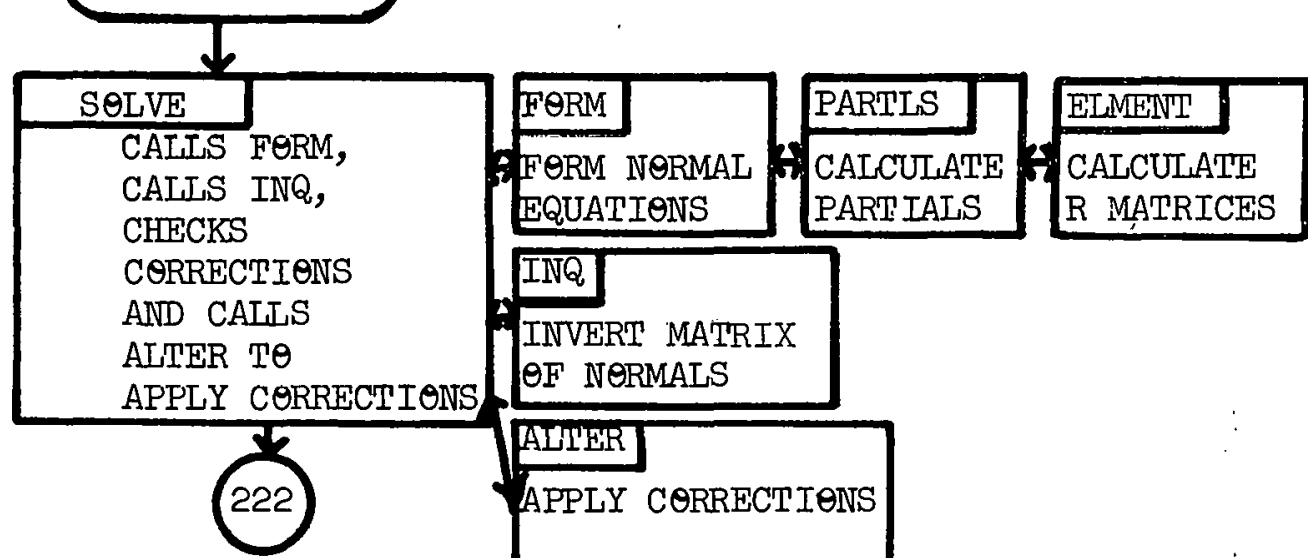

INQ

INVERT MATRIX

OF NORMALS

ALIER

APPLY CORRECTIONS 
SET FLAG TO INDICATE EIVIRY POINT
ENTRY TRANIN(DATA, VARY, KABLE, NSTART, NSTOP, NWORD, IR)

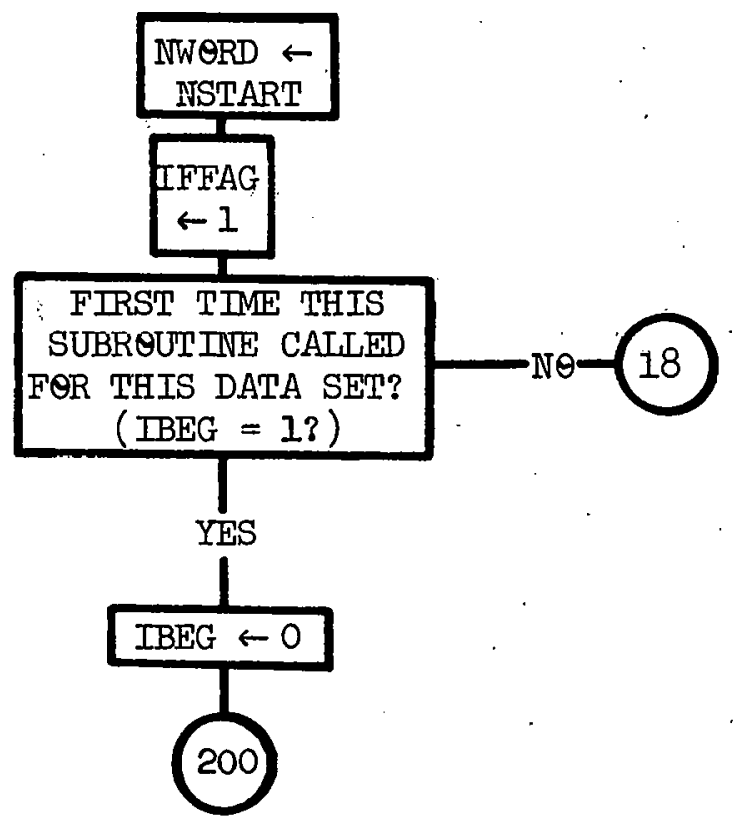

ENTRY BKKIN (DATA, NSTART, NSTOP; NWORD, IR)

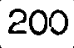

200

INITIALIEE SYMBOLS

TO CHARACTER SET

OF MACHINE $O N$ WHICH

COMPILED

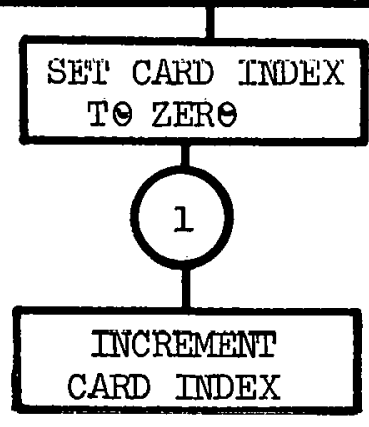


EXAMINE CHARACTER IN COLUMN IW
SET FLQATING

POINIT FLAG

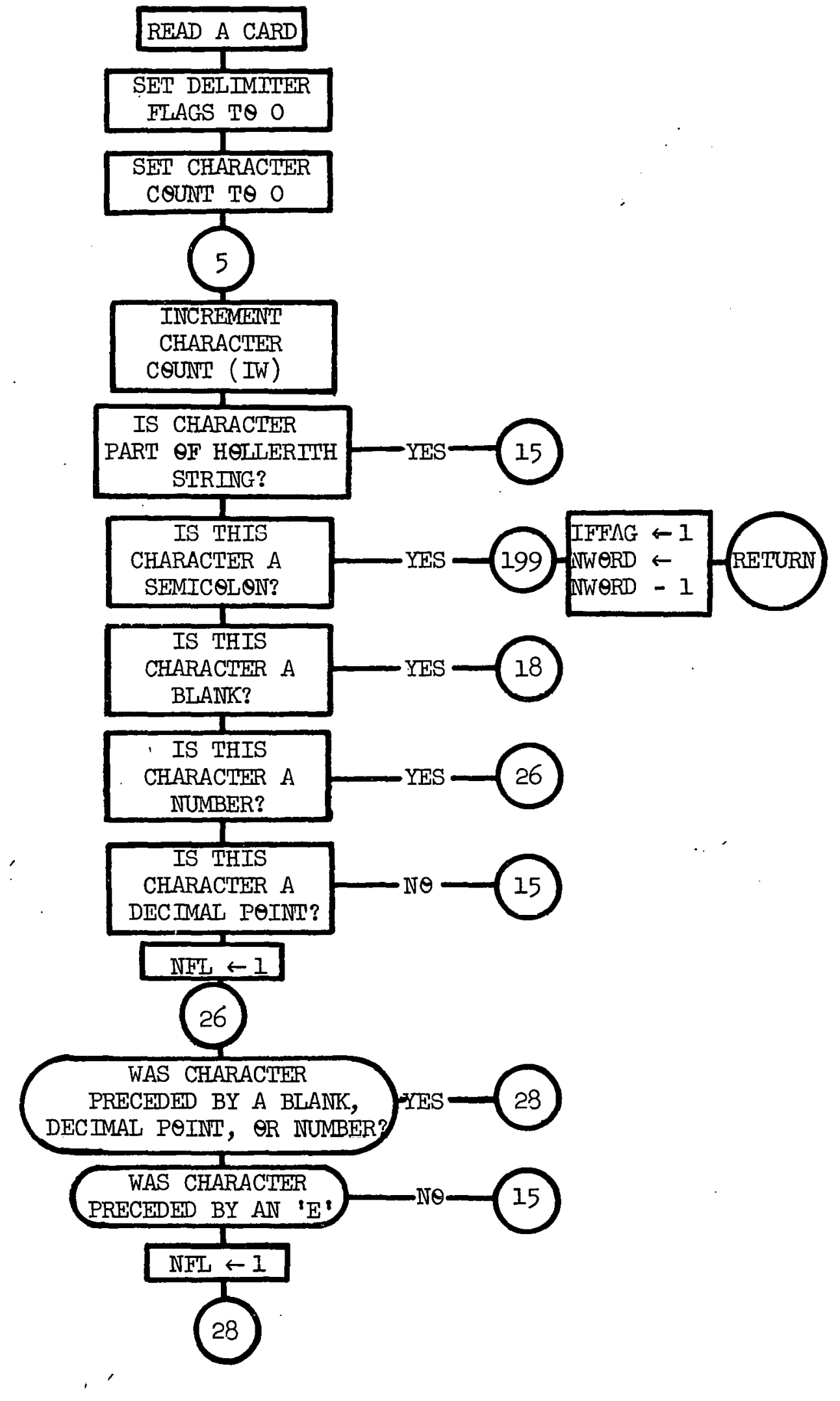


Trta $\therefore \therefore$ CHARACTER -IS PART $\theta F$ NUMERIC FIETD

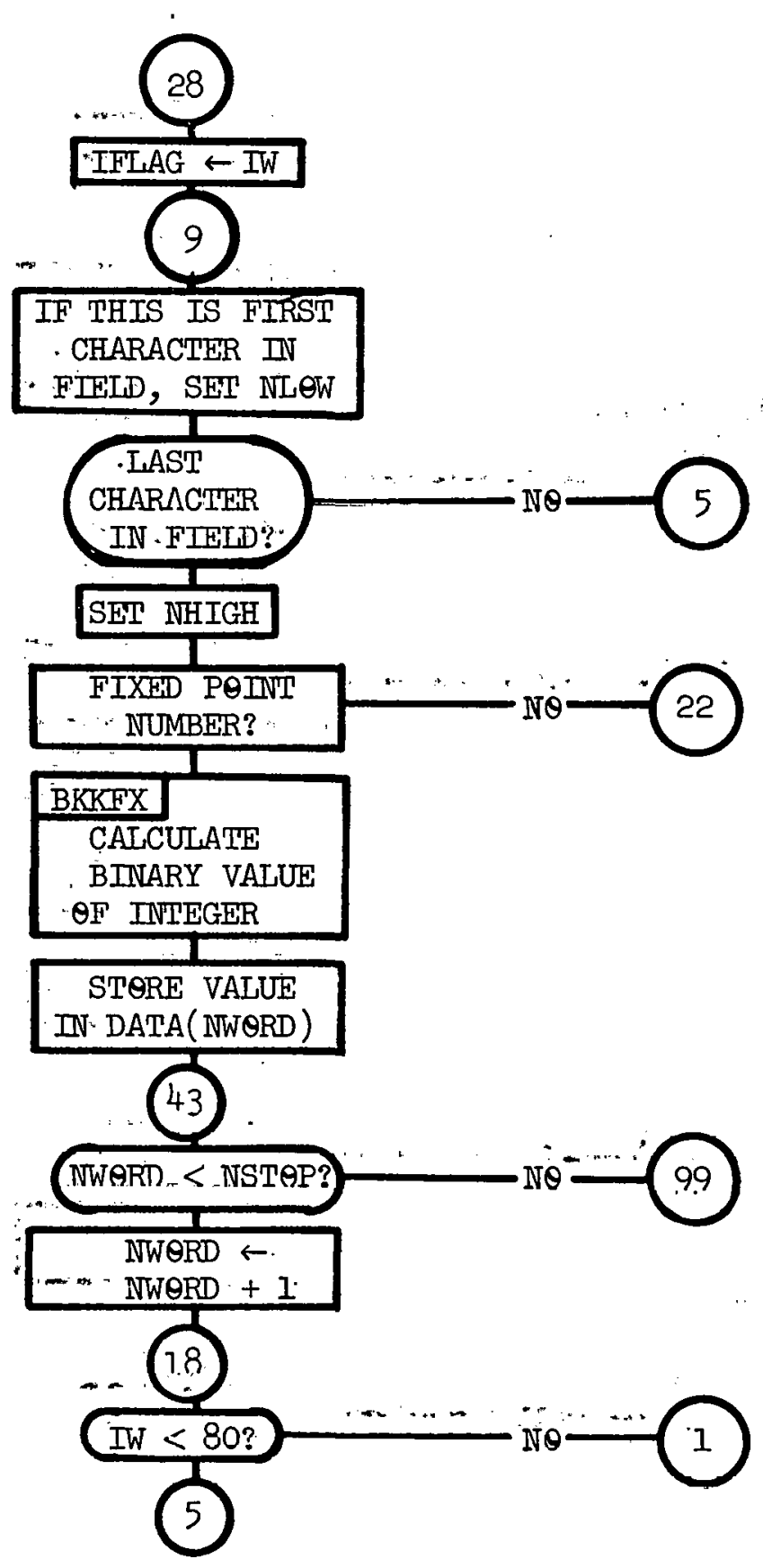

$$
\div \text { F-4 }
$$


FLQATING POINT

NUMBER

DATA

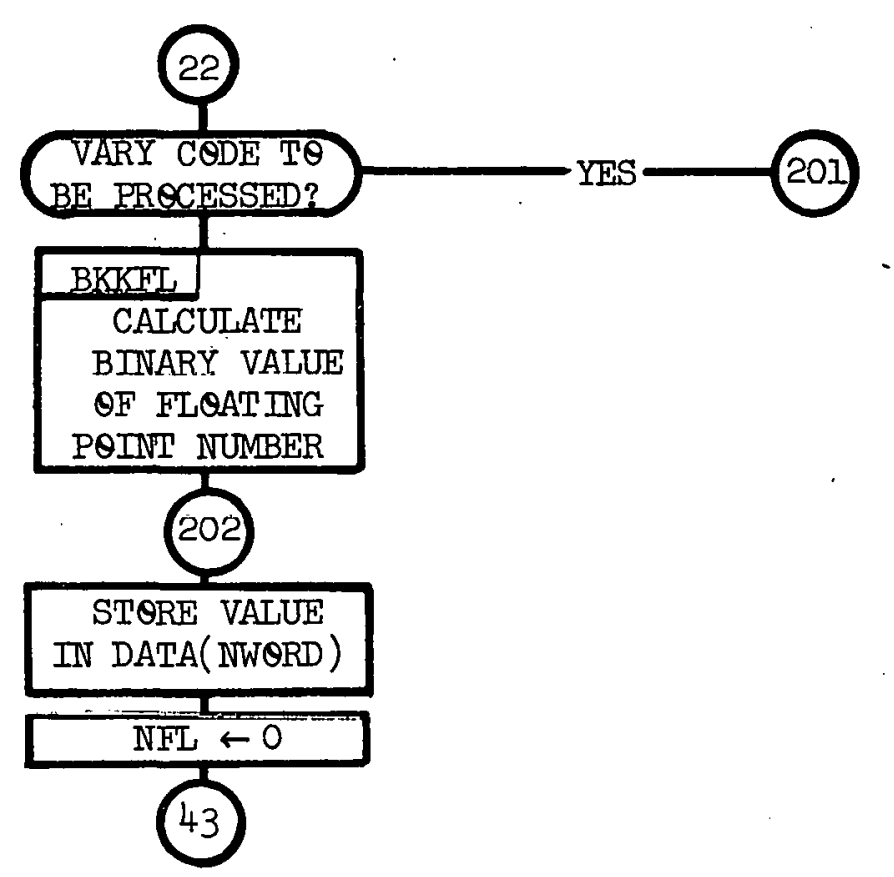

TYPE CODE

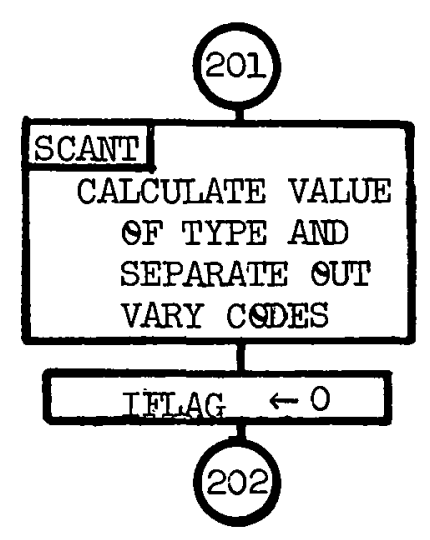


NON-NUMERIC

FIELD

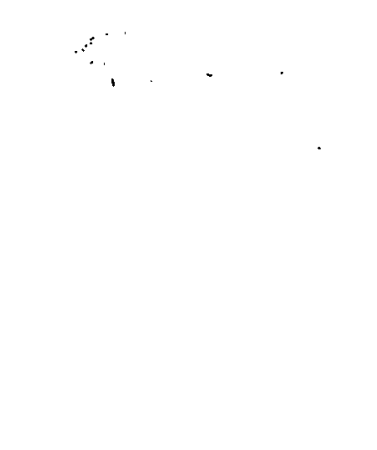

STRIIVG ENCLOSED. IIV QUQTES. STORE

IN DATA ARRAY

\section{CHECK TO SEE IF STRIING WAS TYPE \\ I5 ID OR IAABEL}

(15)

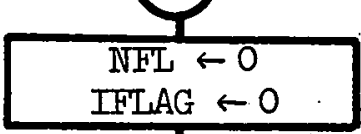

IS CHARACTER A QUETE?

BEGINNING $\theta \mathrm{R}$

ENDIIVG QUOIE? EIDING:

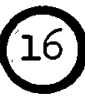

BEGINNING

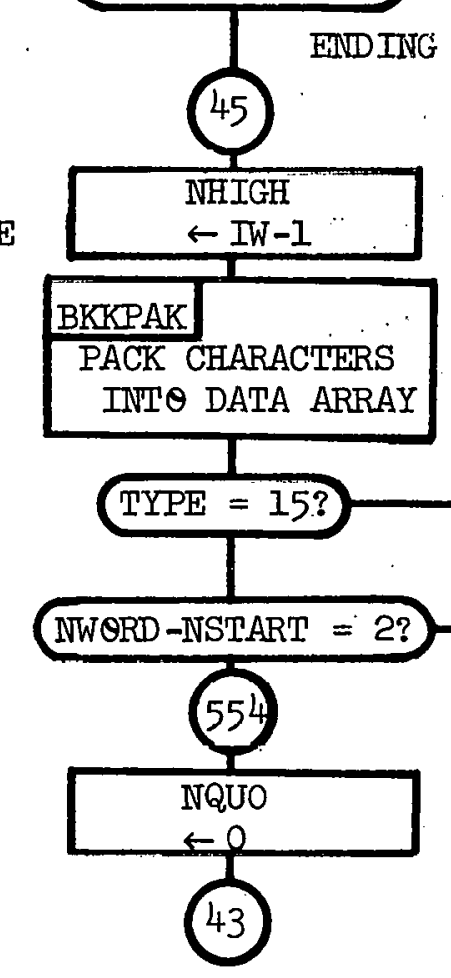

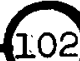

NQUO $\leftarrow 1$

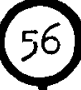

NLOW $\leftarrow$

$I W+I$

18 

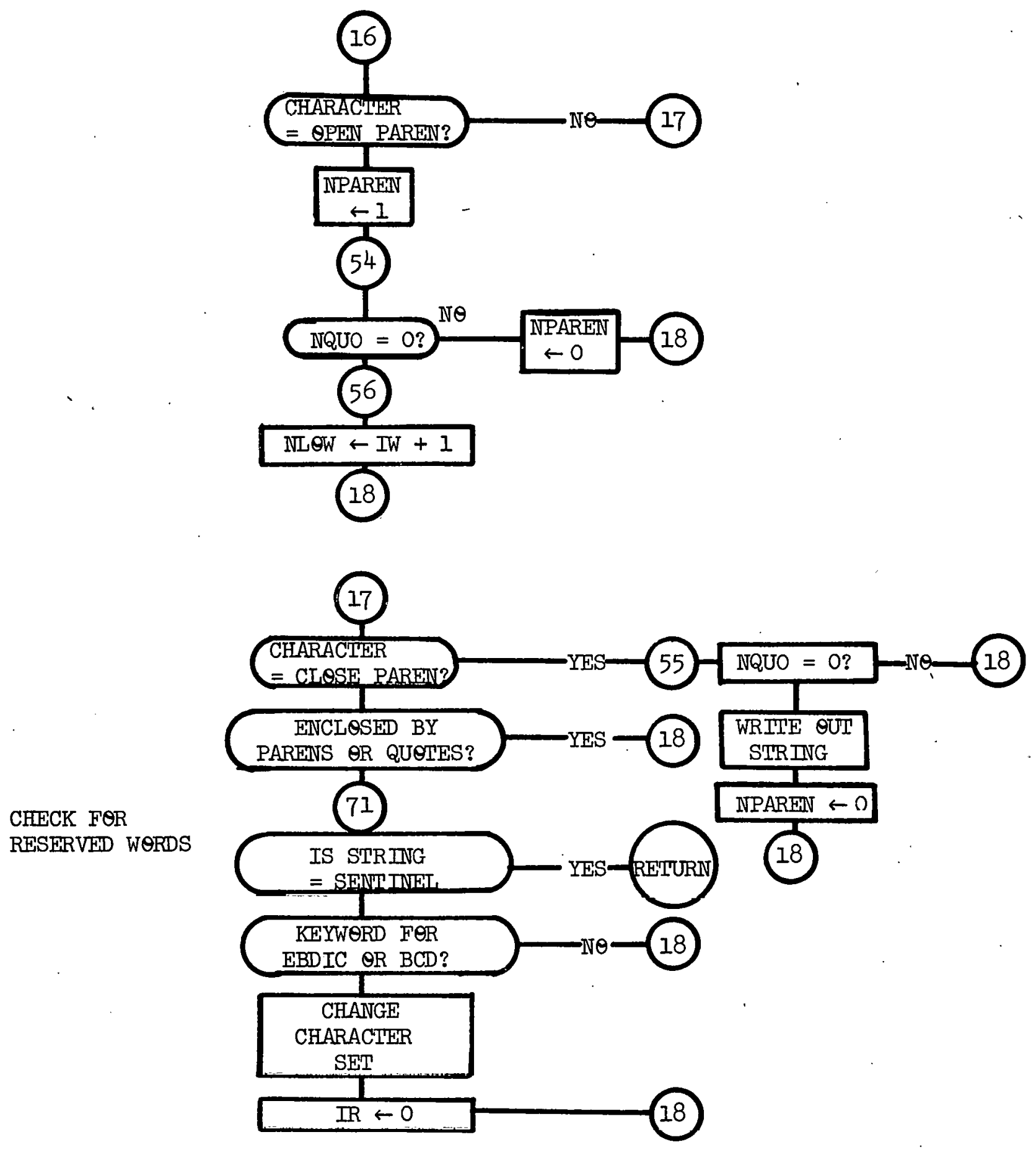


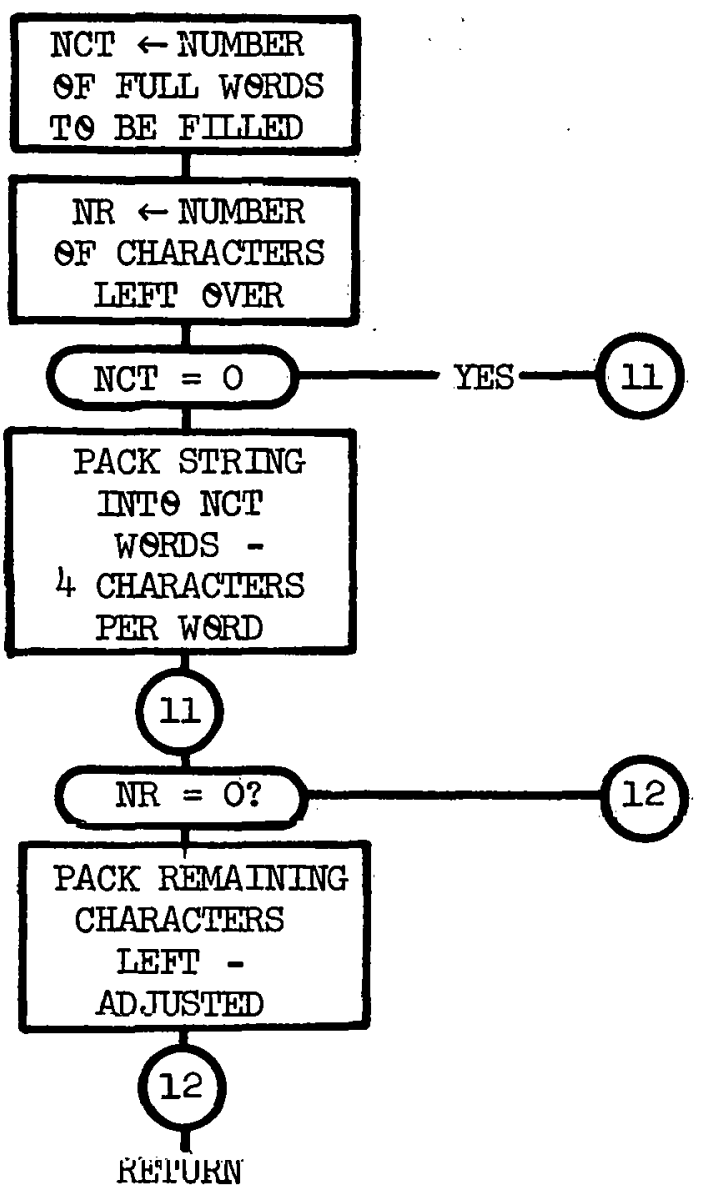


LOQK UP

CHARACTER IN

EBDIC TABLE.

USE CORRESPONDING

DIGIT TO FORM

NUMBER

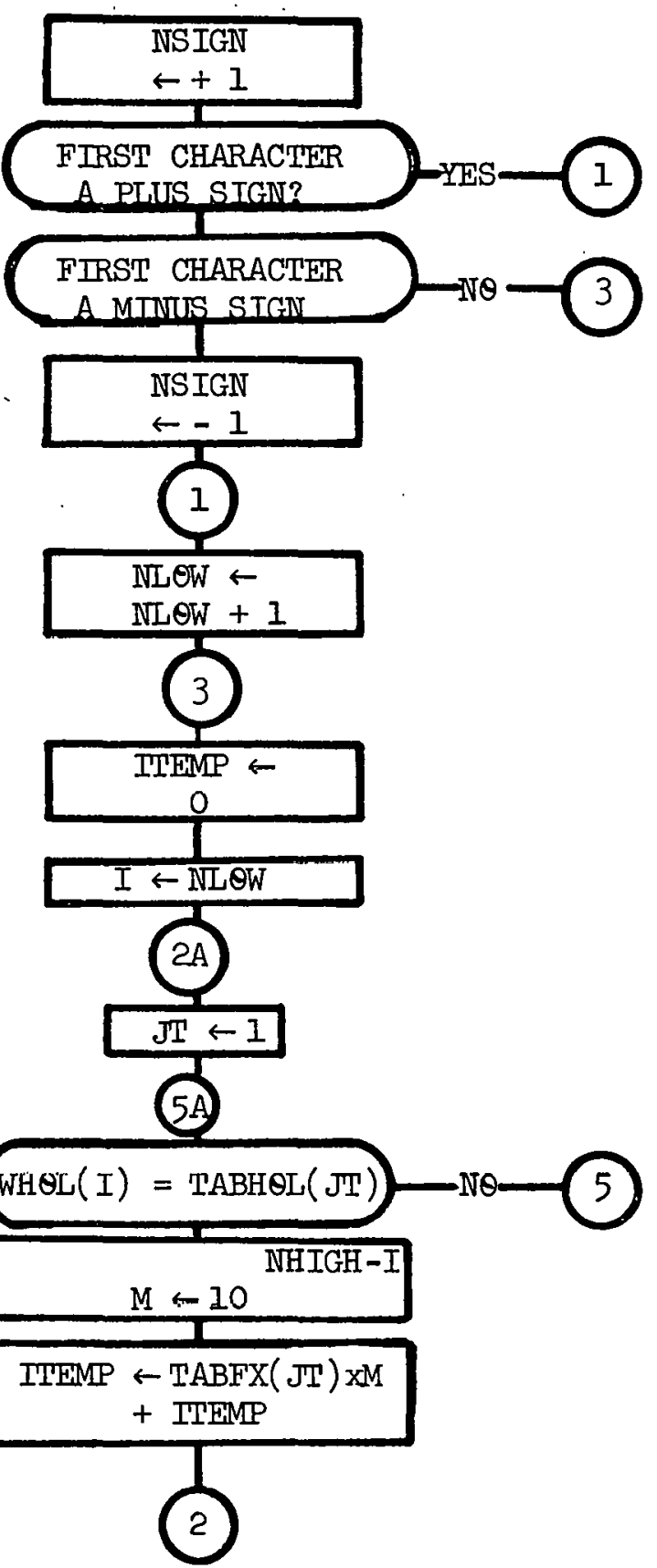


-SUBROUTINE BKKFXX (2)-

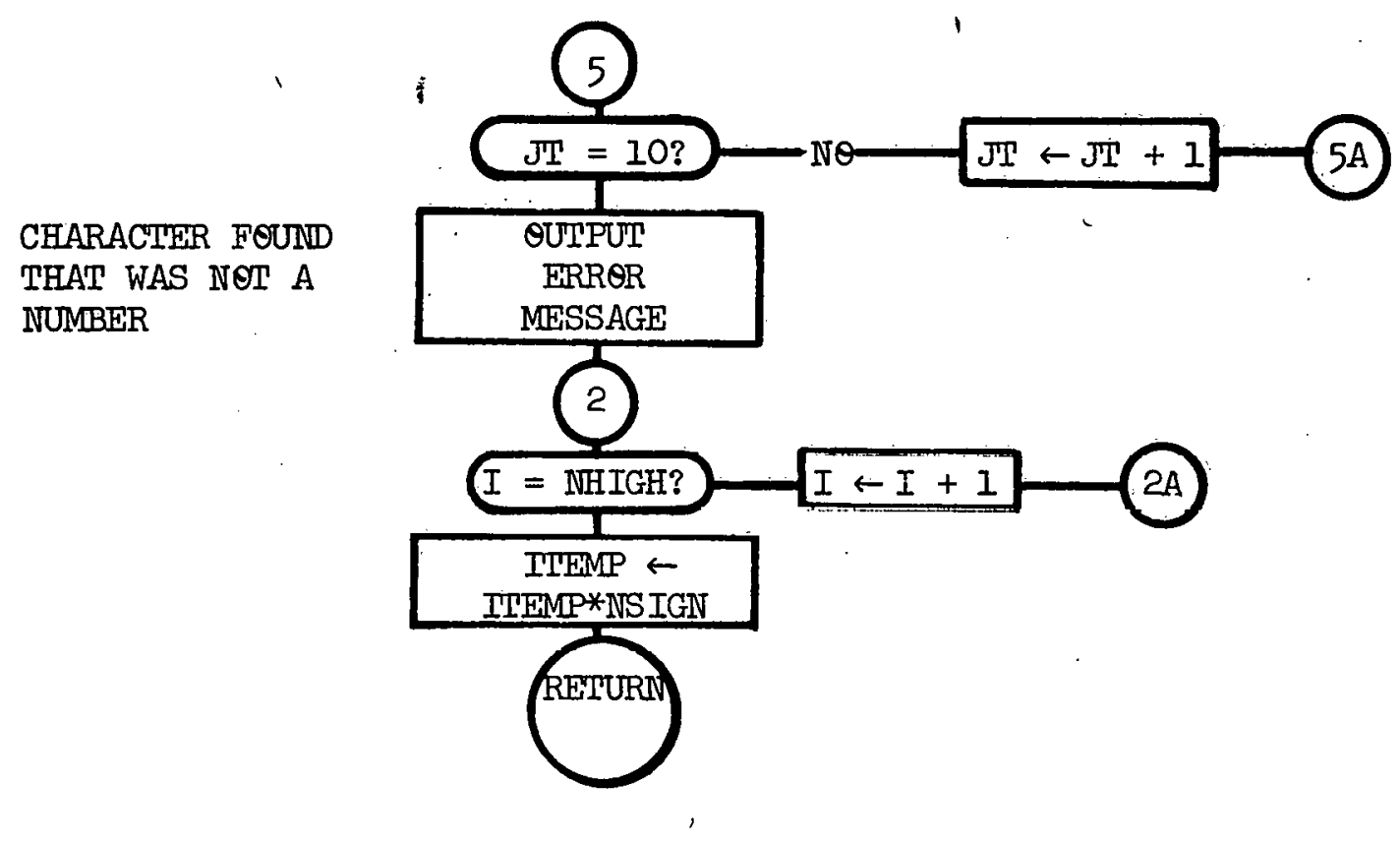



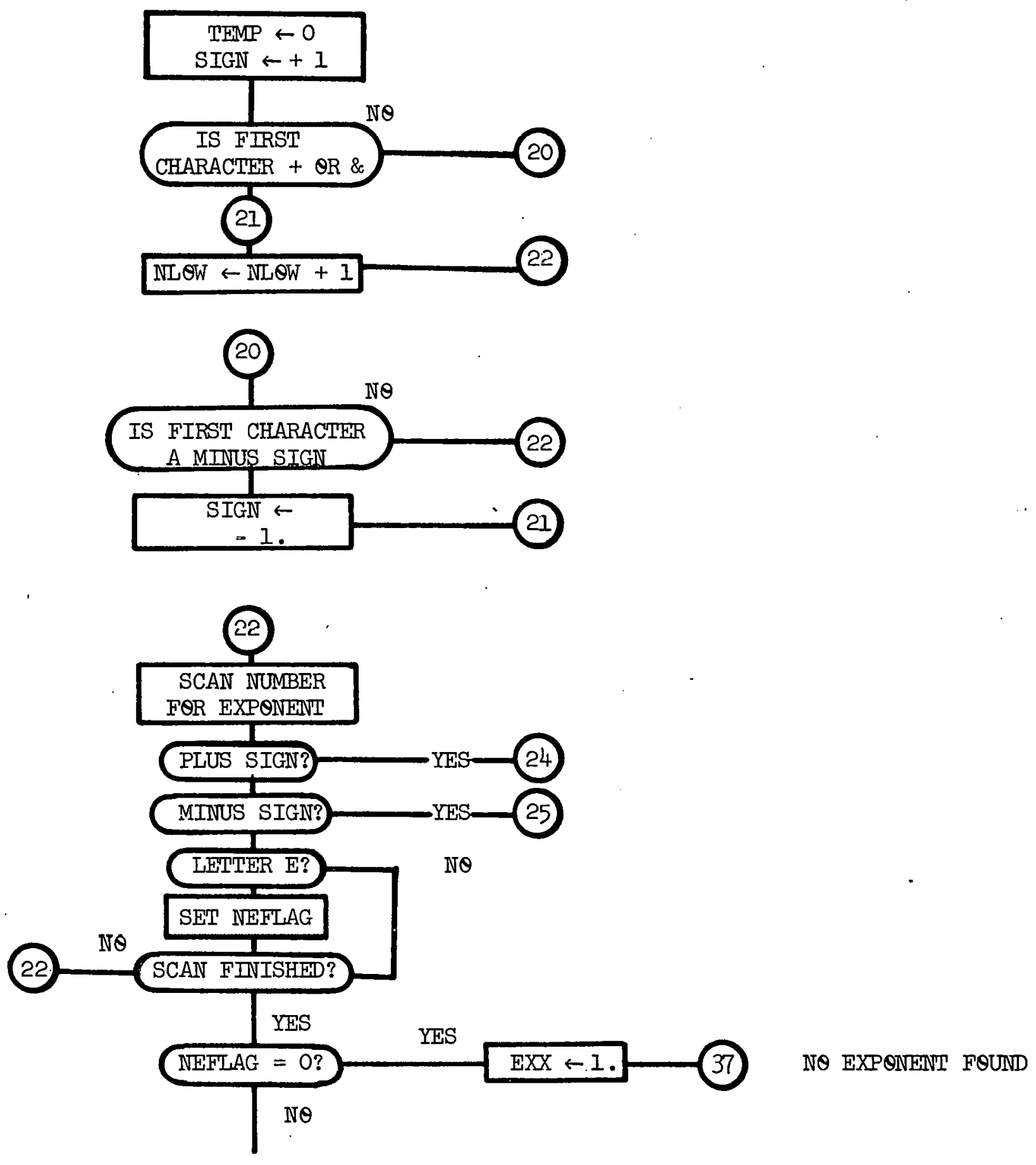
LETTER E FOUND,

BUT NO SIGN

HIGHEST COLUMN

OF FRACTION

FIRST COLUMN

OF EXPONENT

PLUS SIGN

FIRST COLUMN

OF EXPONENT

MINUS SIGN

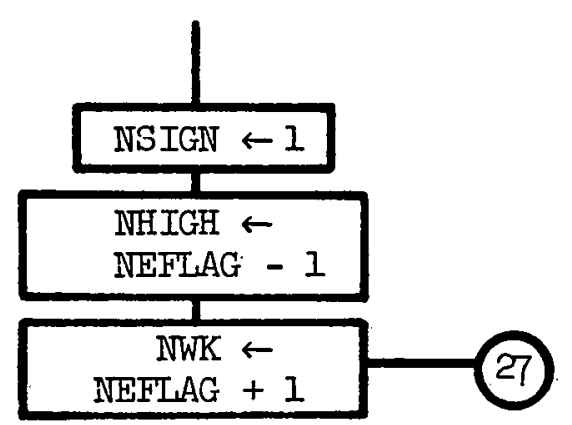

(24)

NSIGN $\leftarrow+1$

NWK $\leftarrow I+I$

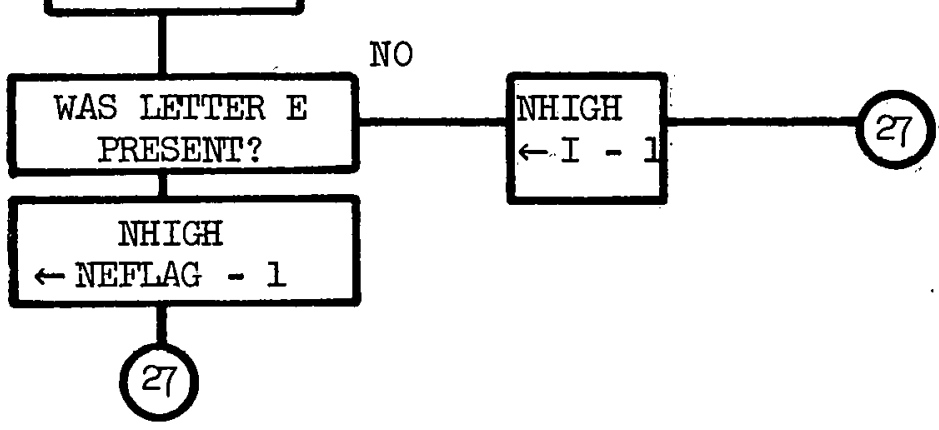

(25)

NSIGN $\leftarrow-1$

$\mathbb{N W K} \leftarrow I+I$

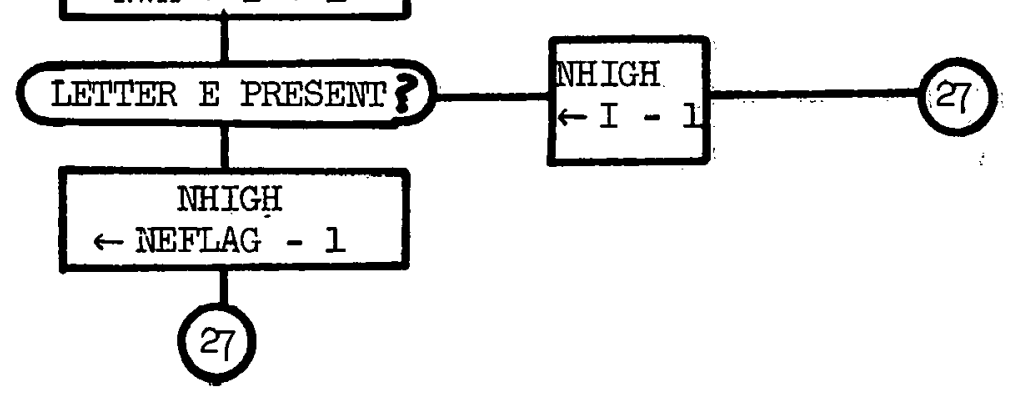


EXPONENT TO

BE EVALUATED

EVALUATE

FRACTION

IN COLUMNS

INLOW TO

NHIGH.

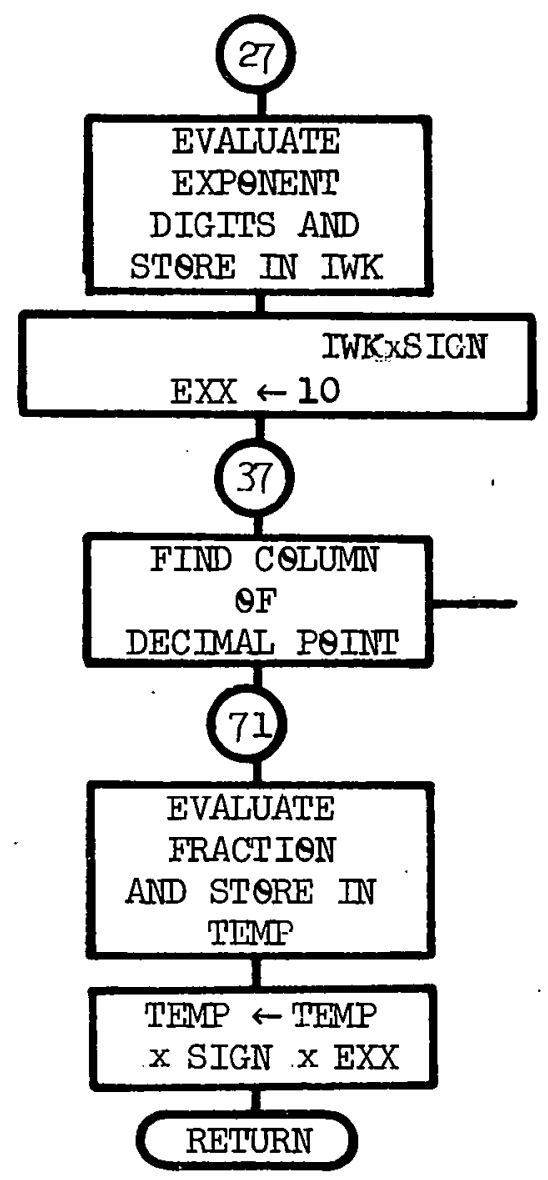

IF NO DECTMAL POINT
OR MORE THAN ONE

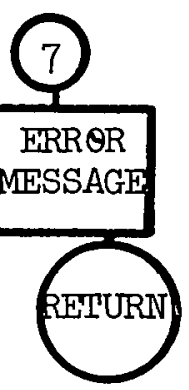


TYPE CODE. SEPARATE TYPE CODE FROM VARY CODES

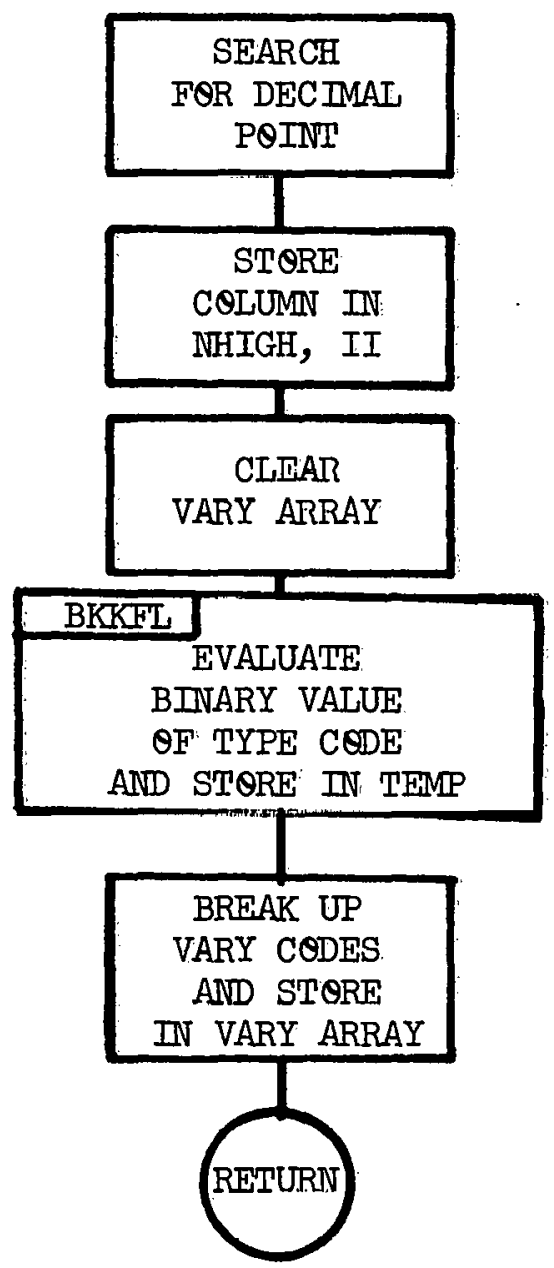




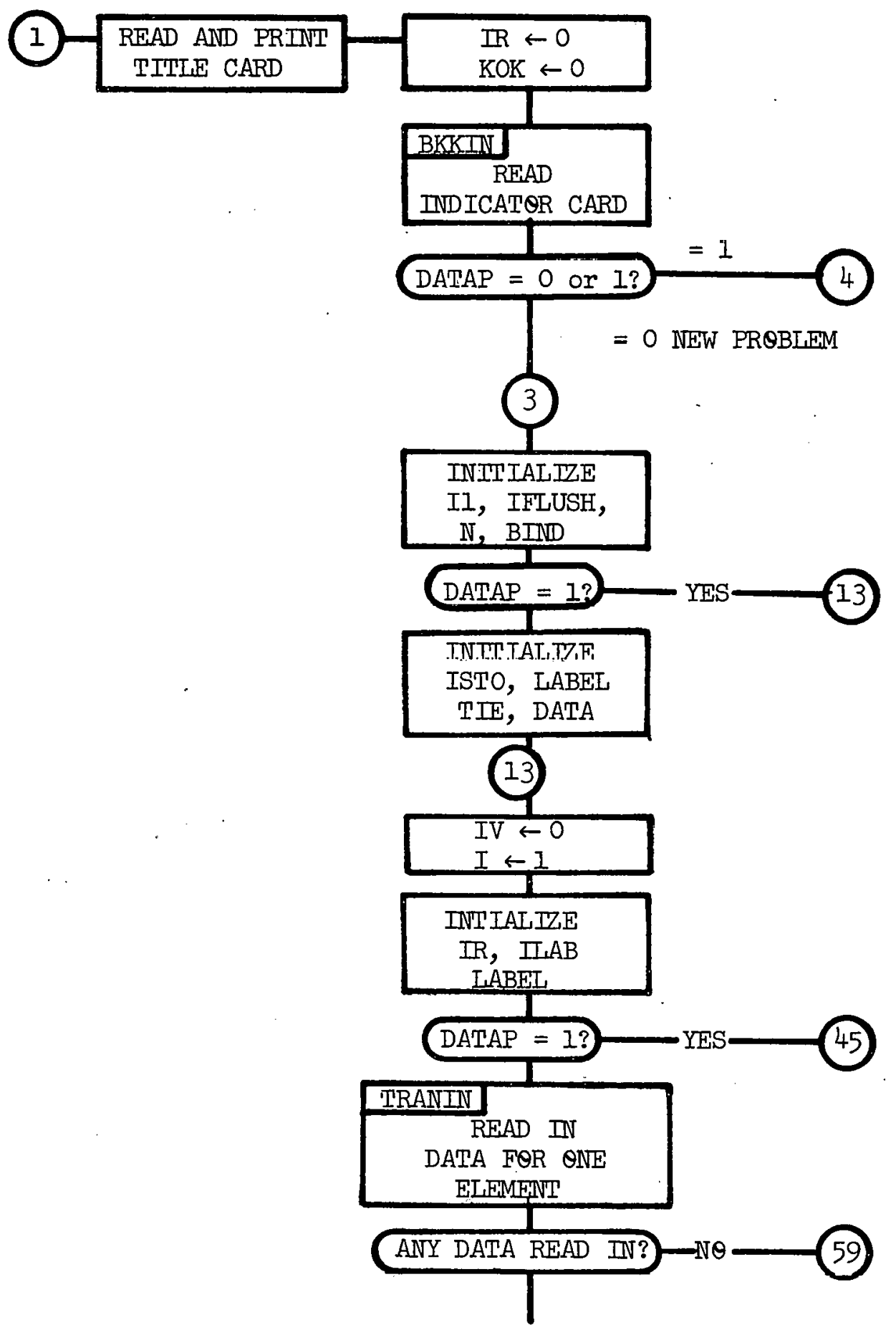


-SUBROUTINE FITTIN (2):-

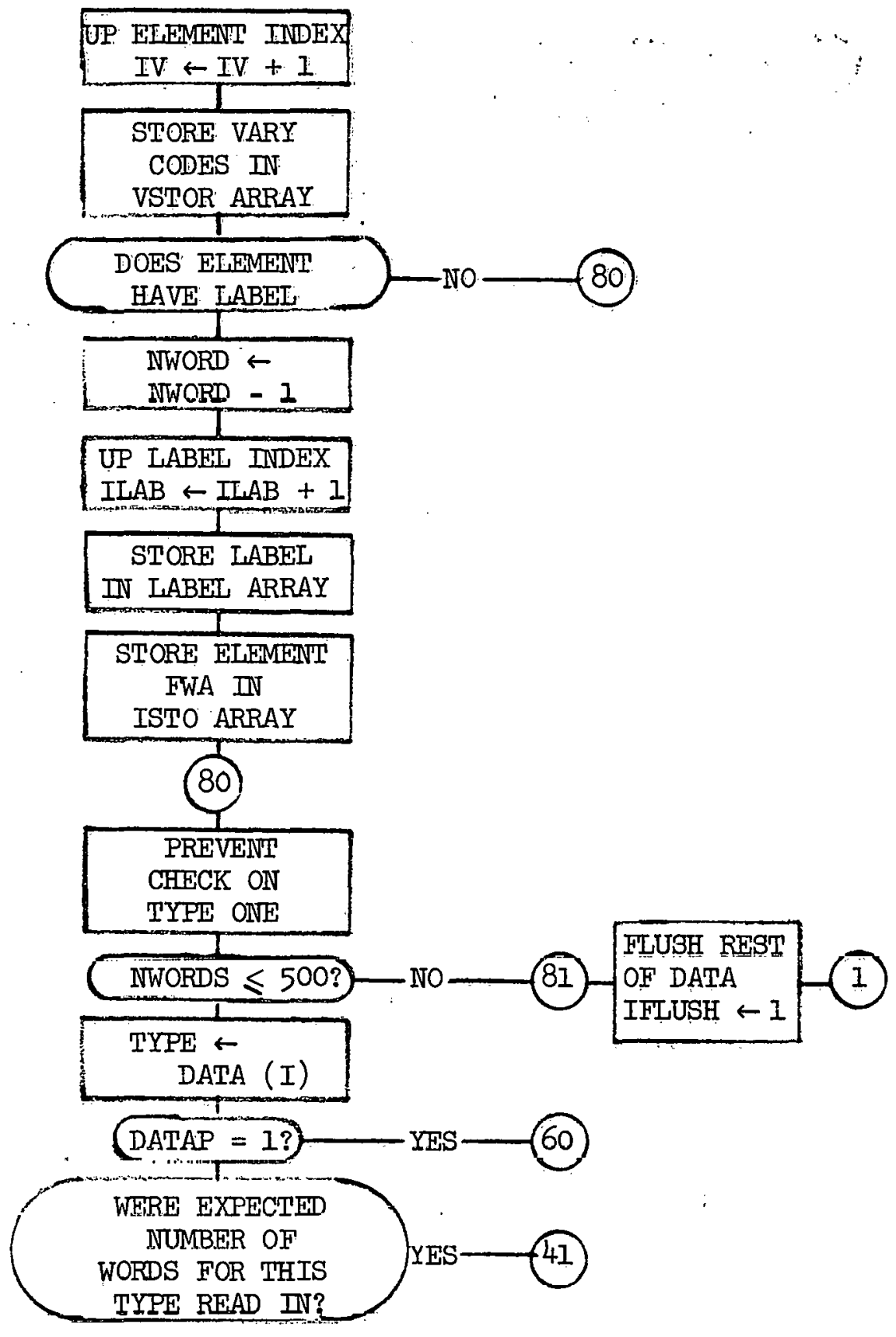


-SUBROUTINE FITTIN (3)-

FEWER WORDS READ IN

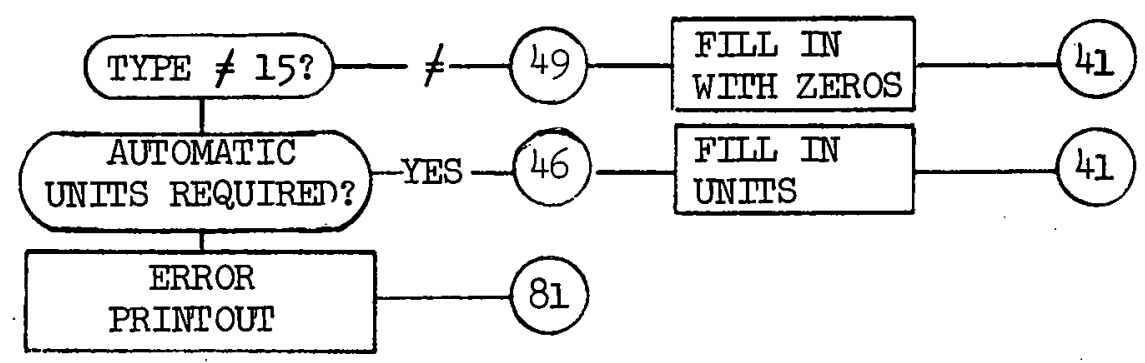

CHECK VARY CODES FOR THIS ELEIMENT AND SET UP TIE ARRAY

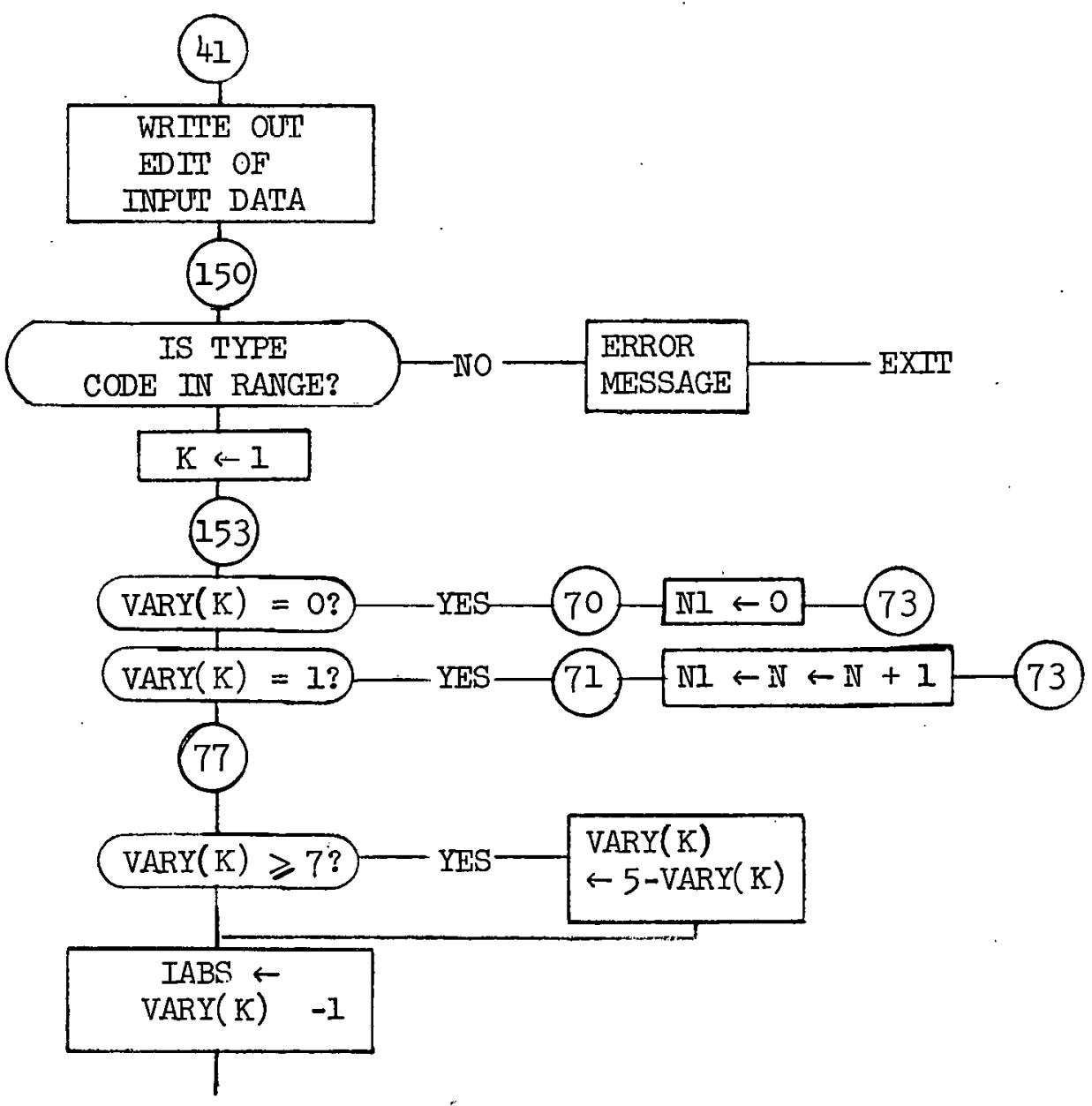




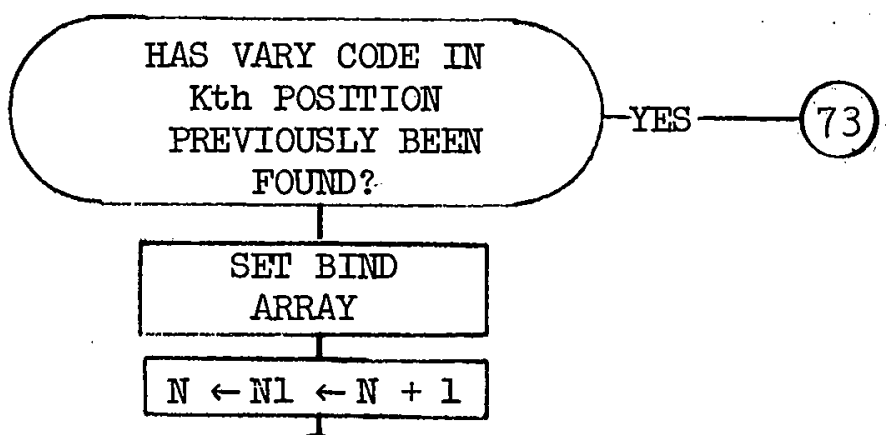

INCREMVENT INDEX OF F.W.A. OF ELEMENTS IV DATA ARRAY

RETURN TO PROCESS ANOTHER ELEMENTI

SET NI TO ZERO SO NO FIITING WILL OCCUR FIRST TIME THROUGH<smiles>[CH+]1CCCCC1</smiles>

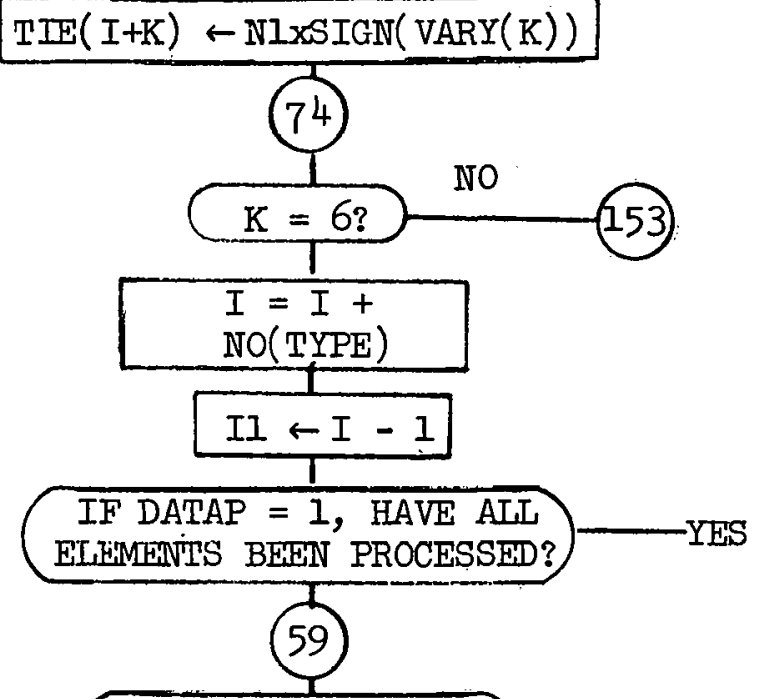

HAS SENTINEL BEEN ENCOUNTPERED?

NNISVE $\leftarrow$ NI $N I \leftarrow N \leftarrow 0$

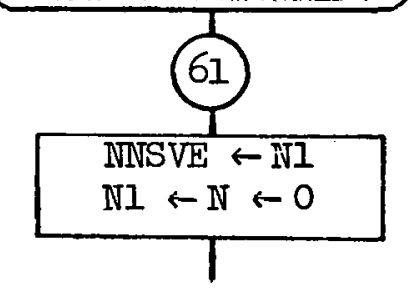


OUTFIT CALLS ELEMENTS WHICH CALCULATES THE R MATRICES FOR THE ELFIMENTS
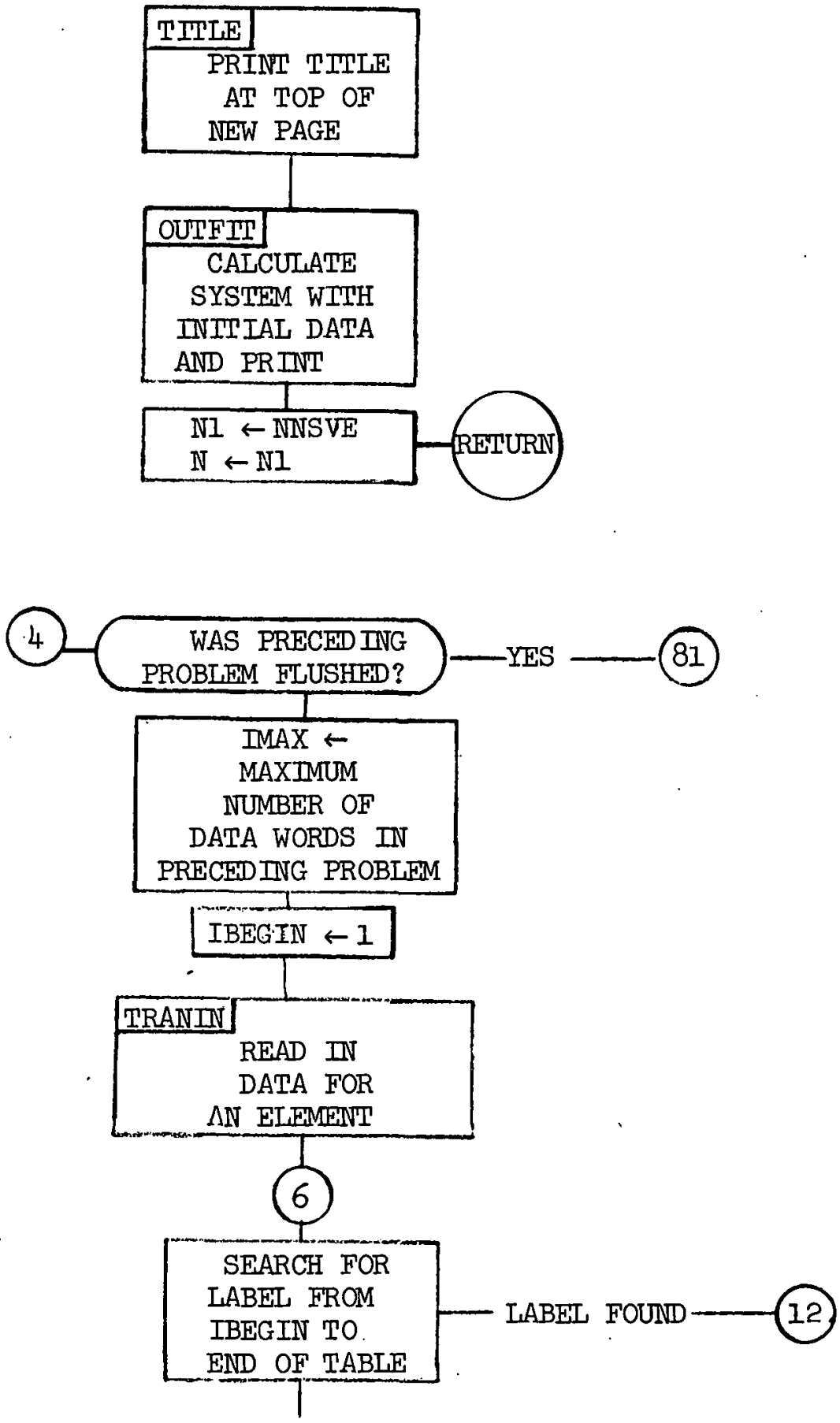

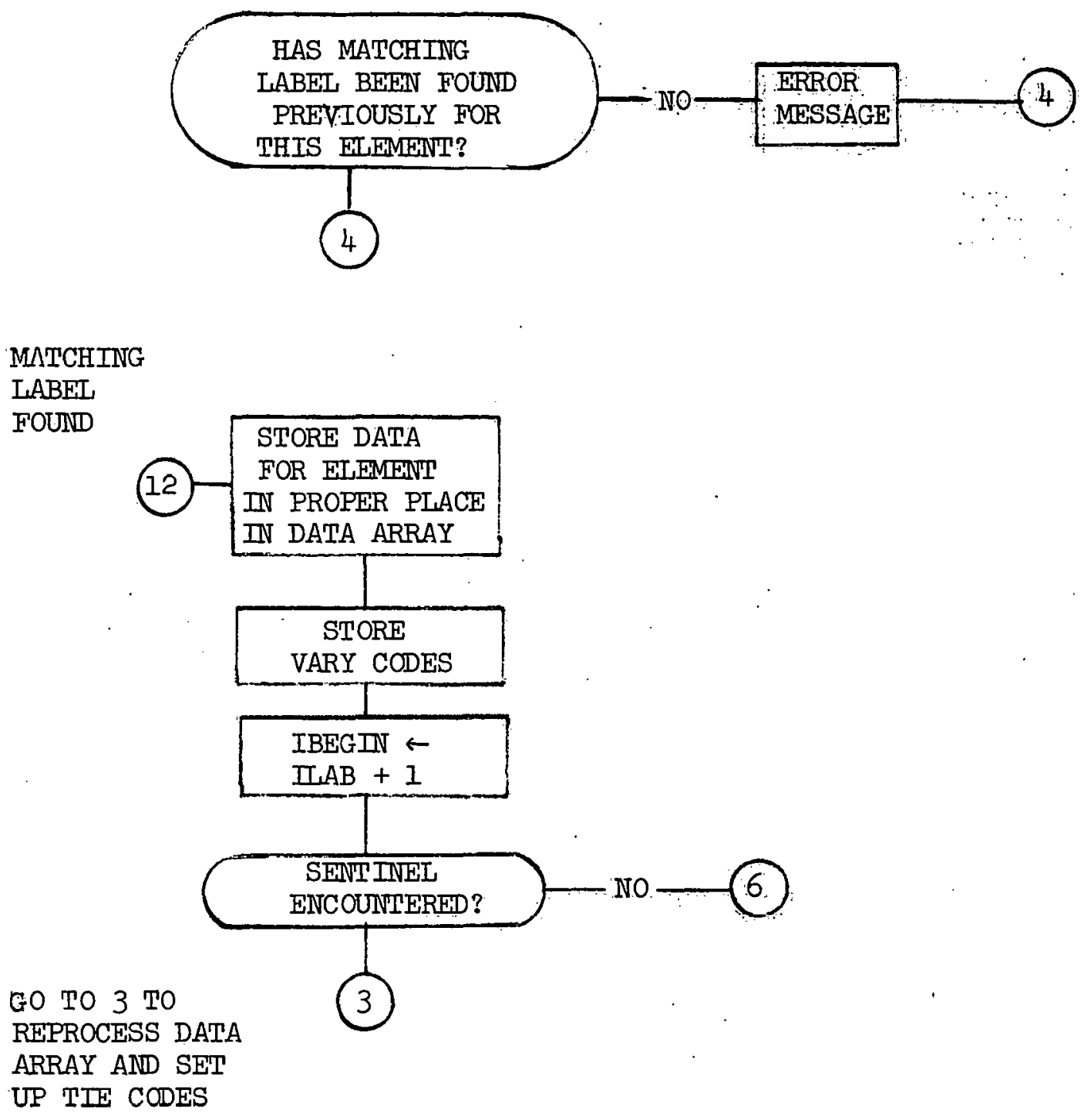


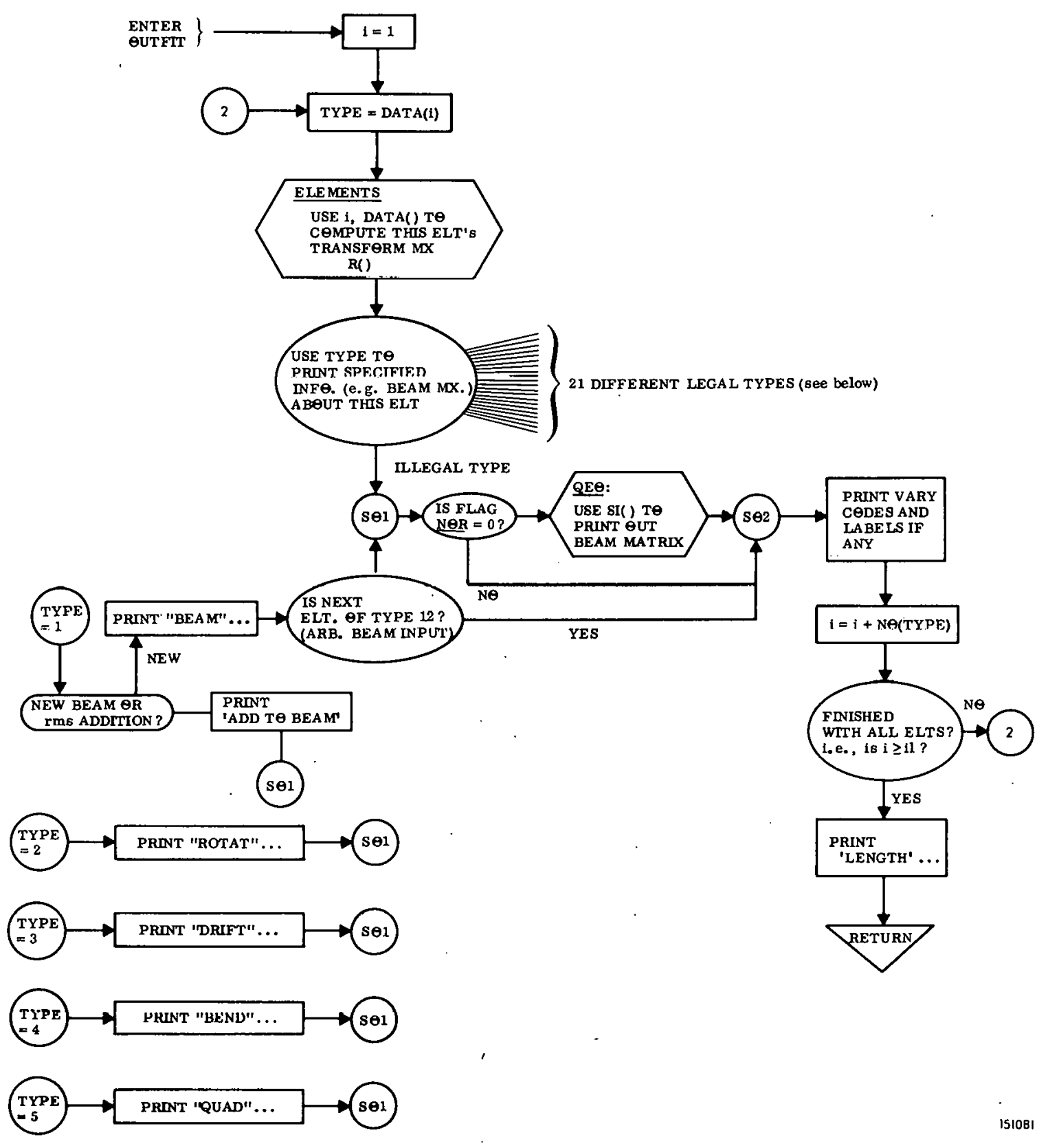



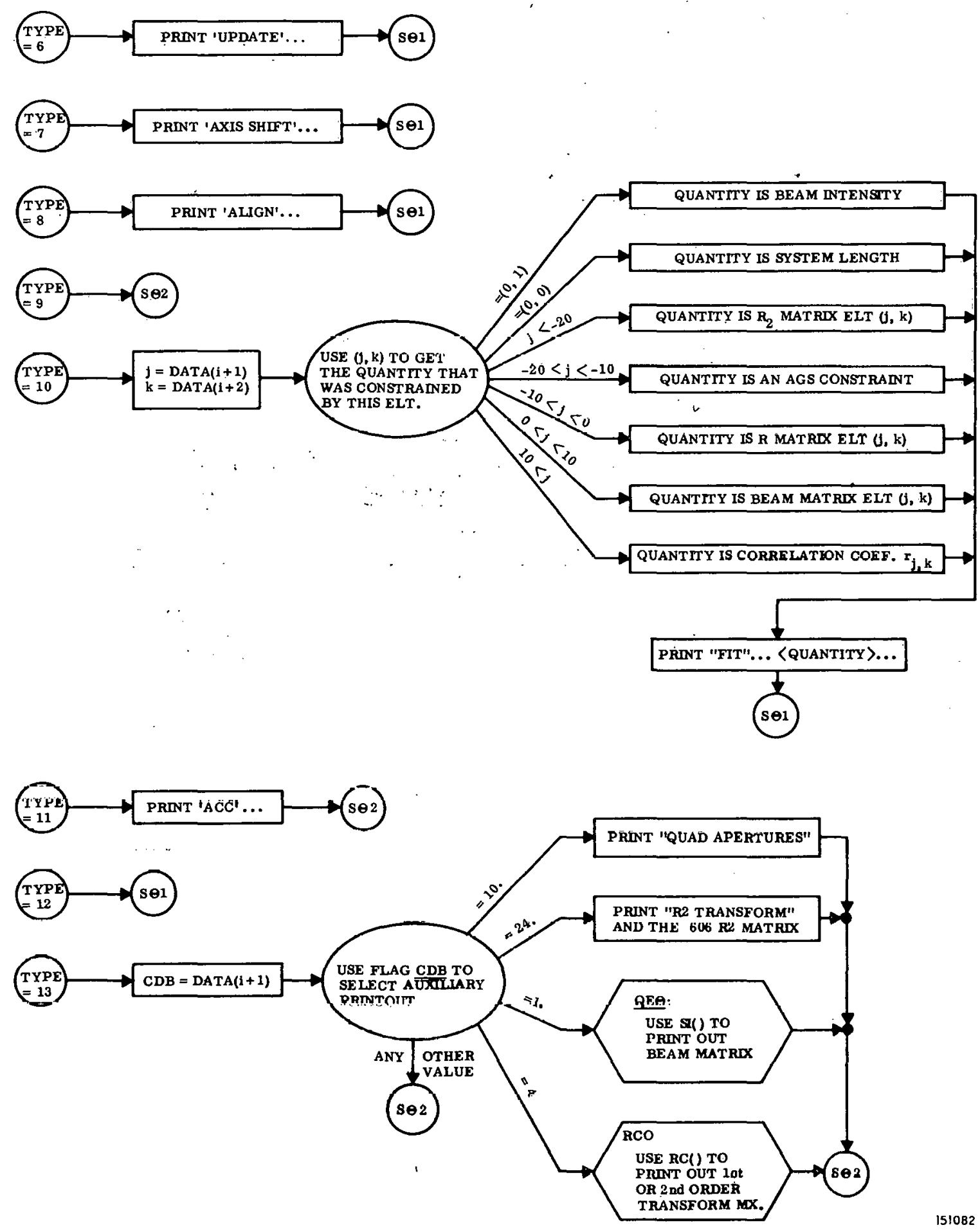
-OUTFIT SUBPROGRAM( 3 )-
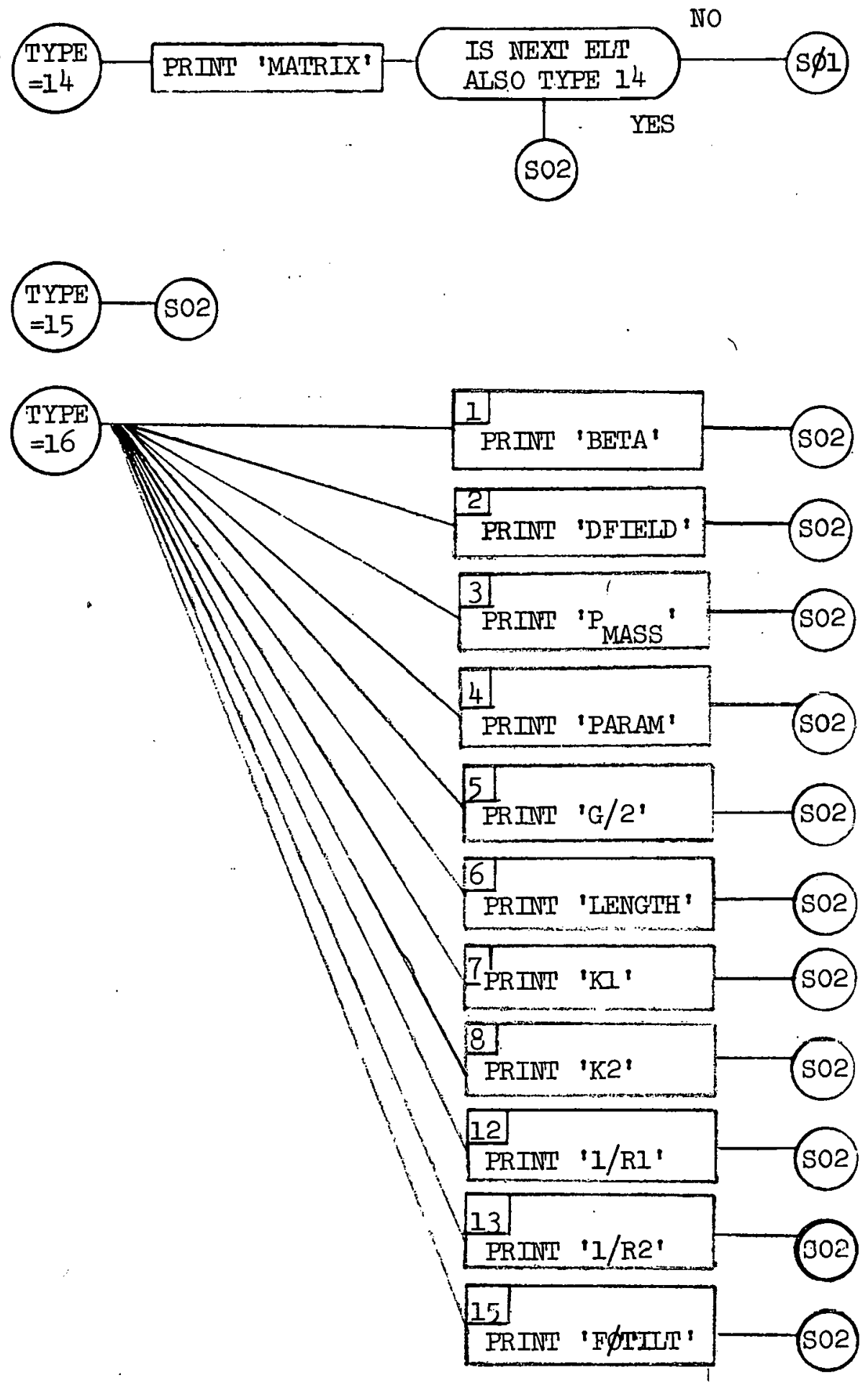
-OUTFIT SUBPROGRAM(4) -

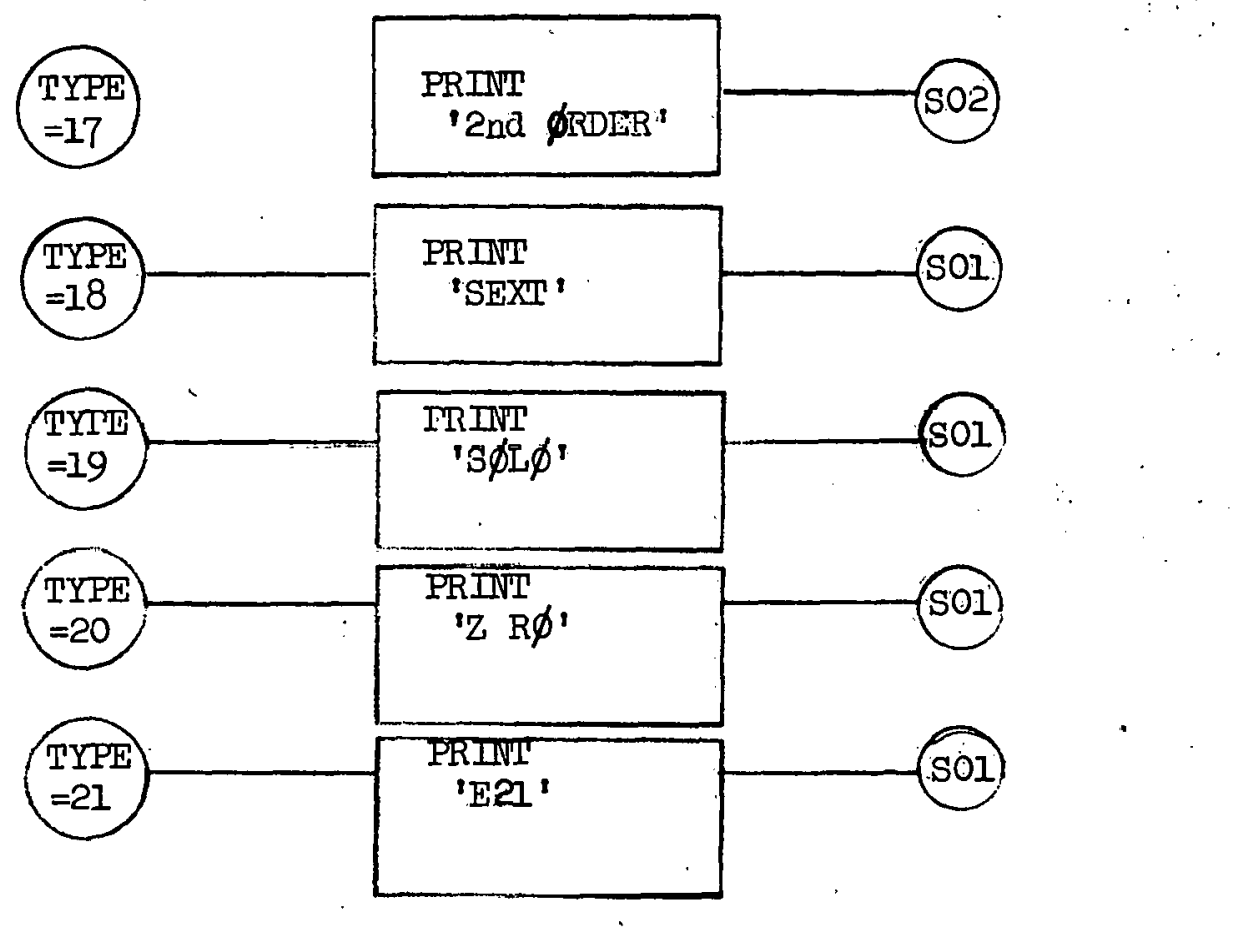




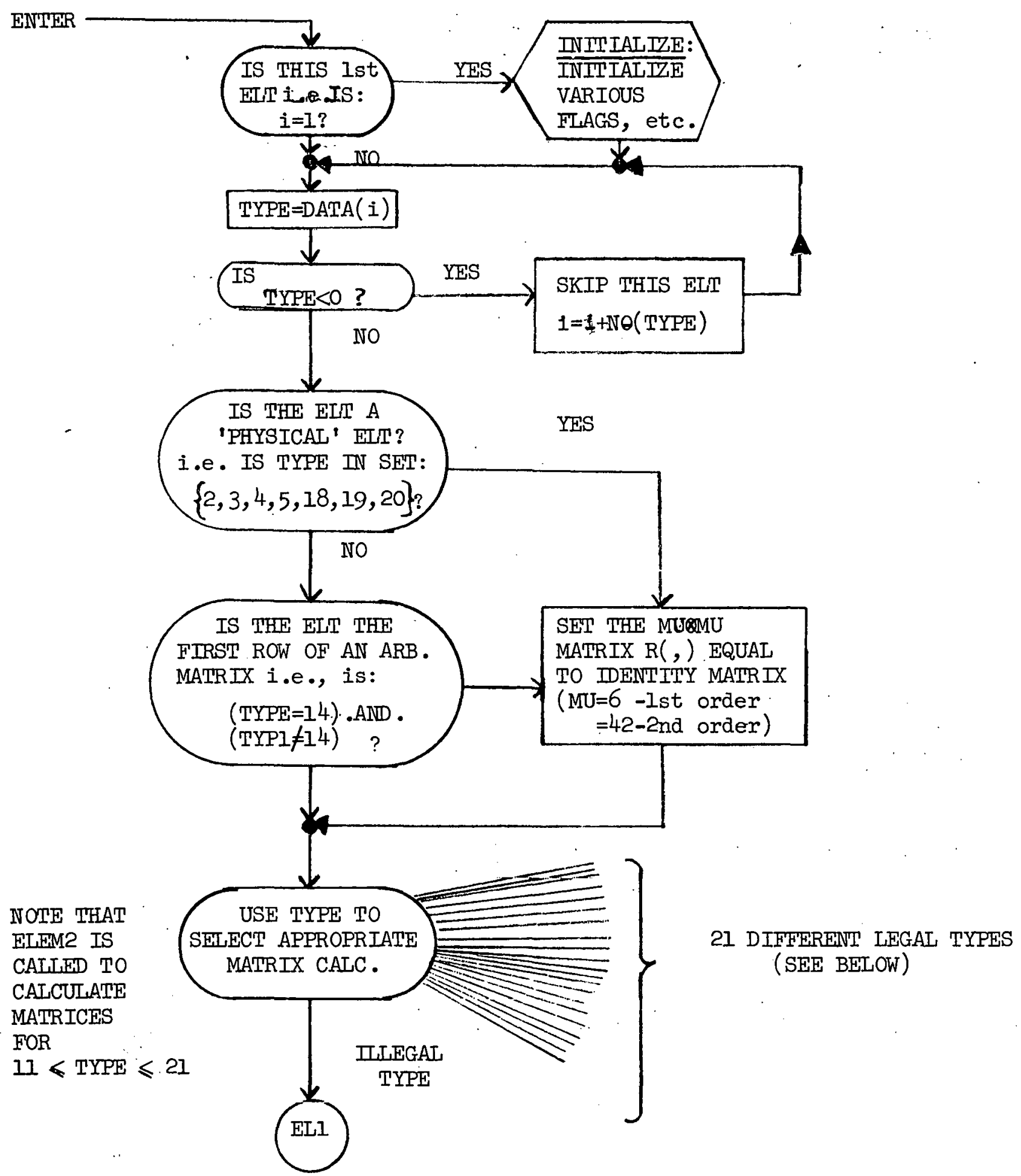


-ELEMENT SUBROUTINE (2)-
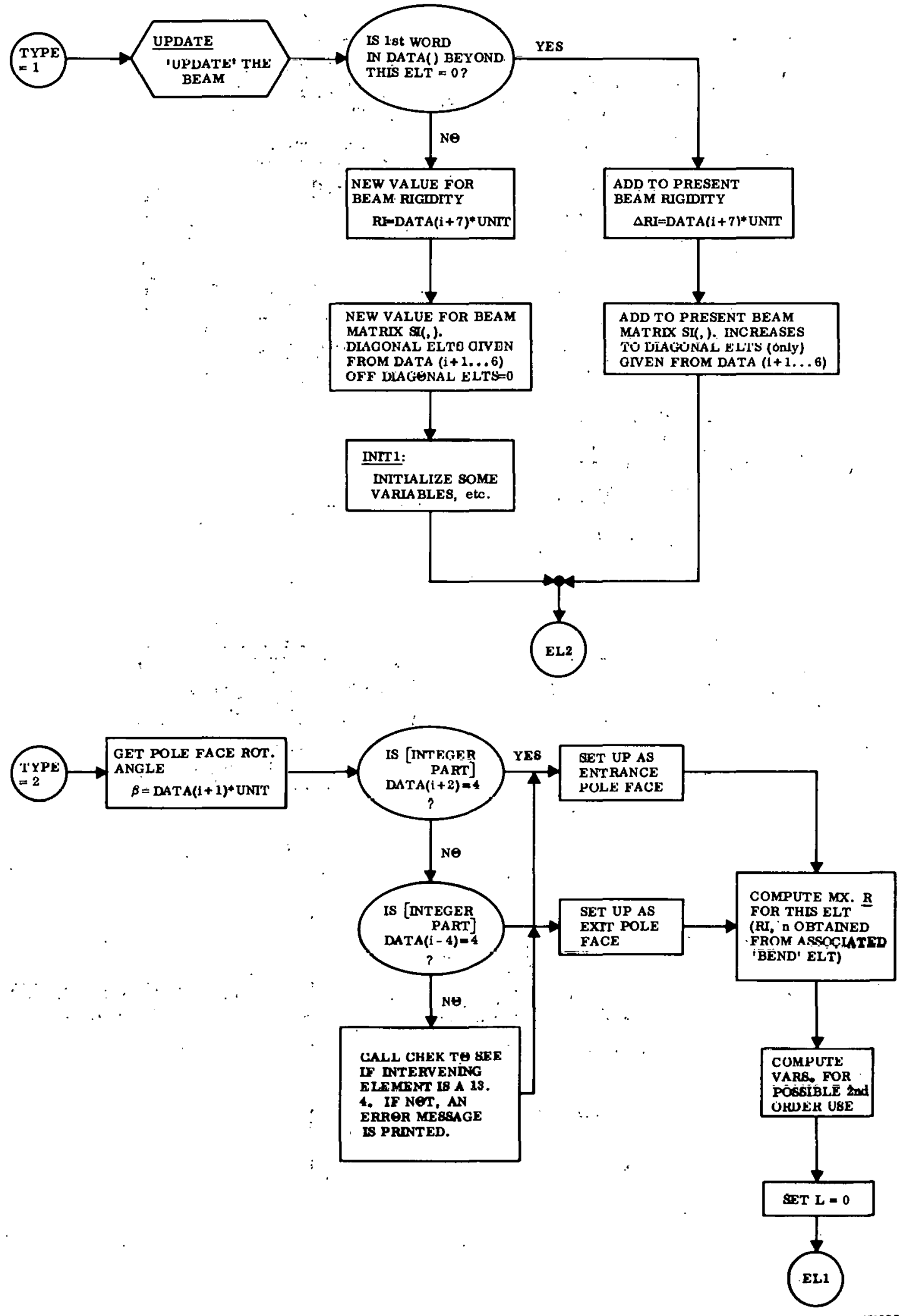

$1510 \mathrm{BJ}$ 
-ELEMENT SUBROUTINE (3ץ-
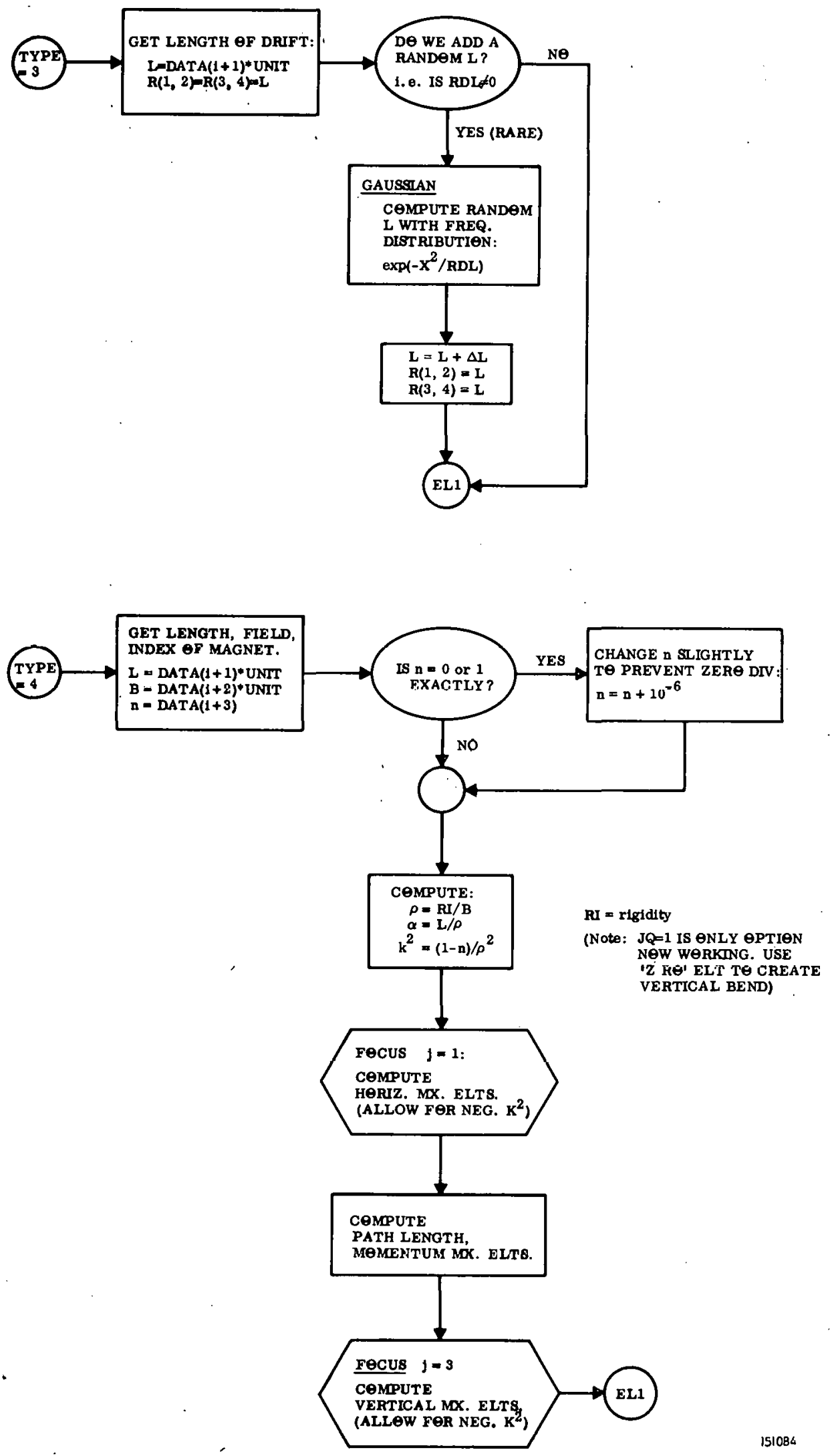


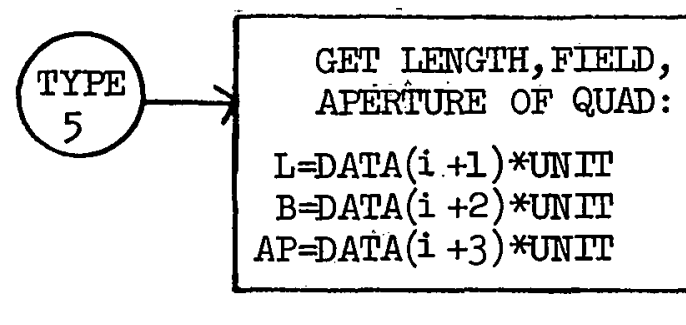

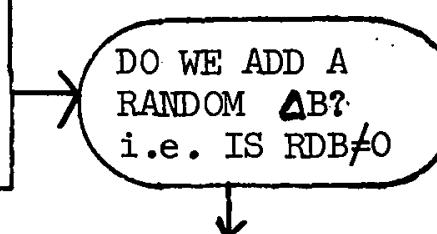

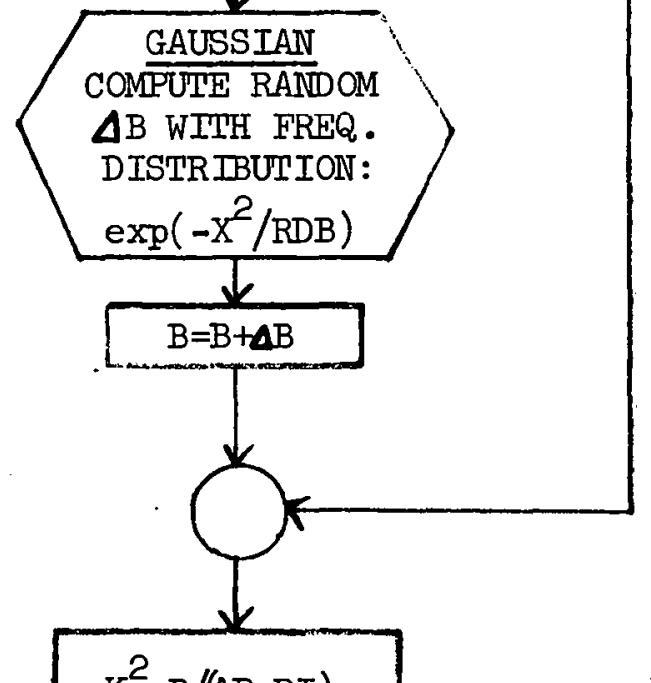

$K^{2}=B /(A P \cdot R I)$

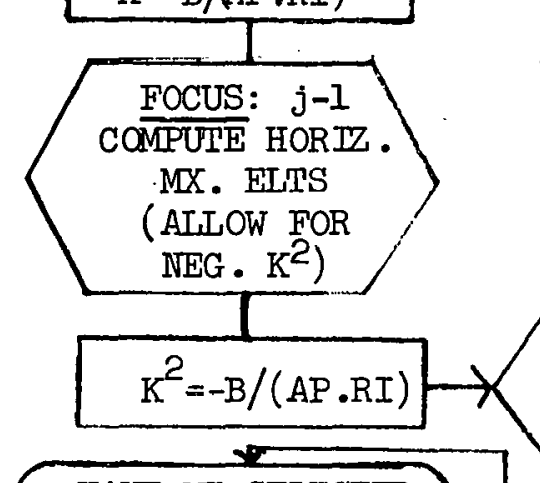

HAVE WE SELECTED "QUAD APERTURES"?<smiles>CC(C)C1CCCCC1</smiles>

FOCUS: $j=3$

COMPUTE VERT.

MX. LLTS (ALLOW

FOR NEG. $\left.\mathrm{K}^{2}\right)$
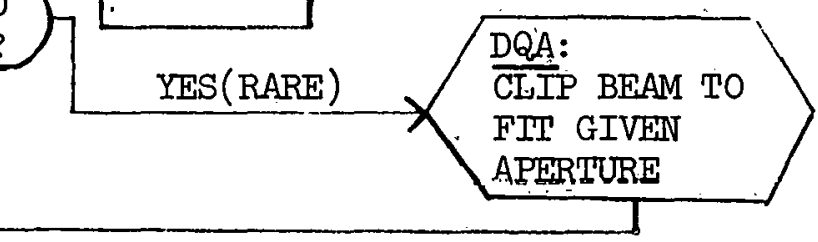

DO WF H.AVF: MON-ZERO CENIRAL TRAJECTORY?

OBTAIN $\left(. \mid x_{0}\right)$ TERMS, THEY ARE NOW lst ORDER WIITH $\chi$ REPLACED BY CENT: TRAJ.

NO
FOLD INIO R(, ) TíHE EFFECT OF NON-ZERO CENT 。 TRAJECTORY:
SET:

$\mathrm{SIP}=0$

ELI 


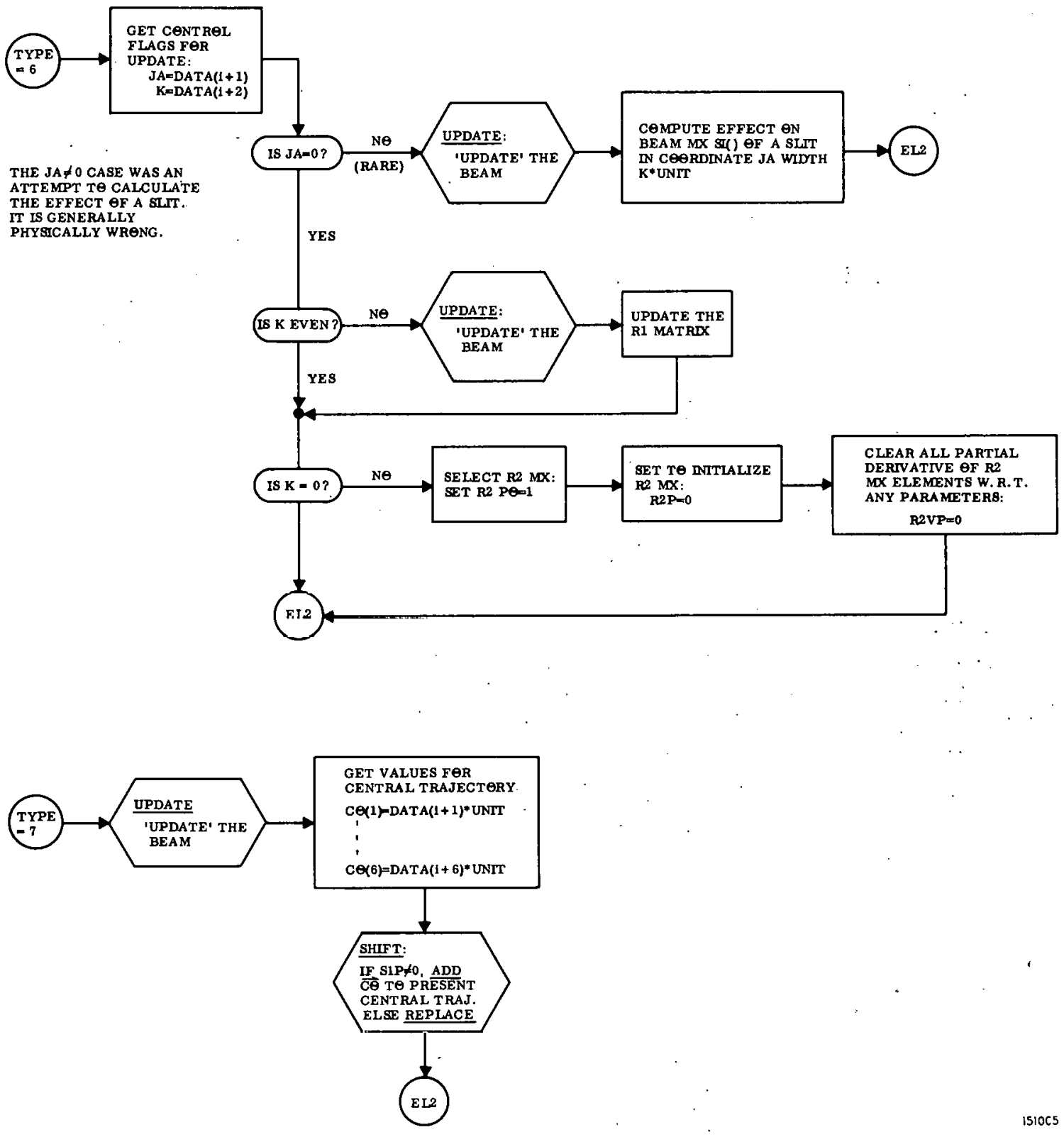




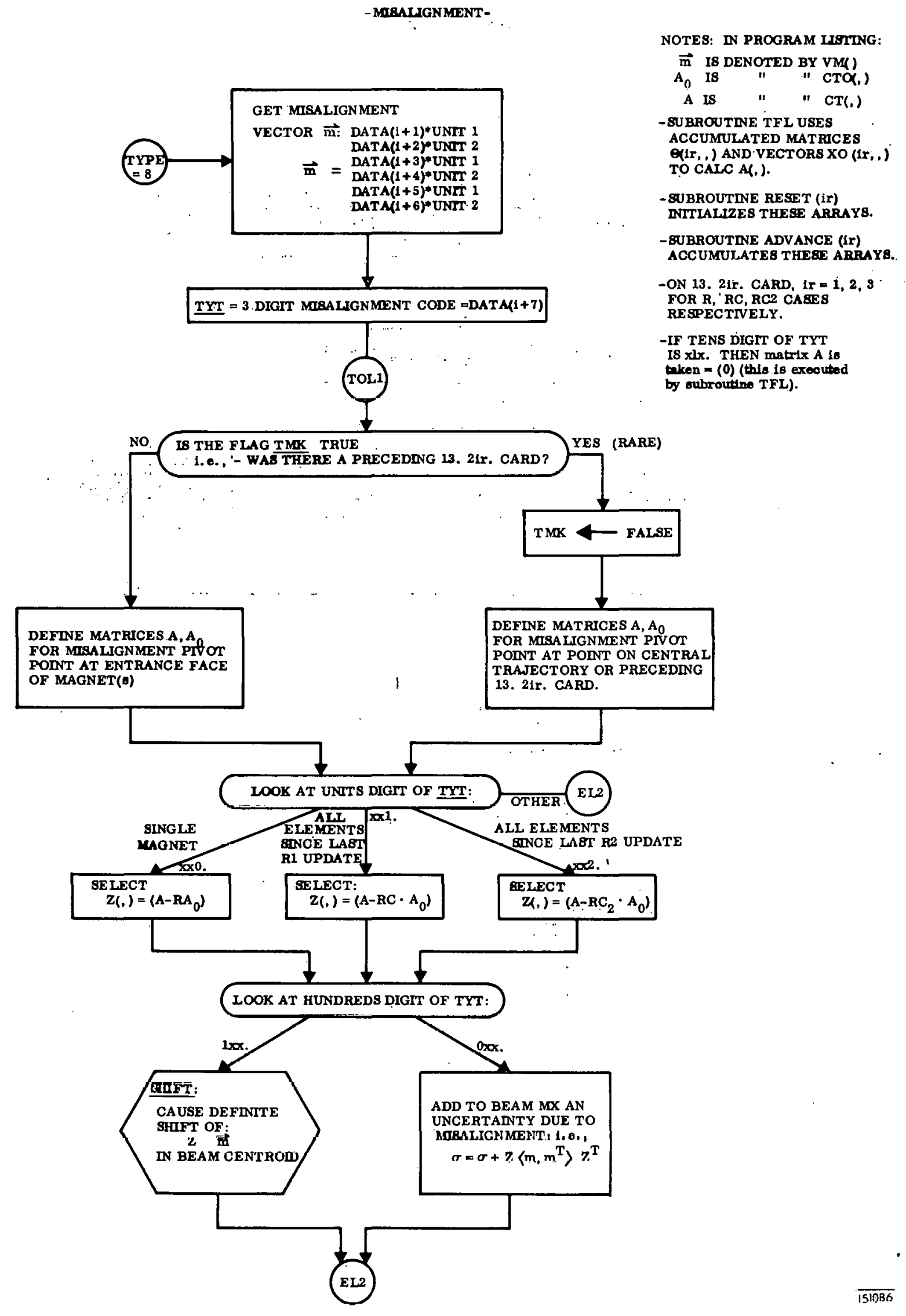


-ELEMENT BUBROUTINE (6)
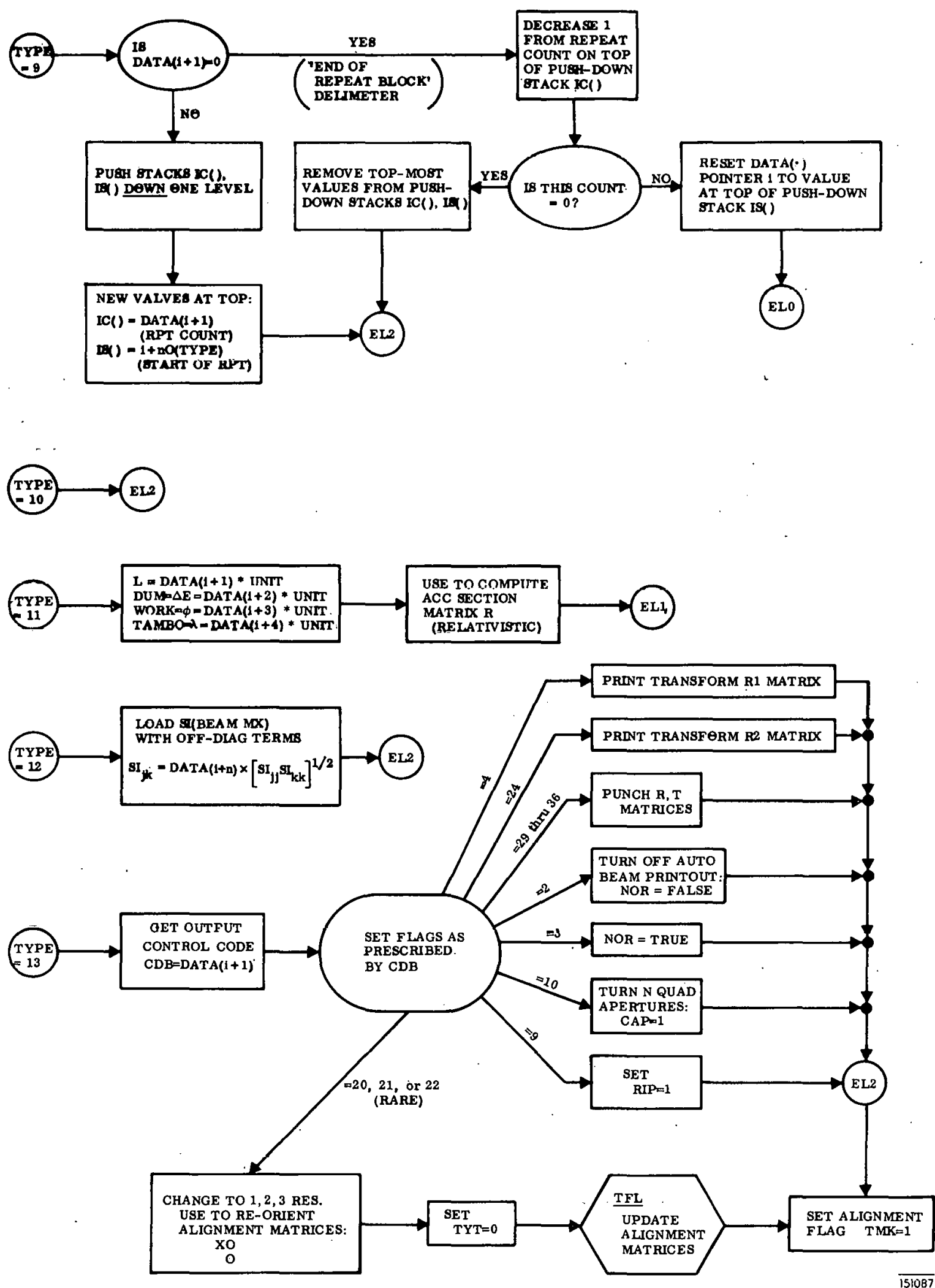

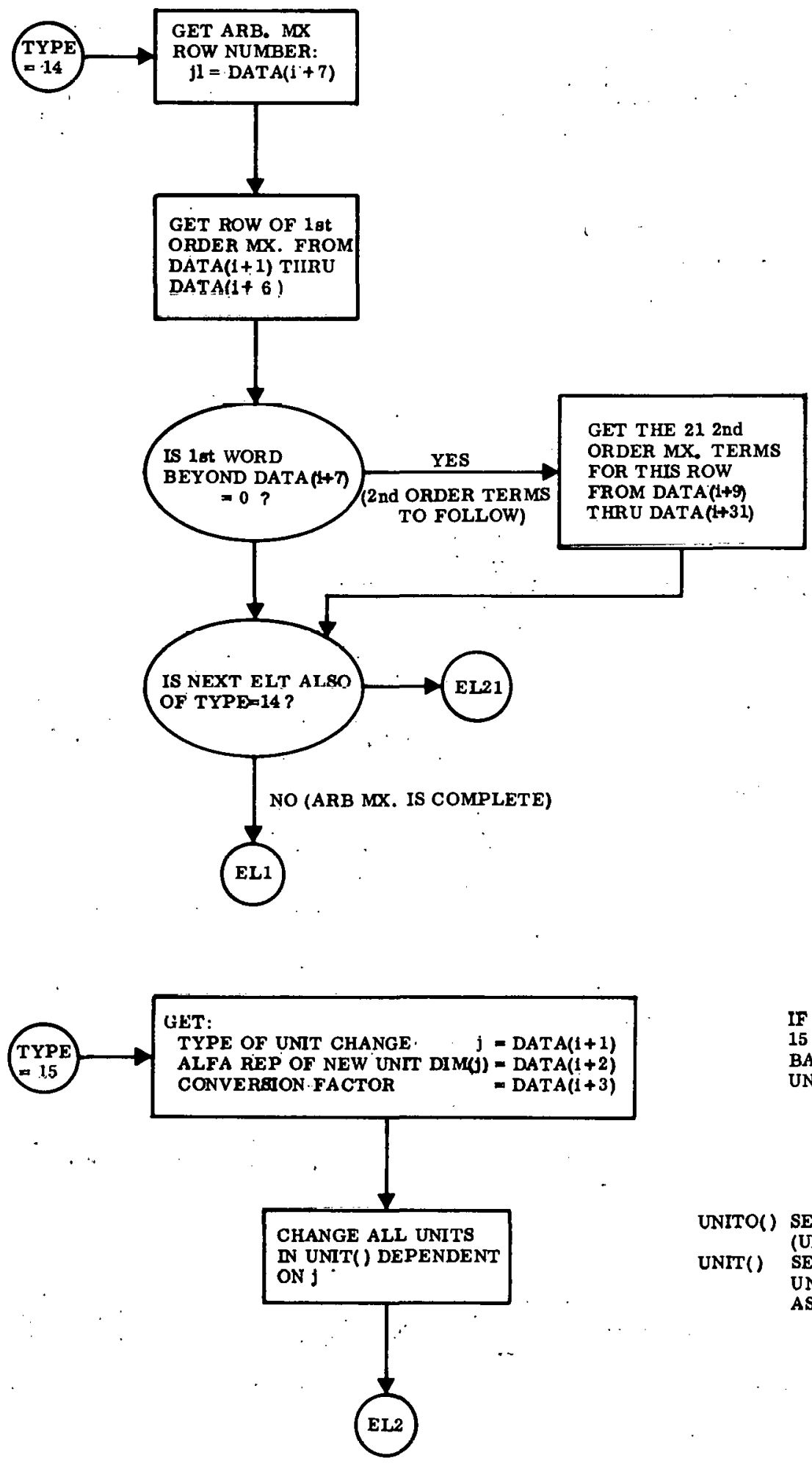

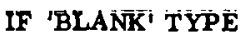
15 CARD, CHANGE BACK TƠ STANDARD UNITS

UNITO() SET OF STANDARD UNITS (UNCHANGEABLE)

UNIT() SET OF USER-CHANGEABLE UNITS, INITLALLY THE SAME AS UNITO() 

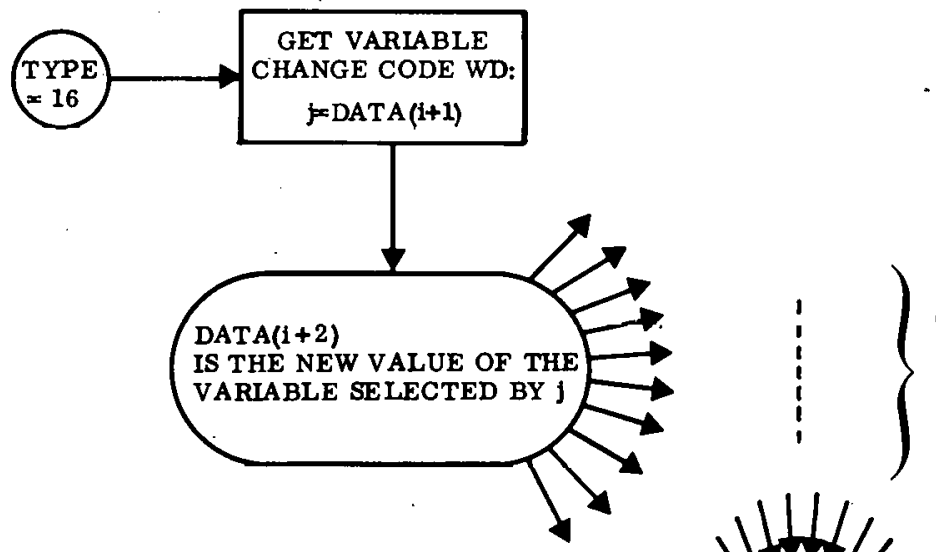

(SEE TRANSPORT MANUAL FOR TABLE OF j VB. SELECTED VARIABLE. e.g. IF $\mathbf{j}=1$ THE DATA $(i+2)$

IS $\beta$, THE 2nd ORDER MAGNET HOMOGENEITY COEFFICIENT)
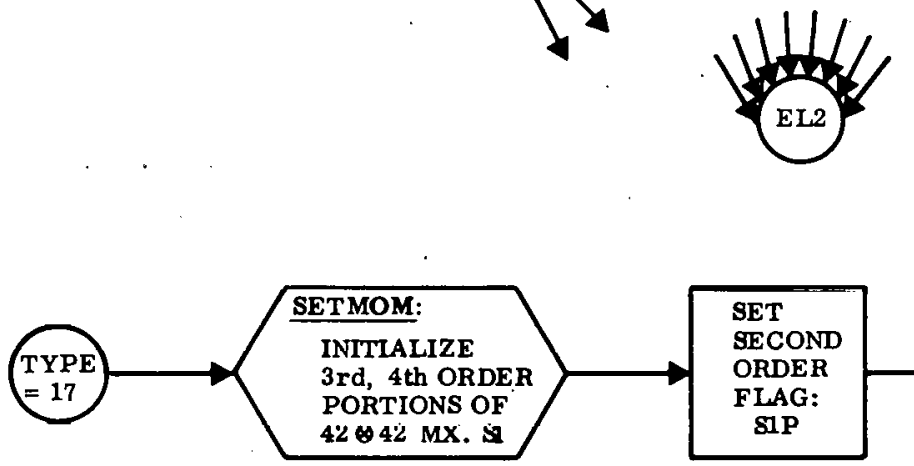

SET MX.

SIZE FOR

2nd ORDER:

MU $=42$
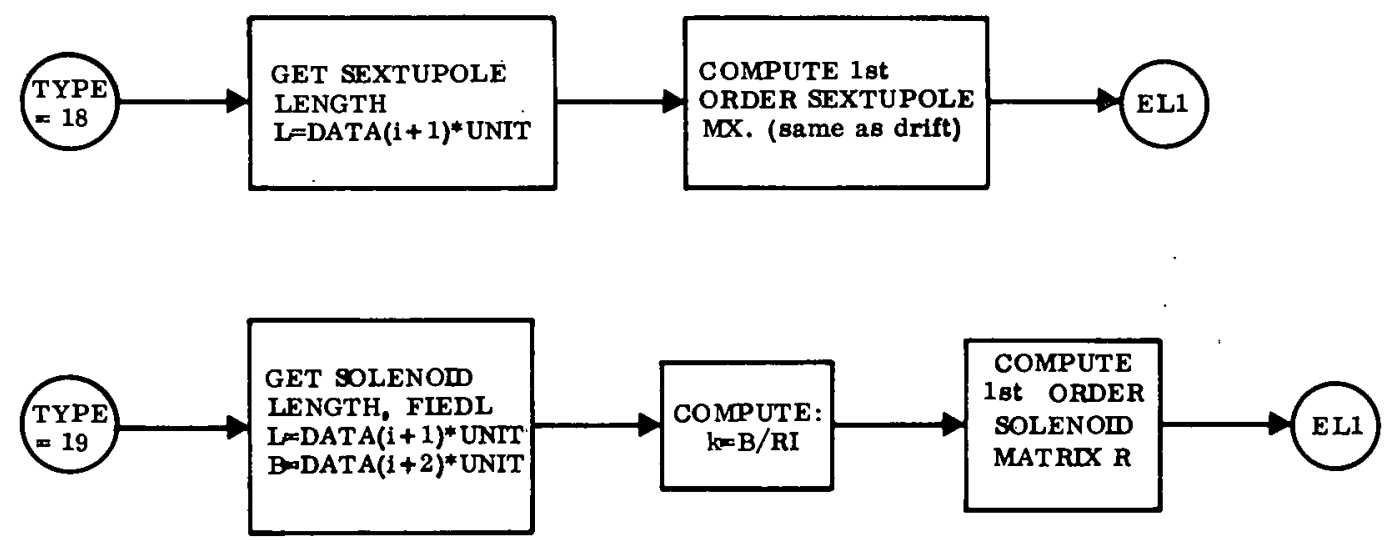
-ELEMENT SUBROUTIINE(9)-

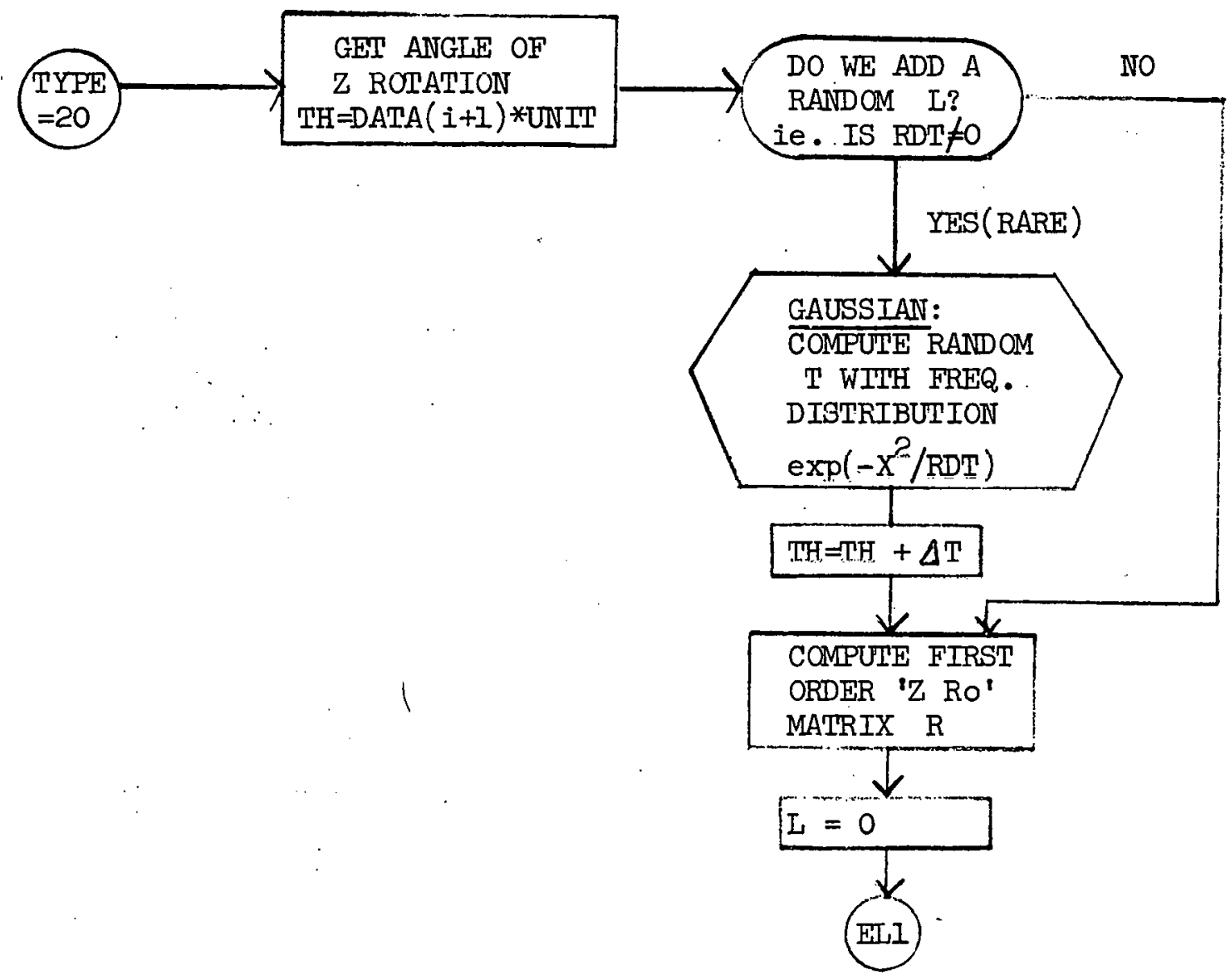




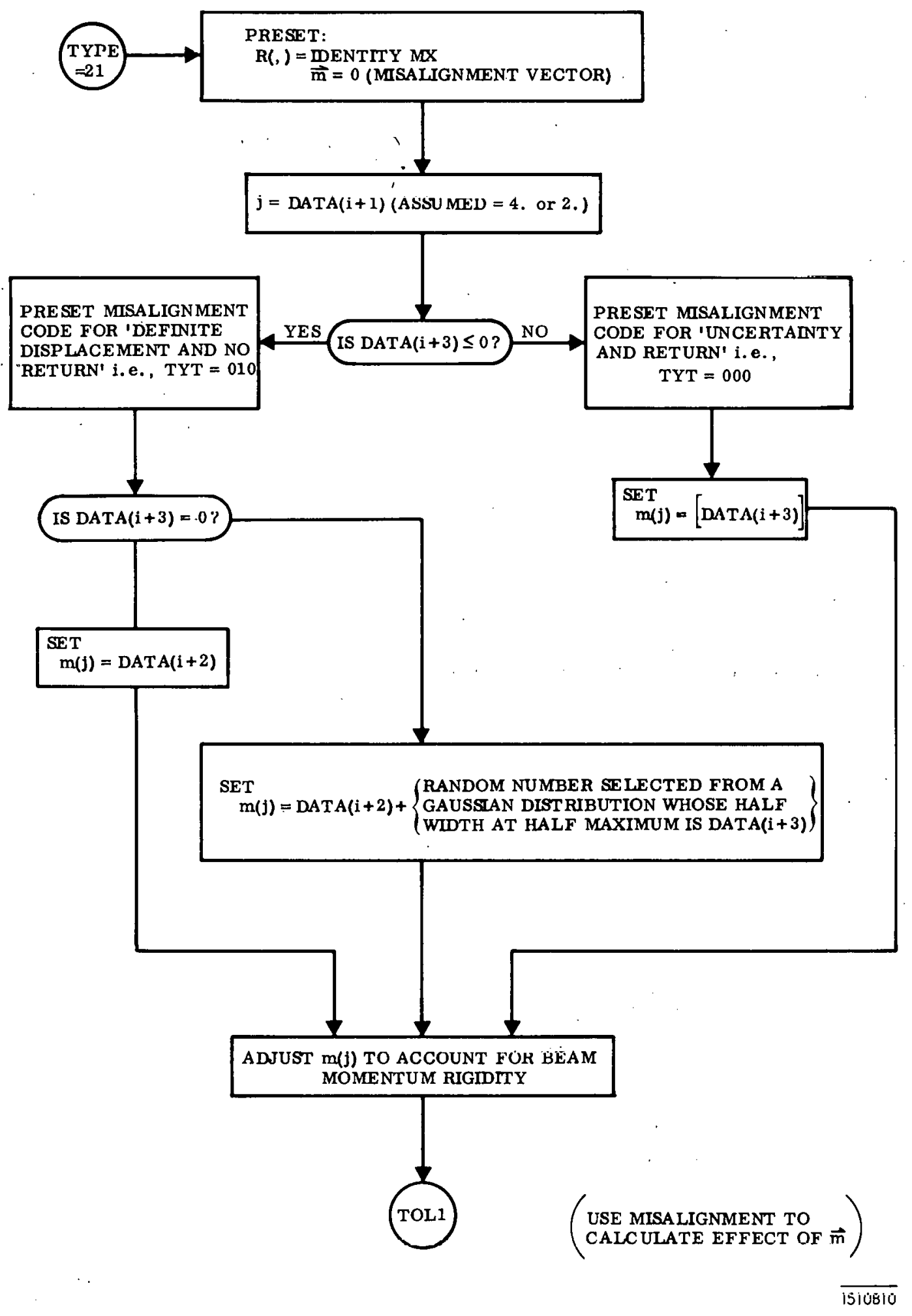




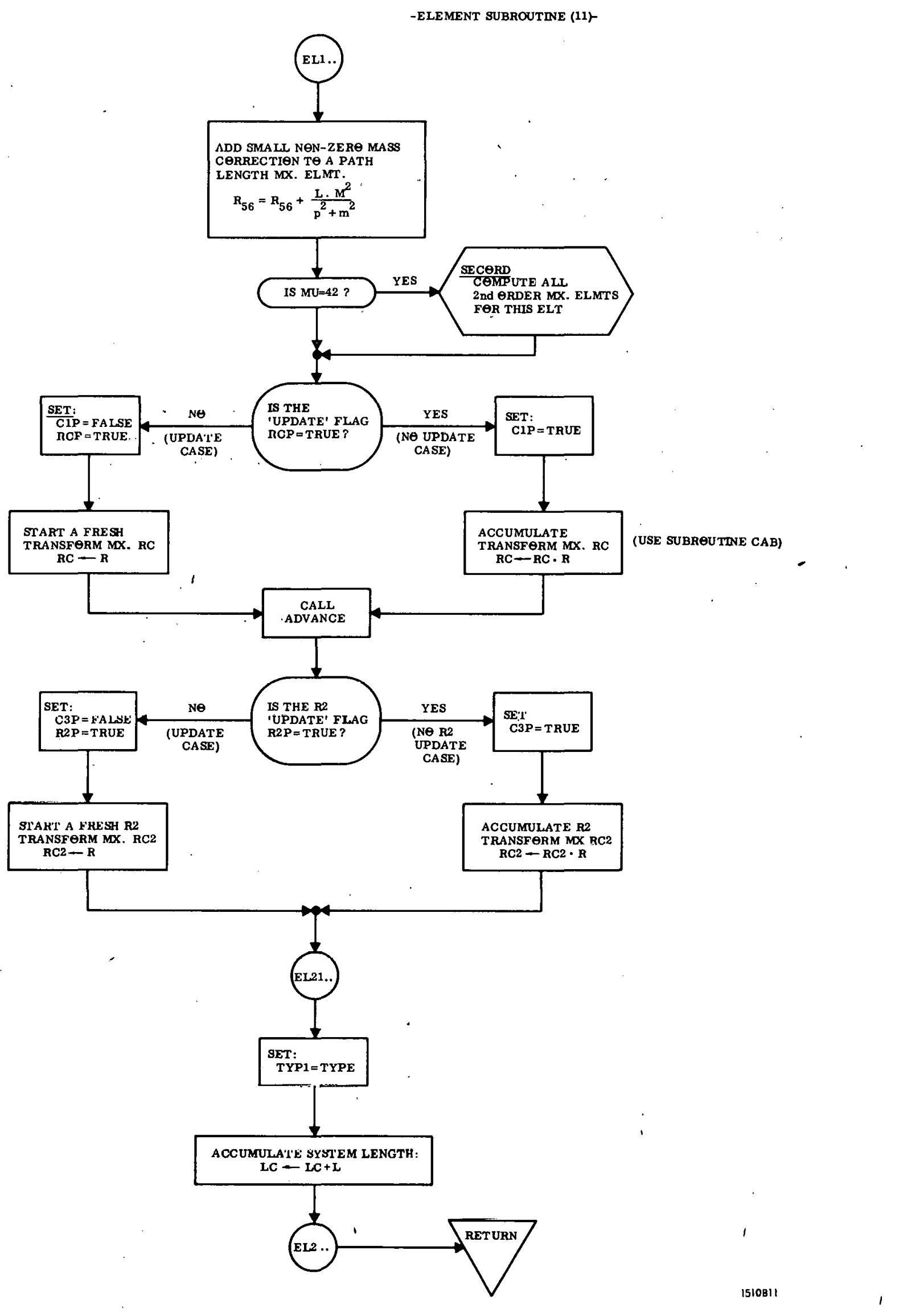


-SOLVE SUBPROGRAM-

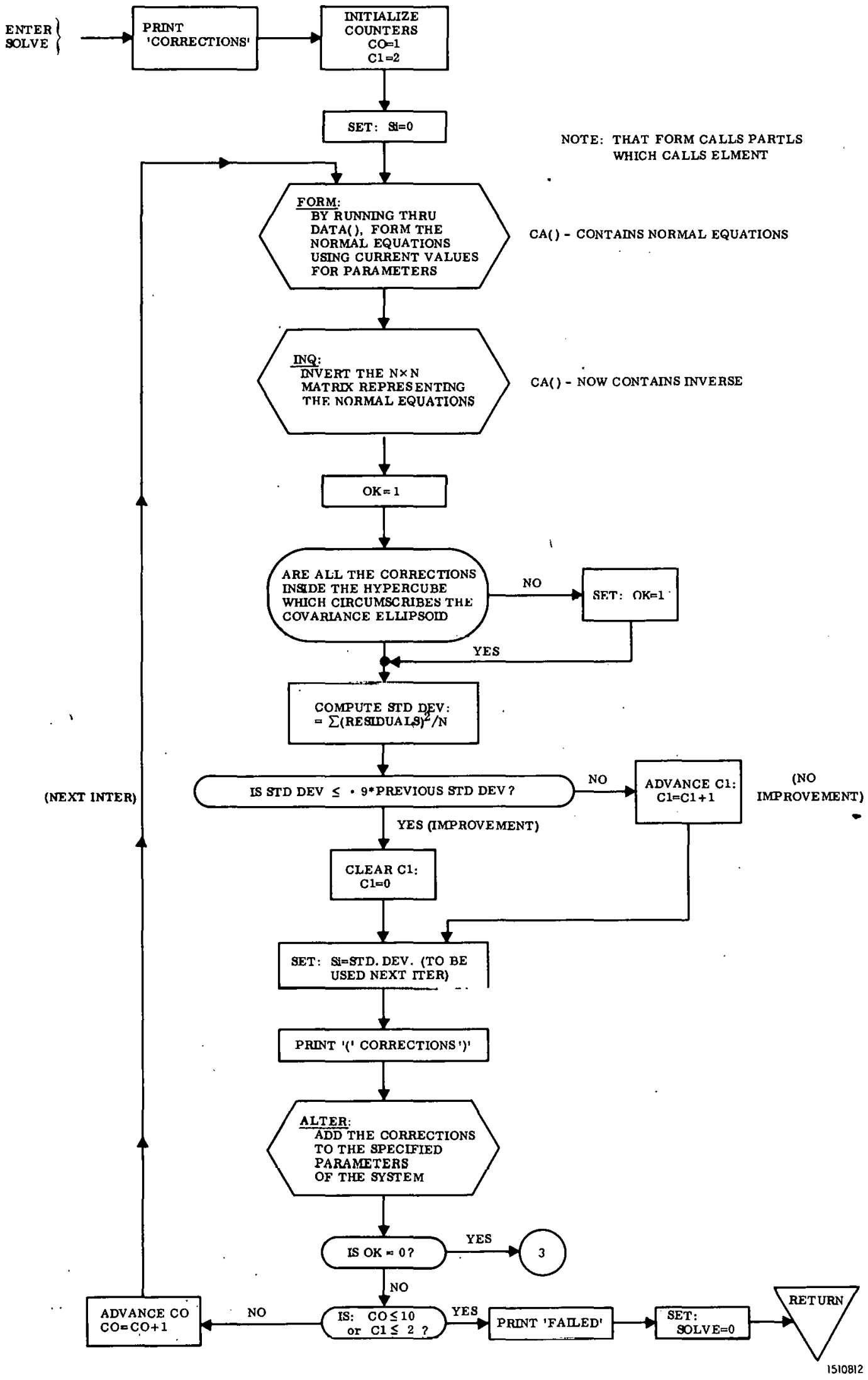


-SOLVE SUBPROGRAM (2)-

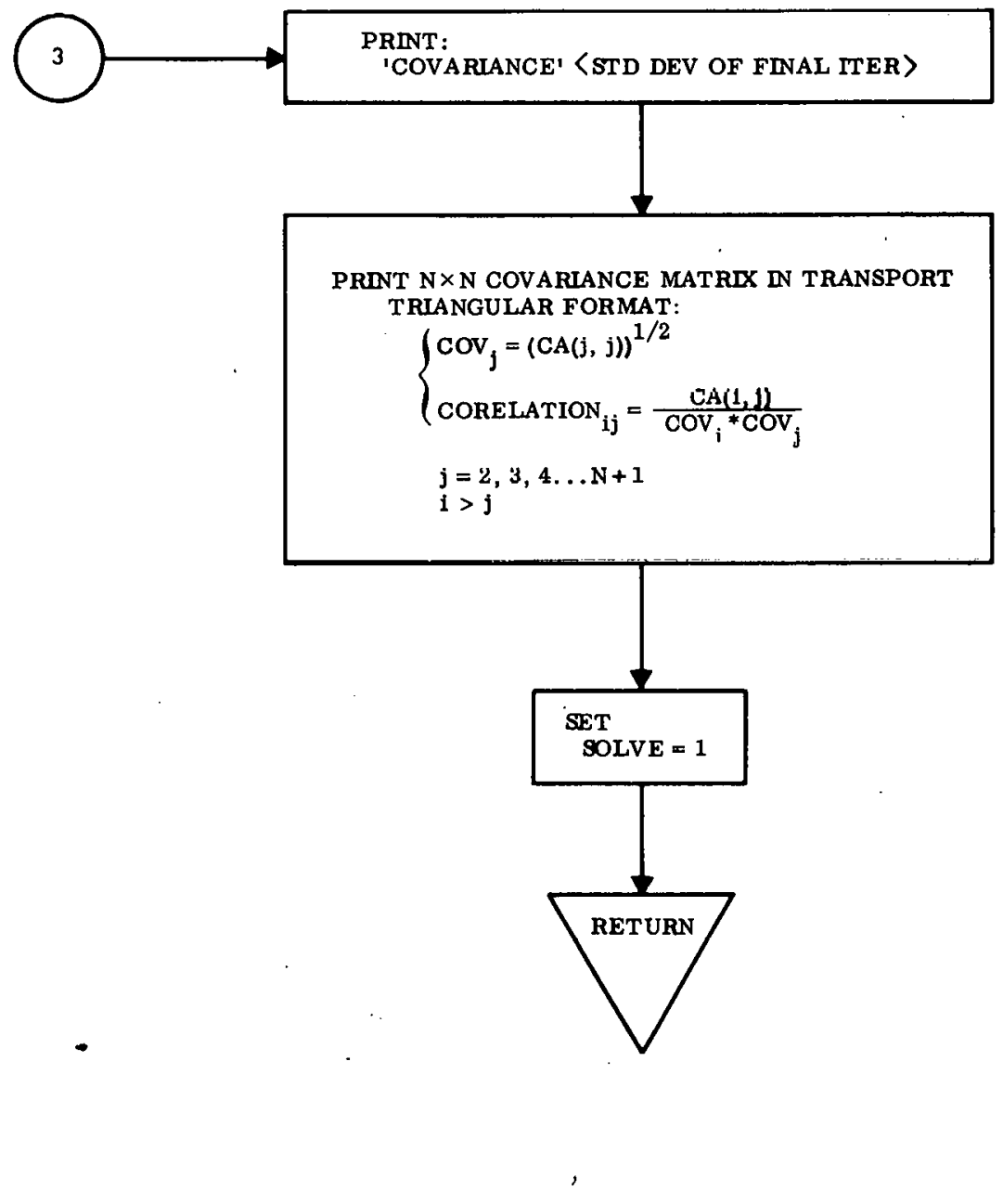

FORMAT OF ARRAY CA(,)

AFTER FORM, BUT BEFORE INQ

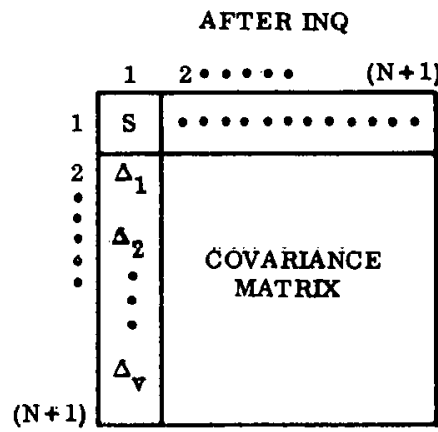

COLUMN VECTOR ON RIGHT STE OF NORMAL EQUATIONS

$S=S U M$ OF SQUARES OF THE LEAST SQUARES RESWUALS $\Delta_{i}=$ CORRECTION FOUND TO ith PARAMETER 
-FORM SUBROUTINE-

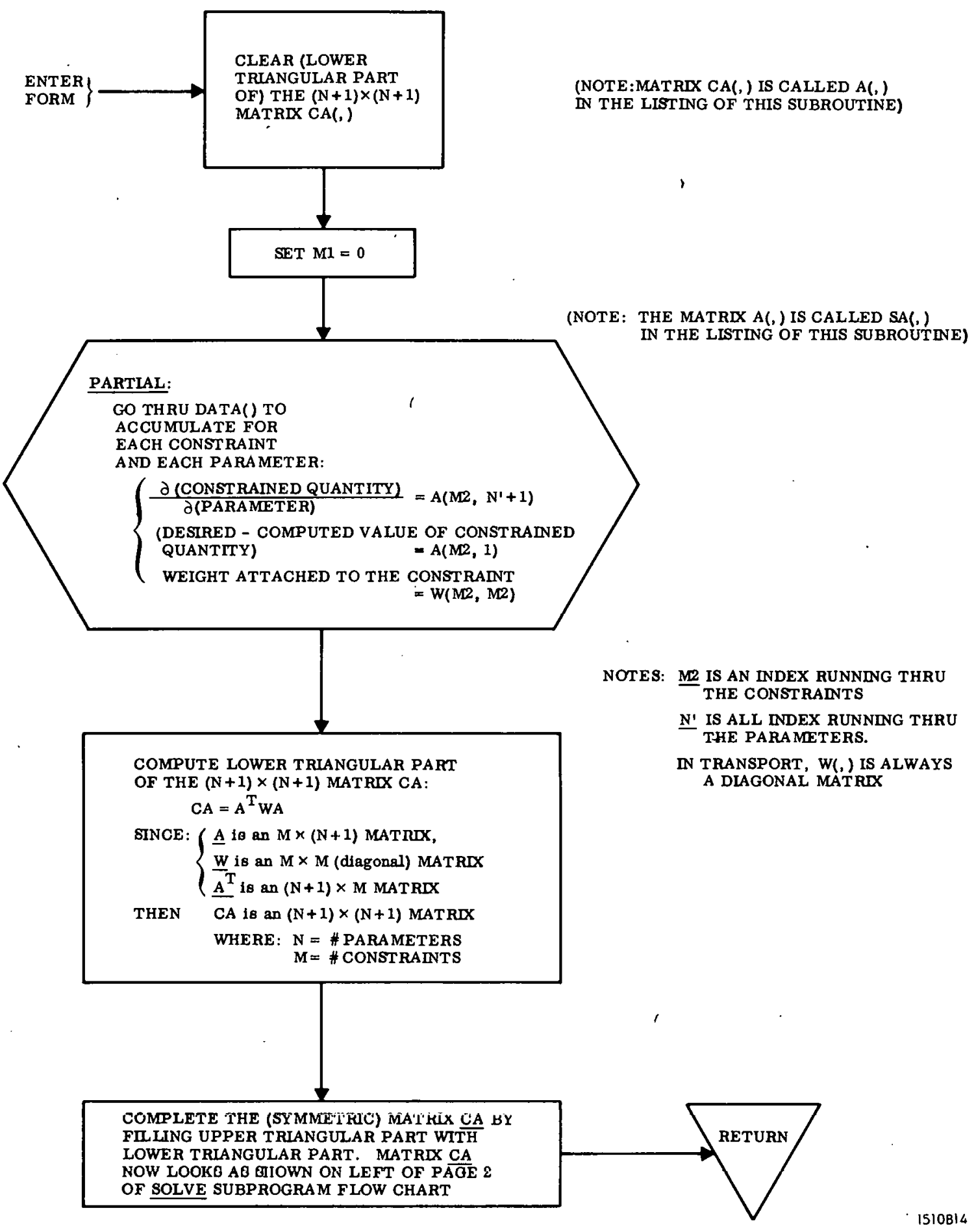




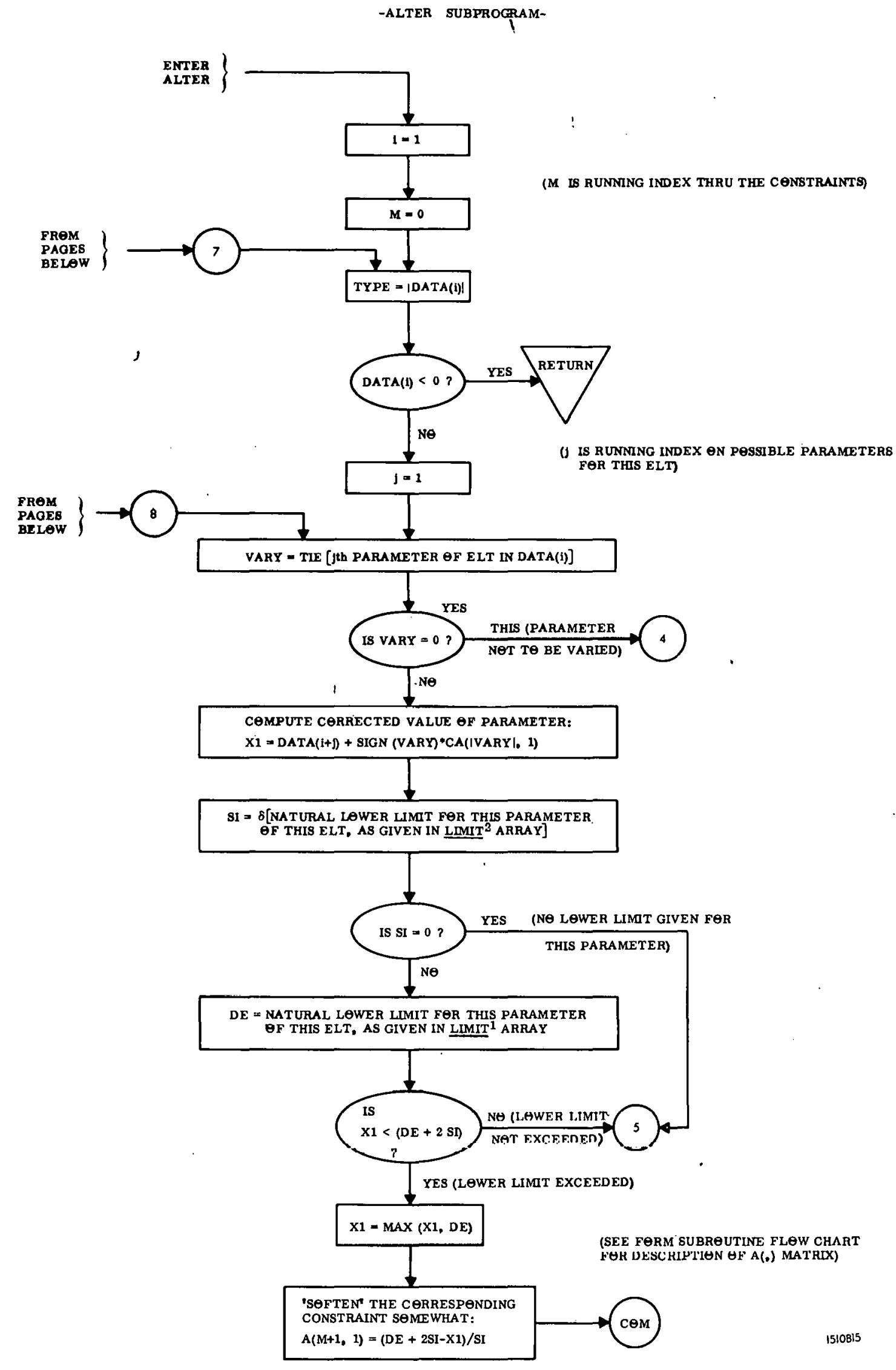


-ALTER SUBROUTINE (2)-

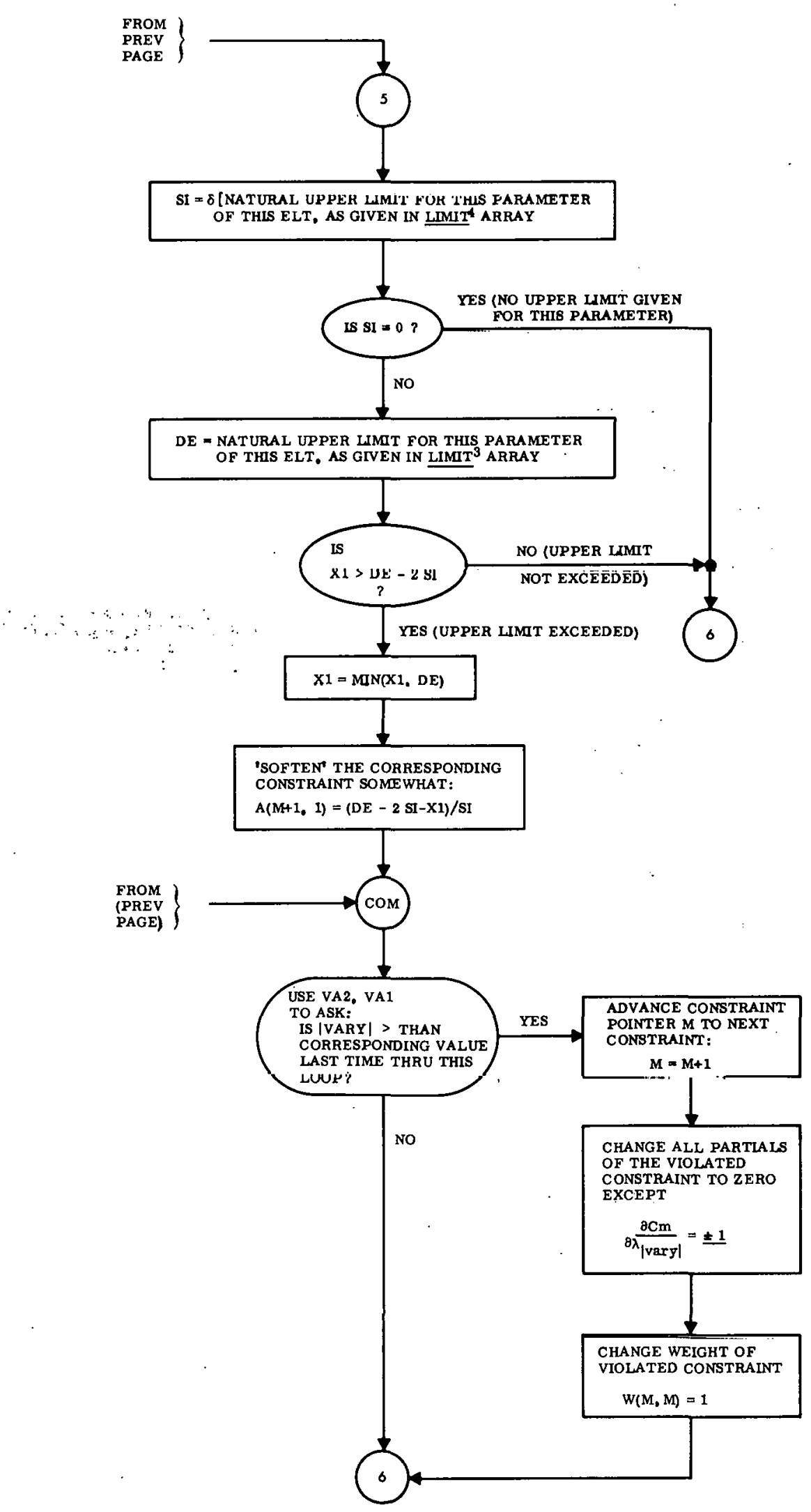

(+ IF VARY >0
- IF VARY $<0)$ 


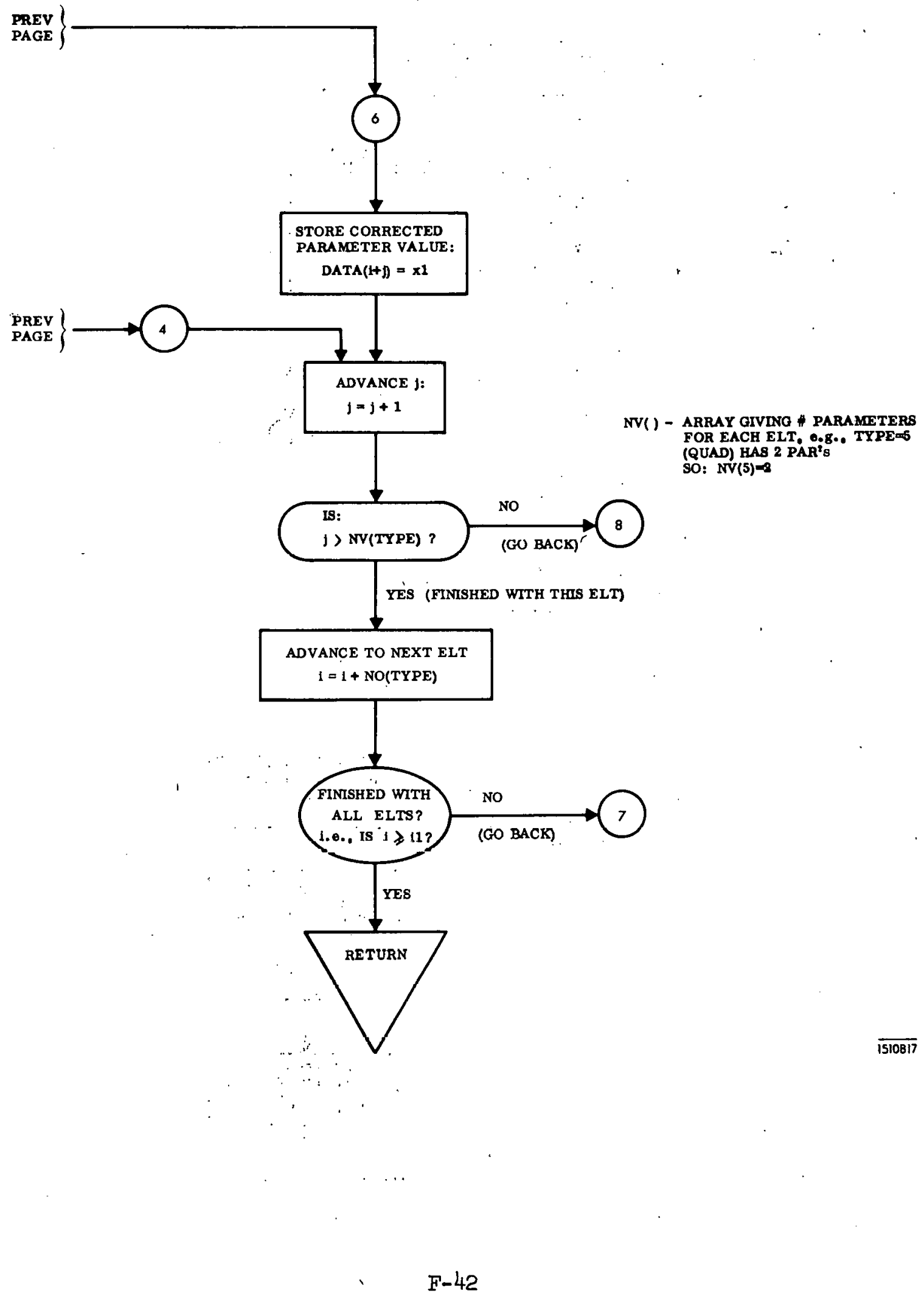




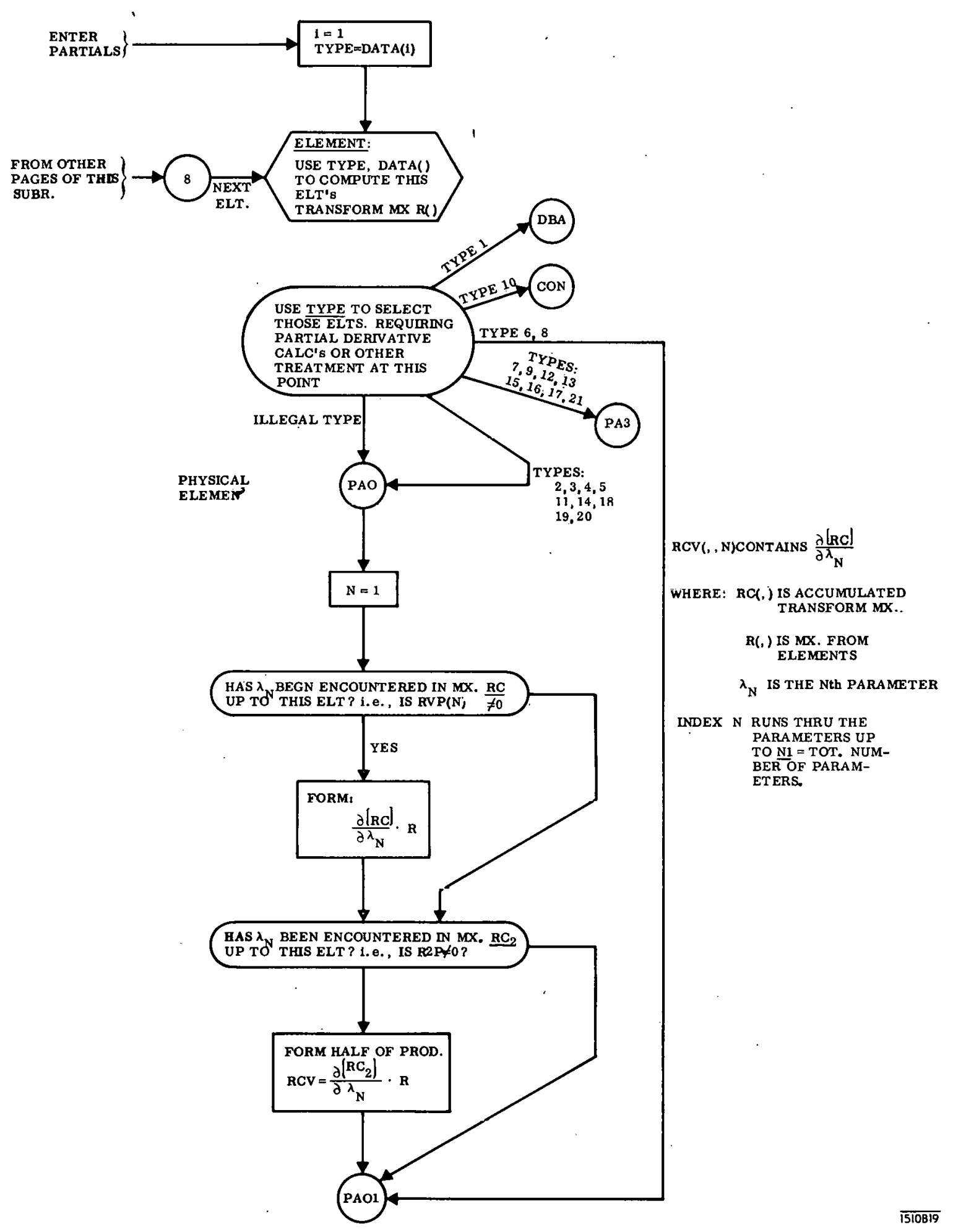



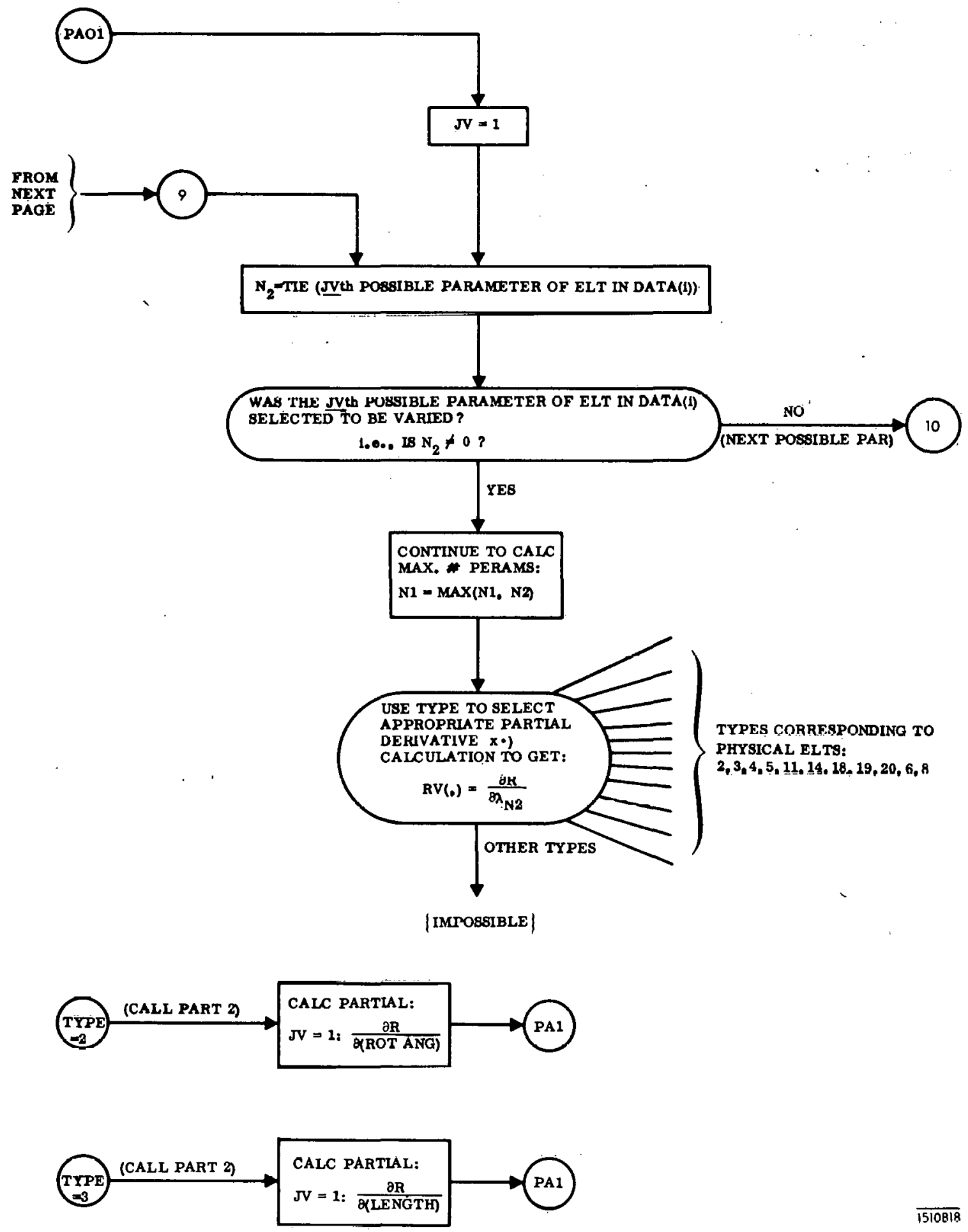

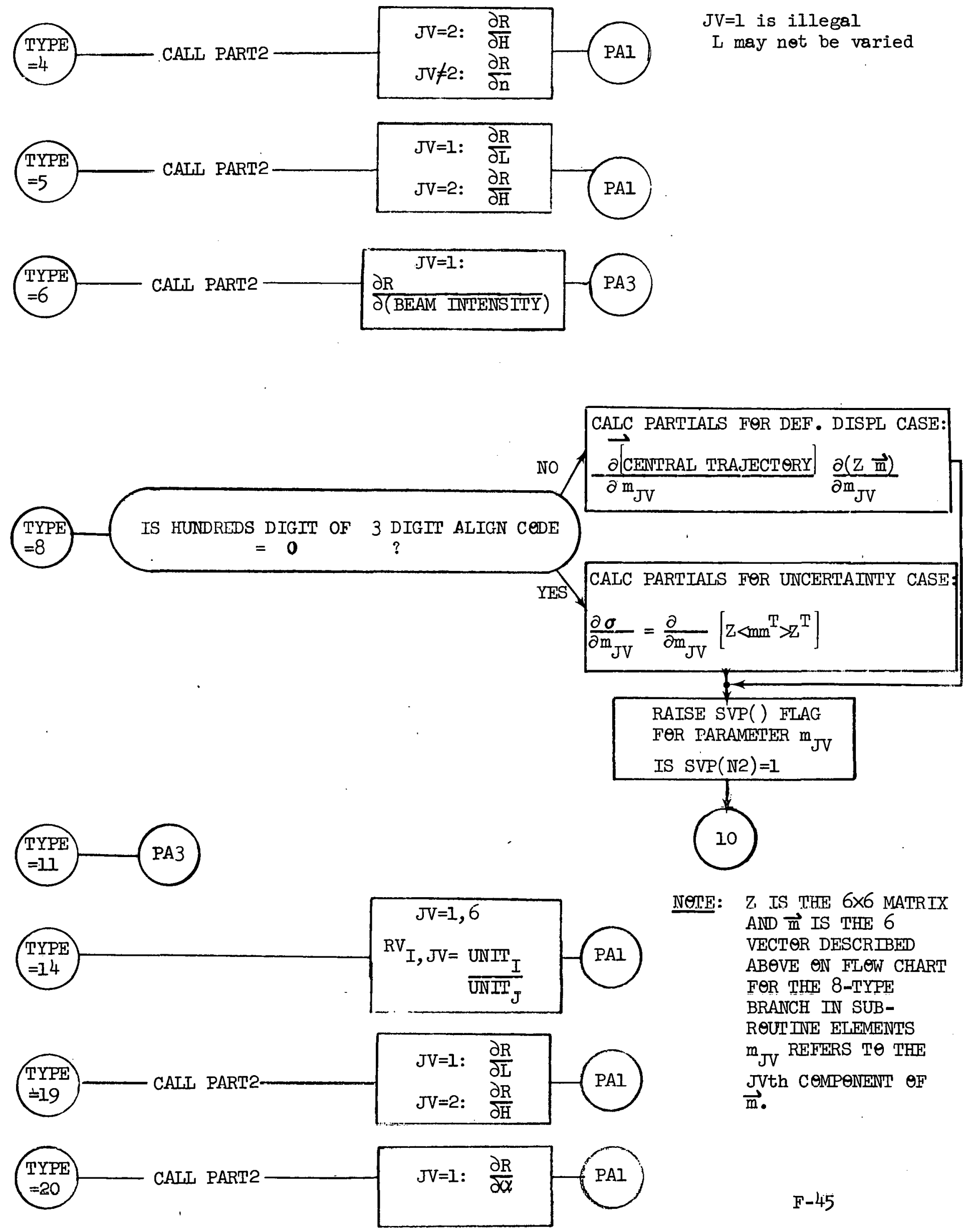
-PARTIALS SUBROUTINE(4)-

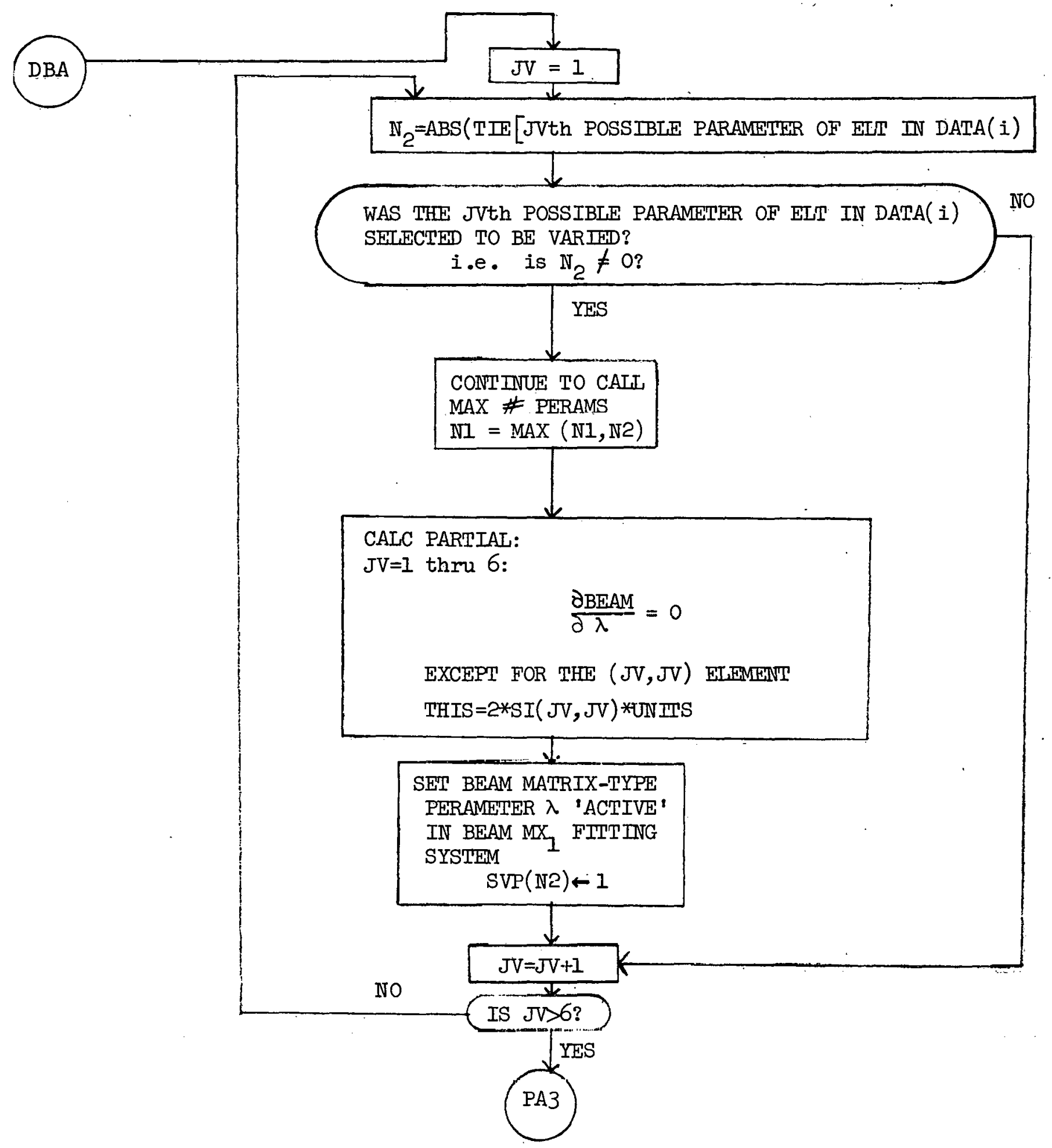



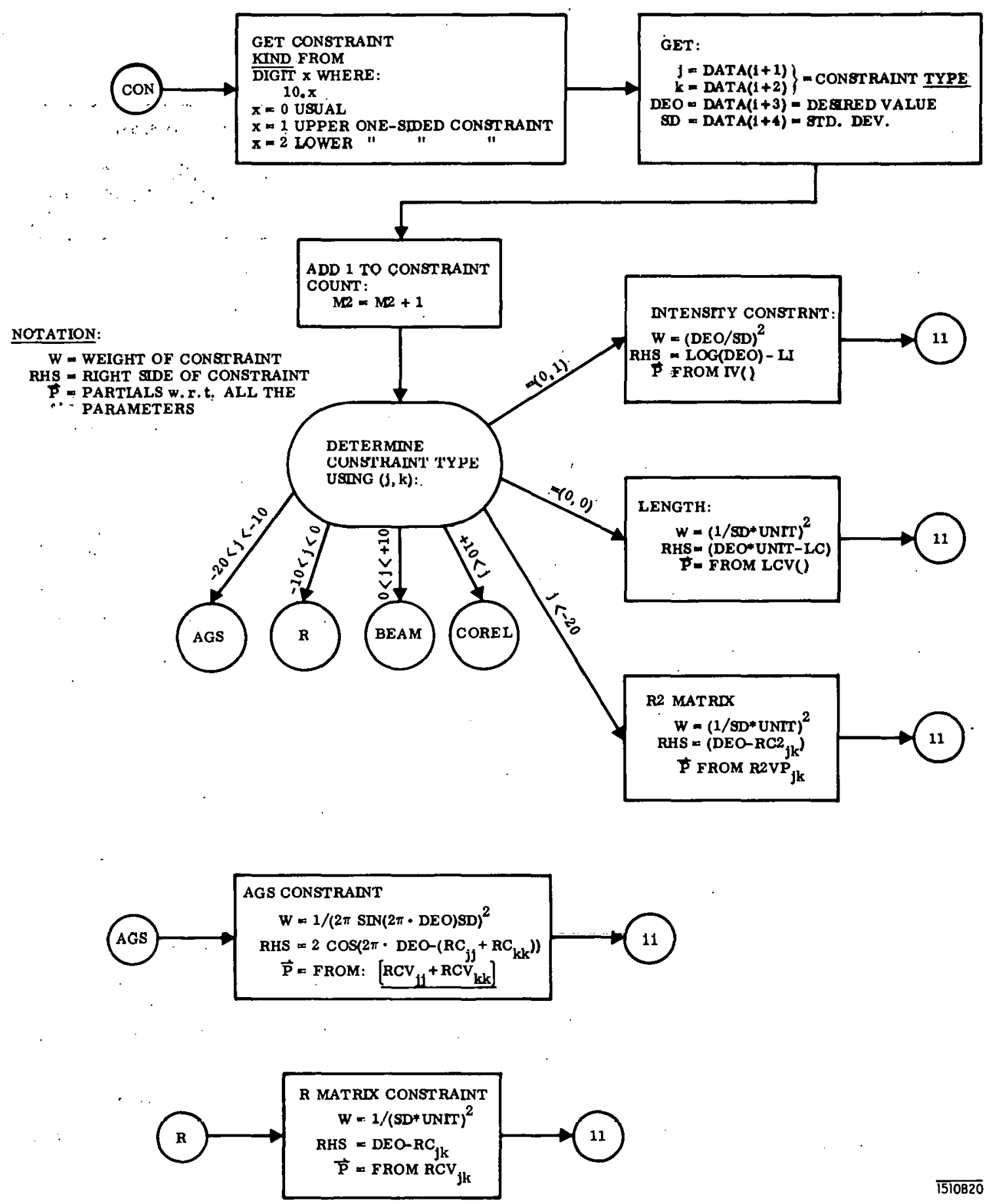


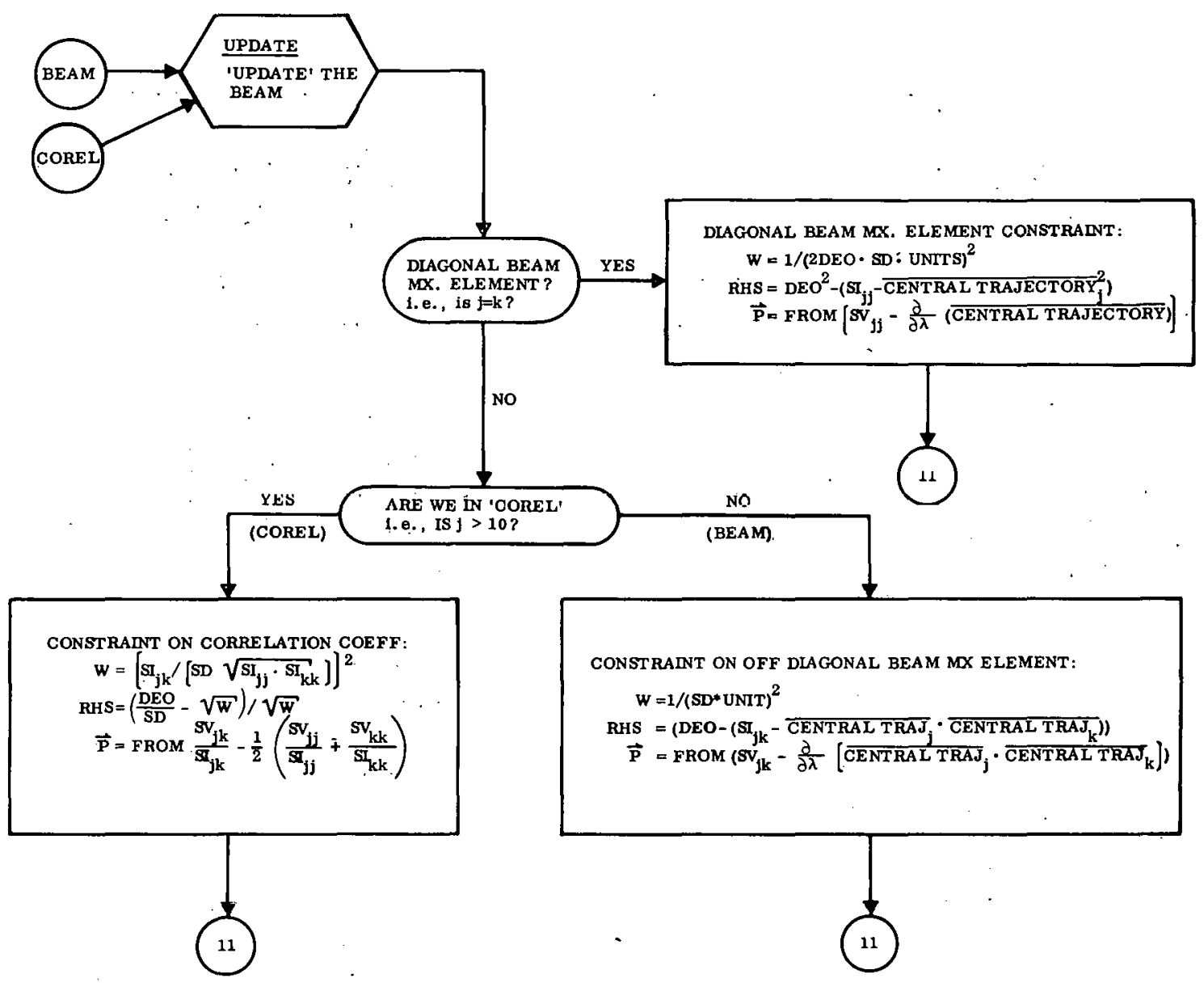

CUMMON RETURN

PT.

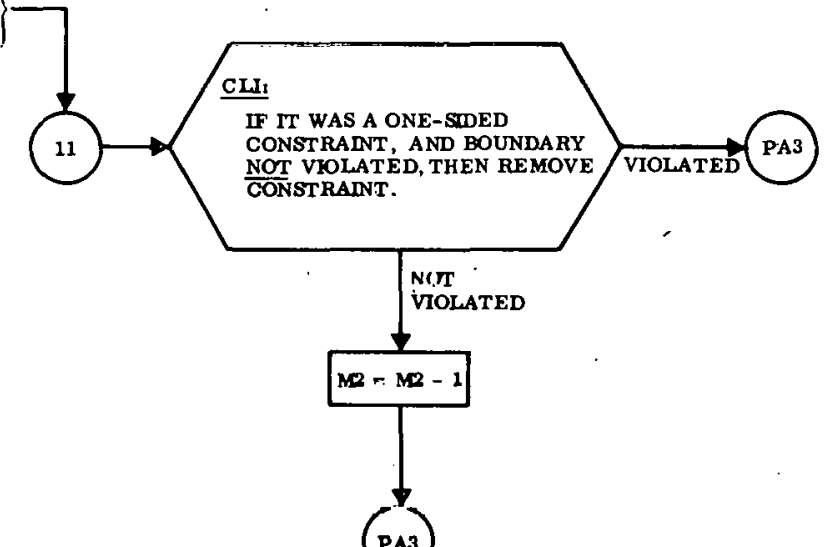

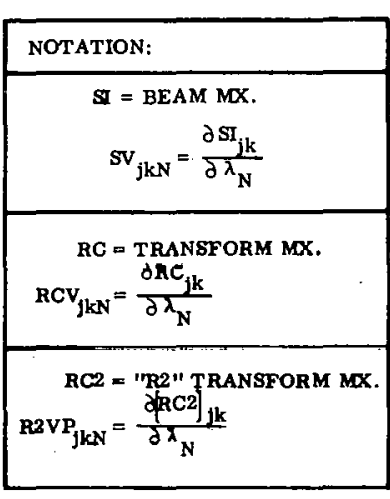




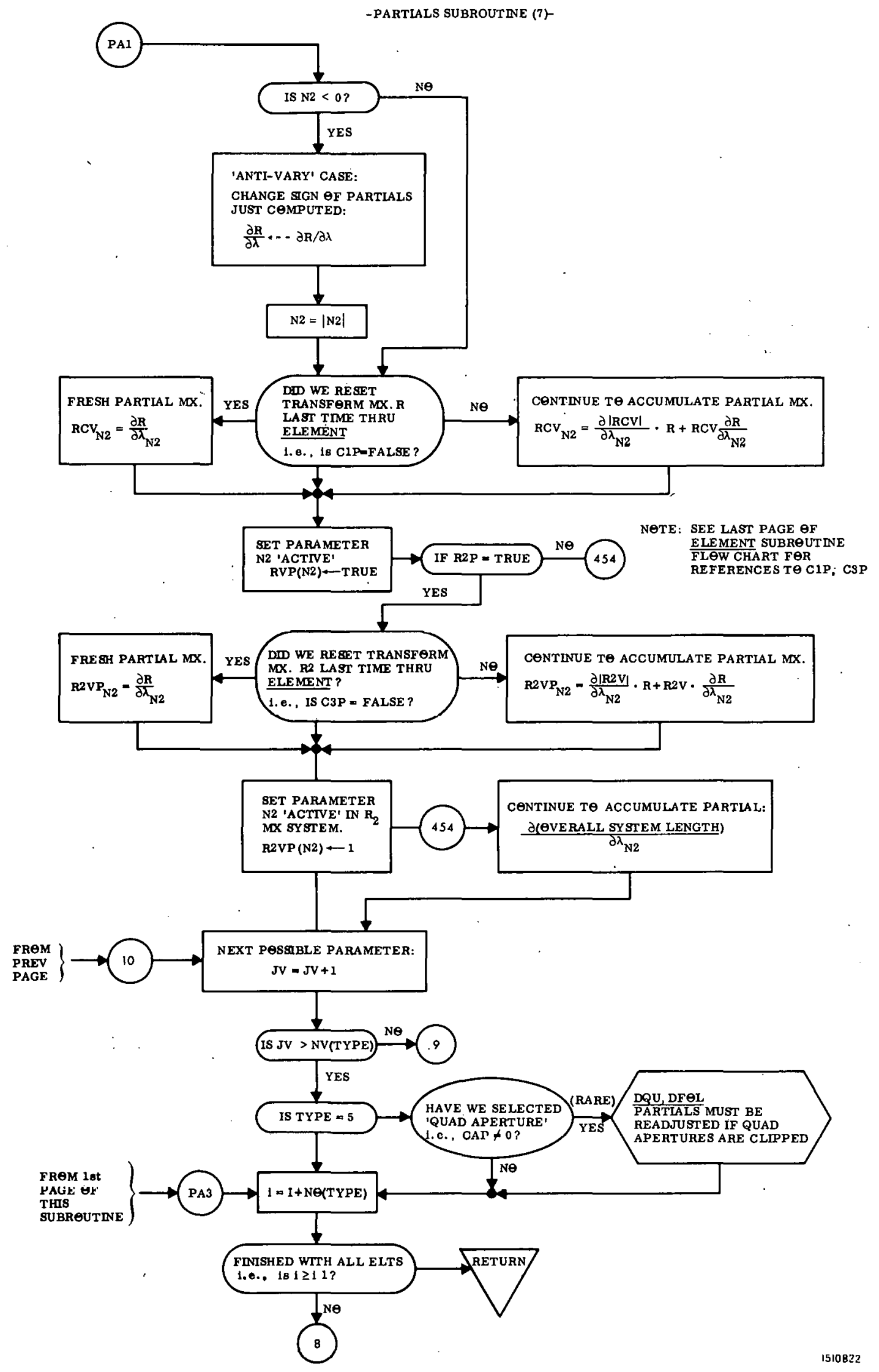


CHECK DATA FOLLOWING

POLE FACE ROTATION TO

SEE IF IS A BEIND OR A

PRINTOUT REQUEST

NO BFIM FOTINTn.

CHECK PRECED ING

DATA

IMPROPER

ELEMEINT

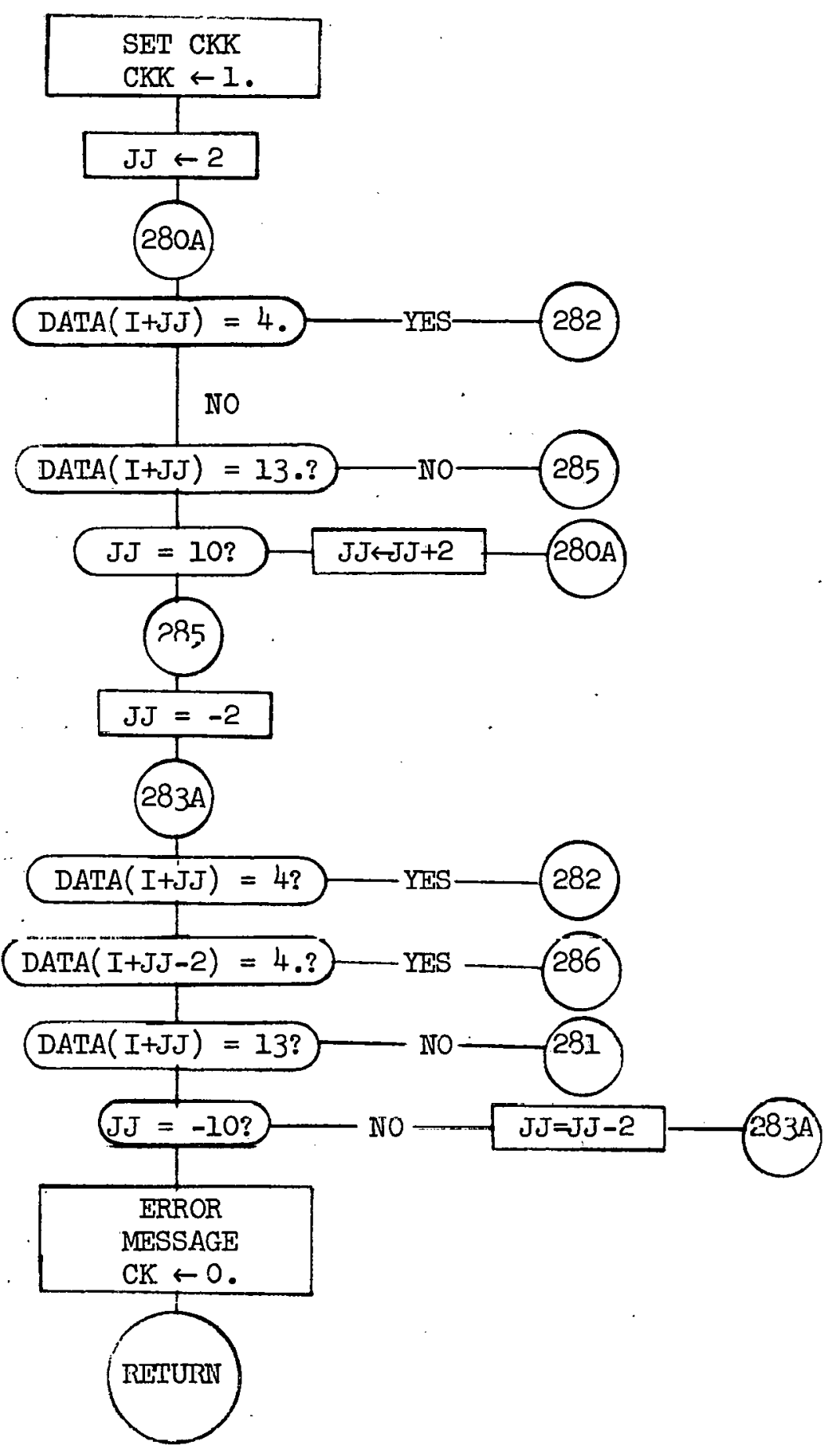


FIEID

SET FAGS FOR POLE FACE ROTATION BEFORE BEND FOR 2ND ORDER CALCULATION
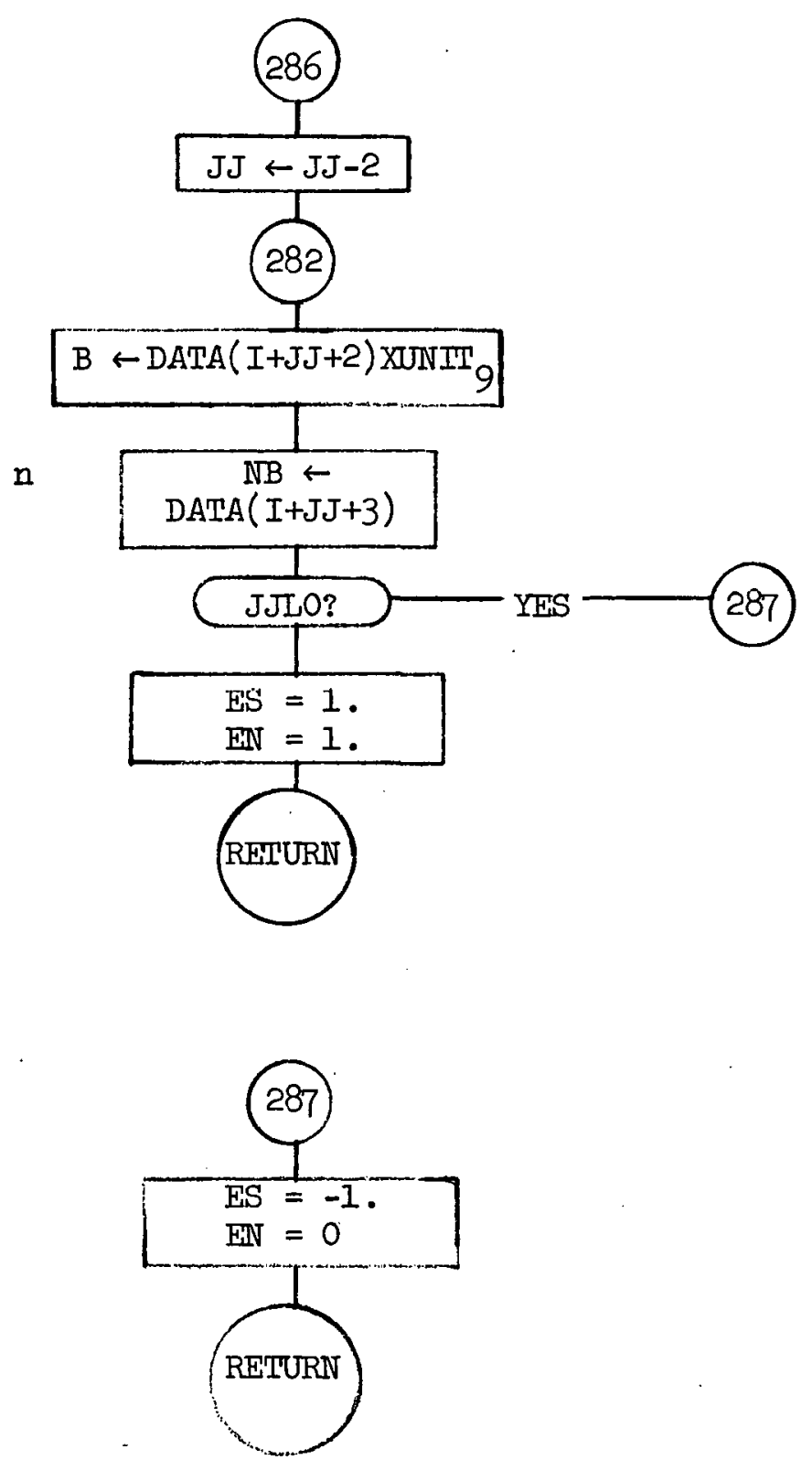


\section{$\therefore$ \\ -SUMMARY-}

MATRX FORMAT

SI:

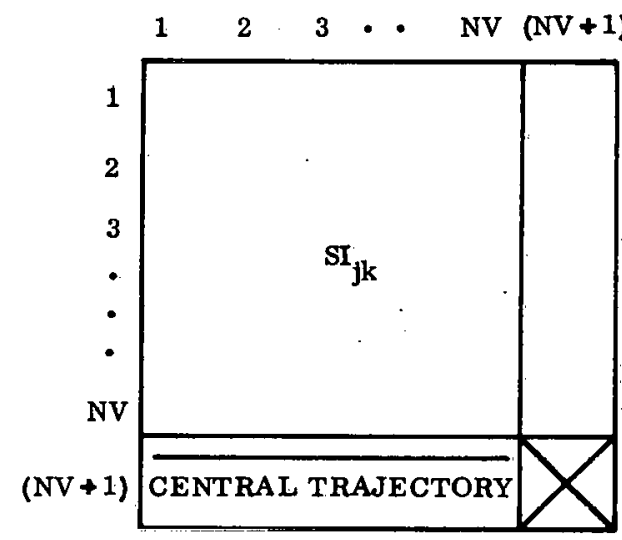

NV $=6$ (FIRST ORDER RUN) $=42$ (SECOND ORDER RUN)

FLAGS:

S1P: 


\section{APPENDIX}

Table of Contents

Introduction

1 - Beam Transport Optics (a set of lectures given to the SLAC technical staff)

Part I

Geometric Light Optics vs. Magnetic Optics

$A-1$

Introduction of Momentum Dispersion into the

$A-1$

Matrix Formalism

A-8

Second-Order Matrix Formalism

$A-12$

Transformations Involving Many Trajectories

$A-13$

Part II

First-Order $3 \times 3$ Matrix Transformation

A -16

Example of a Beam Switchyard

$A-16$

TRANSPORT Notation

$A-17$

Second-Order Matrix

A -22

$A-23$

2 - The First-Order R Matrix and Sigma Matrix (Beam Ellipsoid)

Formulation of TRANEFORT

$A-26$

First-Order Dispersion

A-29

First-Order Path Length

A-29

Achromaticity

Isochronicity

First-Order Imaging Conditions

$A-30$

$A-30$

Focal Lengths

Zero Dispersion

$A-30$

Magnification

$A-31$

$A-32$

First-Order Momentum Resolution

$A-32$

$A-32$

Tirst-Order Phace Ellipee Formalism of TRANSPORT

$A-35$

Description of the Sigma BEAM Matrix

$A-37$

The Phase Ellipse Matrix used by TRANSPORT

Physical Interpretation of Various Projections of the 2-dimensional BEAM Ellipse

The Upright Ellipse

Relationship between a Waist and a Parallel-to-Foint Image

Relationship between a Waist and Point-to-Point Image

A-39

Relationship between a Waist and the Smallest Spot Size

Achievable at a Fixcd Target Position

$A-41$

$A-44$

A -45

A -46

Imaging from an Erect Ellipse to an Erect Ellipse

$A-48$

$A-51$

Relationship between a First-Order Point-to-Point Image and the Minimum Spot Size Achievable at a Fixed Target Position

Orientation of the Major Axes of a Phase Space Ellipse 
Table of Contents - (continued)

3 - Second-Order Calculations and Corrections

A -58

Second-Order Phase Ellipsoid Formalism:

A -59

A Systematic Procedure for Designing High Resolving

Power Beam Transport Systems or Charged Particle

Spectrometers

A -60

I Introduction

A -62

II Theory

Multipole Strengths for Pure Multipole Fields.

A -63

Multipole Strengths for a Non-Uniform Field Expansion

A -65

Multipole Strengths for a Contoured Entrance or Exit

Boundary of a Magnet

A -66

The Description of the Trajectories as a Taylor's Expansion

A-66

A -67

III Solution of the Equation of Motion

A -69

IV Interpretation and usce of the Coupling coefficient

V A Systematic Procedure for Designing High Reșolution

$A-71$ Systems

First-Order Considerations

A -73

First-Order Resolving. Power

$A-73$

Dispersion

A -73

The Selection of the Optical Mode

A -75

Aberrations and their Correction

A -75

$A-75$

References

A -76

4 - Theory of Magnet Alignment Tolerances

A -77

5 - First-Order Parameter Optimization and Covariance

A -80 


\section{APPENDIX}

Introduction

This appendix has been included as an addition to the manual in an attempt to better acquaint the user with 'what TRANSPORT does', and with the notation and mathematical formalism used in a TRANSPORT calculation.

The first section (Beam Transport Optics - Part I and Part II) is a rewrite of two lectures given to members of the SLAC technical staff on the elementary matrix algebra of optics. We include them here for the benefit of the new user who may need a brief refresher course on charged particle optics and/or has a need to become familiar with TRANSPORT notation. The new user should also acquaint himself with the contents of the books and other publications listed under 'references' at the end of the manual. References 1 and 2 are essential if the user is to obtain the maximum value from TRANSPORT.

The second section of this appendix was written to introduce the mathematical formalism of the first-order $R$ matrix and Sigma matrix (phase ellipsoid) beam optics used in a TRANSPORT calculation and to correlate this with the printed output.

Section three discusses second-order calculations and, in particular, a procedure for calculating the "Sextupole" strengths required to minimize and/or eliminate second-order aberrations in a beam transport system.

Section four is a brief derivation of the mathematical formalism used by TRANSPORT for calculating magnet alignment tolerances.

Section five deals with the first-order parameter optimization code of TRANSPORT and Includes a brief explanation of the covariance matrix that is printed after each first-order fit routine. 


\section{Section I}

Beam Transport Optics - Part I

(K. L. Brown)

1. Introduction

A convenient starting point for this lecture is the equation relating the magnetic rigidity of a particle $(B \rho)$ to the particle momentum $P$.

$$
\mathrm{B} \rho=\frac{10^{2}}{2.99793} \mathrm{P} \quad \text { or } \quad \mathrm{B} \rho=33.356 \mathrm{P}
$$

where

$$
\begin{aligned}
& \mathrm{B} \text { is in kilogauss. } \\
& \rho \text { is the bending radius in meters } \\
& P \text { is the particle's momentum in } \mathrm{BeV} / \mathrm{c} \text {. }
\end{aligned}
$$

A note of caution: When using this equation for a TRANSPORT calculation, it is necessary to use at least 5 significant figures for the constant to avoid round-off errors in the reajout.

\section{Geometric Light Optics vis. Magnetic Optics}

To. relate geometrical light optics to charged particle optics, we begin with the thin lens. Figure 1 shows a thin lens with a ray leaving a focal point $A$ at an angle $\theta_{0}$, impinging on the lens at $x_{0} \cdot$ As the ray leaves the lens, it is at $x_{1}$ and going toward a focal point $B$ at an angle of $\theta_{1}$. 


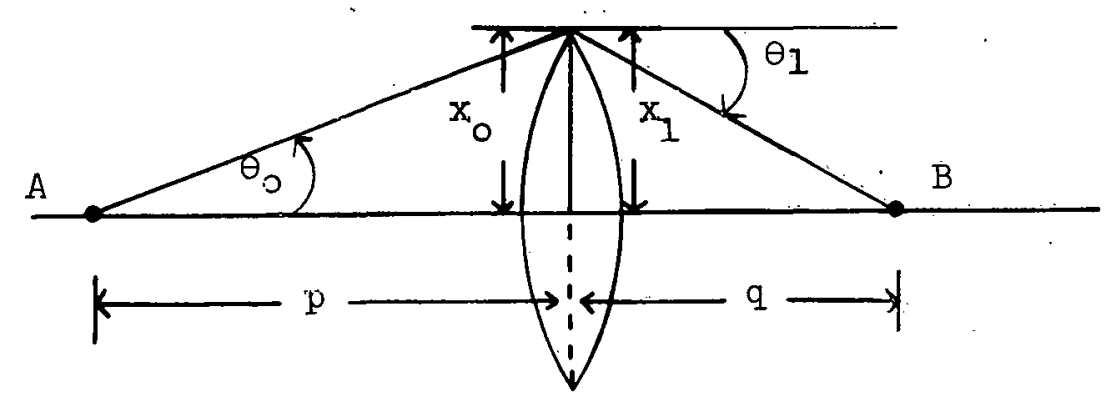

Tigure 1

Thin lens optics says that $1 / p+1 / q=1 / f$. Using this equation it : readily verified that the matrix transformation for the lens action between principal planes is

$$
\left[\begin{array}{c}
x_{1} \\
\theta_{1}
\end{array}\right]=\left[\begin{array}{c|c}
1 & 0 \\
-1 / f & 1
\end{array}\right]\left[\begin{array}{l}
x_{0} \\
\theta_{0}
\end{array}\right]
$$

The transformation for a drift distance $L$ is

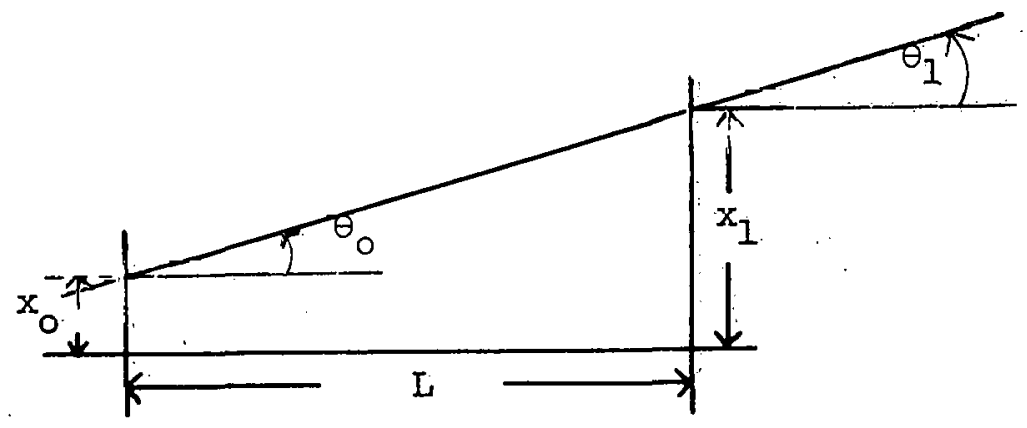

Figure 2

$$
\left[\begin{array}{l}
x_{1} \\
\theta_{1}
\end{array}\right]=\left[\begin{array}{ll}
1 & 1 \\
0 & 1
\end{array}\right]\left[\begin{array}{l}
x_{0} \\
\theta_{0}
\end{array}\right]
$$

Note that the determinant of the matrix in both examples is equal to unity.. This is always the case as will be proved formally later. That this is so is a manifestation of Liouvilie's theorem of conservation of phase space area. 
Consider now a thick lens, as illustrated in Figure 3 .

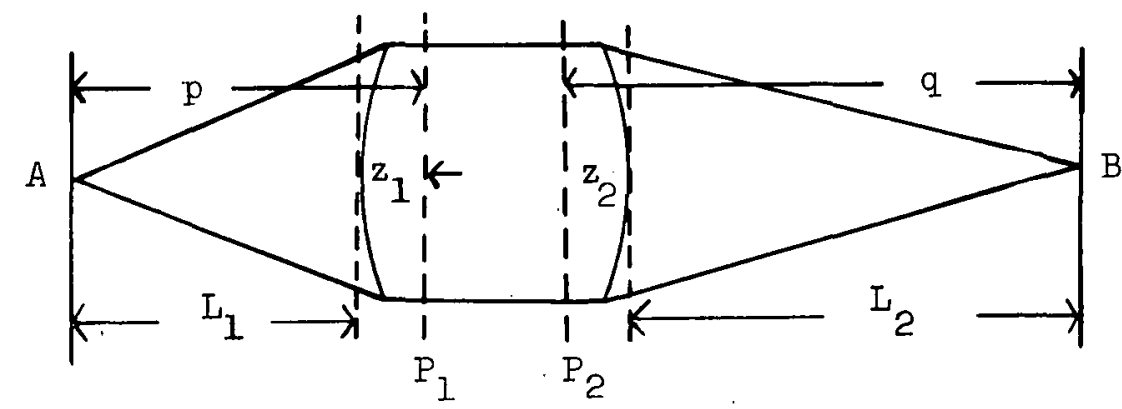

Figure 3

If $I_{1}$ is the object distance to the face of the lens and $L_{2}$ is the corresponding image distance, then, in general,

$$
I / L_{1}+I / L_{2} \cdot \frac{1}{F} I / f
$$

If, however, we introduce two planes $\mathrm{P}_{1}$ and $\mathrm{P}_{2}$ located at a distance $z_{1}$ and $z_{2}$ from the entrance and exit taces of the lens, it is always possible to find $a z_{1}$ and $a_{2} z_{2}$ such that the equation

$$
1 / p+I / q=1 / f \text { is valid. }
$$

where

$$
\begin{aligned}
& p=L_{1}+z_{1} \\
& q=L_{2}+z_{2} .
\end{aligned}
$$

When this is so, $\mathrm{P}_{1}$ and $\mathrm{P}_{2}$ are called the principal planes of the lens. Now, relating the above statement to matrix formalism, the matrix transformation for a thick lens between the input and output faces of the lens has the general form: 


$$
\left[\begin{array}{l}
x_{1} \\
\theta_{1}
\end{array}\right]=\left[\begin{array}{ll}
R_{11} & R_{12} \\
R_{21} & R_{22}
\end{array}\right]\left[\begin{array}{c}
x_{0} \\
\theta_{0}
\end{array}\right]
$$

where as before the $\operatorname{det} R=1$. For a general transformation, $R_{12}$ is not necessarily equal to 0 and $R_{11}$ and $R_{22}$ are not necessarily equal to 1 .

The principal planes may be located by the transformation

$$
\left[\begin{array}{ll}
R_{11} & R_{12} \\
R_{21} & R_{22}
\end{array}\right]=\left[\begin{array}{cc}
1 & z_{2} \\
0 & 1
\end{array}\right]\left[\begin{array}{cc}
1 & 0 \\
-1 / f & 1
\end{array}\right]\left[\begin{array}{ll}
-1 & 2 \\
- & 1 \\
0 &
\end{array}\right]
$$

(2)

Using the relation

$$
\left[\begin{array}{ll}
1 & z \\
0 & 1
\end{array}\right]\left[\begin{array}{cc}
1 & -z \\
0 & 1
\end{array}\right]=\left[\begin{array}{ll}
1 & 0 \\
0 & 1
\end{array}\right]=1 \text { (the unit matrix) }
$$

the previous equation may be manipulated into the form

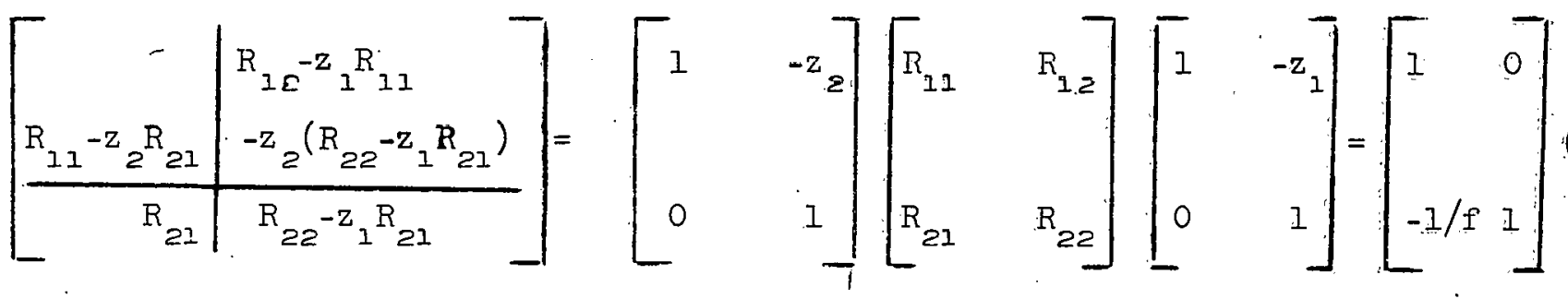

Solving for $z_{I}$ and $z_{2}$, we find

$$
\begin{aligned}
& z_{1}=\frac{R_{22}-1}{R_{21}} \\
& z_{2}=\frac{R_{11}-1}{R_{21}}
\end{aligned}
$$

$A-4$ 
where $z_{1}$ and $z_{2}$ are the location of the principal planes as shown in Fig. 3. The principal planes of any system may be determined by this method. Note that $\mathrm{R}_{21}=-1 / \mathrm{f}$ is not affected by the transformation and that the upper right hand matrix element is zero if $\operatorname{det} R=1$. The principal planes may coincide, may be close together, be far apart; or in many systems, may be located external to all of the elements comprising the system. An example of the latter case is a quadrupole pair.

Some examples of principal plane locations for simple systems follow:

A quadrupole singlet:

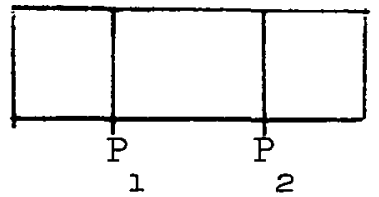

Figure 4

The principal planes in a single quadrupole are located very close to each other and very near the center of the lens. As such, a quadrupole singlet may be considered as a thin lens if the object and image distances are measured to the center of the lens.

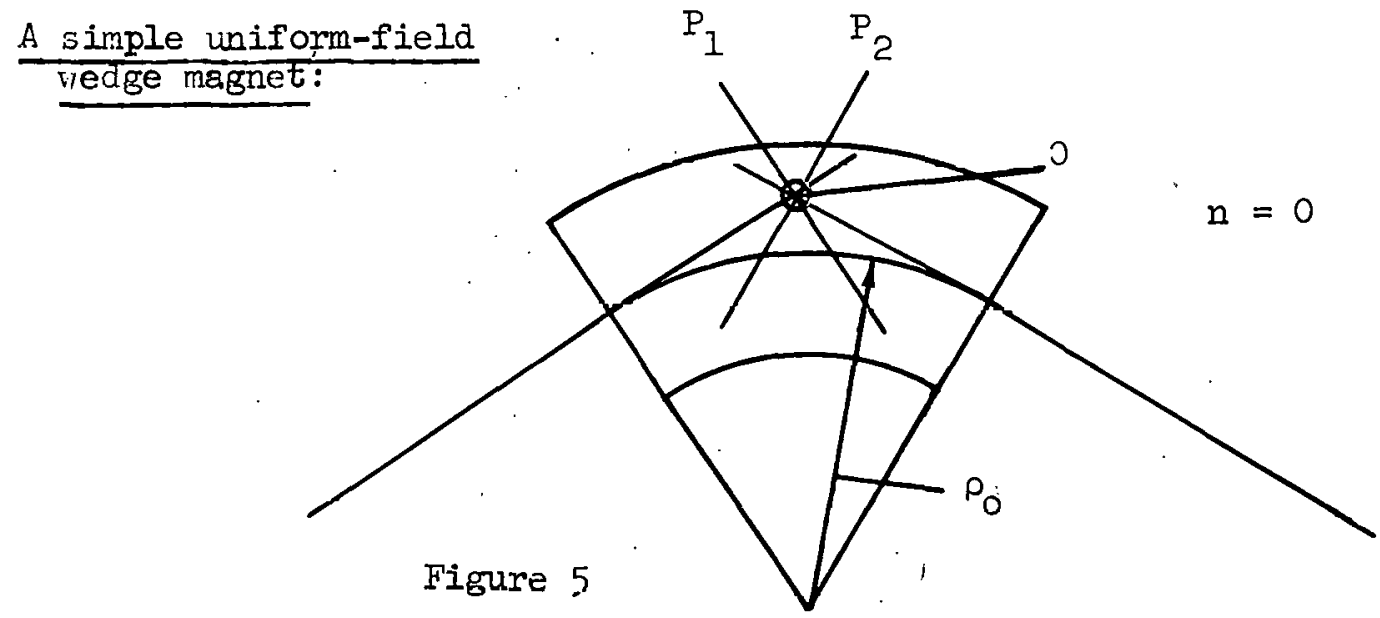

If the optic axis enters and exits perpendicularly to the pole boundary, the principal planes are at the "center" of the magnet, as shown in Figure $h$. From this, we conclude that a simple wedge bending magnet may be considered as a "thin" lens if the object and image distances are measured to the lens center 0 . 
A quadrupole pair:

defocusing focusing

A

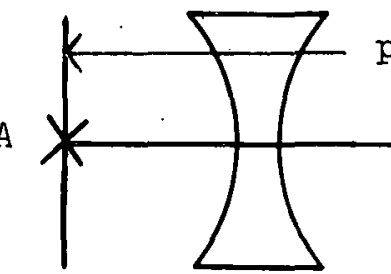

(-)

$x$ plane

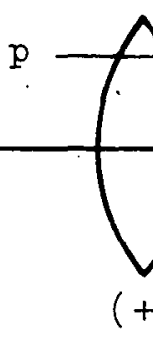

$(+)$

Figure 6

focusing defocusing

A

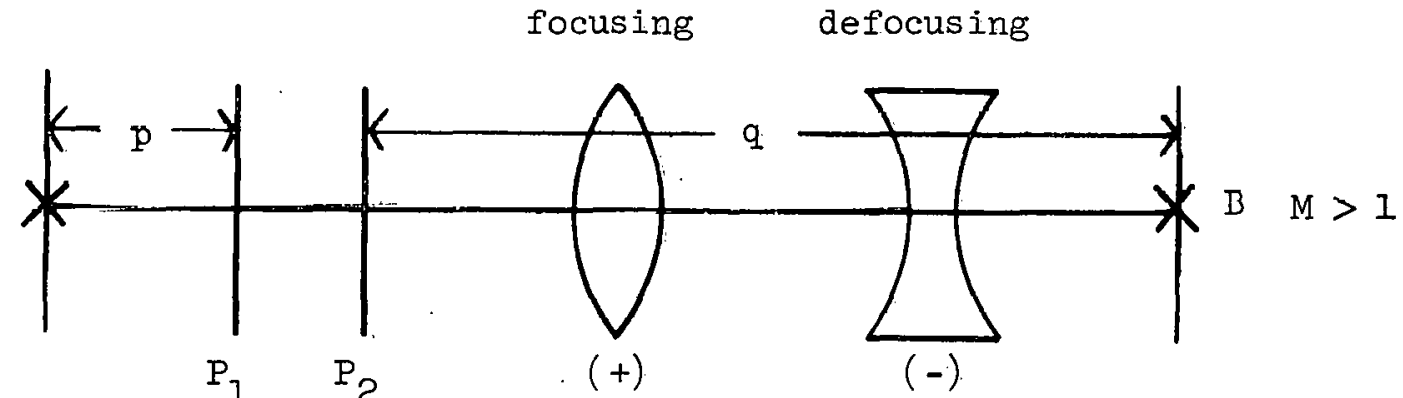

y plane

Figure 7

For a quadrupole pair, the principal planes are displaced toward and, usually, beyond the focusing element of the pair, as shown in Figures 6 and 7 .

For any lens system, no matter how many elements are invclved

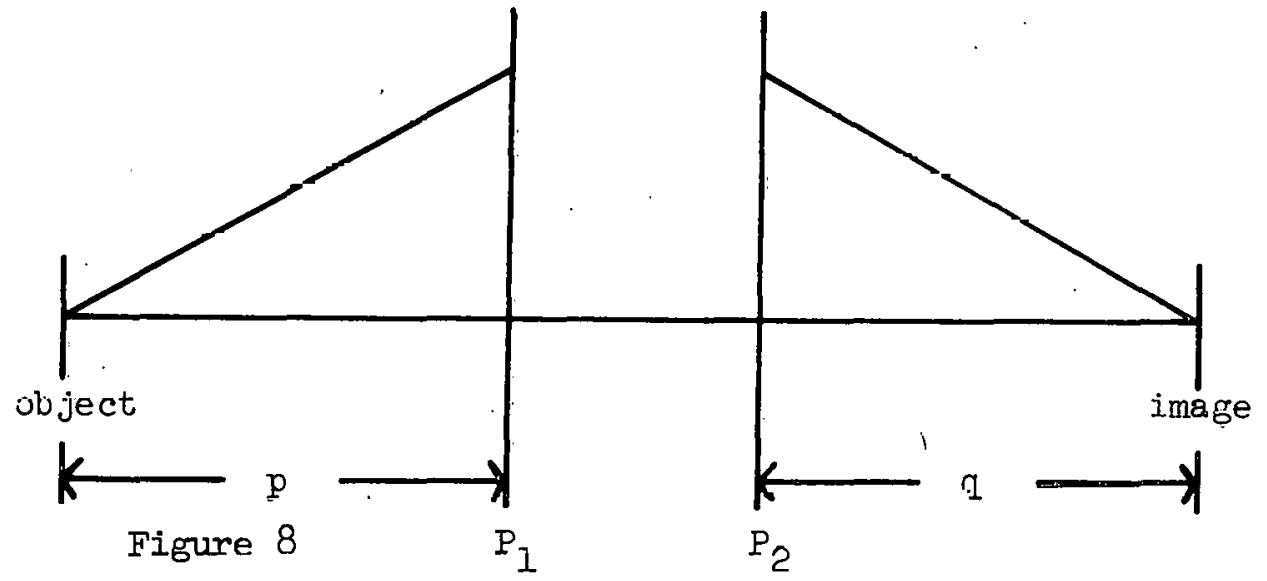

$1 / p+1 / q=1 / f$, if $p$ and $q$ are distances measured to the principal planes. 'l'hen the magnif'ication between object and image planes is $\mathrm{M}=\mathrm{q} / \mathrm{p}$.

Since the quadrupole pair is different in the two planes, (x) and (y), both situations must be examined. The interesting result turns out to be that in the $x$ plane, the principal planes are to the right (Figure 6) and in the $y$ plane, they are to the left (Figure 7). Therefore, in the y plane. 
the magnification is greater than 1 , and in the $x$ plane the magnification is less than 1 . Typically, for a quadrupole pair the ratio of

$$
\mathrm{M}_{\mathrm{y}} / \mathrm{M}_{\mathrm{x}}
$$

may be as high as 20:1 and such cases can be disastrous if not recognized beforehand. This is a first-order image distortion. For example, if the source is a circular spot at $A$, the image at $B$ will appear as a long thin line.

The situation is different for the Quadrupole triplet:

A

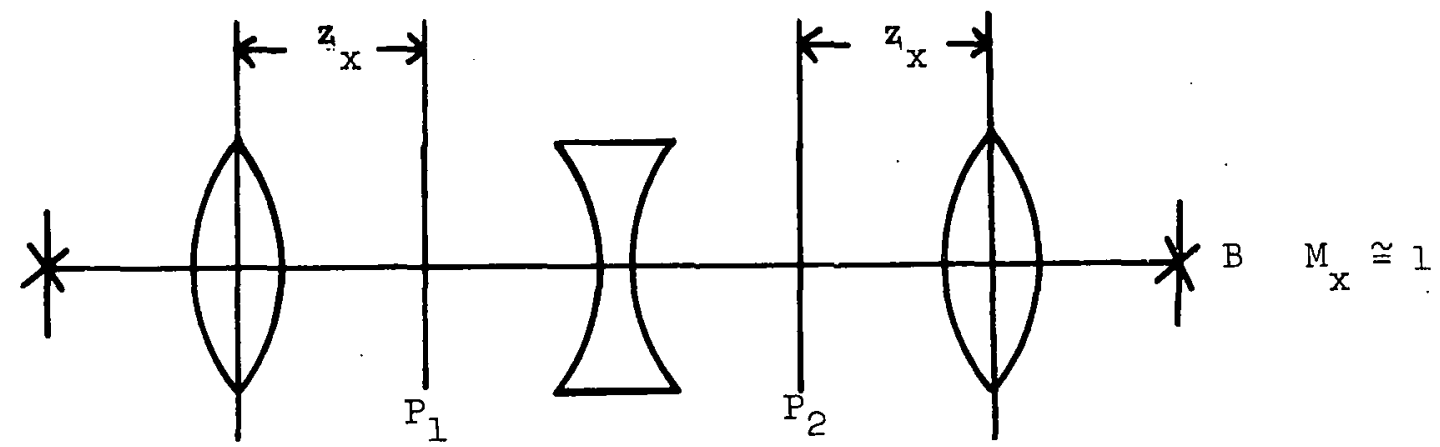

$x$ plane

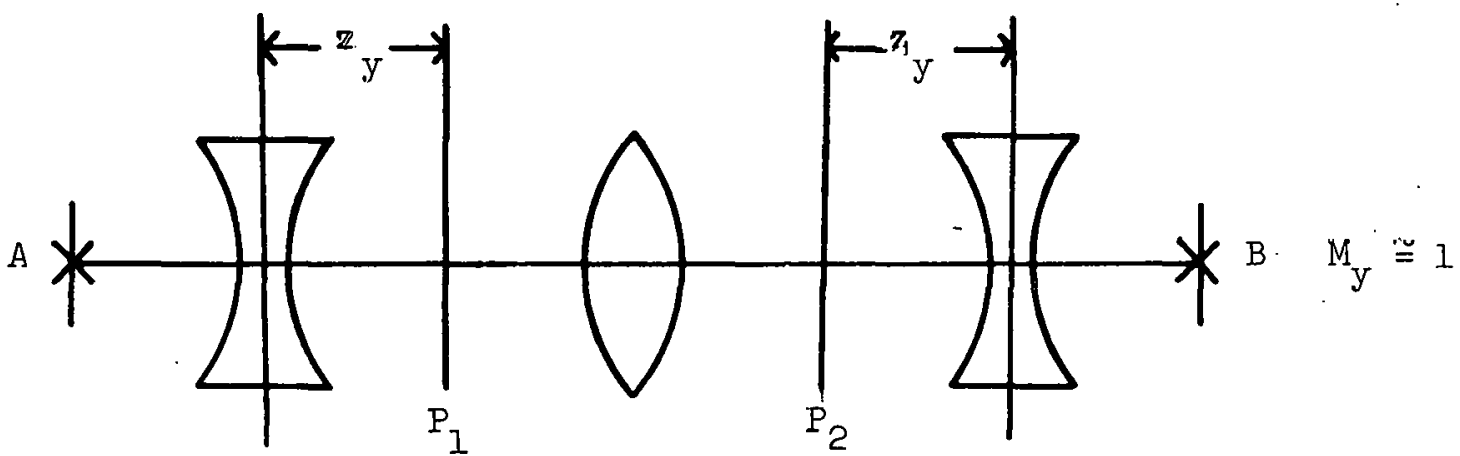

y plane

Figure 10

In the symmetric triplet, as shown in Figures 9 and 10, the principal planes are located symmetrically about the center of the system, although $z_{x}>z_{y}$. This is, perhaps, the dominant reason why quadrupole triplets are-used. The magnification is approximately equal in both planes; consequently, a circular spot can be imaged through the system with much less first-order image distortion than is the case for the doublet. 
3. Introauction of Momentum Dispersion into the Matrix Formalism

The foregoing discussion and examples dealt only with monoenergetic first-order effects. First-orker dispersion may be taken into account by introducing a $3 \times 3$ matrix as follows:

Consider two particles of momentum $p_{0}$ and $p_{0}+\Delta p$ passing through the midplane of a static magnetic field, as illustrated in Figure 11.

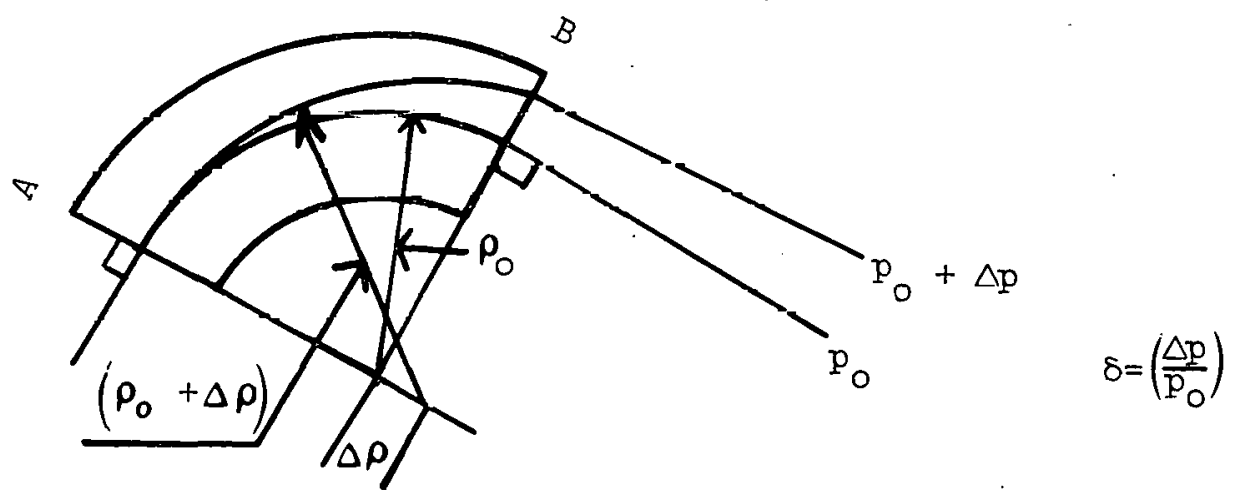

Figure 11

Since the scalar momentum of a particle is constant in a static magnetic field, the transport equation from $A$ to $B$ may be expressed as:

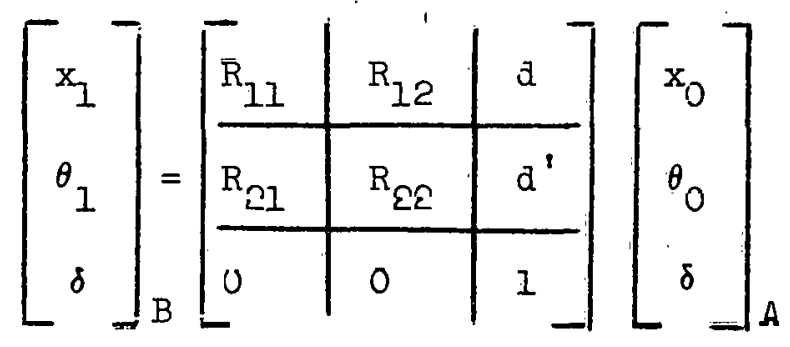

where

$$
\begin{aligned}
\delta & =\Delta \mathrm{p} / \mathrm{p}_{0} \\
\mathrm{~d} & =\text { the spatial monentum dispersion } \\
\mathrm{d}^{\prime} & =\begin{array}{l}
\text { the derivative of the dispersion (the angular } \\
\text { monentum dispersion) }
\end{array}
\end{aligned}
$$

and $\quad 1=a$ carrying term to generate a square matrix and denote a constant momentum. 
The determinant of the matrix $|R|$ is equal to 1 as for the $2 \times 2$ matrix. However, because of the zeros in the bottom row, the fact that $|R|=\left(R_{11} \cdot R_{22}-R_{12} R_{21}\right)=1$ only checks the $2 \times 2$ matrix and not the terms containing $d$ and $d^{\prime}$.

Consider now a general system from an object point $A$ to an image point B.

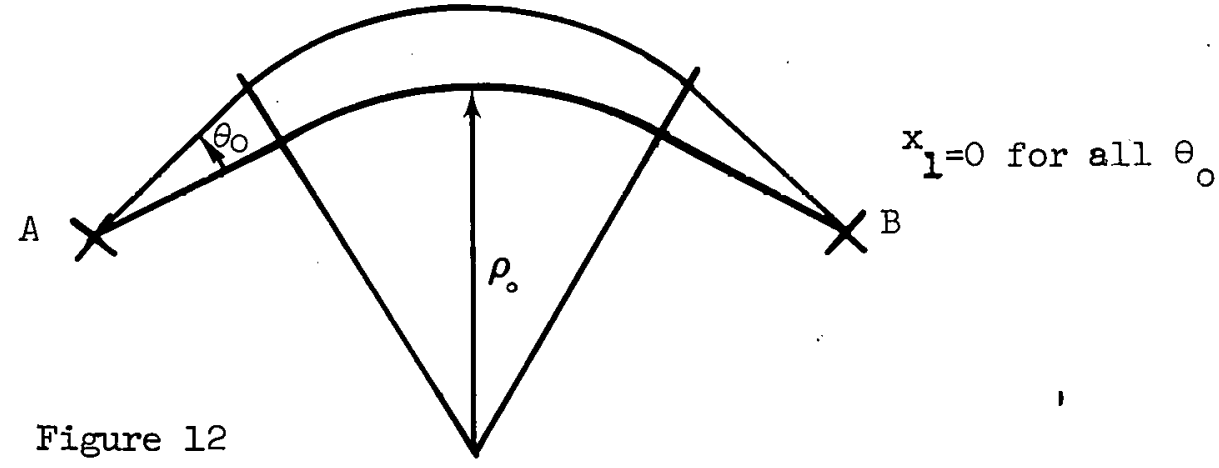

The above matrix equation is still valid for midplane trajectories. If $A$ is a source point and if $R_{12}=0$ (i.e., $x$ is independent of $\theta_{0}$ ), then $B$ is an image point for monoenergetic particles. Under these circumstances:

$$
\begin{aligned}
& \mathrm{R}_{11}=\mathrm{M}_{x} \text { is the } \mathrm{x} \text { plane mannification } \\
& \mathrm{R}_{22}=+1 / \mathrm{M}_{\mathrm{x}} \text { because det } \mathrm{R}=1
\end{aligned}
$$

and

$$
R_{21}=-1 / f_{x}
$$

In fact, $R_{2 I}=-1 / f_{\mathbf{x}}$ for the system between $A$ and $B$, even if $A$ and $B$ are not foci.

It is now convenient to develop a more general definition of the matrix elements $R_{i j}$ and, at the same time, introduce the first-order matrix transformation for the $\mathrm{y}$ (non-bend) plane. Consider, again, a general system where the projection of the central trajectory is allowed to bend. in the $\mathrm{x}$ plane but is a straight line in the $\mathrm{y}$ plane. The $\mathrm{x}$ plane and $\mathrm{y}$ plane matrix transformations may be written as follows: 
For the $\mathrm{x}$ plane $\mathrm{x}_{1}=\mathrm{R}_{\mathrm{x}} \mathrm{x}_{0}$

or

$\left[\begin{array}{l}x_{1} \\ \theta_{2} \\ \delta\end{array}\right]=\left[\begin{array}{l|l|l}c_{x}(t) & s_{x}(t) & a_{x}(t) \\ \hline c_{x}^{\prime}(t) & s_{x}^{\prime}(t) & a_{x}^{\prime}(t) \\ \hline 0 & 0 & 1\end{array}\right]\left[\begin{array}{c}x_{0} \\ \theta_{0} \\ \delta\end{array}\right]$

Similarly, for the $\mathrm{y}$ plane, $\mathrm{y}_{\mathrm{I}}=\mathrm{R}_{\mathrm{y}} \mathrm{y}_{\mathrm{o}}$.

or

$$
\left[\begin{array}{l}
y_{1} \\
\Phi_{1}
\end{array}\right]=\left[\begin{array}{c|c}
c_{y}(t) & s_{y}(t) \\
\hline c_{y}^{\prime}(t) & s_{y}^{\prime}(t)
\end{array}\right]\left[\begin{array}{l}
y_{0} \\
\Phi_{0}
\end{array}\right]
$$

The $c$ and $s$ functions may be defined in terms of their initial conditions. Let $\tau$ be the distance measured along the

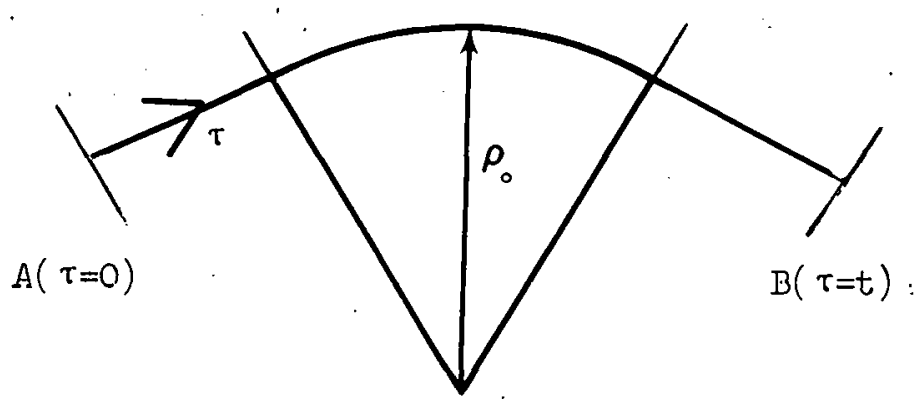

Figure 13

central trajectory. Then:

$$
\begin{aligned}
& s(0)=0 \quad s^{\prime}(0)=1 \text { where } \quad s^{\prime}(\tau)=\frac{d s(\tau)}{d \tau} \\
& c(0)=1 \quad c^{\prime}(0)=0 \quad c^{\prime}(T)=\frac{d c(T)}{d T}=-\frac{1}{f}
\end{aligned}
$$

Within an "ideal" magnet, where the bending radius $\rho_{0}$ is constant, $s$ and c. are sine and cosine or else sinh and cosh functions. Because of 
this, the terminology $\mathrm{s}=\mathrm{a}$ sine-like function and $\mathrm{c}=\mathrm{a}$ cosine-like function has been adopted for describing the general case where $\rho_{0}=\rho_{0}(\tau)$ is a function of $\tau$.

By analogy with previous discussions, we observe that whenever $s(\tau)=0$, we are at an image of point $A$. Also, $c(\tau)$ at the position where $s(\tau)=0$ is the magnification of point $A$ at that image.

$$
c^{\prime}(t)=-1 / f \text { where } f \text { is the focal length of the system between } A
$$

and $B$. The dispersion $d_{x}$ may be derived from the general differential equations of motion of a charged particle in a static magnetic field. (I) The results may always be expressed as a function of $s_{x}$ and $c_{x}$ as follows:

$$
d_{x}(t)=s_{x}(t) \int_{0}^{t} c_{x}(\tau) d \alpha-c_{x}(t) \int_{0}^{t} s_{x}(\tau) d \alpha
$$

and

$$
d_{x}^{\prime}(t)=s_{x}^{\prime}(t) \int_{0}^{t} c_{x}(\tau) d \alpha-c_{x}^{\prime}(t) \int_{0}^{t} s_{x}(\tau) d \alpha
$$

where

$$
d \alpha=\frac{d \tau}{\rho_{\mathrm{o}}(\tau)}
$$

is the differential angle of bend of the central trajectory. At an image point $[s(t)=0]$ note that

$$
d_{x}(t)=-c(t) \int_{0}^{t} s(\tau) d \alpha
$$

This approach to the problem may be generalized to include all of the second-order aberrations of a system. When this is done, it is always

(1) See SIAC-75 for a derivation of these equations. 
possible to express these aberrations as functions of the first-order. matrix elements $c_{x}, s_{x}, d_{x}, c_{y}$ and $s_{y} \cdot$

Having developed the above physical concepts and mathematical tools, we are now in a position to study more complicated systems. As an example, we consider the general system shown in Figure 14.

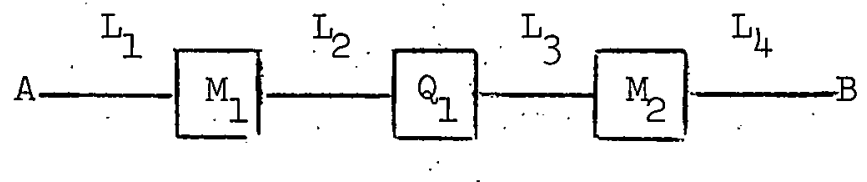

Figure 14

I. $=$ drift elements

= magnetic elements

The matrix formalism states that in the $x$ plane, the transformation from $A$ to $B$. is given by the following matrix equation.

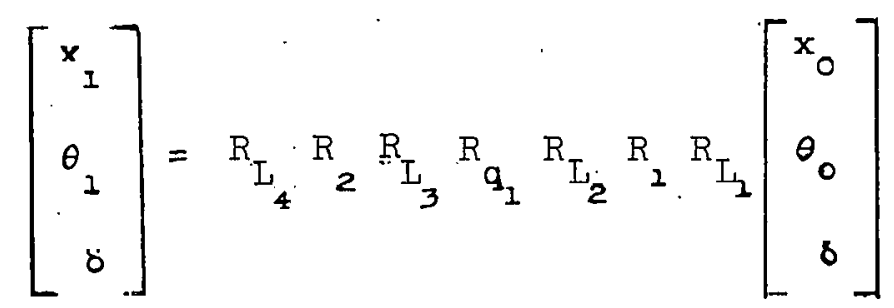

As in all matrix calculations, the order of writing down the elements comprising the system is from right to left. The individual matrix elements must be derived from the solution of the equation of motion within each element. If this has been done, then the calculation for the total system is carried out in the fashion shown by the above equation.

4. Second-Order Matrix Formalism (1)

It is possible to extend the $3 \times 3$ matrix formalism to solve simultaneous sets of power series by generating a second-order matrix equation 
as follows:

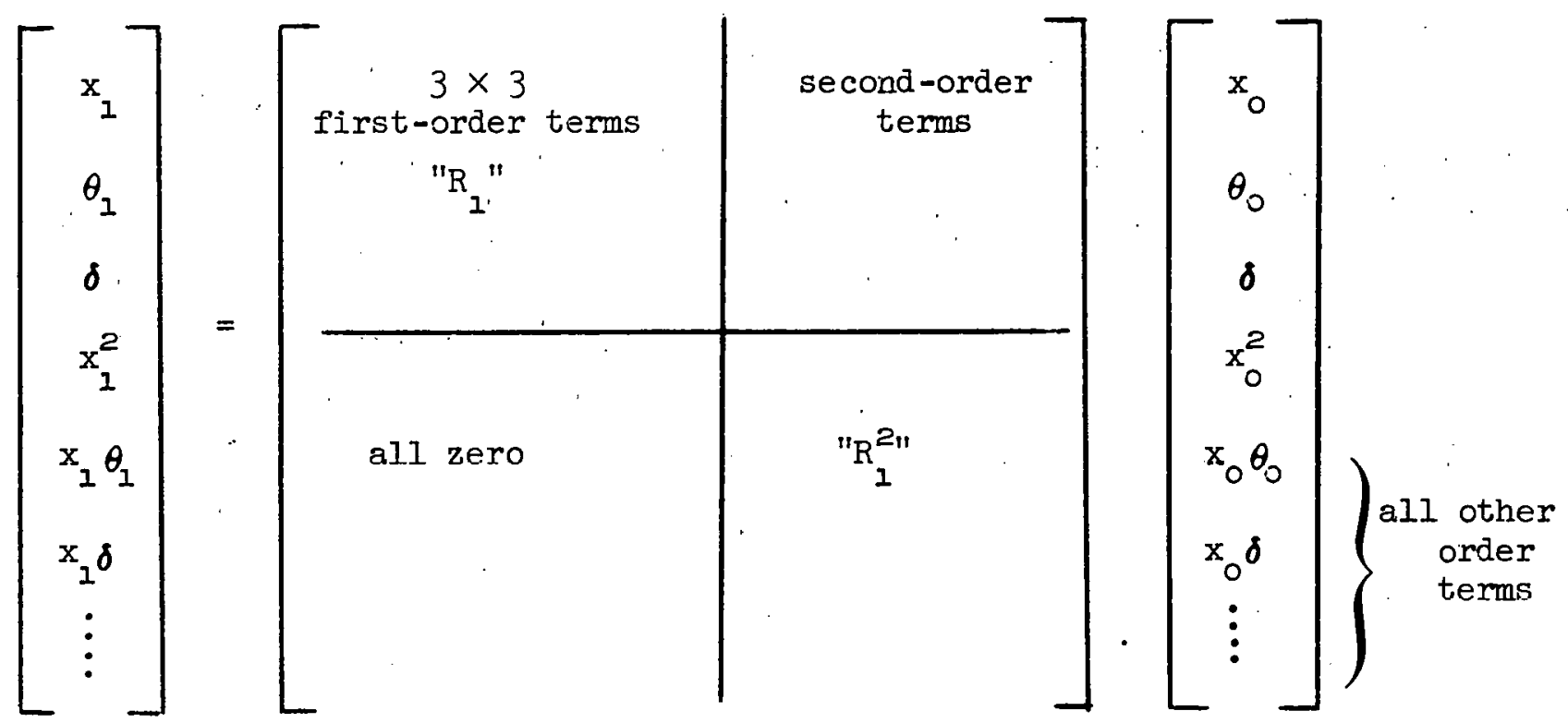

The " $R^{2 "}$ term is obtained by squaring the upper left corner $(3 \times 3)$ matrix so as to obtain second-order equations for $x_{I}^{2}, x_{1} \theta_{1}, x_{1} \delta$, etc., as functions of products of the initial first-order variables $x_{0}, \theta_{0}$, and $\delta$.

This is, then, a convenient mathematical formalism for keeping all the terms desired and dropping those undesired. In the above example, all firstand second-order terms are retained and all higher-order terms are automatically dropped by the matrix multiplication.

\section{Transformations Involving Many Trajectories}

All of the discussion to this point relates to the transformation of a single trajectory (in addition to the central trajectory) through a static magnetic system. We wish now to extend the discussion to include "bundles" of rays. To accomplish this, we take advantage of Liouville's Theorem, which states that the "phase space" is conserved through the system. While the Theorem is strictly true to all orders, a convenient mathematical transfornation 
has only been developed to first-order. A manifestation of Liouville's Theorem is the fact that $|R|=1$.

Now, so long as there is no coupling mechanism between the $x$ plane and $y$ plane of a magnetic-optical system (which is the case if the midplane symmetry prevails throughout the system) then, the phase space area in a given plane is also conserved. Consider a bundle of rays represented by. the parallelogram, shown in Figure 15(a), representing the phase space distribution of the rays at some initial position. If we now look at the phase space distribution of the same bundle after it has drifted down stream, we observe the the $\theta_{\max }$ boundary and the $x$ intercept $x_{i}$ remain unchangeci. In other words, the area of the parallclogram is the same or "phase space area has been conserved."

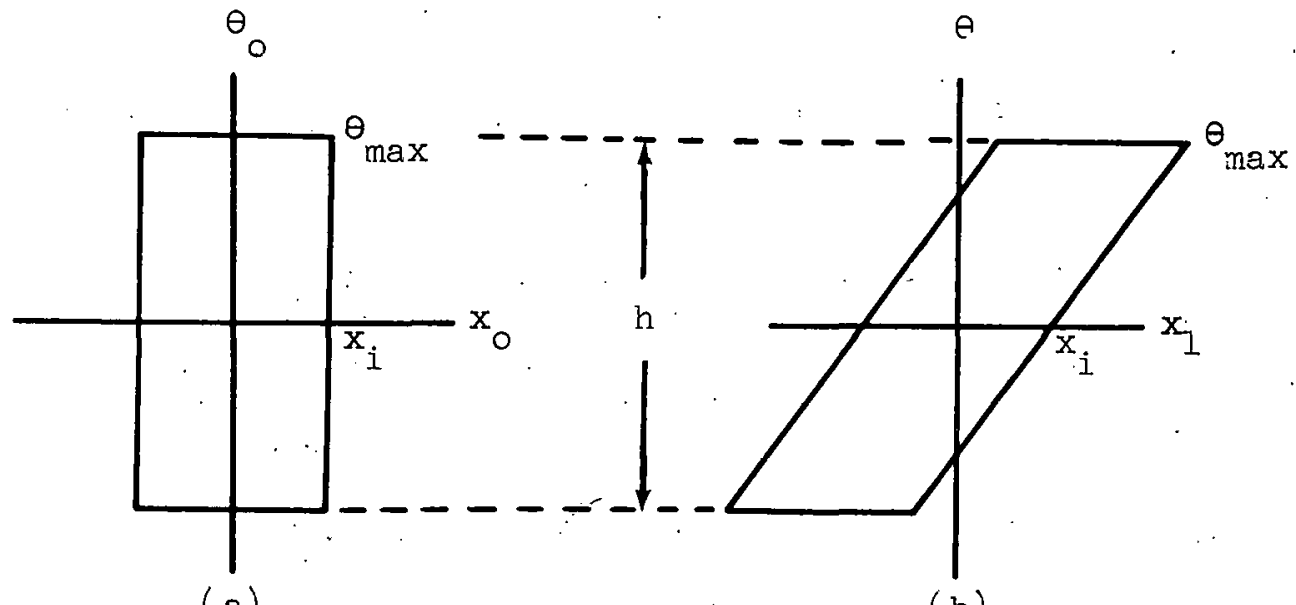

(a)

Figure 15

(b)

For mathematical convenience, the parallelogram is rather difficult to work with and, hence, a. phase ellipse is usually used.

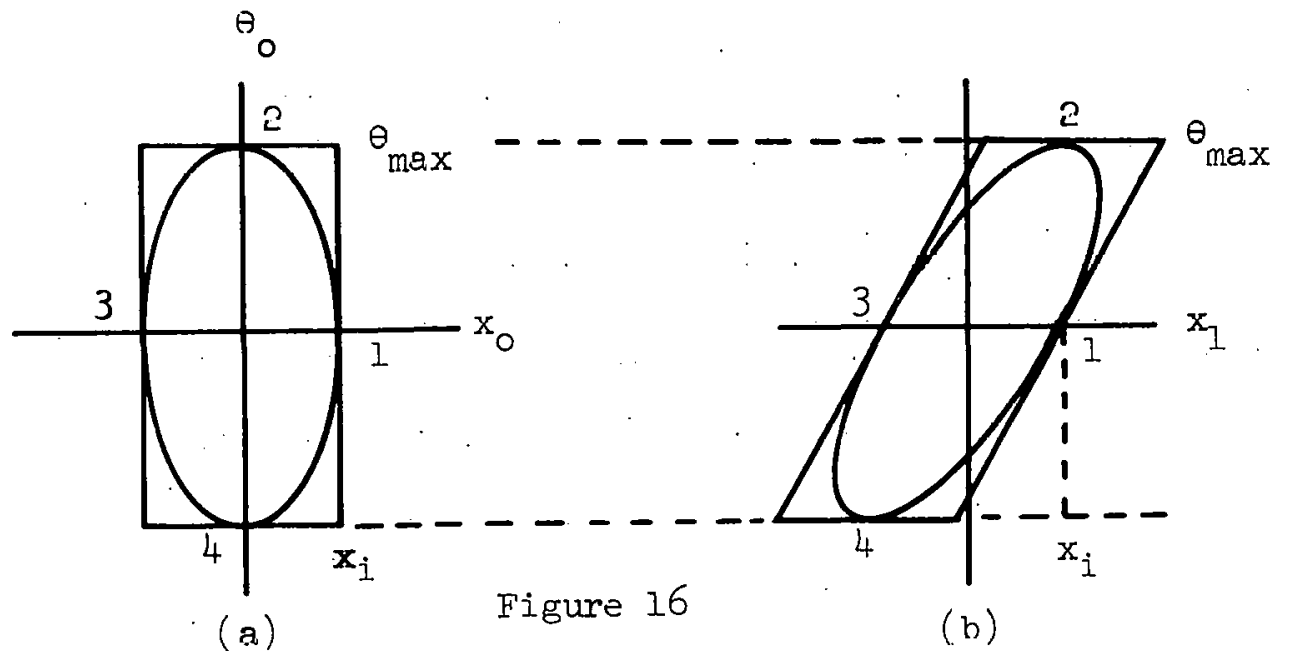


The phase ellipse transformation for a drift distance is illustrated in Figure 16. Figure 16(a) corresponds to a beam which is at its minimum width (a "waist") and Figure 16(b) shows the same beam after it has drifted downstream from the waist position. The physical meaning of this is that particles entering at $\theta=0$ are parallel to the optic axis and, therefore, cannot change their relative positions with respect to the optic axis; that is, all particles on the $x_{0}$ axis act in this manner. Those that enter at a given angle continue at the same angle.

The phase ellipse transformation for a thin lens is illustrated in Figure 17. In passing through a thin lens, $\theta$ changes and the $x$ dimension remains constant for a given trajectory.

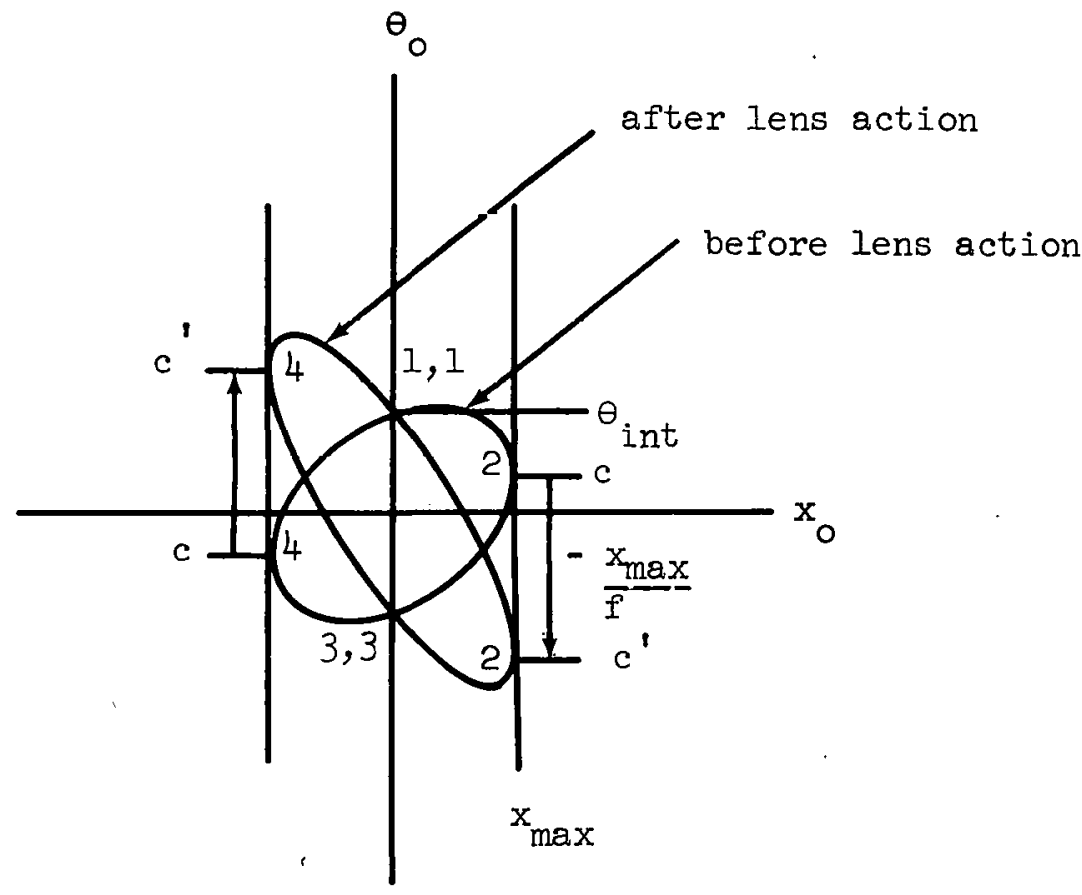

F'igure 17

Stated in other terms, $x_{\max }$ remains constant, as does $\theta_{i}$ (the $\theta$ intercept). It is apparent, in the given example, that the spot size now becomes, or can become, smaller at the new image because $\theta_{\max }$ is larger. This can be related to the physics of the system by saying that the $x$ magnification is less than unity. This fact is observed directly by comparison of the $x$ intercept of the ellipse before and after the lens action. It is interesting to observe that a particle initially at $c$ is transformed to $c^{\prime}$ and that particles entering at $x=0$ do not change their direction $\left({ }_{i}\right.$ is comstant). Tf the particleo are now allowed to drift, the ellipse rotates clockwise; when the ellipse if vertical, the spot size is at a minimum, namely, $x_{\max }=x_{1}$, as was illustrated in Figure 16. 
Beam Transport Optics - Part II

(K. L. Brown)

\section{Introduction}

In Part I, the basic concepts of bean transport optsics were established. Starting from the essentials of geonetric optics, the methods of matrix algebra were introduced with the example of calculating the principal planes of a thick lens. The $3 \times 3$ matrix for the first-order bean transport calculations were introduced to take into account the particle monenta.

\section{First Order Transformation Matrix}

Figure I shows a general region containing a magnetic field.

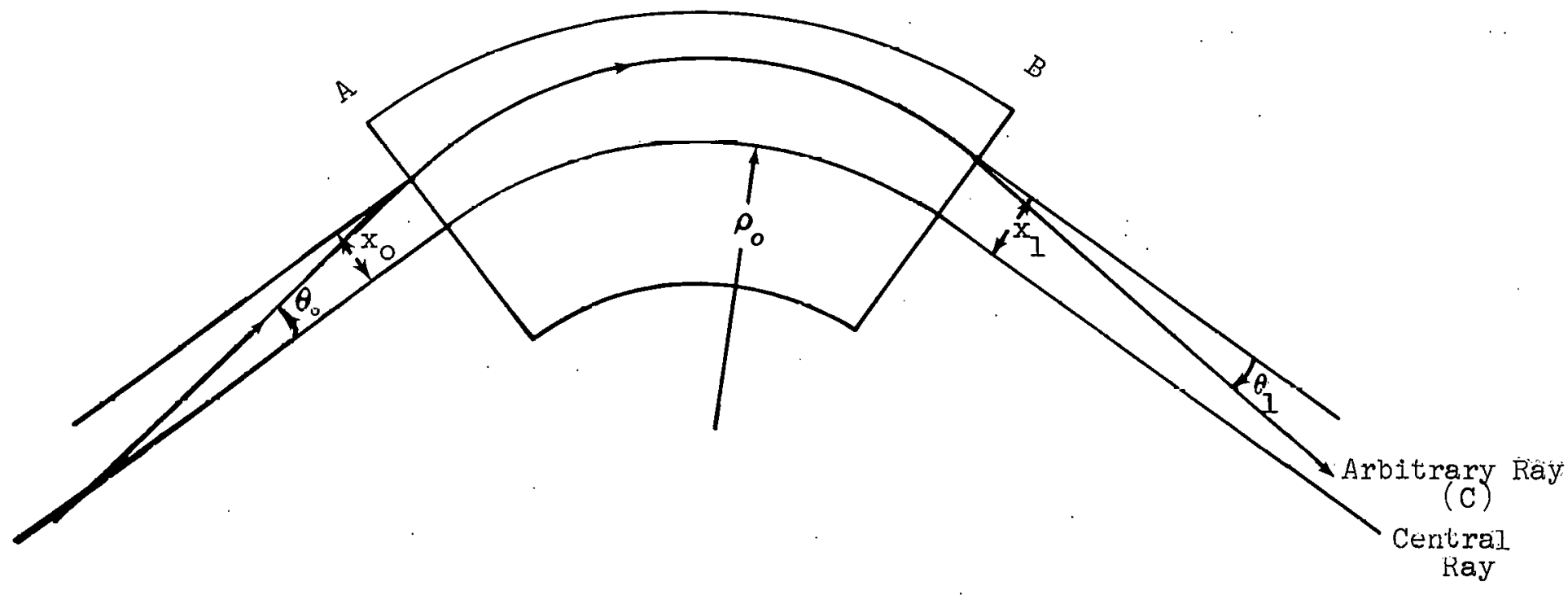

Figure 7: General Magnetic Fiela Cunfiguration

The matrix presents a convenient way of writing the family of equations which describe the transformation from surface A to surface $B$. If $x_{0}, \theta_{0}$ and $\delta$ represent the conditions of a ray entcring the system at $A$, then the conditions of the ray at $B$ are $x_{1}, \theta_{1}$ and $\delta$. Here $x_{0}$ is the distance from the central ray to the ray $c, \theta_{0}$ is the angle between $C$ and the parallel to the central ray and $\delta$ is the ratio $\Delta p / p$ where $\Delta p$ is the difference between the momentum of $\mathrm{C}$ and the momentum of the central ray. 
The linear tranșformation equations are:

$$
\begin{aligned}
x_{1} & =c_{x} x_{0}+s_{x} \theta_{0}+d_{x} \delta \\
\therefore \theta_{I} & =c_{x}^{1} x_{0}+s_{x}^{1} \theta_{0}+d_{x}^{\prime} \delta \\
\delta & =0+0+\delta
\end{aligned}
$$

Expressed as a matrix, Eq. (1) are:

$$
\left[\begin{array}{l}
x_{1} \\
\theta_{1} \\
\delta
\end{array}\right]=\left[\begin{array}{ccc}
c_{x} & s_{x} & d_{x} \\
c_{x}^{\prime} & s_{x}^{\prime} & d_{x}^{\prime} \\
0 & 0 & 1
\end{array}\right]\left[\begin{array}{c}
x_{0} \\
\theta_{0} \\
0
\end{array}\right]
$$

The equation $\delta=\delta$ expresses the fact that the magnetic field cannot change the scalar momentum of the particle. The $\delta$ terms in the $x$ and $\theta$ equations express the momentum dispersion of the system.

If it happens that $A$ is an object point and $B$ is an image point of the system, then $x_{1}$ is independent of $\theta_{0}$, thus $s_{x}=0$. In this case, $c_{x}$ is given by $c_{x}=x_{1} / x_{0}=M_{x}=$ the magnification in $x$ plane, $($ for $\delta=0)$. if $\dot{\theta}_{0}=\delta=0$, then $\theta_{1}=c_{x}^{\prime} x_{0}=-x_{0} / f$ or $c_{x}^{\prime}=-1 / f$. It must always be true that the determinant of the matrix $|R|$, is unity. Thus for this special case of $s_{x}=0$, it follows that $s_{x}^{1}=i / M_{x}$.

\section{Beam Switchyard}

As an example of a system which can be calculated with the matrix melliul, we next conjidcr the beam switchyard of the two-mile accelerator. Tigure 2 showd the three essential. element,s, two bending magnets and a quadrupole lens. In common with many beam transport systems, this one is designed to be achromatic. Mathematically, this means that the matrix elements, $d_{x}$ and $d_{x}^{\prime}$ should be zero, so that there is no $x$ or $\theta$ dependence. on the momentum of the particles. 


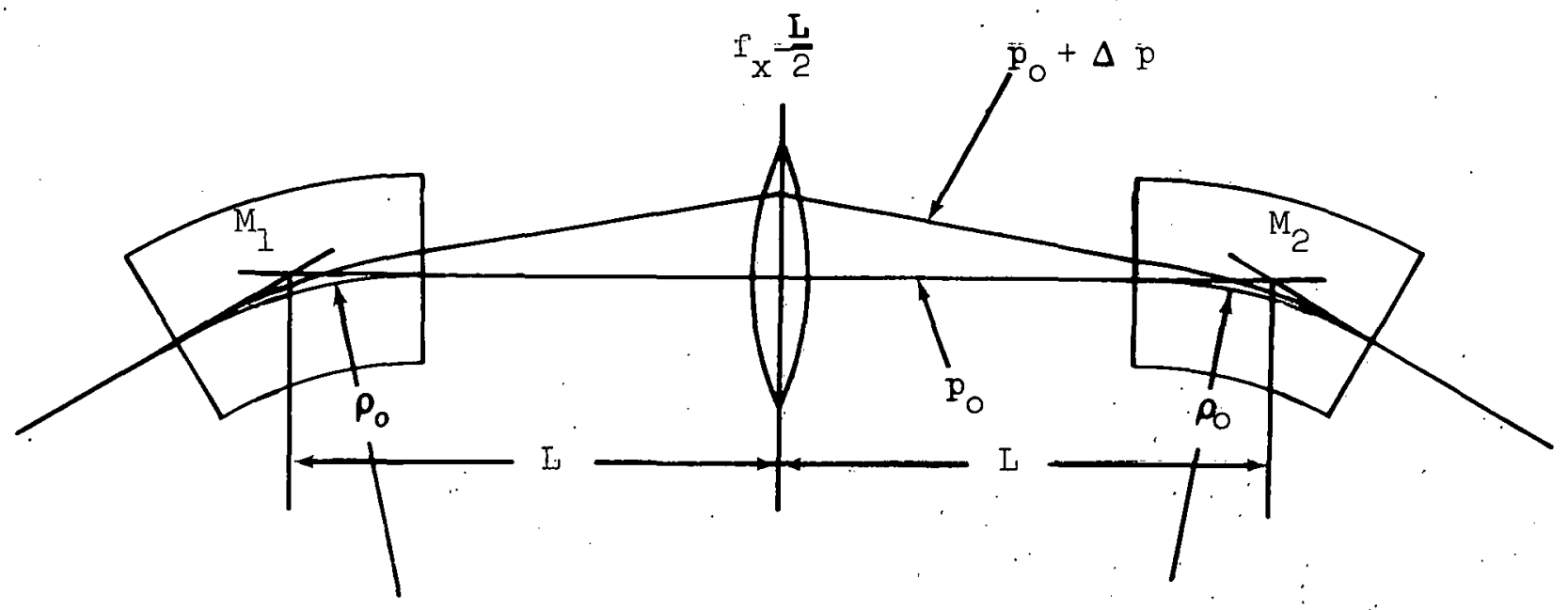

Figure 2: The Essentials of the Beam Switchyard

'As a preliminary step, we will find the matrix expression for a bending' magnet when measured from the principal planes. 'l'he matrix tor a bending magnet, when measured from the ends of the poles, is given by:

$$
R_{\text {bend }}=\left[\begin{array}{c|c|c}
c & s & 1-c \\
\hline-s & c & s \\
\hline 0 & 0 & 1
\end{array}\right]
$$

where $c=\cos \alpha$ and $s=\sin \alpha$ and $\alpha$ is the deflection angle of the central ray. This expression has been normalized by setting the bending radius equal to unity. To restore ordinary units it is only necessary to insert the bending radius wherever a length is needed dimensionally. In this case, the matrix then becomes

$$
R_{\text {bend }}=\left[\begin{array}{c|c|c}
c & \rho s & \rho(1-c) \\
\hline-r / \rho & 0 & 1 \\
\hline 0 & 0, & i
\end{array}\right]
$$

If the distance from the entrance plane to the first principal plane is $z_{1}$ and the distance from the second principal plane to the exit plane is $z_{2}$, we can find the values $z_{1}$ and $z_{2}$ by solving the following matrix 
equation:

$$
\left[\begin{array}{ccc}
1 & -z_{2} & 0 \\
0 & 1 & 0 \\
0 & 0 & 1
\end{array}\right]\left[\begin{array}{ccc}
c & s & 1-c \\
-s & c, & s \\
0 & 0 & 1
\end{array}\right]\left[\begin{array}{ccc}
1 & -z_{1} & 0 \\
0 & 1 & 0 \\
0 & 0 & 1
\end{array}\right]=\left[\begin{array}{ccc}
1 & 0 & d_{x} \\
-1 / 1 & 1 & d_{x}^{\prime} \\
0 & 0 & 1
\end{array}\right]
$$

The matrix multiplication need only be done for the $2 \times 2$ matrices as outlined. To illustrate matrix multiplication the indicated operations will be given below in natural stages as follows:

$$
\begin{aligned}
& {\left[\begin{array}{c|c}
1 & -z_{2} \\
\hline 0 & 1
\end{array}\right]\left[\begin{array}{c|c}
c & -c z_{1}+s \\
\hline-s & +s z_{1}+c
\end{array}\right]=\left[\begin{array}{c|c}
1 & 0 \\
\hline-1 / f & 1
\end{array}\right]} \\
& {\left[\begin{array}{c|c}
c+z_{2}{ }^{s} & \begin{array}{l}
-c z_{1}+s \\
+s z_{1} z_{2}-c z_{2}
\end{array} \\
\hline-s & +s z_{1}+c
\end{array}\right]=\left[\begin{array}{cc}
1 & 0 \\
-1 / f & 1
\end{array}\right]}
\end{aligned}
$$

Note that these transformations do not change the focal length expression, $-I / f=-s$. In order for two matrices to be equal, each individual element must be equal to its counterpart in the other matrix. Thus we have

$$
\begin{aligned}
& c+z_{2} s=1 \\
& c+z_{1} s=1
\end{aligned}
$$

which when solved for $z_{1}$ and $z_{2}$ yield

$$
\mathrm{z}_{2}=(1-\mathrm{c}) / \mathrm{s} \text { and } \mathrm{z}_{1}=(1-\mathrm{c}) / \mathrm{s} \text {. }
$$

If we substitute the trigonometric equivalents, and apply standard identities, we have $z_{2}=z_{1}=\tan (\alpha / 2)$ which can be seen from Figure 3 to indicate that the two principal planes are coincident with the symmetry plane in the middle of the magnet. 


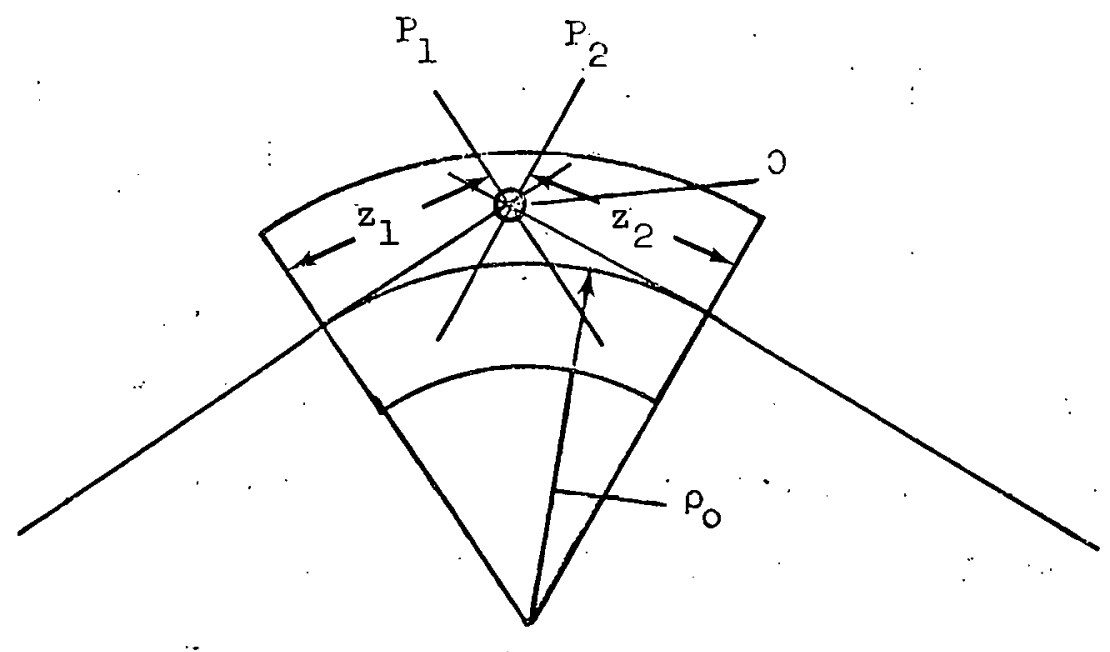

Figure 3: The Principal. Planes of a Simple Rending Magnet are Coincident with the Center Planc

The simplified matrix for a bending magnet measured to the principal planes is then:

$$
\mathrm{R}_{\text {bend }}=\left[\begin{array}{c|c|c}
I & 0 & 0 \\
\hline-s & 1 & s \\
\hline 0 & 0 & 1
\end{array}\right]
$$

To calculate the transformation matrix. for the entire Beam Switchyard system.as shown in Fig. 2, we write the matrices in opposite order from that in which the beam passes through the elements. That this must be true can be seen from the way in which one element alone is calculated by

$$
\left[\begin{array}{l}
x_{1} \\
\theta_{1} \\
\delta
\end{array}\right]=\bar{R}_{1}\left[\begin{array}{c}
x_{0} \\
\theta_{0} \\
\delta
\end{array}\right]
$$

Then for a second element we have

$$
\left[\begin{array}{c}
x_{2} \\
\theta_{2} \\
\delta
\end{array}\right]=R_{2}\left[\begin{array}{c}
x_{1} \\
\theta_{1} \\
\delta
\end{array}\right]=R_{22} R_{1}\left[\begin{array}{c}
x_{0} \\
\theta_{0} \\
\delta
\end{array}\right]
$$

and so forth. 
If we allow the system to be symmetrical, i.e. $s_{1}=s_{2}$ and $L_{1}=I_{2}$; the complete series of matrices for Fig. 2 are

$$
R_{\mathrm{BSY}}=\left[\begin{array}{c|c|c}
1 & 0 & 0 \\
\hline-s & 1 & \mathrm{~s} \\
\hline 0 & 0 & 1
\end{array}\right]\left[\begin{array}{l|l|l}
1 & L & 0 \\
\hline 0 & 1 & 0 \\
\hline 0 & 0 & 1
\end{array}\right]\left[\begin{array}{l|l|l}
1 & 0 & 0 \\
\hline-1 / f & 1 & 0 \\
\hline 0 & 0 & 1
\end{array}\right]\left[\begin{array}{l|l|l}
1 & L & 0 \\
\hline 0 & 1 & 0 \\
\hline 0 & 0 & 1
\end{array}\right]\left[\begin{array}{l|l|l}
I & 0 & 0 \\
\hline-s & 1 & s \\
\hline 0 & 0 & 1
\end{array}\right]
$$

We will show the step-by-step multiplication of the matrices to get the $d_{x}$ and $d_{x}^{\prime}$ terms.

$$
R_{B S Y}=\left[\begin{array}{l|l|l}
1 & L & 0 \\
\hline-s & 1-s L & s \\
\hline 0 & 0 & I
\end{array}\right]\left[\begin{array}{l|l|l}
1 & 0 & 0 \\
\hline-1 / f & 1 & 0 \\
\hline 0 & 0 & I
\end{array}\right]\left[\begin{array}{l|l|l}
1-s L & L & L s \\
\hline-s & 1 & s \\
\hline 0 & 0 & 1
\end{array}\right]
$$$$
R_{\mathrm{BSY}}=\left[\begin{array}{c|c|c}
\left(1-\frac{L}{f}\right) & \\
-L s\left(2-\frac{L}{f}\right) & L\left(2-\frac{L}{f^{\prime}}\right) & \operatorname{Ls}\left(2-\frac{L}{f}\right) \\
\hline(L s-I)\left[2 s+\frac{I}{f}(I-L s)\right] & \begin{array}{c}
\left(I-\frac{L}{f}\right) \\
-L s\left(2-\frac{L}{f}\right)
\end{array} & s(I-L s)\left(2-\frac{L}{f}\right) \\
\hline 0 & 0 & 1
\end{array}\right] .
$$

To obtain the required condition that $d_{x}=d_{x}^{\prime}=0$, we set

$$
\left(2-\frac{L}{f}\right)=0 \text { or } \quad f=\frac{L}{2}
$$

then

$$
R_{B S Y}=\left[\begin{array}{l|l|l}
-I & 0 & 0 \\
\hline-2(I-L s) & -1 & 0 \\
\hline 0 & 0 & 1
\end{array}\right]
$$


Thus the quadrupole acts as a lens to refocus rays from the center of the first bending magnet to the conter of the last one. For the serious, student, it is a worthwhile exercise to do the BSY problem without the simplification which resulted from introducing the principal planes.

\section{TRANSPORT}

As an aid to solving beam transport problems, a computer program TRANSPORT has been developed at SLAC which takes the greatest amount of labor out of this work. The program operates in about the way as the BSY example above was calculated, but with some important exceptions. Most importantly:

1. TRANSPORT has the ability to find the best first-order solution given a certain set of constraints;

2. TRANSPORT also calculates the transformation of a whole family of rays as found in a beam by means of the concept of "phase space" which was introduced in Part I;

3. TRANSPORT can, as an option, calculate the second order effects on the beam. By second order is meant, for example, terms which depend not linearly on the displacement $x_{0}$, but on $x_{0}^{2}$ or $x_{0} \theta_{0}$, etc.

To aid in the discussion of TRANSPORT and of the second order terms we now introduce an abbreviated notation. By writing out the complete equations for $\mathrm{x}$ and $\mathrm{y}$, to second order, we will adequately have displayed the new notation.

$$
\begin{aligned}
x_{1}= & \left(x \mid x_{0}\right) x_{0}+\left(x \mid \theta_{0}\right) \theta_{0}+(x \mid \delta) \delta \\
& +\left(x \mid x_{0}^{2}\right) x_{0}^{2}+\left(x \mid x_{0} \theta_{0}\right) x_{0} \theta_{0}+\left(x \mid x_{0} \delta\right) x_{0} \delta \\
& +\left(x \mid \theta_{0}^{2}\right) \theta_{0}^{2}+\left(x \mid \theta_{0} \delta\right) \theta_{0} \delta+\left(x \mid \delta^{2}\right) \delta^{2} \\
& +\left(x \mid y_{0}^{2}\right) y_{0}^{2}+\left(x \mid y_{0} \Phi_{0}\right) y_{0} \Phi_{0}+\left(x \mid \Phi_{0}^{2}\right) \Phi_{0}^{2} \\
y_{1}= & \left(y \mid y_{0}\right)+\left(y \mid \Phi_{0}\right) \Phi_{0} \\
& +\left(y \mid x_{0} y_{0}\right) x_{0} y_{0}+\left(y \mid x_{0} \Phi_{0}\right) x_{0} \Phi+\left(y \mid \theta_{0} y_{0}\right) \theta_{0} y_{0} \\
& +\left(y \mid \theta_{0} \Phi_{0}\right) \theta_{0} \Phi_{0}+\left(y \mid y_{0} \delta\right) y_{0} \delta+\left(y \mid \delta \Phi_{0}\right) \delta \Phi_{0}
\end{aligned}
$$


The absence of certain terms which might otherwise be expected in Eqs. (14) and (15) is due to the fact that horizontal mid-plane symmetry has been assumed in the derivation. That is, the field on the horizontal mid-plane is normal to the plane. Thus there can be no $(y \mid x)$ or $(y \mid \theta)$ term. Similarly, there can only be even powers of $\mathrm{y}$ and $\phi$, such as $\left(\mathrm{x} \mid \mathrm{y}_{0}^{2}\right)$ and $\left(\mathrm{x} \mid \mathrm{y}_{0} \Phi_{\mathrm{O}}\right)$, in the $\mathrm{x}$ equation. Also, note that there is no $(\mathrm{y} \mid \delta)$ or $\left(\mathrm{y} \mid \delta^{2}\right)$ term if there is mid-plane symmetry.

TRANSPORT uses a numerical notation to signify the six basic coordinates:

$$
\begin{array}{llllll}
\mathrm{x} & \boldsymbol{\theta} & \mathrm{y} & \boldsymbol{\phi} & \ell & 0 \\
1 & 2 & 3 & 4 & 5 & 6
\end{array}
$$

The $\ell$ term has not been introduced here before. Its significance is the preservation of the bunch length of a beam such as the SLAC electron beam. The first order outpit, from TRANSPORT is a $6 \times 6$ matrix printout of the $R$ matrix where the labels are implied by row and column position of the elements. For example the element appearing at the intersection of row 3 and column 4 is the coefficient $\left(y \mid \Phi_{0}\right)$ etc.

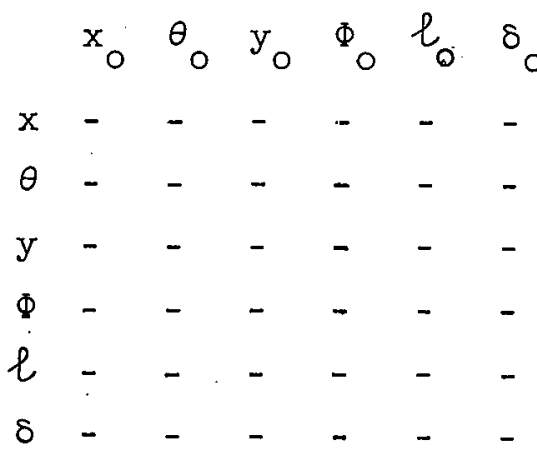

The second order terms are labelied by the convention indicated in Eqs. (14), (15) and (16). For example, $\left(x \mid x_{0}^{2}\right)$ becomes 111 and $\left(x \mid \theta_{0} \delta\right)$ becomes 126.

5. Second Order Matrix

Normally the matrix method is expected only to apply to the solution 
of linear i.e., first order, equations. However, the method has been

extended, to include second order terms in a $12 \times 12$ matrix as discussed in Part I and as shown above.

For a more extensive discussion of the second-order matrix formalism, the reader is referred to SLAC Report Number 75 by K.L. Brown. 


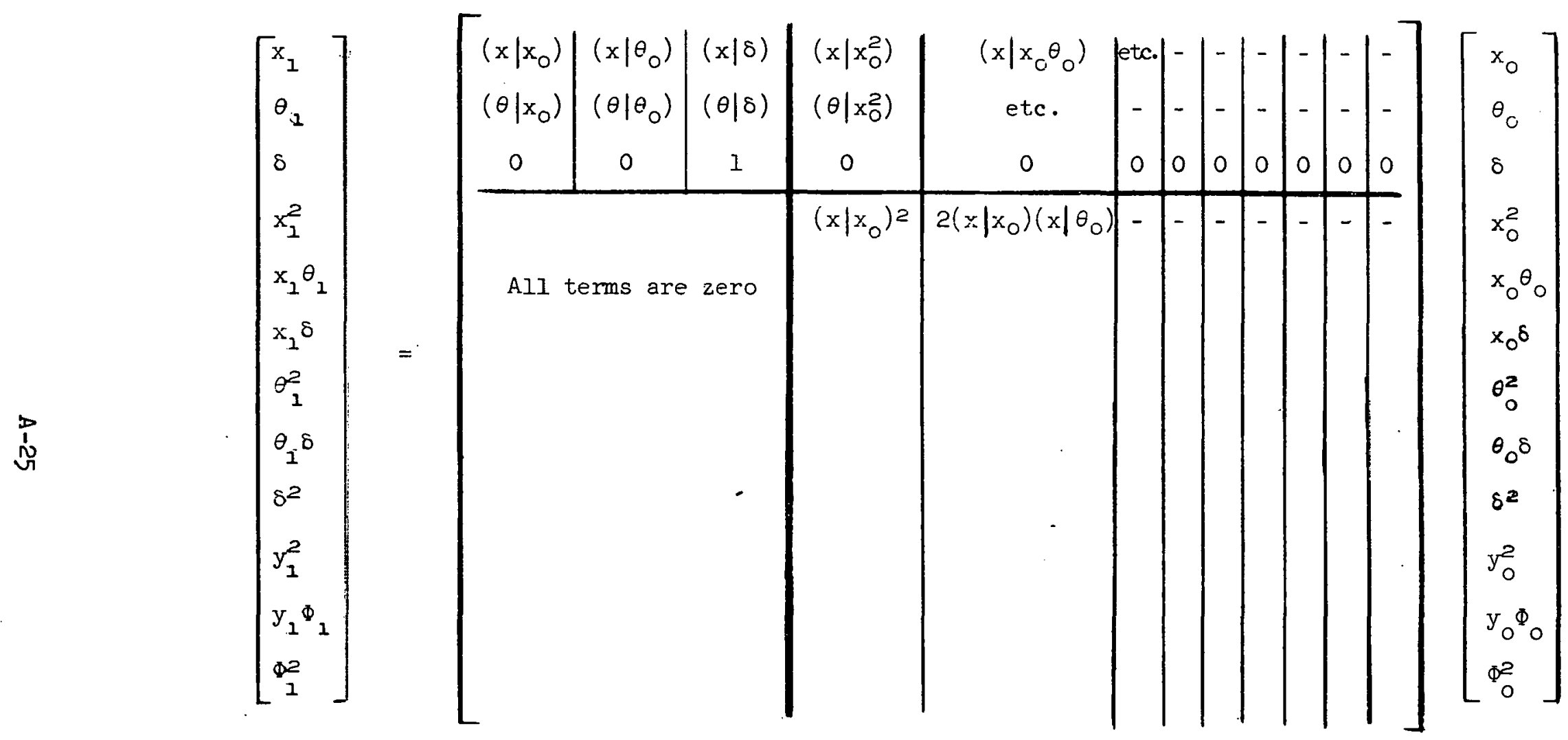

For example, the $x_{1}^{2}$ terms come from squaring Eq. (14). 
Section II

- Beam transport optics may be reduced to a process of matrix multiplication $(1,2)$. To first-order, this is represented by the matrix equation (using the notation of SLAC-75).

$$
x_{i}(t)=\sum_{j=1}^{6} R_{i j} x_{j}(0)
$$

where

$$
x_{1}=x \quad x_{2}=\theta \quad x_{3}=y \quad x_{4}=\varphi \quad x_{5}=\ell \quad \text { and } x_{6}=\delta
$$

The determinant $|R|=1$. This is a direct consequence of the basic equation of motion for a charged particle in a static magnetic field and is a manifestation of Liouville's theorem of conservation of phase space volume. (See SLAC-75, page 41 for a proof that $|R|=1$.)

For static magnetic systems possessing midplane symmetry, the six simultaneous linear equations represented by $\mathrm{Eq}$. (1) may be expanded in matrix form as follows:

$$
\left[\begin{array}{c}
x(t) \\
\theta(t) \\
y(t) \\
\varphi(t) \\
l(t) \\
\delta(t)
\end{array}\right]=\left[\begin{array}{c|c|c|c|c|c}
\mathrm{R}_{11} & \mathrm{R}_{12} & 0 & 0 & 0 & \mathrm{R}_{16} \\
\mathrm{R}_{21} & \mathrm{R}_{22} & 0 & 0 & 0 & \mathrm{R}_{26} \\
0 & 0 & \mathrm{R}_{33} & \mathrm{R}_{34} & 0 & 0 \\
0 & 0 & \mathrm{R}_{43} & \mathrm{R}_{44} & 0 & 0 \\
\mathrm{R}_{51} & \mathrm{R}_{52} & 0 & 0 & 1 & \mathrm{R}_{56} \\
0 & 0 & 0 & 0 & 0 & 1
\end{array}\right]\left[\begin{array}{l}
x_{0} \\
\theta_{0} \\
y_{0} \\
\varphi_{0} \\
l_{0} \\
\delta_{0}
\end{array}\right]
$$


where the transformation is from an initial position $\tau=0$ to a final position $T=t$ measured along the assumed central reference trajectory

Thus at any specified position in a system, an arbitrary charged particle is represented by a vector. (single column matrix), $X$, whose components are the positions, angles, and momentum of the particle with respect to a specified reference trajectory.

$$
\text { i.e. } \quad \mathrm{x}=\left[\begin{array}{c}
\mathrm{x} \\
\theta \\
\dot{\mathrm{y}} \\
\varphi \\
\ell \\
\delta
\end{array}\right]
$$

where:

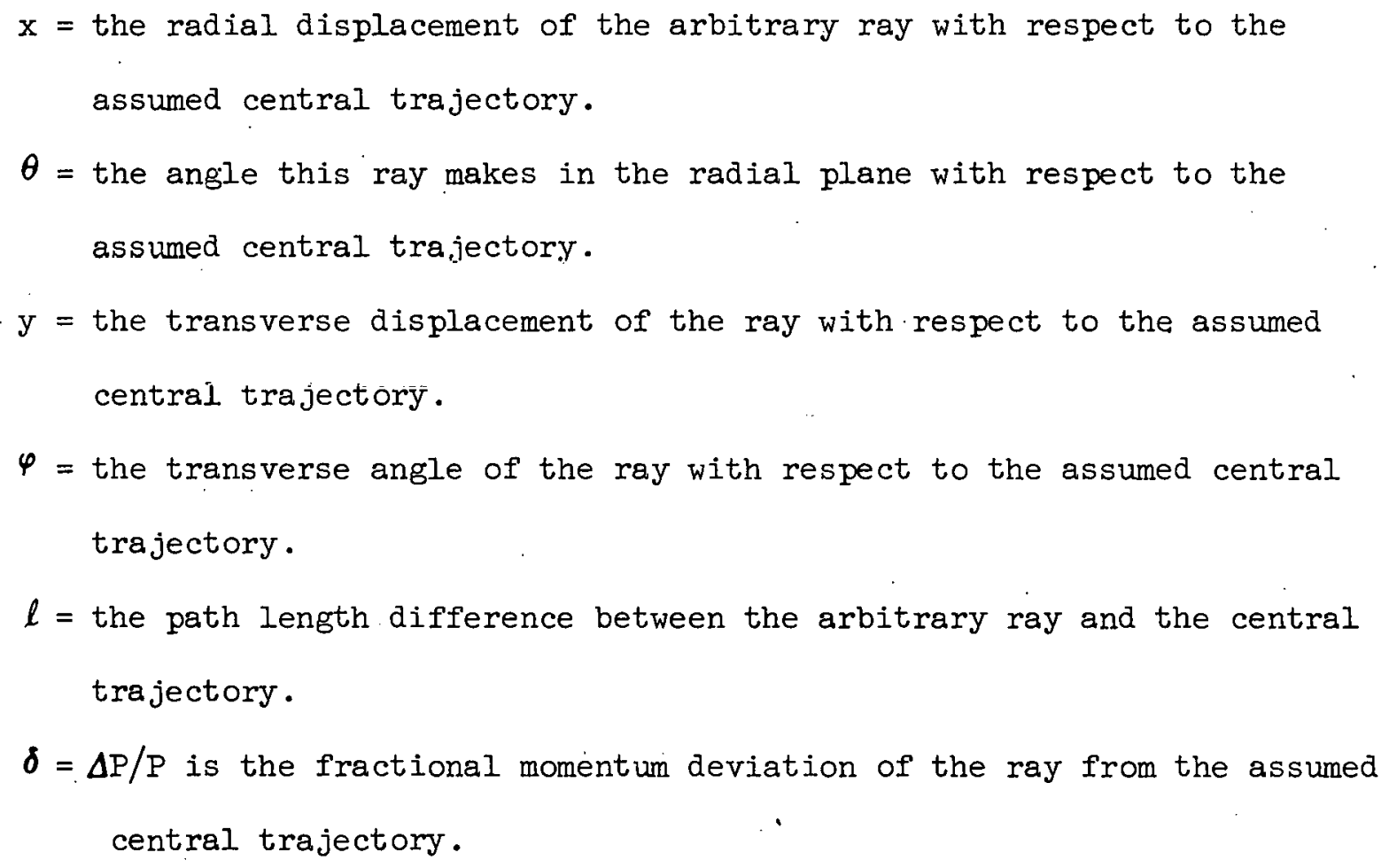


The magnetic lens is represented. by the square matrix, $R$, which describes the action of the magnet on the particle coordinates. Thus the passage of.a charged particle through the system may be represented by the matrix equation:

$$
X(1)=R X(0)
$$

where $X(0)$ is the initial coordinate vector and $X(1)$ is the final coordinate vector of the particle under consideration; $R$ is the transformation matrix for all such particles traversing the system (one particle differing from another only by its initial coordinate vector $X(0))$.

The traversing of several magnets and interspersing orift spaces is described by the same basic equation but with $R$ now being the product matrix $R=R(n) \ldots$ $R(3) R(2) R(1)$ of the individual matrices of the system elements. TRANSPORT calculates and tabulates the product matrix $R$ representing the system.

$$
\text { The zero elements } R_{13}=R_{14}=R_{23}=R_{24}=R_{31}=R_{32}=R_{41}=R_{42}=R_{36}=R_{46}=0
$$

in the $R$ matrix are a direct consequence of midplane symmetry. If midplane symmetry is destroyed, these elements will in general become non-zero. The zero elements in column five occur because the variables $\mathrm{x}, \theta, \mathrm{y}, \varphi$, and $\delta$ are independent of the path length difference $\ell$. The zero's in row six result from the fact that we have restricted the problem to static magnetic fields, i.e., the scalar momentum is a constant of the motion.

In SLAC report 75 (Ref. 1), a physical significance has been attached to the non-zero matrix elements in the first four rows in terms of their identification with characteristic first-order trajectories. We include figures showing these characteristic functions as a convenient reference.

We now wish to relate the elements appearing in column six and those in row five in terms of simple integrals of the characteristic first-order matrix elements. $c_{x}(t)=R_{11}$ and $s_{x}(t)=R_{12}$. In order to do this, we make use of the Green's function integral, Eq. (43), Section II of SLAC-75, and of the expression for the differential path length in curvilinear coordinates 


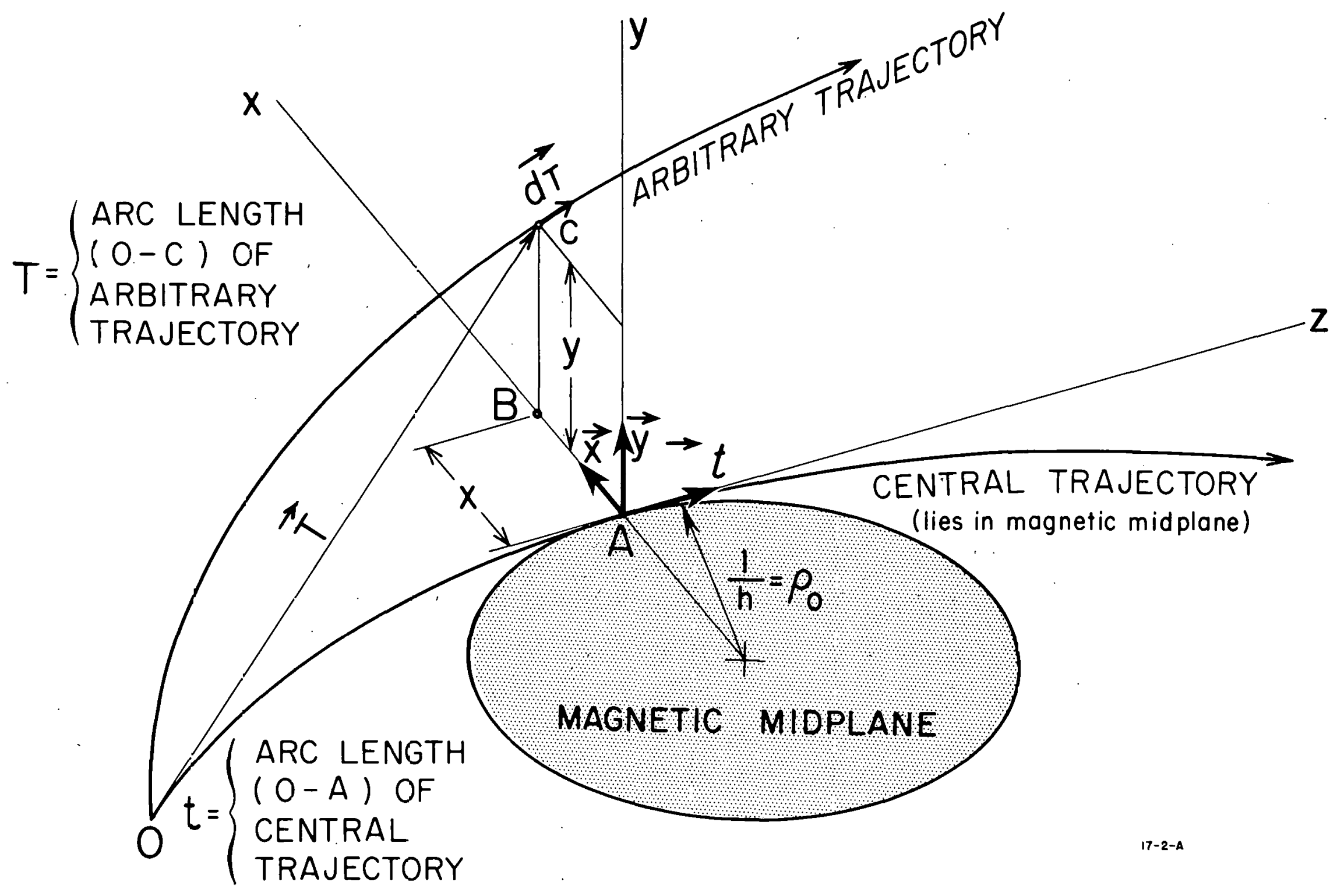

FIG. 1--CURVILINEAF: COORDINATE SYSTEM USED IN DERIVATION OF EQUATIONS OF MOTION. 


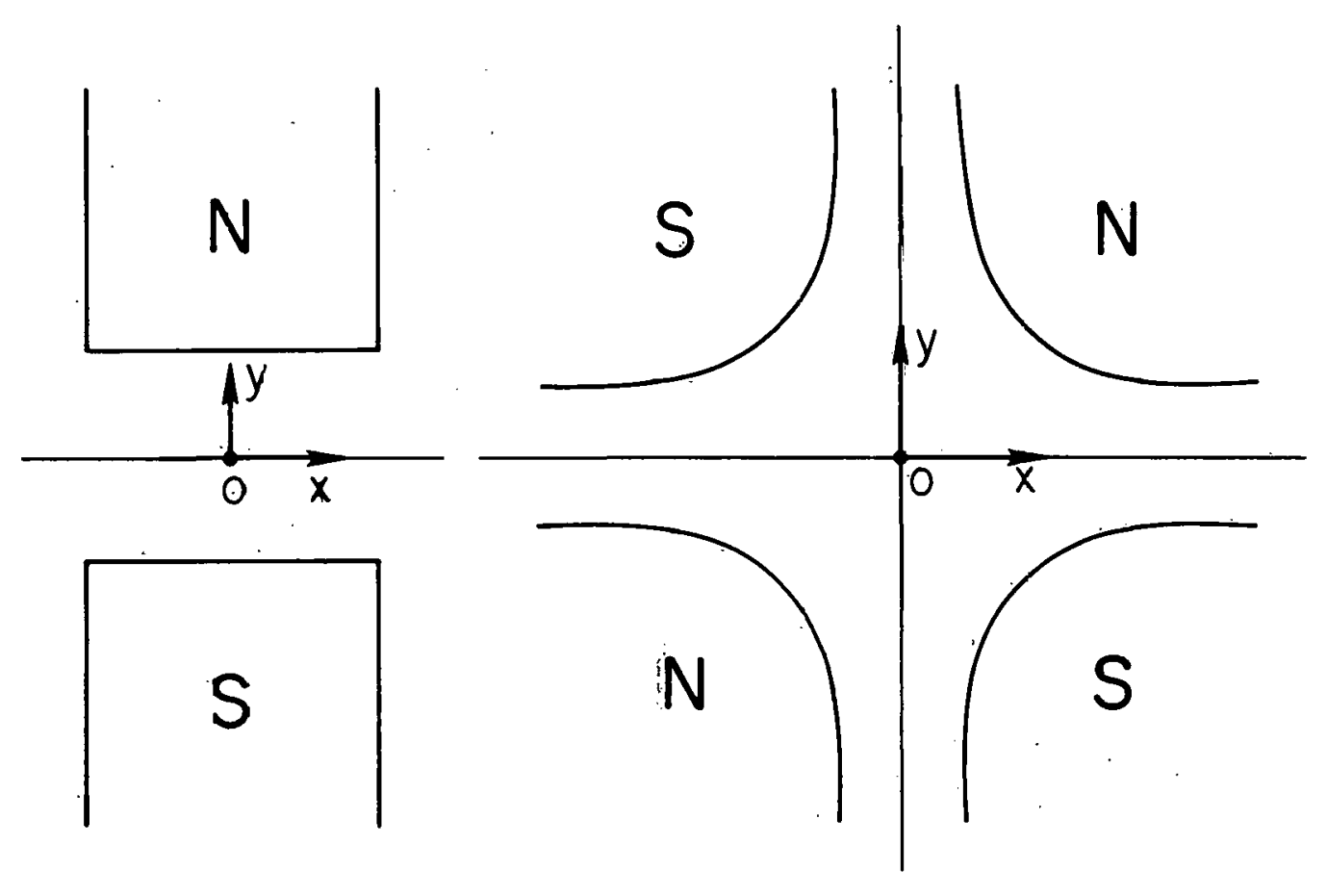

DIFOLE

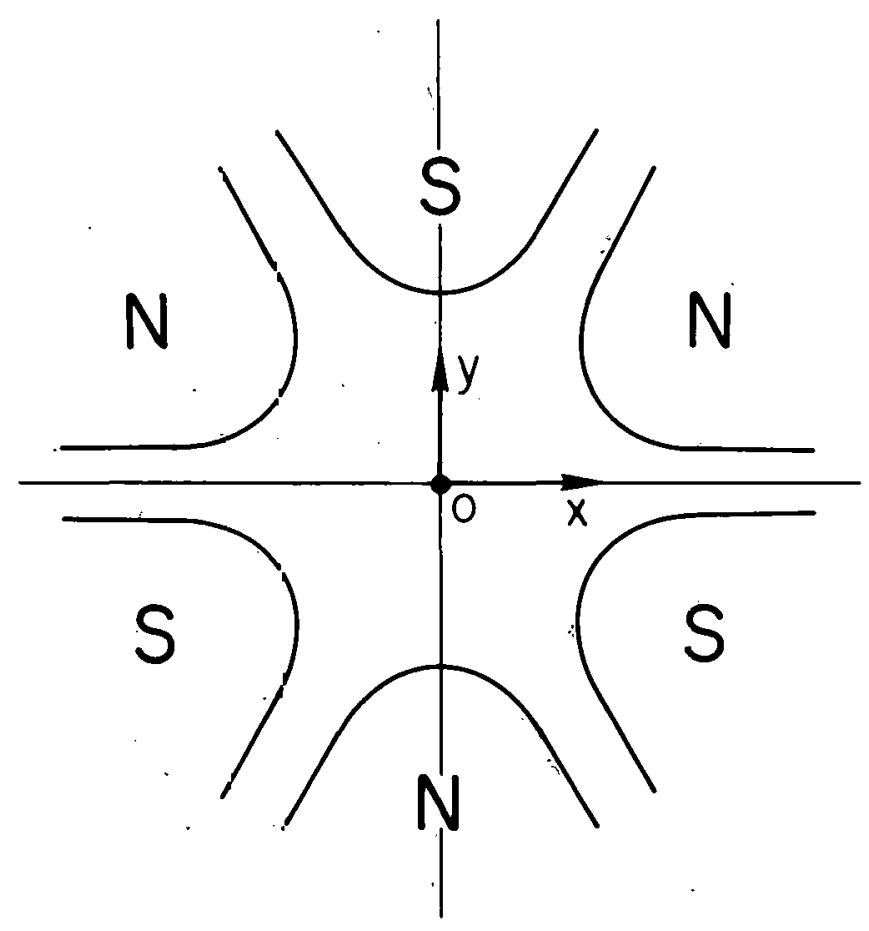

SEXTUPOLE

FIG. 2--TLLUSTRATION OF THE MAGNETIC MIDPLANE (X AXIS) FOR DIPOLE, QUADRUPOLE AND SEXTUPOLE ELEMENTIS: THE MAGNETIC POLARITIES INDICATE MUIT TPOLE ELEMETITS THAT ARE POSITTVE IN RBSPECT TO EQCH OTHER. 


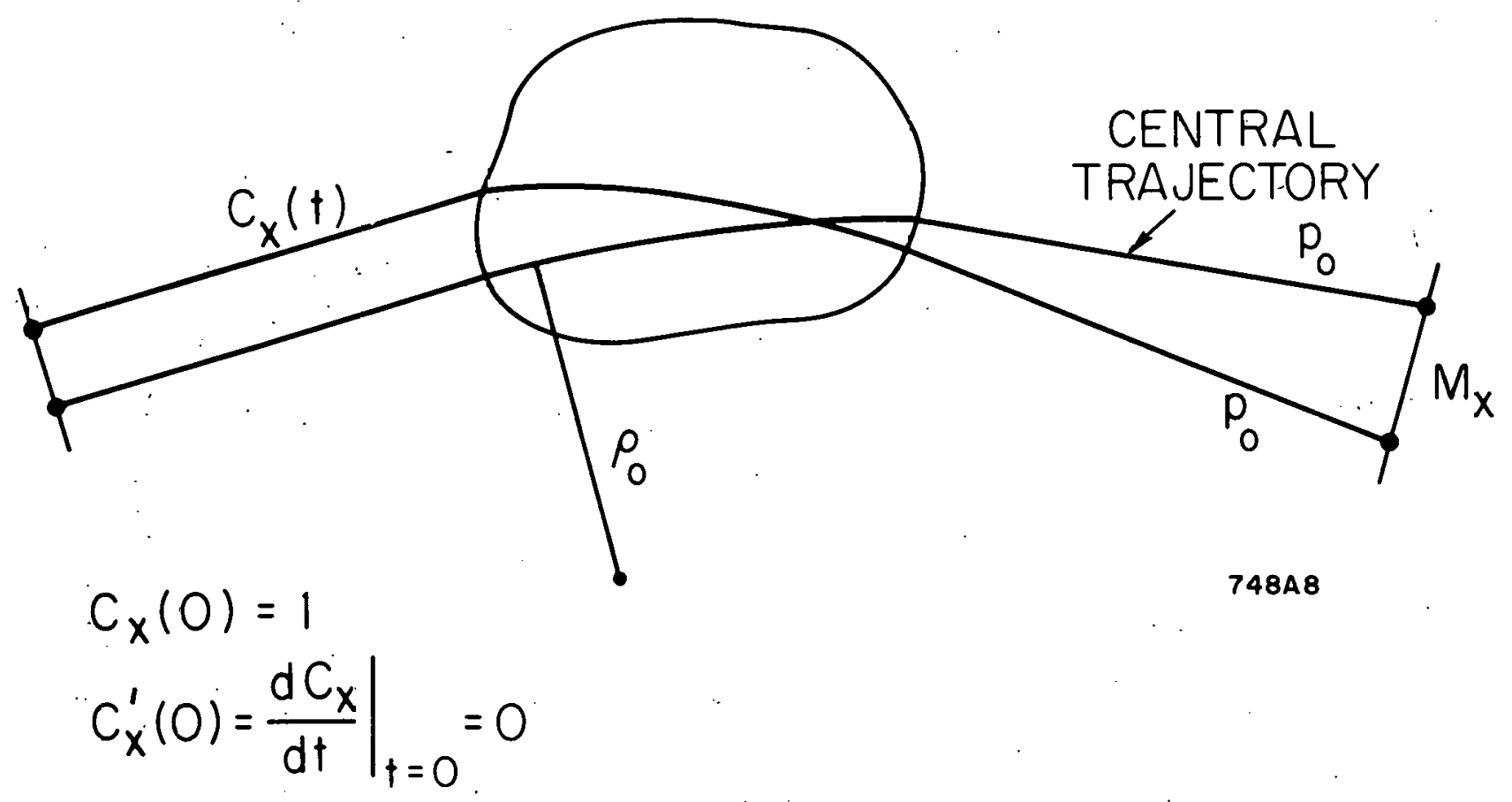

FIG. 3--COSINE-LIKE FUNCTION $c_{x}(t)=R_{1 I}$ IN MAGNETIC MIDPLANE. $\quad c_{x}^{\prime}(t)=R_{2 l}$ : 


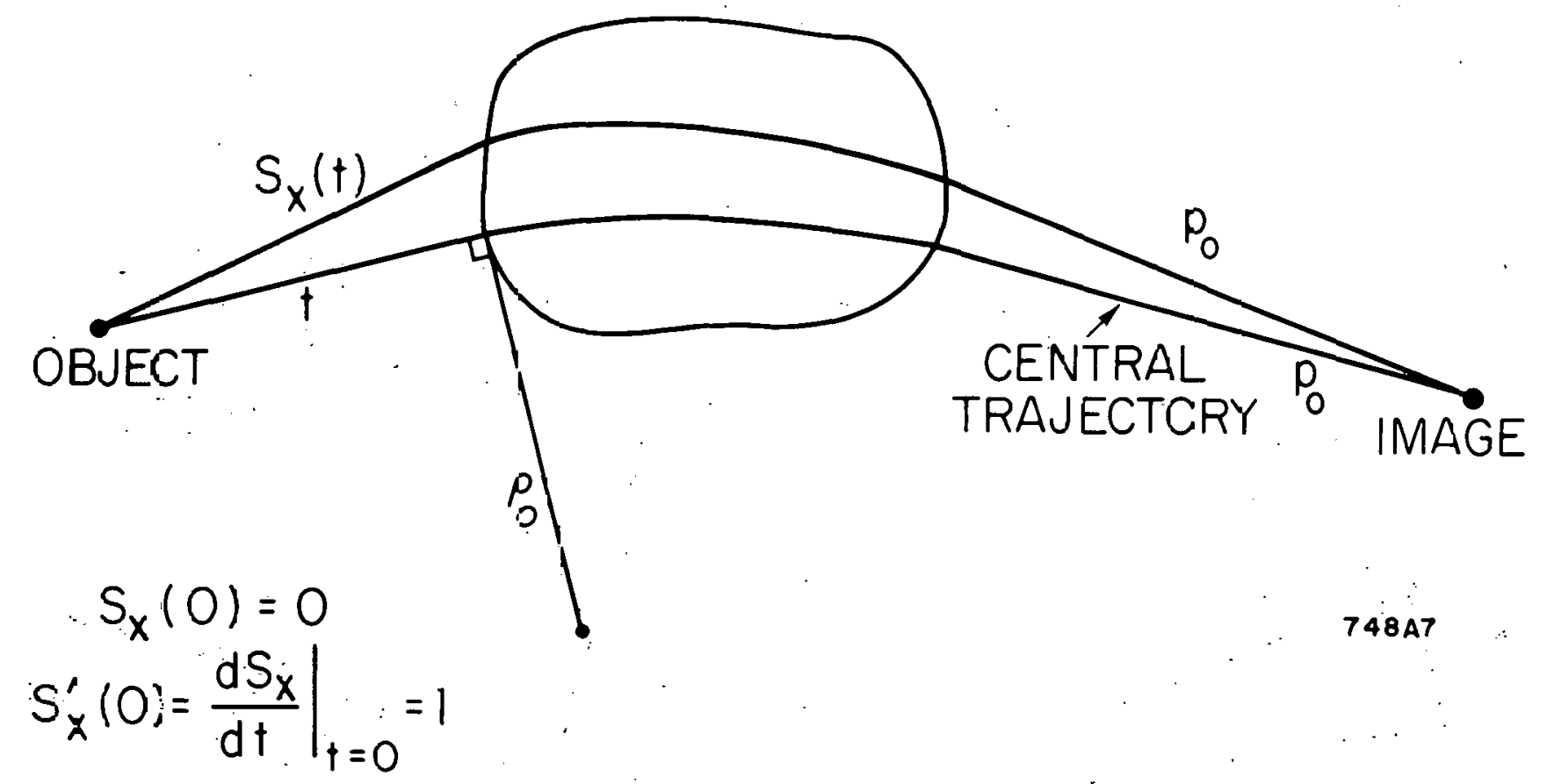

FIG. 4--SINE-LIKE FUNCTICN $s_{x}(t)=\dot{s}_{12}$. IN MAGNETIC MIDFLANE. $\quad s_{x}^{\prime}(t)=R_{22}$. 


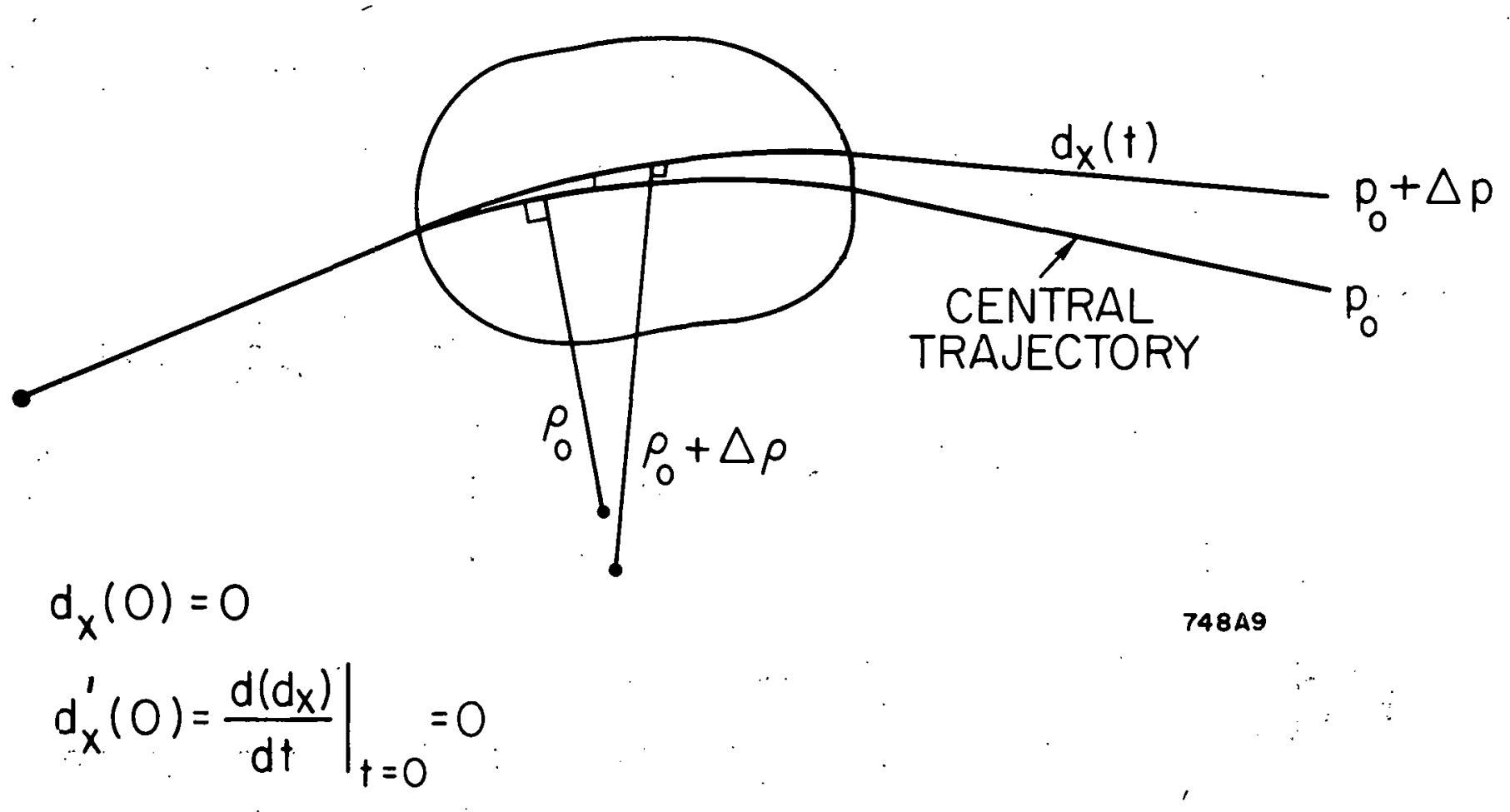

FIG. 5--DISPERSION FUNCTION $d_{x}(t)=R_{i 6}$ IN MAGNEIIO MIDPLANE. $d_{x}^{\prime}(t)=R_{26}$. 


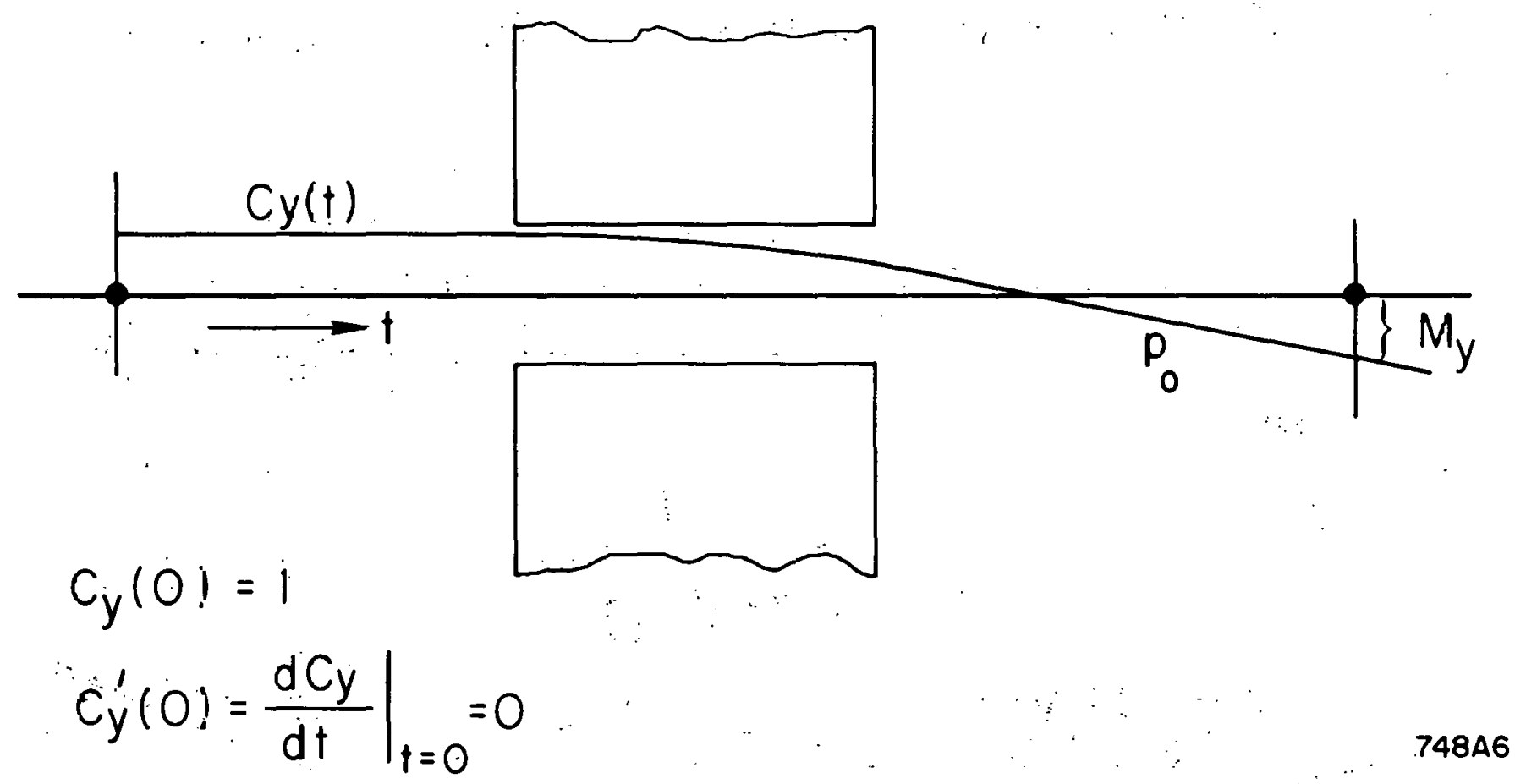

FIG. 6--COSINE-LIKE FUNCTION $c_{y}(t)=R_{33}$ IN THE NON-BEND $(y)$ PLAITE. $\quad c_{y}^{2}(t)=R_{43}$. 


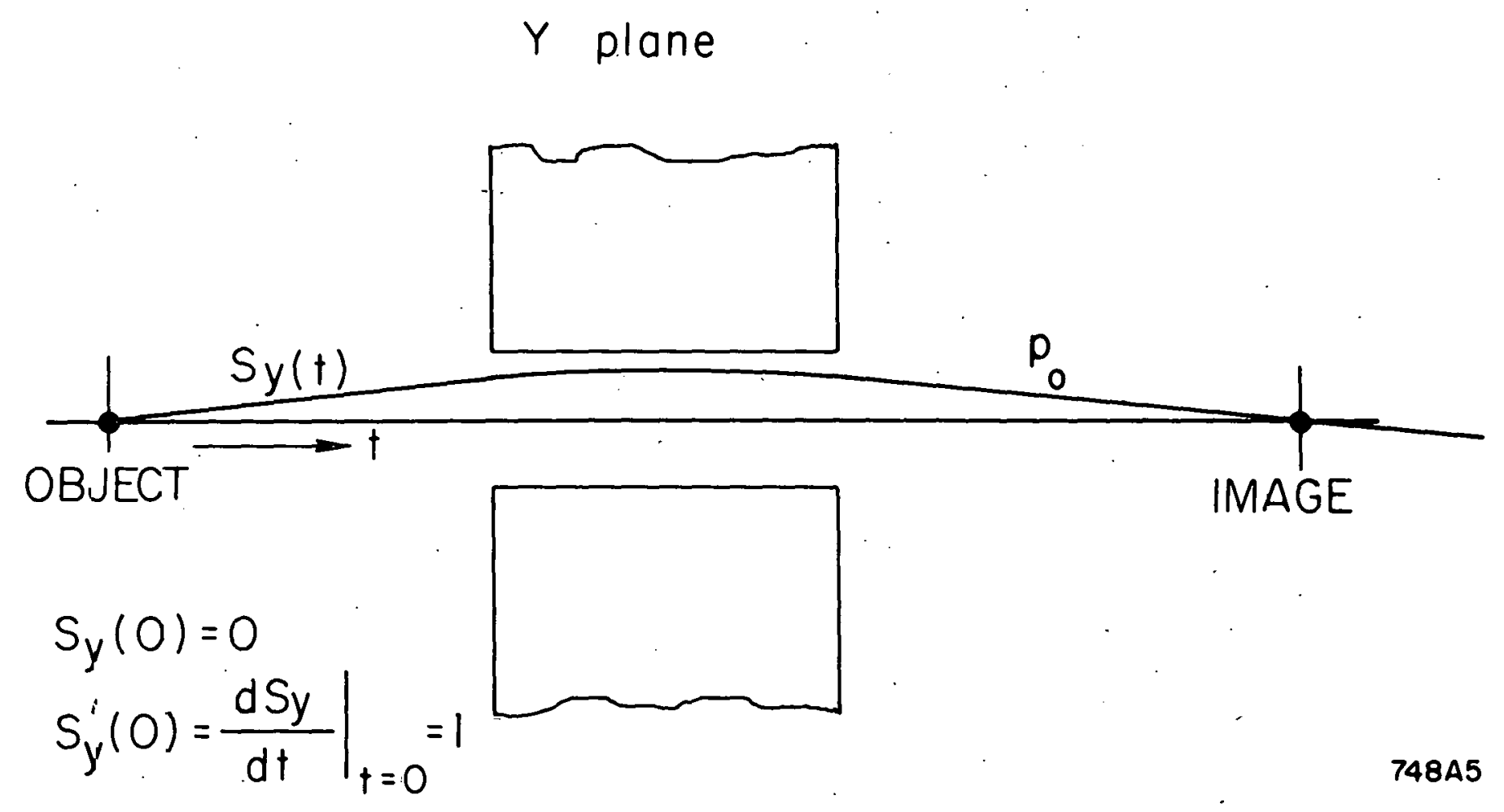

FIG. 7--SDNE-LIKE FUNCTION $s_{y}(t)=R_{34}$ INI NON-BEND $(y)$ PLANE. $\quad s_{y}^{\prime}(t)=R_{44}$. 


$$
\mathrm{dT}=\left[(\mathrm{dx})^{2}+(\mathrm{dy})^{2}+(1+\mathrm{hx})^{2}(\mathrm{dt})^{2}\right]^{1 / 2}
$$

used in the derivation of the equation of'motion.

\section{First-Order Dispersion}

The spatial dispersion $d_{x}(t)=R_{16}$ of a system at position $t$ is derived using the Green's function integral, and the driving term $f(\tau)=h(\tau)=\frac{1}{\rho_{0}(t)}$ for the dispersion ( see Table I of SLAC-75). The result is

$$
a_{x}(t)=R_{16}=s_{x}(t) \int_{0}^{t} c_{x}(\tau) h(\tau) d \tau-c_{x}(t) \int_{0}^{t} s_{x}(v) h(\tau) d \tau
$$

where $\boldsymbol{\tau}$ is the variable of integration. Note that $h(\tau) d \tau=d \alpha$ is the differential angle of bend of the central trajectory at any point in the system. Thus first-order dispersion is generated only in regions where the central trajectory is deflected (i.e., in dipole elements.) The angular dispersion is obtained by direct differentiation of $a_{x}(t)$ with respect to"t; the curvilinear distance along the central trajectory .

$$
d_{x}^{\prime}(t)=R_{26}=s_{x}^{\prime}(t) \int_{0}^{t} c_{x}(\tau) h(\tau) d \tau-c_{x}^{\prime}(t) \int_{n}^{t} s_{x}(\tau) h(\tau) d \tau
$$

where

$$
c_{x}^{\prime}(t)=R_{21} \text { and } s_{s}^{\prime}(t)=R_{22}
$$

First-Order Path Thength

The first-order path length difference is obtained hy expanding and integrating Eq. (4) and retaining only the first-order term, i.e.,

$$
\rho-\ell_{0}=(T-t)=\int_{0}^{t} \cdot x(\tau) \cdot h(\tau) d \tau+\text { higher order terms }
$$


from which

$$
\begin{aligned}
& \ell=x_{0} \int_{0}^{t} c_{x}(\tau) h(\tau) d \tau+\theta_{0} \int_{0}^{t} s_{x}(\tau) h(\tau) d \tau+\ell_{0}+\delta \int_{0}^{t} d_{x}(\tau) h(\tau) d \tau \\
& =R_{51} x_{0}+R_{52} \theta_{0}+\ell_{0}+R_{56} \delta
\end{aligned}
$$

Inspection of Eqs. (5), (6), and (7) yields the following useful theorems:

Achromaticity: A system is defined as being achromatic if $R_{16}=R_{26}=0$, i.e., if $d_{x}(t)=d_{x}^{\prime}(t)=0$. Therefore it follows from Eq's. (5) and (6) that the necessary and sufficient conditions for achromaticity are that

$$
\int_{0}^{t} s_{x}(\tau) h(\tau) d \tau=\int_{0}^{t} c_{x}(\tau) h(\tau) d \tau=0
$$

By comparing Eq. (7) with Eq. (8), we note that if a system is achromatic, all particles of the same momentum will have equal (first-order) path lengths through the system.

Isochronicity: It is somewhat unfortunate that this word has been used in the literature to mean equal path lengths since equal path lengths only imply equal transit times for highly relativistic particles. Nevertheless, from Eq.7, the necessary and sufficient conditions that the first-order path length of all particles (independent of their initial momenta) will be the same through a system are that $R_{51}=R_{52}=R_{56}=0$, i.e., if

$$
\int_{0}^{t} c_{x}(\tau) h(\tau) d \tau=\int_{0}^{t} s_{x}(\tau) h(\tau) d \tau=\int_{0}^{t} d_{x}(\tau) h(\tau) d \tau=0
$$

\section{First-Order Imaging}

First-order point-to-point imaging in the $x$ plane occurs when $x(t)$ is independent of the initial angle $\theta_{0}$. This can only be so when

$$
s_{x}(t)=R_{12}=0 \text {. }
$$


Similarly first-order point-to-point imaging occurs in the y plane when

$$
s_{y}(t)=R_{34}=0
$$

First-order parallel-to-point imaging occurs in the $x$ plane when $x(t)$ is independent of the initial particle position $\mathrm{x}_{0^{\circ}}$ This will occur only if

$$
c_{x}(t)=R_{11}=0
$$

and correspondingly in the y plane, parallel-to-point imaging occurs when

$$
c_{y}(t)=R_{33}=0
$$

A parallel ray entering a system exits parallel to the central trajectory if

$$
c_{x}^{\prime}(t)=R_{21}=0
$$

in the $x$ plane; and if

$$
c_{y}^{\prime}(t)=R_{43}=0
$$

in the $y$ plane.

Point-to-parallel imaging occurs in the $x$ plane if

$$
s_{x}^{\prime}(t)=R_{22}=0 \text {. }
$$

and in the $y$ plane if

$$
s_{y}^{\prime}(t)=R_{44}=0
$$

Focal Lengths

$$
\text { A simple ray diagram of a "thick" lens demonstrates that } R_{21} \text { and } R_{43} \text { have }
$$
the following physical interpretations

$$
c_{x}^{\prime}(t)=R_{21}=-\frac{1}{f_{x}} \text { and } c_{y}^{\prime}(t)=R_{43}=-\frac{1}{f_{y}}
$$

where $f_{x}$ and $f_{y}$ are the system focal lengths in the $x$ and $y$ planes respectively. 
For point-to-point imaging, using Eq's. (5) and (10), the necessary and sufficient condition for zero dispersion at an image is.

$$
d_{x}(t)=R_{i 6}=\int_{0}^{t} s_{x}(\tau) h(\tau) d \tau=0
$$

For parallel-to-point imaging, (i.e., $c_{x}(t)=0$ ), the condition for zero dispersion at the image is

$$
d_{x}(t)=R_{16}=\int_{0}^{t} c_{x}(\tau) h(\tau) d \tau=0 .
$$

\section{Magnification}

For monoenergetic point-to-point imaging in the $x$-plane, the magnification is given by

$$
M_{x}=\frac{x(t)}{x_{0}}=R_{I I}=c_{x}(t)
$$

and in the $\mathrm{y}$ plane by

$$
\mathrm{M}_{\mathrm{y}}=\mathrm{R}_{33}=\mathrm{c}_{\mathrm{y}}(\mathrm{t})
$$

where a negative number means an inverted image.

First-Order Momentum Resolution

For point-to-point imaging the first-order momentum resolving power $\mathrm{R}_{1}$ (not to be confused with the matrix $R$ ) is the ratio of the momentum dispersion to the total image size. Thus if $2 x_{\Omega}$ is the total source size then

$$
R_{1}=\frac{P}{\Delta P}=\left|\frac{R_{16}}{2 x_{0} R_{11}}\right|=\left|\frac{a_{x}(t)}{2 x_{0} c_{x}(t)}\right|
$$


For point-to-point imaging $s_{x}(t)=0$. Using Eq. (5), the dispersion at an image is

$$
d_{x}(t)=-c_{x}(t) \int_{0}^{t} s_{x}(\tau) h(\tau) d \tau
$$

from which the first-order momentum resolving power $\mathrm{R}_{1}$ becomes

$$
2 x_{0} R_{1}=\left|\frac{d_{x}(t)}{c_{x}(t)}\right|=\left|\int_{0}^{t} s_{x}(\tau) h(\tau) d \tau\right|=\left|R_{52}\right|
$$

'Equation ('23) f'or the first-order resolving power of a system may be expressed in a number of useful forms. If we consider a ray (particle) originating at the source with $x_{0}=0$ and $\delta=\frac{\Delta P}{\Gamma}=0$ and lying in the midplane. ( $i . P .$, a mono. energetic point source), the first-order equation representing the midplane displacement, $x$, of this trajectory is

$$
x(t)=s_{x}(t) \theta_{0}
$$

We may then rewrite Equation (23) as follows:

$$
2 x_{0} R_{1}=\int_{0}^{t} s_{x}(\tau) h(\tau) d \tau=\frac{1}{\theta_{0}} \int_{0}^{t} x(\tau) h(\tau) d \tau=\frac{\left(\ell-\ell_{0}\right)}{\theta_{0}}=\left|R_{52}\right|
$$

or we may. also write it in the form

$$
R_{\perp}=\frac{1}{2 x_{0} \theta_{0}} \int_{0}^{t} B \frac{x(t) d \tau}{B \rho_{0}}=\left(\frac{1}{2 x \theta_{0}}\right)\left(\frac{1}{B \rho_{0}}\right) \int_{0}^{t} B d A
$$

where $\int \mathrm{BdA}$ is the magnetic flux inclosed between the central trajectory and the ray represented by $\mathrm{Eq}$. ( 24 ), and $\mathrm{B} p$ is the magnetic rigidity (momentum) of the central trajectory. Please note, however, that if the ray crosses the central trajectory or the sign of $B$ changes, this changes the sign of the integration. 
Some important observations may be made from Eq's. (25) and (26):

1) Resolving particles of different momentum requires that a path length difference must exist between the central trajectory and the trajectory defined by Eq. (22). The greater the path length difference, the greater the resolving power.

2) From Eq. (24), we may define resolving power as the magnetic flux inclosed per unit phase space area per unit momentum (Bo) of the central ray. 
In accelerators and beam transport systems, the behavior of an individua $\perp$ particle is often of less concern than is the behavior of a bundle of particles (the BEAM) of which an individual particle is a member. An extension of the matrix algebra of $\mathrm{Eq}$. (3) provides a convenient means for defining and manipulating this BEAM. TRANSPORT assumes that the bundle of rays constituting a BEAM may correctly be represented in coordinate phase-space by an ellipsoid whose coordinates are the position, angle, and momentum coordinates of the arbitrary rays in the beam about an assumed central trajectory. TRANSPORT is a matrix calculation that truncates the problem to either first- or second-order in a Taylors expansion about the central trajectory. Particles in a BEAM are assumed to lie within the boundaries of the ellipsoid with each point within the ellipsoid representing a possible ray. The sum total of all phase points, the phase space volume, is commonly referred to as the "phase space" occupied by the BEAM. The validity and interpretation of this phase ellipse formalism must be ascertained for each system being designed. However, in general, for charged particle beams in, or emanating, from accelerators, the first-order phase ellipse formalism of TRANSPORT is a reasonable representation of physical reality; but for other applications, such as charged particle spectrometers, caution is in order in its use and interpretation.

The equation of an $n$-dimensional ellipsoid may be written in matrix form as follows:

$$
x(u)^{\mathrm{T}} \overline{\boldsymbol{\sigma}}(u)^{-1} \quad x(u)-1
$$

where $X(0)^{T}$ is the transpose of the coordinate vector $X(0)$, and $\sigma(0)$ is a real, positive definite, symmetric matrix. 
The volume of the n-dimensional ellipsoid defined by sigma is

$\frac{\pi \frac{n}{2}}{\Gamma\left(\frac{n}{2}+1\right)}(\operatorname{det} \sigma)^{1 / 2}$, the area of the projection in one plane is $A=\pi(\operatorname{det} \sigma)^{1 / 2}$.

This is the "phase space" occupied by the beam.

As a particle passes through a system of magnets, it undergoes the matrix transformation of Eq. (3). Combining this transformation with the equation of the initial ellipsoid, and using the identity $\mathrm{RR}^{-1}=I$ (the unity matrix), it follows that:

$$
\mathrm{X}(0)^{\mathrm{T}}\left(\mathrm{R}^{\mathrm{T}} \mathrm{R}^{\mathrm{T}^{-1}}\right) \sigma(0)^{-1}\left(\mathrm{R}^{-1} \mathrm{R}\right) \quad \mathrm{X}(0)=1
$$

from which:

$$
(\operatorname{RX}(0))^{\mathrm{T}}\left(\operatorname{R} \sigma(0) \mathrm{R}^{\mathrm{T}}\right)^{-1}(\mathrm{RX}(0))=1
$$

The equation of the ellipsoid representing the "BEAM" at the end of the system is thus:

$$
\mathrm{X}(1)^{\mathrm{T}} \sigma(1)^{-1} \mathrm{X}(1)=1
$$

where the equation for the sigma matrix at the end may be related to that at the beginning by:

$$
\boldsymbol{\sigma}(1)=\mathrm{R} \boldsymbol{\sigma}(0) \mathrm{R}^{\mathrm{T}}
$$

In addition to calculating the product matrix $R$, TRANSPORT also computes the sigma "BEAM" matrix at the end of each physical element via Eq. (30).

All of the important physical parameters of the BEAM ellipsoid may be expressed as functions of the matrix elements of the sigma matrix at the location 
in question. In particular the square roots of the diagonal elements $\left(\sqrt{\sigma_{i i}}{ }^{\prime} s\right)$ are the projection of the ellipse upon the coordinate axes and thus represent the maximum extent of the BEAM in the various coordinate directions. The correlation between components (the orientation of the ellipse) is determined by the off-diagonal terms (the $\sigma_{i j}$ 's). An illustration of this is given below for a 2 -dimensional ellipse.

Description of the Sigma BEAM Matrix

Consider a 2-dimensional $(x, \theta)$ plane projection of the general 6-dimensional ellipsoid. Let

$$
\sigma=\left[\begin{array}{ll}
\sigma_{11} & \sigma_{21} \\
\sigma_{21} & \sigma_{22}
\end{array}\right]
$$

be a real, positive definite, symmetric matrix; the inverse of which is

$$
\sigma^{-1}=\frac{1}{\epsilon^{2}}\left[\begin{array}{cc}
\sigma_{22} & -\sigma_{21} \\
-\sigma_{21} & \sigma_{11}
\end{array}\right]
$$

where $\epsilon^{2}$ is the determinant of $\sigma$.

The 2-dimensional coordinate vector (column matrix) and its transpose are:

$$
X=\left(\begin{array}{l}
x \\
\theta
\end{array}\right) \text { and } X^{T}=\left(\begin{array}{ll}
x & \theta
\end{array}\right)
$$

The expansion of the matrix equation $X^{\prime \prime} \sigma^{-1} X=1$, is the equation of the ellipse

$$
\sigma_{22} x^{2}-2 \sigma_{21} x \theta+\sigma_{11} \theta^{2}=\epsilon^{2}=\operatorname{det} \cdot \sigma
$$

The $(x, \theta)$ plane BEAM ellipse represented by Eq. (31) is shown in the following figure along with the physical meaning of the sigma matrix elements. 


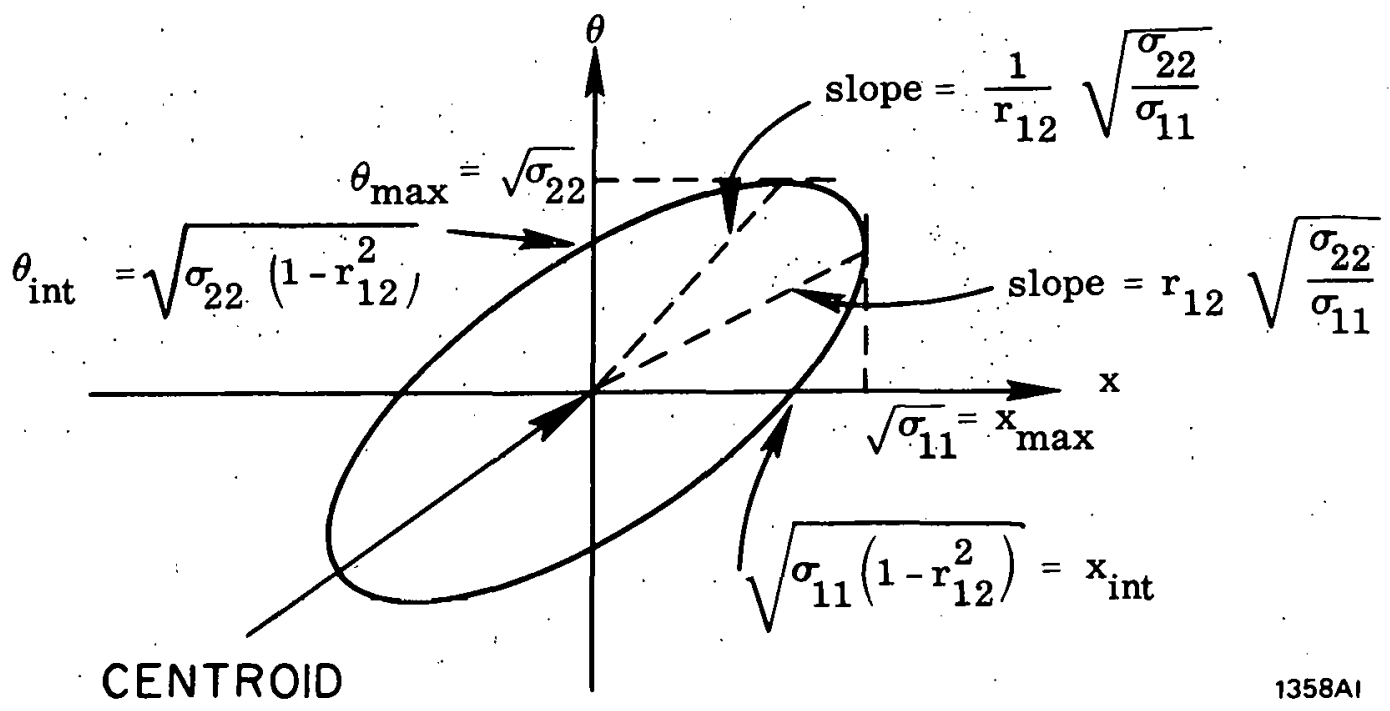

The area of the ellipse is given by:

$$
A=\pi(\operatorname{det} \sigma)^{1 / 2}=\pi x_{\max } \theta_{\text {int }}=\pi x_{\text {int }} \theta_{\max }
$$

A Two Dimensional BEAM Phase Ellipse

The correlation between $x$ and $\theta$ (the orientation of the eliipse) depends upon the off-diagonal term $\sigma_{21}$. This correlation is defined as

$$
r_{21}=r_{12}=\frac{\sigma_{21}}{\sqrt{\sigma_{11} \sigma_{22}}}
$$

So defincd $r$ alwayc falle in the range

$$
-1 \leqslant r \leqslant 1
$$

The correlation, $r$, measures the tilt of the ellipse and the intersection of the ellipse with the coordinate axes. 
Since the $\operatorname{det} R=1$ for all static magnetic beam transport elements, it follows that the determinant of $\sigma(1)$ and $\sigma(0)$ are identical under the transformation of Eq. ( 30$)$. Hence the "phase space" area is an invariant under the transformation of Eq. (30). This is a statement of Liouville's Theorem for the magnetostatic fields employed and results from the fact that the det $R=1$.

It is perhaps worthwhile noting that this 2-dimensional representation of the BEAM matrix has a one to one correspondence with the Courant-Snyder treatment of the theory of the Alternating Gradient Synchrotron* as follows:

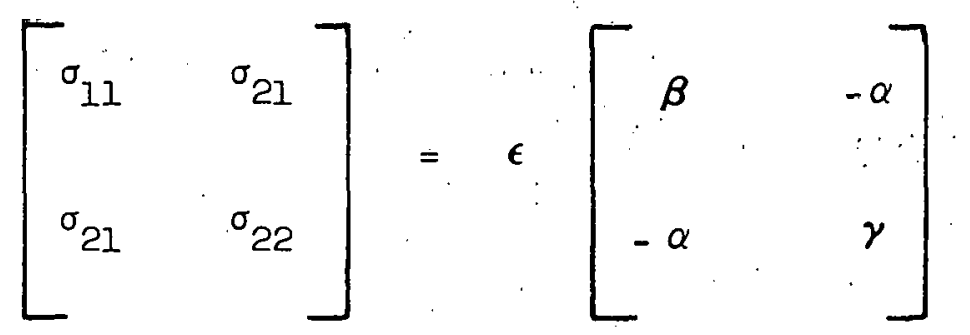

The Phase Ellipse Beam Matrix used by TRANSPORT

For static magnetic systems possessing midplane symmetry, the $(x, \theta)$ plane and $(y, \varphi)$ plane trajectories are decoupled in first-order, i.e. there is no mixing of phase space between the two planes. However for mathematical simplicity and to allow for the possibility of more general systems, the sigma BEAM matrix used in a TRANSPORT calculation has the following general 6-dinensional construction.

\begin{tabular}{c|cccccc} 
& $\mathrm{x}$ & $\theta$ & $\mathrm{y}$ & $\varphi$ & $\ell$ & $\boldsymbol{\delta}$ \\
\hline $\mathrm{x}$ & $\sigma(11)$ & & & & & \\
$\theta$ & $\sigma(21)$ & $\sigma(22)$ & & & & \\
$\mathrm{y}$ & $\sigma(31)$ & $\sigma(32)$ & $\sigma(33)$ & & & \\
$\varphi$ & $\sigma(41)$ & $\sigma(42)$ & $\sigma(43)$ & $\sigma(44)$ & & \\
$\ell$ & $\sigma(51)$ & $\sigma(52)$ & $\sigma(53)$ & $\sigma(54)$ & $\sigma(55)$ & \\
$\delta$ & $\sigma(61)$ & $\sigma(62)$ & $\sigma(63)$ & $\sigma(64)$ & $\sigma(65)$ & $\sigma(66)$
\end{tabular}

* E. D. Courant and H. S. Snyder, "Theory of the Alternating Gradient Synchrotron", Annals of Physics 3, pp 1-48 (1958). 
The matrix is symmetric so that only a triangle of elements is needed.

In the printed output this matrix has a somewhat different format for ease of interpretation:

\begin{tabular}{|c|c|c|c|c|c|c|c|c|}
\hline & & & $\mathrm{x}$ & $\theta$ & & $\mathrm{y}$ & $\varphi$ & $\ell$ \\
\hline $\mathrm{x}$ & $\sqrt{\sigma(11)}$ & $\mathrm{CM}$ & & & & & & \\
\hline$\theta$ & $\sqrt{\sigma(22)}$ & $\mathrm{MR}$ & $\dot{r}(2 I)$ & & & ; & & \\
\hline $\mathrm{y}$ & $\sqrt{\sigma(33)}$ & $\mathrm{CM}$ & $r(31)$ & $r(32)$ & & - & & $\cdot$ \\
\hline$\varphi$ & $\sqrt{\sigma(44)}$ & $\mathrm{MR}$ & $r(41)$ & $r(42)$ & & $r(43)$ & & \\
\hline$\ell$ & $\sqrt{\sigma(55)}$ & CM & $\dot{r}(51)$ & $r(52)$ & & $r(53)$ & $r(54)$ & \\
\hline$\delta$ & $\sqrt{\sigma(66)}$ & $\mathrm{PC}$ & $r(61)$ & $r(62)$ & & $r(63)$ & $r(64)$ & $r(65)$ \\
\hline
\end{tabular}

where: $\quad r(i j)=\frac{\sigma(i j)}{[\sigma(i i) \sigma(j j)]^{1 / 2}}$

As a result of the fact that the matrix is positive definite, the $r(i j)^{i} s$ satisfy the relation

$$
|r(i j)| \leqslant 1
$$

The physical meaning of the $\sqrt{\sigma i i) ' s}$ is as follows:

$\sqrt{\sigma(11)}=x_{\max }=$ The maximum (half) -width of the beam, envelope in the $x$ plane at the point of the printout.

$\sqrt{\alpha 22)}=\theta_{\max }=$ The maximum (half) -angular divergence of the beam envelope in the $\mathrm{x}$ plane.

$\sqrt{\sigma(33)}=y_{\max }=$ The maximum (half)-height of the beam envelope.

$\sqrt{\sigma(44)}=\varphi_{\max }=$ The maximum (half)-angular divergence of the beam envelope in the y plane.

$\sqrt{\sigma 55)}=\ell_{\max }=1 / 2$ the longitudinal extent of the bunch of particles. $\sqrt{\sigma 66)}=\delta=$ The half-width $1 / 2(\Delta \mathrm{P} / \mathrm{P})$ of the momentum interval being transmitted by the system. 
The units appearing next to the $\sqrt{\sigma(i i)}$ 's in the TRANSPORT printout sheet are the units chosen for coordinates $\mathrm{x}, \theta, \mathrm{y}, \varphi, \ell$ and $\delta=\Delta \mathrm{P} / \mathrm{P}$ respectively.

To the immediate left of the listing of the beam envelope size in a TRANSPORT printout, there appears a column of numbers whose values will normally be zero. These numbers are the coordinates of the centroid of the beam phase ellipse (with respect to the initially assumed central trajectory of the system). They may become non-zero under one of three circumstances:

1) When the misalignment (Type Code 8.) is usèd.

2) When a Beam Centroid shift (Type Code 7.) is used.

or 3) When a and-order calculation (Type Code 17.) is used.

Physical Interpretation of Various Projections of the 2-dimensional BEAM Ellipse Consider again Eq. $(30) \cdot \sigma(I)=R \sigma(0) R^{T}$ and expand it in it's most. general form for the 2-dimensional $(x, \theta)$ plane case.

$\sigma(1)=\left(\begin{array}{ll}R_{11} & R_{12} \\ R_{21} & R_{22}\end{array}\right)\left(\begin{array}{ll}\sigma_{11}(0) & \sigma_{21}(0) \\ \sigma_{21}(0) & \sigma_{22}(0)\end{array}\right)\left(\begin{array}{ll}R_{11} & R_{21} \\ R_{12} & R_{22}\end{array}\right)=\left(\begin{array}{ll}\sigma_{11}(1) & \sigma_{21}(1) \\ \vdots & \\ \sigma_{21}(1) & \sigma_{22}(1)\end{array}\right)$

the result is:

$\sigma(1)=\left[\frac{R_{11}^{2} \sigma_{11}(0)+2 R_{11} R_{12} \sigma_{21}(0)+R_{12}^{2} \sigma_{22}(0)}{R_{11} R_{21} \sigma_{11}(0)+\left(R_{11} R_{22}+R_{12} R_{21}\right) \sigma_{21}(0)+R_{12} R_{22} \sigma_{22}(0)}\right]$

In the special case when the initial ellipse is erect i.e., $\sigma_{21}(0)=0$, $\boldsymbol{\sigma}(1)$ reduces to:

$\sigma(1)=\left[\frac{\mathrm{R}_{11}^{2} \sigma_{11}(0)+\mathrm{R}_{12}^{2} \sigma_{22}(0)}{\mathrm{R}_{11} \mathrm{R}_{21 .} \sigma_{11}(0)+\mathrm{R}_{12} \mathrm{R}_{22} \sigma_{22}(0)}\right]$

Similar results are, of course, obtained for the $(y, \varphi)$ plane: 
If an arbitrary beam transport system is reduced to the most elementary first-order form of representing it as an initial drift distance, followed by a lens action between two principal planes, and a final drift distance; then we observe that for the 2-dimensional representation there are only two basic phase ellipse transformations of interest.
(1) An arbitrary DRIFT distance and
(2) A LENS action

Each of these elementary cases are illustrated on Fig. 8 for both a parallelogram as well as elIipse phase space transformations. Note that a DRIFT followed by a LENS action is not necessarily equal to a LENS action followed by a DRIFT; i.e., the matrices do not necessarily commute.

The phase ellipse transformations for a DRIFT and for a LENS action (between principal planes) as shown in Fig. (8) may be readily calculated using the results of $\mathrm{Eq}$. ( 37 ).

The 2-dimensional $R$ matrix representing a drift of distance $L$ is:

$$
R(\text { Drift })=\left(\begin{array}{ll}
1 & I \\
0 & 1
\end{array}\right)
$$

Substituting into $\mathrm{Eq} \cdot(37)$ we find

$$
\sigma(1)=\left[\begin{array}{l|l}
\sigma_{11}(0)+L^{2} \sigma_{22}(0) & L \sigma_{22}(0) \\
\hline L \sigma_{22}(0) & \sigma_{22}(0)
\end{array}\right]=\left[\begin{array}{ll|l}
\sigma_{11}(1) & \sigma_{21}(1) \\
\hline \sigma_{21}(1) & \sigma_{22}(1)
\end{array}\right]
$$

Attaching the physical meaning to the matrix elements yields the following interpretations:

$$
\dot{\sigma}_{11}(1)=\sigma_{11}(0)+L^{2} \sigma_{22}(0)
$$

or

$$
\left(x_{1}^{2}\right)_{\max }=\left(x_{0}^{2}\right)_{\max }+I^{2}\left(\theta_{0}^{2}\right)_{\max }
$$



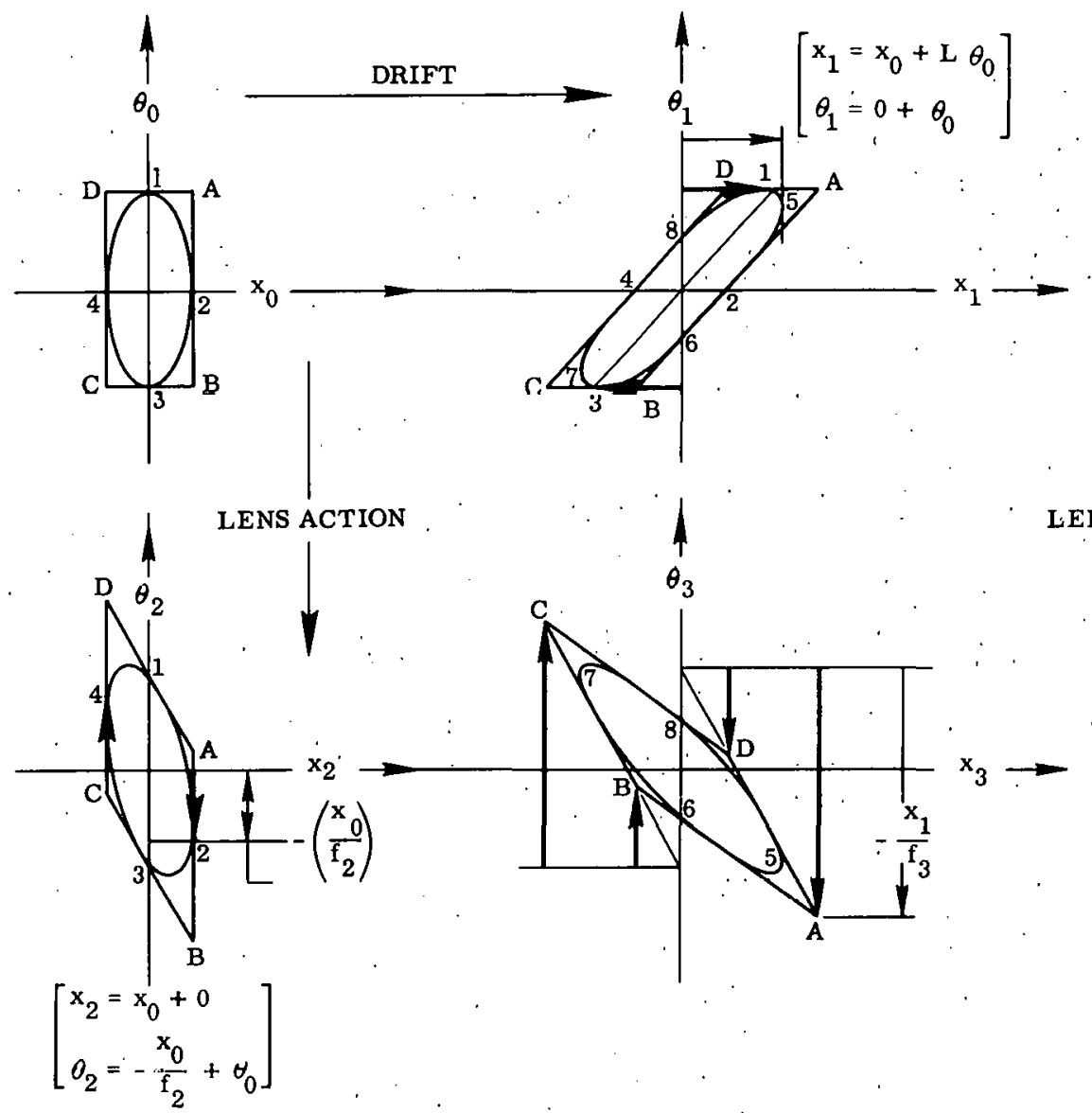

LENS ACTION
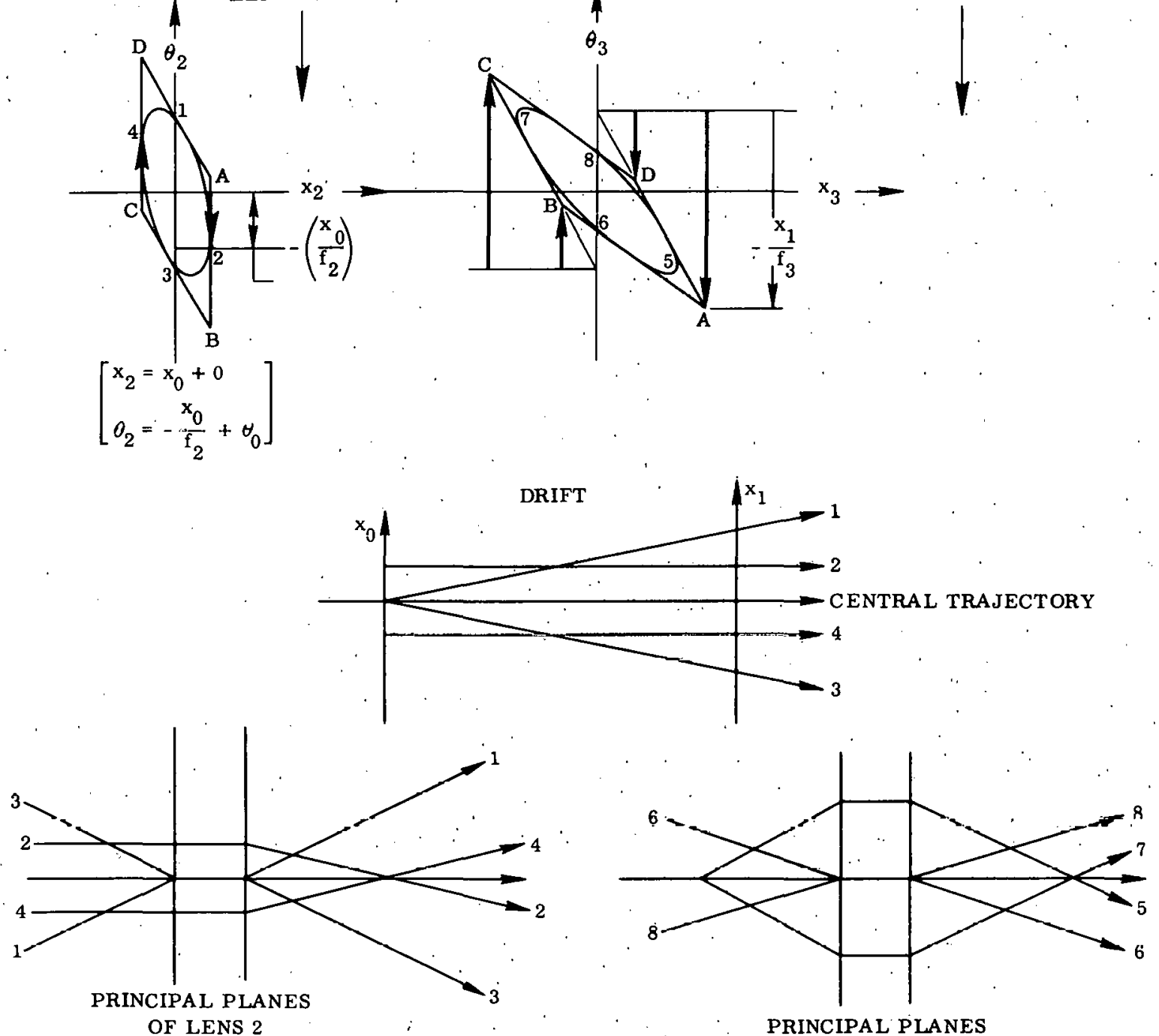

PRINCIPAL PLANES OF LENS 3

135882

Fig. 8 
similarly

$$
\sigma_{22}(1)=\sigma_{22}(0)
$$

or

$$
\left(\theta_{1}^{2}\right)_{\max }=\left(\theta_{0}^{2}\right)_{\max }
$$

Note that this transformation assumes that the initial phase ellipse is erect, i.e., $\sigma_{21}(0)=0$

The 2-dimensional $\mathrm{R}$ matrix'for a lens actions (between principal planes)

is

$$
R(\text { Lens })=\left(\begin{array}{cc}
1 & 0 \\
-\frac{1}{f} & 1
\end{array}\right)
$$

Substitution into Eq. ( 37 ) yields

$$
\sigma(2)=\left[\begin{array}{c|c|c}
\sigma_{11}(0) & -\frac{\sigma_{11}(0)}{f} \\
\hline-\frac{\sigma_{11}(0)}{f} & \frac{\sigma_{11}(0)}{f^{2}}+\sigma_{22}(0)
\end{array}\right]=\left[\begin{array}{ll}
\sigma_{11}(2) & \sigma_{21}(2) \\
\hline \sigma_{21}(2) & \sigma_{22}(2)
\end{array}\right]
$$

Again attaching physical meaning to the matrix elements we have:

$$
\sigma_{11}(2)=\sigma_{11}(0)
$$

or

$$
\left(x_{0}^{2}\right)_{\max }=\left(x_{0}^{2}\right)_{\max }
$$

and

$$
\sigma_{22}(2)=\frac{\sigma_{11}(0)}{f^{2}}+\sigma_{22}(0)
$$

or

$$
\left(\theta_{2}^{2}\right)_{\max }=\frac{1}{f^{2}}\left(x_{0}^{2}\right)_{\max }+\left(\theta_{0}^{2}\right)_{\max }
$$

Note the change in sign of the $\sigma_{21}$ elements for the Drift and the Lens actions indicating the different sense of orientation of the resulting ellipses as illustrated in Fig. 8 . 
A case of particular interest in any 2-dimensional phase ellipse projection (e.g., the $(x, \theta)$ plane or the $(y, \varphi)$ plane) is when the off-diagonal correlation matrix elements are equal to zero; i.e., an erect ellipse. In a field-free region this corresponds to a so-called "waist" in the BEAM as iliustrated in Fir. 9.

It is important to understand correctly the meaning of a waist: For an existing beam, it is the location of the minimum beam size in a given region of the system. Although the waist is the minimum beam size in any given beam, the

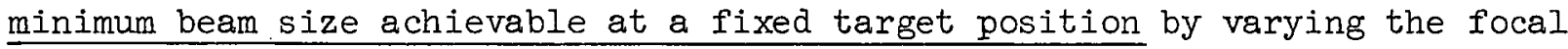
strength of the preceding lens system is not the same as the above defined waist. See Fig. 10. In a field-tree region, the minimum beam spot size achievable at a fixed target position will occur when the preceding lens system is adjusted such that a waist precedes the target position. Only in the limit of zero phase space area do these quantities occur at the same location. A useful criterion that determines the physical proximity of these quantities is the following: If the system has been adjusted f'or the smallest spot size at a t'ixed position and if the size of the beam at the principal planes of the opiical system is large compared to its size at the waist, or at the minimum spot size, then the location of these quantities, the waist and the minimum, will closely coincide; if, on the other hand, the size of the beam does not change substantially throughout the system, then the locations of a waist and the minimum beam size may (and usually will) differ substantially. The numerical proximity of these two quantities will be discussed in greater detail later in the report.

In a fleld free region (i.e., a Drift), the distance to a waist from any location may be readily calculated if the $\sigma$ matrix at the location is known. Using Eq. (36) and the $\mathrm{R}$ matrix for a $\operatorname{Drift}(\mathrm{Eq} .38)$ we have for the $(\mathrm{x}, \theta)$ plane:

$$
\sigma_{21}(I)=\sigma_{21}(0)+I \sigma_{22}(0)=0 \text { (specifying that } \sigma(I) \text { shall be at a waist) }
$$




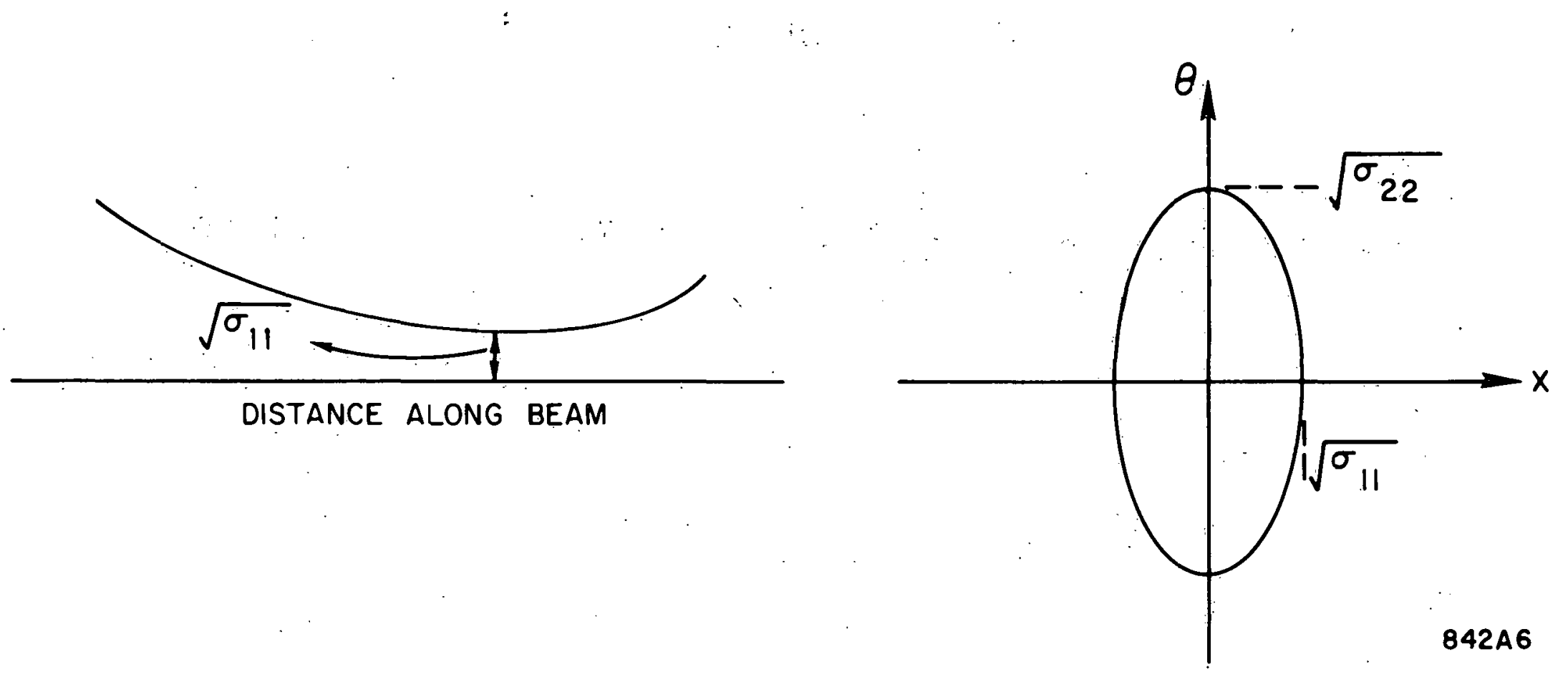

FIG. 9--BEAM WAIST. 


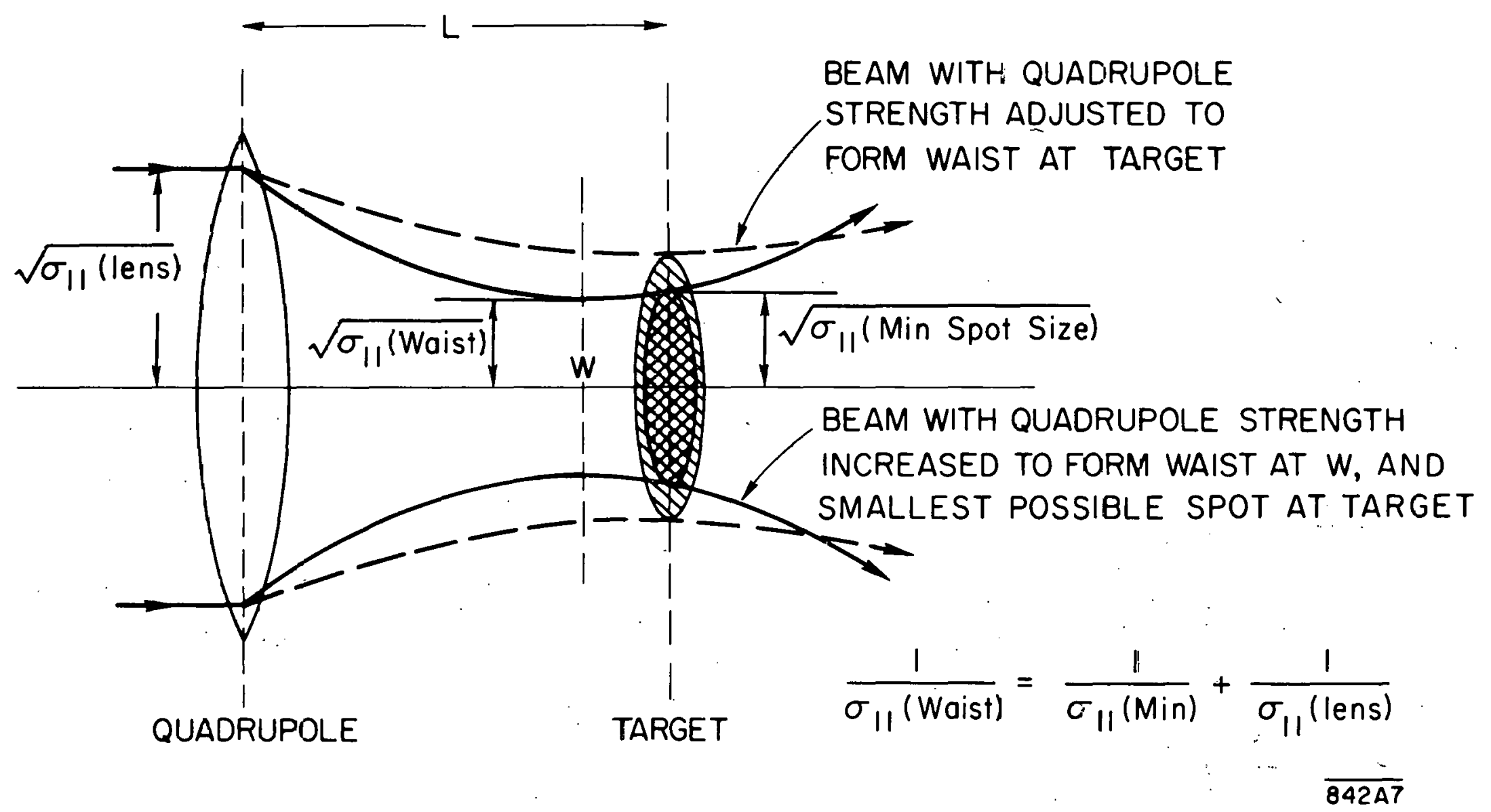

Fig. 10 
or the distance to the waist is:

$$
L=-\frac{\sigma_{21}(0)}{\sigma_{22}(0)}=-r_{21} \sqrt{\frac{\sigma_{11}}{\sigma_{22}}}
$$

Similarly for the $(\mathrm{y}, \varphi)$ plane the distance to a waist is:

$$
I=-\frac{\sigma_{43}(0)}{\sigma_{44}(0)}=-r_{43} \sqrt{\frac{\sigma_{33}}{\sigma_{44}}}
$$

Relationship between a Waist and a Parallel-to-Point Image

A parallel-to-point image in the $(x, \theta)$ plane occurs when $R_{11}=0$. The $R$ matrix corresponding to this is

$$
R=\left(\begin{array}{c|c}
0 & R_{12} \\
\hline R_{21} & R_{22}
\end{array}\right)=\left(\begin{array}{c|c}
0 & s_{x} \\
\hline c_{x} & s_{x}^{\prime}
\end{array}\right)=\left(\begin{array}{c|c}
0 & f_{x} \\
\hline-\frac{1}{f_{x}} & s_{x}^{\prime}
\end{array}\right)
$$

Since $|R|=1, R_{12} R_{21}=-1$ for this situation.

If we assume an erect ellipse $\sigma(0)$ as the beginning of the system, the final beam matrix $\sigma(1)$ is given by substitution of $\mathrm{Eq}$. (48) into $\mathrm{Eq}$. (37) as follows:

$$
\sigma(1)=\left[\begin{array}{l|l}
\mathrm{R}_{12}^{2} \sigma_{22}(0) & \mathrm{R}_{12^{2} \mathrm{R}_{22} \sigma_{22}(0)} \\
\hline \mathrm{R}_{12} \mathrm{R}_{22} \sigma_{22}(0) & \mathrm{R}_{21}^{2} \sigma_{11}(0)+\mathrm{R}_{22}^{2} \sigma_{22}(0)
\end{array}\right]
$$

for parallel-to-point imaging.

Several conclusions may be extracted from this result: The first observation is that a waist and a parallel-to-point image will coincide if $R_{11}=R_{22}=0$. This is equivalent to requiring that the object and image distances (measured to the principal planes) are both equal to the focal length $f$ of the system. 
The distance to a waist in this example is:

$$
I=-\frac{\sigma_{21}(1)}{\sigma_{22}(1)}=-\frac{R_{22} R_{12} \sigma_{22}(0)}{R_{21}^{2} \sigma_{11}(0)+R_{22}^{2} \sigma_{22}(0)}=-\frac{s_{x} s^{\prime} \sigma_{22}(0)}{\left(c_{x}^{\prime}\right)^{2} \sigma_{11}(0)+\left(s_{x}^{\prime}\right)^{2} \sigma_{22}(0)}
$$

If $s_{x^{\prime} x} s^{\prime}=R_{1,2} R_{22}=0$, a waist and a parallel-to-point image coincide. If $\mathrm{s}_{\mathrm{x}} \dot{\mathrm{s}}_{\mathrm{x}}^{i}=\mathrm{R}_{12} \mathrm{R}_{22}>0$, the waist precedes the image; and if $\mathrm{s}_{\mathrm{x}}{ }^{\prime}{ }_{\mathrm{x}}=\mathrm{R}_{12} \mathrm{R}_{22}<0$; the waist follows the image; unless $\sigma_{22}(0)=0$ (zero phase space area) in which case a waist and an image always coincide.

The size of the beam at the image is:

$$
\left(x_{1}^{2}\right)_{\max }=\sigma_{11}(I)=R_{12}^{2} \sigma_{22}(0)=\dot{f}^{2}\left(\theta_{0}^{2}\right)_{\max }
$$

independent of the source size $x_{0}$ and of the object distance.

The size of the beam at the waist is:

$$
(\text { size at waist })^{2}=\frac{|\sigma(0)|}{\sigma_{22}(1)}=\frac{\sigma_{11}(0) \sigma_{22}(0)}{R_{21}^{2} \sigma_{11}(0)+R_{22}^{2} \sigma_{22}(0)}
$$

If $R_{22}=s_{x}^{\prime}=0$, the two sizes are equal as expected, otherwise the size at the waist is always smaller.

\section{Relationship between a Waist and a Point-to-Point Image}

A point-to-point first-order image in the $(x, \theta)$ plane occurs when $R_{12}=s_{x}=0$. The $R$ matrix representing this case is:

$$
R=\left(\begin{array}{c|c}
R_{11} & 0 \\
\hline R_{21} & R_{22}
\end{array}\right)=\left(\begin{array}{c|c}
c_{x} & 0 \\
\hline c_{x}^{\prime} & s_{x}^{\prime}
\end{array}\right)=\left(\begin{array}{c|c}
M & 0 \\
\hline-\frac{1}{f_{x}} & \frac{1}{M}
\end{array}\right)
$$

where $|R|=I=R_{11} R_{22}$, and $M$ is the magnification. 
If we again assume an erect ellipse $\sigma(0)$ as the beginning, the final beam matrix $\sigma(1)$ is given by Eq. (37) as:

$$
\sigma(1) .=\left[\begin{array}{l|l}
R_{11}^{2} \sigma_{11}(0) & R_{11} R_{21} \sigma_{11}(0) \\
\hline R_{11} R_{21} \sigma_{11}(0) & R_{21}^{2} \sigma_{11}(0)+R_{22}^{2} \sigma_{22}(0)
\end{array}\right]
$$

for point-to-point imaging.

Our first observation is that except for a zero source size, an image and a waist will coincide only if $R_{12}=R_{21}=0$. Clearly this is not possible with a single lens; at least two lenses are needed. Such an optical situation is as follows:

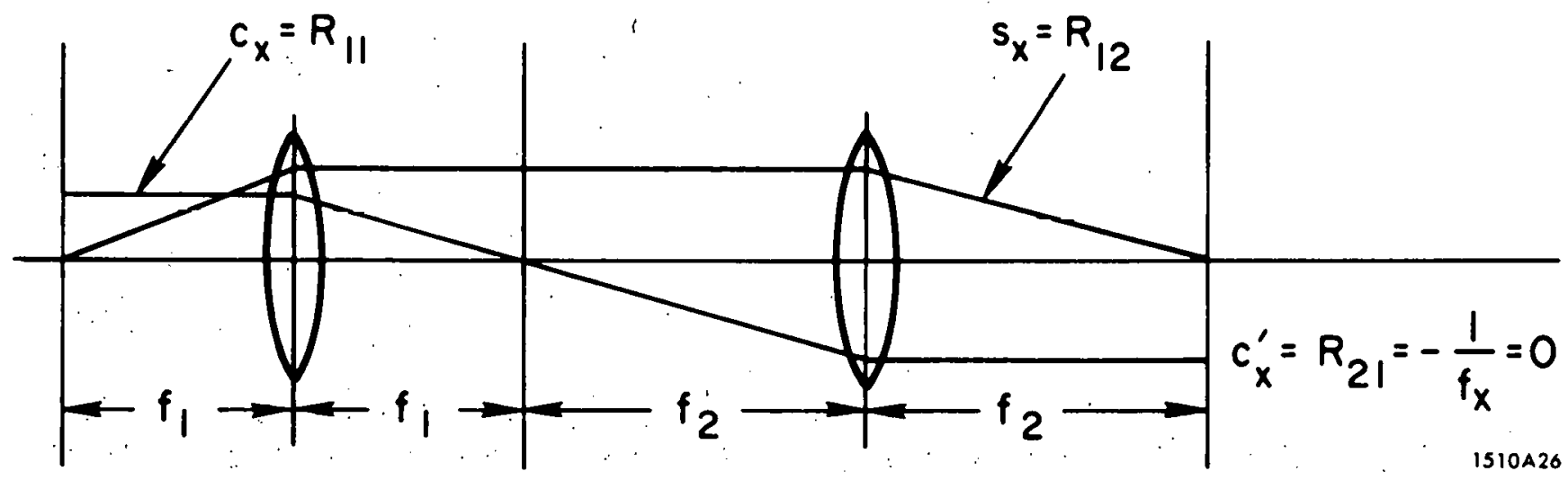

The distance to a waist is

$$
L=-\frac{\sigma_{21}(1)}{\sigma_{22}(1)}=-\frac{R_{11} R_{21} \sigma_{11}(0)}{R_{21}^{2} \sigma_{11}(0)+R_{22}^{2} \sigma_{22}(0)}
$$

So if $R_{11} R_{21}=c_{x} c^{\prime}=0$, a waist and a point-to-point image coincide. If $c_{x}{ }^{\prime \prime}{ }_{x}^{\prime}>0$, the waist precedes the image and if $c_{x} c_{x}^{\prime}<0$, the waist follows the inage." 
The sizê of the beam at the image is

$$
\left(x_{1}^{2}\right)_{\max }=\sigma_{11}(1)=R_{11}^{2 \cdot} \sigma_{11}(0)=\left(M x_{0}\right)^{2}
$$

and the size of the beam at the waist is:

$$
\text { (size at waist) }{ }^{2}=\frac{|\sigma(0)|}{\sigma_{22}(1)}=\frac{\sigma_{11}(0) \sigma_{22}(0)}{R_{21}^{2} \sigma_{11}(0)+R_{22}^{2} \sigma_{22}(0)}
$$

Thus if $R_{2 l}=0$, the two sizes are equal since $|R|=R_{11} R_{\varepsilon \varepsilon}=1$. Otherwise the size at the waist is smaller than the image size.

Relationship between a Waist and the Smallest Spot Size Achievable at a Fixed Target Position

Consider the following general situation:

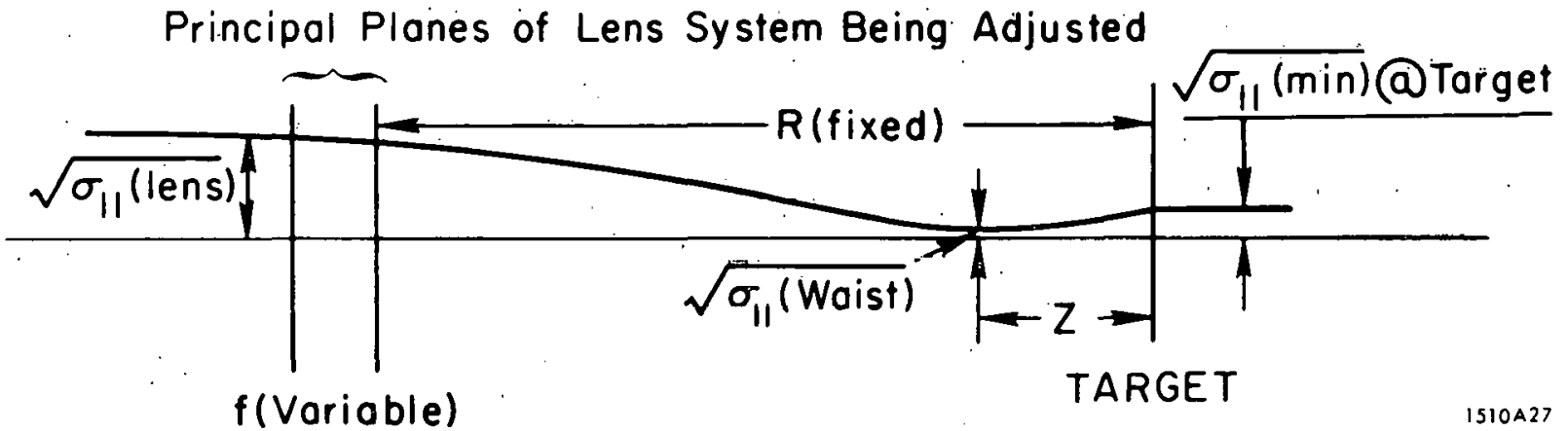

Assume that the size of the beam $\sqrt{\sigma_{11}(\text { lens })}$ at the principal planes of the lens

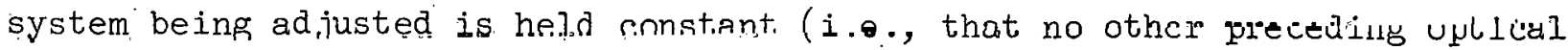
elements of the system are being varied); and that the remainder of the system may be represented by a general matrix $R$ which is also held constant. The focal length $f$ is then varied until a minimum spot size $\sqrt{\sigma_{11}(\min )}$ is achieved at the target location. The sigma beam matrix at the target position then has the 
following unique form independent of the orientation of the initial beam ellipse at the lens.

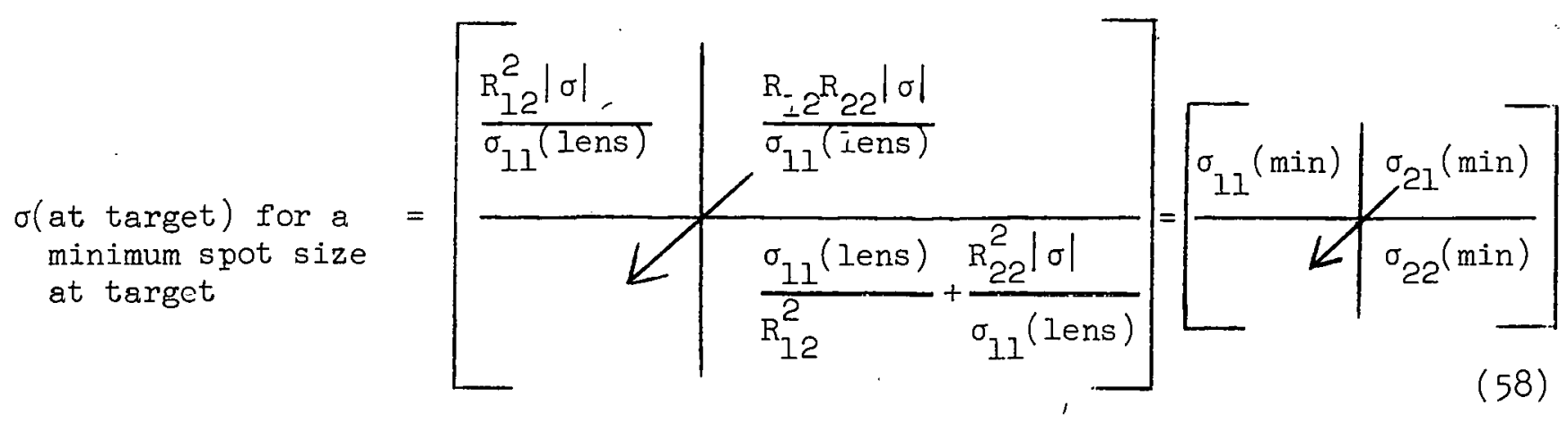

thus

$$
\sigma_{11}(\min )=\frac{R_{12}^{2}|\sigma|}{\sigma_{11}(\text { lens })}
$$

or

$$
x_{\min }=\frac{\mathrm{R}_{12}|\sigma|^{1 / 2}}{x(\text { lens })}
$$

If the position of the waist and the minimum beam size both fall within the same field-free region, then the distance to the waist from the target is:

$$
\mathrm{Z}=-\frac{\sigma_{21}(\min )}{\sigma_{22}(\mathrm{~min})}=-\frac{\mathrm{R}_{12} \mathrm{R}_{22}}{\mathrm{R}_{22}^{2}+\frac{\sigma_{11}(\text { Iens })}{\sigma_{11}(\min )}}=-\mathrm{R}_{12} \mathrm{R}_{22} \frac{\sigma_{11} \text { (waist) }}{\sigma_{11} \text { (lens) }}
$$

So if $s_{x} s^{\prime}=R_{12} R_{22}=0$, the waist and the minimum spot size coincide. If. $R_{12} R_{22}>0$, the waist precedes the target; and if $R_{12} R_{22}<0$, the waist occurs after the target position.

If the waist and the target positions fall within the same field-free region, the following simple relationship exists between the beam size at the lens $\sqrt{\sigma_{11}(\text { lens })}$, at the waist $\sqrt{\sigma_{11}(\text { waist })}$, and at the target $\sqrt{\sigma_{11}(\min )}$. 


$$
\frac{1}{\sigma_{11}(\text { waist })}=\frac{1}{\sigma_{11}(\min )}+\frac{\mathrm{R}_{22}^{2}}{\sigma_{11}(\text { lens })}
$$

If now the lens system is readjusted to form a waist at the target position as shown by the dotted lines in Fig. 10, the relative size of this waist and the minimum spot size achieved by the previous lens setting is:

$$
\frac{\sigma_{11}(\text { min at target })}{\sigma_{11}(\text { waist at target })}=1-\frac{R_{22}^{2} \sigma_{11} \text { (waist at target) }}{\sigma_{11}(\text { lens })}
$$

Again we observe that the two quantities approach each other if the size nf the beam at the lens is large compared to the beam size at the target.

There are several cases of special interest that may be derived from the above equations:

I) If $\mathrm{R}_{22}=0$ at the target position, then a minimum spot size at the target is also a waist. This corresponds to point-to-parallel imaging from the principal planes of the variable lens system to the target position. Beyond the last lens in the field-free region preceding the target, $R_{12}=$ a constant if $\mathrm{K}_{22}=0$; thus we conclude from $\mathrm{Eq}$. (59) that in this field-free region, the minimum spot size achievable at a target is a waist and is independent of the target position. Such a system is a "Zoom" lens.

2) If there are no lenses beyond the variable lens system, i.e., $R$ is an entirely field-free region ( a drift), then $R$ is of the form:

$$
R=\left(\begin{array}{ll}
1 & I \\
0 & 1
\end{array}\right)=\left(\begin{array}{ll}
R_{11} & R_{12} \\
R_{21} & R_{22}
\end{array}\right)
$$

In this situation $R_{12} R_{22}=I$ is a positive quantity, consequently the waisli always precedes a minimum spot size at a target. A case of particular interest is when the minimum spot size achievable is equal to the initial beam size at the lens. It then follows from Eq. (60) that $\mathrm{Z}=-\mathrm{L} / 2$, i.e., a waist occurs midway between the 
lens and the target. From Eq. (61), the ratio of the size of the beam at the lens and at the waist is:

$$
\frac{x(\text { Iens })}{x(\text { waist })}=\sqrt{\frac{\sigma_{11}(\text { lens })}{\sigma_{11}(\text { waist })}}=\sqrt{2}
$$

Combining this result with Eq. (59),

$$
I=R_{12}=\frac{x(\text { lens }) x(\min )}{|\sigma|^{1 / 2}}=\frac{x^{2}(\text { lens })}{x(\text { waist }) \theta(\text { waist })}
$$

or

$$
L=\frac{2 x(\text { waist })}{\theta(\text { waist })}
$$

where $L$ is the longest distance a beam can drift without exceeding its initial size at the lens.

Imaging from an Erect Ellipse to an Erect Ellipse

The general sigma matrix for imaging from an erect ellipse to an erect ellipse may be derived by inspection from $\overline{\mathrm{Eq}} .(36)$ by setting $\sigma_{21}(1)=\sigma_{2 l}(0)=0$. The result is:

$$
\sigma(1)=\left[\begin{array}{c|c}
R_{11}^{2} \sigma_{11}(0)+R_{12}^{2} \sigma_{22}(0) & 0 \\
\hline 0 & R_{21}^{2} \sigma_{11}(0)+R_{22}^{2} \sigma_{22}(0)
\end{array}\right]=\left[\begin{array}{c|c}
\sigma_{11}(1) & 0 \\
\hline 0 & \sigma_{22}(1)
\end{array}\right]
$$

For symmetric magnetic systems $R_{11}=R_{22}$. Using this property and the fact that $|R|=1$, it follows that $R_{12} R_{21}=\left(R_{11}^{2}-1\right)$. So for symmetric magnetic systems Eq. (65) reduces to:

$$
\sigma(1)=\left[\begin{array}{c|c}
{\left[-\frac{R_{12}|\sigma|}{R_{21}}\right]^{1 / 2}} & 0 \\
0 & {\left[-\frac{R_{21}|\sigma|}{R_{12}}\right]^{1 / 2}}
\end{array}\right]=\left[\begin{array}{c|c|c|}
\sigma_{11}(1) & 0 \\
0 & \sigma_{22}(1)
\end{array}\right]
$$


The above equations may be used to calculate the optimum design parameters for periodic beam transport systems.

Example No. 1:

Consider a unit-cell of a periodic focusing array consisting of focusing elements only as indicated below.

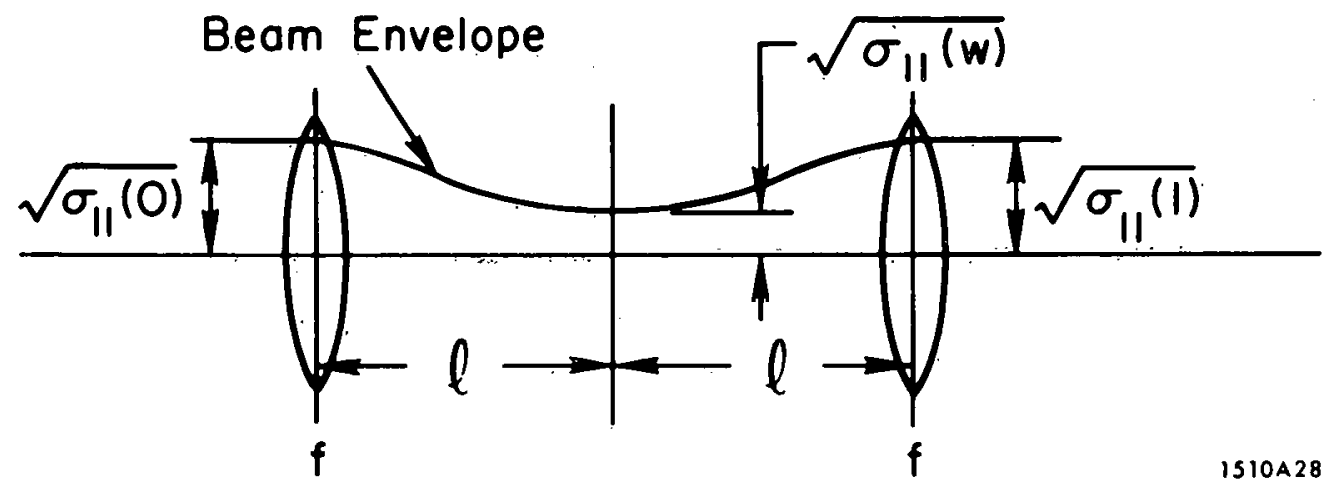

The $R$ matrix for the unit-cell, i.e., from the principal planes of the first lens to the principal planes of the second lens, is

$$
R=\left[\begin{array}{c|c}
R_{11} & 2 \ell \\
\hline-\frac{1}{f^{\prime}}+\frac{\ell}{\partial f^{2}} & R_{11}
\end{array}\right]
$$

If now we require that the beam envelope possess symmetry coiucident with the lens s. mmetry i.e., that erect ellipses occur at the principal pianes of eacis lens and a waist midway in between, and furthermore that the beam size at the second lens. be kept to a minimum and equal to the beam size at the first lens: then substituting Eq.(67) 1nto Eq. (66) and setting $\sigma_{11}(1)$ to be a minimum yields:

$$
\begin{aligned}
& \sqrt{\sigma_{11}(1)}=\sqrt{\sigma_{11}(0)} \\
& f=\ell=\frac{1}{2} \sqrt{\frac{\sigma_{11}(0)}{\sigma_{22}(0)}}=\sqrt{\frac{\sigma_{11}(w)}{\sigma_{22}(w)}}
\end{aligned}
$$


where $\sigma_{11}(0)$ and $\sigma_{22}(0)$ are measured at the principal planes of the first lens.

and finally

$$
\frac{x(\max )}{x(\min )}=\sqrt{\frac{\sigma_{11}(0)}{\sigma_{11}(w)}}=\sqrt{2}
$$

Note that the ratio of the maximum to the minimum beam size (Eq. 69) is independent of the phase space area and of the lens spacing.

Example No. 2:

If the unit cell is a FODO array as follows:

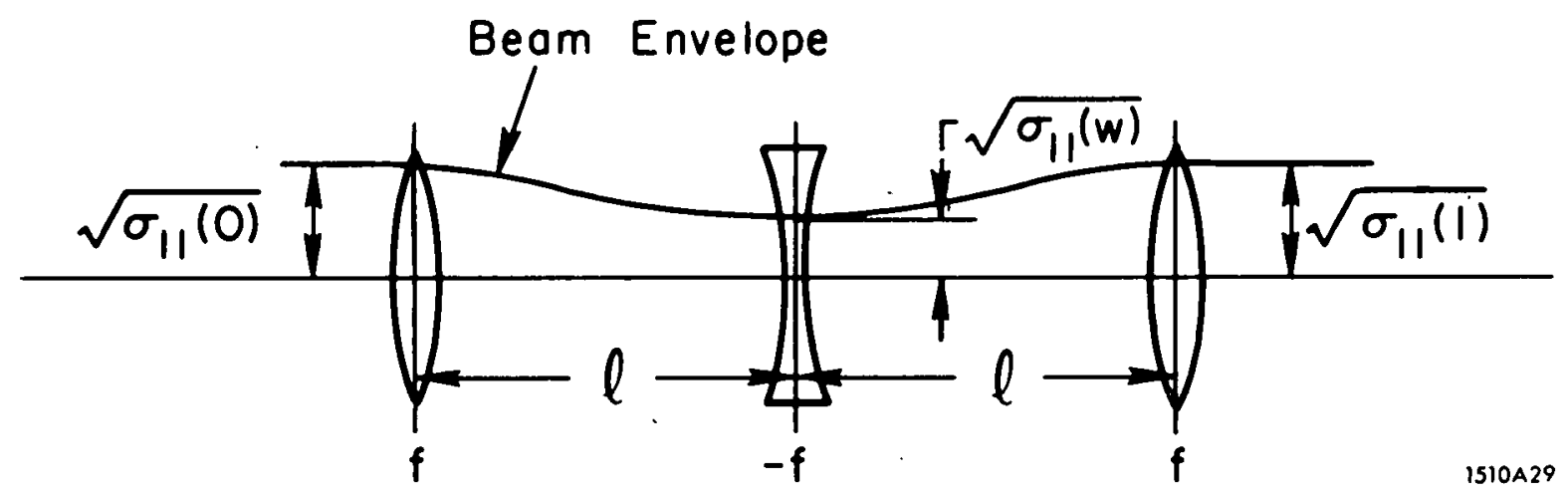

The $R$ matrix for the unit cell(from the principal planes of the first lens to the principal planes of the third lens) is:

$$
R=\left[\begin{array}{l|c}
\mathrm{R}_{11} & \ell\left(2+\frac{\bar{l}}{\mathrm{f}}\right) \\
\hline-\frac{\ell}{4 \mathrm{f}^{2}}\left(2-\frac{\ell}{\mathrm{f}}\right) & \mathrm{R}_{11}
\end{array}\right],
$$

If we now impose the symmetry requirements that erect eilipses occur at the principal planes of each lens; and that the beam size $\sqrt{\sigma_{11}(1)}$ at lens 3 be a minimum and equal to the beam size $\sqrt{\sigma_{11}(0)}$ at lens 1 , then it follows that:

$$
\begin{aligned}
& \sqrt{\sigma_{11}(1)}=\sqrt{\sigma_{11}(0)} \\
& \frac{\ell}{I}=\frac{4}{1+\sqrt{5}} \cong 1.236
\end{aligned}
$$




$$
\frac{\sigma_{11}(0)}{\sigma_{11}(w)}=-\frac{3+\sqrt{5}}{1-\sqrt{5}} \cong 4.23
$$

or

$$
\frac{x(\max )}{x(\min )}=\sqrt{\frac{\sigma_{11}(0)}{\sigma_{11 .}(w)}} \cong 2.055
$$

and finally

$$
\ell \cong 0.3003 \sqrt{\frac{\sigma_{11}(0)}{\sigma_{22}(0)}}
$$

where $\sigma_{11}(0)$ and $\sigma_{22}(0)$ are measured at the principal planes of the first lens in the FODO array.

For a FODO quadrupole array where the field strength is held constant for all elements rather than the focal lengths, the results are somewhat different than those above. This case may be readily calculated via TRANSPORT using the above results as initial guesses in the calculation.

Relationship between a First-Order Point-to-Point Image and the Minimum Spot Size Achievable at a Fixed Target Position

This problem is not as easy to explore as were the preceding ones because the question arises "the first-order image of what?" If, however, we restrict the discussion to a thick or thin lens system that dos not have intermediate images between the source and the image under consideration, then the following comments are applicable.

The ratio of the minimum beam size to the size of a first-order image at a fixed target position may be calculated using Eq.'s (56) and (59). From Eq. (59) we have:

$$
\sigma_{11}(\min )=\frac{L^{2}|\sigma|}{\sigma_{11}(\text { lens })}
$$

and fron Eq. (56) the size of a first-order image at the target position is:

$$
\begin{aligned}
\sqrt{\sigma_{11}(\text { image })}=|M| & \sqrt{\sigma_{11} \text { (object) }}=\left(\frac{L}{p}\right) \sqrt{\sigma_{11} \text { (ob ject) }} \\
& A-54
\end{aligned}
$$


where $M$ is the magnification of the first-order image, $p$ is the object distance measured to the principal planes, and $L$ is the distance to the target measured from the principal planes.

$$
\begin{aligned}
& \text { The ratio of sizes is } \\
& \frac{\sigma_{11}(\min )}{\sigma_{11}(\text { lst order image) }}=\frac{p^{2}|\sigma|}{\sigma_{11}(\text { object }) \sigma_{11} \text { (lens) }}
\end{aligned}
$$

Using Eq. (36), we may write

$$
\sigma_{11}(\text { lens })=\sigma_{11} \text { (object) }+2 p \sigma_{21} \text { (object) }+p^{2} \sigma_{22} \text { (object) }
$$

and since

$$
|\sigma|=\sigma_{11} \text { (object) } \sigma_{22}(\text { object })-\sigma_{21}{ }^{2} \text { (object) }
$$

it follows that the first-order image will coincide with the smallest spot size only if the orientation of the initial beam ellipse at the object is such that

$$
\mathrm{p} \sigma_{21}(\text { object })=-\sigma_{11} \text { (object) }
$$

or if $\sigma_{11}$ (object) $=0$ 1.e., for a point source.

For an erect ellipse at the source and the lens adjusted to provide a minimum spot size at the target, it can be shown that the first-order image will always follow the target position (the minimum spot size) by a distance

$$
Z=L|M| \frac{\sigma_{1 I} \text { (object) }}{\sigma_{1 I}(\text { lens })}=L|M| \frac{x_{0}^{2}}{x^{2}(\text { lens })}
$$

where $\dot{L}$ is the distance to the target position from the principal planes of the lens - system, $x_{0}$ is the source size, and $M$ is the magnification of the first-order image. Again we observe that the ratio of the beam size at the source and the beam size at the "Iens" is the criterion determining the proximity of these two quantities.

\section{Orientation of the Major Axes of a Phase Space Ellipse}

The matrix equation for a coordinate rotation as shown in Fig. Il is 


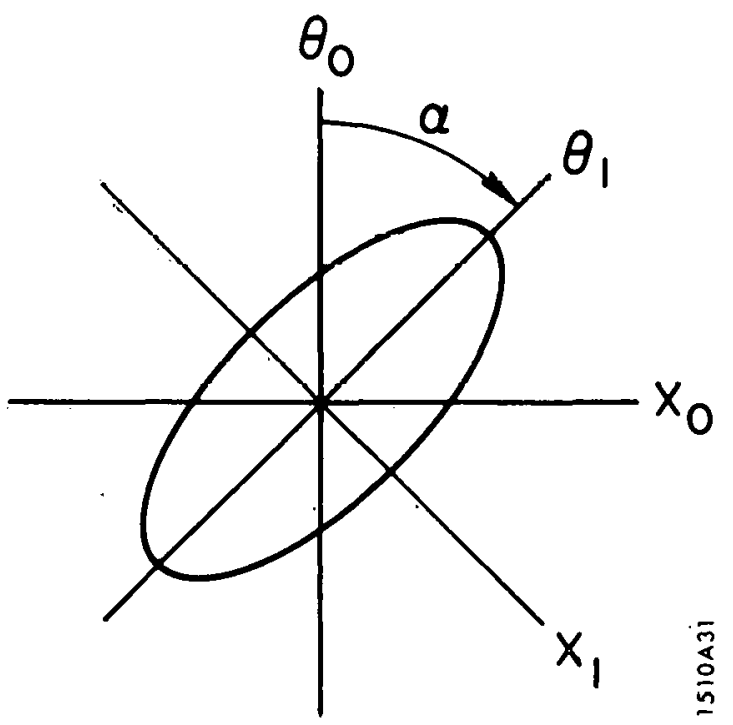

Fig. 11 


$$
\left(\begin{array}{l}
x_{1} \\
\theta_{1}
\end{array}\right)=\left(\begin{array}{cc}
\cos \alpha \cdot & -\sin \alpha \\
\sin \alpha & \cos \alpha
\end{array}\right)\left(\begin{array}{l}
x_{0} \\
\theta_{0}
\end{array}\right)
$$

or

$$
\mathrm{X}_{1}=\mathrm{M} \mathrm{X}_{\mathrm{O}}
$$

The equation of an ellipse in either set of coordinates is

$$
\mathrm{X}^{\mathrm{T}} \sigma^{-1} \mathrm{X}=1 \quad \mathrm{w}=\left(\begin{array}{l}
\mathrm{x} \\
\theta
\end{array}\right) ; \mathrm{X}^{\mathrm{T}}=(\mathrm{x} \theta)
$$

and the transformation from $\sigma(0)$ to $\sigma(1)$ is

$$
\sigma(1)=\mathrm{M} \sigma(0) \mathrm{M}^{\mathrm{T}}
$$

provided $|M|=1$, which it does.

If we assume a general ellipse for $\sigma(0)$ and an "erect" ellipse for $\sigma(1)$, i.e.,

$$
\sigma(0)=\left[\begin{array}{c|c}
\sigma_{11}(0) & \sigma_{21}(0) \\
\hline \sigma_{21}(0) & \sigma_{22}(0)
\end{array}\right] \text { and } \sigma(1)=\left[\begin{array}{c|c}
\sigma_{11}(1) & 0 \\
\hline 0 & \sigma_{22}(1)
\end{array}\right]
$$

It follows from Eq. (72) that:

$$
\because \sigma_{21}(1)=0=M_{11} M_{21} \sigma_{11}(u)+\left(M_{11} M_{22}+M_{21} M_{12}\right) \sigma_{12}(0)+M_{12} M_{22} \sigma_{22}(0)
$$

from which

$$
\operatorname{Tan} 2 \alpha^{\prime}=\frac{2 \sigma_{21}(0)}{\sigma_{22}(0)-\sigma_{11}(0)}
$$

or using the definition

$$
r_{21}=\frac{\sigma_{21}}{\sqrt{\sigma_{1,1} \sigma_{22}}}
$$


an alternate form of expressing the ellipse orientation is

$$
\operatorname{Tan} 2 x^{\prime}=\frac{2 r_{21} \sqrt{\sigma_{11} \sigma_{22}}}{\sigma_{22}-\sigma_{11}}=\frac{2 r_{21}}{\sqrt{\frac{\sigma_{22}}{\sigma_{11}}}-\sqrt{\frac{\sigma_{11}}{\sigma_{22}}}}
$$

Clearly $\alpha$ is dependent upon the units chosen for $\alpha_{11}$ and $\sigma_{2 C}$ except in the obvious case of $\alpha=0$; i.e., an erect ellipse. 


\section{Section III}

TRANSPORT has the capability of calculating the second-order matrix elements (aberration coefficients) of any static-magnetic beam transport system composed of combinations of bending magnets, quadrupoles, solenoids, sextupoles and interspersed drift spaces. It is assumed that mid-plane symmetry prevails for any given magnetic element in a system (except for solenoids) but not necessarily for the system as a whole. The notation used in a TRANSPORT printout is described in reference 1 (SLAC-75) beginning on page 46. The subscript notation is the same as that used for first-order where the subscript 1 means $x, 2$ means $\theta, \quad 3$ means $y$, 4 means $\varphi, 5$ means $\ell$, and 6 means $\delta$.

The symbol $\mathrm{R}_{i j}$ has been used to signify a first-order matrix element and the symbol $T_{i j k}$ will be used to signify a second-order matrix element. Thus we may write the second-order Taylor expansion representing the deviation of an arbitrary trajectory from the central trajectory as:

$$
x_{i}(t)=\sum_{j=1}^{6} R_{i j} x_{j}(0)+\sum_{j=1}^{6} \sum_{k=1}^{6} T_{i j k} x_{j}(0) x_{k}(0)
$$

where $\mathrm{x}_{1}=\mathrm{x} \quad \mathrm{x}_{2}=\theta \quad \mathrm{x}_{3}=\mathrm{y} \quad \mathrm{x}_{4}=\varphi \quad \mathrm{x}_{5}=\ell$ and $\mathrm{x}_{6}=\delta$ denotes the subscript notiation. In an actual computer printout, the $\mathrm{T}_{i j l k}$ 's are abbreviated as ( $i j k$ ); for example. $\mathrm{T}_{126}=\left(\mathrm{x} \mid \theta_{0} \delta\right)$. would appear in a printout as (1 26) followed by the computed value of the aberration coefficient for the system being designed.

In order to modify the magnitude of any given aberration coefficient, it is necessary to introduce multipole component(s) of the magnetic field of order equal to or less than the order of the aberration. Thus sextupole, quadrupole and dipole components of the field may all be used to modify any given second-order aberration. But, in practice, the second-order aberrations are usualiy winimized by only introducing sextupole components so as not to disturb the l'irst-order optics of the system. It should always be kept in mind, however, that it may be 
beneficial to go back and change the first-order solution (optical mode) so as to provide a more favorable situation for correcting aberrations; a wise selection of the tirst-order optical mode may in many instances be the deciding factor between the success or failure of a design.

For a fixed location of a sextupole component, the partial derivative of any second-order aberration coefficient $\mathrm{T}_{i j k}$ with respect to the strength $\mathrm{S}_{2}$. of a given sextupole component is a constant. i.e:,

$$
\frac{\partial T_{i j k}}{\partial S_{2}}=\text { a constant }=\text { the coupling coefficient of } S_{2} \text { to } T_{i j k}
$$

Thus minlmizing a selected group of aberrations is a straight forward problem. of solving a set of simultaneous hinear equations once the coupling coefficients are known.

The coupling coefficients may be derived directly either by using two successive TRANSPORT muns (with and without unity strength sextupoles) or by direct calculation via the coupling coefficient equations derived later in this section (see equation 19). Eventually TRANSPORT may have a built-in second-order fitting routine; but until this happens, each user will have to devise his own least squares program for second-order aberration minimization.

\section{Second-Order Phase Ellipsoid Formalism}

It will be noted by the user, that a second-order TRANSPORT calculitiinn modifies the phase-ellipsoi.d prințout. In a second-order run, TRANSPORT. calculates and prints out the second-moments of the phase space distribution function in the $\sqrt{v_{i i}}$ columns. In addition, it also calculates and prints out the new coordinates of the centroid (first-moment) of the phase space distribution function and tabulates this result to the left of the $\sqrt{\sigma_{i i}}$ columns in the same manner as it does for a magnet misalignment run.

Caution should be used in the use and interpretation of the second-order phase ellipsoid results especially if it is known or suspected that the phase space 
distribution resulting from a second-order run is not symmetrical about the beam centroid. To be certain of the situation in any given design, it would be wise to calculate the actual distribution function by sampling of a large number of trajectories using the first-and second-order matrix elements of a representative TRANSPORT run as the basis for the calculation.

A Systematic Procedure for Designing High Resolving Power Beam Transport Systems or Charged Particle Spectrometers

The following is a report submitted to the Third International Magnet Symposium held in Hamburg, Germany - May 1970. It is a general description of a suggested procedure for designing systems to any order and includes the derivation of the coupling coefficient of an nth-order multipole to any nth-order aberration coefficient: The report also derives the multipole strengths for the three techniques for introducing multipole components into a system: namely, 1) pure multipole fields, 2) non-uniform fields, and 3) contoured entrance or exit boundaries of magnets.

The notation used in this report is identical to TRANSPORT notation except for the following:

Replace $x^{\prime}$ and $y^{\prime}$ in the report by $\theta$ and $\varphi$ respectively to convert to TRANSPORT. notation . 
REPORT SUBMITTED TO THE THIRD INTERNATIONAL MAGNET SYMPOSIUM

HELD IN HAMBURG, GERMANY-May 1970

by

Karl L. Brown

Stanford Linear. Accelerator lenter

Stanford, California

By extrapolating the systematics of the general first-and second-order theory of beam transport optics $(1,2,3)$ to include higher order multipole terms, it has been possible to evolve a simple, step by step, procedure for the design of high resolving power static-magnetic beam transport systems. The choice of the appropriate dipole and quadrupole elements for a given system may be determined once the resolving power, solid angle, momentum range and detector system of the instrument have been specified. The partial derivat,ive of any nth-order aberration coefficient with respect to an nth-order multipole component located anywhere in the system has been derived. From this "coupling. coefficient", the strength and the optimum location of multipole element(s) to correct or modify a given aberration or group of aberrations is uniquely determined. 


\section{Introduction}

Within the last two decades; significant advances have been made in the understanding of charged particle optics. Perhaps the first major contribution was the development of the theory of the Alternating Gradient Synchrotron (A.G.S.) by Courant, Livingston, and Snyder(4) which led to the first-order matrix algebra. formulation of beam-transport optics. Subsequent to this a second-order matrix algebra was developed by Brown, Belbeoch, and Bounin(5); followed by the development at SLAC of the digital computer program called TRANSPORT(6) that is widely used today in many laboratories for solving first-and second-order staticmagnetic beam transport problems.. In principle, the second-order matrix formalism may be extended to any order, but in practice this approach has proved to be too cumbersome. Thus beyond second-order it has been'more efficient to use computer ray-tracing programs which integrate the basic differential equation of motion of the charged particles through the known or assumed magnetic fields. The fundamental difficulty with ray-tracing has been the required computational time to complete a design involving the minimization of many higher-order aberrations.

In this report, 'we will outline a systematic procedure for the design of high-resolution systems based upon the extrapolation of the fiirst- and secondorder theory $(1,2,3)$ to include higher-order multipole components. A general equation has been derived for the coupling coefficient of an nth-order multipole to any given nth-order aberration coefficient. As will be shown later, these coupling coefficients are a function only of the characteristic first-order trajectories (matrix elements) introduced and defined in References 1 and 2 .

Given this information, a systematic procedure for designing high resolution beam transport systems is as follows:

1) Find a satisfactory first-order solution to the problem using TRANSPORT or its equivalent.

2) Calculate and make the necessary corrections to the second-order aberrations by introducing sextupole components into the system. The "best" locations and strengths of the sextupole components required may be selected via the coupling coefficients for the aberrations to be minimized.

3) Calculate and make the necessary corrections (via ray-tracing) to the third-order aberrations by introducing octupole components into the system. (Note that an nth-order multipole couples with terms of order $n$ or higher but not with terms of order lower than $n$. Thus an octupole component will not disturb the first-and second-order solutions already found from steps 1 and 2.)

4) Repeat the above procedure up to the multipole order desired or needed to achieve the design objectives.

, If the design requires a solution to nth-order and m multipoles at each order are necessary to minimize the aberrations, the number of computer runs previously needed to complete a design was at least $(n+m)^{2}$. Having a knowledge of the coupling coefficients, after the first-order design has been selected, now (in principle) reduces the number of computer runs required to $n$. Since ray-tracing is very time consuming, this is indeed a significant saving. 


\section{Theory*}

The following results are applicable to static-magnetic charged particle optical systems possessing median plane.symmetry. As. in Ref. I, we shall use a right-handed curvilinear coordinate $\operatorname{system}(x, y, t)$ where $z$ and $y$ are the transverse coordinates. $\mathrm{x}$ is the outward normal distance in the median plane away from the central trajectory, $y$ is the perpendicular distance from the median plane, $t$ is the distance along the central trajectory, and $h=h(t)$ is the curvature of the central trajectory.

The existence of the median plane requires that the scalar potential $\varphi$ be an odd function of $y$, i.e., $\varphi(x, y, t)=-\varphi(x,-y, t)$. The most general form of $\varphi$ may therefore, be expressed as follows:

$$
\varphi(x, y, t)=\sum_{m=0}^{\infty} \sum_{n=0}^{\infty} A_{2 m+1, n} \frac{x^{n}}{n !} \frac{y^{2 m+1}}{(2 m+1) !}
$$

where the coefficients $A_{2 m+1, n}$ are functions of $t$.

In this coordinate system, the differential line element dT is given by

$$
d T^{2}=d x^{2}+d y^{2}+(1+h x)^{2} d t^{2}
$$

The Laplace equation has the form

$\nabla^{2} \varphi=\frac{1}{(1+h x)} \frac{\partial}{\partial x}\left[(1+h x) \frac{\partial \varphi}{\partial x}\right]+\frac{\partial^{2} \varphi}{\partial y^{2}}+\frac{1}{(1+h x)} \frac{\partial}{\partial t}\left[\frac{1}{(1+h x)} \frac{\partial \varphi}{\partial t}\right]=0$

Substitution of (1) into (2) gives the following recursion formula for the coefficients:

$$
\begin{aligned}
{ }^{-A_{2 m+3, n}}= & A_{2 m+1 ; n}^{\prime \prime}+n A_{2 m+1, n-1}^{\prime \prime}-n^{\prime} A_{2 m+1, n-1}^{\prime}+A_{2 m+1, n+2} \\
& +(3 n+1) h A_{2 m+1, n+1}+n(3 n-1) h^{2} A_{2 m+1, n}+n(n-1)^{2} h^{3} A_{2 m+1, n-1} \\
& +3 n h A_{2 m+3, n-1}+3 n(n-1) h^{2} A_{2 m+3, n-2}+n(n-1)(n-2) h^{3} A_{2 m+3, n-3}
\end{aligned}
$$

where prime means $\frac{d}{d t}$, and where it is understood that all coefficients A with one or more negative subscripts are zero. 'lhis recursion formula expresses all

* The notation used in this report follows that used in Ref. 1 unless otherwise indicated. 
the coefficients in terms of the midplane field ${ }_{i y}(x, 0, t)$ :

where

$$
A_{1, n}=\left(\frac{\partial^{n} B}{\partial x^{n}}\right)_{\substack{x=0 \\ y=0}}=\text { functions of } t
$$

Since $\varphi$ is an odd function of $y$, on the median plane we have $B_{x}=B_{t}=0$. The normal (in $x$ direction). derivatives of $B_{y}$ on the reference curve defines $B_{y}$ over the entire median plane, hence the magnetic field $\vec{B}$ over the whole space. "The components of the field are expressed in terms of $\varphi$ explicitly by $\vec{B}=\vec{\nabla} \varphi$ or

$$
\begin{aligned}
& B_{x}=\frac{\partial \varphi}{\partial x}=\sum_{m=0}^{\infty} \sum_{n=0}^{\infty} A_{2 m+1, n+1} \frac{x^{n}}{n !} \frac{y^{2 m+1}}{(2 m+1) !} \\
& B_{y}=\frac{\partial \varphi}{\partial y}=\sum_{m=0}^{\infty} \sum_{n=0}^{\infty} A_{2 m+1, n} \cdot \frac{x^{n}}{n !} \frac{y^{2 m}}{(2 m) !} \\
& B_{t}=\frac{1}{(1+h x)} \cdot \frac{\partial \varphi}{\partial t}=\frac{1}{(1+h x)} \sum_{m=0}^{\infty} \sum_{n=0}^{\infty} A_{2 m+1, n}^{i} \frac{x^{n}}{n !} \cdot \frac{y^{2 m+1}}{(2 m+1)} !
\end{aligned}
$$

The expression for the magnetic field on the midplane is

$$
B_{y}(x, o, t)=\sum_{n=0}^{\infty} A_{1, n} \frac{x^{n}}{n !}
$$

At this point we deviate from the notation and formalism of Ref. 1 and introduce $K_{n}(t)$, the multipole strength per unit length; and $S_{n}$, the total multipole strength of a static-magnetic field.

We rewrite equation (6) as

$$
B_{y}(x, 0, t)=B \rho \cdot \sum_{n=0}^{\infty} K_{n}(t) x^{n}
$$

Where $B \rho=\frac{B}{h}=\frac{P_{0}}{e}$ is the magnetic rigidity of a particle of momentum $P_{0}$ and charge e along the central trajectory; from which

$$
K_{n}(t)=\left(\frac{1}{B p}\right)\left(\frac{1}{n !}\right)\left(A_{I, n}(t)\right)=\left(\frac{1}{B p}\right)\left(\frac{1}{n !}\right)\left(\frac{\partial^{n} B_{y}}{\partial x^{n}}\right)_{x=y=0}
$$


We define $S_{n}$ as

$$
s_{n}=\int_{0}^{L} K_{n}(t) d t
$$

$S_{n}$ so defined is the strength of the nth-order multipole component of a field over the interval of integration.

Multipole Strengths for Pure Multipole Fields

Consider the scalar potential of an nth-order $[2(n+1)$ pole $]$ pure multipole element:

$$
\varphi=\frac{B_{0} r^{n+1}}{(n+1) a^{n}}[\sin (n+1) \theta]
$$

where

$$
x=r \cos \theta \text { and } y=r \sin \theta
$$

$B_{0}$ is the field at the pole and $a$ is the radial distance to the pole from the central trajectory.

Expanding $\varphi$ as a function of $x$ and $y$ and differentiating, we have

$$
B_{y}=\frac{\partial \varphi}{\partial y}=\frac{B_{0}}{a^{n}}\left[x^{n}+\cdots \cdot \cdot \cdot\right]
$$

From which

$$
K_{n}=\left(\frac{B_{0}}{a^{n}}\right)\left(\frac{1}{B \rho}\right)
$$

and

$$
S_{n}=\left(\frac{B_{i}}{a^{n}}\right)\left(\frac{L}{R p}\right)
$$

Where $L$ is the length of the multipole element. 
For a dipole $n=0$ and the dipole strength is

$$
\mathrm{S}_{\mathrm{O}}=\frac{\mathrm{L}}{\rho}=\alpha \text { (The angle of bend of the central trajectory) }
$$

For a quadrupole $n=1$ and

$$
S_{i}:=\left(\frac{B_{0}}{a}\right)\left(\frac{L}{B p}\right)
$$

For a sextupole $n=2$ and

$$
S_{2}=\left(\frac{B_{0}}{a}\right)\left(\frac{L}{B p}\right)
$$

etc. for higher-order multipoles.

Multipole Strengths for a Non-Uniform Field Expansion

From the midplane field expansion of a non-uniform magnetic field

$$
B_{y}(x, o, t)=B_{y}(0, o, t)\left[1-n h x+\beta(h x)^{2}+\gamma(h x)^{3}+\cdots \cdots \cdot\right]
$$

the multipole strength factors are:

$$
K_{0}=n, \quad K_{1}=-n h^{2}, \quad K_{2}=\beta h^{3}, \quad \text { etc. }
$$

and $S_{n}$ evaluated over the length $I$ of the central trajectory is:

$$
\begin{aligned}
& S_{0}=h L=\alpha \text { as before, } \\
& S_{1}=-n h^{2} L \text {, and } S_{2}=\beta h^{3} L, \text { etc. }
\end{aligned}
$$

Multipole Strengths for a Contoured Entrance or Exit Boundary of a Magnet

A third method of introducing multipole components is via a curved entrance or exit boundary of a magnet. To calculate the multipole strengths in this case, we integrate equation (7), holding $x$ constant, ac follows:

$$
\int_{0}^{L} B_{y}(x, o, t) d t=B p \sum x^{n} \int_{0}^{L} K_{n}(t) d t=B p \sum S_{n} x^{n}
$$


To relate this to the field boundary, we assume $B_{y}$ to be a constant inside the effective field boundary and zero outside (i.e., we ignore the finite extent of the fringing field). In this sharp-cutoff approximation, the field boundary $\mathrm{z}=\mathrm{Z}(\mathrm{x})$ is :

$$
\mathrm{z}=\frac{\mathrm{I}}{\mathrm{B}_{\mathrm{y}}} \int_{0}^{\mathrm{L}} \mathrm{B}_{\mathrm{y}}(\mathrm{x}, \mathrm{o}, \mathrm{t}) \mathrm{dt}=\frac{1}{\mathrm{~h}} \sum \mathrm{s} \mathrm{x}^{\mathrm{n}}=-\mathrm{x} \tan \beta+\frac{\mathrm{s}_{2}}{\mathrm{~h}} \mathrm{x}^{2}+\cdots
$$

where $h=\frac{1}{\rho}$ and $\beta$ is the angle of rotation of the entrance or exit face of the magnet at $x=0$. A positive $\beta$ implies radial ( $x$ ) defocusing and transverse (y) focusing.: We note that:

$$
S_{1}=-h \tan \beta=\text { The "quadrupole strength" }
$$

The radius of curvature of the boundary is related to the sextupole strength as follows:

$$
\frac{1}{R}=\frac{z^{\prime \prime}}{\left(1+z^{\prime 2}\right)^{3 / 2}}=\frac{2 S_{2}}{h \sec ^{3} \beta}
$$

or

$$
S_{2}=\frac{h \sec ^{3} \beta}{2 R}=\text { The "sextupole strength" }
$$

From equation (13), we note that a positive multipole component of the field increases the $\int$ Bdt for a positive $x$; thus a positive sextupole is represented. by a concave surface of the entrance or exit boundary.

The Description of the Trajectories as a Taylor's Expansion

The deviation of an arbitrary trajectory from the central trajectory is described by expressing $x$ and $y$ as functions of $t$. The expressions wil1. also contain $x_{0}, y_{0}, x_{0}^{1}, y_{o}^{\prime}$ and $\delta$, where the subscript 0 indicates that the quantity is evaluated at $t=0$. The prime (') denotes the derivative with respect to $t$, and $\delta=\frac{\Delta P}{P_{O}}$ is the fractional momentum deviation of the ray from that of the central trajectory. These five initial boundary values will have the value zero for the central trajectory itself. $x$ and $y$ are expressed as a five-fold Taylor expansion using these initial boundary values. The expansions are written: 


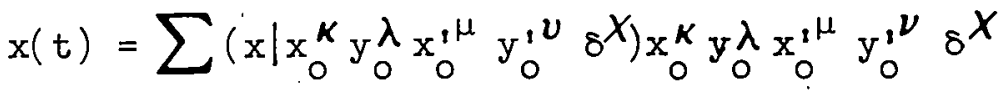

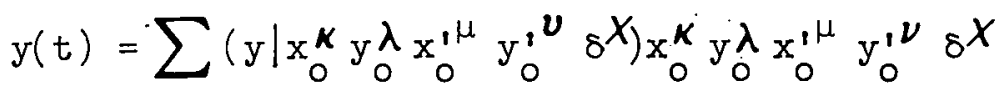

Here, the parentheses are symbols for the Taylor coefficients; the first part of the symbol identifies the coordinate represented by the expansion, and the second indicates the term in question. These coefficients are functions of $t$ to be determined. The symbol $\sum$ indicates summation over zero and all positive integer values of the exponents $\kappa, \lambda, \mu, \nu, X ;$. The constant term is zero, and the terms that would indicate a coupling between the coordinates $\mathrm{x}$ and $\mathrm{y}$ are also zero; this results from the midplane symmetry. Thus we have

$$
\begin{aligned}
& (x \mid 1)=(y \mid 1)=0 \\
& \left(x \mid y_{0}\right)=\left(y \mid x_{0}\right)=0 \\
& \left(x \mid y_{0}^{\prime}\right)=\left(y \mid x_{0}^{\prime}\right)=0
\end{aligned}
$$

Here, the first line is a consequence of choosing the central trajectory as the reference axis, while the second and third lines follow directly from considerations of median plane symmetry.

Since they will appear often in the formalism, it is convenient to introduce the following abbreviations for the first-order Taylor coefficients:

$$
\begin{array}{ll}
\left(x \mid x_{0}\right)=c_{x}(t) \quad\left(x \mid x_{0}^{i}\right)=s_{x}(t) \quad(x \mid \delta)=d_{x}(t) \\
\left(y \mid y_{0}\right)=c_{y}(t) \quad\left(y \mid y_{0}^{1}\right)=s_{y}(t)
\end{array}
$$

When the transverse position of an arbitrary trajectory at position $t$ is written as a first-order Taylor's expansion as a function of the initial boundary conditions, the above five quantities are just the coefficients appearing in the expansion for the transverse coordinates $\mathrm{x}$ and $\mathrm{y}$ as follows:

$$
x(t)=c_{x}(t) x_{0}+s_{x}(t) x_{0}^{1}+d_{x}(t) \delta+\text { higher-order terms }
$$

and

$$
y(t)=c_{y}(t) y_{0}+s_{y}(t) y_{0}^{\prime}+\text { higher-order terms }
$$


III. Solution of the Equations of Motion

The general differential equation of motion of a charged particle in a static-magnetic field valid to all orders in $\mathrm{x}$ and $\mathrm{y}$ and their derivatives as derived in Ref. 1, equation ( 5 ) is:

$$
\begin{aligned}
& \hat{x}\left\{\left[x^{\prime \prime}-h(I+h x)\right]-\frac{x^{\prime}}{\left(T^{\prime}\right)^{2}}\left[x^{\prime} x^{\prime \prime}+y^{\prime} y^{\prime \prime}+(I+h x)\left(h x^{\prime}+h^{\prime} x\right)\right]\right\} \\
& +\hat{y}\left\{y^{\prime \prime}-\frac{y^{\prime}}{\left(T^{\prime}\right)^{2}}\left[x^{\prime} x^{\prime \prime}+y^{\prime} y^{\prime \prime}+(I+h x)\left(h x^{\prime}+h^{\prime} x\right)\right]\right\} \\
& \quad+\hat{t}\left\{\left(2 h x^{\prime}+h^{\prime} x\right)-\frac{(1+h x)}{\left(T^{\prime}\right)^{2}}\left[x^{\prime} x^{\prime \prime}+y^{\prime} y^{\prime \prime}+(1+h x)\left(h x^{\prime}+h^{\prime} x\right)\right]\right\} \\
& =\frac{e}{P} T^{\prime}\left(\vec{T}^{\prime} \times \vec{B}\right)=\frac{e}{P} T^{\prime}\left\{\hat { x } \left[y^{\prime} B_{t}-(I+h x) B y+\hat{y}\left[(1+h x) B_{x}-x^{\prime} B_{t}\right]\right.\right.
\end{aligned}
$$

If this equation is solved to nth-order for the Taylor's coefficients of equation (15), it will be observed that the result has the remarkably simple form:

$$
\begin{aligned}
& \left(x_{i} \mid x_{0}^{\kappa_{y} \lambda_{x}{ }^{\prime} \mu_{j}, \nu} \nu_{o} X\right)= \pm\left[\frac{n !}{\kappa ! \lambda ! \mu ! \nu ! X !}\right] \int_{0}^{t} G_{i}(t, \tau) c_{x}^{\kappa}(\tau) c_{y}^{\lambda}(\tau) s_{x}^{\mu}(\tau) s_{y}^{\nu}(\tau) d_{x}^{X}(\tau) K_{n}(\tau) d \tau \\
& + \text { Termis containing } K_{\sigma}, \cdots \ldots K_{n-1}
\end{aligned}
$$

where the variable of integration is $T$ and $n=(\kappa+\lambda+\mu+\nu+X)$.

The $x_{i}$ have the following meaning:

$$
x_{1} \otimes x(t) \quad x_{2}=x^{\prime}(t) \quad x_{3}=y(t) \quad x_{1}=y^{\prime}(t)
$$

$c_{x}, c_{y}, s_{x}, s_{y}$, and $d_{x}$ are defined by rquation (17) and in general are turctions of the variable of integration $T$ over the interval of integration. $K_{n}$ is def'ined by equation (8) and in general is also a function of $\tau$.

The $G_{i}$ 's are Green's functions where: 


$$
\begin{aligned}
& G_{1}(t, \tau)=\left(x(t) \mid x^{\prime}(\tau)\right)=s_{x}(t) c_{x}(\tau)-c_{x}(t) s_{x}(\tau) \\
& G_{2}(t, \tau)=\left(x^{\prime}(t) \mid x^{\prime}(\tau)\right)=s_{x}^{\prime}(t) c_{x}(\tau)-c_{x}^{\prime}(t) s_{x}(\tau) \\
& G_{3}(t, \tau)=\left(y(t) \mid y^{\prime}(\tau)\right)=s_{y}(t) c_{y}(\tau)-c_{y}(t) s_{y}(\tau) \\
& G_{4}(t, \tau)=\left(y^{\prime}(t) \mid y^{\prime}(\tau)\right)=s_{y}^{\prime}(t) c_{y}(\tau)-c_{y}^{\prime}(t) s_{y}(\tau)
\end{aligned}
$$

Note that the $G_{i}$ 's are just first-order Taylor's coefficients measured from the location $(\tau)$. of the multipole component to the end of the system ( $t)$.

Thus we see that the coupling coefficient to an nth-order multipole is a function only of the first-order matrix elements $c_{x}, c_{y}, s_{x}, s_{y}, d_{x}$ and their derivatives with respect to $t$.

From median-plane symmetry considerations, the allowed aberrations are those with $\mathrm{y}$ and/or $\mathrm{y}^{\prime}$ appearing an even number of times in the Taylor coefficient. For example $\left(x \mid x_{0}^{2}\right),\left(x \mid y_{0} y_{0}^{1}\right)$ and $\left(y \mid y_{0}^{2} y_{0}^{1}\right)$ are allowed aberrations; whereas $\left(x \mid y_{0}\right),\left(x \mid x_{0}^{2} y_{0}^{1}\right)$ or $\left(y \mid y_{0}^{2}\right)$ are not allowed and are therefore equal to zero.

The minus sign is used when $\mathrm{y}$ and/or $\mathrm{y}^{\prime}$ appear $0,4,8,12 \ldots$ times and the plus sign is used when $\mathrm{y}$ and/or $\mathrm{y}^{\prime}$ appear $2,6,10 \cdots \cdots$ times. For example for the coefficients $\left(x \mid x_{0}^{2}\right)$ and $\left(y \mid y_{0}^{3}\right)$, the minus sign is applicable; whereas for the coefficients $\left(x \mid y_{0}^{2}\right)$ and $\left(y^{\prime} \mid y_{0}^{3} y_{0}^{2}\right)$ the plus sign is applicable.

Equation (19) is derived by observing in the pattern of the solution of the differential equation that an nth-order aberration term containing the nth-order multipole strength factor $K_{n}$ cannot include multipole strength factors of lower order than $\mathrm{n}$; or stated physically, an nth-order multipole cannot couple to aberrations (terms) of order lower than $n$. This fact allows the recursion formula equation (3) to be reduced to the simple form

$$
A_{2 m+3, n}=-A_{2 m+1, n+2}
$$

in so far as it applies to the derivation of nth-order terms containing only $K_{n}$. As a consequence, the scalar potential for deriving these terms assumes the simplied form

$$
\varphi(x, y, t)=\sum_{m=0}^{\infty} \sum_{n=0}^{\infty}(-1)^{m} A_{1,2 m+n} \frac{x^{n}}{n !} \frac{y^{2 m+1}}{(2 m+1) !}
$$


From which, it follows that

$$
B_{x}(x, y, t)=\left(\frac{F_{o}}{e}\right) \sum_{m=0}^{\infty} \sum_{n=0}^{\infty}(-1)^{m} K_{2 m+n+1} \frac{(2 m+n+1 .) !}{n !(2 m+1) !} x^{n} y^{2 m+1}
$$

and

$$
B_{y}(x, y, t)=\left(\begin{array}{l}
P \\
\frac{\mathrm{o}}{e}
\end{array}\right) \sum_{m=0}^{\infty} \sum_{n=0}^{\infty}(-1)^{m} K_{2 m+n} \frac{(2 m+n) !}{n ! 2 m !} x^{n} y^{2 m}
$$

For terms containing only $\mathrm{K}_{\mathrm{n}}$, the basic differential equations assume the form:

$$
\begin{aligned}
& x^{\prime \prime}+\cdots \cdot=-\frac{e}{P} B_{y} \\
& y^{\prime \prime}+\cdots \cdot=\frac{e}{P} B_{x}
\end{aligned}
$$

Substituting the Taylor's expansion of equation (15) and solving for the nth-order terms using a conventional Green's function solution (see Ref. 1) yields equation (19) above.

IV. Interpretation and Use of Equation (19).

For most practical cases of interest, $K_{n}$ will be a constant over the interval of integration. In this event we may define the coupling coefficient of an nth-order multipole to an nth-order aberration as the partial derivative of equation (19) with respect to the $K_{n}$ in question as follows:

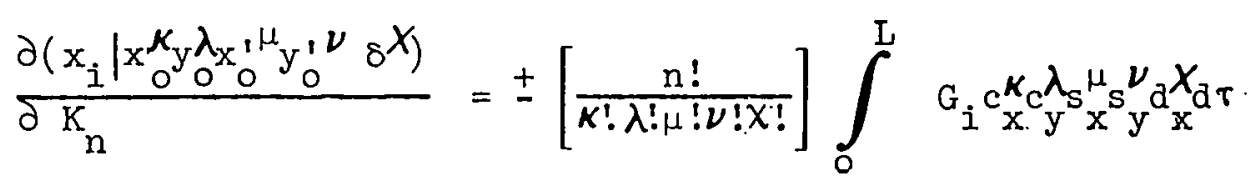

where now the interval of integration is over the multipole length I represented by $\mathrm{K}_{\mathrm{n}}$. For a distributed multipole component (such as in a non-uniform field bending magnet), equation (25) is used.

In many cases where a curved entrance or exit pole contour is used or a short multipole magnet is used such that the characteristic first-order functions $c_{x}, c_{y}, s_{x}, s_{y}$ and $d_{x}$ are essentially constants over the interval of integration (the length of one multipole), then the coupling coefficient is best defined as the partial derivative of equation (19) with respect to $\mathrm{s}_{\mathrm{n}}$ as follows: 


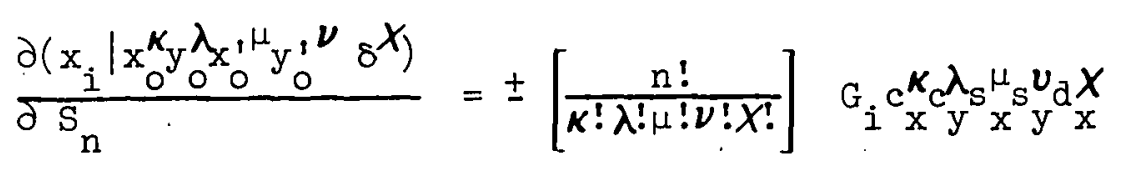

Examples

Assume a situation where the end of the system is a point-to-point image or the origin (i.e., $s_{x}(t)=0$ ), then using equation 26 , the coupling coefficients of a sextupole of strength $\mathrm{S}_{2}$ to various second order aberration coefficients are:

$$
\begin{aligned}
& \frac{\partial\left(x \mid x_{0}^{\prime} \delta\right)}{\partial s_{2}}=c_{x}(t) s_{x}^{2} d x \\
& \frac{\partial\left(x \mid x_{0}^{\prime 2}\right)}{\partial s_{2}}=c_{x}(t) s_{x}^{3} \\
& \frac{\partial\left(x_{x} y_{0} y_{0}^{\prime}\right)}{\partial s_{2}}=-2 c_{x}(t) c_{y} s_{x} s_{y}
\end{aligned}
$$

et.c. Where the Green's function ùsed in these examples is

$$
G_{1}=s_{x}(t) c_{x}-c_{x}(t) s_{x}=-c_{x}(t) s_{x} \quad \text { (since } s_{x}(t)=\underset{\text { for point-to-point }}{0} \text { imaging) }
$$

The aberration and $c_{x}(t)$ are evaluated at the end of the system. $c_{x}(t)$ is equal to the magnification $\mathrm{M}_{x}$ in the examples given. The remaining coefficients $c_{y}, s_{x}, s_{y}$ and $d_{x}$ are evaluated at the location of the sextupole $s_{2}$. 'ine above results are in agreement with Table VII of Ref. I.

To illustrate a more complex example, consider the fourth-order aberration coefficient $\left(y \mid \mathrm{y}_{\mathrm{O}}^{2} \mathrm{y}_{0}^{\prime} \delta\right)$ and assume parallel-to-point imaging in the $\mathrm{y}$ coordinate (i.e., $c_{y}(t)=0$ ). The appropriate Green's function is:

$$
G_{3}=s_{y}(t) c_{y}-c_{y}(t) s_{y}=s_{y}(t) c_{y}
$$

and the coupling coefficient to a fourth-order multipole of strength $\mathrm{S}_{4}$ is:

$$
\frac{\partial\left(y \mid y_{0}^{2} y_{0}^{i} \delta\right)}{\partial s_{4}}=-\left(\frac{4 !}{2 !}\right) \quad s_{y}(t) c_{y}^{3} s_{y} d^{x}
$$


where again the aberration coefficient $\left(y \mid y_{0}^{2} y_{0}^{\prime} \delta\right)$ and $s_{y}(t)$ are evaluated at the end of the optical system and $c y, s_{y}$, and $d_{x}$ are evaluated at the location of the fourth-order multipole $S_{4}$.

\section{A Systematic Procedure for Designing High Resolution Systems}

Pirst-Order Considerations

In many respects, the determination of a satisfactory first-order magneticoptical design is more difficult to achieve than is the subsequent higher-order design. This is true not only because the basic equipment configuration is dominated by first-order optical considerations but also because the choice of the first-order optics affects the magnitude of all higher-order aberrations and the ease with which these aberrations may be minimized by introducing multipole components into the design.

The dominating design parameters that must be clearly specified in order to evolve a first-order design are the momentum resolving power; the spatial resolution of the particle detector system to be used (this determines the momentum dispersion required); the required phase space acceptance (the solid angle, the source size, and the momentum range) of the instrument, and the first-order imaging requirements in both the $\mathrm{x}$ and $\mathrm{y}$ coordinates.

Given the above specifications (assuming they are self-consistent), the optical mode and physical configuration of the instrument may be determined. Often, more than one theoretical solution exists; in which case the choice is usually resolved by practical or economic considerations. In other cases, no solution is evident and the basic specifications must be modified accordingly. In any event, the following equations and discussion are applicable to the solution of the problem.

\section{1) First-Order Resolving Power}

A general equation for the first-order resolving power has been derived in References ( $1, \%$, and 3 ). For point-to-point imaging the first-order momentum resolving power $R_{1}$ is defined as the ratio of the momentum dispersion at the image plane ton the total imaec size. Thus if $2 x_{0}$ is the total source size then from Reference 1 we have:

$$
R_{1}=\frac{P}{\triangle P}=\left|\frac{a_{x}(t)}{2 x_{0} c_{x}(t)}\right|=\frac{1}{2 x_{0}}\left|\int_{0}^{t} s_{x}(\tau) h(\tau) d \tau\right|
$$

Note that $h(\tau) d \tau=d \alpha$ is the differential angle of bend of the central trajectory of the optical system. 
Equation (29) may be expressed in a number of useful forms. If we consider a particle originating at the source with $x_{0}=0$ and $\delta=\frac{\triangle P}{P_{0}}=0$ and lying in the midplane (i.e., a monoenergetic point source), the first-order equation of its trajectory is

$$
x(\tau)=s_{x}(\tau) x_{0}^{\prime}
$$

We may then rewrite equation (29) as follows:

$$
R_{1}=\frac{1}{2 x_{0} x_{0}^{\prime}}\left|\int_{0}^{t} x(\tau) h(\tau) d \tau\right|=\frac{\left(\ell-l_{0}\right)}{2 x_{0} x_{0}^{\prime}}
$$

where $\left(\boldsymbol{l}-\boldsymbol{\ell}_{0}\right)$ is the path length difference between the trajectory described by equation (30) and the central trajectory. Or we may also write equation (31) in the form

$$
R_{1}=\frac{1}{2 x_{0} x_{0}^{\prime}}\left|\int_{0}^{t} \frac{B x(\tau) d \tau}{B \rho}\right|=\left(\frac{1}{2 x_{0} x_{0}^{\prime}}\right)\left(\frac{1}{B \rho}\right)\left|\int_{0}^{t} B d A\right|
$$

where $\int \mathrm{BdA}$ is the magnetic flux enclosed between the central trajectory and the trajectory described by equation (30), and $\mathrm{BP}$ is the magnetic rigidity of the central trajectory. Please note, however, that if the trajectory of equation (30) crosses the central trajectory or the sign of B changes, this changes the sign of the integration. From equation (32) we may define resolving power as the magnetic flux enclosed per unit phase space area $\left(2 x_{0} x_{0}^{i}\right)$, per unit momentum $(B p)$ of the central ray.

In any given design, one or more of the above equations may be used as a guide toward arhieving the required resolving power. One of the design decisions that must be made is the appropriate choice of the dipole magnet parameters (width and length) to achieve the required $\int B d A$. From first-order considerations, this choice is dominated primarily by practical and economic factors. However, a study of the nature of the origin of aberrations (see for example Ref. 1) suggests that it is advisable to keep the amplitude of $\mathrm{s}_{\mathrm{x}}$ small. In order to simul.taneously satisfy this requirement and meet the required resolving power $R_{1}$, we see from equation (29) that the total angle of bend $\alpha$ of the central trajectory should be chosen as large as is practical. Also, in general, the focal plane angle tends to be more normal to the optic axis for larger $\alpha$ - a property usually desired in most designs. 
2) Dispersion

From Reference 1, 2, or 3; for point-to-point imaging $\left(s_{x}(t)=0\right)$ the dispersion at the image plane is

$$
d_{x}(t)=-c_{x}(t) \int_{0}^{t} s_{x}(\tau) h(\tau) d \tau
$$

where $c_{x}(t)$ is the magnification at the image plane.

The dispersion and hence the magnification in the design of a spectro'meter is dominated almost entirely by a compromise between the spatial resolution of the particle detectors used at the image plane and the momentum range to be covered by the instrument; or in the case of a momentum defining (analyzing) system, by the acceptable momentum-defining slit spacings.

3) The Selection of the Optical Mode

By optical mode, we mean the type of imaging (e.g., point-to-point or parallel-to-point, etc.) required at the image plane in both the $x$ and $y$ coordinates, and the number of intermediate images imposed between the source and image planes. The imaging requirements at the image plane are usually dominated by the physics to be performed by the instrument and the nature of the particle detectors used. However often (especially at low energies) the imaging in the y plane may be unimportant as far as the physics requirements are concerned which in turn provides some additional flexibility in the optics design.

A study of the coupling coefficients to the aberration coefficients (equation 19) shows the not surprising result, that multipoles located at intermediate images in a system do not couple to aberrations in the plane in which the intermediate image occurs. Hence it often proves beneficial to intentionally create an intermediate image in the $\mathrm{y}$ plane of an optical system so as to achieve some degree of "orthogonality" in the minimizing of $x$ and $y$ aberrations .

The considerations of 1), 2), and 3) above are the determining factors in the selection of the first-order solution of a system design.

The optical mode and dispersion of the system are determined to a great extent by the choice of the quadrupole components chosen to achieve the fireuorder imaging although it is clear that the dipole elements also influence the first-order imaging to a greater or lesser extent depending upon the total angle of bend of the system.

4) Aberrations and their Correction

A study of the source of second-and higher-order aberrations (see for example Ref. 1) suggests that it is advisable to maintain the characteristic 
first-order functions $c_{x}, s_{x}, d_{x}$ and $c_{y}, s_{y}$ and their derivatives as small as is feasible through the magnetic elements of a system when choosing the firstorder design. This procedure will tend to reduce the initial size of the aberrations and hence simplify the problem of minimizing them by the addition of multipole components to the system design.

The procedure for minimizing aberrations has already been outlined in the Introduction and as such will not be repeated here. The "key" to the minimization procedure is the coupling coefficient given by the integral expression in equation (19). The "best" location for the correcting multipole is where the coupling coefficient has its maximum value.

The preferred method of introducing the multipole components, i.e., via pure multipoles, contoured entrance or exit boundaries, or non-uniform fields is a combination of practical and economical considerations and, of course, personal taste and experience. All three methods have been used with pure multipoles dominating the situation for higher energy physics and the other two methods dominating medium and low-energy physics applications. All three techniques should be considered in any given design situation to be certain that an important economic or practical advantage has not been ignored.

\section{REFERENCES}

(1) "A First- and Second-Order Matrix Theory for the Design of Beam' Trans port Systems and Charged Particle Spectrometers" SLAC Report No. 75 by K. I. Brown or Advances in Particle Physics Interscience 1, 71-134 (1967).

(2)' "A General First- and Second-Order Theory of Beam Transport Optics", Proc. of the International Symposium on Magnet Technology (1965); p. 141.

(3). "Some First- and Second-Order Magnetic Optics Theorems Applicable to the Design of Beam Transport Systems and Charged Particle Spectrometers", Proc. of the International Symposium on Magnet Technology (1967); p. 40.

(4) E. D. Courant and H. S. Snyder, "Theory of the Alternating Gradient Synchrotron", Annals of Physics 3, pp. 1-48 (1958).

(5) K. L. Brown (with R. Belbeoch and P. Bounin), "First- and Second-Order Magnetic Optics Matrix Equations for the Midplane of Uniform-Field Wedge Magnets, Rev. Sci. Instr. 35, 481 (1964).

(6) K. I. Brown; B. K. Kear, S. K. Howry, "TRANSPORT/360 A Computer Program for Designing Beam Transport Systems", SLAC Report No. 91, Stanford Linear Accelerator Center, Stanford University, Stanford, California (1970). 
When a charged particle passes through a perfectly aligned magnet the transformation may be described by the matrix equation

$$
X(1)=R X(0)
$$

The ray $X(0)=0$ is the central trajectory and passes undisturbed along the axis of the magnet. A misalignment causes some other ray to play the role of the central trajectory thru the magnet. Suppose that this ray relative to the magnet axis is described by a vector displacement $D(O)$ at the entrance face and by $D(I)$ at the exit face of the magnet (see figure below).

Beam displacement due to magnet misalignment

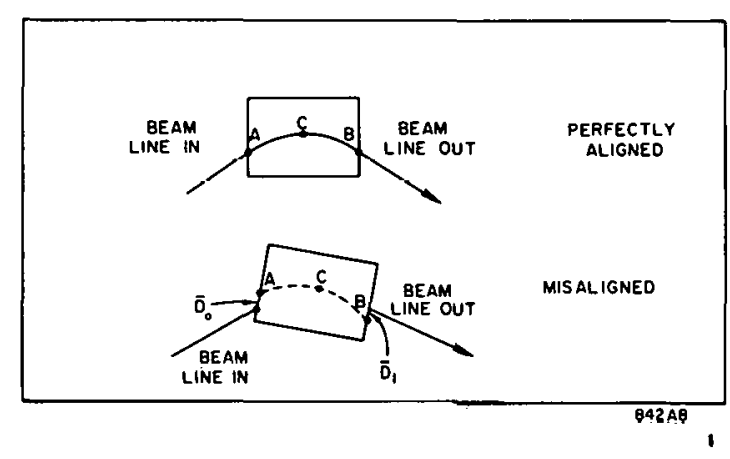

Then the transformation through the misaligned magnet is described to first-order by

$$
X(1)-D(1)=K[X(0)-D(0)]
$$

The problem now is to express the 6-vectors $D(0)$ and $D(1)$ in terms of numbers readily measured as misalignments.

A rigid magnet has six degrees of treedom, three translational and three rotational. These are conveniently represented by the six quantities

$$
\mathrm{m}=\left(\begin{array}{c}
\delta \mathrm{x} \\
\theta_{\mathrm{x}} \\
\delta \mathrm{y} \\
\theta_{\mathrm{y}} \\
\delta \mathrm{z} \\
\theta_{\mathrm{z}}
\end{array}\right)
$$


where $\delta \mathrm{x}, \delta \mathrm{y}, \delta \mathrm{z}$ are the displacements in the $\mathrm{x}, \mathrm{y}$, and $\mathrm{z}$ directions and $\theta_{\mathrm{x}}, \theta_{\mathrm{y}}, \theta_{\mathrm{z}}$ are the rotations about the $x, y$, and $z$ axes, respectively. The origin of the $x y z$ coordinate system, called the pivot, is the point about which the misalignments are measured. Assune that the pivot is at some point $C$ along the central ray $X=0$ (see Figure (1) above). At $\mathrm{C}$ the $\mathrm{x}, \mathrm{y}, \mathrm{z}$ axes of the misalignment coordinate system colncide with the $x, y$ TRANSPORT beam coordinates and the beam direction $\mathbf{z}$ respectively.

Since misalignment is a rigid transformation, the 6-vectors $D(O)$ and $D(I)$ are uniquely determined by $\mathrm{m}$, along with the length and curvature of the circular arc $A C B$. For small $|\mathrm{m}|$ the relationship is

$$
\begin{aligned}
& D(0)=A_{0} m \\
& D(1)=A_{1} m
\end{aligned}
$$

where

$$
A=\left(\begin{array}{cccccc}
\cos \alpha & 0 & 0 & \rho \sin \alpha & \sin \alpha & 0 \\
0 & 0 & 0 & 1 & 0 & 0 \\
0 & -\rho \sin \alpha & 1 & 0 & 0 & -\rho(1-\cos \alpha) \\
0 & -\cos \alpha & 0 & 0 & 0 & -\sin \alpha \\
-\sin \alpha & 0 & 0 & -\rho(1-\cos \alpha) & \cos \alpha & 0 \\
0 & 0 & 0 & 0 & 0 & 0
\end{array}\right)
$$

Define $\alpha_{0}$ as the angle of bend from the pivot to the entrance face of the magnet and $\alpha_{1}$ as the angle of bend from the pivot to the exit face of the magnet. $\rho$ is the radius of curvature of arc $A C B$.

In TRANSPORT, the pivot is chosen as the magnet entrance face, so $\alpha_{0}=0$ and $\alpha_{1}=\alpha$ the full angle of bend. In this case $A_{0}$ reduces to:

$$
A_{0} \text { (for pivot at entrance face) }=\left(\begin{array}{cccccc}
1 & 0 & 0 & 0 & 0 & 0 \\
0 & 0 & 0 & 1 & 0 & 0 \\
0 & 0 & 1 & 0 & 0 & 0 \\
0 & -1 & 0 & 0 & 0 & 0 \\
0 & 0 & 0 & 0 & 1 & 0 \\
0 & 0 & 0 & 0 & 0 & 0
\end{array}\right)
$$


Matrices $A_{7}$ and $A_{n}$ for quadrupoles are obtained by going to the

Iimits:

$$
\alpha_{0}=\alpha_{1}=0
$$

and $\alpha_{1} \rho=I$, the effective length of the quadrupole. For the pivot at the entrance face of the quadrupole, $A_{0}$ is the same as for a bending magnet and $A_{1}$ becomes:

$A_{1}$ (for quad with pivot at entrance face) $=\left(\begin{array}{cccccc}1 & 0 & 0 & I & 0 & 0 \\ 0 & 0 & 0 & 1 & 0 & 0 \\ 0 & -I & 1 & 0 & 0 & 0 \\ 0 & -1 & 0 & 0 & 0 & 0 \\ 0 & 0 & 0 & 0 & 1 & 0 \\ 0 & 0 & 0 & 0 & 0 & 0\end{array}\right)$.

We now can write the transformation of a ray through a misaligned magnet about the pivot point as:

$$
X(1)=R X(0)+\left(A_{1}-R A_{0}\right) m
$$

This completes the model for a known misalignment m. Consider now an uncertainty in the magnet position and let the covariance matrix $\left\langle\mathrm{mm}^{\mathrm{T}}\right\rangle$ measure the resulting distribution of magnet positions, where $\mathrm{m}^{\mathrm{T}}$ is the trainspose of $\mathrm{m}$. If the position of the reference particle upon entering the magnet is $\left\langle x^{T}\right\rangle_{0}(d u e$ to, say, uncertainties introduced by misalignments of preceding magnets) and if there is no correlation of errors between any two magnets, then from Eq. (8) we concluale that:

$$
\left\langle X x^{T}\right\rangle_{1}=R\left\langle X x^{T}\right\rangle_{0} R^{T}+\left(A-R A_{0}\right)\left\langle m^{T}\right\rangle\left(A-R A_{0}\right)^{T}
$$

Thio cquation expresses the sesullanl alsheliuliun lif uncerlatnly upon leaving the magnet. This model for misalignment has been implemented in TRANSPORT. It enables one to introduce a displacement uncertainty $\delta x$, of a magnet and trace its effect through the system. The computer can then be used to zenerate tables of misalignment effects of all the magnets as described in the TRANSPORT manual under Type Code 8. 


\section{FIRST-ORDER PARAMETER OPTIMIZATION AND COVARIANCE}

Section $V$

To optimize the selected parameters, TRANSPORT uses the method of nonlinear least squares, differential correction, a good description of which is found in Ref. (7) below pages, 390-393. A useful by-product of this method is the covariance matrix C, printed by the program at the successful conclusion of any run involving parameter fitting. In many applications $C$ may be used to estimate tolerances on the fitted parameters subject to the specified tolerances (i.e., the standard deviations) of the constraints.

The covariance matrix $\mathrm{C}$ is symmetric. This admits a geometrical interpretation as an ellipsoid, and is printed in the same suggestive format as is the beam ellipsoid $\sigma$, except that in this case the dimension is equal to $N$, the number of parameters varied. The center of the ellipsoid is at

$$
\overrightarrow{\lambda^{0}}=\left(\lambda_{1}^{0}, \lambda_{2}^{0} \cdot \cdot \lambda_{N}^{0}\right),
$$

the $\mathbb{N}$ values found by TRANSPORT to be the best estimate of the varied parameters. The equation of the covariance ellipsoid is

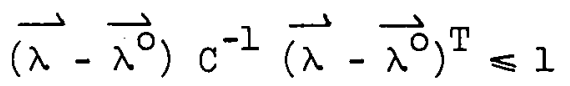

where $\left(\mathrm{C}_{11}\right)^{1 / 2}$, the first diagonal element printed, measures the maximum extent of the ellipsoid along the $\lambda_{1}$ axis (the first varied parameter) in the same sense that $\left(\sigma_{11}\right)^{1 / 2}$ measures the maximum extent of the beam ellipsoid along the $x$ axis. The off diagonal elements are normalized so that they are $\leqslant 1$ in magnitude, in analogy with the $r_{i j}$ of the beam matrix, and can be interpreted as measures of the orientation of the covariance ellipsoid.

(7) SOLMITZ Analysis of Experiment in Particle Physics, Ann. Rev. Nuc. Sci., Vol 14, 1964. 
The best estimates (or optimized values) of the varied parameters $\lambda$ are precisely those that minimize the quantity:

$$
x^{2}=\sum_{K=1}^{M}\left[\frac{\xi_{K}-f_{K}\left(\lambda_{1} \cdots \lambda_{N}\right)}{S_{K K}}\right]^{2}
$$

where:

$$
\begin{aligned}
\mathrm{M}= & \text { number of contraints } \\
f_{\mathrm{K}}= & \text { a function selected by the code digits }(i, j) \text { on the constraint definition } \\
& \text { (Type Code } 10 .) \text { For example, } i=-1, j=1 \text { means that the transform } \\
& \text { matrix element } \mathrm{R}_{11} \text { is to be constrained. } \\
\xi_{\mathrm{K}}= & \text { the desired value of the selected function. } \\
\mathrm{S}_{\mathrm{KK}}= & \text { the desired accuracy of fit ( } i . e ., \text { the standard deviation). }
\end{aligned}
$$

In our notation this minimum is expressed by:

$$
x_{\min }^{2}=\sum_{\mathrm{K}=1}^{\mathrm{M}}\left[\frac{\xi_{\mathrm{K}}-\mathrm{f}_{\mathrm{K}}\left(\lambda_{1}^{0} \ldots \lambda_{\mathrm{N}}^{0}\right)}{\mathrm{S}_{\mathrm{KK}}}\right]^{2}
$$

$x_{\text {min }}^{2}$ is printed at the successful conclusion of any run involving parameter fitting. Whether or not the optimization $\left(x_{\min }^{2}, \overrightarrow{\lambda^{0}}\right)$ is 'acceptable' depends on each application and must be evaluated by the user. Values of $\chi_{\min }^{2} \sim(M-N)$ are sometimes (but not necessarily) regarded as 'good'. In particular if $M=N$, then an exact solution, $x_{\text {min }}^{2}=0$ should be found by TRANSPORT.

If the resulting fit is acceptable; then the following interpretation may be put on the covariance matrix C: Let the parameters be changed to values $\vec{\lambda}$ near the optimum $\overrightarrow{\lambda^{0}}$, such that they stay within the ellipsoid defined by:

$$
\overrightarrow{\left(\lambda-\overrightarrow{\lambda^{0}}\right)} \mathrm{C}^{-1} \overrightarrow{\left(\lambda-\overrightarrow{\lambda^{0}}\right)^{\mathrm{T}} \leqslant 1}
$$

Then the resulting deviation of the specified constrained quantities is. bounded by:

$$
\sum_{K=1}^{M}\left[\frac{\xi_{K}-f_{K}(\lambda)}{S_{K K}}\right]^{\prime 2} \leqslant x_{\text {min }}^{2}+1
$$


This interpretation is strictly true if the constrained functions $f_{K}$ are linear in the parameters $\vec{\lambda}$. In the non-linear case, it is an approximation valid only in some neighborhood of $\overrightarrow{\lambda^{0}}$.

Example:

On the following page is an example of a TRANSPORT data deck and the resulting covariance fit of a first-order run. We have ask for a point-to-point image in both the $\mathrm{x}$ and $\mathrm{y}$ planes by varying the fields of the quadrupole triplet. The following definitions and solution are applicable:

$$
\begin{aligned}
& \mathrm{B}_{1}=\lambda_{1}, \mathrm{~B}_{2}=\lambda_{2}, \mathrm{~B}_{1}^{0}=\lambda_{1}^{0}, \mathrm{~B}_{2}^{0}=\lambda_{2}^{0}, \mathrm{f}_{1}=\mathrm{R}_{12}, \mathrm{f}_{2}=\mathrm{R}_{34}, \xi_{1}=0, \xi_{2}=0 \\
& \mathrm{~S}_{11}=0.005, \mathrm{~S}_{22}=0.005 \text { and } \mathrm{N}=2 .
\end{aligned}
$$

From the data deck and the TRANSPORT printout shown on the following page, we learn that:

$$
\left.\begin{array}{l}
\frac{x_{\text {min }}^{2}}{N}=0.8 \\
B_{1}^{0}=-7.4096 \mathrm{Kg}, B_{2}^{0}=6.1577 \mathrm{Kg} \\
\sqrt{C_{11}}=0.079 \\
r_{12}=-0.883 \quad \sqrt{C_{22}}=0.038
\end{array}\right\} \text { Covariance matrix information }
$$

The ellipsoid $\left(\overrightarrow{B-B^{0}}\right) C^{-1}\left(\overrightarrow{B-B^{0}}\right)^{T} \leqslant 1.0$ can then be constructed as shown:

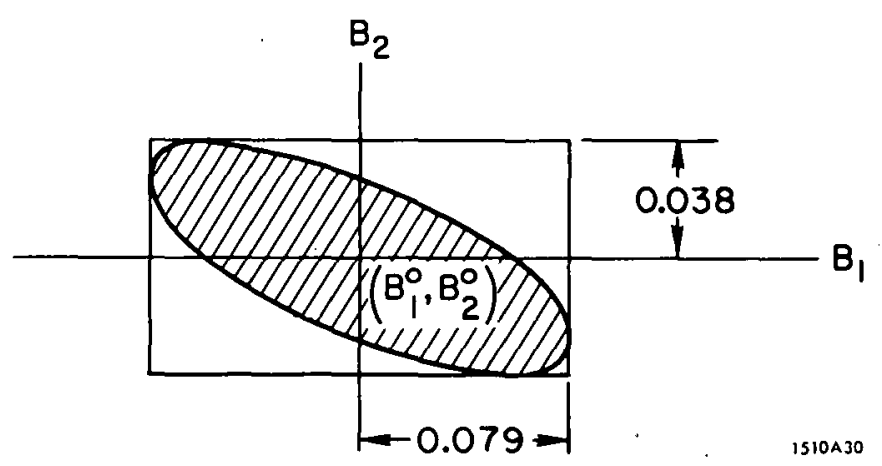

*Note: the printout format is:

COVARIANCE (FIT $\left.x_{\min }^{2} / \mathbb{N}\right)$

$\sqrt{\mathrm{C}_{11}}$

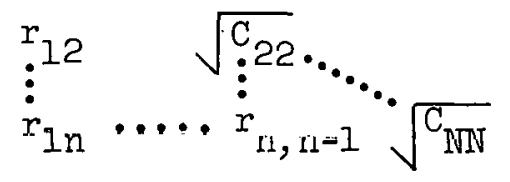


1. 'USF TRIPLET TO FORM POINT-TO-POINT IMAGE AT TARGFT'

2. 0

3. 13.2 .

4. 15. 1. 'I!'; 15.8. 'FT';

5. $\quad 1.0 .0 .0 .0 .0 .0 .1 .05 ;$.

6. 3.012 . :

7. $5.021 .5-8.04 .0{ }^{\prime} \cap 11^{\prime} ;$

8. $\quad 3.00 .5$;

9. $5.013 .0^{\prime} 7.04 .0$ ' 22 ';

10. $\quad 3.00 .5$;

11. $5.021 .5-8.4 .01011^{\prime}$;

12. 3.07 .0 ;

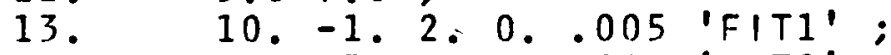

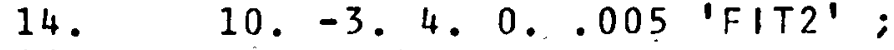

15. SENTINEL

16. $/$ *

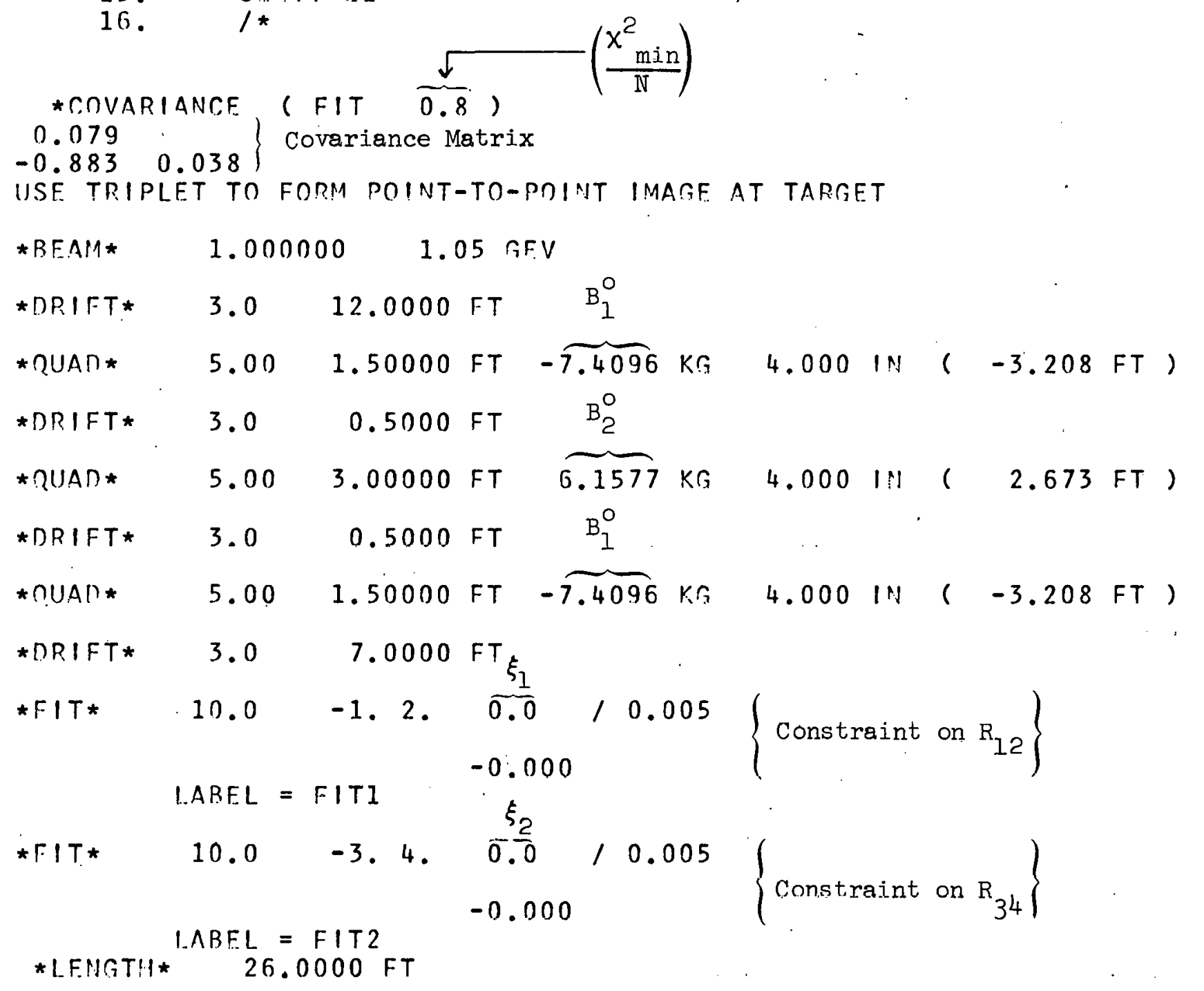


So long as $B_{1}$ and $B_{2}$ fall inside the shaded area (this is the tolerance requirement), then the ellipsoid representing the corresponding deviations of the matrix elements $R_{12}$ and $R_{34}$ is using Eq. (5):

$$
\left(\frac{0-R_{12}}{0.005}\right)^{2}+\left(\frac{0-R_{34}}{0.005}\right)^{2} \leqslant x_{\min }^{2}+1.0=2.6
$$

or:

$$
R_{12}^{2}+R_{34}^{2} \leqslant 2.6(0.005)^{2}
$$

Note that it is not enough to prescribe tolerances $\left|\Delta \mathrm{B}_{1}\right|<.079$ and $\left|\Delta \mathrm{B}_{2}\right|$ $<.038$, since there is unshaded area inside the rectangle defined by these values. The strongly tilted covariance. ellipse (i.e., $\left|r_{12}\right| \sim 1$ ) suggests that the triplet power supplies should be designed so that any drift in magnetic field $B_{1}$ causes a compensating drift in the magnetic field $B_{2}$ so as to stay inside the shaded area shown in Figure. 1. 